\title{
Physical functioning in patients with chronic cardiopulmonary diseases
}

Citation for published version (APA):

Barreto de Mesquita, R. (2017). Physical functioning in patients with chronic cardiopulmonary diseases. [Doctoral Thesis, Maastricht University]. Datawyse / Universitaire Pers Maastricht. https://doi.org/10.26481/dis.20170914rbdm

Document status and date:

Published: 01/01/2017

DOI:

10.26481/dis.20170914rbdm

Document Version:

Publisher's PDF, also known as Version of record

\section{Please check the document version of this publication:}

- A submitted manuscript is the version of the article upon submission and before peer-review. There can be important differences between the submitted version and the official published version of record.

People interested in the research are advised to contact the author for the final version of the publication, or visit the DOI to the publisher's website.

- The final author version and the galley proof are versions of the publication after peer review.

- The final published version features the final layout of the paper including the volume, issue and page numbers.

Link to publication

\footnotetext{
General rights rights.

- You may freely distribute the URL identifying the publication in the public portal. please follow below link for the End User Agreement:

www.umlib.nl/taverne-license

Take down policy

If you believe that this document breaches copyright please contact us at:

repository@maastrichtuniversity.nl

providing details and we will investigate your claim.
}

Copyright and moral rights for the publications made accessible in the public portal are retained by the authors and/or other copyright owners and it is a condition of accessing publications that users recognise and abide by the legal requirements associated with these

- Users may download and print one copy of any publication from the public portal for the purpose of private study or research.

- You may not further distribute the material or use it for any profit-making activity or commercial gain

If the publication is distributed under the terms of Article $25 \mathrm{fa}$ of the Dutch Copyright Act, indicated by the "Taverne" license above, 
Physical functioning in patients with chronic cardiopulmonary diseases 
(C) copyright Rafael Mesquita, Maastricht 2017

Printing: Datawyse | Universitaire Pers Maastricht

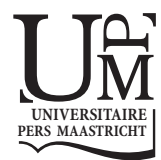

ISBN 9789461597373 


\title{
Physical functioning in patients with chronic cardiopulmonary diseases
}

\author{
DISSERTATION \\ to obtain the degree of Doctor at Maastricht University, \\ on the authority of the Rector Magnificus, Prof.dr. Rianne M. Letschert \\ in accordance with the decision of the Board of Deans, \\ to be defended in public \\ on Thursday 14 September 2017, at 16:00 hours \\ by
}

Rafael Barreto de Mesquita 


\section{SUPERVISOR}

Prof. dr. M.A. Spruit

Prof. dr. E.F.M. Wouters

\section{CO-SUPERVISOR}

Prof. dr. F. Pitta, State University of Londrina, Brazil

\section{ASSESSMENT COMMITTEE}

Prof. dr. M.K.C. Hesselink (chairman)

Prof. dr. R.A. de Bie

Dr. J.B.J. Bussmann, Erasmus MC University Medical Center, the Netherlands

Prof. E.M. Clini, University of Modena, Italy

Prof. dr. L.W. van Rhijn

\section{$+$}




\section{CONTENTS}

$\begin{array}{lll}\text { Chapter } 1 \quad \text { General Introduction } & 7\end{array}$

Chapter 2 What is the impact of impaired left ventricular ejection fraction in 31 COPD after adjusting for confounders?

Chapter 3 Objectively identified comorbidities in COPD: impact on pulmonary rehabilitation outcomes

Chapter 4 Reliability, construct validity and determinants of 6-minute walk 83 test performance in patients with chronic heart failure

Chapter 5 Within-day test-retest reliability of the Timed "Up \& Go" test in 103 patients with advanced chronic organ failure

Chapter 6 Measurement properties of the Timed Up \& Go test in patients 121 with COPD

Chapter $7 \quad$ Physical activity patterns and clusters in 1001 patients with COPD 147

Chapter $8 \quad$ Changes in physical activity and sedentary behaviour following 201 pulmonary rehabilitation in patients with COPD

Chapter 9 Activity levels and exercise motivation in COPD patients and their 235 resident loved ones

$\begin{array}{lll}\text { Chapter } 10 & \text { General Discussion } & 269\end{array}$

$\begin{array}{ll}\text { Summary } & 291\end{array}$

$\begin{array}{ll}\text { Samenvatting } & 297\end{array}$

$\begin{array}{ll}\text { Valorisation } & 303\end{array}$

$\begin{array}{ll}\text { Acknowledgments } & 309\end{array}$

$\begin{array}{ll}\text { Curriculum vitae } & 313\end{array}$

List of publications $\quad 315$ 



\section{CHAPTER 1}

General Introduction 


\section{Chronic cardiopulmonary diseases: a major health problem}

According to the World Health Organisation (WHO), chronic cardiopulmonary diseases are amongst the top major causes of mortality during the past decade [1]. Ischaemic heart disease, one of the main causes of chronic heart failure (CHF), leads the ranking with around 7.4 million deaths in 2012, which represents around $13.2 \%$ of all deaths in the world [1]. Chronic obstructive pulmonary disease (COPD) ranks third in the list with 3.1 million deaths, which represents around $5.6 \%$ of all deaths [1]. In the Netherlands, estimates from the WHO suggest that there are over one million patients with lung diseases and approximately 23000 die every year of related causes, making lung diseases the fourth most common cause of death [2].

Chronic obstructive pulmonary disease is defined as a preventable and treatable disease characterised by persistent and usually progressive airflow limitation [3]. Heart failure is defined as a clinical syndrome characterised by typical symptoms (e.g., breathlessness) that may be accompanied by signs caused by a structural and/or functional cardiac abnormality [4]. 'Chronic' heart failure is used for patients who have had heart failure for some time [4]. Historically, COPD and CHF have been described in terms of the function of the main organ affected. Indeed, COPD is characterised and qualified according to the degree of airflow limitation (i.e., reduced forced expiratory volume in the first second $\left(\mathrm{FEV}_{1}\right) /$ forced vital capacity (FVC) ratio) [3], while CHF has been frequently characterised by a reduction in the amount of blood pumped from the left ventricle of the heart (i.e., reduced left ventricular ejection fraction (LVEF)) [4]. Nevertheless, over the last decades clinicians and scientists have observed that patients with COPD and/or CHF often present with extra-pulmonary and extra-cardiac manifestations, respectively, which contribute importantly to the severity of these diseases. Features such as systemic inflammation and oxidative stress have been reported for both diseases, which may contribute to other systemic manifestations, such as peripheral muscle abnormalities that lead to a shift in muscle fibres from oxidative to glycolytic energy metabolism [5]. Although causality has yet not been proved, there is evidence supporting this assumption. For instance, activation of nuclear factor-kappa B (a prototypical pro-inflammatory signalling pathway) has been observed in the skeletal muscle of patients with COPD [6], which may be sufficient for the induction of muscle atrophy. In patients with heart failure, the potential negative impact of nuclear factor-kappa B activation has been suggested previously [7]. Further, findings from animal studies suggest that the inhibition of nuclear factor-kappa B leads to faster restoration of normal muscle architecture and associates with markers of muscle regeneration [8]. Systemic manifestations in patients with COPD or CHF may also complicate already existing comorbidities, such as malnutrition, osteoporosis, depression, and diabetes $[9,10]$. These findings help explaining, at least in part, the high prevalence of comorbidities in patients with these conditions [11, 
12]. Importantly, comorbidities in both conditions have been shown to associate with increased hospitalisations and mortality $[13,14]$.

Chronic obstructive pulmonary disease has been reported as common comorbid condition in patients with $\mathrm{CHF}$, but the opposite is also true. In the Netherlands, the reported prevalence of COPD in patients with CHF varies between $19 \%$ and $25 \%[15,16]$, whilst a similar value is reported for the prevalence of CHF in patients with COPD, i.e., $20.5 \%$ [17]. In spite of these findings, the presence of one disease as comorbid condition of the other is often ignored and as a consequence the comorbid condition is often underdiagnosed. A recent multicentre, cross-sectional study conducted in nine European countries observed that underdiagnosis of airflow limitation compatible with COPD was present in $82.9 \%$ of patients with ischaemic heart disease with CHF [18]. Given that patients with co-existing CHF and COPD were found to have a worse prognosis [19], an early diagnosis of chronic airflow limitation in those patients with CHF seems crucial.

One of the reasons why COPD and CHF often co-exist in the same patient is because some risk factors, such as cigarette smoking, advanced age, and systemic inflammation, are shared by these two diseases [20]. Indeed, associations between low-grade systemic inflammation and an increased risk of cardiac injury have been reported in patients with moderate-to-severe airflow obstruction [21]. Besides having some shared risk factors, patients with COPD and patients with CHF often present with similar symptoms, such as exertional breathlessness, nocturnal cough, and fatigue [22], which can in fact complicate the diagnose of one of the conditions. For example, Schellenbaum and colleagues [23] used data from a population-based cohort to investigate the impact of two different diagnostic criteria for congestive heart failure. The authors observed that, depending on the diagnostic criteria used, a different prevalence of COPD was found (13\% vs. 6\%). Symptoms such as exertional breathlessness and fatigue can be very debilitating in patients with chronic cardiopulmonary diseases. When present they can force patients into a downward spiral that links symptom-induced progressive inactivity to accelerating physical deconditioning and disability [24]. The final result is reduced physical capacity (e.g., reduced exercise tolerance, functional mobility) and a physically inactive and/or sedentary lifestyle, which are often accompanied by impaired healthrelated quality of life and mood disturbances. These consequences ultimately can lead to higher morbidity and premature mortality.

\section{Impairments in physical capacity in patients with chronic cardiopulmonary diseases}

Physical capacity (fitness) can be defined as the ability to carry out daily tasks with vigour and alertness, without undue fatigue and with energy to enjoy pursuits and to meet 


\section{CHAPTER 1}

emergencies [25]. Physical capacity can be operationalised as a set of measurable attributes such as exercise tolerance, muscular strength and endurance, body composition, functional mobility and balance [25]. Impaired physical capacity is a common feature in patients with COPD or CHF. Nevertheless, this feature does not seem to be an exclusive consequence of the impairments in the main affected organ. Previous research observed that resting lung function impairments in patients with COPD [26] and resting cardiac function impairments in patients with CHF [27] had a poor ability to predict exercise intolerance in these populations (Figure 1). Additionally, exercise intolerance has been observed even after therapies that aimed to restore these indexes of primary organ failure, such as medications or lung/heart transplantation [28-31]. Based on these findings it is clear that factors other than organ failure may contribute to the exercise intolerance found in patients with COPD or CHF.

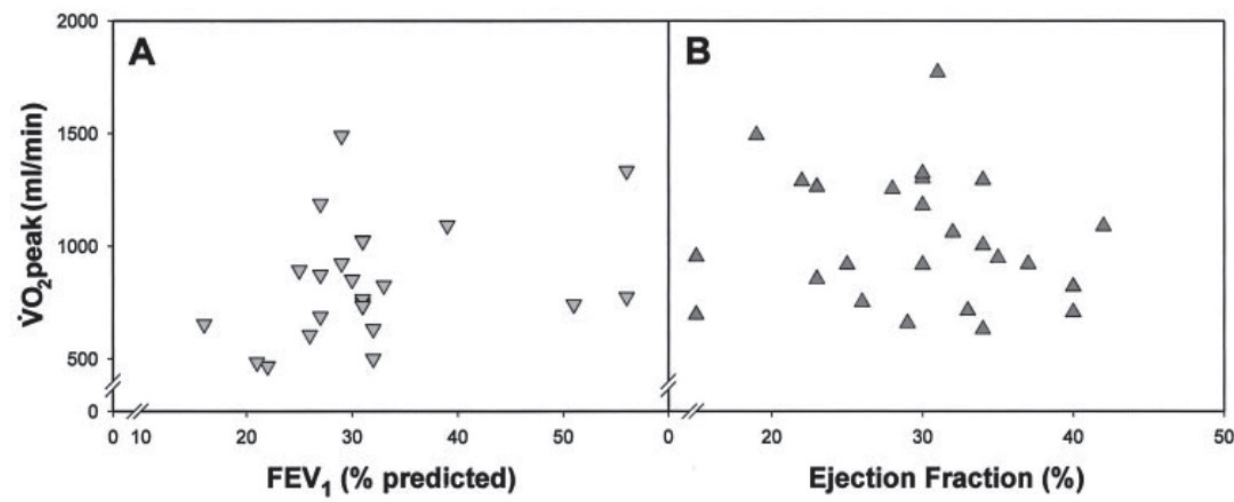

Figure 1 Relationship between peak oxygen uptake ( $\mathrm{V} \mathrm{O}_{2}$ peak) during maximal exercise and forced expiratory volume in the first second $\left(\mathrm{FEV}_{1}\right)$ in patients with chronic obstructive pulmonary disease (Figure $\mathrm{A}$ ) and between $\mathrm{VO}_{2}$ peak and left ventricular ejection fraction in patients with chronic heart failure (Figure B). There were no significant correlations. Reprinted from Chest, Vol 123, Gosker HR, Lencer NH, Franssen FM, van der Vusse GJ, Wouters EF, Schols AM, Striking similarities in systemic factors contributing to decreased exercise capacity in patients with severe chronic heart failure or COPD, pp. 1416-24, Copyright (2003), with permission from Elsevier.

In patients with COPD and/or CHF, exercise intolerance appears to be multifactorial in origin, with cardiovascular, pulmonary, peripheral and psychological factors involved in both diseases in different ways [32-35].

\section{Exercise intolerance in patients with COPD}

Dynamic hyperinflation is one of the main factors limiting exercise in patients with COPD. It refers to the temporary increase in operating lung volumes above their resting value [36]. To meet the increased metabolic demand related to exercise, patients with COPD increase their minute ventilation, first by increments in tidal volume and then by increments in respiratory rate. As these patients are characterised by expiratory flow 
limitation, which is the pathophysiological hallmark of the disease, the increase in minute ventilation will lead to a decrease in expiratory time and consequently to dynamic lung hyperinflation [37]. The role of dynamic hyperinflation on exercise tolerance is confirmed by studies that observed improved exercise endurance after treatment with bronchodilators, which improve dynamic airway function and enhance lung emptying [38]. Hyperinflation, both dynamic and chronic, can place the inspiratory muscles of patients with COPD at a mechanical disadvantage [39], which will lead to respiratory muscle dysfunction that can further compromise the patients' exercise tolerance. The mechanical disadvantage imposed by hyperinflation will compromise the capacity of the inspiratory muscles to generate pressure and this happens through different mechanisms, such as worsening of the length-tension relationship and increase in the elastic recoil of the thoracic cage [40]. Other factors can also contribute to respiratory muscle dysfunction in patients with COPD, such as malnutrition and prolonged use of glucocorticoids [40]. Gas exchange abnormalities are also common in patients with COPD and normally result in hypoxaemia and hypercapnia [3]. Hypoxia leads to the increase in pulmonary ventilation through augmenting peripheral chemoreceptor output and stimulation of lactic acid production [41]. By counteracting these mechanisms, supplemental oxygen therapy during exercise has been shown to increase exercise tolerance in patients with COPD [42].

Cardiac dysfunction can also contribute to exercise intolerance in patients with COPD [43]. An increase in the right ventricular afterload can be observed as a consequence of an elevated pulmonary vascular resistance which results from direct vascular injury and/or remodelling [44], hypoxic vasoconstriction [45], and/or increased effective pulmonary vascular resistance due to an increase in the number of erythrocytes [46]. Chronic right ventricle overload can then lead to right ventricular hypertrophy and failure. Additionally, right ventricular dysfunction can also compromise the function of the left ventricle through septal shifts. These findings combined may reduce the ability of the heart to meet the exercise demands.

Lower-limb muscle dysfunction is a factor that has been increasingly recognised as a contributor to exercise intolerance in COPD. Quadriceps force is an important determinant of exercise tolerance in COPD [47]. Hamilton and co-workers [48] identified the perception of leg effort as the main limiting symptom during exercise in 40 to $45 \%$ of patients with COPD. This was corroborated in a landmark experimental study by Saey et al. [49] who observed that exercise tolerance was not enhanced after acute bronchodilation in patients who developed contractile fatigue after exercise, but only in those who did not develop this condition. Lower-limb muscle dysfunction in patients with COPD may be attributable to inactivity-induced deconditioning, systemic inflammation, oxidative stress, smoking, blood gas disturbances, nutritional impairment, hypoxaemia, hypercapnia, low anabolic hormone levels, aging, and corticosteroid use [50]. These 
factors will lead to morphological and structural changes, such as mitochondrial dysfunction, a shift in fibre type distribution mainly from type I to type IIx, a reduction in the number of capillaries per muscle fibre and a loss of muscle mass, which will then translate into functional changes, such as muscle weakness and reduced endurance [50]. Of note, previous findings suggest considerable inter-individual heterogeneity in muscle dysfunction in patients with COPD. For example, Seymour and Spruit et al. [51] observed a prevalence of quadriceps weakness of around $30 \%$ in patients with COPD with mild to moderate airflow limitation (Global Initiative for Chronic Obstructive Lung Disease (GOLD) grades 1 or 2), which was not statistically different when compared to the prevalence in those with the most severe airflow limitation (38\% in GOLD grade 4). This suggests that exercise tolerance may be affected differently depending on the degree of muscle dysfunction. Although most of the abovementioned findings have been observed in patients with moderate to very severe COPD, recent findings have shown that even patients with mild disease can have considerable physiological impairment, such as inflammation and dysfunction of the pulmonary microvasculature and small airway dysfunction, which will contribute to exercise intolerance and poor perceived health status [52].

Exercise intolerance in patients with COPD is often accompanied by impaired quality of life, although the strength of these relationships has been found to vary between studies. For example, while Oga et al. [53] observed moderate correlation $\left(r_{s}=-0.56\right)$ between the performance on the 6-minute walk test (6MWT) and health-related quality of life scores, Brown et al. [54] observed a weak correlation ( $r=-0.26)$ between exercise tolerance and health-related quality of life assessed with the same measures. Associations with mood disorders have also been reported. Spruit et al. [55] identified the presence of depressive symptoms as a significant clinical determinant of a poor 6minute walk distance (6MWD) in a large $(n=1795)$ multinational cohort of patients with clinically stable COPD.

\section{Exercise intolerance in patients with $\mathrm{CHF}$}

In patients with $\mathrm{CHF}$, cardiovascular factors are among the main contributors to exercise intolerance, not only due to their direct influence on exercise tolerance but also due to their contribution to pulmonary and peripheral impairments. The capacity of the patients to perform exercise will depend on the ability of the heart to augment its output to the exercising muscles [56]. This capacity is obviously compromised in patients with CHF. At peak exercise, patients with systolic CHF may achieve less than half of the maximal cardiac output attained by healthy subjects, and this occurs mainly due to a minimal increase in stroke volume which is accompanied by a lower maximal heart rate achieved at a lower workload [57]. A reduced maximal cardiac output is usually observed secondary to a failure to increase left ventricular systolic emptying and thus 
augmenting LVEF. This derives from a combination of impaired intrinsic contractility, reduced $\beta$-adrenergic responsiveness, elevated systemic vascular resistance and a blunted peripheral arterial vasodilator response to exercise [56].

Ventilatory abnormalities can also play a role in limiting exercise in patients with CHF. These patients are characterised by excessive ventilation during exercise when compared to healthy controls [58]. An increased ventilatory response has been reported to occur due to early metabolic acidosis, abnormal ventilation-perfusion relationships, subclinical interstitial pulmonary oedema, diastolic dysfunction, increased airway resistance, and stimulation of chest wall and/or lung mechanoreceptors [59]. Less explored in the literature, respiratory muscle weakness is also thought to explain the exercise intolerance of patients with CHF. Maximal oxygen consumption during exercise was found to be correlated with maximal inspiratory pressure, a measure of respiratory muscle strength, in patients with heart failure [60]. Maximal inspiratory pressure is reduced in these patients when compared to healthy subjects and this feature has been associated with premature mortality [61]. Previous findings have shown that inspiratory muscle training in these patients was able to lead to a $17 \%$ increase in peak oxygen uptake during an incremental exercise test [62].

Hormonal changes (e.g., neuroendocrine activation) can also contribute to exercise intolerance in patients with CHF [35]. These hormonal changes initially occur as a compensatory response to heart damage in order to maintain an adequate circulatory support. However, when chronically maintained these changes become detrimental (e.g., proarrhythmic and profibrotic effects, sodium-water retention) leading to the overt clinical picture of CHF [33]. Impairments in endothelial function have also been reported as a limiting factor to exercise in patients with CHF [33]. Abnormalities in endothelial synthesis of nitric oxide (NO), which is responsible for peripheral arterial vasodilation during exercise, is one of the main factors involved [33]. Flow-induced NO-mediated vasodilatation during exercise seems to be blunted in patients with heart failure. Katz and colleagues [63] observed that the forearm vasodilatation response to handgrip exercise was significantly reduced after NO synthase inhibition in normal individuals, but not in patients with heart failure. Importantly, peripheral muscle training has been shown to significantly enhance endothelium-dependent vasodilation in the skeletal muscle circulation of these patients [64]. Alterations of excitatory reflexes, namely the chemoreflex and the ergoreflex, have been suggested as central mechanisms in the origin of exercise limitation in patients with CHF [34]. These patients may present with an increased sensitivity of these excitatory reflexes, which associates with abnormalities in the haemodynamic, ventilatory and autonomic responses to exercise and ultimately lead to premature exercise termination [34]. 
Lower-limb muscle dysfunction can also play an important role in the origin of exercise intolerance in patients with CHF. Indeed, as previously highlighted by Gosker and coworkers $[5,65]$, there are striking similarities between COPD and CHF with respect to the muscular alterations underlying the impairments in muscle function. Previous studies have shown that biochemical and histological changes in the lower-limb muscles of patients with $\mathrm{CHF}$ are better related to the degree of exercise intolerance than haemodynamic parameters [66]. Examples of these changes include a decrease in aerobic enzyme content in the muscles [67], muscle fibre atrophy across all fibre types [68], and a shift in fibre type distribution [68]. More specifically, a shift from slow-twitch type I oxidative fibres to fast-twitch type II glycolytic fibres is usually observed [68]. Patients with $\mathrm{CHF}$, similarly to those with COPD, may also show impaired exercise tolerance due to anxiety, poor motivation, or abnormal symptom perception. Moreover, as is the case for patients with COPD, impairments in exercise tolerance in patients with CHF have often been associated with impairments in health-related quality of life and mood status. In patients with advanced heart failure, Westlake et al. [69] identified the performance on the 6MWT as an independent predictor of the mental health component of health-related quality of life. Additionally, in a recent meta-analysis including patients with different heart conditions, depressive symptoms were found to be inversely correlated to measures of exercise tolerance [70].

\section{Exercise intolerance in patients with co-existing COPD and CHF}

Exercise intolerance is a common finding in patients with COPD or CHF and multiple factors are related to this feature, as previously described. Whereas impairments in exercise tolerance can be found in patients with one of these diseases alone, it seems reasonable to hypothesize that patients with co-existing COPD and CHF would present with even greater impairments. This is corroborated by Miller et al. [71] who reported worse health-related quality of life and functional exercise tolerance, and more breathlessness in patients with COPD and self-reported heart trouble, as reported by the authors, when compared to those without self-reported heart trouble. Similarly, Putcha et al. [72] observed a higher likelihood of worse functional exercise tolerance and worse dyspnoea in patients with COPD and self-reported CHF compared to those with no selfreported CHF. Despite the importance of these findings, some limitations can be identified in the studies. The diagnosis of the cardiac condition in both studies was based on non-objective measures (e.g., medical history, self-report), which are less accurate than echocardiography, and in the former study differences in relevant characteristics were observed between the groups under comparison, such as differences in body composition, which might have compromised some of the results.

To date, the additional impact of objectively assessed cardiac impairments, such as an impaired LVEF, on physical and psychological status in matched groups of patients with 
COPD has never been studied. Identifying and understanding the impact of cardiac impairments on the physical and psychological status in patients with COPD could contribute to a better management and treatment of patients with this disease. Interestingly, previous research observed a negative impact of objectively assessed lung impairments compatible with COPD on physical health outcomes, such as peak oxygen uptake during a maximal incremental cardiopulmonary exercise test, in matched groups of patients with heart failure [73].

\section{Comorbidities and exercise training}

Exercise training is the corner stone in the treatment of exercise intolerance in patients with cardiopulmonary diseases. Exercise training in these populations has been consistently shown to increase exercise tolerance $[74,75]$, improve health-related quality of life [74, 76], and decrease morbidity [76, 77]. Nevertheless, previous research suggested that the benefits of exercise training in patients with cardiopulmonary diseases may be hindered by the presence of comorbidities, but the results are conflicting. In a retrospective analysis including patients with COPD undergoing pulmonary rehabilitation (PR), Crisafulli et al. [78] observed a decreased likelihood of having a meaningful change in 6MWD in patients with comorbid metabolic disease. Some years later, the same group was not able to replicate these results in a prospective analysis, but observed a decreased likelihood of having a meaningful 6MWD change in patients with comorbid osteoporosis [79]. In patients with heart failure, Mentz et al. [80] reported that COPD had no impact on the changes in exercise tolerance or health status after an aerobic exercise training programme. These findings highlight the need for more studies investigating comorbidities and exercise training outcomes in patients with cardiopulmonary diseases. Moreover, comorbidities in the mentioned studies were identified using selfreport and/or medical history, which can be less accurate, and only the impact of individual comorbidities or arbitrarily grouped comorbidities were studied, while patients with COPD may present with clusters of comorbidities. Based on 13 objectively diagnosed comorbidities in patients with COPD, Vanfleteren et al. [81] were the first to report the presence of five clusters of comorbidities: less comorbidity, cardiovascular, cachectic, metabolic, and psychological. The authors observed comparable airflow limitation, functional exercise performance and systemic inflammation between the clusters, but differences were observed in terms of health status [81]. To date, it remains unknown whether and to what extent these clusters of comorbidities would negatively influence the patients' ability to experience meaningful improvements in exercise tolerance and health status after established interventions such as PR.

\section{Measurement of exercise intolerance}

In many previous studies of patients with chronic cardiopulmonary diseases, exercise intolerance was measured through the maximal incremental cardiopulmonary exercise 
test, which has been recognised as the gold standard [82]. While this test provides detailed information on the cardiovascular, pulmonary, and muscular systems [59], it requires state-of-the-art equipment and skilled staff, which makes it impractical in the context of routine clinical practice on a broad scale. Alternatively, field exercise tests such as the 6MWT are easy to administer and inexpensive to complete [83]. In patients with cardiopulmonary diseases, the 6MWT has been shown to provide important clinical information. Hernandes et al. [84] observed that the 6MWT was reproducible in patients with COPD, but there was an average learning effect of $27 \mathrm{~m}$ between two tests performed on subsequent days. Spruit et al. [85] reported that this test is able to identify subsets of the COPD population at higher risk of exacerbation-related admission or death. In a more recent prospective study, variables other than the distance covered, such as the work or speed developed during the test, were also found to have important prognostic value [86]. Moreover, another recent study revealed that the 6MWT is a useful tool to prescribe high-intensity exercise in patients with COPD [87]. Regarding patients with cardiac diseases, Rostagno et al. [88] observed a worse survival rate in patients with congestive heart failure who had a 6MWD lower than 300 metres. In the prospective, observational study by Tallaj and co-workers [89], the distance covered during the test was used to prescribe intensity of exercise training during cardiac rehabilitation (CR). Moreover, the 6MWT can also be used to evaluate the responses to treatments, both pharmacological [90] and non-pharmacological [91] treatments, in patients with different cardiac conditions. Despite the wide use of the 6MWT in cardiac patients, to date, a detailed analysis of the measurements properties of this test is lacking in the literature, as are the possible determinants of the distance performed during this test. These data could contribute to increase the uptake of the 6MWT in clinical practice in patients with cardiac diseases, including CHF.

Despite being commonly used in clinical practice, the 6MWT might be too demanding for certain patients, which might prevent them from performing two tests as suggested in international guidelines [83]. Indeed, previous research has shown that $10 \%$ of patients with COPD entering a PR programme [84] and $16 \%$ of patients with cardiac disease entering a CR programme [92] were unable to complete a second 6MWT. The reasons for not completing the second 6MWT were not reported for patients with COPD [84] and included intermittent claudication, chest pain, arthritic pain, or lack of assessor or patient time for patients with cardiac disease [92]. As an alternative, a metabolically less demanding test for more disabled patients may be the Timed Up \& Go (TUG) test. In these patients the results of the TUG test could be useful to infer exercise intolerance. The TUG test is an effective method of measuring functional mobility [93]. In this simple performance test subjects are requested to stand up from a chair, walk a distance of $3 \mathrm{~m}$ at their usual speed, turn and walk back to the chair to sit down again [93]. The time to perform this task is recorded and used for analysis [93]. Despite its simplicity, the TUG test has been shown to predict morbidity and mortality in different 
populations [94-96]. This test has been even recommended by the American Geriatrics Society and British Geriatrics Society [97] as a gait and balance assessment tool and by the British Geriatrics Society, Age UK and Royal College of General Practitioners [98] as a measure for the recognition of frailty. To date, the TUG test has been used scarcely in patients with COPD or CHF. Nevertheless, these patients often present with lower-limb muscle dysfunction, exercise intolerance, and fatigue $[65,99,100]$, especially in advanced stages of the disease, which can compromise functional mobility and balance, and consequently increase the risk for falling. Before recommending the use of the TUG test in daily clinical practice in these target groups of patients, its measurement properties, such as reliability, validity and responsiveness, need to be determined.

\section{Physical inactivity and sedentarism in patients with chronic cardiopulmonary diseases}

There are robust data showing that lack of physical activity is a major risk factor for morbidity and premature mortality in the general population [101, 102]. Indeed, physical inactivity has been identified as the fourth leading risk factor for global mortality and it is estimated to be one of the principal causes of burden of diseases such as breast and colon cancer, type 2 diabetes, and ischaemic heart disease [103]. Despite the wellestablished benefits of a physically active lifestyle, the amount of physically inactive subjects around the world is substantial. Estimates based on self-reported data showed that $23.3 \%$ of adults worldwide are physically inactive [104], that is, are not achieving at least 150 minutes of moderate-intensity aerobic activity or 75 minutes of vigorousintensity aerobic activity per week, or an equivalent combination [105]. If objectively measured physical activity data are taken into account, which are more accurate, an even higher prevalence of $53.1 \%$ is observed [106].

One of the explanations as to why a high percentage of physically inactive adult subjects has been observed in previous studies is due to subjects spending the majority of their waking time being sedentary $[106,107]$. Recently, there is developing interest on sedentary behaviour, which is considered to be a distinct concept from physical activity. Physical activity is defined as any bodily movement produced by skeletal muscles that results in energy expenditure [25], usually $>1.5$ metabolic equivalents of task (METs). Sedentary behaviour, in turn, is defined as any waking behaviour characterised by an energy expenditure $\leq 1.5 \mathrm{METs}$ while in a sitting or reclining posture [108]. An integrated and comprehensive view of these two concepts is provided by Hill et al. [109] (Figure 2). Previous data suggest that engaging in physical activity might not be enough to prevent the detrimental consequences of a sedentary lifestyle. In a cross-sectional analysis, Healy et al. [110] observed positive associations between television-viewing time and a number of metabolic risk variables even in healthy adults who met physical activity 
guidelines. These findings were corroborated in another cross-sectional study that observed detrimental linear associations of sedentary time with metabolic risk variables, such as waist circumference, HDL-cholesterol, and C-reactive protein, independently of confounders including moderate-to-vigorous exercise [111].

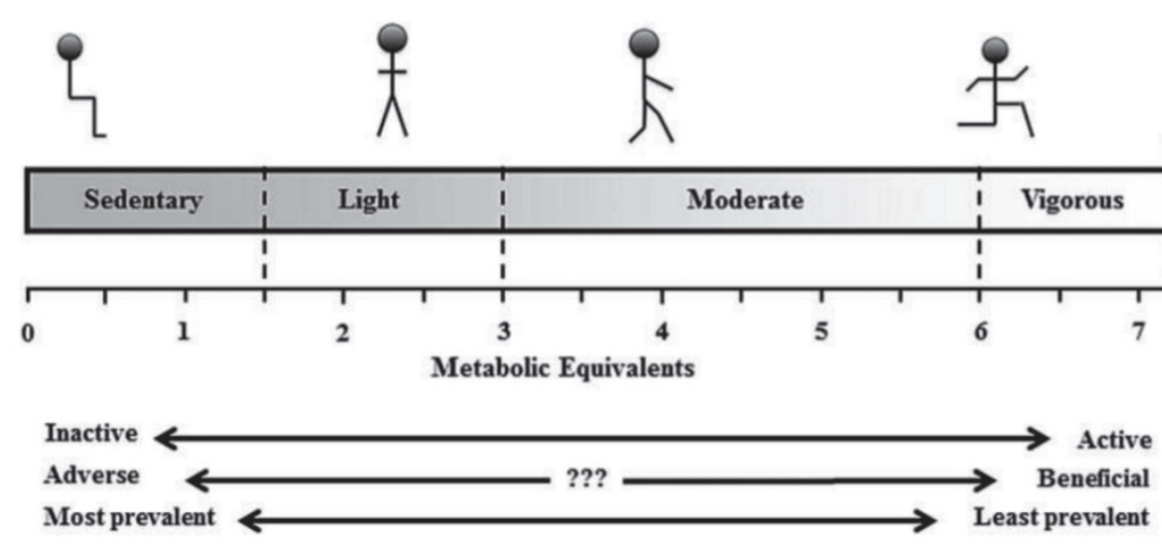

Figure 2 An integrated and comprehensive view of activity and inactivity. Reprinted from Internal Medicine Journal, Vol 45, Hill K, Gardiner PA, Cavalheri V, Jenkins SC, Healy GN, Physical activity and sedentary behaviour: applying lessons to chronic obstructive pulmonary disease, pp. 474-82, Copyright (2015), with permission from John Wiley and Sons.

Patients with chronic cardiopulmonary diseases often present with symptoms, such as breathlessness and fatigue [3,112], which compromises their performance on activities of daily living ( $A D L)$ and lead to sedentarism. Indeed, to date, there is strong evidence showing that patients with chronic cardiopulmonary diseases are more physically inactive than healthy age-matched subjects [113-115]. Although less well established, current evidence also suggests that patients with cardiopulmonary diseases are more sedentary than healthy controls [109].

In one of the first studies investigating objectively-measured physical activity in patients with COPD, Schönhofer and co-workers [116] observed that activity counts measured with a pedometer were three times lower in those with COPD when compared to healthy controls. Some years later, using a more advanced device (i.e., a triaxial accelerometer) Pitta et al. [117] observed that these low levels of physical activity seem to reduce even more during and after a hospitalisation for an exacerbation of the disease. These findings are especially important when considering that physical inactivity has been associated with greater morbidity and mortality in COPD $[118,119]$. Interestingly, patients with COPD appear to not only move less, but also waste more energy for specific tasks when compared to healthy controls. Vaes et al. [120] observed that patients with COPD used a significantly higher proportion of their peak aerobic capacity and ventilation to perform $\mathrm{ADL}$ than did healthy subjects. In patients with $\mathrm{CHF}$, Van den 
Berg-Emons et al. [114] observed a lower mean duration of movement-related activities when compared to healthy controls. Moreover, patients with $\mathrm{CHF}$ also appear to waste more energy for specific tasks. Spruit et al. [121] observed that patients with CHF performing pre-defined simple ADL consumed oxygen at a higher proportion of their peak aerobic capacity when compared to healthy subjects. As is the case for healthy subjects and patients with COPD, a physically inactive lifestyle in patients with CHF also associates with premature mortality [122].

Many previous studies investigating activity levels in patients with cardiopulmonary diseases have presented their results as a total amount or as an average of multiple measurement days $[114,117,123,124]$. Nevertheless, other studies have shown that more detailed analyses can also provide useful information. In patients with Parkinson's disease, Rochester and colleagues [125] investigated physical activity hourly patterns, which are a graphic representation of the temporal trends of physical activity accumulated at different intensities over the course of a day. Based on these measures, the authors observed that patients with Parkinson's disease have a delayed morning start and a reduced peak of activity when compared to healthy controls. More recently, Bellettiere et al. [126] observed less sedentary time during morning hours in women compared to men when investigating activity hourly patterns in older adults living in retirement communities. Another detailed analysis able to provide useful information is cluster analysis, which can be defined as multivariate analysis techniques that seeks to organise information about variables with the aim of classifying heterogeneous groups of subjects into relatively homogeneous subgroups or 'clusters' [127]. In a cross-sectional study in middle-aged Chinese adults, Lee and co-workers [128] used this type of analysis to identify subgroups of subjects with distinct characteristics. Two clusters were identified and male subjects in the least active cluster showed higher body fat percentage and older age than those in the active cluster [128]. In another cross-sectional study, De Bourdeaudhuij and co-workers [129] identified four clusters with different patterns in 10- to 12 -year-old children. In girls, the cluster with high moderate to vigorous-intensity physical activity (MVPA) and low sedentary time had a significantly lower body mass index and waist circumference, and the lowest percentage of overweight compared with the other three clusters (i.e., low MVPA and high sedentary time, low MVPA and low sedentary time, and moderate to high MVPA and moderate sedentary time) [129]. In patients with cardiopulmonary diseases, a study investigating activity hourly patterns and using cluster analysis could provide a better understanding of how these patients accumulate physical activity, which can inform strategies to improve physical activity in these populations. 


\section{Improving physical activity and counteracting sedentary behaviour}

Rehabilitation programmes based on exercise training have a great potential to increase daily physical activity and reduce sedentary behaviour in patients with cardiopulmonary diseases as they include components aimed at increasing exercise tolerance and improving psychological factors $[130,131]$. Nevertheless, these programmes have been shown to result in none or modest overall improvements in outcomes of physical activity and/or sedentary behaviour in these populations. In patients with COPD, Pitta et al. [132] observed no significant improvements in time spent walking, standing, sitting or lying down after a 3-month comprehensive PR programme, despite improvements in exercise tolerance, quadriceps force and health-related quality of life. Similarly, Van den Berg-Emons et al. [133] observed that an aerobic exercise training programme failed to improve the duration of dynamic activities (e.g., walking, cycling) or the number of walking periods in patients with $\mathrm{CHF}$, despite improvements in exercise tolerance and quadriceps muscle strength. Although most previous studies reported none or minor differences when comparing pre and post measures of physical activity and sedentary behaviour, a broad range in the change in physical activity and sedentary behaviour appears to exist. For example, a subgroup of patients may present increased physical activity and reduced sedentary behaviour levels following a rehabilitation programme. Identifying potential groups with different patterns of change in physical activity and sedentary behaviour following rehabilitation might help clinicians to determine which groups may benefit the most from this intervention and which could potentially benefit from additional strategies aimed at improving physical activity and sedentary behaviour, such as behavioural interventions.

One of the main goals in the management of COPD and CHF is to adopt a healthier lifestyle, which includes regular participation in physical activity $[3,4]$. In patients with COPD, a recent systematic review identified different interventions that can be used to help achieve this goal [134]. Nevertheless, the results are heterogeneous and it is not clear whether the improvements, in case those interventions actually led to improvements, are clinically relevant [134]. Family members or loved ones could play an important role in improving patients' physical activity, for instance, by encouraging patients to have joint daily walks or to engage more in household activities. Family-based interventions have shown to be useful to achieve healthy behaviours in other populations. In children, Viitasalo et al. [135] observed that an individualised and family-based lifestyle intervention aimed at decreasing cardiometabolic risk in children by increasing physical activity, decreasing sedentary behaviour and enhancing diet quality lead to significant improvements in physical activity and attenuated the increase in sedentary behaviour in comparison to the control group, which received verbal and written advice on health improving physical activity and diet. In Hispanic adult patients with diabetes, a family-based intervention programme focusing on diabetes education had beneficial 
effects on haemoglobin A1c, systolic blood pressure, diabetes self-efficacy, diabetes knowledge, and physical and mental components of health-related quality of life [136]. Moreover, family members significantly lowered their body mass index and improved their diabetes knowledge after the intervention [136]. Family-based interventions may be useful in patients with cardiopulmonary diseases. Nevertheless, before considering family-based lifestyle interventions for these patients and their family members, a better understanding of the physical activity and sedentary behaviour levels, as well as exercise motivation of the patients and their family members is necessary in order to inform interventions with greater chances of successful outcomes.

\section{Aims of the present thesis}

The aims of the present thesis were:

1. To compare health outcome measures between patients with COPD with and without impaired LVEF after adjusting for relevant confounders;

2. To investigate the impact of both individual as well as clusters of objectively identified comorbidities on changes in exercise tolerance and health status after PR in patients with COPD;

3. To investigate the reliability, construct validity, and determinants of the distance covered in the 6MWT in patients with CHF;

4. To investigate the within-day test-retest reliability of the TUG test in patients with advanced chronic organ failure, including advanced COPD and CHF;

5. To investigate the construct validity of the TUG test in patients with COPD; to identify characteristics related to an abnormal TUG time; and to examine the responsiveness of the TUG to PR;

6. To describe physical activity measures and hourly patterns in patients with COPD after stratification for generic and COPD-specific characteristics; and to identify clusters of patients based on multiple physical activity measures;

7. To describe groups of patients with COPD according to patterns of change in physical activity and sedentary behaviour after a PR programme;

8. To compare physical activity levels and exercise motivation between patients with COPD and their resident loved ones; to compare the same outcome measures in patients after stratification for the physical activity level of the loved ones; and to predict the likelihood of being physically active in patients with a physically active resident loved one. 


\section{Outline of the present thesis}

The aims of the present thesis were addressed through three main themes: the investigation of the impact of comorbidities on relevant outcome measures in patients with COPD (Chapters 2 and 3); the investigation of measurement properties of field physical capacity tests in patients with COPD or CHF (Chapters 4, 5 and 6); and the investigation of patterns of physical activity and sedentary behaviour in patients with COPD (Chapters 7, 8 and 9). These three main themes were generally discussed in a broader perspective in Chapter 10, which also presents the main limitations of the previous chapters, gives future directions, and provides concluding remarks.

In order to address the impact of comorbidities on relevant outcome measures in patients with COPD, Chapter 2 investigates the impact of impaired LVEF on measures of physical and psychological health. In a sample of more than 500 patients with COPD, those with impaired LVEF were identified and pair-wise matched for relevant confounders to a group of patients without impaired LVEF. A comprehensive number of measures were compared between these two groups, including exercise tolerance measures. Chapter 3 investigates the impact of objectively identified comorbidities on PR outcomes in patients with COPD. Specifically, the impact of 13 individual comorbidities, as well as of 5 clusters of comorbidities, on changes in functional exercise capacity and health status after a comprehensive PR programme was investigated. This chapter explores whether individual comorbidities or clusters of comorbidities can prevent patients with COPD from experiencing meaningful improvements following a PR programme.

In order to address the measurement properties of field physical capacity tests in patients with COPD or CHF, Chapter 4 investigates the reliability and the construct validity of the 6MWT in patients with CHF entering a rehabilitation programme. Moreover, potential determinants of the best 6MWD are explored among a comprehensive number of characteristics. Chapter 5 investigates the within-day test-retest reliability of the TUG test in patients with advanced chronic organ failure, namely COPD, CHF, and chronic renal failure recruited from the outpatient clinics of one academic hospital and six general hospitals. Chapter 6 adds to the previous chapter by investigating the construct validity of the TUG test and its responsiveness to a comprehensive PR programme in patients with COPD. Furthermore, this chapter also includes a sample of subjects without COPD, which allowed the definition of a cut-off point to identify abnormal TUG times. Characteristics related to an abnormal TUG time in the patient group are also investigated.

In order to address patterns of physical activity and sedentary behaviour in patients with COPD, Chapter 7 investigates patterns and clusters of physical activity using histori- 
cal data of 1001 patients with COPD from 10 countries (the United Kingdom, Ireland, the Netherlands, Germany, Switzerland, Italy, Spain, the United States of America, Brazil, and Australia). The main focus of this chapter is on clusters of physical activity and how they could inform interventions aimed at improving physical activity. Chapter 8 complements the previous chapter by investigating the impact of a PR programme on physical activity and sedentary behaviour in patients with COPD. Specifically, this chapter explores groups of patients with different patterns of change in physical activity and sedentary behaviour following PR, and the characteristics related to these groups. Chapter 9 adds to the two previous chapters by exploring the potential of physically active resident loved ones as a source of support to the adoption of a more physically active lifestyle by patients. This chapter investigates objectively assessed physical activity and sedentary behaviour in patients with COPD and their resident loved ones. 


\section{REFERENCES}

1. World Health Organization. The top 10 causes of death. Fact sheet 310. Available at: http://www.who.int/mediacentre/factsheets/fs310/en/. Accessed June 24, 2016.

2. Chronic respiratory diseases. Data and statistics. Available at: http://www.euro.who.int/en/healthtopics/noncommunicable-diseases/chronic-respiratory-diseases/data-and-statistics. Accessed June 24, 2016.

3. Vestbo J, Hurd SS, Agusti AG, Jones PW, Vogelmeier C, Anzueto A, Barnes PJ, Fabbri LM, Martinez FJ, Nishimura $M$, et al: Global strategy for the diagnosis, management, and prevention of chronic obstructive pulmonary disease: GOLD executive summary. Am J Respir Crit Care Med 2013, 187:347-365.

4. Ponikowski P, Voors AA, Anker SD, Bueno H, Cleland JG, Coats AJ, Falk V, Gonzalez-Juanatey JR, Harjola VP, Jankowska EA, et al: 2016 ESC Guidelines for the diagnosis and treatment of acute and chronic heart failure: The Task Force for the diagnosis and treatment of acute and chronic heart failure of the European Society of Cardiology (ESC). Developed with the special contribution of the Heart Failure Association (HFA) of the ESC. Eur J Heart Fail 2016, 18:891-975.

5. Gosker HR, Wouters EF, van der Vusse GJ, Schols AM: Skeletal muscle dysfunction in chronic obstructive pulmonary disease and chronic heart failure: underlying mechanisms and therapy perspectives. Am J Clin Nutr 2000, 71:1033-1047.

6. Agusti A, Morla M, Sauleda J, Saus C, Busquets X: NF-kappaB activation and iNOS upregulation in skeletal muscle of patients with COPD and low body weight. Thorax 2004, 59:483-487.

7. Gordon JW, Shaw JA, Kirshenbaum LA: Multiple facets of NF-kappaB in the heart: to be or not to NFkappaB. Circ Res 2011, 108:1122-1132.

8. Messina S, Bitto A, Aguennouz M, Minutoli L, Monici MC, Altavilla D, Squadrito F, Vita G: Nuclear factor kappa-B blockade reduces skeletal muscle degeneration and enhances muscle function in Mdx mice. Exp Neurol 2006, 198:234-241.

9. Barnes PJ, Celli BR: Systemic manifestations and comorbidities of COPD. Eur Respir J 2009, 33:1165-1185.

10. Cavailles A, Brinchault-Rabin G, Dixmier A, Goupil F, Gut-Gobert C, Marchand-Adam S, Meurice JC, Morel H, Person-Tacnet C, Leroyer C, Diot P: Comorbidities of COPD. Eur Respir Rev 2013, 22:454-475.

11. Lange P, Mogelvang R, Marott JL, Vestbo J, Jensen JS: Cardiovascular morbidity in COPD: A study of the general population. COPD 2010, 7:5-10.

12. Dahlstrom U: Frequent non-cardiac comorbidities in patients with chronic heart failure. Eur J Heart Fail 2005, 7:309-316.

13. Mannino DM, Thorn D, Swensen A, Holguin F: Prevalence and outcomes of diabetes, hypertension and cardiovascular disease in COPD. Eur Respir J 2008, 32:962-969.

14. Braunstein JB, Anderson GF, Gerstenblith G, Weller W, Niefeld M, Herbert R, Wu AW: Noncardiac comorbidity increases preventable hospitalizations and mortality among Medicare beneficiaries with chronic heart failure. J Am Coll Cardiol 2003, 42:1226-1233.

15. Bouvy ML, Heerdink ER, Leufkens HG, Hoes AW: Predicting mortality in patients with heart failure: a pragmatic approach. Heart 2003, 89:605-609.

16. van der Wel MC, Jansen RW, Bakx JC, Bor HH, Olderikkert MG, van Weel C: Non-cardiovascular comorbidity in elderly patients with heart failure outnumbers cardiovascular co-morbidity. Eur J Heart Fail 2007, 9:709-715.

17. Rutten FH, Cramer MJ, Grobbee DE, Sachs AP, Kirkels JH, Lammers JW, Hoes AW: Unrecognized heart failure in elderly patients with stable chronic obstructive pulmonary disease. Eur Heart J 2005, 26:18871894.

18. Franssen FM, Soriano JB, Roche N, Bloomfield PH, Brusselle G, Fabbri LM, Garcia-Rio F, Kearney MT, Kwon N, Lundback B, et al: Lung Function Abnormalities in Smokers With Ischemic Heart Disease. Am J Respir Crit Care Med 2016, 194:568-576.

19. De Blois J, Simard S, Atar D, Agewall S, Norwegian Heart Failure R: COPD predicts mortality in HF: the Norwegian Heart Failure Registry. J Card Fail 2010, 16:225-229. 
20. Chhabra SK, Gupta M: Coexistent chronic obstructive pulmonary disease-heart failure: mechanisms, diagnostic and therapeutic dilemmas. Indian J Chest Dis Allied Sci 2010, 52:225-238.

21. Sin DD, Man SF: Why are patients with chronic obstructive pulmonary disease at increased risk of cardiovascular diseases? The potential role of systemic inflammation in chronic obstructive pulmonary disease. Circulation 2003, 107:1514-1519.

22. Hawkins NM, Petrie MC, Jhund PS, Chalmers GW, Dunn FG, McMurray JJ: Heart failure and chronic obstructive pulmonary disease: diagnostic pitfalls and epidemiology. Eur J Heart Fail 2009, 11:130-139.

23. Schellenbaum GD, Rea TD, Heckbert SR, Smith NL, Lumley T, Roger VL, Kitzman DW, Taylor HA, Levy D, Psaty BM: Survival associated with two sets of diagnostic criteria for congestive heart failure. Am J Epidemiol 2004, 160:628-635.

24. Polkey MI, Moxham J: Attacking the disease spiral in chronic obstructive pulmonary disease: an update. Clin Med (Lond) 2011, 11:461-464.

25. Caspersen CJ, Powell KE, Christenson GM: Physical activity, exercise, and physical fitness: definitions and distinctions for health-related research. Public Health Rep 1985, 100:126-131.

26. Cotes JE, Zejda J, King B: Lung function impairment as a guide to exercise limitation in work-related lung disorders. Am Rev Respir Dis 1988, 137:1089-1093.

27. Steele IC, Moore A, Nugent AM, Riley MS, Campbell NP, Nicholls DP: Non-invasive measurement of cardiac output and ventricular ejection fractions in chronic cardiac failure: relationship to impaired exercise tolerance. Clin Sci (Lond) 1997, 93:195-203.

28. Grove A, Lipworth BJ, Reid P, Smith RP, Ramage L, Ingram CG, Jenkins RJ, Winter JH, Dhillon DP: Effects of regular salmeterol on lung function and exercise capacity in patients with chronic obstructive airways disease. Thorax 1996, 51:689-693.

29. Miller MM: Current trends in the primary care management of chronic congestive heart failure. Nurse Pract 1994, 19:64-70.

30. Grover FL, Fullerton DA, Zamora MR, Mills C, Ackerman B, Badesch D, Brown JM, Campbell DN, Chetham P, Dhaliwal A, et al: The past, present, and future of lung transplantation. Am J Surg 1997, 173:523-533.

31. Beller GA: Selecting patients with ischemic cardiomyopathy for medical treatment, revascularization, or heart transplantation. J Nucl Cardiol 1997, 4:S152-157.

32. Pepin V, Saey D, Laviolette L, Maltais F: Exercise capacity in chronic obstructive pulmonary disease: mechanisms of limitation. COPD 2007, 4:195-204.

33. Piepoli MF, Guazzi M, Boriani G, Cicoira M, Corra U, Dalla Libera L, Emdin M, Mele D, Passino C, Vescovo G, et al: Exercise intolerance in chronic heart failure: mechanisms and therapies. Part I. Eur J Cardiovasc Prev Rehabil 2010, 17:637-642.

34. Piepoli MF, Guazzi M, Boriani G, Cicoira M, Corra U, Dalla Libera L, Emdin M, Mele D, Passino C, Vescovo $\mathrm{G}$, et al: Exercise intolerance in chronic heart failure: mechanisms and therapies. Part II. Eur J Cardiovasc Prev Rehabil 2010, 17:643-648.

35. Vogiatzis I, Zakynthinos S: The physiological basis of rehabilitation in chronic heart and lung disease. J Appl Physiol (1985) 2013, 115:16-21.

36. O'Donnell DE: Hyperinflation, dyspnea, and exercise intolerance in chronic obstructive pulmonary disease. Proc Am Thorac Soc 2006, 3:180-184.

37. O'Donnell DE, Revill SM, Webb KA: Dynamic hyperinflation and exercise intolerance in chronic obstructive pulmonary disease. Am J Respir Crit Care Med 2001, 164:770-777.

38. O'Donnell DE, Fluge T, Gerken F, Hamilton A, Webb K, Aguilaniu B, Make B, Magnussen H: Effects of tiotropium on lung hyperinflation, dyspnoea and exercise tolerance in COPD. Eur Respir J 2004, 23:832840.

39. Sinderby C, Spahija J, Beck J, Kaminski D, Yan S, Comtois N, Sliwinski P: Diaphragm activation during exercise in chronic obstructive pulmonary disease. Am J Respir Crit Care Med 2001, 163:1637-1641.

40. Laghi F, Tobin MJ: Disorders of the respiratory muscles. Am J Respir Crit Care Med 2003, 168:10-48.

41. Somfay A, Porszasz J, Lee SM, Casaburi R: Effect of hyperoxia on gas exchange and lactate kinetics following exercise onset in nonhypoxemic COPD patients. Chest 2002, 121:393-400. 
42. Emtner M, Porszasz J, Burns M, Somfay A, Casaburi R: Benefits of supplemental oxygen in exercise training in nonhypoxemic chronic obstructive pulmonary disease patients. Am J Respir Crit Care Med 2003, 168:1034-1042.

43. Matthay RA, Berger HJ, Davies RA, Loke J, Mahler DA, Gottschalk A, Zaret BL: Right and left ventricular exercise performance in chronic obstructive pulmonary disease: radionuclide assessment. Ann Intern Med 1980, 93:234-239.

44. Santos S, Peinado VI, Ramirez J, Melgosa T, Roca J, Rodriguez-Roisin R, Barbera JA: Characterization of pulmonary vascular remodelling in smokers and patients with mild COPD. Eur Respir J 2002, 19:632-638.

45. Voelkel NF, Tuder RM: Hypoxia-induced pulmonary vascular remodeling: a model for what human disease? J Clin Invest 2000, 106:733-738.

46. Chetty KG, Brown SE, Light RW: Improved exercise tolerance of the polycythemic lung patient following phlebotomy. Am J Med 1983, 74:415-420.

47. Gosselink R, Troosters T, Decramer M: Peripheral muscle weakness contributes to exercise limitation in COPD. Am J Respir Crit Care Med 1996, 153:976-980.

48. Hamilton AL, Killian KJ, Summers E, Jones NL: Muscle strength, symptom intensity, and exercise capacity in patients with cardiorespiratory disorders. Am J Respir Crit Care Med 1995, 152:2021-2031.

49. Saey D, Debigare R, LeBlanc P, Mador MJ, Cote CH, Jobin J, Maltais F: Contractile leg fatigue after cycle exercise: a factor limiting exercise in patients with chronic obstructive pulmonary disease. Am J Respir Crit Care Med 2003, 168:425-430.

50. Maltais F, Decramer M, Casaburi R, Barreiro E, Burelle Y, Debigare R, Dekhuijzen PN, Franssen F, GayanRamirez G, Gea J, et al: An official American Thoracic Society/European Respiratory Society statement: update on limb muscle dysfunction in chronic obstructive pulmonary disease. Am J Respir Crit Care Med 2014, 189:e15-62.

51. Seymour JM, Spruit MA, Hopkinson NS, Natanek SA, Man WD, Jackson A, Gosker HR, Schols AM, Moxham J, Polkey MI, Wouters EF: The prevalence of quadriceps weakness in COPD and the relationship with disease severity. Eur Respir J 2010, 36:81-88.

52. O'Donnell DE, Neder JA, Elbehairy AF: Physiological impairment in mild COPD. Respirology 2016, 21:211223.

53. Oga T, Nishimura K, Tsukino M, Hajiro T, Ikeda A, Mishima M: Relationship between different indices of exercise capacity and clinical measures in patients with chronic obstructive pulmonary disease. Heart Lung 2002, 31:374-381.

54. Brown CD, Benditt JO, Sciurba FC, Lee SM, Criner GJ, Mosenifar Z, Shade DM, Slivka WA, Wise RA, National emphysema Treatment Trial Research G: Exercise testing in severe emphysema: association with quality of life and lung function. COPD 2008, 5:117-124.

55. Spruit MA, Watkins ML, Edwards LD, Vestbo J, Calverley PM, Pinto-Plata V, Celli BR, Tal-Singer R, Wouters EF, Evaluation of CLtIPSEsi: Determinants of poor 6-min walking distance in patients with COPD: the ECLIPSE cohort. Respir Med 2010, 104:849-857.

56. Pina IL, Apstein CS, Balady GJ, Belardinelli R, Chaitman BR, Duscha BD, Fletcher BJ, Fleg JL, Myers JN, Sullivan MJ, et al: Exercise and heart failure: A statement from the American Heart Association Committee on exercise, rehabilitation, and prevention. Circulation 2003, 107:1210-1225.

57. Sullivan MJ, Cobb FR: Central hemodynamic response to exercise in patients with chronic heart failure. Chest 1992, 101:340S-346S.

58. Myers J, Salleh A, Buchanan N, Smith D, Neutel J, Bowes E, Froelicher VF: Ventilatory mechanisms of exercise intolerance in chronic heart failure. Am Heart J 1992, 124:710-719.

59. ATS/ACCP Statement on cardiopulmonary exercise testing. Am J Respir Crit Care Med 2003, 167:211-277.

60. Nanas S, Nanas J, Kassiotis C, Alexopoulos G, Samakovli A, Kanakakis J, Tsolakis E, Roussos C: Respiratory muscles performance is related to oxygen kinetics during maximal exercise and early recovery in patients with congestive heart failure. Circulation 1999, 100:503-508.

61. Meyer FJ, Borst MM, Zugck C, Kirschke A, Schellberg D, Kubler W, Haass M: Respiratory muscle dysfunction in congestive heart failure: clinical correlation and prognostic significance. Circulation 2001, 103:2153-2158. 
62. Dall'Ago P, Chiappa GR, Guths H, Stein R, Ribeiro JP: Inspiratory muscle training in patients with heart failure and inspiratory muscle weakness: a randomized trial. J Am Coll Cardiol 2006, 47:757-763.

63. Katz SD, Krum H, Khan T, Knecht M: Exercise-induced vasodilation in forearm circulation of normal subjects and patients with congestive heart failure: role of endothelium-derived nitric oxide. J Am Coll Cardiol 1996, 28:585-590.

64. Katz SD, Yuen J, Bijou R, LeJemtel TH: Training improves endothelium-dependent vasodilation in resistance vessels of patients with heart failure. J Appl Physiol (1985) 1997, 82:1488-1492.

65. Gosker HR, Lencer NH, Franssen FM, van der Vusse GJ, Wouters EF, Schols AM: Striking similarities in systemic factors contributing to decreased exercise capacity in patients with severe chronic heart failure or COPD. Chest 2003, 123:1416-1424.

66. Nicoletti I, Cicoira M, Zanolla L, Franceschini L, Brighetti G, Pilati M, Zardini P: Skeletal muscle abnormalities in chronic heart failure patients: relation to exercise capacity and therapeutic implications. Congest Heart Fail 2003, 9:148-154.

67. Sullivan MJ, Green HJ, Cobb FR: Altered skeletal muscle metabolic response to exercise in chronic heart failure. Relation to skeletal muscle aerobic enzyme activity. Circulation 1991, 84:1597-1607.

68. Drexler $\mathrm{H}$, Riede $\mathrm{U}$, Munzel $\mathrm{T}$, Konig H, Funke $\mathrm{E}$, Just $\mathrm{H}$ : Alterations of skeletal muscle in chronic heart failure. Circulation 1992, 85:1751-1759.

69. Westlake C, Dracup K, Creaser J, Livingston N, Heywood JT, Huiskes BL, Fonarow G, Hamilton M: Correlates of health-related quality of life in patients with heart failure. Heart Lung 2002, 31:85-93.

70. Papasavvas T, Alhashemi M, Micklewright D: Association Between Depressive Symptoms and Exercise Capacity in Patients With Heart Disease: A META-ANALYSIS. J Cardiopulm Rehabil Prev 2016.

71. Miller J, Edwards LD, Agusti A, Bakke P, Calverley PM, Celli B, Coxson HO, Crim C, Lomas DA, Miller BE, et al: Comorbidity, systemic inflammation and outcomes in the ECLIPSE cohort. Respir Med 2013, 107:13761384.

72. Putcha N, Han MK, Martinez CH, Foreman MG, Anzueto AR, Casaburi R, Cho MH, Hanania NA, Hersh CP, Kinney GL, et al: Comorbidities of COPD have a major impact on clinical outcomes, particularly in African Americans. Chronic Obstr Pulm Dis (Miami) 2014, 1:105-114.

73. Guazzi M, Myers J, Vicenzi M, Bensimhon D, Chase P, Pinkstaff S, Arena R: Cardiopulmonary exercise testing characteristics in heart failure patients with and without concomitant chronic obstructive pulmonary disease. Am Heart J 2010, 160:900-905.

74. McCarthy B, Casey D, Devane D, Murphy K, Murphy E, Lacasse Y: Pulmonary rehabilitation for chronic obstructive pulmonary disease. Cochrane Database Syst Rev 2015:CD003793.

75. Jonsdottir S, Andersen KK, Sigurosson AF, Sigurosson SB: The effect of physical training in chronic heart failure. Eur J Heart Fail 2006, 8:97-101.

76. Taylor RS, Sagar VA, Davies EJ, Briscoe S, Coats AJ, Dalal H, Lough F, Rees K, Singh S: Exercise-based rehabilitation for heart failure. Cochrane Database Syst Rev 2014:CD003331.

77. Puhan M, Scharplatz M, Troosters T, Walters EH, Steurer J: Pulmonary rehabilitation following exacerbations of chronic obstructive pulmonary disease. Cochrane Database Syst Rev 2009:CD005305.

78. Crisafulli E, Costi S, Luppi F, Cirelli G, Cilione C, Coletti O, Fabbri LM, Clini EM: Role of comorbidities in a cohort of patients with COPD undergoing pulmonary rehabilitation. Thorax 2008, 63:487-492.

79. Crisafulli E, Gorgone P, Vagaggini B, Pagani M, Rossi G, Costa F, Guarriello V, Paggiaro P, Chetta A, de Blasio $F$, et al: Efficacy of standard rehabilitation in COPD outpatients with comorbidities. Eur Respir J 2010, 36:1042-1048.

80. Mentz RJ, Schulte PJ, Fleg JL, Fiuzat M, Kraus WE, Pina IL, Keteyian SJ, Kitzman DW, Whellan DJ, Ellis SJ, O'Connor CM: Clinical characteristics, response to exercise training, and outcomes in patients with heart failure and chronic obstructive pulmonary disease: findings from Heart Failure and A Controlled Trial Investigating Outcomes of Exercise TraiNing (HF-ACTION). Am Heart J 2013, 165:193-199.

81. Vanfleteren LE, Spruit MA, Groenen M, Gaffron S, van Empel VP, Bruijnzeel PL, Rutten EP, Op 't Roodt J, Wouters EF, Franssen FM: Clusters of comorbidities based on validated objective measurements and systemic inflammation in patients with chronic obstructive pulmonary disease. Am J Respir Crit Care Med 2013, 187:728-735. 
82. ERS Task Force, Palange P, Ward SA, Carlsen KH, Casaburi R, Gallagher CG, Gosselink R, O'Donnell DE, Puente-Maestu L, Schols AM, et al: Recommendations on the use of exercise testing in clinical practice. Eur Respir J 2007, 29:185-209.

83. Holland AE, Spruit MA, Troosters T, Puhan MA, Pepin V, Saey D, McCormack MC, Carlin BW, Sciurba FC, Pitta F, et al: An official European Respiratory Society/American Thoracic Society Technical Standard: field walking tests in chronic respiratory disease. Eur Respir J 2014, 44:1428-1446.

84. Hernandes NA, Wouters EF, Meijer K, Annegarn J, Pitta F, Spruit MA: Reproducibility of 6-minute walking test in patients with COPD. Eur Respir J 2011, 38:261-267.

85. Spruit MA, Polkey MI, Celli B, Edwards LD, Watkins ML, Pinto-Plata V, Vestbo J, Calverley PM, Tal-Singer R, Agusti A, et al: Predicting outcomes from 6-minute walk distance in chronic obstructive pulmonary disease. J Am Med Dir Assoc 2012, 13:291-297.

86. Andrianopoulos V, Wouters EF, Pinto-Plata VM, Vanfleteren LE, Bakke PS, Franssen FM, Agusti A, MacNee W, Rennard SI, Tal-Singer R, et al: Prognostic value of variables derived from the six-minute walk test in patients with COPD: Results from the ECLIPSE study. Respir Med 2015.

87. Rodrigues A, Di Martino M, Nellessen AG, Hernandes NA, Neder JA, Pitta F: Is the six-minute walk test a useful tool to prescribe high-intensity exercise in patients with chronic obstructive pulmonary disease? Heart Lung 2016, 45:550-556.

88. Rostagno C, Olivo G, Comeglio M, Boddi V, Banchelli M, Galanti G, Gensini GF: Prognostic value of 6minute walk corridor test in patients with mild to moderate heart failure: comparison with other methods of functional evaluation. Eur J Heart Fail 2003, 5:247-252.

89. Tallaj JA, Sanderson B, Breland J, Adams C, Schumann C, Bittner V: Assessment of functional outcomes using the 6-minute walk test in cardiac rehabilitation: comparison of patients with and without left ventricular dysfunction. J Cardiopulm Rehabil 2001, 21:221-224.

90. Zugck C, Krüger C, Dürr S, Gerber SH, Haunstetter A, Hornig K, Kübler W, Haass M: Is the 6-minute walk test a reliable substitute for peak oxygen uptake in patients with dilated cardiomyopathy? Eur Heart J 2000, 21:540-549.

91. Araya-Ramirez F, Briggs KK, Bishop SR, Miller CE, Moncada-Jimenez J, Grandjean PW: Who is likely to benefit from phase II cardiac rehabilitation? J Cardiopulm Rehabil Prev 2010, 30:93-100.

92. Bellet RN, Francis RL, Jacob JS, Healy KM, Bartlett HJ, Adams L, Morris NR: Repeated six-minute walk tests for outcome measurement and exercise prescription in outpatient cardiac rehabilitation: a longitudinal study. Arch Phys Med Rehabil 2011, 92:1388-1394.

93. Podsiadlo D, Richardson S: The timed "Up \& Go": a test of basic functional mobility for frail elderly persons. J Am Geriatr Soc 1991, 39:142-148.

94. Robinson TN, Wallace JI, Wu DS, Wiktor A, Pointer LF, Pfister SM, Sharp TJ, Buckley MJ, Moss M: Accumulated frailty characteristics predict postoperative discharge institutionalization in the geriatric patient. J Am Coll Surg 2011, 213:37-42.

95. Soubeyran P, Fonck M, Blanc-Bisson C, Blanc JF, Ceccaldi J, Mertens C, Imbert Y, Cany L, Vogt L, Dauba J, et al: Predictors of early death risk in older patients treated with first-line chemotherapy for cancer. J Clin Oncol 2012, 30:1829-1834.

96. Laflamme GY, Rouleau DM, Leduc S, Roy L, Beaumont E: The Timed Up and Go test is an early predictor of functional outcome after hemiarthroplasty for femoral neck fracture. J Bone Joint Surg Am 2012, 94:1175-1179.

97. Panel on Prevention of Falls in Older Persons, American Geriatrics Society and British Geriatrics Society. Summary of the Updated American Geriatrics Society/British Geriatrics Society clinical practice guideline for prevention of falls in older persons. J Am Geriatr Soc 2011, 59:148-157.

98. Turner G, Clegg A, British Geriatrics S, Age UK, Royal College of General P: Best practice guidelines for the management of frailty: a British Geriatrics Society, Age UK and Royal College of General Practitioners report. Age Ageing 2014, 43:744-747.

99. Spruit MA, Franssen FM, Rutten EP, Wagers SS, Wouters EF: Age-graded reductions in quadriceps muscle strength and peak aerobic capacity in COPD. Rev Bras Fisioter 2012, 16:148-156. 
100.Clark A, Rafferty D, Arbuthnott K: Relationship between isokinetic muscle strength and exercise capacity in chronic heart failure. Int J Cardiol 1997, 59:145-148.

101.Lee IM, Shiroma EJ, Lobelo F, Puska P, Blair SN, Katzmarzyk PT, Lancet Physical Activity Series Working G: Effect of physical inactivity on major non-communicable diseases worldwide: an analysis of burden of disease and life expectancy. Lancet 2012, 380:219-229.

102. Wen CP, Wai JP, Tsai MK, Yang YC, Cheng TY, Lee MC, Chan HT, Tsao CK, Tsai SP, Wu X: Minimum amount of physical activity for reduced mortality and extended life expectancy: a prospective cohort study. Lancet 2011, 378:1244-1253.

103.WHO: Global health risks: mortality and burden of disease attributable to selected major risks. Geneva: World Health Organization; 2009.

104.Sallis JF, Bull F, Guthold R, Heath GW, Inoue S, Kelly P, Oyeyemi AL, Perez LG, Richards J, Hallal PC, Lancet Physical Activity Series 2 Executive C: Progress in physical activity over the Olympic quadrennium. Lancet 2016, 388:1325-1336.

105.Global recommendations on physical activity for health. Geneva: World Health Organization, 2011. Available at: http://www.who.int/dietphysicalactivity/factsheet_recommendations/en/index.html. Acessed June 24, 2016.

106. Marsaux CF, Celis-Morales C, Hoonhout J, Claassen A, Goris A, Forster H, Fallaize R, Macready AL, NavasCarretero S, Kolossa S, et al: Objectively Measured Physical Activity in European Adults: Cross-Sectional Findings from the Food4Me Study. PLoS One 2016, 11:e0150902.

107. Hansen BH, Kolle E, Dyrstad SM, Holme I, Anderssen SA: Accelerometer-determined physical activity in adults and older people. Med Sci Sports Exerc 2012, 44:266-272.

108. Sedentary Behaviour Research N: Letter to the editor: standardized use of the terms "sedentary" and "sedentary behaviours". Appl Physiol Nutr Metab 2012, 37:540-542.

109. Hill K, Gardiner PA, Cavalheri V, Jenkins SC, Healy GN: Physical activity and sedentary behaviour: applying lessons to chronic obstructive pulmonary disease. Intern Med J 2015, 45:474-482.

110. Healy GN, Dunstan DW, Salmon J, Shaw JE, Zimmet PZ, Owen N: Television time and continuous metabolic risk in physically active adults. Med Sci Sports Exerc 2008, 40:639-645.

111. Healy GN, Matthews CE, Dunstan DW, Winkler EA, Owen N: Sedentary time and cardio-metabolic biomarkers in US adults: NHANES 2003-06. Eur Heart J 2011, 32:590-597.

112. McMurray JJ, Adamopoulos S, Anker SD, Auricchio A, Bohm M, Dickstein K, Falk V, Filippatos G, Fonseca C, Gomez-Sanchez MA, et al: ESC Guidelines for the diagnosis and treatment of acute and chronic heart failure 2012: The Task Force for the Diagnosis and Treatment of Acute and Chronic Heart Failure 2012 of the European Society of Cardiology. Developed in collaboration with the Heart Failure Association (HFA) of the ESC. Eur Heart J 2012, 33:1787-1847.

113.Vorrink SN, Kort HS, Troosters T, Lammers JW: Level of daily physical activity in individuals with COPD compared with healthy controls. Respir Res, 12:33.

114.van den Berg-Emons H, Bussmann J, Balk A, Keijzer-Oster D, Stam H: Level of activities associated with mobility during everyday life in patients with chronic congestive heart failure as measured with an "activity monitor". Phys Ther 2001, 81:1502-1511.

115. Hoodless DJ, Stainer K, Savic N, Batin P, Hawkins M, Cowley AJ: Reduced customary activity in chronic heart failure: assessment with a new shoe-mounted pedometer. Int J Cardiol 1994, 43:39-42.

116.Schonhofer B, Ardes P, Geibel M, Kohler D, Jones PW: Evaluation of a movement detector to measure daily activity in patients with chronic lung disease. Eur Respir J 1997, 10:2814-2819.

117.Pitta F, Troosters T, Probst VS, Spruit MA, Decramer M, Gosselink R: Physical activity and hospitalization for exacerbation of COPD. Chest 2006, 129:536-544.

118.Garcia-Aymerich J, Lange P, Benet M, Schnohr P, Anto JM: Regular physical activity reduces hospital admission and mortality in chronic obstructive pulmonary disease: a population based cohort study. Thorax 2006, 61:772-778.

119. Waschki B, Kirsten A, Holz O, Muller KC, Meyer T, Watz H, Magnussen H: Physical activity is the strongest predictor of all-cause mortality in patients with COPD: a prospective cohort study. Chest 2011, 140:331342. 
120.Vaes AW, Wouters EF, Franssen FM, Uszko-Lencer NH, Stakenborg KH, Westra M, Meijer K, Schols AM, Janssen PP, Spruit MA: Task-related oxygen uptake during domestic activities of daily life in patients with COPD and healthy elderly subjects. Chest 2011, 140:970-979.

121.Spruit MA, Wouters EF, Eterman RM, Meijer K, Wagers SS, Stakenborg KH, Uszko-Lencer NH: Task-related oxygen uptake and symptoms during activities of daily life in CHF patients and healthy subjects. Eur J Appl Physiol 2011, 111:1679-1686.

122.Doukky R, Mangla A, Ibrahim Z, Poulin MF, Avery E, Collado FM, Kaplan J, Richardson D, Powell LH: Impact of Physical Inactivity on Mortality in Patients With Heart Failure. Am J Cardiol 2016, 117:1135-1143.

123. Pitta F, Troosters T, Spruit MA, Probst VS, Decramer M, Gosselink R: Characteristics of physical activities in daily life in chronic obstructive pulmonary disease. Am J Respir Crit Care Med 2005, 171:972-977.

124.van den Berg-Emons RJ, Bussmann JB, Balk AH, Stam HJ: Factors associated with the level of movementrelated everyday activity and quality of life in people with chronic heart failure. Phys Ther 2005, 85:13401348.

125.Rochester L, Jones D, Hetherington V, Nieuwboer A, Willems AM, Kwakkel G, van Wegen E: Gait and gaitrelated activities and fatigue in Parkinson's disease: what is the relationship? Disabil Rehabil 2006, 28:1365-1371.

126. Bellettiere J, Carlson JA, Rosenberg D, Singhania A, Natarajan L, Berardi V, LaCroix AZ, Sears DD, Moran K, Crist K, Kerr J: Gender and Age Differences in Hourly and Daily Patterns of Sedentary Time in Older Adults Living in Retirement Communities. PLoS One 2015, 10:e0136161.

127.Wardlaw AJ, Silverman M, Siva R, Pavord ID, Green R: Multi-dimensional phenotyping: towards a new taxonomy for airway disease. Clin Exp Allergy 2005, 35:1254-1262.

128. Lee PH, Yu YY, McDowell I, Leung GM, Lam TH: A cluster analysis of patterns of objectively measured physical activity in Hong Kong. Public Health Nutr 2013, 16:1436-1444.

129.De Bourdeaudhuij I, Verloigne M, Maes L, Van Lippevelde W, Chinapaw MJ, Te Velde SJ, Manios Y, Androutsos O, Kovacs E, Dossegger A, Brug J: Associations of physical activity and sedentary time with weight and weight status among 10- to 12-year-old boys and girls in Europe: a cluster analysis within the ENERGY project. Pediatr Obes 2013, 8:367-375.

130.Spruit MA, Singh SJ, Garvey C, Zuwallack R, Nici L, Rochester C, Hill K, Holland AE, Lareau SC, Man WD, et al: An official american thoracic society/european respiratory society statement: key concepts and advances in pulmonary rehabilitation. Am J Respir Crit Care Med 2013, 188:e13-64.

131.Sagar VA, Davies EJ, Briscoe S, Coats AJ, Dalal HM, Lough F, Rees K, Singh S, Taylor RS: Exercise-based rehabilitation for heart failure: systematic review and meta-analysis. Open Heart 2015, 2:e000163.

132.Pitta F, Troosters T, Probst VS, Langer D, Decramer M, Gosselink R: Are patients with COPD more active after pulmonary rehabilitation? Chest 2008, 134:273-280.

133.van den Berg-Emons R, Balk A, Bussmann H, Stam H: Does aerobic training lead to a more active lifestyle and improved quality of life in patients with chronic heart failure? Eur J Heart Fail 2004, 6:95-100.

134. Mantoani LC, Rubio N, McKinstry B, MacNee W, Rabinovich RA: Interventions to modify physical activity in patients with COPD: a systematic review. Eur Respir J 2016, 48:69-81.

135. Viitasalo A, Eloranta AM, Lintu N, Vaisto J, Venalainen T, Kiiskinen S, Karjalainen P, Peltola J, Lampinen EK, Haapala EA, et al: The effects of a 2-year individualized and family-based lifestyle intervention on physical activity, sedentary behavior and diet in children. Prev Med 2016, 87:81-88.

136. Hu J, Wallace DC, McCoy TP, Amirehsani KA: A family-based diabetes intervention for Hispanic adults and their family members. Diabetes Educ 2014, 40:48-59. 


\section{CHAPTER 2}

What is the impact of impaired left ventricular ejection fraction in COPD after adjusting for confounders?

Rafael Mesquita, Frits M.E. Franssen, Sarah Houben-Wilke, Nicole H.M.K. Uszko-Lencer, Lowie E.G.W. Vanfleteren, Yvonne M.J. Goërtz, Fabio Pitta, Emiel F.M. Wouters, and Martijn A. Spruit

International Journal of Cardiology 2016; 225: 365-370 


\section{ABSTRACT}

Background: It remains unknown whether and to what extent impaired left ventricular ejection fraction (LVEF) affects physical and psychological status in COPD. We aimed to compare health outcome measures between COPD patients with and without impaired LVEF after adjusting for age, sex, BMI and $\mathrm{FEV}_{1}$.

Methods: Impaired LVEF was defined as values < 50\%. 85 COPD patients with impaired LVEF and 85 COPD patients with normal LVEF were matched for sex, age, BMI and FEV ${ }_{1}$. Exercise capacity, quadriceps muscle function, functional mobility, inflammatory status, health status, care dependency, and mood disorders were cross-sectionally assessed.

Results: Patients with impaired LVEF had shorter 6-minute walk distance (mean -59 (95\% confidence interval: -94, -25) m), lower symptom-limited peak oxygen uptake ($131(-268,7) \mathrm{ml} / \mathrm{min})$, weaker quadriceps muscles $(-12(-20,-3) \mathrm{Nm})$ and had more symptoms of anxiety $(+2(1,3)$ points $)$ and depression $(+1(0,2)$ points) than those with normal LVEF (all $\mathrm{P}<0.05)$. Health status was not statistically different between groups $(P>0.05)$.

Conclusions: Impaired LVEF has a clear impact on physical and psychological status in patients with COPD, even after adjusting for confounders. This reinforces the importance of assessing and treating cardiac problems in COPD. 


\section{INTRODUCTION}

Cardiac problems are common and associated with important morbidity and mortality in patients with chronic obstructive pulmonary disease (COPD) [1, 2]. Previous reports suggest a prevalence between 14 and $33 \%$ of cardiac diseases in patients with COPD [3]. Lower-limb muscle weakness, exercise intolerance, impaired body composition and reduced daily activity level can be found in patients with COPD or heart failure compared to healthy peers [4]. Therefore, it seems reasonable to hypothesize that the presence of cardiac problems in patients with COPD would have an additional negative impact on physical and psychological status. Indeed, COPD patients with a medical history of ischaemic heart disease were found to have worse quality of life, lower 6-minute walk distance, and more breathlessness than COPD patients without ischaemic heart disease [5].

Other studies have also suggested a negative impact of self-reported cardiac diseases on health outcome measures in patients with $\operatorname{COPD}[2,6,7]$. Nevertheless, the diagnosis of the cardiac condition in most of these studies was based on non-objective measures (e.g., medical history, self-reports), which is less accurate than echocardiography, and in some studies relevant characteristics differed between groups, which may explain the significant differences in exercise capacity $[2,6]$. To date, the additional impact of objectively assessed cardiac impairments, such as an impaired left ventricular ejection fraction (LVEF), on physical and psychological status in matched groups of patients with COPD has never been studied. Identifying and understanding the impact of cardiac impairments on COPD patients' physical and psychological status may contribute to a better management and treatment of patients with COPD.

The aim of this study was to compare physical and psychological status between COPD patients with and without impaired LVEF referred for pulmonary rehabilitation (PR). A priori, we hypothesised that COPD patients with impaired LVEF will have worse physical and psychological status compared with matched COPD patients with normal LVEF. Although this hypothesis may seem axiomatic, to the best of our knowledge no study has tested it before.

\section{METHOD}

\subsection{Study design and participants}

This is a cross-sectional analysis with data from the COPD, health status and comorbidities (Chance) study [8]. In brief, the Chance study was designed to investigate the impact of cardiovascular comorbidities on health status in patients with COPD. Eligibility 
criteria were: age 40-85 years, COPD diagnosis according to the Global initiative for chronic Obstructive Lung Disease (GOLD) guidelines [9], no recent exacerbation (previous 4 weeks) or condition influencing health status not related to symptoms of COPD, and referral for PR at CIRO, in Horn, the Netherlands [10]. Echocardiographic assessment was standardised and part of the study protocol [8], but only patients with a complete echocardiographic assessment were included in the current analyses. Of the 518 patients with COPD who volunteered to partake in the Chance study between April 2012 and September 2014, only four patients were excluded due to unreliable echocardiographic data. Among the 514 patients with complete and reliable echocardiographic data, 85 had impaired LVEF (i.e., LVEF <50\%). Of the remaining 429 patients, 85 COPD patients with a LVEF $\geq 50 \%$ were selected and pairwise-matched (1:1) for sex, age, body mass index (BMI) and forced expiratory volume in the first second $\left(F^{\prime} V_{1}\right)$ with the group of 85 COPD patients with impaired LVEF. COPD patients with normal LVEF were also free of other echocardiographic abnormalities, such as left ventricular hypertrophy and increased right ventricular (RV) systolic pressure. The Chance study was registered at the Dutch Trial Register (NTR 3416) and conforms to the ethical guidelines of the 1975 Declaration of Helsinki as reflected in a priori approval by the Medical Ethical Committee of the Maastricht University Medical Center + (MUMC+), Maastricht, the Netherlands (METC 11-3-070). Informed consent was obtained from each participant.

\subsection{Outcome measures}

Demographics, anthropometrics, clinical data, resting arterial blood gases, comorbidities (chart-based and objectively assessed), post-bronchodilator lung function, and physical and psychological health measures were assessed as part of the baseline assessment at CIRO [10]. Resting echocardiography was done as part of the Chance study [8]. For all details about each measurement, please see appendix A, supplementary data.

Physical health measures: Measures of exercise capacity, quadriceps muscle function, functional mobility, body composition, and inflammatory status were included. Functional exercise capacity was assessed with the 6-minute walk test performed twice; the best distance was used for analyses [11]. Maximal exercise capacity was assessed using a symptom-limited cardiopulmonary incremental cycle test [12]. Moreover, a constant work-rate cycling endurance test was performed at 75\% of the peak work rate [13]. Quadriceps muscle strength and endurance was assessed using a Biodex computerized dynamometer [14]. The Timed Up \& Go (TUG) test was used to assess functional mobility $[15,16]$. Body composition was assessed with fat-free mass (FFM) measured with whole-body dual-energy X-ray absorptiometry. In addition, fat-free mass index (FFMI) was calculated as FFM divided by squared height. Finally, serum C-reactive protein was 
measured in duplicate samples by high-sensitivity particle-enhanced immunoassay for the assessment of inflammatory status.

Psychological health measures: Measures of health status, care dependency, and symptoms of anxiety and depression were included. Health status was assessed with two COPD-specific valid and reliable instruments: the COPD Assessment Test (CAT; scores range from 0 (optimal) to 40 (worst) points) [17] and the COPD-specific version of the St. George's Respiratory Questionnaire (SGRQ-C; scores range from 0 (optimal) to 100 (worst) points) [18]. Care dependency was assessed with the care dependency scale (scores range from 15 (worst) to 75 (best) points) [19]. Symptoms of anxiety and depression were assessed using the Hospital Anxiety and Depression scale, consisting of 14 questions distributed in two subscales: 7 for symptoms of anxiety and 7 for symptoms of depression (scores in each subscale range from 0 (optimal) to 21 (worst) points) [20].

\subsection{Statistical analysis}

Normality in continuous data distribution was checked with the Shapiro-Wilk test. Data were expressed as mean \pm standard deviation (SD) or median (interquartile range), as appropriate. Categorical data were expressed as relative frequency. As the groups were matched, paired t-test or Wilcoxon test was used to compare patients with and without impaired LVEF. An unpaired t-test or Mann-Whitney test was used to compare patients without impaired LVEF who were included in this analysis with those who were not included. Categorical variables were compared by the chi-square or Fisher's exact test. Statistical significance was considered as $\mathrm{P}<0.05$ and the analyses were performed using SPSS 17.0 (SPSS, Chicago, Illinois, United States of America (USA)) or GraphPad Prism 5 (GraphPad Software, La Jolla, California, USA).

\section{RESULTS}

\subsection{General characteristics}

COPD severity in both groups ranged from mild to very severe, but most patients had moderate or severe disease (Table 1). The average resting blood gas values were normal. Patients with and without impaired LVEF were similar in terms of demographics, smoking status, proportion of long-term oxygen therapy users, exacerbation history, and lung function, except for lung diffusing capacity which was worse in patients with impaired LVEF (Table 1). The degree of static hyperinflation (based on the ratio between residual volume and total lung capacity) was also similar between groups. COPD patients with impaired LVEF had worse renal function (Table 1) and higher levels of $\mathrm{N}$ - 


\section{CHAPTER 2}

terminal pro-B-type natriuretic peptide, worse overall cardiac function, reported more often myocardial infarct and congestive heart failure, and were using more often cardiac medication compared to patients with normal LVEF (Table 2). Furthermore, patients with impaired LVEF had more anaemia and symptoms of anxiety than patients with normal LVEF (Figure 1), as well as a worse Charlson index score $(2(1-3)$ versus $1(1-2)$ points, respectively; $\mathrm{P}<0.0001)$. Compared with patients with normal LVEF who were not included in the current study (i.e., remaining patients of the Chance study; $n=344$ ), patients with normal LVEF who were included in the current analyses were more often male and used more inhaled corticosteroids and less inhaled corticosteroid + longacting b2-agonist $(\mathrm{P}<0.05$ for all comparisons; Table A.2.1 in appendix A, supplementary data). Other demographic and lung function characteristics were similar between these groups. 
Table 1 Demographics, clinical data, and lung function in COPD patients with and without impaired LVEF

\begin{tabular}{|c|c|c|c|c|c|}
\hline \multirow[t]{2}{*}{ Characteristic } & \multicolumn{2}{|r|}{$\begin{array}{c}\text { COPD with } \\
\text { normal LVEF }\end{array}$} & \multicolumn{2}{|r|}{$\begin{array}{l}\text { COPD with } \\
\text { impaired LVEF }\end{array}$} & \multirow[t]{2}{*}{ P-value } \\
\hline & $\mathrm{n}$ & & $\mathrm{n}$ & & \\
\hline Age, years & 85 & $65(58-72)$ & 85 & $68(60-74)$ & 0.10 \\
\hline Male, \% & 85 & 77 & 85 & 77 & 1.00 \\
\hline Smoking status N/F/C, \% & 85 & $1 / 82 / 17$ & 85 & $1 / 82 / 17$ & 1.00 \\
\hline Long-term oxygen therapy, \% & 85 & 18 & 85 & 22 & 0.44 \\
\hline Exacerbation history ( $\geq 2$ previous year), $\%$ & 85 & 48 & 85 & 55 & 0.36 \\
\hline $\mathrm{BMI}, \mathrm{kg} \cdot \mathrm{m}^{-2}$ & 85 & $25.6(22.0-28.7)$ & 85 & $26.2(21.5-30.0)$ & 0.43 \\
\hline $\mathrm{FEV}_{1}, \%$ predicted & 85 & $45(33-58)$ & 85 & $46(32-62)$ & 0.83 \\
\hline GOLD $1 / 2 / 3 / 4, \%$ & 85 & $8 / 27 / 45 / 20$ & 85 & $11 / 35 / 36 / 18$ & 0.26 \\
\hline GOLD A/B/C/D, \% & 85 & $5 / 18 / 3 / 74$ & 81 & $2 / 21 / 5 / 72$ & 0.92 \\
\hline $\mathrm{RV}, \%$ predicted & 82 & $149(118-195)$ & 80 & $145(111-185)$ & 0.33 \\
\hline TLC, \% predicted & 82 & $117(103-125)$ & 80 & $113(99-125)$ & 0.18 \\
\hline $\mathrm{RV} / \mathrm{TLC}, \%$ & 82 & $50 \pm 11$ & 80 & $51 \pm 13$ & 0.87 \\
\hline$D_{\text {Lco, }} \%$ predicted & 78 & $50(39-63)$ & 77 & $45(36-55)$ & $0.04^{*}$ \\
\hline $\mathrm{Hb}, \mathrm{mmol} / \mathrm{L}$ & 84 & $9.0 \pm 0.7$ & 83 & $8.8 \pm 0.9$ & 0.10 \\
\hline $\mathrm{eGFR}, \mathrm{ml} / \mathrm{min}$ & 85 & $74(60-89)$ & 84 & $66(51-85)$ & $0.03^{*}$ \\
\hline \multicolumn{6}{|l|}{ Arterial blood gases } \\
\hline $\mathrm{PaO}_{2}, \mathrm{kPa}$ & 84 & $9.8(8.8-10.6)$ & 83 & $9.8(8.9-10.5)$ & 0.56 \\
\hline $\mathrm{PaCO}_{2}, \mathrm{kPa}$ & 85 & $5.2(4.8-5.6)$ & 84 & $5.0(4.6-5.5)$ & 0.08 \\
\hline \multicolumn{6}{|l|}{ Lung medication } \\
\hline SABA, $\%$ & 85 & 39 & 85 & 44 & 0.53 \\
\hline LAMA, \% & 85 & 65 & 85 & 75 & 0.13 \\
\hline ICS, \% & 85 & 27 & 85 & 22 & 0.48 \\
\hline Combination ICS/LABA, \% & 85 & 59 & 85 & 66 & 0.34 \\
\hline
\end{tabular}

Data expressed as relative frequency, mean \pm SD or median (IQR). N: never smoker; F: former smoker; C: current smoker; BMI: body mass index; $\mathrm{FEV}_{1}$ : forced expiratory volume in the first second; GOLD: global initiative for chronic obstructive lung disease; RV: residual volume; TLC: total lung capacity; $D_{\text {Lco: diffusing }}$ capacity for carbon monoxide; $\mathrm{Hb}$ : Haemoglobin; eGFR: estimated glomerular filtration rate; $\mathrm{PaO}_{2}$ : resting arterial oxygen tension; $\mathrm{PaCO}_{2}$ : resting arterial carbon dioxide tension; $\mathrm{kPa}$ : kilopascal; $\mathrm{SABA}$ : short-acting $\beta 2$ agonist; LAMA: long-acting muscarinic antagonist; ICS: inhaled corticosteroid; LABA: long-acting b2-agonist. ": $\mathrm{P}$-value $<0.05$. 
Table 2 Cardiovascular status in COPD patients with and without impaired LVEF

\begin{tabular}{|c|c|c|c|c|c|}
\hline \multirow{2}{*}{ Characteristic } & \multicolumn{2}{|c|}{ COPD with normal LVEF } & \multicolumn{2}{|c|}{ COPD with impaired LVEF } & \multirow{2}{*}{ P-value } \\
\hline & $\mathrm{n}$ & & $\mathrm{n}$ & & \\
\hline Systolic blood pressure, $\mathrm{mm} \mathrm{Hg}$ & 82 & $141 \pm 24$ & 81 & $136 \pm 28$ & 0.21 \\
\hline Diastolic blood pressure, $\mathrm{mm} \mathrm{Hg}$ & 82 & $80(70-90)$ & 81 & $80(69-86)$ & 0.05 \\
\hline History of myocardial infarct, \% & 85 & 8 & 85 & 28 & $0.001^{*}$ \\
\hline History of congestive heart failure, $\%$ & 85 & 0 & 85 & 17 & $<0.0001^{*}$ \\
\hline NT-proBNP, pg/mL & 79 & $82.2(43.2-161.9)$ & 79 & $361.9(133.1-1288.0)$ & $<0.0001^{*}$ \\
\hline NYHA I/II/III/IV, \% & 69 & $7 / 52 / 36 / 5$ & 73 & $8 / 38 / 43 / 11$ & 0.14 \\
\hline \multicolumn{6}{|l|}{ Cardiac medication } \\
\hline ACE-I/ARB, \% & 85 & 17 & 85 & 37 & $0.003^{*}$ \\
\hline$\beta$-blockers, \% & 85 & 13 & 85 & 35 & $0.001^{*}$ \\
\hline Diuretics, \% & 85 & 12 & 85 & 35 & $<0.0001^{*}$ \\
\hline \multicolumn{6}{|l|}{ Echocardiographic findings } \\
\hline LVEF, \% & 85 & $63(60-66)$ & 85 & $45(35-49.5)$ & $<0.0001^{*}$ \\
\hline LA dimension, $\mathrm{mm}$ & 84 & $34.5 \pm 4.7$ & 83 & $39.6 \pm 8.1$ & $<0.0001^{*}$ \\
\hline LA dimension $>40 \mathrm{~mm}, \%$ & 84 & 9 & 83 & 47 & $<0.0001^{*}$ \\
\hline LVEDD, $\mathrm{mm}$ & 85 & $42 \pm 4$ & 85 & $49 \pm 8$ & $<0.0001^{*}$ \\
\hline LVESD, mm & 85 & $28(26-30)$ & 85 & $39(33-43)$ & $<0.0001^{*}$ \\
\hline E-wave/A-wave & 82 & $0.8(0.7-0.9)$ & 65 & $0.7(0.6-0.8)$ & $<0.0001^{*}$ \\
\hline Deceleration time of the E-wave, ms & 53 & $192 \pm 30$ & 49 & $196 \pm 40$ & 0.61 \\
\hline IVS diameter, mm & 85 & $8(8-9)$ & 84 & $9(8-10)$ & $<0.0001^{*}$ \\
\hline $\mathrm{RV}$ diameter, $\mathrm{mm}$ & 79 & $35(32-39)$ & 80 & $36(33-39)$ & 0.24 \\
\hline RV diameter $>42$ mm, \% & 79 & 3 & 80 & 11 & $0.03^{*}$ \\
\hline RVSP, $\mathrm{mm} \mathrm{Hg}$ & 66 & $30(25-31)$ & 63 & $30(25-38)$ & 0.07 \\
\hline TR velocity, $\mathrm{m} / \mathrm{s}$ & 66 & $2.7(2.5-2.8)$ & 63 & $2.7(2.5-3.1)$ & 0.14 \\
\hline TAPSE, mm & 83 & $23(21-25)$ & 82 & $21(17-23)$ & $<0.0001^{*}$ \\
\hline TAPSE $<16 \mathrm{~mm}, \%$ & 83 & 0 & 82 & 11 & 0.001 \\
\hline Valvular abnormalities, \% & 76 & 1 & 78 & 19 & $<0.0001^{*}$ \\
\hline Wall motion abnormalities, $\%$ & 81 & 0 & 75 & 81 & $<0.0001^{*}$ \\
\hline
\end{tabular}

Data expressed as relative frequency, mean \pm SD or median (IQR). NT-proBNP: N-terminal pro-B-type natriuretic peptide; NYHA: New York Heart Association; ACE-I/ARB: angiotensin-converting enzyme inhibitor/angiotensin receptor blocker; LVEF: left ventricular ejection fraction; LA: left atrium; LVEDD: left ventricular end-diastolic dimension; LVESD: left ventricular end-systolic dimension; IVS: interventricular septum; RV: right ventricular; RVSP: right ventricular systolic pressure; TR: tricuspid regurgitation; TAPSE: tricuspid annular plane systolic excursion. : : P-value $<0.05$. 


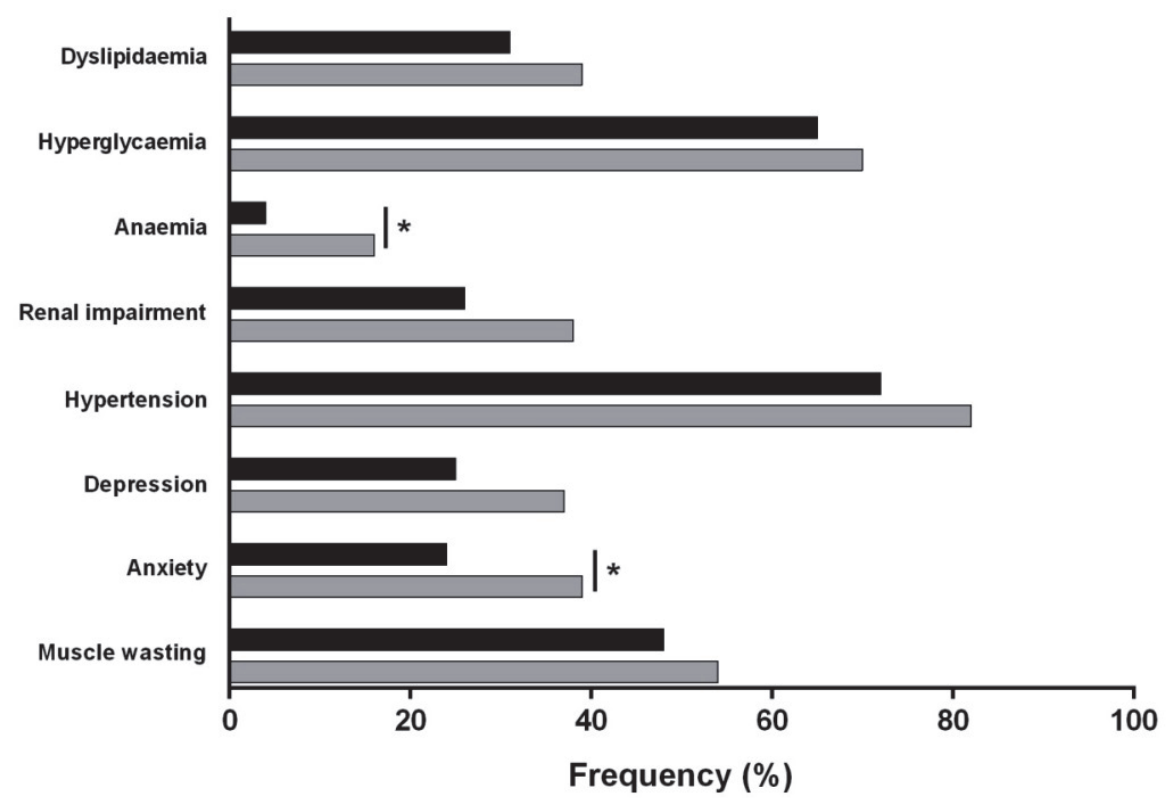

Figure 1 Objectively assessed comorbidities in COPD patients with or without impaired LVEF (grey and black columns, respectively). ${ }^{*}: \mathrm{P} \leq 0.05$.

\subsection{Physical health measures}

Functional and maximal exercise capacity was worst in patients with impaired LVEF (Table 3). The mean difference in 6-minute walk distance (6MWD) between groups (-59 $(95 \% \mathrm{Cl}-94,-25) \mathrm{m}$ ) exceeded the clinically relevant cut-off of $30 \mathrm{~m} \mathrm{[11]} \mathrm{and} \mathrm{the} \mathrm{pro-}$ portion of patients with a 6MWD $<350 \mathrm{~m}$ was significantly higher in the impaired LVEF group ( $36 \%$ vs. $9 \%, \mathrm{P}<0.0001$ ). Borg dyspnoea scores, $\mathrm{SpO}_{2}$, and peak cardiovascular and ventilatory responses during the maximal exercise capacity test were similar between groups (Table 3). Quadriceps peak torque and total work were worst in patients with impaired LVEF (Table 3). TUG time was also worst (i.e., longer) in patients with impaired LVEF, and more patients in this group had an abnormal TUG time (i.e., $>11 \mathrm{~s}$ ) [16]. Similar C-reactive protein levels were found between groups (Table 3). Furthermore, similar FFM (median (IQR) 53.0 (44.0 - 57.1) vs. 50.1 (43.5 - 56.0) kg, P=0.64) and FFMI (mean \pm SD $17.4 \pm 2.1$ vs. $17.3 \pm 2.4 \mathrm{~kg} \cdot \mathrm{m}-2, \mathrm{P}=0.76$ ) were also found between patients with normal LVEF and those with impaired LVEF, respectively, and there was no difference in the proportion of patients with a FFMI $<5^{\text {th }}$ percentile of reference values [21] (48\% vs. 54\%, respectively; $P=0.39)$. 


\section{CHAPTER 2}

Table 3 Exercise capacity, quadriceps muscle function, functional mobility, and inflammatory status in COPD patients with and without impaired LVEF

\begin{tabular}{|c|c|c|c|c|}
\hline \multirow[t]{2}{*}{ Characteristic } & & $\begin{array}{l}\text { COPD with } \\
\text { normal LVEF }\end{array}$ & $\begin{array}{c}\text { COPD with } \\
\text { impaired LVEF }\end{array}$ & \multirow[t]{2}{*}{ P-value } \\
\hline & $\mathrm{n}$ & & $\mathrm{n}$ & \\
\hline
\end{tabular}

\section{Exercise capacity}

6-minute walk test

6MWD, $\mathrm{m}$

$\begin{array}{lcccc}85 & 456 \pm 96 & 84 & 397 \pm 137 & 0.0009^{*} \\ 85 & 88(84-93) & 84 & 90(86-93) & 0.08 \\ 85 & 5(4-7) & 84 & 6(4-7) & 0.08 \\ 85 & 4(3-7) & 84 & 4(3-7) & 0.05 \\ 85 & 19 & 84 & 37 & 0.009^{*}\end{array}$

$\mathrm{SpO}_{2}$ post, \%

Borg dyspnoea post, points

Borg leg fatigue post, points

Rollator, \%

$$
67(51-95) \quad 80
$$

$60(41-84)$

$0.04^{*}$

Peak $\mathrm{VO}_{2}, \mathrm{ml} / \mathrm{min}$

68

$1083(892-1311) \quad 65$

$914(683-1256)$

$0.04^{*}$

HR post, bpm

84

$119(109-132)$

$120(106-137)$

0.90

Peak VE, \% MVV

67

$$
87 \pm 26
$$

65

$$
80 \pm 23
$$

$\mathrm{SpO}_{2}$ post, \%

83

$$
91(88-94) \quad 75
$$

$$
93(90-95)
$$

84

$$
7(7-9)
$$$$
7(5-9)
$$

84

$$
5(3-7)
$$$$
7(4-8)
$$

$0.02^{*}$

Constant work rate test

Cycle time, $\mathrm{s}$

82

82

$289(184-370)$

76

$207(165-314)$

0.08

81

$$
93(91-95)
$$

75

$93(91-95)$

0.94

Borg dyspnoea post, points

80

$7(6-9)$

$8(7-9)$

0.43

80

$7(4-9)$

$7(5-8)$

0.14

Quadriceps muscle function

Peak torque, $\mathrm{Nm}$

77

$$
107 \pm 33
$$

76

$96 \pm 37$

$0.009^{*}$

Total work, Joules

76

$1868 \pm 682$

$1620 \pm 737$

$0.006^{*}$

Functional mobility

TUG time, $\mathrm{s}$

$\begin{array}{ccccc}83 & 9.4(8.2-10.8) & 81 & 10.8(8.8-13.0) & 0.002^{*} \\ 83 & 20 & 81 & 44 & 0.001^{*}\end{array}$

TUG time >11 s [16], \%

Inflammatory status

CRP, mg/L

$85 \quad 2.75(0.88-7.44)$

$84 \quad 2.99(1.00-9.42)$

0.33

Data expressed as relative frequency, mean $\pm \mathrm{SD}$, or median (IQR). 6MWD: 6-minute walk distance; $\mathrm{SpO}_{2}$ : oxyhaemoglobin saturation; $\mathrm{VO}_{2}$ : oxygen uptake; HR: heart rate; VE: minute ventilation; MVV: estimated maximal voluntary ventilation; Nm: Newton-metre; TUG: timed up \& go; CRP: C-reactive protein. ${ }^{*}$ : P-value $<0.05$. 


\subsection{Psychological health measures}

Mean scores on the CAT and SGRQ-C were not statistically different between groups (Table 4). Care dependency and the proportion of care dependent patients (i.e., those with a score $\leq 68$ points [22]) were also similar between groups (Table 4). COPD patients with impaired LVEF had more symptoms of anxiety and depression than those with normal LVEF (Table 4).

Table 4 Health status, care dependency, and symptoms of anxiety and depression in COPD patients with and without impaired LVEF

\begin{tabular}{|c|c|c|c|c|c|}
\hline \multirow[t]{2}{*}{ Characteristic } & \multicolumn{2}{|r|}{$\begin{array}{l}\text { COPD with } \\
\text { normal LVEF }\end{array}$} & \multicolumn{2}{|r|}{$\begin{array}{c}\text { COPD with } \\
\text { impaired LVEF }\end{array}$} & \multirow[t]{2}{*}{ P-value } \\
\hline & $\mathrm{n}$ & & $\mathrm{n}$ & & \\
\hline \multicolumn{6}{|l|}{ Health status } \\
\hline CAT, points & 85 & $22(17-26)$ & 81 & $22(18-27)$ & 0.33 \\
\hline SGRQ-C symptoms, points & 85 & $58.9 \pm 20.3$ & 80 & $63.3 \pm 18.4$ & 0.09 \\
\hline SGRQ-C activity, points & 85 & $83.6(66.5-91.8)$ & 80 & $84.2(75.5-100)$ & 0.09 \\
\hline SGRQ-C impact, points & 85 & $46.6 \pm 21.3$ & 80 & $51.4 \pm 21.3$ & 0.15 \\
\hline SGRQ-C total score, points & 85 & $59.6(45.5-74.0)$ & 80 & $64.8(52.9-74.0)$ & 0.06 \\
\hline \multicolumn{6}{|l|}{ Care dependency } \\
\hline CDS score, points & 81 & $73(70-75)$ & 80 & $72(67-74)$ & 0.10 \\
\hline CDS score $\leq 68$ points, $\%$ & 81 & 21 & 80 & 31 & 0.14 \\
\hline \multicolumn{6}{|c|}{ Symptoms of anxiety and depression } \\
\hline HADS Anxiety, points & 84 & $6(4-9)$ & 79 & $8(5-12)$ & $0.007^{*}$ \\
\hline HADS Depression, points & 84 & $7(4-10)$ & 79 & $7(5-12)$ & $0.02^{*}$ \\
\hline
\end{tabular}

Data expressed as relative frequency, mean $\pm \mathrm{SD}$, or median (IQR). CAT: COPD assessment test; SGRQ-C: COPD-specific version of the St. George's respiratory questionnaire; CDS: care dependency scale; HADS: hospital anxiety and depression scale. ${ }^{*}$ : P-value $<0.05$.

\section{DISCUSSION}

We demonstrated for the first time that COPD patients with impaired LVEF have worse exercise capacity, quadriceps muscle function, and functional mobility than matched COPD patients with normal LVEF. Moreover, patients with impaired LVEF had more symptoms of anxiety and depression than patients with normal LVEF. These findings have clinical importance for the management of COPD patients, especially when considering the high prevalence of cardiac problems among these patients [1].

Cardiovascular comorbidities are associated with high mortality in patients with COPD, even in the early stages of the disease [23]. Similarly, poor exercise capacity is also asso- 
ciated with high mortality in COPD [24]. In our study patients with COPD and impaired LVEF were found to have impaired functional and maximal exercise capacity, which suggests that these patients may have a very poor prognosis. Even a simple functional test such as the TUG test was able to demonstrate the impact of an impaired LVEF, with patients with impaired LVEF showing the longest TUG time and having more often a TUG time higher than $11 \mathrm{~s}$, which has been associated with worse health outcome measures [16]. Low peak oxygen uptake $\left(\mathrm{VO}_{2}\right)$ during the maximal exercise test was also found in COPD patients with impaired LVEF, but this does not seem to be a consequence of poor effort during the test since both groups responded similarly in terms of heart rate, ventilation, and perceived dyspnoea and fatigue.

Oliveira et al. [7] recently observed lower cycle endurance time in COPD patients with heart failure compared to age-, sex-, and FEV - $_{1}$ atched COPD patients without heart failure. Although the cycle time in our study was not statistically different between groups (Table 3), the $\mathrm{P}$-value for this comparison approached significance (i.e., $\mathrm{P}=0.08$ ) and the difference between groups was similar to the one found by Oliveira and colleagues (i.e., $30 \%$ lower in COPD with heart failure). Reduced cardiac output and muscle blood flow in COPD patients with heart failure was also observed by these authors [7]. Unfortunately, these outcomes were not investigated in our study but they might have contributed to the differences in exercise tolerance observed in our study. Nevertheless, the influence of other potential factors cannot be excluded. Systemic inflammation could also be suspected as a cause of worse exercise capacity [25]. Nevertheless, similar levels of C-reactive protein were found between the groups. Miller et al. [2] found results similar between COPD patients with and without cardiac problems, but they also found significant differences in other inflammatory markers. This suggests that one inflammatory marker is probably not enough when investigating systemic inflammation in COPD patients with cardiac comorbidities.

COPD patients with impaired LVEF presented with worse lung diffusing capacity compared to those with normal LVEF. Reduced diffusing capacity can be found in COPD patients mainly due to a loss of alveoli (i.e., emphysema), which results in a lower surface area for diffusion [26]. Cardiac diseases are also known to cause reduced diffusing capacity mainly through an increase in lung capillary pressures and fluids in the interstitial and/or alveolar spaces [27]. Both factors may have contributed to a reduced diffusing capacity in COPD patients with impaired LVEF. Nevertheless, since the degree of static hyperinflation was similar between groups, we believe the latter factor may have played a more important role. Patients with COPD have shown to present with RV dysfunction [28], which associates with worse survival [29]. We observed a higher proportion of patients with RV enlargement ( $11 \%$ vs. 3\%, $\mathrm{P}=0.03$ ) and reduced TAPSE - suggestive of RV systolic dysfunction - (11\% vs. $0 \%, \mathrm{P}=0.001)$ among those with impaired LVEF. Although one could hypothesise a possible influence of RV abnormalities on LV function, 
this cannot be confirmed due to the lack of other parameters for a proper evaluation of RV dimensions and function [30].

In our study we observed that not only quadriceps muscle strength but also quadriceps muscle endurance was reduced in COPD patients with impaired LVEF, which possibly also contributes to worse exercise capacity. Corroborating this hypothesis, Gosker et al. [4] observed significant correlations between quadriceps muscle strength and peak $\mathrm{VO}_{2}$ in both COPD and heart failure patients. Several structural changes of the quadriceps can contribute to worse quadriceps function in COPD, such as mitochondrial dysfunction, poor oxidative capacity and shift in fiber-type [31]. However, to date it is not clear whether these factors are more pronounced in COPD patients with impaired LVEF or not. Important to mention, to the best of our knowledge this is the first study to investigate quadriceps muscle function in matched COPD patients with and without objectively assessed cardiac impairments.

Despite impairments in exercise capacity, functional mobility and quadriceps muscle function, COPD patients with and without impaired LVEF had statistically similar health status, contradicting previous findings $[2,5]$. Nevertheless, the mean difference between groups in the SGRQ-C total score (i.e., $-4.7(95 \% \mathrm{Cl}-0.5,9.8)$ points) seems to be clinically relevant as it exceeded -4 points [32], but the p-value just did not reached statistical significance ( $P=0.06)$. In the study of Miller et al. [2] COPD patients with cardiac problems had a statistically higher SGRQ-C total score (i.e., worse health status) than those without cardiac problems, and the difference between groups (i.e., -4.6 points) was similar to the one found in our study. Nevertheless, their sample size was much larger than ours, which may help explain the lack of statistical difference in our analyses. Additionally, in our study we used COPD-specific instruments for the assessment of health status, which focus mainly on the impact of respiratory symptoms on daily life activities. Maybe generic instruments or even cardiac-specific instruments could have shown a more pronounced difference between groups. These hypotheses have to be tested in future studies. Despite having statistically similar health status, COPD patients with impaired LVEF had more symptoms of anxiety and depression than COPD patients with normal LVEF. These findings are of importance since psychological factors have been associated with high mortality in patients with COPD [33].

Interestingly, we observed that only less than half of the patients with impaired LVEF were on treatment with cardiac medications, which may suggest that cardiac diseases were underdiagnosed among these patients. Moreover, considering all the above mentioned deficits related to an impaired LVEF in patients with COPD we believe that interventions such as PR might be particularly beneficial in this subgroup of patients. Cardiovascular comorbidities do not seem to preclude COPD patients from obtaining significant and clinically meaningful improvements after PR [34]. Indeed, we believe that 
COPD patients with cardiovascular comorbidities might benefit even more from PR since exercise - the cornerstone of PR -, is also an important component of cardiac rehabilitation [35].

Strengths of this study include matching the groups for factors that could influence the results, such as sex, age, $\mathrm{BMI}$, and $\mathrm{FEV}_{1}$. In previous studies the groups were not matched $[2,6]$, which could have influenced some of the analyses. Another strength is that we used an objective measure for the assessment of cardiac function, namely echocardiography. This is particularly important as recent findings have shown poor agreement between chart-based or self-reported comorbidities and objectively assessed comorbidities [36]. Moreover, in our study we also included different physical and psychological health outcome measures, which allowed us to have a more comprehensive view of the physical and psychological status of the patients.

\subsection{Study limitations}

Our findings need to be interpreted in light of the limitations. Firstly, the lack of longitudinal measurements prevents us from further explaining the impact of impaired LVEF in patients with COPD. Secondly, a proper diagnosis of heart failure could not be established as symptoms of heart failure were not systematically investigated. Nevertheless, since patients with impaired LVEF were more often using cardiac medication and had higher levels of $\mathrm{N}$-terminal pro-B-type natriuretic peptide, we believe they may represent the population of COPD patients with heart failure. Another limitation is that the potential impact of heart failure with preserved ejection fraction was not investigated. The patients in our study were recruited at a tertiary care pulmonary rehabilitation setting as part of the Chance study [8], which could have compromised the external validity of our findings. Nevertheless, comparable characteristics, such as age, BMI and $\mathrm{FEV}_{1}$, were observed when we compared the patients included in our study with those from pulmonary rehabilitation studies from different parts of the world [37-39]. Finally, the number of multiple comparison tests performed can be viewed as a limitation. Nonetheless, multiple findings in the same direction rather than a single statistically significant result are suggestive that these are not due to chance alone.

\section{CONCLUSIONS}

In summary, this study demonstrates that an impaired LVEF has a clear negative impact on physical and psychological status in patients with COPD, even after matching for sex, age, $\mathrm{BMI}$ and $\mathrm{FEV}_{1}$. This reinforces the importance of assessing and treating cardiac comorbidities in COPD. 


\section{REFERENCES}

1. N.M. Hawkins, M.C. Petrie, P.S. Jhund, G.W. Chalmers, F.G. Dunn, J.J. McMurray, Heart failure and chronic obstructive pulmonary disease: diagnostic pitfalls and epidemiology, Eur. J. Heart Fail. 11 (2) (2009) 130139.

2. J. Miller, L.D. Edwards, A. Agusti, P. Bakke, P.M. Calverley, B. Celli, H.O. Coxson, C. Crim, D.A. Lomas, B.E. Miller, S. Rennard, E.K. Silverman, R. Tal-Singer, J. Vestbo, E. Wouters, J.C. Yates, W. Macnee, Evaluation of COPD longitudinally to identify predictive surrogate endpoints (ECLIPSE) investigators. Comorbidity, systemic inflammation and outcomes in the ECLIPSE cohort, Respir. Med. 107 (9) (2013) 1376-1384.

3. D.W. Mapel, D. Dedrick, K. Davis, Trends and cardiovascular co-morbidities of COPD patients in the Veterans Administration Medical System, 1991-1999, COPD 2 (1) (2005) 35-41.

4. H.R. Gosker, N.H. Lencer, F.M. Franssen, G.J. van der Vusse, E.F. Wouters, A.M. Schols, Striking similarities in systemic factors contributing to decreased exercise capacity in patients with severe chronic heart failure or COPD, Chest 123 (5) (2003) 1416-1424.

5. A.R. Patel, G.C. Donaldson, A.J. Mackay, J.A. Wedzicha, J.R. Hurst, The impact of ischemic heart disease on symptoms, health status, and exacerbations in patients with COPD, Chest 141 (4) (2012) 851-857.

6. N. Putcha, H. MK, M. CH, F. MG, A. AR, R. Casaburi, C. MH, H. NA, H. CP, K. GL, M. BJ, S. RM, L. SM, T. BM, W. AA, B. SP, B. TH, B. RP, R. JW, C. JL, D. Everett, H. JE, L. DA, S. ER, S. EK, C. JD, W. RA, R. EA, H. NN, the COPDGene ${ }^{\circledR}$ Investigators. Comorbidities of COPD have a major impact on clinical outcomes, particularly in African Americans, Chron. Obstruct. Pulmon. Dis. 1 (1) (2014) 105-114.

7. M.F. Oliveira, A. FF, A. MC, A. Souza, S. PA, M. WM, A. Mazzuco, A. Borghi-Silva, M. LA, R. Santos, H. DM, F. Mancuso, D. Almeida, O.'.D. DE, N. JA, Heart failure impairs muscle blood flow and endurance exercise tolerance in COPD, COPD 13 (4) (2016) 407-415.

8. D.E. Smid, S. Wilke, P.W. Jones, J.W. Muris, E.F. Wouters, F.M. Franssen, M.A. Spruit, Impact of cardiovascular comorbidities on COPD assessment test (CAT) and its responsiveness to pulmonary rehabilitation in patients with moderate to very severe COPD: protocol of the chance study, BMJ Open 5 (7) (2015) e007536.

9. J. Vestbo, S.S. Hurd, A.G. Agusti, P.W. Jones, C. Vogelmeier, A. Anzueto, P.J. Barnes, L.M. Fabbri, F.J. Martinez, M. Nishimura, R.A. Stockley, D.D. Sin, R. Rodriguez-Roisin, Global strategy for the diagnosis, management, and prevention of chronic obstructive pulmonary disease: GOLD executive summary, Am. J. Respir. Crit. Care Med. 187 (4) (2013) 347-365.

10. M.A. Spruit, I. Vanderhoven-Augustin, P.P. Janssen, E.F. Wouters, Integration of pulmonary rehabilitation in COPD, Lancet 371 (9606) (2008) 12-13.

11. A.E. Holland, M.A. Spruit, T. Troosters, M.A. Puhan, V. Pepin, D. Saey, M.C. McCormack, B.W. Carlin, F.C. Sciurba, F. Pitta, J.Wanger, N.Macintyre, D.A. Kaminsky, B.H. Culver, S.M. Revill, N.A. Hernandes, V. Andrianopoulos, C.A. Camillo, K.E. Mitchell, A.L. Lee, C.J. Hill, S.J. Singh, An official European Respiratory Society/American Thoracic Society Technical Standard: field walking tests in chronic respiratory disease, Eur. Respir. J. 44 (6) (2014) 1428-1446.

12. ATS/ACCP Statement on cardiopulmonary exercise testing, Am. J. Respir. Crit. Care Med. 167 (2) (2003) 211-277.

13. A. van 't Hul, R. Gosselink, G. Kwakkel, Constant-load cycle endurance performance: test-retest reliability and validity in patientswith COPD, J. Cardpulm. Rehabil. 23 (2) (2003) 143-150.

14. M.J. Sillen, F.M. Franssen, J.M. Delbressine, N.H. Uszko-Lencer, L.E. Vanfleteren, E.P. Rutten, E.F. Wouters, M.A. Spruit, Heterogeneity in clinical characteristics and comorbidities in dyspneic individuals with COPD GOLD D: findings of the DICES trial, Respir.Med. 107 (8) (2013) 1186-1194.

15. R. Mesquita, D.J. Janssen, E.F. Wouters, J.M. Schols, F. Pitta, M.A. Spruit, Within-day test-retest reliability of the timed up \& go test in patients with advanced chronic organ failure, Arch. Phys. Med. Rehabil. 94 (11) (2013) 2131-2138. 
16. R. Mesquita, S. Wilke, D.E. Smid, D.J. Janssen, F.M. Franssen, V.S. Probst, E.F. Wouters, J.W. Muris, F. Pitta, M.A. Spruit, Measurement properties of the timed up \& go test in patients with COPD, Chron. Respir. Dis. (2016), http://dx.doi.org/10.1177/1479972316647178.

17. P.W. Jones, G. Harding, P. Berry, I. Wiklund, W.H. Chen, L.N. Kline, Development and first validation of the COPD assessment test, Eur. Respir. J. 34 (3) (2009) 648-654.

18. M. Meguro, E.A. Barley, S. Spencer, P.W. Jones, Development and validation of an improved, COPDspecific version of the St. George Respiratory Questionnaire, Chest 132 (2) (2007) 456-463.

19. A. Dijkstra, L.J. Tiesinga, W.T. Goossen, T.W. Dassen, Further psychometric testing of theDutch care dependency scale on two different patient groups, Int. J.Nurs. Pract. 8 (6) (2002) 305-314.

20. A.S. Zigmond, R.P. Snaith, The hospital anxiety and depression scale, Acta Psychiatr. Scand. 67 (6) (1983) 361-370.

21. F.M. Franssen, E.P. Rutten, M.T. Groenen, L.E. Vanfleteren, E.F. Wouters, M.A. Spruit, New reference values for body composition by bioelectrical impedance analysis in the general population: results from the UK Biobank, J. Am. Med. Dir. Assoc. 15 (6) (2014) (448 e441-446).

22. D.J. Janssen, E.F. Wouters, J.M. Schols, M.A. Spruit, Care dependency independently predicts two-year survival in outpatients with advanced chronic organ failure, J. Am. Med. Dir. Assoc. 14 (3) (2013) 194-198.

23. C.E. Berry, R.A. Wise, Mortality in COPD: causes, risk factors, and prevention, COPD, 7 (5) (2010) 375-382.

24. M.A. Spruit, M.I. Polkey, B. Celli, L.D. Edwards, M.L. Watkins, V. Pinto-Plata, J. Vestbo, P.M. Calverley, R. Tal-Singer, A. Agusti, H.O. Coxson, D.A. Lomas, W. MacNee, S. Rennard, E.K. Silverman, C.C. Crim, J. Yates, E.F. Wouters, Predicting outcomes from 6-minute walk distance in chronic obstructive pulmonary disease, J. Am. Med. Dir. Assoc. 13 (3) (2012) 291-297.

25. P.J. Barnes, B.R. Celli, Systemic manifestations and comorbidities of COPD, Eur. Respir. J. 33 (5) (2009) 1165-1185.

26. K.L. Bailey, The importance of the assessment of pulmonary function in COPD, Med. Clin North Am. 96 (4) (2012) 745-752.

27. J.L. Siegel, A. Miller, L.K. Brown, A. DeLuca, A.S. Teirstein, Pulmonary diffusing capacity in left ventricular dysfunction, Chest 98 (3) (1990) 550-553.

28. R. Sabit, C.E. Bolton, A.G. Fraser, J.M. Edwards, P.H. Edwards, A.A. Ionescu, J.R. Cockcroft, D.J. Shale, Subclinical left and right ventricular dysfunction in patients with COPD, Respir. Med. 104 (8) (2010) 11711178 .

29. M.I. Burgess, N. Mogulkoc, R.J. Bright-Thomas, P. Bishop, J.J. Egan, S.G. Ray, Comparison of echocardiographic markers of right ventricular function in determining prognosis in chronic pulmonary disease, J. Am. Soc. Echocardiogr. 15 (6) (2002) 633-639.

30. L.G. Rudski, W.W. Lai, J. Afilalo, L. Hua, M.D. Handschumacher, K. Chandrasekaran, S.D. Solomon, E.K. Louie, S. NB, Guidelines for the echocardiographic assessment of the right heart in adults: a report from the American Society of Echocardiography endorsed by the European Association of Echocardiography, a registered branch of the European Society of Cardiology, and the Canadian Society of Echocardiography, J. Am. Soc. Echocardiogr. 23 (7) (2010) 685-713 (quiz 786-688).

31. F. Maltais, M. Decramer, R. Casaburi, E. Barreiro, Y. Burelle, R. Debigare, D. PN, F. Franssen, G. GayanRamirez, J. Gea, G. HR, R. Gosselink, M. Hayot, H. SN, W. Janssens, P. MI, J. Roca, D. Saey, S. AM, S. MA, M. Steiner, T. Taivassalo, T. Troosters, I. Vogiatzis, W. PD, ATS/ERS Ad Hoc committee on limb muscle dysfunction in COPD. An official American Thoracic Society/European Respiratory Society statement: update on limb muscle dysfunction in chronic obstructive pulmonary disease, Am. J. Respir. Crit. Care Med. 189 (9) (2014) e15-e62.

32. P.W. Jones, St. George's Respiratory Questionnaire: MCID, COPD 2 (1) (2005) 75-79.

33. J.N. de Voogd, J.B. Wempe, G.H. Koeter, K. Postema, E. van Sonderen, A.V. Ranchor, J.C. Coyne, R. Sanderman, Depressive symptoms as predictors of mortality in patients with COPD, Chest 135 (3) (2009) 619-625.

34. R. Mesquita, L.E. Vanfleteren, F.M. Franssen, J. Sarv, Z. Taib, M.T. Groenen, S. Gaffron, P.L. Bruijnzeel, F. Pitta, E.F. Wouters, M.A. Spruit, Objectively identified comorbidities in COPD: impact on pulmonary rehabilitation outcomes, Eur. Respir. J. 46 (2) (2015) 545-548. 
35. A. Mezzani, L.F. Hamm, A.M. Jones, P.E. McBride, T.Moholdt, J.A. Stone, A. Urhausen, M.A. Williams, Aerobic exercise intensity assessment and prescription in cardiac rehabilitation: a joint position statement of the European Association for Cardiovascular Prevention and Rehabilitation, the American Association of Cardiovascular and Pulmonary Rehabilitation, and the Canadian Association of Cardiac Rehabilitation, J. Cardiopulm. Rehabil. Prev. 32 (6) (2012) 327-350.

36. F.J. Triest, F.M. Franssen, M.A. Spruit, M.T. Groenen, E.F. Wouters, L.E. Vanfleteren, Poor agreement between chart-based and objectively identified comorbidities of COPD, Eur. Respir. J. 46 (5) (2015) 14921495.

37. G.W. Bisca, M. Proenca, A. Salomao, N.A. Hernandes, F. Pitta, Minimal detectable change of the London chest activity of daily living scale in patients with COPD, J. Cardiopulm. Rehabil. Prev. 34 (3) (2014) 213216.

38. H. Demeyer, C. Burtin, M. Hornikx, C.A. Camillo, H. Van Remoortel, D. Langer, W. Janssens, T. Troosters, The minimal important difference in physical activity in patients with COPD, PLoS ONE 11 (4) (2016) e0154587.

39. L. Laviolette, J. Bourbeau, S. Bernard, Y. Lacasse, V. Pepin, M.J. Breton, M. Baltzan, M. Rouleau, F. Maltais, Assessing the impact of pulmonary rehabilitation on functional status in COPD, Thorax 63 (2) (2008) 115121.

Reprinted from International Journal of Cardiology, Vol. 225, Mesquita R, Franssen FME, Houben-Wilke S, Uszko-Lencer NHMK, Vanfleteren LEGW, Goërtz YMJ, Pitta F, Wouters EFM, Spruit MA, What is the impact of impaired left ventricular ejection fraction in COPD after adjusting for confounders?, pp. 365-70, Copyright (2016), with permission from Elsevier. 


\section{APPENDIX A, SUPPLEMENTARY DATA}

\section{A.1 METHODS}

\section{A.1.1 Detailed information about outcome measures}

As part of demographics we assessed age, sex, and smoking status, while body weight and height were assessed as part of anthropometrics, and body mass index (BMI) was calculated as weight per squared height $\left(\mathrm{kg} \cdot \mathrm{m}^{-2}\right)$ [1]. Clinical data included the use of long-term oxygen therapy (yes or no), the use of walking aids in daily life (yes or no), medications in use, the number of exacerbations in the last year, and the degree of disease severity according to the New York Heart Association (NYHA) classification, and the 2007 [2] and 2011 [3] Global Initiative for Chronic Obstructive Lung Disease (GOLD) classifications. The number of exacerbations in the last year was analysed as $<$ or $\geq 2$, as this cut-off has been associated with risk of future exacerbations [3]. Furthermore, a sample of venous blood was collected in the fasted state for the assessment of haematology, chemistry, and inflammatory status. Serum samples were stored at $-80 \mathrm{oC}$ prior to their analysis. Haemoglobin, glucose, creatinine, high density lipoprotein (HDL), triglycerides, N-terminal pro-B-type natriuretic peptide (NT-proBNP) and C-reactive protein (CRP) levels were quantified. A sample of arterial blood was also collected, for the assessment of blood gases. Arterial oxygen tension $\left(\mathrm{PaO}_{2}\right)$ and arterial carbon dioxide tension $\left(\mathrm{PaCO}_{2}\right)$ were measured with a blood gas analyser (GEM4000, Instrumentation Laboratory, Peachtree City, USA). Blood pressures (systolic and diastolic) were measured early in the morning by a trained medical technician after 15 minutes of supine rest. Three measurements were performed separated by 5 -minute intervals. The three measurements were averaged and the averaged value was used for analysis.

Comorbidities were assessed using self-reports (i.e., chart-based) and objective measurements. Chart-based comorbidities were assessed using the Charlson comorbidity index, which is composed by 19 categories of comorbidities [4]. The total score reflects the cumulative increased likelihood of 1-year mortality [5]. Higher scores can be translated as a higher burden of comorbidities. History of myocardial infarct and congestive heart failure were based on data from this instrument. Objectively assessed comorbidities included dyslipidaemia, hyperglycaemia, anaemia, renal impairment, hypertension, symptoms of depression, symptoms of anxiety, and muscle wasting. Dyslipidaemia was defined as either a triglyceride level $>1.7 \mathrm{mmol} / \mathrm{L}$, or a $\mathrm{HDL}$ cholesterol level $<1.03$ $\mathrm{mmol} / \mathrm{L}$ in males or $<1.29 \mathrm{mmol} / \mathrm{L}$ in females [6]. Hyperglycaemia was defined as a fasting glucose level $>5.6 \mathrm{mmol} / \mathrm{L}$, as suggested by the American Diabetes Association [7]. 
Anaemia was defined as a haemoglobin level $<13 \mathrm{~g} / \mathrm{dL}(8.1 \mathrm{mmol} / \mathrm{L})$ in males or $<12$ $\mathrm{g} / \mathrm{dL}(7.5 \mathrm{mmol} / \mathrm{L})$ in females, as suggested by the World Health Organization [8]. Renal impairment was defined as an estimated glomerular filtration rate (eGFR) $<60 \mathrm{ml} / \mathrm{min}$, which according to the National Kidney Foundation Kidney Disease Outcome Quality Initiative (NKF KDOQI) guidelines corresponds to stage 3 chronic kidney disease [9]. eGFR was calculated based on sex, age, body weight and creatinine levels using the Cockcroft-Gault formula [10]. Hypertension was defined as a systolic blood pressure $\geq 140 \mathrm{~mm} \mathrm{Hg}$ or a diastolic pressure $\geq 90 \mathrm{~mm} \mathrm{Hg}$, according to the guidelines of the European Society of Hypertension (ESH)/European Society of Cardiology (ESC) [11], or by the use of antihypertensive drugs. Symptoms of anxiety or depression were defined as a score $\geq 10$ points in the anxiety or depression subscales of the Hospital Anxiety and Depression Scale (HADS), respectively. This cut-off has been defined as abnormally high and may indicate clinically relevant symptoms [12]. Further details about the HADS are provided in paragraphs ahead. Finally, muscle wasting was defined as a fat-free mass index (FFMI) below the $5^{\text {th }}$ percentile of reference values according to sex, age, and body mass index (BMI) [13].

Lung function was assessed with post-bronchodilator spirometry, plethysmography and the single breath method for diffusing capacity for carbon monoxide $\left(D_{L c o}\right)$. Postbronchodilator spirometry (Masterlab ${ }^{\circledR}$, Viasys, Germany) was used to measure forced vital capacity (FVC) and forced expiratory volume in the first second $\left(F E V_{1}\right)$, to then calculate the $\mathrm{FEV}_{1} / \mathrm{FVC}$ ratio. This test followed international recommendations [14] and the results were compared to reference values [15]. The degree of airflow limitation was used to classify the subjects according to the 2007 GOLD classification [2]: GOLD 1 $\left(\mathrm{FEV}_{1} \geq 80 \%\right.$ predicted); GOLD $2\left(50 \% \leq \mathrm{FEV}_{1}<80 \%\right.$ predicted); GOLD $3\left(30 \% \leq \mathrm{FEV}_{1}<\right.$ $50 \%$ predicted); and GOLD 4 ( $\mathrm{FEV}_{1}<30 \%$ predicted). Whole-body plethysmography was performed to measure residual volume (RV) and total lung capacity (TLC), to then calculate the RV/TLC ratio. The single breath method (Masterlab ${ }^{\circledR}$, Jaeger, Germany) was used to measure $D_{L C O}$.

Cardiac function was assessed by resting echocardiography (My Lab, Esaote), which was performed by an experienced echocardiographer. Standard views were used to obtain subcostal, parasternal and apical views. The following parameters were measured: left ventricular ejection fraction (LVEF), left atrium (LA) dimension, left ventricular enddiastolic dimension (LVEDD), left ventricular end-systolic dimension (LVESD), the ratio between $\mathrm{E}$-wave and A-wave of mitral inflow, deceleration time of E-wave, interventricular septum (IVS) diameter, right ventricular (RV) diameter; right ventricular systolic pressure (RVSP), tricuspid regurgitation (TR) velocity, tricuspid annular plane systolic excursion (TAPSE), valvular abnormalities, and relevant wall motion abnormalities. TR velocity was calculated based on RVSP values according to the modified Bernoulli equation: RVSP $=4 \times(\text { TR velocity })^{2}$ [16]. A LVEF $<50 \%$ was defined as impaired LVEF compati- 
ble with heart failure. A LA dimension $>40 \mathrm{~mm}$ was defined as suggestive of LA enlargement [17], while a RV diameter $>42 \mathrm{~mm}$ was defined as suggestive of RV enlargement [18]. A TAPSE $<16 \mathrm{~mm}$ was defined as suggestive of RV systolic dysfunction [18]. Moreover, an IVS $\geq 10 \mathrm{~mm}$ was used to identify patients with left ventricular hypertrophy (LVH), while a RVSP $\geq 40 \mathrm{mmHg}$ was used to identify patients with increased RVSP suggestive of pulmonary hypertension. Due to diagnostic challenges (i.e., suboptimal echocardiographic images), parameters of diastolic dysfunction have not been included in the current analyses.

As part of the assessment of physical health measures, the following health outcomes were investigated: functional, maximal, and submaximal exercise capacity; quadriceps muscle strength and endurance; functional mobility; body composition; and inflammatory status.

Functional exercise capacity was assessed using the 6-minute walk test, which was performed according to the European Respiratory Society (ERS) / American Thoracic Society (ATS) guidelines [19]. The cut-off point of 350 meters was used as it has been shown to predict exacerbations and death $[20,21]$. This test was performed twice to account for a possible learning effect [22], and the best distance was used for analysis. Maximal exercise capacity was assessed using a symptom-limited cardiopulmonary incremental test (+10 watts per minute) performed on an electrically, braked cycle ergometer (Carefusion, Houten, the Netherlands). This test was performed in accordance with the guidelines of the ATS and the American College of Chest Physicians (ACCP) [23] and the results were compared to reference values $[24,25]$. The peak minute ventilation (VE) achieved during to test was compared to the estimated maximal voluntary ventilation calculated as $\mathrm{FEV}_{1}$ (in litres) x 40. Submaximal exercise capacity was assessed with a constant work-rate cycling endurance test performed at $75 \%$ of the peak work rate identified during the symptom-limited incremental test, as previously suggested [26]. Oxyhaemoglobin saturation $\left(\mathrm{SpO}_{2}\right)$, and perceived dyspnoea and leg fatigue (modified Borg scale [27]) were measured before and after all exercise capacity tests. Moreover, heart rate $(\mathrm{HR})$ was measured before and after the symptom-limited incremental test.

Quadriceps muscle function was assessed in terms of strength and endurance using a Biodex (Biodex System 4 Pro, Biodex Medical Systems, Inc., New York, USA) [28]. This method has been shown to be reliable in patients with COPD [29]. Peak torque in Newton-metre $(\mathrm{Nm})$ and total work in Joules were measured isokinetically for the assessment of muscle strength and endurance, respectively. Thirty volitional maximal contractions at an angular velocity of $90^{\circ}$ per second were performed twice, and the highest values were considered for analysis [28]. 
Functional mobility was assessed using the Timed Up \& Go (TUG) test. Participants were required to stand up from a chair, walk at a comfortable and safe speed a distance of 3 metres, turn and walk back to the chair to sit down again, as described in original protocol [30]. Participants were instructed to wear their usual footwear, and walking aids and/or oxygen were allowed, if necessary. The time in seconds was recorded and used as the main outcome of analysis. Three trials were performed by the same assessor and the best trial (i.e., shortest duration) was used for analysis [31]. The cut-off point of $11 \mathrm{~s}$ was used as it has been shown to associate with worse health outcomes [32].

Body composition was assessed with fat-free mass (FFM). FFM was calculated as the sum of lean and mineral bone mass, and measured by whole-body dual energy X-ray absorptiometry using a Lunar Prodigy system (GE Healthcare, Piscataway, NJ, USA) [33].

Inflammatory status was assessed by serum CRP measured in duplicate samples by high-sensitivity particle-enhanced immunoassay (COBAS Mira ${ }^{\circledR}$, Radiometer, Copenhagen).

As part of the assessment of psychological health measures, the following health outcomes were investigated: health status, care dependency, and symptoms of anxiety and depression.

Health status was assessed with two valid and reliable disease-specific instruments: the COPD assessment test (CAT), and the COPD-specific version of the St. George's Respiratory Questionnaire (SGRQ-C). The CAT is a questionnaire that comprises 8 items raising questions regarding symptoms, energy, sleep and activity [34]. Scores can range from 0 (no impact) to 40 points (severe impact) [34]. The SGRQ-C comprises 40 items distributed in three domains: symptoms, activities and impact [35]. Scores per domain and a total score are provided and can range from 0 (optimal) to 100 points (worst) [35].

Care dependency was assessed by the care dependency scale (CDS), which is composed of 15 items related to basic and instrumental activities of daily living [36]. The final score can range from 15 (worst) to 75 (best) points [36]. Patients with a score $\leq 68$ points can be considered as care dependent [37].

Symptoms of anxiety and depression were assessed with the HADS, as mentioned earlier. This is a valid and reliable self-administered questionnaire widely used for screening clinically relevant symptoms of anxiety and/or depression [12]. This questionnaire consists of 14 questions distributed in two subscales, 7 for the assessment of anxiety (HADS anxiety subscale) and 7 for the assessment of depression (HADS depression subscale) [12]. The total score in each subscale can range from 0 (optimal) to 21 (worst) [12]. 


\section{CHAPTER 2}

Based on the degree of airflow limitation (GOLD grades), the number of exacerbations in the previous year, and symptoms as assessed by the CAT questionnaire, patients were classified into GOLD groups A, B, C, or D, according to the 2011 GOLD classification [3]. GOLD grades 1-4 or exacerbations in the previous year were used to classify patients as low (group A or B) or high (group C or D) risk. Patients classified as GOLD grade 1 or 2 , or with $<2$ exacerbations in the previous year, were classified as low risk, whilst those GOLD grade 3 or 4 , or with $\geq 2$ exacerbations in the previous year, were classified as high risk; the criterion with the worse score was used for analysis [3]. The CAT questionnaire was used to classify patients as less (group A or C) or more (group B or D) symptoms [3]. Patients with a CAT score $<10$ points were classified as less symptoms, whilst those with a score $\geq 10$ points were classified as more symptoms [3]. 


\section{A.2 RESULTS}

Table A.2.1 Demographics, clinical data, and lung function in COPD patients without impaired LVEF who were included in the study and those who were not included

\begin{tabular}{|c|c|c|c|c|c|}
\hline \multirow[t]{2}{*}{ Characteristic } & \multicolumn{2}{|c|}{$\begin{array}{l}\text { COPD with normal } \\
\text { LVEF included in } \\
\text { the study }\end{array}$} & \multicolumn{2}{|c|}{$\begin{array}{l}\text { COPD with normal } \\
\text { LVEF not included in } \\
\text { the study }\end{array}$} & \multirow[t]{2}{*}{$P$-value } \\
\hline & $\mathrm{n}$ & & $n$ & & \\
\hline Age, years & 85 & $65(58-72)$ & 344 & $63(57-71)$ & 0.28 \\
\hline Male, \% & 85 & 77 & 332 & 45 & $<0.0001^{*}$ \\
\hline Smoking status N/F/C, \% & 85 & $1 / 82 / 17$ & 332 & $1 / 74 / 25$ & 0.11 \\
\hline Long-term oxygen therapy, \% & 85 & 18 & 344 & 26 & 0.10 \\
\hline Exacerbation history ( $\geq 2$ previous year), \% & 85 & 48 & 344 & 59 & 0.07 \\
\hline $\mathrm{FEV}_{1}, \%$ predicted & 85 & $45(33-58)$ & 344 & $46(32-64)$ & 0.47 \\
\hline GOLD $1 / 2 / 3 / 4, \%$ & 85 & $8 / 27 / 45 / 20$ & 344 & $6 / 38 / 35 / 21$ & 0.54 \\
\hline GOLD A/B/C/D, \% & 85 & $5 / 18 / 3 / 74$ & 324 & $2 / 21 / 3 / 74$ & 0.81 \\
\hline $\mathrm{RV}, \%$ predicted & 82 & $149(118-195)$ & 329 & $159(129-189)$ & 0.22 \\
\hline TLC, \% predicted & 82 & $117(103-125)$ & 329 & $120(108-130)$ & 0.11 \\
\hline $\mathrm{RV} / \mathrm{TLC}, \%$ & 82 & $50(42-59)$ & 329 & $52(44-60)$ & 0.16 \\
\hline $\mathrm{D}_{\mathrm{LcO}}, \%$ predicted & 78 & $50(39-63)$ & 316 & $46(37-60)$ & 0.09 \\
\hline \multicolumn{6}{|l|}{ Arterial blood gases } \\
\hline $\mathrm{PaO}_{2}, \mathrm{kPa}$ & 84 & $9.8(8.8-10.6)$ & 340 & $9.4(8.4-10.4)$ & 0.07 \\
\hline $\mathrm{PaCO}_{2}, \mathrm{kPa}$ & 85 & $5.2(4.8-5.6)$ & 340 & $5.2(4.7-5.7)$ & 0.94 \\
\hline \multicolumn{6}{|l|}{ Lung medication } \\
\hline SABA, \% & 85 & 39 & 344 & 46 & 0.25 \\
\hline LAMA, \% & 85 & 65 & 344 & 72 & 0.20 \\
\hline ICS, \% & 85 & 27 & 344 & 18 & $0.046^{*}$ \\
\hline Combination ICS/LABA, \% & 85 & 59 & 344 & 71 & $0.03^{*}$ \\
\hline
\end{tabular}

Data expressed as relative frequency, mean \pm SD or median (IQR). See table 1 for definition of abbreviations. : P-value $<0.05$. 


\section{References}

1. Keys A, Fidanza F, Karvonen MJ, Kimura N, Taylor HL. Indices of relative weight and obesity. Journal of chronic diseases 1972: 25(6): 329-343.

2. Rabe KF, Hurd S, Anzueto A, Barnes PJ, Buist SA, Calverley P, Fukuchi Y, Jenkins C, Rodriguez-Roisin R, van Weel C, Zielinski J. Global strategy for the diagnosis, management, and prevention of chronic obstructive pulmonary disease: GOLD executive summary. American journal of respiratory and critical care medicine 2007: 176(6): 532-555.

3. Vestbo J, Hurd SS, Agusti AG, Jones PW, Vogelmeier C, Anzueto A, Barnes PJ, Fabbri LM, Martinez FJ, Nishimura M, Stockley RA, Sin DD, Rodriguez-Roisin R. Global strategy for the diagnosis, management, and prevention of chronic obstructive pulmonary disease: GOLD executive summary. American journal of respiratory and critical care medicine 2013: 187(4): 347-365.

4. Charlson ME, Pompei P, Ales KL, MacKenzie CR. A new method of classifying prognostic comorbidity in longitudinal studies: development and validation. Journal of chronic diseases 1987: 40(5): 373-383.

5. Charlson M, Szatrowski TP, Peterson J, Gold J. Validation of a combined comorbidity index. Journal of clinical epidemiology 1994: 47(11): 1245-1251.

6. Alberti KG, Zimmet P, Shaw J, Group IDFETFC. The metabolic syndrome - a new worldwide definition. Lancet 2005: 366(9491): 1059-1062.

7. American Diabetes A. Diagnosis and classification of diabetes mellitus. Diabetes care 2010: 33 Suppl 1: S62-69.

8. Nutritional anaemias. Report of a WHO scientific group. World Health Organization technical report series 1968: 405: 5-37.

9. Kdoqi. KDOQI Clinical Practice Guidelines and Clinical Practice Recommendations for Diabetes and Chronic Kidney Disease. American journal of kidney diseases : the official journal of the National Kidney Foundation 2007: 49(2 Suppl 2): S12-154.

10. Cockcroft DW, Gault MH. Prediction of creatinine clearance from serum creatinine. Nephron 1976: 16(1): 31-41.

11. Mansia G, De Backer G, Dominiczak A, Cifkova R, Fagard R, Germano G, Grassi G, Heagerty AM, Kjeldsen SE, Laurent S, Narkiewicz K, Ruilope L, Rynkiewicz A, Schmieder RE, Struijker Boudier HA, Zanchetti A, European Society of H, European Society of C. 2007 ESH-ESC Guidelines for the management of arterial hypertension: the task force for the management of arterial hypertension of the European Society of Hypertension (ESH) and of the European Society of Cardiology (ESC). Blood pressure 2007: 16(3): 135-232.

12. Zigmond AS, Snaith RP. The hospital anxiety and depression scale. Acta Psychiatr Scand 1983: 67(6): 361370.

13. Franssen FM, Rutten EP, Groenen MT, Vanfleteren LE, Wouters EF, Spruit MA. New reference values for body composition by bioelectrical impedance analysis in the general population: results from the UK Biobank. Journal of the American Medical Directors Association 2014: 15(6): 448 e441-446.

14. Miller MR, Hankinson J, Brusasco V, Burgos F, Casaburi R, Coates A, Crapo R, Enright P, van der Grinten CP, Gustafsson P, Jensen R, Johnson DC, Maclntyre N, McKay R, Navajas D, Pedersen OF, Pellegrino R, Viegi G, Wanger J. Standardisation of spirometry. The European respiratory journal 2005: 26(2): 319-338.

15. Knudson RJ, Burrows B, Lebowitz MD. The maximal expiratory flow-volume curve: its use in the detection of ventilatory abnormalities in a population study. The American review of respiratory disease 1976: 114(5): 871-879.

16. Yock PG, Popp RL. Noninvasive estimation of right ventricular systolic pressure by Doppler ultrasound in patients with tricuspid regurgitation. Circulation 1984: 70(4): 657-662.

17. Hirata T, Wolfe SB, Popp RL, Helmen CH, Feigenbaum H. Estimation of left atrial size using ultrasound. American heart journal 1969: 78(1): 43-52.

18. Rudski LG, Lai WW, Afilalo J, Hua L, Handschumacher MD, Chandrasekaran K, Solomon SD, Louie EK, Schiller NB. Guidelines for the echocardiographic assessment of the right heart in adults: a report from the American Society of Echocardiography endorsed by the European Association of Echocardiography, a 
registered branch of the European Society of Cardiology, and the Canadian Society of Echocardiography. Journal of the American Society of Echocardiography : official publication of the American Society of Echocardiography 2010: 23(7): 685-713; quiz 786-688.

19. Holland AE, Spruit MA, Troosters T, Puhan MA, Pepin V, Saey D, McCormack MC, Carlin BW, Sciurba FC, Pitta F, Wanger J, Maclntyre N, Kaminsky DA, Culver BH, Revill SM, Hernandes NA, Andrianopoulos V, Camillo CA, Mitchell KE, Lee AL, Hill CJ, Singh SJ. An official European Respiratory Society/American Thoracic Society Technical Standard: field walking tests in chronic respiratory disease. The European respiratory journal 2014: 44(6): 1428-1446.

20. Cote CG, Pinto-Plata V, Kasprzyk K, Dordelly LJ, Celli BR. The 6-min walk distance, peak oxygen uptake, and mortality in COPD. Chest 2007: 132(6): 1778-1785.

21. Spruit MA, Polkey MI, Celli B, Edwards LD, Watkins ML, Pinto-Plata V, Vestbo J, Calverley PM, Tal-Singer R, Agusti A, Coxson HO, Lomas DA, MacNee W, Rennard S, Silverman EK, Crim CC, Yates J, Wouters EF. Predicting outcomes from 6-minute walk distance in chronic obstructive pulmonary disease. Journal of the American Medical Directors Association 2012: 13(3): 291-297.

22. Hernandes NA, Wouters EF, Meijer K, Annegarn J, Pitta F, Spruit MA. Reproducibility of 6-minute walking test in patients with COPD. The European respiratory journal 2011: 38(2): 261-267.

23. American Thoracic Society (ATS)/American College of Chest Physicians (ACCP). ATS/ACCP Statement on cardiopulmonary exercise testing. American journal of respiratory and critical care medicine 2003: 167(2): 211-277.

24. Jones NL, Makrides L, Hitchcock C, Chypchar T, McCartney N. Normal standards for an incremental progressive cycle ergometer test. The American review of respiratory disease 1985: 131(5): 700-708.

25. Hansen JE, Sue DY, Wasserman K. Predicted values for clinical exercise testing. The American review of respiratory disease 1984: 129(2 Pt 2): S49-55.

26. van 't Hul A, Gosselink R, Kwakkel G. Constant-load cycle endurance performance: test-retest reliability and validity in patients with COPD. Journal of cardiopulmonary rehabilitation 2003: 23(2): 143-150.

27. Borg GA. Psychophysical bases of perceived exertion. Medicine and science in sports and exercise 1982: 14(5): 377-381.

28. Sillen MJ, Franssen FM, Delbressine JM, Uszko-Lencer NH, Vanfleteren LE, Rutten EP, Wouters EF, Spruit MA. Heterogeneity in clinical characteristics and co-morbidities in dyspneic individuals with COPD GOLD D: findings of the DICES trial. Respiratory medicine 2013: 107(8): 1186-1194.

29. Mathur S, Makrides L, Hernandez P. Test-retest relibility of isometric and isokinetic torque in patients with chronic obstructive pulmonary disease. Physiotherapy Canada 2004: 56: 94-101.

30. Podsiadlo D, Richardson S. The timed "Up \& Go": a test of basic functional mobility for frail elderly persons. Journal of the American Geriatrics Society 1991: 39(2): 142-148.

31. Mesquita R, Janssen DJ, Wouters EF, Schols JM, Pitta F, Spruit MA. Within-day test-retest reliability of the timed up \& go test in patients with advanced chronic organ failure. Archives of physical medicine and rehabilitation 2013: 94(11): 2131-2138.

32. Mesquita R, Wilke S, Smid DE, Janssen DJ, Franssen FM, Probst VS, Wouters EF, Muris JW, Pitta F, Spruit MA. Measurement properties of the Timed Up \& Go test in patients with COPD. Chronic Respiratory Disease 2016, http://dx.doi.org/10.1177/1479972316647178.

33. Graat-Verboom L, Spruit MA, van den Borne BE, Smeenk FW, Martens EJ, Lunde R, Wouters EF. Correlates of osteoporosis in chronic obstructive pulmonary disease: An underestimated systemic component. Respiratory medicine 2009: 103(8): 1143-1151.

34. Jones PW, Harding G, Berry P, Wiklund I, Chen WH, Kline Leidy N. Development and first validation of the COPD Assessment Test. The European respiratory journal 2009: 34(3): 648-654.

35. Meguro M, Barley EA, Spencer S, Jones PW. Development and Validation of an Improved, COPD-Specific Version of the St. George Respiratory Questionnaire. Chest 2007: 132(2): 456-463.

36. Dijkstra A, Tiesinga L, Goossen WT, Dassen TW. Further psychometric testing of the Dutch Care Dependency Scale on two different patient groups. Int J Nurs Pract 2002: 8(6): 305-314.

37. Dijkstra A, Tiesinga LJ, Plantinga L, Veltman G, Dassen TW. Diagnostic accuracy of the care dependency scale. Journal of advanced nursing 2005: 50(4): 410-416. 



\section{CHAPTER 3}

Objectively identified comorbidities in COPD: impact on pulmonary rehabilitation outcomes

Rafael Mesquita*, Lowie E.G.W. Vanfleteren*, Frits M.E. Franssen, Janeli Sarv, Ziad Taib, Miriam T.J. Groenen, Swetlana Gaffron, Piet L.B. Bruijnzeel, Fabio Pitta, Emiel F.M. Wouters, and Martijn A. Spruit

'Both authors contributed equally.

\section{Published as a research letter in the}

European Respiratory Journal 2015; 46: 545-548 


\title{
CHAPTER 3
}

\begin{abstract}
It remains unclear whether and to what extent changes in pulmonary rehabilitation (PR) outcomes are influenced by comorbidities in chronic obstructive pulmonary disease (COPD). We aimed to investigate the impact of both individual as well as clusters of objectively identified comorbidities on changes in exercise capacity and health status after PR in COPD patients.
\end{abstract}

In a prospective study, 213 participants (59\% men, age $64 \pm 7$ years, FEV $151 \pm 17 \%$ predicted) had assessed their exercise capacity (6-min walk test (6MWT) and constant work rate test (CWRT)) and health status (St. George Respiratory Questionnaire [SGRQ]) before and after a PR programme. Thirteen comorbidities and five comorbidity clusters (based on the thirteen comorbidities) were studied at baseline.

194 participants completed the PR programme. Overall, exercise capacity and health status improved significantly and meaningfully. Individual comorbidities did not influence these improvements, but when comorbidity clusters were considered only the "psychologic" cluster showed a higher likelihood of achieving a meaningful improvement in the 6MWT (OR 3.12, 95\% Cl 1.29-7.55, $\mathrm{P}=0.01)$.

Comorbidities do not prevent COPD patients from improving exercise capacity and health status after PR. COPD patients should not be withheld from rehabilitation programmes based on stable comorbidities. 


\section{INTRODUCTION}

Patients with chronic obstructive pulmonary disease (COPD) often present with other chronic conditions [1-4], which are associated with increased morbidity and mortality [5-7]. Indeed, as also acknowledged by the 2013 version of the Global Initiative for Chronic Obstructive Lung Disease strategy document, comorbidities importantly contribute to the overall disease severity in individual patients [8].

Pulmonary rehabilitation (PR) is a comprehensive intervention recognized as a core component in the treatment of patients with COPD [9]. Comorbidities have been suggested to affect outcomes of PR in this population, but conflicting results have been found. In a large retrospective study, Crisafulli and colleagues [10] found that the presence of metabolic and heart diseases was negatively and positively associated with meaningful improvements in exercise capacity following PR, respectively. In a subsequent prospective study, the same group observed that only the presence of osteoporosis was associated with poorer rehabilitation outcomes [11]. Two other studies support the association between the change in exercise capacity after PR and metabolic disease, but the direction of this association was divergent between the studies [12, 13]. Contradictory results were also observed regarding improvements in health status and the presence of comorbidities [10-14]. Most previous studies, however, have used charts reviewing to identify comorbid conditions. Furthermore, only the impact of individual comorbidities or arbitrarily grouped comorbidities was studied [10-14].

Recently, Vanfleteren et al. [15] identified five distinct comorbidity clusters in patients with COPD, based on 13 objectively diagnosed comorbidities. The impact of these individual comorbidities or of the comorbidity clusters on changes in widely used outcomes after PR, however, remains unknown. Therefore, the aim of the present study was to investigate the impact of both individual as well as clusters of objectively identified comorbidities on changes in functional exercise capacity (i.e., walking and cycling) and health status after PR in patients with COPD. The baseline data of this study have been published previously [15].

\section{MATERIALS AND METHODS}

\section{Study design and participants}

This study is part of the $\mathrm{CIRO}$ CO-morbidity (CIROCO) study, an observational prospective single-centre study performed at $\mathrm{ClRO}+$, Centre of Expertise for Chronic Organ Failure, in Horn, the Netherlands. Participants were recruited between November 2007 and November 2010 and submitted to an inpatient or outpatient PR programme. The 
following inclusion criteria were used: Global Initiative for Chronic Obstructive Lung Disease grades $2-4$ [8], 40 to 80 years of age, clinically stable state, and a smoking history of $\geq 10$ pack years or relevant occupational exposure. Participants were considered ineligible if they had any disease and/or condition that could compromise the proposed assessments; detailed exclusion criteria can be found in the supplemental material. All participants gave written informed consent and the study was approved by the local ethics and review boards (MEC 10-3-067).

\section{Measurements and intervention}

Participants' demographic, smoking status and long-term oxygen use were documented at study entry. Moreover, the following assessments were performed: lung function, body composition, subclinical atherosclerosis, cardiac ischaemia, blood pressure, functional exercise capacity, dyspnoea, and psychological and health status. A sample of venous blood was obtained in fasting state for the assessment of haematology and chemistry. Details and references of these assessments can be found in the supplemental material. Functional exercise capacity (6-min walk test [6MWT] and constant work rate cycling test [CWRT]) and health status (St. George's Respiratory Questionnaire [SGRQ]) were selected as the primary outcomes of this study, and then the results on these outcomes were investigated before and after PR. These outcomes were chosen as they have shown to be the most widely used outcomes in PR [16]. An improvement above the minimum important difference (MID) was considered meaningful according to the following thresholds: an increase in 6-min walk distance (6MWD) of $30 \mathrm{~m}$ or more [17]; an increase in CWRT time of $100 \mathrm{~s}$ or more [18]; a decrease in SGRQ total score of 4 points or more [19].

According to diagnostic criteria established by relevant international societies (see Vanfleteren et al. [15]), thirteen comorbidities were objectively investigated during initial evaluation for PR: chronic kidney disease, anaemia, hypertension, obesity, underweight, muscle wasting, hyperglycaemia, dyslipidaemia, osteoporosis, symptoms of anxiety and depression, atherosclerosis and myocardial infarction. Based on these conditions, five comorbidity clusters were previously identified: cluster 1 (less comorbidity), cluster 2 (cardiovascular), cluster 3 (cachectic), cluster 4 (metabolic), and cluster 5 (psychologic) [15]. In addition, self-reported comorbidities were assessed by the Charlson comorbidity index [20]; details can be found in the supplemental material.

After the abovementioned assessments participants were enrolled in an 8-week inpatient (5 days.week ${ }^{-1}$ ) or 14-week outpatient ( 3 days.week $^{-1}$ ) comprehensive PR programme [21]. Briefly, both types of programme were implemented by an interdisciplinary team and consisted of high-intensity progressive exercise training (i.e., endurance treadmill walking and cycling and strength training of lower and upper extremities), 
nutritional support, occupational therapy and psychological counselling, if indicated, besides educational sessions in groups.

\section{Statistics}

Data for quantitative variables were presented as mean \pm standard deviation or mean (95\% confidence intervals), whilst data for discrete variables were presented as absolute and/or relative frequency. Chi-square or Fisher's exact test was used for categorical variables. Student's t test was used for comparisons between participants who did or did not complete the PR programme (i.e., those with or without final assessment, respectively). Within- and between-group comparisons were performed based on 95\% confident intervals. Logistic regression models adjusted for age, gender, forced expiratory volume in the first second and the baseline value in each outcome measure were built to identify the predictive role of comorbidities on meaningful improvements in exercise capacity and health status after PR. Statistical significance was considered as $\mathrm{P}<0.05$ and data analysis was carried out using SPSS 17.0 (SPSS, Chicago, Illinois) or SAS version 8.2 (SAS Institute, Cary, NC).

\section{RESULTS}

\section{General characteristics}

255 patients with COPD were recruited, but 213 were eligible [15]. Of the 213 participants, 13 lacked acceptable quality carotid intima media thickness measurement, 6 did not fill out the questionnaire for symptoms of anxiety and depression, and 11 lacked an acceptable ECG. Table 1 summarises the baseline characteristics, the distribution of individual comorbidities and the clusters identified. Details on the frequency of comorbidities in each cluster were provided by Vanfleteren et al. [15]. 
CHAPTER 3

Table 1 Baseline characteristics of the participants

\begin{tabular}{ll}
\hline Characteristic Value \\
\hline
\end{tabular}

N

213

Demographics, anthropometrics and clinical data

Age, years

$64 \pm 7$

Male, n (\%)

126 (59)

BMI, $\mathrm{kg} / \mathrm{m}^{2}$

$26.2 \pm 5.1$

mMRC dyspnoea grade

$2.1 \pm 1.1$

Current smoker, n (\%)

Pack-years

$46 \pm 26$

Long-term oxygen therapy, n (\%)

Charlson comorbidity index ${ }^{\dagger}$

$0.6 \pm 0.9$

Pulmonary function

$\mathrm{FEV}_{1}, \mathrm{~L}$

$1.40 \pm 0.54$

$\mathrm{FEV}_{1}, \%$ predicted

$51 \pm 17$

$\mathrm{FEV}_{1} / \mathrm{FVC}, \%$

$40 \pm 11$

ITGV, \% predicted

$148 \pm 33$

$\mathrm{D}_{\mathrm{LCO}}, \%$ predicted

$56 \pm 17$

Functional outcomes

6MWD, $\mathrm{m}$

$470 \pm 106$

CWRT, s*

$353 \pm 269$

SGRQ total score

$51 \pm 18$

SGRQ symptoms score

$55 \pm 21$

SGRQ activity score

$68 \pm 22$

SGRQ impact score

$40 \pm 20$

Objectively identified comorbidities

Number of comorbidities per participant

$4 \pm 2$

Chronic kidney disease, $\mathrm{n}(\%)$

47 (22)

Anaemia, n (\%)

$11(5)$

Hypertension, n (\%)

103 (48)

Obesity, n (\%)

$50(23)$

Underweight, n (\%)

$30(14)$

Muscle wasting, n (\%)

Hyperglycaemia, n (\%)

$116(54)$

Dyslipidaemia, n (\%)

Osteoporosis, n (\%)

66 (31) 
Symptoms of anxiety, n (\%)

Symptoms of depression, $\mathrm{n}(\%)$

Atherosclerosis, $\mathrm{n}(\%)$

106 (53)

Myocardial infarction, $\mathrm{n}(\%)$

Clusters of objectively identified comorbidities

Cluster 1: less comorbidity, n (\%)

Cluster 2: cardiovascular, n (\%)

Cluster 3: cachectic, n (\%)

Cluster 4: metabolic, n (\%)

Cluster 5: psychologic, n (\%)

20 (9)

Data expressed as mean \pm standard deviation for quantitative variables, and absolute and relative frequency for discrete variables. BMI: body mass index; mMRC: modified medical research council; FEV ${ }_{1}$ : forced expiratory volume in the first second; FVC: forced vital capacity; ITGV: intrathoracic gas volume; $D_{\text {Lco: diffusing }}$ capacity for carbon monoxide; 6MWD: 6-minute walk distance; CWRT: constant work rate test; SGRQ: St. George's respiratory questionnaire. ${ }^{*} n=202$; + COPD is not included in the score.

\section{Changes after pulmonary rehabilitation}

19 participants (9\%) dropped out the PR programme. This group of participants had more current smokers ( $26 \%$ versus $47 \% ; \mathrm{P}=0.05$ ) and lower baseline scores in the SGRQ activity domain ( $69 \pm 22$ versus $58 \pm 18$ points; $P=0.03$ ) compared with those who completed the programme (online supplementary table S1). Amongst completers, 57 participants (29\%) were submitted to inpatient rehabilitation and 137 (71\%) to outpatient rehabilitation. The proportion of participants submitted to inpatient or outpatient rehabilitation was similar amongst clusters $(P=0.37)$.

On average, the 6MWD (mean change $(95 \% \mathrm{Cl}), 30(23,38) \mathrm{m})$, cycle endurance time (mean change $(95 \% \mathrm{Cl}), 202(155,248) \mathrm{s})$, and health status (mean change $(95 \% \mathrm{Cl}),-4.0$ $(-6.0,-2.0))$ improved significantly in the whole sample. Changes in exercise capacity and health status after PR are shown in Tables 2 (individual comorbidities) and 3 (comorbidity clusters). Results according to the SGRQ symptoms, activity and impact sub-scores can be found in the online supplementary table S2. Results of logistic regression models are summarized in Figures 1 (individual comorbidities) and 2 (comorbidity clusters). 
A) $6 \mathrm{MWT}$

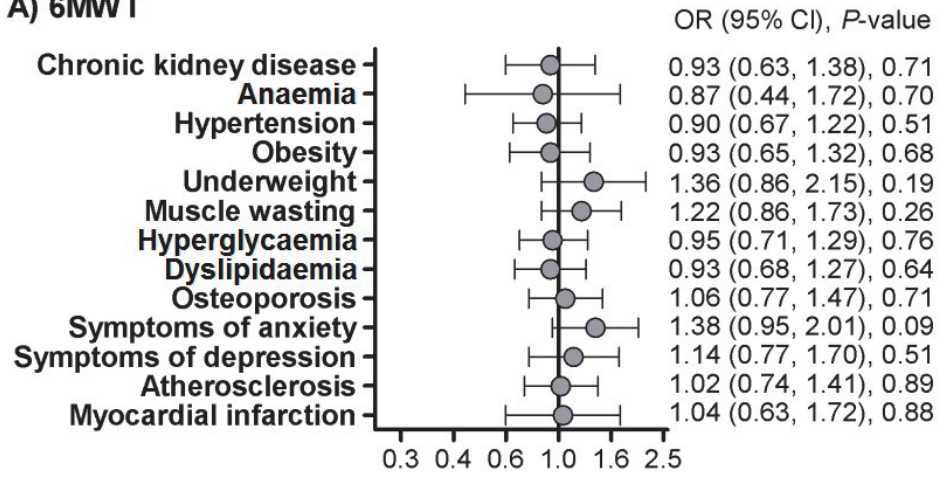

Odds ratio

B) CWRT

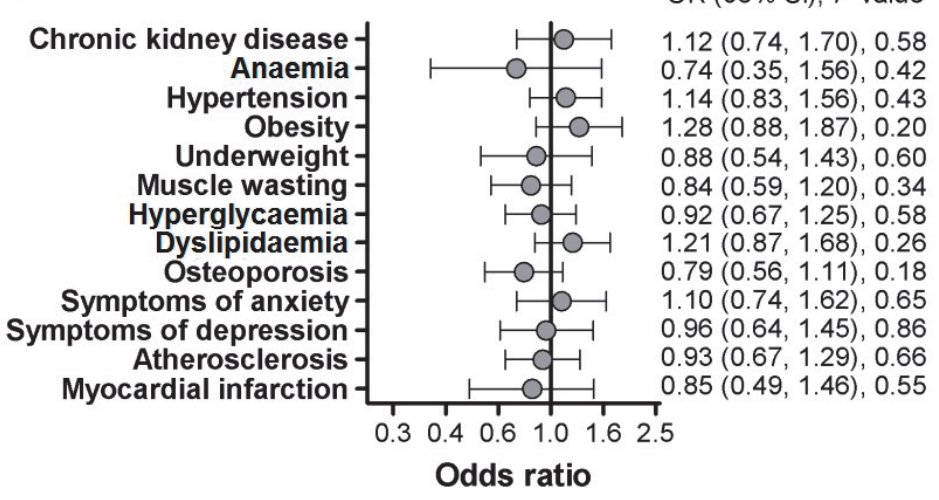

C) SGRQ

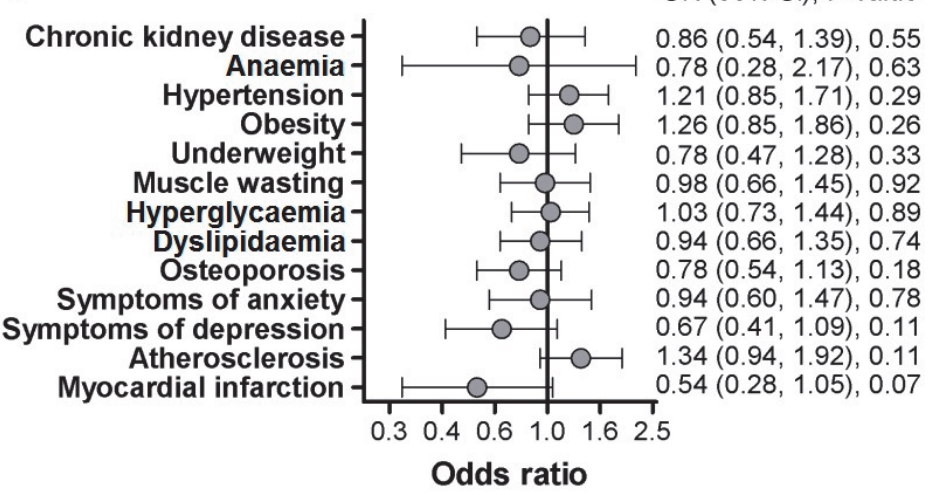

Figure 1 Predictive role of individual objectively identified comorbidities on meaningful improvements in (A) 6min walk test (6MWT, $n=187$ due to missing data), (B) constant work rate test (CWRT, $n=174$ due to missing data), and (C) St. George Respiratory Questionnaire ( $S G R Q, n=151$ due to missing data) after pulmonary rehabilitation. Models adjusted for age, gender, $\mathrm{FEV}_{1} \%$ predicted and the baseline value in each outcome measure. 


\section{A) 6MWT}

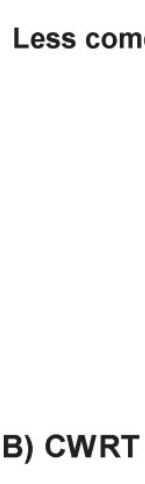

OR (95\% Cl), $P$-value

1.00

$0.82(0.45,1.50), 0.51$

$1.12(0.60,2.10), 0.72$

$0.57(0.29,1.15), 0.12$

$3.12(1.29,7.55), 0.01$

Odds ratio

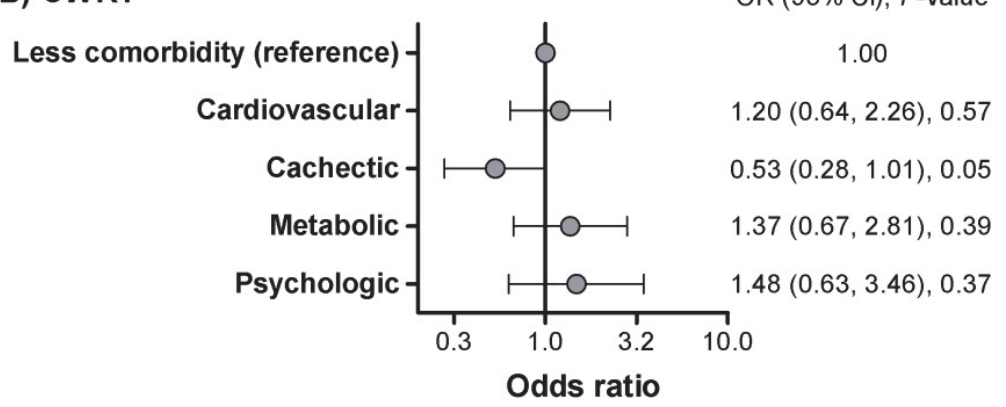

C) SGRQ

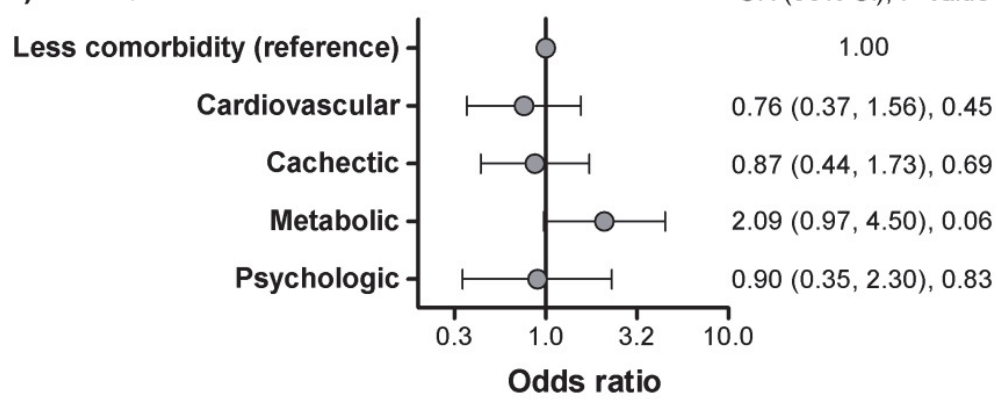

Figure 2 Predictive role of clusters of objectively identified comorbidities on meaningful improvements in (A) 6-min walk test ( $6 M W T, n=187$ due to missing data), (B) constant work rate test (CWRT, $n=174$ due to missing data), and (C) St. George Respiratory Questionnaire (SGRQ, $n=151$ due to missing data) after pulmonary rehabilitation. Models adjusted for age, gender, $\mathrm{FEV}_{1} \%$ predicted and the baseline value in each outcome measure. 


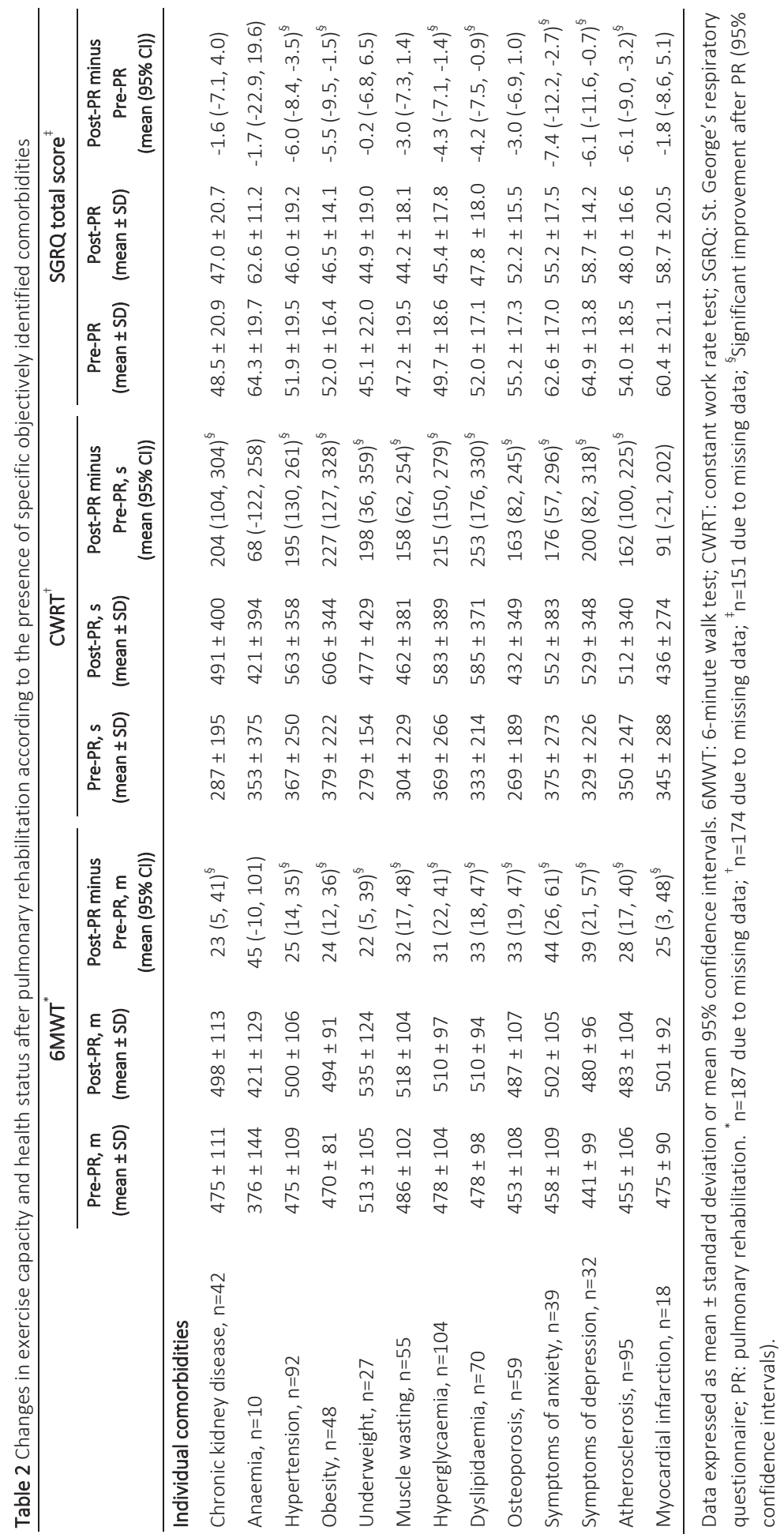




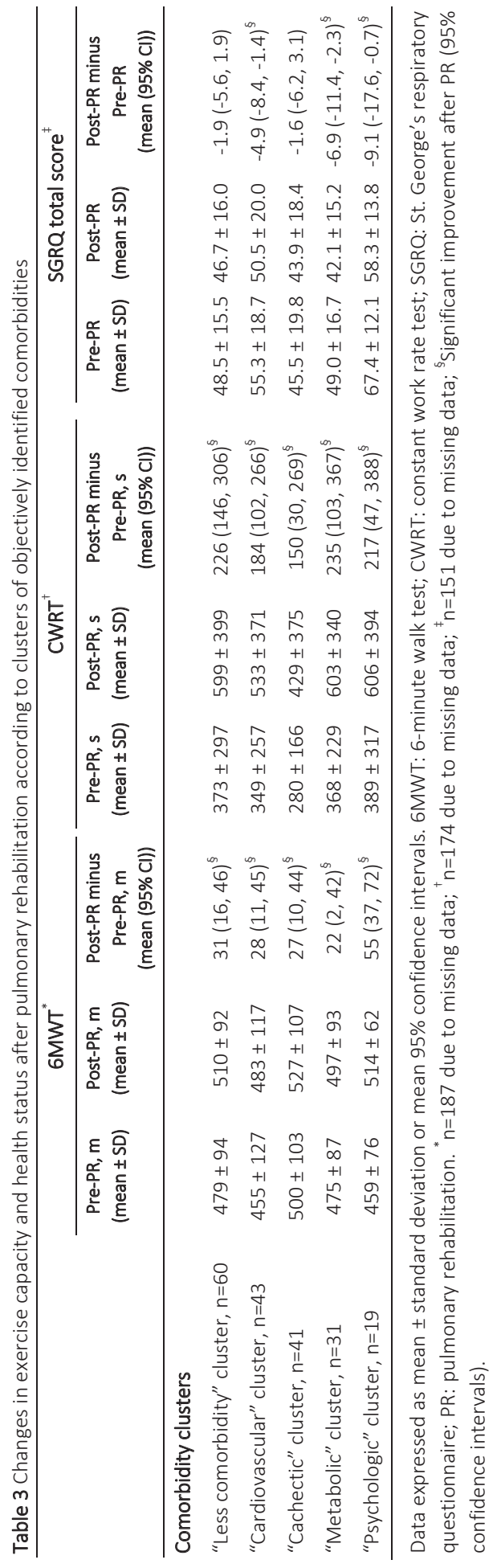




\section{PR outcomes and self-reported comorbidities}

Irrespective of the final score in the Charlson index, on average, significant and meaningful improvements were found after PR in both the 6MWT and the CWRT (online supplementary table S3). However, only participants with no comorbidity improved statistically and meaningfully in the SGRQ (online supplementary table S3). No statistical significance was found in any of the measures when the Charlson score was taken into account in logistic regression models (online supplementary figure S1).

\section{PR outcomes and individual comorbidities}

Significant improvements in both the 6MWT and the CWRT were found in each individual comorbidity, with the exception of anaemia and myocardial infarction (Table 2). Participants with chronic kidney disease, underweight, muscle wasting, osteoporosis, anaemia or myocardial infarction, on average did not improve in the SGRQ (Table 2). A logistic regression analysis revealed that none of the comorbidities was able to predict clinically meaningful changes in any of the outcome measures (Figure 1). The proportion of participants with an improvement $\geq$ MID for the 6MWT, CWRT and SGRQ ranged from $40 \%$ to $59 \%, 38 \%$ to $65 \%$, and $31 \%$ to $56 \%$, respectively.

No statistical significance was found in any of the measures when the number of comorbidities (none, one, or more than one comorbidity) was taken into account in logistic regression models ( $\mathrm{P}>0.05$ for all; online supplementary figure $\mathrm{S} 2$ ).

\section{PR outcomes and comorbidity clusters}

Significant improvements in the 6MWT and CWRT were found in all comorbidity clusters (Table 3). The proportion of participants with an improvement $\geq$ MID for the 6MWT and CWRT ranged from $38 \%$ to $74 \%$, and $40 \%$ to $66 \%$, respectively. Compared to the 'less comorbidity' cluster, participants from the 'psychologic' cluster had a higher likelihood of achieving the MID for the 6MWT, whilst subjects from the 'cachectic' cluster had a tendency towards a lower likelihood of achieving the MID for the CWRT (Figure 2).

SGRQ improved only in the 'cardiovascular', 'metabolic' and 'psychologic' clusters (Table 3). The proportion of participants with an improvement $\geq$ MID for the SGRQ ranged from $40 \%$ to $63 \%$. In a logistic regression model considering the 'less comorbidity' cluster as reference, the analyses for the SGRQ did not reach statistical significance (Figure 2). 


\section{DISCUSSION}

The present study showed that COPD patients with one or more objectively identified comorbidities following PR are able to obtain significant improvements in exercise capacity and health status. Moreover, the likelihood of achieving meaningful improvements in 6MWD, CWRT time or SGRQ scores was generally not influenced by the presence of individual or combinations of comorbidities.

Most of the studies published so far investigating the impact of comorbidities on PR outcomes have focused on either self-reported comorbidities or comorbidities retrieved from medical records [22]. In our study, self-reported comorbidities were not able to predict meaningful improvements in terms of exercise capacity or health status. Selfreported comorbidities might provide reasonably accurate estimates, but objective measures should be preferred for a conclusive diagnosis.

Previous studies using self-reported measures or data from medical records have suggested that COPD patients with metabolic disease have a higher or lower likelihood of achieving the MID for the 6MWT [10-13]. When assessing comorbidities in an objective manner, we were not able to corroborate any of these findings. Different factors can help to explain the differences between the studies, but probably the main one is that in our study comorbidities were assessed using objective measures. Whilst subjects in the "metabolic" cluster, on average, did not reach the MID for the 6MWT, the same cluster had the highest mean improvement in the CWRT. This contrasting response suggests that excessive body weight may play an important role as cycling can be considered a non-weight bearing exercise and walking a weight bearing exercise.

Still regarding changes in the 6MWT, surprisingly, the 'psychologic' cluster had the highest proportion of participants reaching the MID for this test and was the only one associated with a higher likelihood of achieving meaningful improvements compared to the 'less comorbidity' cluster. One could argue that participants of the 'psychologic' cluster' have lower baseline 6MWD compared to those of the 'less comorbidity' cluster, and that this lead to greater improvements as a result of the regression-toward-themean phenomenon. Nevertheless, Vanfleteren et al. [15] already showed that no statistical difference was found when comparing the baseline 6MWD between clusters. Moreover, in the present study these baseline values were taken into account in the logistic regression models that were built, thus correcting the models for a potential influence of the baseline values.

The 'psychologic' cluster is characterised by a higher proportion of participants with baseline symptoms of anxiety and depression [15]. Previously, von Leupoldt et al. [23] and Withers et al. [24] showed that more symptoms of anxiety and depression, in gen- 
eral, are associated with reduced exercise capacity. Indeed, in our study we found a statistically significant negative correlation between baseline scores of depression and 6MWD ( $r=-0.15$; $P=0.04$; results not shown). We believe that more symptoms of anxiety and/or depression may lead to excessive sensitiveness to dyspnoea and this can work as a limiting factor for exercise. As suggested by Maurer et al. [25], the multidisciplinary and comprehensive approache adopted in pulmonary rehabilitation programmes may help to desensitize patients who are excessively sensitive to dyspnoea, which in turn can translate as greater improvements in terms of exercise capacity. We reason that the same is true for the current study [21].

Participants in the 'cachectic' cluster had a tendency towards a lower likelihood of achieving the MID for the CWRT, but not for the 6MWT, although both tests can be used to assess exercise capacity. Man et al. [26] observed an enhanced fatigue response after cycling compared to walking. As the 'cachectic' cluster was characterised by more participants with muscle wasting compared to other clusters [15], we believe participants of this cluster were more influenced by the CWRT than by the 6MWT. Important to mention that cachexia itself was not investigated in this study. Cachexia has been defined as an ongoing process of active weight loss and accelerated muscle protein degradation or muscle wasting [27]. The 'cachectic' cluster was given this name for reasons of clinical recognition of the cachectic phenotype in its participants, as these participants simultaneously showed low muscle mass, low bone mass and destruction of lung parenchyma [15].

Individual comorbidities generally did not affect improvements in health status, although patients with underweight, muscle wasting, osteoporosis, or chronic kidney disease were not improving significantly or in a clinically relevant magnitude. Consistently, participants in the 'cachectic' cluster, on average, did not improve their health status after PR. The reasons are not completely clear. Although the 'cachectic' cluster had less impaired health status at baseline when compared to the whole group [15], after running a logistic regression analysis corrected for SGRQ baseline results this cluster was not associated with the likelihood of achieving meaningful improvements (Figure 2). Interestingly, we also found that the 'less comorbidity' cluster did not show significant and clinically meaningful improvements in SGRQ scores, illustrating again the limited impact of comorbidities on widely used PR outcomes.

Previous PR trials in patients with COPD have used the presence of comorbidities as a criterion for excluding participants. Nevertheless, our study has shown that patients with COPD and comorbidities can show significant improvements after PR. Exercise has been suggested as the cornerstone of PR [9], and this strategy has been used as a therapy not only in COPD but also in other chronic diseases, such as cardiovascular and metabolic diseases $[28,29]$. Therefore, patients with COPD and such comorbidities may 
benefit even more from PR. McNamara et al. [30] showed that even subjects with more physically debilitating comorbidities can benefit from PR. In addition, a comprehensive PR programme implements a rigorous assessment of the patients' comorbidities and a multidisciplinary approach. In this context, PR might be an optimal setting for the assessment and treatment of comorbidities, both pharmacologically and nonpharmacologically.

This is the first study to investigate the impact of objectively assessed comorbidities and the combinations of comorbidities (i.e., clusters) on PR outcomes. Objective assessments provide more accurate results, and the organization in clusters is probably more realistic as multimorbidity is frequently found in patients with COPD [2]. Moreover, a prospective design was used in our study, supporting more accurate assessments, whilst most previous studies have used retrospective designs [22].

Despite the strengths of our study, some limitations are acknowledged. Participants were recruited from a single center and no Global Initiative for Chronic Obstructive Lung Disease grade 1 patient was included, possibly compromising the generalizability of our results. Nevertheless, Vanfleteren et al. [15] have already shown that the participants from our cohort have similar characteristics compared to participants from other cohorts. Some analyses might have been underpowered, and this may constitute another limitation. Future studies should take care to ensure sufficiently powered analyses. Due to the limited sample size, we believe that the current findings are more hypothesis-generating than definitive.

In summary, comorbidities do not seem to preclude patients with COPD from showing significant and clinically meaningful improvements in exercise capacity and health status after pulmonary rehabilitation. COPD patients should not be withheld from rehabilitation programmes based on stable comorbidities. 


\section{REFERENCES}

1. Vanfleteren LE, Franssen FM, Uszko-Lencer NH, et al. Frequency and relevance of ischemic electrocardiographic findings in patients with chronic obstructive pulmonary disease. Am J Cardiol 2011; 108: 16691674.

2. Divo M, Cote $\mathrm{C}$, de Torres JP, et al. Comorbidities and risk of mortality in patients with chronic obstructive pulmonary disease. Am J Respir Crit Care Med 2012; 186: 155-161.

3. Janssen DJ, Spruit MA, Leue C, et al. Symptoms of anxiety and depression in COPD patients entering pulmonary rehabilitation. Chron Respir Dis 2010; 7: 147-157.

4. Graat-Verboom L, Wouters EF, Smeenk FW, et al. Current status of research on osteoporosis in COPD: a systematic review. Eur Respir J 2009; 34: 209-218.

5. Barnes PJ, Celli BR. Systemic manifestations and comorbidities of COPD. Eur Respir J 2009; 33: 1165-1185.

6. Patel AR, Donaldson GC, Mackay AJ, et al. The impact of ischemic heart disease on symptoms, health status, and exacerbations in patients with COPD. Chest 2012; 141: 851-857.

7. Aryal S, Diaz-Guzman E, Mannino DM. Prevalence of COPD and comorbidity. Eur Respir Monogr 2013; 59: $1-12$.

8. Vestbo J, Hurd SS, Agusti AG, et al. Global strategy for the diagnosis, management, and prevention of chronic obstructive pulmonary disease: GOLD executive summary. Am J Respir Crit Care Med 2013; 187 : 347-365.

9. Spruit MA, Singh SJ, Garvey C, et al. An official american thoracic society/european respiratory society statement: key concepts and advances in pulmonary rehabilitation. Am J Respir Crit Care Med 2013; 188: e13-64.

10. Crisafulli E, Costi S, Luppi $F$, et al. Role of comorbidities in a cohort of patients with COPD undergoing pulmonary rehabilitation. Thorax 2008; 63: 487-492.

11. Crisafulli E, Gorgone P, Vagaggini B, et al. Efficacy of standard rehabilitation in COPD outpatients with comorbidities. Eur Respir J 2010; 36: 1042-1048.

12. Walsh JR, McKeough ZJ, Morris NR, et al. Metabolic disease and participant age are independent predictors of response to pulmonary rehabilitation. J Cardiopulm Rehabil Prev 2013; 33: 249-256.

13. Walsh JR, Morris NR, McKeough ZJ, et al. A simple clinical measure of quadriceps muscle strength identifies responders to pulmonary rehabilitation. Pulm Med 2014; 2014: 782702.

14. Carreiro A, Santos J, Rodrigues F. Impact of comorbidities in pulmonary rehabilitation outcomes in patients with chronic obstructive pulmonary disease. Rev Port Pneumol 2013; 19: 106-113.

15. Vanfleteren LE, Spruit MA, Groenen M, et al. Clusters of comorbidities based on validated objective measurements and systemic inflammation in patients with chronic obstructive pulmonary disease. Am J Respir Crit Care Med 2013; 187: 728-735.

16. Spruit MA, Pitta F, Garvey C, et al. Differences in content and organisational aspects of pulmonary rehabilitation programmes. Eur Respir J 2014; 43: 1326-1337.

17. Holland AE, Spruit MA, Troosters $T$, et al. An official European Respiratory Society/American Thoracic Society Technical Standard: field walking tests in chronic respiratory disease. Eur Respir J 2014; 44: 14281446.

18. Laviolette L, Bourbeau J, Bernard S, et al. Assessing the impact of pulmonary rehabilitation on functional status in COPD. Thorax 2008; 63: 115-121.

19. Jones PW. St. George's Respiratory Questionnaire: MCID. COPD 2005; 2: 75-79.

20. Charlson $M E$, Pompei $P$, Ales $K L$, et al. A new method of classifying prognostic comorbidity in longitudinal studies: development and validation. J Chronic Dis 1987; 40: 373-383.

21. Spruit MA, Vanderhoven-Augustin I, Janssen PP, et al. Integration of pulmonary rehabilitation in COPD. Lancet 2008; 371: 12-13.

22. Hornikx M, Van Remoortel H, Demeyer $\mathrm{H}$, et al. The influence of comorbidities on outcomes of pulmonary rehabilitation programs in patients with COPD: a systematic review. Biomed Res Int 2013; 2013: 146148. 
23. von Leupoldt A, Taube K, Lehmann K, et al. The impact of anxiety and depression on outcomes of pulmonary rehabilitation in patients with COPD. Chest 2011; 140: 730-736.

24. Withers NJ, Rudkin ST, White RJ. Anxiety and depression in severe chronic obstructive pulmonary disease: the effects of pulmonary rehabilitation. J Cardiopulm Rehabil 1999; 19: 362-365.

25. Maurer J, Rebbapragada V, Borson S, et al. Anxiety and depression in COPD: current understanding, unanswered questions, and research needs. Chest 2008; 134: 43S-56S.

26. Man WD, Soliman MG, Gearing J, et al. Symptoms and quadriceps fatigability after walking and cycling in chronic obstructive pulmonary disease. Am J Respir Crit Care Med 2003; 168: 562-567.

27. Evans WJ, Morley JE, Argiles J, et al. Cachexia: a new definition. Clin Nutr 2008; 27: 793-799.

28. Mezzani A, Hamm LF, Jones AM, et al. Aerobic exercise intensity assessment and prescription in cardiac rehabilitation: a joint position statement of the European Association for Cardiovascular Prevention and Rehabilitation, the American Association of Cardiovascular and Pulmonary Rehabilitation, and the Canadian Association of Cardiac Rehabilitation. J Cardiopulm Rehabil Prev 2012; 32: 327-350.

29. O'Hagan C, De Vito G, Boreham CA. Exercise prescription in the treatment of type 2 diabetes mellitus : current practices, existing guidelines and future directions. Sports Med 2013; 43: 39-49.

30. McNamara RJ, McKeough ZJ, McKenzie DK, et al. Water-based exercise in COPD with physical comorbidities: a randomised controlled trial. Eur Respir J 2013; 41: 1284-1291. 


\section{ONLINE SUPPLEMENT}

\section{METHODS}

\section{Detailed exclusion criteria}

Patients were excluded if they met the following exclusion criteria: history of asthma or any other significant respiratory disease, diagnosis of alpha-1 antitrypsin deficiency, any previous lung surgery, any active inflammatory disease, acute myocardial infarction within the last 6 months, any known bone disease other than osteoporosis, current or recently (i.e., $<5$ years before the study) treated malignant disease, exacerbation requiring oral corticosteroid or hospitalization in the previous 4 weeks; high-dose (i.e., >10 mg daily), long term systemic corticosteroid therapy; inability to walk; any blood transfusion 4 weeks prior to the first visit; blood donor in the last 3 months prior to the first visit; suspicion of alcohol or drug abuse or any other condition associated with poor compliance in the investigator's opinion; and known infectious transmissible disease: Human Immune-deficiency virus (HIV), hepatitis B or C.

\section{Details of the assessment of outcome measures}

\section{Lung function}

Post-bronchodilator forced expiratory volume in the first second $\left(\mathrm{FEV}_{1}\right)$ and forced vital capacity (FVC) were collected using standardised spirometry (MasterScreen ${ }^{\circledR}$ Body, Carefusion, Germany). Static lung volumes (intrathoracic gas volume [ITGV]) and carbon monoxide transfer factor ( $D_{\mathrm{LcO}}$, single breath hold method) were determined in all patients (MasterScreen ${ }^{\circledR}$ Body, Carefusion, Germany).

\section{Body composition}

Height was measured to the nearest $0.1 \mathrm{~cm}$, whilst body weight was assessed with an electronic beam scale with digital readout to the nearest $0.1 \mathrm{~kg}$ after emptying the bladder and with the subjects standing barefoot and wearing light indoor clothing. Body mass index (BMI) was calculated as body weight in kilograms per squared height in meters $\left(\mathrm{kg} / \mathrm{m}^{2}\right)$ [1]. Fat free mass (FFM) was assessed using an overnight-fasting bioelectrical impedance assessment (Bodystat ${ }^{\circledR}$, United Kingdom). Dual-energy X-ray Absorptiometry (DEXA) had to be used with two patients instead due to total hip replacement $(n=1)$ or presence of iron after sternal surgery $(n=1)$. FFM index (FFMI) was calcu- 
lated as FFM in kilograms per squared height in meters [2]. DEXA scan (Lunar Prodigy ${ }^{\circledR}$, Ge-Lunar) was used to determine hip, lumbar spine and whole-body bone mineral density (BMD).

\section{Sub-clinical atherosclerosis}

A high-resolution B-mode ultrasound with a 10- $\mathrm{MHz}$ linear transducer (Art.LabEsaotePicus, Pie-medical Netherlands/Italy) was used for the assessment of carotid intima media thickness (c-IMT), which was thus quantified semi-automatically, reducing the interobserver variability [3]. C-IMT measurements were performed with the patients in a supine position and throughout $10-\mathrm{mm}$ segments across the far wall of the left and right common carotid arteries, at a point most proximal to the carotid bifurcation free of visual plaques. The probe was moved to obtain measurements of the common carotid artery at 4 angles on both sides $\left(180^{\circ}, 150^{\circ}, 120^{\circ}\right.$, and $\left.90^{\circ}\right)$. For each measured segment mean and maximum IMT values were acquired automatically throughout the $10-\mathrm{mm}$ vessel length. The average of segmental maximum carotid IMT values was determined as carotid IMT per patient.

\section{Cardiac ischaemia}

The cardiac infarction injury score (CIIS) was scored by a cardiologist based on a resting electrocardiogram (ECG). This cardiologist was blinded for patient's medical history and outcome measures. CIIS is an electrocardiographic classification system developed as a diagnostic tool to determine the presence of myocardial infarctions. It is based on the power of certain electrocardiographic characteristics to discriminate between myocardial infarction patients and healthy individuals. These characteristics are weighted and combined into a single score [4].

\section{Blood pressure}

Peripheral blood pressure was measured early in the morning by a trained medical technologist, blinded for clinical outcomes. Patients were instructed to rest for 15 minutes in supine position and after that three measurements were performed, with 5minute intervals between each pair of measurements. Mean values were calculated.

\section{Functional exercise capacity}

The 6-min walk test (6MWT) and the constant work rate test (CWRT) were assessed as measures of functional exercise capacity. The 6MWT was performed according to the guidelines of the European Respiratory Society (ERS) and American Thoracic Society (ATS) [5]. Two tests were performed at the initial assessment to account for a possible 


\section{CHAPTER 3}

learning effect [6], and the best test was used for analysis. The CWRT was performed at $75 \%$ of the pre-determined peak cycling load [7], based on a symptom-limited cardiopulmonary incremental cycle test [8] and following the guidelines of the ATS and the American College of Chest Physicians [9].

\section{Dyspnoea}

The modified Medical Research Council (mMRC) dyspnoea scale was used for the assessment of self-perceived dyspnoea in relation to physical disability [10].

\section{Psychological status}

Symptoms of anxiety and depression were assessed by using the Hospital Anxiety and Depression Scale (HADS), a validated and reliable self-administered instrument widely used in medically ill patients for screening clinically relevant symptoms of anxiety and/or depression [11]. HADS consists of 14 questions distributed in two subscales, an anxiety subscale (HADS-A, 7 questions) and a depression subscale (HADS-D, 7 questions). Total scores for each subscale can range from 0 (optimal) to 21 (worst) points.

\section{Health status}

Health status was assessed by using the St. George's Respiratory Questionnaire (SGRQ), which consists of a 50-item questionnaire divided in three domains (symptoms, activities and impact) [12]. Scores per domain and a total score are provided and they can range from 0 (optimal) to 100 points (worst) [12].

\section{Laboratory analysis}

A venous blood sample was collected from all patients in the fasted state. Serum samples were stored at $-80^{\circ} \mathrm{C}$ prior to their analysis. Haemoglobin, leukocytes, glucose, creatinine, high density lipoprotein (HDL), and triglycerides were determined in all subjects.

\section{Self-reported comorbidities}

Self-reported comorbidities were assessed by the Charlson comorbidity index [13], which is composed of 19 categories of comorbidities. The total score reflects the cumulative increased likelihood of 1-year mortality [14], and higher scores can be translated as a higher burden of comorbidities. The total score was not adjusted for age and did not include COPD as a comorbidity, as done in previous studies [15-17]. 


\section{RESULTS}

Supplementary Table S1 Baseline characteristics of completers and non-completers after pulmonary rehabilitation

\begin{tabular}{|c|c|c|c|}
\hline Variable & Completers & Non-completers & P-value \\
\hline$N$ & 194 & 19 & - \\
\hline Age, years & $63 \pm 7$ & $66 \pm 8$ & 0.10 \\
\hline Male, (\%) & 59 & 58 & 0.91 \\
\hline $\mathrm{BMI}, \mathrm{kg} / \mathrm{m}^{2}$ & $26.3 \pm 5.2$ & $25.5 \pm 4.0$ & 0.50 \\
\hline mMRC dyspnoea grade & $2.1 \pm 1.1$ & $1.7 \pm 0.9$ & 0.13 \\
\hline Current smoker, (\%) & 26 & 47 & 0.05 \\
\hline Pack-years & $47 \pm 26$ & $40 \pm 16$ & 0.27 \\
\hline Long-term oxygen therapy, (\%) & 17 & 21 & 0.54 \\
\hline $\mathrm{FEV}_{1}, \mathrm{~L}$ & $1.40 \pm 0.54$ & $1.41 \pm 0.58$ & 0.91 \\
\hline $\mathrm{FEV}_{1}, \%$ predicted & $51 \pm 17$ & $53 \pm 17$ & 0.72 \\
\hline $\mathrm{FEV}_{1} / \mathrm{FVC}, \%$ & $40 \pm 11$ & $42 \pm 12$ & 0.31 \\
\hline ITGV, \% predicted & $148 \pm 34$ & $143 \pm 30$ & 0.52 \\
\hline $\mathrm{D}_{\mathrm{LCO}}, \%$ predicted & $56 \pm 17$ & $53 \pm 15$ & 0.44 \\
\hline Charlson comorbidity index ${ }^{\dagger}$ & $0.6 \pm 0.9$ & $0.6 \pm 0.6$ & 0.80 \\
\hline Number of comorbidities & $4 \pm 2$ & $4 \pm 2$ & 0.75 \\
\hline 6MWD, $\mathrm{m}$ & $473 \pm 105$ & $449 \pm 125$ & 0.35 \\
\hline CWRT, s* & $356 \pm 270$ & $321 \pm 264$ & 0.59 \\
\hline SGRQ total score & $51.9 \pm 17.7$ & $45.7 \pm 14.5$ & 0.15 \\
\hline SGRQ symptoms score & $55.1 \pm 20.9$ & $55.5 \pm 18.0$ & 0.95 \\
\hline SGRQ activity score & $69.3 \pm 22.0$ & $58.1 \pm 18.3$ & 0.03 \\
\hline SGRQ impact score & $40.2 \pm 20.0$ & $35.8 \pm 19.4$ & 0.36 \\
\hline
\end{tabular}

Data expressed as mean \pm standard deviation for quantitative variables, and relative frequency for discrete variables. See Table 1 for definition of abbreviations. ${ }^{*} \mathrm{n}=202$; ${ }^{\dagger}$ This index score does not include COPD as a comorbidity. 


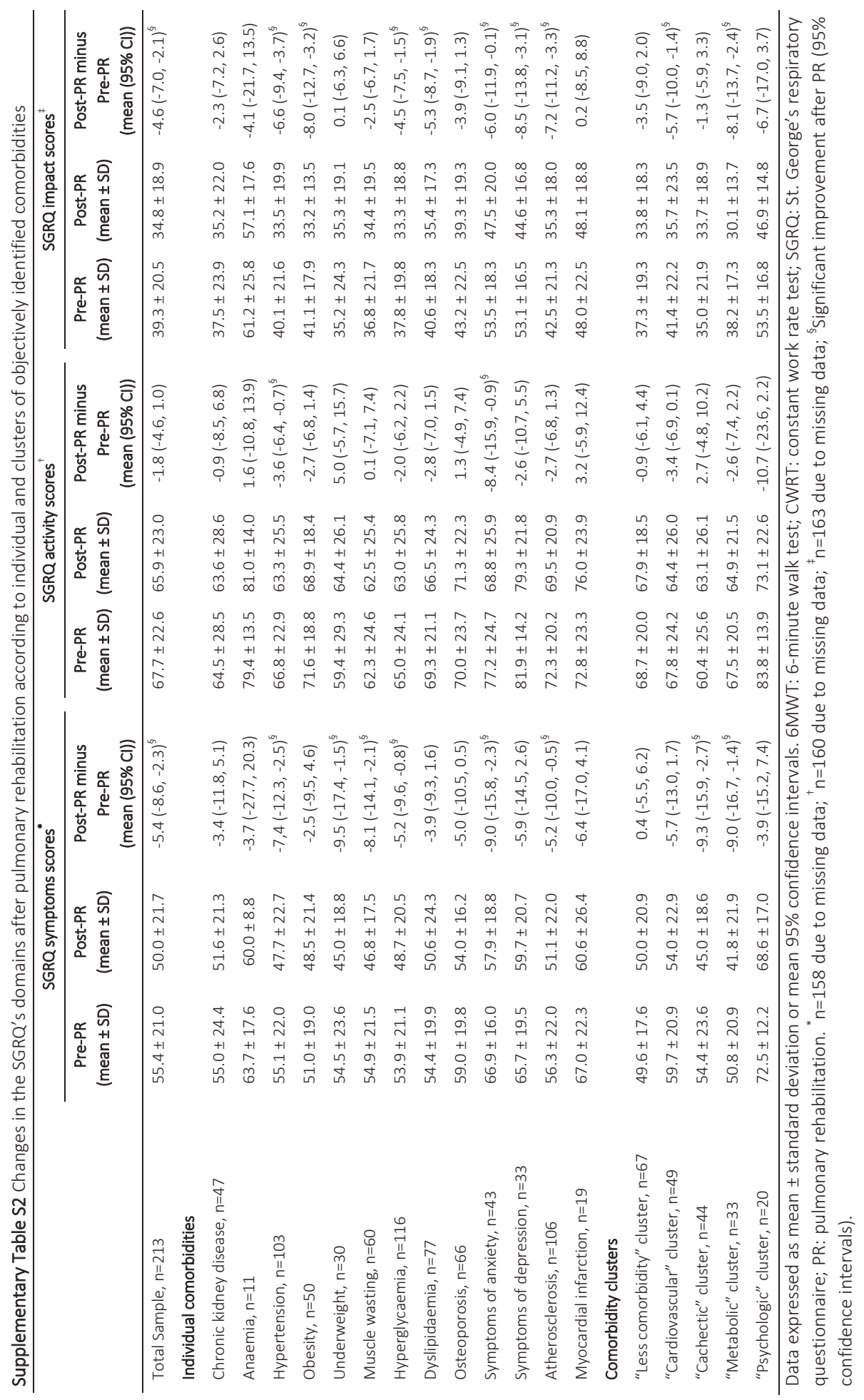




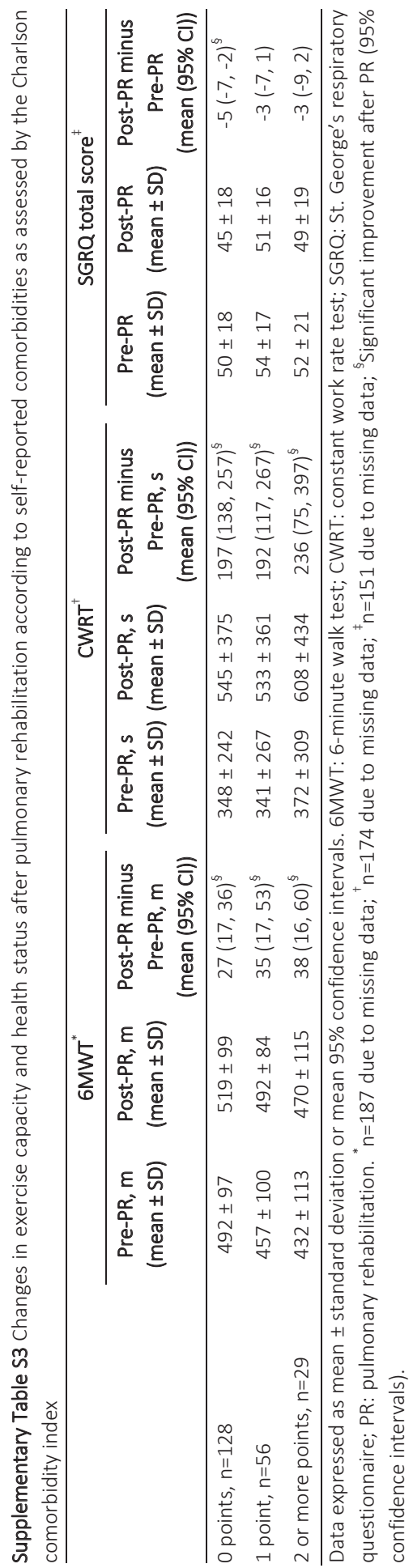




\section{A) 6MWT}

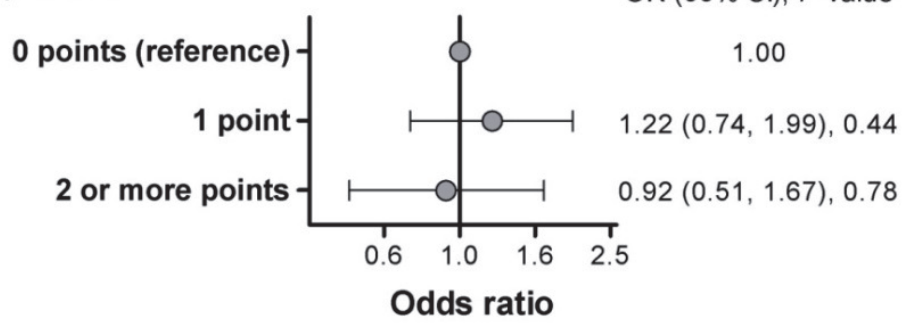

B) CWRT

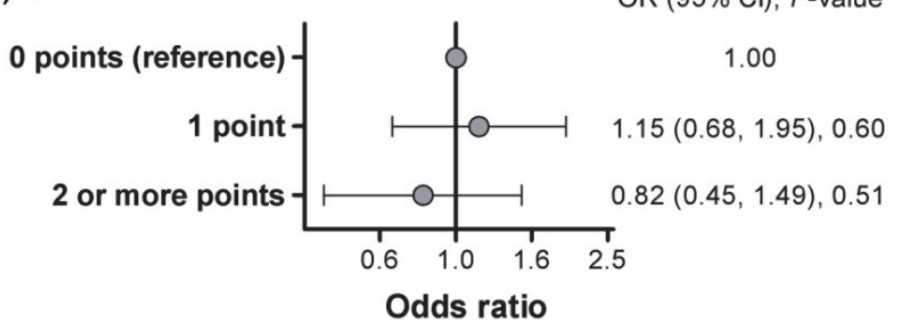

C) SGRQ

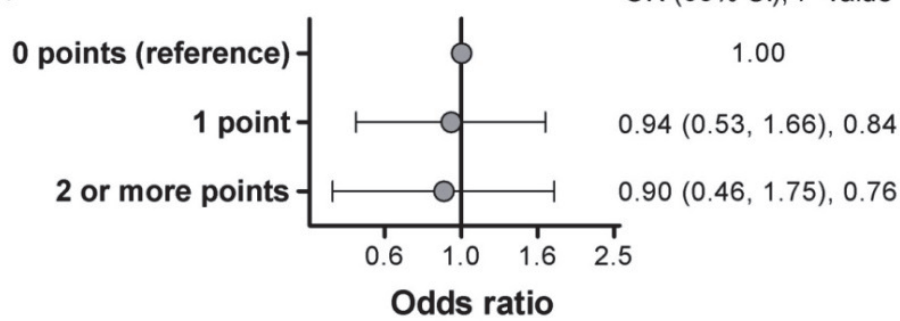

Supplementary Figure S1 Predictive role of self-reported comorbidities as assessed by the Charlson comorbidity index on meaningful improvements in (A) 6-min walk test (6MWT, $n=187$ due to missing data), (B) constant work rate test (CWRT, $n=174$ due to missing data), and (C) St. George Respiratory Questionnaire (SGRQ, $\mathrm{n}=151$ due to missing data) after pulmonary rehabilitation. Models adjusted for age, gender, $\mathrm{FEV}_{1} \%$ predicted and the baseline value in each outcome measure. 
A) 6MWT

OR $(95 \% \mathrm{Cl}), P$-value

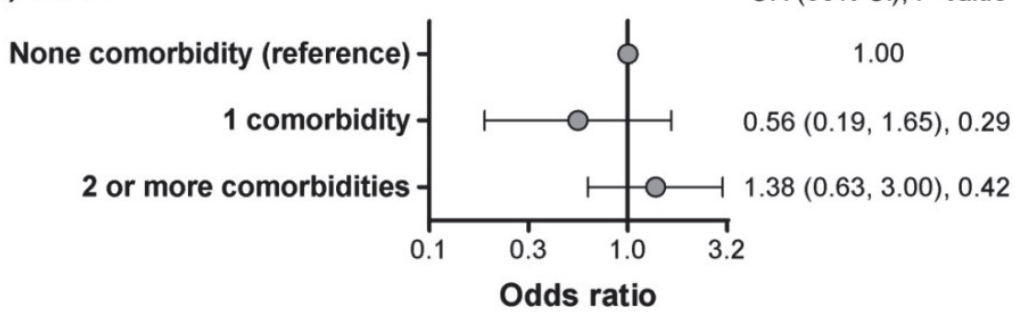

B) CWRT

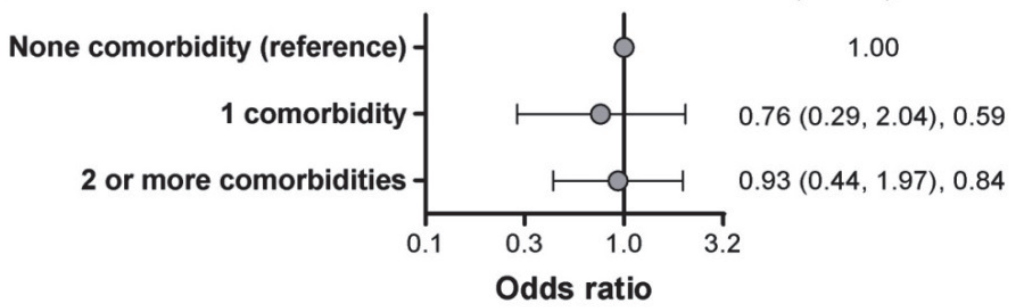

C) SGRQ

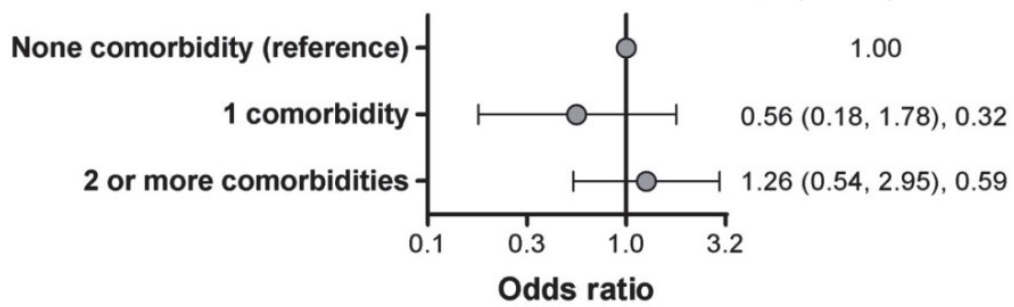

Supplementary Figure S2 Predictive role of the number of objectively identified comorbidities on meaningful improvements in (A) 6-min walk test (6MWT, $n=187$ due to missing data), (B) constant work rate test (CWRT, $\mathrm{n}=174$ due to missing data), and (C) St. George Respiratory Questionnaire (SGRQ, $n=151$ due to missing data) after pulmonary rehabilitation. Models adjusted for age, gender, $\mathrm{FEV}_{1} \%$ predicted and the baseline value in each outcome measure. 


\section{References}

1. Keys A, Fidanza F, Karvonen MJ, et al. Indices of relative weight and obesity. J Chronic Dis 1972; 25: 329343.

2. Schols AM, Wouters EF, Soeters PB, et al. Body composition by bioelectrical-impedance analysis compared with deuterium dilution and skinfold anthropometry in patients with chronic obstructive pulmonary disease. Am J Clin Nutr 1991; 53: 421-424.

3. Rossi AC, Brands PJ, Hoeks AP. Automatic recognition of the common carotid artery in longitudinal ultrasound B-mode scans. Med Image Anal 2008; 12: 653-665.

4. Rautaharju PM, Warren JW, Jain U, et al. Cardiac infarction injury score: an electrocardiographic coding scheme for ischemic heart disease. Circulation 1981; 64: 249-256.

5. Holland AE, Spruit MA, Troosters T, et al. An official European Respiratory Society/American Thoracic Society Technical Standard: field walking tests in chronic respiratory disease. Eur Respir J 2014; 44 :14281446.

6. Hernandes NA, Wouters EF, Meijer K, et al. Reproducibility of 6-minute walking test in patients with COPD. Eur Respir J 2011; 38: 261-267.

7. van 't Hul A, Gosselink R, Kwakkel G. Constant-load cycle endurance performance: test-retest reliability and validity in patients with COPD. J Cardiopulm Rehabil 2003; 23: 143-150.

8. Sillen MJ, Franssen FM, Delbressine JM, et al. Heterogeneity in clinical characteristics and co-morbidities in dyspneic individuals with COPD GOLD D: findings of the DICES trial. Respir Med 2013; 107: 1186-1194.

9. American Thoracic Society, American College of Chest Physicians. ATS/ACCP Statement on cardiopulmonary exercise testing. Am J Respir Crit Care Med 2003; 167: 211-277.

10. Bestall JC, Paul EA, Garrod R, et al. Usefulness of the Medical Research Council (MRC) dyspnoea scale as a measure of disability in patients with chronic obstructive pulmonary disease. Thorax 1999; 54: 581-586.

11. Zigmond AS, Snaith RP. The hospital anxiety and depression scale. Acta Psychiatr Scand 1983; 67: 361370.

12. Jones PW, Quirk FH, Baveystock CM, et al. A self-complete measure of health status for chronic airflow limitation. The St. George's Respiratory Questionnaire. Am Rev Respir Dis 1992; 145: 1321-1327.

13. Charlson ME, Pompei $\mathrm{P}$, Ales $\mathrm{KL}$, et al. A new method of classifying prognostic comorbidity in longitudinal studies: development and validation. J Chronic Dis 1987; 40: 373-383.

14. Charlson M, Szatrowski TP, Peterson J, et al. Validation of a combined comorbidity index. J Clin Epidemiol 1994; 47: 1245-1251.

15. Marti S, Munoz X, Rios J, et al. Body weight and comorbidity predict mortality in COPD patients treated with oxygen therapy. Eur Respir J 2006; 27: 689-696.

16. Crisafulli E, Costi S, Luppi F, et al. Role of comorbidities in a cohort of patients with COPD undergoing pulmonary rehabilitation. Thorax 2008; 63: 487-492.

17. Crisafulli E, Gorgone P, Vagaggini B, et al. Efficacy of standard rehabilitation in COPD outpatients with comorbidities. Eur Respir J 2010; 36: 1042-1048. 


\section{CHAPTER 4}

Reliability, construct validity and determinants of 6 -minute walk test performance in patients with chronic heart failure

Nicole H.M.K. Uszko-Lencer ${ }^{*}$, Rafael Mesquita ${ }^{*}$, Eefje Janssen, Christ Werter, Hans-Peter Brunner-La Rocca, Fabio Pitta, Emiel F.M. Wouters, and Martijn A. Spruit

${ }^{*}$ Both authors contributed equally.

International Journal of Cardiology 2017; 240: 285-290 


\begin{abstract}
Background: In-depth analyses of the measurement properties of the 6-minute walk test (6MWT) in patients with chronic heart failure (CHF) are lacking. We investigated the reliability, construct validity, and determinants of the distance covered in the 6MWT (6MWD) in CHF patients.
\end{abstract}

Methods: 337 patients were studied (median age 65 years, 70\% male, ejection fraction $35 \%)$. Participants performed two 6MWTs on subsequent days. Demographics, anthropometrics, clinical data, ejection fraction, maximal exercise capacity, body composition, lung function, and symptoms of anxiety and depression were also assessed. Construct validity was assessed in terms of convergent, discriminant and known-groups validity. Stepwise linear regression was used.

Results: 6MWT was reliable $(I C C=0.90, P<0.0001)$. The learning effect was $31 \mathrm{~m}(95 \% \mathrm{Cl}$ $27,35 \mathrm{~m}$ ). Older age ( $\geq 65$ years), lower lung diffusing capacity ( $<80 \%$ predicted) and higher NYHA class (NYHA III) were associated with a lower likelihood of a meaningful increase in the second test (OR 0.45-0.56, $\mathrm{P}<0.05$ for all). The best 6MWD had moderate-to-good correlations with peak exercise capacity $\left(r_{s}=0.54-0.69\right)$ and no-to-fair correlations with body composition, lung function, ejection fraction, and symptoms of anxiety and depression ( $\left.r_{s}=0.04-0.49\right)$. Patients with higher NYHA classes had lower 6MWD. 6MWD was independently associated with maximal power output during maximal exercise, estimated glomerular filtration rate and age (51.7\% of the variability).

Conclusion: 6MWT was found to be reliable and valid in patients with mild-to-moderate CHF. Maximal exercise capacity, renal function and age were significant determinants of the best 6MWD. These findings strengthen the clinical utility of the 6MWT in CHF. 


\section{INTRODUCTION}

The 6-minute walk test (6MWT) is a standardized field test to evaluate functional exercise performance in persons with a variety of chronic conditions (1). Subjects are instructed to walk as far as possible in 6 minutes on a flat and hard surface. The 6MWT is believed to mimic, at least in part, daily functionality, and is generally well tolerated and safe $(2,3)$.

The 6-minute walk distance (6MWD) has important prognostic value in patients with chronic heart failure (CHF) $(4,5)$. Indeed, a $6 \mathrm{MWD}<300 \mathrm{~m}$ was associated with a higher 1-year mortality (4-6), whilst a 6MWD $<468 \mathrm{~m}$ was associated with an increased hospitalization rate (7). Therefore, $6 \mathrm{MWT}$ has also been recommended to monitor the disease course and to assess the efficacy of interventions (8).

Measurement properties of the 6MWT, such as reliability and construct validity, have been studied in $\mathrm{CHF}(3,9)$. Indeed, $6 \mathrm{MWT}$ has been shown to be reliable and to associate positively with peak aerobic capacity in patients with $\operatorname{CHF}(2,4,10)$. Nonetheless, considerable variability can be found among studies. For instance, the amount of improvement between first and second 6MWTs was reported to vary between $5 \%$ and $33 \%$ (11). This is probably a consequence of the learning effect related to this test, i.e., patients achieving a better 6MWD during the second test. However, it remains unclear which clinical characteristics can determine the extent of learning effect between two 6MWTs. Correlation coefficients between 6MWD and peak oxygen consumption $\left(\mathrm{V}_{2}\right)$ and/or maximum power during symptom-limited exercise tests also varied considerably between studies, ranging from 0.59 to 0.93 (12-14). Other parameters were also investigated in previous studies, but most focused on a limited set of parameters (e.g., quality of life, New York Heart Association [NYHA] class) (15). Common comorbidities such as chronic renal failure, chronic lung failure, muscle loss and/or obesity may also affect the performance in the 6MWT in patients with CHF. To date, in-depth analyses of the measurement properties of the 6MWT and determinants of $6 \mathrm{MWD}$ in patients with $\mathrm{CHF}$ have not been performed.

Therefore, in a convenience sample of patients with CHF entering a rehabilitation program we aimed: 1) to investigate the reliability of the 6MWT between two tests performed on subsequent days, quantifying the learning effect between tests and studying possible determinants of this learning effect; 2 ) to evaluate the construct validity of the 6MWT; and 3) to identify the clinical determinants of the best 6MWD. 


\section{METHODS}

In this retrospective observational study, 344 patients with CHF entering a rehabilitation program at CIRO, in Horn, the Netherlands, between 2005 and 2013 were screened for eligibility. The data used in this study were collected during the routine prerehabilitation assessment. Patients were included in the current analysis if they met the following criteria: diagnosis of CHF according to the European Society of Cardiology (ESC) guidelines (15); clinical stability (no decompensations/hospitalizations in the last 3 months); and two 6MWTs performed on subsequent days. Seven patients performed the 6MWT once only and therefore were excluded. In total, 337 patients could be included (Table 1). The current retrospective analyses are institutional review board exempt due to the use of de-identified, pre-existing data. The Board of Directors of CIRO approved the use of de-identified patients' records.

\section{6-min walk test}

The 6MWT was performed according to international guidelines (1). Patients were instructed to walk at their own pace while attempting to cover the greatest distance possible. Standardized encouragement was provided every minute by telling the patients either "You are doing well" or "Keep up the good work" and informing them the remaining time (17). Two 6MWTs were conducted on subsequent days in a triangular walking course of $125 \mathrm{~m}$. The tests were supervised by qualified staff that walked behind the patient. Patients were permitted to stop (if required) during the test, but were instructed to resume walking once able. 6MWD was registered in meters and patients were allowed to use their walking aids during the tests. Before, during and after the 6MWTs, heart rate $(\mathrm{HR})$ and oxygen saturation $\left(\mathrm{SpO}_{2}\right)$ were measured by pulse oximetry (Model 2500, Nonin Medical, Inc., Minneapolis, MN, USA), whilst perceived dyspnea and leg fatigue were assessed with the modified Borg scale, a 0-10 point scale concerning dyspnea and leg fatigue ( $0=$ no symptoms; $10=$ worst symptoms). Oxygen desaturation during the 6MWT was defined as a decline of $4 \%$ or more in $\mathrm{SpO}_{2}$ and/or an end $\mathrm{SpO}_{2}$ of $<88 \%$ (18). 6MWD reference values were calculated according to Troosters et al. (19).

\section{CHF severity}

The NYHA functional classification was used to evaluate the functional limitation due to CHF (20). The left ventricular ejection fraction (LVEF) was measured by resting echocardiography (MyLab, Esaote, Italy). The estimated glomerular filtration rate (eGFR) was calculated using the Cockcroft-Gault formula. eGFR is used as a measure of renal function, which in $\mathrm{CHF}$ is known to be an independent prognostic marker $(21,22)$. Based on their eGRF patients can be classified into one out of 4 groups: normal, $\geq 90 \mathrm{~mL} / \mathrm{min}$; 
mildly depressed eGFR, 60 to $89 \mathrm{~mL} / \mathrm{min}$; moderately depressed eGFR, 30 to 59 $\mathrm{mL} / \mathrm{min}$; and severely depressed eGFR, $<30 \mathrm{~mL} / \mathrm{min}(22)$.

\section{Other measurements}

Demographics (sex and age), anthropometrics (body weight and height), and the use of walking aids (rollator or cane) were assessed. Resting lung function (Masterlab ${ }^{\circledR}$, Jaeger, Würzburg, Germany) was measured by post-bronchodilator spirometry, with the forced expiratory volume in the first second $\left(\mathrm{FEV}_{1}\right)$ and $\mathrm{FEV}_{1} /$ forced vital capacity (FVC) ratio (23); whole-body plethysmography, with residual volume (RV), total lung capacity (TLC) and the ratio between RV and TLC; and the single-breath method, with diffusion capacity of the lung for carbon monoxide $\left(D_{L C O}\right)$. Obstructive spirometry was defined as a $\mathrm{FEV}_{1} / \mathrm{FVC}$ ratio $<0.70$. Maximal exercise capacity was assessed with a symptom-limited incremental (+10 watts per minute) cardiopulmonary exercise test (CPET) (24) performed on a cycle ergometer (Carefusion, Houten, The Netherlands; Oxycon $\beta$, Jaeger, Würzburg, Germany). Maximal exercise effort was verified with a maximal respiratory quotient (RQ) greater than one (25). For the assessment of body composition, a whole body scan was performed by DEXA method (Lunar Prodigy system; GE Healthcare, Piscataway, NJ, USA). This method measures the amount of lean mass and bone mineral density, which combined equal the amount of fat free mass (FFM). The body mass index (BMI) was calculated as body weight $(\mathrm{kg})$ divided by height squared $\left(\mathrm{m}^{2}\right)$, while the fat free mass index (FFMI) was calculated as FFM (in $\mathrm{kg}$ ) divided by height squared $\left(\mathrm{m}^{2}\right.$ ). Lower-limb lean muscle mass (LL-LMM) was also analyzed. Participants were classified as having normal or abnormal low FFMI based on the fifth percentile of previously defined reference values (26). The Charlson comorbidity index was used to assess selfreported comorbidities (27). Symptoms of anxiety and depression were investigated with the Hospital Anxiety and Depression scale (HADS) (28).

\section{Statistical analyses}

Statistical analyses were performed using SPSS 21.0 (IBM Inc., Chicago, IL, USA), and Graph Pad Prism 6 (Graph Pad Software Inc., La Jolla, CA, USA). Categorical data were expressed as absolute and/or relative frequency, and continuous data as mean \pm SD or median (IQR). Shapiro-Wilk test was used to test normality in data distribution. To test the reliability of 6MWT, the two-way random intra-class correlation coefficient with single measures $\left(\mathrm{ICC}_{2,1}\right)$ was calculated. The Kappa coefficient was used to test the agreement between the two 6MWTs for the proportion of patients stratified according to the following cut-off points: 200,300, and $400 \mathrm{~m}$. These values are close to the mean values of patients with NYHA classes I/II, III and IV, respectively (29). To analyze the agreement between two 6MWTs, the Bland and Altman plot was used. The paired t-test was used to test differences between two 6MWTs. The unpaired t-test, one-way ANO- 
VA (post-hoc Tukey), or chi square was used to compare groups. Logistic regression analysis adjusted for age, sex, BMI, Charlson scores, and LVEF was used to investigate the determinants of a change between the two 6MWTs above the upper limit of a clinically meaningful change in CHF (i.e., $\geq 39.4 \mathrm{~m}$ (30)). Construct validity was investigated in terms of convergent, discriminant, and known-groups validity. Construct validity refers to the degree to which a test measures what it claims, or purports, to be measuring. Convergent validity shows that measures that should be related are in reality related; discriminant validity shows that measures that should not be related are indeed not related; and known-groups validity shows that an instrument or test is able to demonstrate different scores for groups known to vary on the variable being measured (31). To investigate convergent and discriminant validity, Pearson or Spearman correlation coefficients were calculated. For convergent validity test, we hypothesized that the best 6MWD would correlate positively and at least moderately ( $r>0.50)$ (31) with measures of maximal exercise capacity (i.e., maximal power output and $\mathrm{V}_{2}$ ). Moreover, little-tofair correlations $(r<0.50)$ (31) would be found with measures of body composition, lung function, LVEF, and symptoms of anxiety and depression, supporting discriminant validity. For known-groups validity, patients with different NYHA classes were compared. Patients with higher NYHA classes would have shorter 6MWDs. Finally, a stepwise multiple linear regression model was built to identify the parameters that independently explained the variance in the best 6MWD. Only variables with a correlation coefficient of at least 0.20 were considered for analysis. In case of multicollinearity (i.e., $r \geq 0.70$ ) or singularity between two independent variables, only the variable with the strongest correlation coefficient with the dependent variable was kept in the model. All tests were carried out as two-tailed with a chosen significance level of $P \leq 0.05$.

\section{RESULTS}

\section{Characteristics of the patients}

Demographic and clinical characteristics of 337 patients with mild-to-moderate CHF are summarized in Table 1. The majority of patients were male, with overweight-to-obese BMI, severely impaired LVEF, and with symptoms compatible with NYHA functional class II. Only a minority of patients used a rollator (5\%) or cane (1\%). Fifteen percent had a pacemaker and $42 \%$ had an implantable cardioverter defibrillator. Moreover, $44 \%$ had an obstructive spirometry and $72 \%$ had a mildly-to-severely depressed renal function. The median maximal RQ during the CPET was 1.13 , which is $>1$ and therefore suggestive of maximal effort (25). 
Table 1 Characteristics of patients with chronic heart failure

\begin{tabular}{|c|c|c|}
\hline Characteristic & $\begin{array}{c}\text { Patients } \\
\mathrm{n}\end{array}$ & Value \\
\hline Age, years & 337 & $65(54-73)$ \\
\hline Male sex, $\mathrm{n}(\%)$ & 337 & $235(70)$ \\
\hline Rollator, n (\%) & 334 & $18(5)$ \\
\hline Cane, n (\%) & 329 & $4(1)$ \\
\hline Body weight, kg & 336 & $85.1(74.1-95.7)$ \\
\hline $\mathrm{BMI}, \mathrm{kg} \cdot \mathrm{m}^{-2}$ & 336 & $28.4(25.5-32.2)$ \\
\hline $\mathrm{BMI}<18.5 / 18.5-24.99 / 25-29.99 / \geq 30 \mathrm{~kg} \cdot \mathrm{m}^{-2}, \mathrm{n}(\%)$ & 336 & $2(1) / 75(22) / 136(40) / 123(37)$ \\
\hline FFM, kg & 312 & $56.0(47.8-62.4)$ \\
\hline $\mathrm{FFMI}, \mathrm{kg} \cdot \mathrm{m}^{-2}$ & 311 & $18.6 \pm 2.4$ \\
\hline LL-LMM, kg & 312 & $16.9(14.3-18.9)$ \\
\hline LVEF, \% & 337 & $35(27-45)$ \\
\hline LVEF $\geq 55$ / 45-54 / 30-44 / <30 \%, n (\%) & 337 & $37(11) / 51(15) / 149(44) / 100$ (30) \\
\hline Pacemaker, n (\%) & 317 & $47(15)$ \\
\hline Implantable cardioverter defibrillator, n (\%) & 334 & $141(42)$ \\
\hline NYHA I / II / III, n (\%) & 311 & $23(7) / 195(63) / 93$ (30) \\
\hline $\mathrm{FEV}_{1}, \mathrm{~L}$ & 335 & $2.60(2.09-3.34)$ \\
\hline $\mathrm{FEV}_{1}, \%$ pred & 334 & $91 \pm 22$ \\
\hline $\mathrm{FEV}_{1} / \mathrm{FVC}$ ratio, $\%$ & 332 & $71(64-78)$ \\
\hline$R V, L$ & 327 & $2.16(1.75-2.53)$ \\
\hline RV, \% pred & 326 & $94(80-109)$ \\
\hline TLC, L & 327 & $5.90(5.05-6.97)$ \\
\hline TLC, \% pred & 326 & $95 \pm 15$ \\
\hline RV/TLC ratio, \% & 327 & $35(31-42)$ \\
\hline$D_{L C O}, L$ & 316 & $5.92(4.87-7.34)$ \\
\hline $\mathrm{D}_{\mathrm{LCO}}, \%$ pred & 315 & $69(59-79)$ \\
\hline eGFR, $\mathrm{ml} / \mathrm{min}$ & 275 & $71(52-93)$ \\
\hline Charlson Index 1 / 2 / >2, n (\%) & 337 & $136(40) / 107(32) / 94(28)$ \\
\hline HADS anxiety, points & 323 & $6(3-9)$ \\
\hline HADS depression, points & 323 & $5(2-8)$ \\
\hline Maximal power output CPET, watts & 336 & $100(74-130)$ \\
\hline $\mathrm{VO}_{2} \max \mathrm{CPET}, \mathrm{ml} / \mathrm{min}$ & 328 & $1293(1034-1663)$ \\
\hline $\mathrm{VO}_{2} \max C P E T, \mathrm{ml} / \mathrm{min} / \mathrm{kg}$ & 326 & $15.5(12.8-18.6)$ \\
\hline
\end{tabular}

Data are expressed as absolute and relative frequencies, mean \pm SD or median (IQR). BMI: body mass index; FFM: fat-free mass; FFMI: fat-free mass index; LL: lower-limbs; LMM: lean muscle mass; LVEF: Left Ventricle Ejection Fraction; NYHA: New York Heart Association; FEV ${ }_{1}$ : forced expiratory volume in 1 second; FVC: forced vital capacity; RV: residual volume; TLC: total lung capacity; $\mathrm{D}_{\mathrm{Lco}}$ : diffusing capacity of the lung for carbon 
monoxide; eGRF: estimated glomerular filtration rate; HADS: Hospital Anxiety and Depression Scale; CPET: cardiopulmonary exercise test; $\mathrm{VO}_{2}$ max: maximal oxygen consumption.

\section{Reliability of the 6MWT}

During the two 6MWTs patients walked, on average, $473 \mathrm{~m}(95 \% \mathrm{Cl} 462,484 \mathrm{~m})$ and $504 \mathrm{~m}$ (95\% Cl 492, $516 \mathrm{~m}$ ), respectively (mean change: $31 \mathrm{~m}$ (95\% Cl 27, $35 \mathrm{~m}$ ) or $7 \%$ (95\% Cl 6, $8 \%$ ); $\mathrm{P}<0.0001$ ). Two hundred eighty-one patients (83\%) walked further in the second test, with 134 (40\% of total) having an increase $>39.4 \mathrm{~m}$, which is the upper limit of a clinically meaningful change in 6MWD for patients with CHF (30). Bland and Altman plots confirmed that the majority of patients increased their 6MWD during the second 6MWT (Figures $1 \mathrm{~A}$ and $1 \mathrm{~B}$ ). The limits of agreement between first and second 6MWTs ranged from -42 to $104 \mathrm{~m}$ in absolute values (Figure $1 \mathrm{~A}$ ) and from -12 to $25 \%$ in percentage of improvement (Figure 1B). The ICC showed good reliability between the two 6MWTs (ICC=0.90 (95\% Cl 0.63, 0.96), P<0.0001). Substantial agreement (32) was found for the proportion of participants with a 6MWD lower than 200, 300, and $400 \mathrm{~m}$ between the two 6MWTs (Kappa coefficients of $0.75,0.78$, and 0.72 , respectively). Logistic regression models showed that patients with older age, lower lung diffusing capacity or higher NYHA class had a lower likelihood of having a meaningful increase in 6MWD in the second 6MWT (Figure A.1.1 in appendix A, Supplementary data), while no other parameter reached statistical significance. These characteristics did not importantly influence the reliability of the $6 \mathrm{MWT}$ in terms of ICC values $(0.87 \leq \mathrm{ICC} \leq 0.90$; Table A.1.1 in appendix A, Supplementary data). Nevertheless, poorer agreement for the proportion of patients with a poor 6MWD (i.e., <300 $\mathrm{m}(4-6))$ was observed in older patients (i.e., $\geq 65$ years) and in patients with NYHA class I or II (Kappa coefficients of 0.56 and 0.50 , respectively; Table A.1.1 in appendix A, Supplementary data).
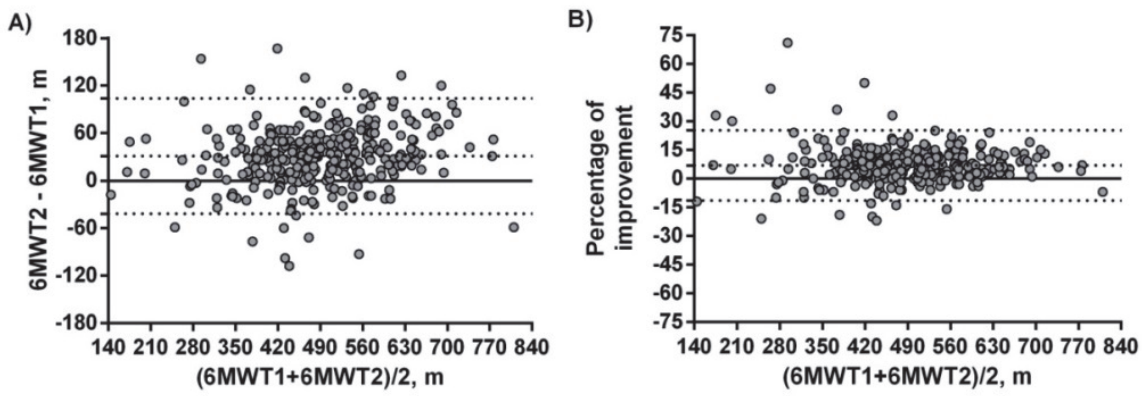

Figure 1 Bland and Altman plots of the difference between two 6-minute walk tests (6MWTs) expressed in absolute values (A) and in percentage of improvement (B) plotted against the mean value between these tests in patients with CHF. The central continuous line corresponds to the zero line; the central dotted line corresponds to the average difference between the two 6MWTs (Figure 1A: $31 \mathrm{~m}$; Figure 1B: 7 \%); and the lower 
and upper dotted lines correspond to the lower (Figure 1A: -42 m; Figure 1B: -12 \%) and upper (Figure 1A: 104 m; Figure 1B: $25 \%$ ) limits of agreement, respectively.

\section{Construct validity of 6MWD}

The average 6MWD on the best test was $509 \mathrm{~m}$ (95\% Cl 497, $520 \mathrm{~m}$ ), which corresponds to $79 \%$ predicted $(95 \% \mathrm{Cl} 77,80 \%$ predicted). Table 2 presents the correlation coefficient between the best 6MWD and other outcome measures. Moderate-to-good correlations (i.e., $r>0.50$ ) were found only with measures of maximal exercise capacity. The strongest correlation was found with maximal power output $\left(r_{s}=0.69, P<0.0001\right.$; Figure A.1.2 in appendix A; Supplementary data). No-to-fair correlations were found with measures of body composition, lung function, LVEF, and with symptoms of anxiety and depression (i.e., $\left.r_{s}=0.04-0.49\right)$. There was no significant correlation with LVEF $\left(r_{s}=-\right.$ $0.05, \mathrm{P}=0.37$; Figure A.1.3 in appendix A; Supplementary data). A lower mean 6MWD

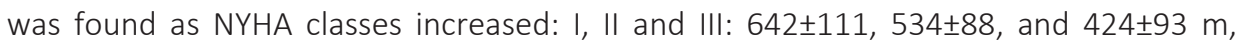
respectively $(\mathrm{P}<0.0001$ for all comparisons), supporting known-groups validity.

Table 2 Correlations between best 6-minute walk distance and other health outcome measures in patients with chronic heart failure

\begin{tabular}{|c|c|c|c|}
\hline Outcome measure & $\begin{array}{c}\text { Patients } \\
\mathrm{n}\end{array}$ & $r_{s}(95 \% \mathrm{Cl})$ & P-value \\
\hline Age, years & 337 & $-0.40(-0.49,-0.31)$ & $<0.0001$ \\
\hline $\mathrm{FEV}_{1}, \mathrm{~L}$ & 335 & $0.49(0.40,0.57)$ & $<0.0001$ \\
\hline $\mathrm{FEV}_{1} / \mathrm{FVC}$ ratio, $\%$ & 332 & $0.12(0.00,0.22)$ & 0.04 \\
\hline$R V, L$ & 327 & $0.04(-0.07,0.15)$ & 0.49 \\
\hline TLC, L & 327 & $0.42(0.33,0.51)$ & $<0.0001$ \\
\hline RV/TLC ratio, \% & 327 & $-0.37(-0.46,-0.27)$ & $<0.0001$ \\
\hline$D_{L C O}, L$ & 316 & $0.41(0.31,0.50)$ & $<0.0001$ \\
\hline Body weight, kg & 336 & $0.11(-0.00,0.22)$ & 0.045 \\
\hline $\mathrm{BMI}, \mathrm{kg} \cdot \mathrm{m}^{-2}$ & 336 & $-0.11(-0.22,-0.00)$ & 0.04 \\
\hline FFM, kg & 312 & $0.31(0.20,0.41)$ & $<0.0001$ \\
\hline $\mathrm{FFMI}, \mathrm{kg} \cdot \mathrm{m}^{-2}$ & 311 & $0.11(-0.00,0.22)^{*}$ & 0.06 \\
\hline LL-LMM, kg & 312 & $0.36(0.26,0.46)$ & $<0.0001$ \\
\hline LVEF, \% & 337 & $-0.05(-0.16,0.06)$ & 0.37 \\
\hline eGFR, $\mathrm{ml} / \mathrm{min}$ & 275 & $0.32(0.20,0.42)$ & $<0.0001$ \\
\hline HADS anxiety, points & 323 & $-0.06(-0.17,0.05)$ & 0.27 \\
\hline HADS depression, points & 323 & $-0.16(-0.26,-0.04)$ & 0.005 \\
\hline Maximal power output CPET, watts & 336 & $0.69(0.63,0.75)$ & $<0.0001$ \\
\hline $\mathrm{VO}_{2} \max \mathrm{CPET}, \mathrm{ml} / \mathrm{min}$ & 328 & $0.54(0.46,0.62)$ & $<0.0001$ \\
\hline $\mathrm{VO}_{2} \max \mathrm{CPET}, \mathrm{ml} / \mathrm{min} / \mathrm{kg}$ & 326 & $0.58(0.50,0.65)$ & $<0.0001$ \\
\hline
\end{tabular}

See Table 1 for definition of abbreviations. : Pearson correlation coefficient. 


\section{Determinants of the best 6MWD}

All the variables with a correlation coefficient of at least 0.20 or -0.20 were considered in a stepwise multiple linear regression model: age, FEV $1, T L C, R V / T L C$ ratio, $D_{L C O}, F F M$, LL-LMM, eGFR, maximal power output, $\mathrm{V} \mathrm{O}_{2}$ max in $\mathrm{ml} / \mathrm{min}$ and $\mathrm{V}_{2}$ max in $\mathrm{ml} / \mathrm{min} / \mathrm{kg}$ (Table 2). RV/TLC ratio and $\mathrm{VO}_{2} \max$ in $\mathrm{ml} / \mathrm{min}$ had to be excluded due to singularity with TLC and $\mathrm{VO}_{2}$ max in $\mathrm{ml} / \mathrm{min} / \mathrm{kg}$, respectively. Moreover, $\mathrm{FEV}_{1}$ and $\mathrm{VO}_{2}$ max in $\mathrm{ml} / \mathrm{min} / \mathrm{kg}$ had to be excluded due to multicollinearity with maximal power output $\left(r_{s}=0.71, P<0.0001\right.$ for both) and FFM due to multicollinearity with LL-LMM $\left(r_{s}=0.96\right.$; $\mathrm{P}<0.0001)$. The following variables were then included in the model: age, TLC, $\mathrm{D}_{L C O}, \mathrm{LL}-$ LMM, eGFR, and maximal power output during the CPET. As there was a difference in 6MWD between male and female patients $(525 \pm 110$ vs $470 \pm 95 \mathrm{~m}$, respectively; $\mathrm{P}<0.0001$ ), sex was also included in the model. The results revealed that only maximal power output during the CPET, eGFR, and age independently influenced the best distance covered in the 6MWT, accounting for $51.7 \%$ of the variance (Table 3 ).

Table 3 Stepwise multiple linear regression analysis with the best 6MWD as the dependent variable

\begin{tabular}{lccccc}
\hline & \multicolumn{2}{c}{$\begin{array}{c}\text { Unstandardized coeffi- } \\
\text { cients }\end{array}$} & $\begin{array}{c}\text { Standardized } \\
\text { coefficients }\end{array}$ & P-value & $\begin{array}{c}\text { Part } \\
\text { correlation }\end{array}$ \\
\hline Constant & B & Standard error & Beta & NA & NA \\
Maximal power output, watts & 1.898 & 0.139 & 0.763 & $<0.0001$ & 0.601 \\
eGFR, ml/min & -0.695 & 0.197 & -0.225 & 0.001 & -0.154 \\
Age, years & -1.290 & 0.509 & -0.149 & 0.01 & -0.111 \\
\hline
\end{tabular}

eGFR: estimated glomerular filtration rate.

\section{DISCUSSION}

The 6MWT was found to be reliable, but a learning effect of $31 \mathrm{~m} \mathrm{(95 \%} \mathrm{Cl} \mathrm{27,} 35 \mathrm{~m}$ ) was observed and older age, lower lung diffusing capacity and a higher NYHA class among a comprehensive number of possible determinants were associated with a lower likelihood of a meaningful increase in the second 6MWT. Moderate-to-good correlations with measures of maximal exercise capacity supported the convergent validity of the test. Moreover, maximal power output during the CPET, estimated glomerular filtration rate and age were identified as determinants of the best distance covered during the $6 \mathrm{MWT}$, explaining more than half of its variance.

The 6MWT showed to be reliable when performed twice on subsequent days (ICC=0.90 [95\% Cl 0.63, 0.96]; $\mathrm{P}<0.0001)$, which is consistent with earlier reports by Cahalin et al. (4), O'Keeffe et al. (33), and Hamilton et al. (2). Although good reliability was observed, 
we also observed a significant increase of $31 \mathrm{~m}(95 \% \mathrm{Cl} 27,35 \mathrm{~m})$ between the two tests. In fact, $83 \%$ of the patients would have a false low 6MWD if only one test was used and $40 \%$ of the patients improved more than $39.4 \mathrm{~m}$, which represents a clinically meaningful change in patients with CHF (30). Interestingly, substantial agreement was found (Kappa coefficient of 0.78 ) between the two 6MWTs for the proportion of patients with a poor 6MWD (i.e., <300 m (4-6)). This suggests that if the aim is to identify patients with a poor 6MWD, one test may be enough. Nevertheless, as lower Kappa coefficient values were observed in older patients (i.e., $\geq 65$ years) and in patients with NYHA classes I or II, so in order to have an accurate identification of a poor 6MWT performance in these subgroups two tests might be necessary.

In a population of CHF patients, Adsett et al. (34) observed no learning effect in patients with a poor 6MWD in the first test (i.e., <300 m). Our findings seem to suggest the contrary, i.e., an association between a poor distance in the first test and a greater change in the second test (Figure 1B). However, when we tested whether a poor distance in the first test (i.e., <300 m) could predict a clinically meaningful change in the second test, the result did not reach statistical significance (Figure 2). In patients with chronic obstructive pulmonary disease, BMI, Charlson comorbidity index, and a poor distance in the first test (i.e., $<350 \mathrm{~m}$ ) were identified as significant determinants of a clinically meaningful change in the second test (35). In turn, we observed that older age, lower lung diffusing capacity and higher NYHA classification associated with a lower likelihood of a meaningful increase in the second 6MWT. These findings suggest that despite presenting with similar symptoms, patients with COPD or CHF have different factors influencing their exercise capacity.

One could argue that anxiety and depression could play an important role in changes in the second test, as could muscle atrophy and obesity. However, this was not corroborated by our findings. Abnormalities in lung function are a common finding in patients with heart failure (36), but the only parameter to associate significantly with a meaningful increase in the second test was $D_{L C O}$. A low $D_{L C O}$ associated with a lower likelihood of a meaningful increase, suggesting that patients with a low $D_{\text {Lco }}$ show less variation between the first and second 6MWT. $D_{\text {LCO }}$ impairments have been reported to be a limiting factor to exercise performance in patients with heart failure (37). We believe that a reduced $D_{L c o}$ led to limited exercise reserve, which then prevented patients from showing relevant increases in the second test. Interestingly, LVEF was not identified as a significant determinant of a clinically meaningful increase. Moreover, this parameter did not correlate strongly with the best 6MWD (Figure A.1.3 in appendix A; Supplementary data). So, clinicians cannot determine the patient's exercise tolerance (i.e., 6MWD) based on resting echocardiography. 
Strong significant correlations with the best 6MWD were found only with outcomes from the CPET. This is in line with previous studies which found strong correlations with peak $\mathrm{V} \mathrm{O}_{2}$ and $\mathrm{VO}_{2} / \mathrm{VCO}_{2}$ slope $(4,13,38)$. Next to that, significant correlations were also found with age, Charlson index for comorbidities, measures of lung function (e.g., $\mathrm{FEV}_{1}, \mathrm{RV} / \mathrm{TLC}$, and $\mathrm{D}_{\mathrm{LCO}}$ ), body composition (e.g., BMI, LL-LMM), and symptoms of anxiety and depression in our study. Interestingly, several lung function parameters were found to correlate positively with the best 6MWD. As mentioned earlier, disturbances in lung function are well known in patients with heart failure and often undiagnosed (36).

Similarly to what we found, Stanojevic et al. (39) also found significant correlations between eGFR and 6MWD in patients with CHF ( $r=0.39, P<0.001)$. Kidney function (i.e., eGFR) is probably a mirror for cardiac function, as they also found LVEF and NYHA class to be significant predictors of eGFR. In our sample, together with maximal power output and age, eGFR explained $51.7 \%$ of the variability in the best 6MWD. Renal insufficiency has been suggested as a limiting factor to exercise capacity in patients with heart failure (40). Interestingly, we observed that eGFR associated positively with the best 6MWD when tested with correlation coefficients (Table 2), but negatively when tested in a multiple linear regression model (Table 3). We believe this happened due to a 'partial multicollinearity' of eGFR with maximal power output during the CPET and age $\left(r_{s}=53\right.$ and $r_{s}=-65$, respectively; $P<0.0001$ for both). When these two variables were removed from the model a positive association was observed between eGFR and the best 6MWD (results not shown). Overall, the 6MWT - and thus exercise tolerance - is related to several extra-cardiac features. So, comorbidities partly determine the exercise intolerance in patients with CHF.

\section{Strengths and limitations}

Although several authors have already studied the reliability of 6MWT, not much was known about the determinants of meaningful increase and the determinants of the best 6MWD. Our sample consisted of a considerable number of CHF patients with a clinically representative disease severity. Importantly, patients showed a wide range of LVEF values (please see Table 1 and Figure A.1.3 in appendix A, Supplementary data), thus encompassing CHF with preserved LVEF (typically considered as $\geq 50 \%$ ), but also CHF with reduced LVEF (typically considered as $<40 \%$ ) and the newly defined of CHF with mid-range LVEF (with LVEF in the range of 40-49\%) (16). These characteristics may contribute to the external validity of the findings. We studied a large amount of possible relevant determinants, including lung function, renal function, obesity, muscle atrophy, and symptoms of anxiety and depression. To ensure the reliability of the results the participants walked always in the same course when performing the 6MWT. So we could exclude the influence of the length and layout of the track on the outcome of the 6MWT (41). The 6MWTs were performed on the same time of the day and the staff 
who executed the test were all familiar with it, and instructions and encouragement were standardized by the guidelines of the ERS/ATS (1). All these factors contribute to ensure reliable results.

Potential limitations to this research have to be acknowledged. Patients included in this study were entering a rehabilitation program, were selected by their cardiologist and should be motivated to work on their illness. Moreover, they had relatively good functional status as demonstrated by high body mass index, low number of comorbidities, and fairly good performance during the CPET. These factors may limit the external validity of our findings. Moreover, other variables that could have helped to explain the improvement between two tests as well as the best 6MWD, such as daily physical activity level and balance, were not available.

\section{CONCLUSION}

In conclusion, 6MWT was proven to be reliable and valid in patients with CHF. Nonetheless, patients of older age, lower lung diffusing capacity, and higher NYHA class showed a lower likelihood of having a clinically meaningful increase in the second 6MWT. Lung function, renal function, obesity, muscle atrophy, and symptoms of anxiety and depression were not determinants of a better second test. Determinants of the best 6MWD were the maximal power output during a maximal exercise test, the estimated glomerular filtration rate and age. These findings should be taken into account by researchers and clinicians when performing and interpreting the 6MWT in patients with mild-tomoderate $\mathrm{CHF}$ in order to have an accurate assessment of their patients' functional exercise performance. 


\section{REFERENCES}

1. A.E. Holland, M.A. Spruit, T. Troosters, M.A. Puhan, V. Pepin, D. Saey, et al., An official European Respiratory Society/American Thoracic Society technical standard: field walking tests in chronic respiratory disease, Eur. Respir. J. 44 (6) (Dec 2014) 1428-1446.

2. D.M. Hamilton, R.G. Haennel, Validity and reliability of the 6-minute walk test in a cardiac rehabilitation population, J. Cardpulm. Rehabil. 20 (3) (May-Jun 2000) 156-164.

3. S. Solway, D. Brooks, Y. Lacasse, S. Thomas, A qualitative systematic overviewof the measurement properties of functional walk tests used in the cardiorespiratory domain, Chest 119 (1) (Jan 2001) 256-270.

4. L.P. Cahalin, M.A. Mathier, M.J. Semigran, G.W. Dec, T.G. DiSalvo, The six-minute walk test predicts peak oxygen uptake and survival in patients with advanced heart failure, Chest 110 (2) (Aug 1996) 325-332.

5. V. Bittner, D.H. Weiner, S. Yusuf, W.J. Rogers, K.M. McIntyre, S.I. Bangdiwala, et al., Prediction of mortality and morbidity with a 6-minute walk test in patients with left ventricular dysfunction. SOLVD investigators, JAMA 270 (14) (Oct 13 1993) 1702-1707.

6. C. Rostagno, G. Olivo, M. Comeglio, V. Boddi, M. Banchelli, G. Galanti, et al., Prognostic value of 6minutewalk corridor test in patients with mild to moderate heart failure: comparison with other methods of functional evaluation, Eur. J. Heart Fail. 5 (3) (Jun 2003) 247-252.

7. K. Wegrzynowska-Teodorczyk, E. Rudzinska, M. Lazorczyk, K. Nowakowska, W. Banasiak, P. Ponikowski, et al., Distance covered during a six-minute walk test predicts long-term cardiovascular mortality and hospitalisation rates in men with systolic heart failure: an observational study, J. Physiother. 59 (3) (Sep 2013) 177-187.

8. J.J. McMurray, S. Adamopoulos, S.D. Anker, A. Auricchio, M. Bohm, K. Dickstein, et al., ESC guidelines for the diagnosis and treatment of acute and chronic heart failure 2012: the Task Force for the Diagnosis and Treatment of Acute and Chronic Heart Failure 2012 of the European Society of Cardiology. Developed in collaboration with the Heart Failure Association (HFA) of the ESC, Eur. J. Heart Fail. 14 (8) (Aug 2012) 803869.

9. R.N. Bellet, L. Adams, N.R.Morris, The 6-minute walk test in outpatient cardiac rehabilitation: validity, reliability and responsiveness - a systematic review, Physiotherapy 98 (4) (2012 Dec) 277-286.

10. G. Roul, P. Germain, P. Bareiss, Does the 6-minute walk test predict the prognosis in patients with NYHA class II or III chronic heart failure? Am. Heart J. 136 (3) (1998 Sep) 449-457.

11. R.N. Bellet, R.L. Francis, J.S. Jacob, K.M. Healy, H.J. Bartlett, L. Adams, et al., Repeated six-minute walk tests for outcome measurement and exercise prescription in outpatient cardiac rehabilitation: a longitudinal study, Arch. Phys. Med. Rehabil. 92 (9) (2011 Sep) 1388-1394.

12. C. Opasich, G.D. Pinna, A. Mazza, O. Febo, R. Riccardi, P.G. Riccardi, et al., Six-minute walking performance in patients with moderate-to-severe heart failure; is it a useful indicator in clinical practice? Eur. Heart J. 22 (6) (Mar 2001) 488-496.

13. M. Guazzi, K. Dickstein, M. Vicenzi, R. Arena, Six-minute walk test and cardiopulmonary exercise testing in patients with chronic heart failure: a comparative analysis on clinical and prognostic insights, Circ. Heart Fail. 2 (6) (Nov 2009) 549-555.

14. Á.R.M. Kristjánsdóttir, M. Einarsson, B. Torfason, A comparison of the 6-minute walk test and symptom limited graded exercise test for phase II cardiac rehabilitation of older adults, J. Geriatr. Phys. Ther. 27 (2004) 65-69.

15. C. Demers, R.S. McKelvie, A. Negassa, S. Yusuf, R.P.S. Investigators, Reliability, validity, and responsiveness of the six-minute walk test in patients with heart failure, Am. Heart J. 142 (4) (2001 Oct) 698-703.

16. P. Ponikowski, A.A. Voors, S.D. Anker, H. Bueno, J.G. Cleland, A.J. Coats, et al., 2016 ESC guidelines for the diagnosis and treatment of acute and chronic heart failure: the Task Force for the Diagnosis and Treatment of Acute and Chronic Heart Failure of the European Society of Cardiology (ESC) developed with the special contribution of the Heart Failure Association (HFA) of the ESC, Eur. Heart J. 37 (27) (Jul 14 2016) 2129-2200. 
17. ATS statement: guidelines for the six-minute walk test, Am. J. Respir. Crit. Care Med. 166 (1) (Jul 12002 ) 111-117.

18. A.B. Chatterjee, R.W. Rissmiller, K. Meade, C. Paladenech, J. Conforti, N.E. Adair, et al., Reproducibility of the 6-minute walk test for ambulatory oxygen prescription, Respiration 79 (2) (2010) 121-127.

19. T. Troosters, R. Gosselink, M. Decramer, Six minute walking distance in healthy elderly subjects, Eur. Respir. J. 14 (2) (Aug 1999) 270-274.

20. Heart Failure Society of America. NYHA classification - the stages of heart failure HFSA; 2002 ([updated 12-06-2011]).

21. D.L. Dries, D.V. Exner, M.J. Domanski, B. Greenberg, L.W. Stevenson, The prognostic implications of renal insufficiency in asymptomatic and symptomatic patients with left ventricular systolic dysfunction, J. Am. Coll. Cardiol. 35 (3) (Mar 1 2000) 681-689.

22. F.A. McAlister, J. Ezekowitz, M. Tonelli, P.W. Armstrong, Renal insufficiency and heart failure prognostic and therapeutic implications from a prospective cohort study, Circulation 109 (8) (2004) 1004-1009.

23. M.R. Miller, J. Hankinson, V. Brusasco, F. Burgos, R. Casaburi, A. Coates, et al., Standardisation of spirometry, Eur. Respir. J. 26 (2) (Aug 2005) 319-338.

24. American Thoracic Society, American College of Chest Physicians, ATS/ACCP statement on cardiopulmonary exercise testing, Am. J. Respir. Crit. Care Med. 167 (2) (Jan 15 2003) 211-277.

25. S.A. Plowman, Exercise Physiology for Health, Fitness, and Performance, Lippincott Williams \&Wilkins, Philadelphia, 2008.

26. F.M. Franssen, E.P. Rutten, M.T. Groenen, L.E. Vanfleteren, E.F. Wouters, M.A. Spruit, New reference values for body composition by bioelectrical impedance analysis in the general population: results from the UK Biobank, J. Am. Med. Dir. Assoc. 15 (6) (Jun 2014) 448 e1-6.

27. M. Charlson, T.P. Szatrowski, J. Peterson, J. Gold, Validation of a combined comorbidity index, J. Clin. Epidemiol. 47 (11) (Nov 1994) 1245-1251.

28. A.S. Zigmond, R.P. Snaith, The hospital anxiety and depression scale, Acta Psychiatr. Scand. 67 (6) (Jun 1983) 361-370.

29. J. Yap, F.Y. Lim, F. Gao, L.L. Teo, C.S. Lam, K.K. Yeo, Correlation of the New York Heart Association classification and the 6-minute walk distance: a systematic review, Clin. Cardiol. 38 (10) (Oct 2015) 621-628.

30. M.J. Shoemaker, A.B. Curtis, E. Vangsnes, M.G. Dickinson, Clinically meaningful change estimates for the six-minute walk test and daily activity in individuals with chronic heart failure, Cardiopulm. Phys. Ther. J. 24 (3) (2013 Sep) 21-29.

31. L.D.W.M. Portney, Foundations of Clinical Research: Applications to Practice, Upper Saddle River, Prentice Hall, 2008.

32. J.R. Landis, G.G. Koch, The measurement of observer agreement for categorical data, Biometrics 33 (1) (Mar 1977) 159-174.

33. S. O'Keeffe, M. Lye, C. Donnellan, D. Carmichael, Reproducibility and responsiveness of quality of life assessment and six minute walk test in elderly heart failure patients, Heart 80 (4) (1998) 377-382.

34. J. Adsett, R. Mullins, R. Hwang, A. Hogden, E. Gibson, K. Houlihan, et al., Repeat six- minute walk tests in patients with chronic heart failure: are they clinically necessary? Eur. J. Cardiovasc. Prev. Rehabil. 18 (4) (Aug 2011) 601-606.

35. N.A. Hernandes, E.F. Wouters, K. Meijer, J. Annegarn, F. Pitta, M.A. Spruit, Reproducibility of 6-minute walking test in patients with COPD, Eur. Respir. J. 38 (2) (2011 Aug) 261-267.

36. K.K. Iversen, J. Kjaergaard, D. Akkan, L. Kober, C. Torp-Pedersen, C. Hassager, et al., The prognostic importance of lung function in patients admitted with heart failure, Eur. J. Heart Fail. 12 (7) (Jul 2010) 685691.

37. M.D. Kraemer, S.H. Kubo, T.S. Rector, N. Brunsvold, Bank AJ, Pulmonary and peripheral vascular factors are important determinants of peak exercise oxygen uptake in patients with heart failure, J. Am. Coll. Cardiol. 21 (3) (Mar 1 1993) 641-648.

38. D.E. Forman, J.L. Fleg, D.W. Kitzman, C.A. Brawner, A.M. Swank, R.S. McKelvie, et al., 6-min walk test provides prognostic utility comparable to cardiopulmonary exercise testing in ambulatory outpatients with systolic heart failure, J. Am. Coll. Cardiol. 60 (25) (2012 Dec 25) 2653-2661. 


\section{CHAPTER 4}

39. D. Stanojevic, S. Apostolovic, R. Jankovic-Tomasevic, S. Salinger-Martinovic, M. Pavlovic, M. Zivkovic, et al., Prevalence of renal dysfunction and its influence on functional capacity in elderly patients with stable chronic heart failure, Vojnosanit. Pregl. 69 (10) (Oct 2012) 840-845.

40. P.A. McCullough, B.A. Franklin, E. Leifer, G.C. Fonarow, Impact of reduced kidney function on cardiopulmonary fitness in patients with systolic heart failure, Am. J. Nephrol. 32 (3) (2010) 226-233.

41. F. Sciurba, G.J. Criner, S.M. Lee, Z.Mohsenifar, D. Shade, W. Slivka, et al., Six-minute walk distance in chronic obstructive pulmonary disease: reproducibility and effect of walking course layout and length, Am. J. Respir. Crit. Care Med. 167 (11) (Jun 1 2003) 1522-1527.

Reprinted from International Journal of Cardiology, Vol. 240, Uszko-Lencer NHMK, Mesquita R, Janssen E, Werter C, Brunner-La Rocca HP, Pitta F, Wouters EFM, Spruit MA, Reliability, construct validity and determinants of 6-minute walk test performance in patients with chronic heart failure, pp. 285-90, Copyright (2017), with permission from Elsevier. 


\section{SUPPLEMENTARY DATA}

\section{A.1 RESULTS}

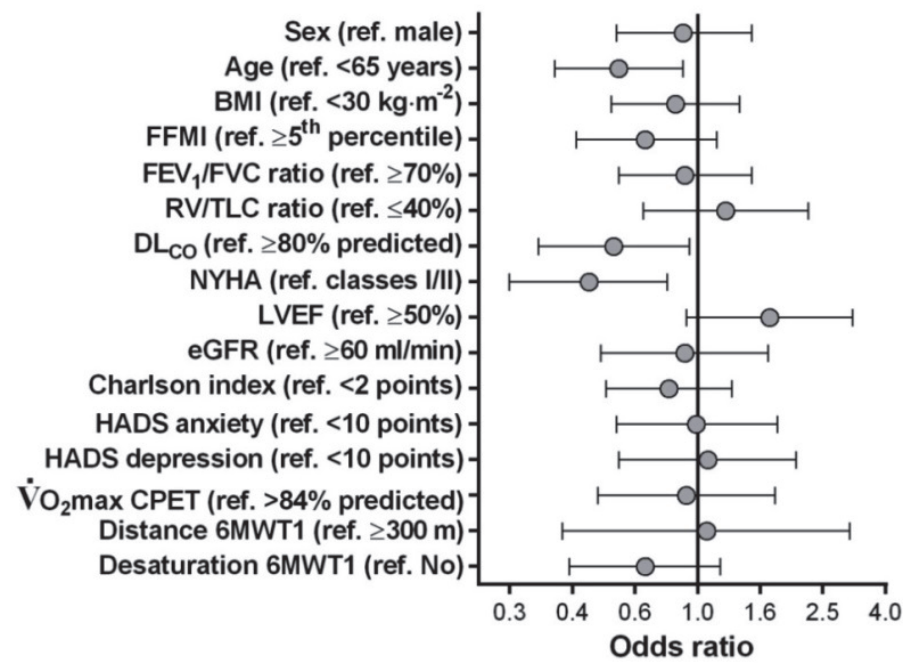

OR ( $95 \% \mathrm{Cl}), P$-value

$0.90(0.55,1.49), 0.69$

$0.56(0.35,0.90), 0.02^{*}$

$0.85(0.53,1.36), 0.49$

0.68 (0.41, 1.15), 0.15

$0.91(0.56,1.49), 0.71$

$1.23(0.67,2.26), 0.50$

$0.54(0.31,0.94), 0.03^{*}$

$0.45(0.25,0.80), 0.007^{\star}$

$1.70(0.92,3.12), 0.09$

0.91 (0.49, 1.68), 0.75

0.81 (0.51, 1.29), 0.37

$0.99(0.55,1.80), 0.99$

$1.08(0.56,2.06), 0.83$

$0.92(0.48,1.77), 0.80$

1.07 (0.37, 3.06), 0.90

$0.68(0.39,1.18), 0.17$

Figure A.1.1 Relationship between patient characteristics and changes in walked distance in patients with chronic heart failure. Models adjusted for age, sex, BMI, Charlson scores, and left ventricular ejection fraction (in analyses considering any of these variables as independent variable, only the four other variables were used for adjustment). BMI: body mass index; FFMI: fat-free mass index; FEV ${ }_{1}$ : forced expiratory volume in the

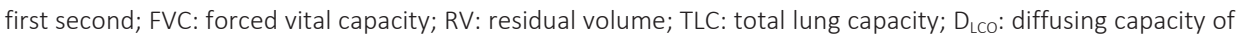
the lung for carbon monoxide; NYHA: New York Heart Association; LVEF: Left Ventricle Ejection Fraction; eGFR: estimated glomerular filtration rate; HADS: Hospital Anxiety and Depression scales; $\mathrm{VO}_{2}$ max: maximal oxygen consumption; CPET: cardiopulmonary exercise test. ${ }^{*}: \mathrm{P}<0.05$. 
CHAPTER 4

Table A.1.1 Intraclass correlation coefficient and Kappa coefficient after stratification for age, lung diffusing capacity, and New York Heart Association classes

\begin{tabular}{lcccc}
\hline \multicolumn{1}{c}{ Characteristic } & Subgroup & Patients $n$ & ICC $(95 \% \mathrm{Cl})^{*}$ & $\begin{array}{c}\text { Kappa coefficient for the cut- } \\
\text { off of }<300 \mathrm{~m}\end{array}$ \\
\hline Total sample & - & 337 & $0.90(0.63,0.96)$ & 0.78 \\
Age & $<65$ years & 170 & $0.89(0.75,0.94)$ & 0.85 \\
& $\geq 65$ years & 167 & $0.90(0.34,0.96)$ & 0.56 \\
& $<80 \%$ predicted & 238 & $0.90(0.69,0.95)$ & 0.77 \\
DLCO & $\geq 80 \%$ predicted & 77 & $0.89(0.34,0.96)$ & 0.66 \\
& I/II & 218 & $0.87(0.34,0.95)$ & 0.50 \\
NYHA & III & 93 & $0.89(0.80,0.93)$ & 0.80
\end{tabular}

ICC: intraclass correlation coefficient; Cl: confidence intervals; $\mathrm{D}_{\mathrm{Lco}}$ : diffusing capacity of the lung for carbon monoxide; NYHA: New York Heart Association. . : P<0.0001 for all ICC values. 


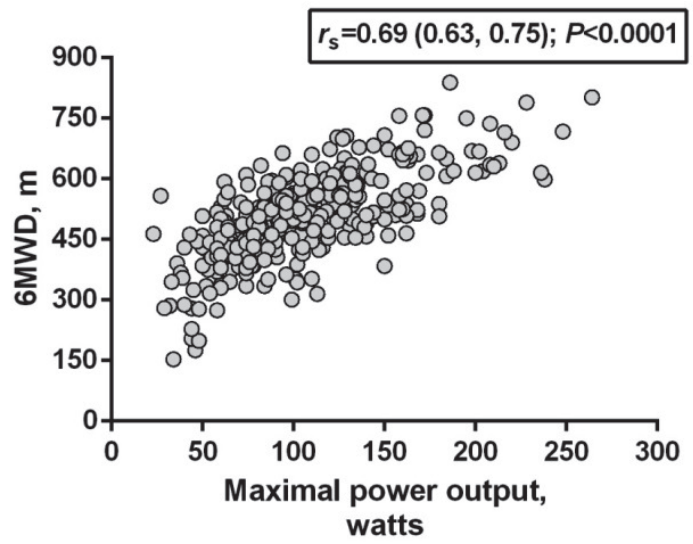

Figure A.1.2 Correlation between 6-minute walk distance (6MWD) and maximal power output during the symptom-limited incremental cardiopulmonary exercise test in patients with chronic heart failure.

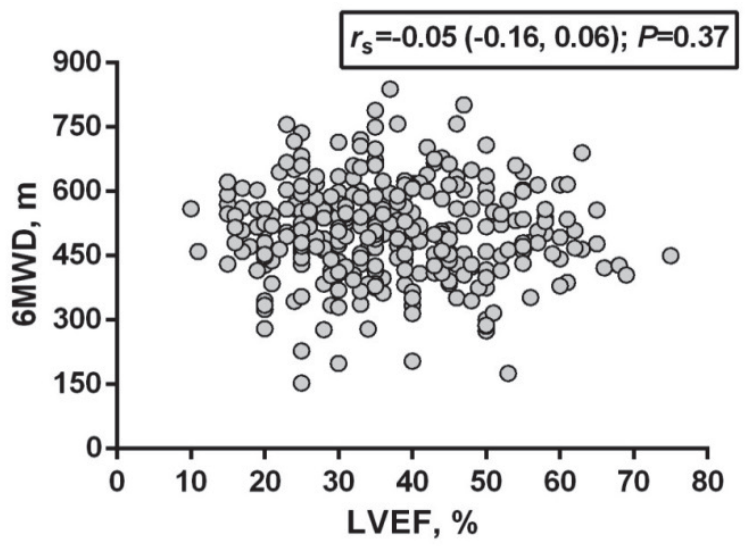

Figure A.1.3 Correlation between 6-minute walk distance (6MWD) and left ventricular ejection fraction (LVEF) in patients with chronic heart failure. 



\section{CHAPTER 5}

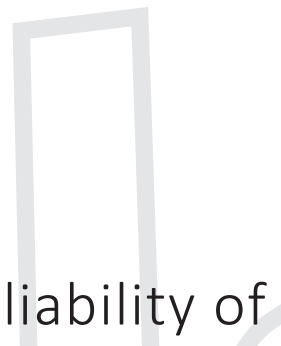

the Timed "Up \& Go" test in patients with advanced chronic organ failure

Rafael Mesquita, Daisy J.A. Janssen, Emiel F.M. Wouters, Jos M.G.A. Schols, Fabio Pitta, and Martijn A. Spruit 


\title{
CHAPTER 5
}

\begin{abstract}
Objective: To investigate the within-day test-retest reliability of the Timed "Up \& Go" (TUG) test in patients with advanced Chronic Obstructive Pulmonary Disease (COPD), Chronic Heart Failure (CHF), and Chronic Renal Failure (CRF).

Design: Cross-sectional.
\end{abstract}

Setting: Patients' home environment in the South-East region of the Netherlands.

Participants: 235 subjects (64\% men, median [IQR] age 70 [61-77] years; body mass index [BMI] 25.6 [22.8-29.4] $\mathrm{kg} \cdot \mathrm{m}^{-2}$ ) with advanced COPD ( $\left.\mathrm{n}=95\right), \mathrm{CHF}(\mathrm{n}=68)$ or CRF $(n=72)$.

Interventions: Not applicable.

Main outcome measure: Time to complete the TUG test. Three trials were performed on the same day and by the same assessors. The Intraclass Correlation Coefficient (ICC), $\mathrm{K}$ coefficient, Standard Error of Measurement (SEM), and absolute and relative Minimal Detectable Change (MDC) values were calculated.

Results: Good agreement was observed, in general, for both the total sample and subgroups (COPD, CHF, and CRF) with ICC values ranging from 0.85 to 0.98 , and $\mathrm{k}$ coefficients from 0.49 to 1.00 . However, statistical improvement occurred in the total sample from the first to the second trial with large limits of agreement (mean difference [95\% $\mathrm{Cl}]-0.97$ [+3.00 to -4.94$]$ seconds, $p<0.01)$. The third trial added little or no information to the first two trials. For the total sample, values of SEM around 1.6 seconds, MDC $_{95 \%}$ around 4.5 seconds, and $\mathrm{MDC}_{95 \%} \%$ around 35\% were found between the first two trials, with close values found for the subgroups.

Conclusions: The TUG test is reliable in patients with advanced COPD, CHF, or CRF after two trials. Values of SEM and MDC may be used in daily clinical practice with these populations to define what is expected and what represents true change in repeated measures. 


\section{INTRODUCTION}

The Timed "Up \& Go" (TUG) test is a performance test that was first described in 1991 by Podsiadlo and Richardson ${ }^{1}$ to assess functional mobility in frail elderly. In this test the subjects are requested to stand up from a chair, walk at their usual speed a distance of three meters, turn and walk back to the chair and sit down again. The time to perform this task is recorded and usually used as the main outcome of analysis. Since its first description, the TUG test has been used to assess balance, walking ability and risk of falling in a variety of populations (e.g., elderly, patients with neurological diseases, patients with orthopedical problems $)^{2-4}$. The widespread use of this test is probably due to its simplicity and low cost. Moreover, the TUG test has been show to be reliable in subjects with different conditions, presenting Intraclass Correlation Coefficient (ICC) values that range from 0.69 to $0.99^{1,2,4-6}$, indicating moderate to good agreement ${ }^{7}$.

To date, the TUG test has been used scarcely in patients with advanced chronic organ failure, such as Chronic Obstructive Pulmonary Disease (COPD), Chronic Heart Failure $(\mathrm{CHF})$, or Chronic Renal Failure (CRF) ${ }^{8-10}$. Nevertheless, these patients often present lower-limb muscle dysfunction, exercise intolerance, and fatigue ${ }^{11-15}$ - especially in advanced disease - which compromise functional mobility, balance, and consequently increase the risk for falling ${ }^{16-18}$.

Before recommending its use in daily clinical practice in these target groups of patients, the reliability of the TUG test needs to be determined in patients with advanced COPD, CHF or CRF. The ICC is considered to be a measure of relative consistency, but for a better understanding of a test's reliability, both relative and absolute consistency should be considered. The Standard Error of Measurement (SEM) can be used for absolute consistency ${ }^{19}$. It allows to calculate the boundaries around which the true scores of a subject lie in repeated measures ${ }^{19}$. Moreover, it seems necessary to calculate the Minimal Detectable Change (MDC), defined as the smallest difference needed to be considered real ${ }^{19}$, to define whether and to what extent a true change in TUG test may occur.

Therefore, the aim of this study was to investigate the within-day test-retest reliability of the TUG test in patients with advanced COPD, CHF or CRF by providing ICC and SEM values, as well as to calculate its MDC values. 


\section{CHAPTER 5}

\section{METHODS}

\section{Study design and participants}

The present study is part of a longitudinal observational study concerning palliative care needs of patients with advanced COPD, CHF or CRF ${ }^{20-30}$. For the present study, only the results from the baseline assessment were considered. The study received the approval from the Medical Ethical Committee of the Maastricht University Medical Center + (MUMC+), Maastricht, the Netherlands (MEC 07-3-054), and all patients gave written informed consent.

Patients were conveniently recruited from the outpatient clinics of one academic hospital and six general hospitals in the South-East of the Netherlands. They were considered eligible if they were clinically stable and had one of the following diseases in advanced stages: COPD with Global Initiative for Chronic Obstructive Lung Disease (GOLD) grades III or IV ${ }^{31}$; CHF with New York Heart Association (NYHA) classes III or IV; or CRF requiring dialysis. Patients were excluded from this analysis if, for any reason, they did not perform all three trials of the TUG test.

Patients with advanced COPD, CHF or CRF were pooled together in the present study for the fact that these conditions lead to similar signs and symptoms, such as lower-limb muscle dysfunction, exercise intolerance, and fatigue ${ }^{11-15}$, which can compromise functional mobility, balance, and consequently increase the risk for falling ${ }^{16-18}$. Nevertheless, separate analyses for each disease were also provided in order to have specific pictures of each chronic disease.

\section{Outcome measures}

The following patient characteristics were assessed in the patients' home environment, or if CRF patients preferred at the dialysis department: demographics (sex and age), anthropometrics (weight, height, and Body Mass Index [BMI]), dialysis type in CRF patients, smoking status (never, former or current), regular use of walking aids, selfreported poor health, and symptoms of dyspnoea and fatigue using the modified Borg scale ${ }^{32}$.

In addition, the following outcomes were assessed:

The timed "up \& go" test: The test was performed according to the original protocol described by Podsiadlo and Richardson ${ }^{1}$. The subjects were requested to stand up from a regular chair, walk at their usual speed a distance of three meters, turn and walk back to the chair and sit down again. The time in seconds to perform this task was recorded 
by ordinary stopwatches and used as the main outcome of analysis. The timing started when the participant got up from the chair and ended when the participant touched the chair when sitting back after the walk. Three trials were performed by the same assessor and with sufficient resting time (until symptoms recovery) between the trials. The assessors (one physician and two research nurses) were previously trained to apply the test. During the trials, all participants wore their own usual footwear, and walking aids and oxygen were allowed if necessary.

Podsiadlo and Richardson ${ }^{1}$ classified TUG test results in three categories: 1) $<20$ seconds - functionally independent; 2) 20-29 seconds - referred here as somewhat functionally independent and; 3) >29 seconds - functionally dependent. These categories were also considered for analysis.

Marker of diseases: For the diagnosis and severity classification of COPD, forced expiratory volume in the first second $\left(\mathrm{FEV}_{1}\right)$ and forced vital capacity (FVC) were measured by spirometry according to international criteria $^{33}$ and considering previous reference values $^{34}$. N-terminal pro-B type natriuretic peptide (NT-proBNP) levels were measured as a systemic biomarker of heart failure ${ }^{35}$ and creatinine levels as a marker of kidney's failure.

Comorbidities: The Charlson comorbidity index ${ }^{36}$ was used to assess current selfreported comorbidities. This instrument is composed by 19 categories of comorbidities and the total score reflects the cumulative increased likelihood of one-year mortality ${ }^{37}$. Higher scores can be translated as higher burden of comorbidities.

Care dependency: To assess care dependency the Care Dependence Scale (CDS) was used. It is composed by 15 items related to basic and instrumental activities of daily living $^{38}$. The final score ranges from 15 (worst) to 75 (best) points, and patients $\leq 68$ points can be considered as care dependent ${ }^{39}$.

Generic health status: Generic health status was assessed with the EuroQol-5 Dimensions (EQ-5D) questionnaire. This instrument is composed by five items (mobility, selfcare, usual activity, pain/discomfort and anxiety/depression) with three levels each (no problems, some problems and extreme problems $)^{40}$. A final index score ranging from 0.59 (worst) to 1.0 (best) is provided ${ }^{40}$.

\section{Statistical Analysis}

Data were described in absolute numbers, relative frequency, mean (SD) or median (interquartile range, IQR), as appropriate. To assess reliability in both the total sample and subgroups, the model two of ICC (two-way random effect model) ${ }^{41}$ with single rat- 
ing $\left(\mathrm{ICC}_{2,1}\right)$ and the $\mathrm{k}$ coefficient were used to determine the level of agreement, while the SEM was used to assess response stability. Considering the SEM, the absolute $\left(\mathrm{MDC}_{95 \%}\right)$ and relative $\left(\mathrm{MDC}_{95 \%} \%\right)$ values of $\mathrm{MDC}$ at the $95 \%$ confidence level were calculated. The following formulas were used ${ }^{42,43}$ : SEM $=$ SD of baseline assessment $\times \sqrt{(1-\mathrm{ICC})} ; \mathrm{MDC}_{95 \%}=1.96 \times \sqrt{2} \times \mathrm{SEM}$ and $\mathrm{MDC}_{95 \%} \%=\left(\mathrm{MDC}_{95 \%} \div\right.$ mean of two trials) $\times 100$. The $95 \%$ limits of agreement with the plots of Bland and Altman ${ }^{44}$ were also calculated to visually evaluate trends and agreement between the trials. Chi-square test, paired student's t test, one-way Analysis of Variance (ANOVA, post hoc Tukey), repeated measures ANOVA (post hoc Tukey), or Kruskal-Wallis test (post hoc Dunn) were used for comparisons, while the coefficient of Pearson was used for correlations, as appropriate. Complete-case analysis was used to deal with missing values, which

means that analyses were restricted to individuals with complete data only ${ }^{45}$. Missing values were observed in thirty subjects, which were excluded for not presenting one of the three TUG trials. The level of statistical significance was considered as $p<0.01$ and all analyses were performed using the Statistical Package of Social Science (SPSS) $17.0^{\mathrm{a}}$ or GraphPad Prism $5^{\mathrm{b}}$.

\section{RESULTS}

\section{Characteristics of the subjects}

From the 265 subjects initially included in the main study ${ }^{20-28}$, thirty were excluded from the current analysis because they were not able to perform three TUG trials. Table 1 presents the general characteristics of the 235 elderly subjects included. In general, the majority of patients had COPD, were men and former smoker. Almost half of the patients were regular users of walking aids and dependent in personal care. The median $\mathrm{BMI}$ was normal-to-overweight, and the majority of patients scored two or more points on the Charlson comorbidity index. EQ-5D scores showed moderate impairment in heath status, and most of the patients referred no or some problems in its dimensions. Most COPD patients (72\%) were classified as GOLD grade IV, while $93 \%$ of the CHF patients were classified as NYHA class III. In the subgroup of patients with CRF, 96\% were on haemodialysis. 
Table 1 General characteristics of the subjects

\begin{tabular}{|c|c|c|c|c|c|}
\hline \multirow[t]{2}{*}{ Characteristic } & \multirow{2}{*}{$\begin{array}{c}\text { Total sample } \\
(n=235)\end{array}$} & \multicolumn{3}{|c|}{ Subgroups } & \multirow[b]{2}{*}{$p$-value } \\
\hline & & $\begin{array}{l}\text { COPD } \\
(n=95)\end{array}$ & $\begin{array}{c}\mathrm{CHF} \\
(\mathrm{n}=68)\end{array}$ & $\begin{array}{c}\text { CRF } \\
(n=72)\end{array}$ & \\
\hline Gender (\%, M/F) & $64 / 36$ & $61 / 39$ & $71 / 29$ & $63 / 37$ & 0.77 \\
\hline Age (years) & $70(61-77)$ & $66(61-71)^{*}$ & $78(72-82)$ & $64(54-75)^{*}$ & $<0.0001$ \\
\hline $\mathrm{BMI}\left(\mathrm{kg} \cdot \mathrm{m}^{-2}\right)$ & $\begin{array}{c}25.6 \\
(22.8-29.4)\end{array}$ & $\begin{array}{c}25.3 \\
(21.6-30.1)\end{array}$ & $\begin{array}{c}26.8 \\
(25.3-31.9)\end{array}$ & $\begin{array}{c}24.3 \\
(21.8-26.6)^{*}\end{array}$ & 0.0001 \\
\hline $\mathrm{FEV}_{1}(\%$ pred $)$ & $63(37-86)$ & $33(26-42)^{*}+t$ & $76(64-90)$ & 84 (71-99) & $<0.0001$ \\
\hline NT-proBNP $\left(\mathrm{pmol} \cdot \mathrm{L}^{-1}\right)$ & $\begin{array}{c}86.7 \\
(17.6-317.5)\end{array}$ & $\begin{array}{c}12.8 \\
(7.7-29.5)^{*}+\dagger\end{array}$ & $\begin{array}{c}156.6 \\
(54.1-334.6)\end{array}$ & $\begin{array}{c}373.0 \\
(159.2-880.4)^{*}\end{array}$ & $<0.0001$ \\
\hline Creatinine $\left(\mu \mathrm{mol} \cdot \mathrm{L}^{-1}\right)$ & $\begin{array}{c}124.0 \\
(84.0-525.5)\end{array}$ & $\begin{array}{c}80.0 \\
(70.0-101.3)^{*}+\dagger\end{array}$ & $\begin{array}{c}129.0 \\
(100.0-171.0)\end{array}$ & $\begin{array}{c}745.0 \\
(549.3-892.5)^{*}\end{array}$ & $<0.0001$ \\
\hline Charlson index (points) & $3(2-5)$ & $2(1-3)^{*}+\dagger$ & $4(3-5)$ & $4(2-5)$ & $<0.0001$ \\
\hline \multicolumn{6}{|l|}{ Smoking status } \\
\hline (\%, Never/Former/Current) & $19 / 63 / 18$ & $11 / 65 / 24$ & $19 / 71 / 10$ & $31 / 54 / 15$ & 0.02 \\
\hline Walking aid (\%) & 47 & $53+$ & 69 & $19^{*}$ & $<0.0001$ \\
\hline Poor health (\%) & 13 & 17 & 13 & 8 & 0.11 \\
\hline Borg Dyspnea (points) & $3(1-5)$ & $4(3-6)^{+}$ & $4(2-5)$ & $0.5(0-2)^{*}$ & $<0.0001$ \\
\hline Borg Fatigue (points) & $4(3-6)$ & $4(3-6)$ & $4(3-7)$ & $3(2-6)$ & 0.08 \\
\hline Care dependence (\%) & 40 & $43^{+}$ & 60 & $17^{*}$ & 0.002 \\
\hline \multicolumn{6}{|l|}{$E Q-5 D$} \\
\hline Index score (points) & $\begin{array}{c}0.69 \\
(0.52-0.81)\end{array}$ & $\begin{array}{c}0.64 \\
(0.27-0.80)^{\dagger}\end{array}$ & $\begin{array}{c}0.59 \\
(0.42-0.73)\end{array}$ & $\begin{array}{c}0.80 \\
(0.67-0.88)^{*}\end{array}$ & $<0.0001$ \\
\hline \multicolumn{6}{|l|}{ Dimensions (\%, NP/SP/EP) } \\
\hline Mobility & $24 / 76 / 0$ & $17 / 83 / 0+$ & $10 / 90 / 0$ & $46 / 54 / 0^{*}$ & $<0.0001$ \\
\hline Self-care & $57 / 36 / 7$ & $43 / 48 / 9+$ & $40 / 47 / 13$ & $90 / 10 / 0^{*}$ & $<0.0001$ \\
\hline Usual activities & $28 / 59 / 13$ & $19 / 61 / 20+$ & $18 / 68 / 14$ & $49 / 47 / 4^{*}$ & $<0.0001$ \\
\hline Pain/discomfort & $51 / 41 / 8$ & $56 / 35 / 9$ & $37 / 56 / 7$ & $58 / 35 / 7$ & 0.73 \\
\hline Anxiety/depression & $66 / 28 / 6$ & $61 / 29 / 10^{+}$ & $59 / 37 / 4$ & $79 / 20 / 1^{*}$ & 0.006 \\
\hline
\end{tabular}

Data expressed as absolute number, relative frequency, or median (IQR). M: male; F: female; NP: no problems; SP: some problems; EP: extreme problems. ${ }^{*}: p<0.01$ vs $C H F ;{ }^{\dagger}: p<0.01$ vs CRF.

\section{Reliability of the Timed “Up \& Go" test}

Table 2 presents the results of the TUG test in the first, second, and third trials for the total sample and the subgroups. In the total sample, one hundred seventy-nine patients (76\%) presented the best result (i.e., shortest duration) in one of the first two trials. When only these trials were considered, sixty-six subjects (28\%) had exactly the same result, thirty-nine (17\%) were slower, and one hundred thirty subjects (55\%) were faster during the second trial compared to the first trial; the mean (SD) improvement in the latter group was -2.2 (1.9) seconds. When the lowest of the three trials was compared to the lowest of the first two trials, despite statistical significance $(p<0.01)$, no clinically relevant difference was observed (12.1 [5.3] vs 12.3 [5.4] seconds, respectively). 


\section{CHAPTER 5}

Table 2 Results of the TUG test in each trial for the total sample and stratified for disease

\begin{tabular}{|c|c|c|c|c|c|}
\hline & $1^{\text {st }}$ trial & $2^{\text {nd }}$ trial & $3^{\text {rd }}$ trial & $\begin{array}{l}\text { Difference } \\
1^{\text {st }} \text { and } 2^{\text {nd }} \\
\text { trialst }\end{array}$ & $\begin{array}{l}\text { Difference } \\
2^{\text {nd }} \text { and } 3^{\text {rd }} \\
\text { trialst }\end{array}$ \\
\hline \multicolumn{6}{|l|}{ Total sample $(n=235)$} \\
\hline Absolute (seconds) & $13.5(6.2)$ & $12.6(5.6)^{*}$ & $12.7(5.7)^{*}$ & $-1.0(2.0)$ & $0.1(1.2)$ \\
\hline Classification (\%, & $88 / 10 / 2$ & $90 / 8 / 2$ & $90 / 8 / 2$ & $1 \uparrow$ and $5 \downarrow$ & $2 \uparrow$ and $1 \downarrow$ \\
\hline \multicolumn{6}{|l|}{$\operatorname{COPD}(n=95)$} \\
\hline Absolute (seconds) & $11.8(4.5)$ & $11.0(3.5)^{*}$ & $11.0(3.6)^{*}$ & $-0.8(2.1)$ & $0.1(1.1)$ \\
\hline SFI, FD) & $/ 5 / 1$ & $95 / 5 / 0$ & $95 / 5 / 0$ & $1 \uparrow$ and $3 \downarrow$ & $0 \uparrow$ and $0 \downarrow$ \\
\hline \multicolumn{6}{|l|}{$\mathrm{CHF}(\mathrm{n}=68)$} \\
\hline Absolute (seconds) & $17.4(8.0)$ & $16.1(7.5)^{*}$ & $16.6(7.6)$ & $-1.3(2.4)$ & $0.4(1.5)$ \\
\hline Classi & & & & $3 \uparrow a$ & $6 \uparrow$ \\
\hline \multicolumn{6}{|l|}{$\operatorname{CRF}(n=72)$} \\
\hline Absolute (seconds) & $12.2(4.2)$ & $11.3(3.9)^{*}$ & $11.2(3.8)^{*}$ & $-0.9(1.5)$ & $-0.2(1.1)$ \\
\hline Classification (\%, FI, SFI, FD) & $94 / 6 / 0$ & $96 / 4 / 0$ & $99 / 1 / 0$ & $0 \uparrow$ and $1 \downarrow$ & $0 \uparrow$ and $3 \downarrow$ \\
\hline \multicolumn{6}{|c|}{$\begin{array}{l}\text { Data expressed as relative frequency or mean (SD). Fl: functionally independent }(<20 \text { seconds); SFI: somewhat } \\
\text { functionally independent ( } 20-29 \text { seconds); FD: functionally dependent }(>29 \text { seconds })^{1} . *: p<0.01 \text { vs } 1^{\text {st }} \text { trial; }+: \\
\text { last minus former trial; } \uparrow: \text { moved one category forward; } \downarrow: \text { moved one category backward. No subject } \\
\text { moved more than one category. }\end{array}$} \\
\hline \multicolumn{6}{|c|}{$\begin{array}{l}\text { The ICC among the three trials in the total sample was } 0.94(95 \% \mathrm{Cl} 0.92-0.96) \\
(p<0.0001) \text {. Table } 3 \text { presents the ICC between two trials (i.e., first and second, and sec- } \\
\text { ond and third), as well as the } \mathrm{k} \text { coefficient, SEM and MDC values between them, both } \\
\text { for the total sample and subgroups. The Bland and Altman plots for the same pairs of } \\
\text { trials are depicted in Figure } 1 \text {, the correlation coefficients for these plots were: A) } r=- \\
0.30(p<0.0001) ; B) r=0.10(p=0.13), C) r=-0.49(p<0.0001) ; D) r=0.08(p=0.46) ; E) r=- \\
0.24(p=0.05) ; F) r=0.05(p=0.72) ; G) r=-0.16(p=0.19) \text { and; H) } r=-0.07(p=0.55) \text {. }\end{array}$} \\
\hline
\end{tabular}


Table $3 \mathrm{k}$ coefficient, ICC, SEM and MDC values of the TUG test between the first and the second, and the second and the third trials, for the total sample and stratified for disease

\begin{tabular}{|c|c|c|c|c|}
\hline & \multirow[t]{2}{*}{ Total sample } & \multicolumn{3}{|c|}{ Subgroups } \\
\hline & & COPD & $\mathrm{CHF}$ & CRF \\
\hline \multicolumn{5}{|l|}{$1^{\text {st }}$ and $2^{\text {nd }}$ trials } \\
\hline k coefficient ${ }^{*}$ & 0.68 & 0.62 & 0.63 & 0.85 \\
\hline ICC $(95 \% \mathrm{Cl})+$ & $0.93(0.87-0.96)$ & $0.85(0.76-0.91)$ & $0.94(0.87-0.97)$ & $0.91(0.78-0.96)$ \\
\hline SEM (seconds) & 1.63 & 1.76 & 1.97 & 1.24 \\
\hline $\mathrm{MDC}_{95 \%}$ (seconds) & 4.52 & 4.88 & 5.46 & 3.44 \\
\hline $\mathrm{MDC}_{95 \%} \%(\%)$ & 35 & 43 & 33 & 29 \\
\hline \multicolumn{5}{|l|}{$2^{\text {nd }}$ and $3^{\text {rd }}$ trials } \\
\hline k coefficient ${ }^{*}$ & 0.83 & 1.00 & 0.80 & 0.49 \\
\hline ICC $(95 \% \mathrm{Cl})+$ & $0.98(0.97-0.98)$ & $0.95(0.93-0.97)$ & $0.98(0.97-0.99)$ & $0.96(0.94-0.98)$ \\
\hline SEM (seconds) & 0.79 & 0.79 & 1.06 & 0.78 \\
\hline $\mathrm{MDC}_{95 \%}$ (seconds) & 2.19 & 2.19 & 2.94 & 2.16 \\
\hline $\mathrm{MDC}_{95 \%} \%(\%)$ & 17 & 20 & 18 & 19 \\
\hline
\end{tabular}

: for the classification in FI, SFI or FD (see Table 1 for definition of abbreviations) ${ }^{1}$; †: $p<0.0001$ for all ICC values. 

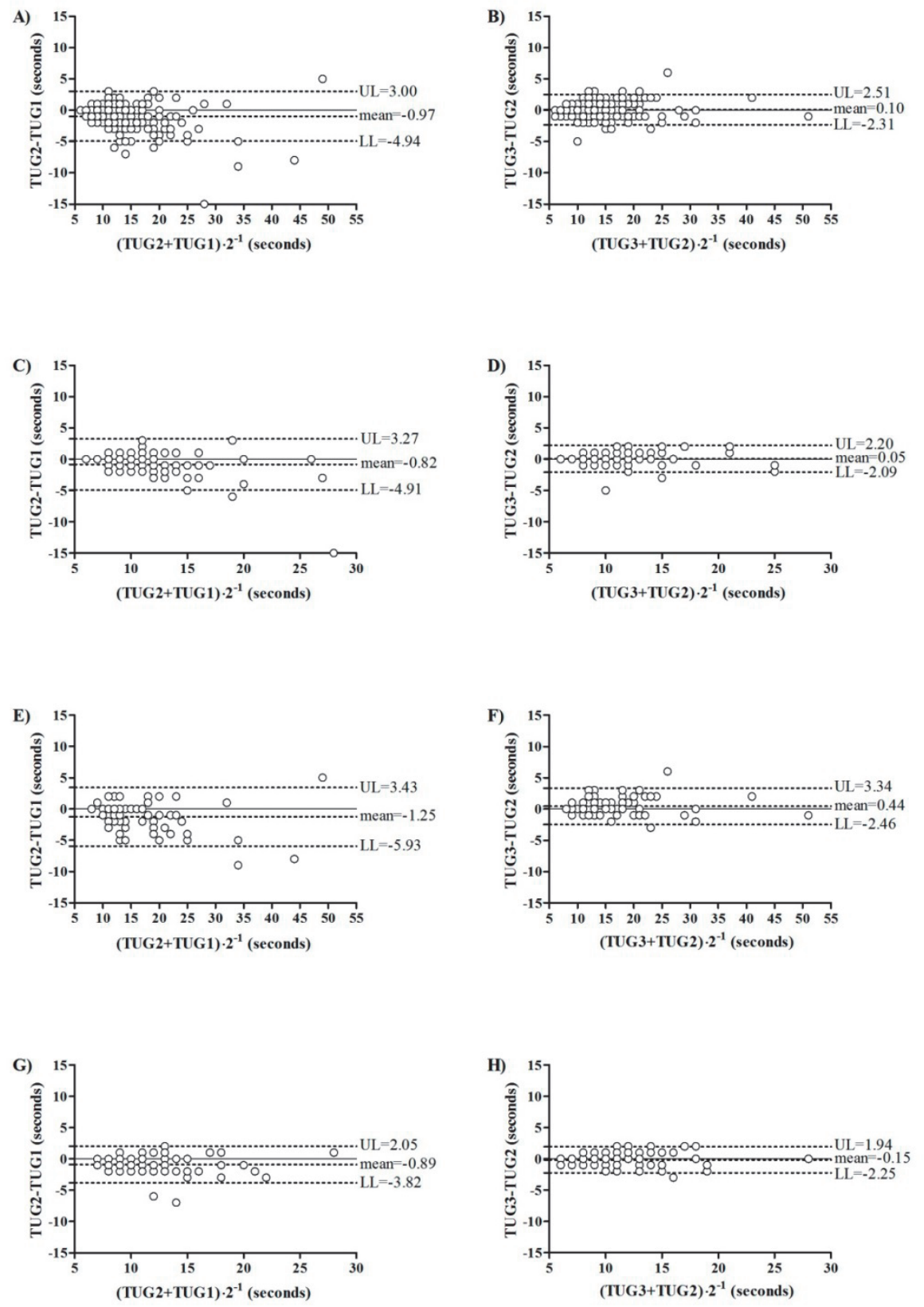

Figure 1 Bland and Altman plots of the difference against the mean for the first and second (A, $C, E$, and $G)$, and second and third $(B, D, F$, and $H$ ) trials. $A$ and $B$ : Total sample; $C$ and $D$ : COPD subgroup; $E$ and $F$ : $C H F$ subgroup; $G$ and $H$ : CRF subgroup. The central dotted line corresponds to the average difference between two trials (mean), whereas the lower dotted line corresponds to the lower limit (LL) and the upper dotted line to the upper limit (UL) of the $95 \%$ limits of agreement. 


\section{DISCUSSION}

This study has shown that there is high agreement in the TUG test results when the test was performed on the same day and in patients with advanced chronic organ failure (ICCs ranging from 0.85 to 0.98 ). Considering the classification proposed by Podsiadlo and Richardson ${ }^{1}$, good agreement was also found in general, but with a wider range of values (k coefficients from 0.49 to 1.00). Despite good agreement, the result of the TUG test significantly improved from the first to the second trial, but not from the second to the third. Furthermore, moderate to high values of SEM, $\mathrm{MDC}_{95 \%}$, and $\mathrm{MDC}_{95 \%} \%$ were found between the first two TUG test trials.

The TUG test is a performance test widely used and many studies have investigated its reliability in various populations ${ }^{1,2,4-6}$. Fewer studies, however, have focused on testretest reliability within the same day. In patients with Parkinson disease, Brusse et al. ${ }^{6}$ found an ICC value of 0.94 between two TUG test trials performed in the same day after one practice trial. Steffen and coworkers ${ }^{46}$ using a similar design found an ICC value of 0.97 in community-dwelling elderly. Other studies also found good agreement (i.e., ICC $>0.757$ ) for test-retest analyses, but considering between-days protocols ${ }^{4,5,42}$. Together with the present findings, these results show that the TUG test is consistently reliable in test-retest analyses, either when considered different populations or protocols. This is probably the first study to also report the level of agreement of the categorical classification ${ }^{1}$ of TUG test's results. Reasonable ? coefficients were observed, indicating moderate to excellent agreement ${ }^{47}$. Surprisingly, despite the fact that the patients had advanced diseases, the vast majority of them was classified as functionally independent, based on the results of the TUG test. In contrast, almost half of the patients were classified as care dependent, based on the CDS scores. Therefore, the functionality classification as proposed by Podsiadlo and Richardson ${ }^{1}$ may not be valid in patients with advanced chronic organ failure.

Despite good agreement, statistically significant improvement was found from the first to the second TUG test trial. Furthermore, the 95\% limits of agreement analysis with the plot of Bland and Altman between these trials revealed a range of approximately 8 seconds, which is actually similar to the TUG test results of some healthy subjects ${ }^{1}$. Most subjects (76\%) of the total sample presented their best result in one of the first two trials. Moreover, no difference was observed between the second and the third trials and a narrower 95\% limits of agreement interval was observed between them. Morris et al. ${ }^{48}$ also found no statistical difference in the comparison of the second to the third trial, either in healthy subjects or in patients with Parkinson disease. In addition, these authors found that the first trial was statistically higher than the second trial in both samples, and that its removal enhanced the reliability of the TUG test ${ }^{48}$. Therefore, we suggest that, in patients with advanced COPD, CHF or CRF, the TUG test needs to be 
performed twice to account for possible learning effects, but the third trial seems to be not necessary.

The ICC is a measure of relative consistency, which means that it concerns the consistency of subjects' position or rank in the group relative to others ${ }^{19}$. Measures of absolute consistency (i.e., the consistency of individual scores ${ }^{19}$ ) are also necessary. An example of such measure is the SEM, which in our study ranged from 1.24 to 1.97 seconds between the first and the second TUG test trials, showing close values among the different diseases. Based on a confidence interval, the SEM values can be used as an estimate of repeated measures ${ }^{7}$, which means that if a subject exceeds this interval in a repeated measure, a further one might be necessary. The SEM is considered to be a fixed characteristic of a measure, regardless of the population under investigation ${ }^{19}$. This probably explains why other studies with different populations and designs have found similar results, with SEM values ranging between 1.30 and 1.75 seconds $^{4,5,42}$.

Absolute and relative values of MDC were also calculated. The MDC can be translated as the minimal change necessary to represent a true change ${ }^{7}$. Both absolute and relative values are relevant in clinical practice, but the MDC\% is probably more useful as it considers the observed score of the subject ${ }^{43}$. Some authors have considered as an acceptable value of MDC\% a result of less than $30 \%{ }^{42,49}$, and higher values were found in the present study between the first two trials. Patients with COPD, CHF or CRF are well known to present clinical features that may impair functional mobility and balance, such as fatigue, muscle dysfunction, and exercise intolerance ${ }^{11-15}$ - especially in advanced diseases. The higher MDC\% values found in the present study may be explained by the presence of these characteristics. Unfortunately, muscle function and exercise tolerance were not accessed. Previous studies have reported similar to higher values of absolute MDC (3.5 to 11 seconds) 52, 43, 50, however subjects with diseases that directly interfere in the gait pattern (e.g., stroke, Parkinson disease) were assessed, probably leading to greater variability and, consequently, higher MDC $_{95 \%}$ values. The MDC values represent the minimum change detectable in repeated measures beyond the threshold of error ${ }^{7}$, having no relationship with prospective assessments, such as after an intervention. Studies which used the TUG test before and after an intervention have found a difference between these two moments which varies from -0.40 to $-1.5 s^{8,51,52}$. These values are well below the MDC values found in the present study. Therefore, a prospective validation of the absolute and relative MDC values found is necessary.

\section{Study limitations}

This study used a convenience sample and included only patients with advanced COPD, $\mathrm{CHF}$ or CRF, which could limit its external validity. Therefore, the present findings may not be directly applicable for patients with COPD, CHF or CRF in less severe stages. For 
this purpose, a replication of the present study using patients with these characteristics is advised. Nevertheless, in the present study different diseases were considered in a relatively large and heterogeneous sample, increasing the external validity of the results. Obviously, the calculated MDC values are specific for the populations assessed and need to be validated prospectively in future studies.

\section{CONCLUSION}

This is the first study to investigate the reliability of the TUG test in patients with clinically stable, but advanced COPD, CHF or CRF. The TUG test is reliable after only two trials, but relevant improvement occurs from the first to the second trial. The third trial adds little or no information to the previous ones. Therefore, we suggest doing only two TUG test trials in patients with these chronic diseases in advanced stage.

Values of SEM around 1.6 seconds, of $\mathrm{MDC}_{95 \%}$ around 4.5 seconds and of $\mathrm{MDC}_{95 \%} \%$ around $35 \%$ between the first two trials were identified. These values may be useful in daily clinical practice to define what can be expected and what represents true change in repeated measures in patients with advanced COPD, CHF or CRF like those assessed in the present study. Obviously, the current results need to be corroborated in future studies by other research groups. 


\section{REFERENCES}

1. Podsiadlo D, Richardson S. The timed "Up \& Go": a test of basic functional mobility for frail elderly persons. J Am Geriatr Soc 1991;39:142-8.

2. Flansbjer UB, Holmback AM, Downham D, Patten C, Lexell J. Reliability of gait performance tests in men and women with hemiparesis after stroke. J Rehabil Med 2005;37:75-82.

3. Okumiya K, Matsubayashi K, Nakamura T, Fujisawa M, Osaki Y, Doi Y, Ozawa T. The timed "up \& go" test is a useful predictor of falls in community-dwelling older people. J Am Geriatr Soc 1998;46:928-30.

4. Resnik L, Borgia M. Reliability of outcome measures for people with lower-limb amputations: distinguishing true change from statistical error. Phys Ther 2011;91:555-65.

5. Dal Bello-Haas V, Klassen L, Sheppard MS, Metcalfe A. Psychometric Properties of Activity, Self-Efficacy, and Quality-of-Life Measures in Individuals with Parkinson Disease. Physiother Can 2011;63:47-57.

6. Brusse KJ, Zimdars S, Zalewski KR, Steffen TM. Testing functional performance in people with Parkinson disease. Phys Ther 2005;85:134-41.

7. Portney LD, Watkins MP. Foundations of clinical research: applications to practice. 3rd ed. Upper Saddle River: Prentice Hall; 2008.

8. Witham MD, Crighton LJ, Gillespie ND, Struthers AD, McMurdo ME. The effects of vitamin D supplementation on physical function and quality of life in older patients with heart failure: a randomized controlled trial. Circ Heart Fail 2010;3:195-201.

9. Beauchamp MK, Hill K, Goldstein RS, Janaudis-Ferreira T, Brooks D. Impairments in balance discriminate fallers from non-fallers in COPD. Respir Med 2009;103:1885-91.

10. Taskapan H, Baysal O, Karahan D, Durmus B, Altay Z, Ulutas O. Vitamin D and muscle strength, functional ability and balance in peritoneal dialysis patients with vitamin D deficiency. Clin Nephrol 2011;76:110-6.

11. Spruit MA, Franssen FM, Rutten EP, Wagers SS, Wouters EF. Age-graded reductions in quadriceps muscle strength and peak aerobic capacity in COPD. Rev Bras Fisioter 2012;16:148-56.

12. Gosker HR, Lencer NH, Franssen FM, van der Vusse GJ, Wouters EF, Schols AM. Striking similarities in systemic factors contributing to decreased exercise capacity in patients with severe chronic heart failure or COPD. Chest 2003;123:1416-24.

13. van den Ham EC, Kooman JP, Schols AM, Nieman FH, Does JD, Franssen FM, Akkermans MA, Janssen PP, van Hooff JP. Similarities in skeletal muscle strength and exercise capacity between renal transplant and hemodialysis patients. Am J Transplant 2005;5:1957-65.

14. Seymour JM, Spruit MA, Hopkinson NS, Natanek SA, Man WD, Jackson A, Gosker HR, Schols AMWJ, Moxham J, Polkey MI, Wouters EFM. The prevalence of quadriceps weakness in COPD and the relationship with disease severity. Eur Respir J 2010;36:81-8.

15. Waschki B, Spruit MA, Watz H, Albert PS, Shrikrishna D, Groenen M, Smith C, Man WD-C, Tal-Singer R, Edwards LD, Calverley PMA, Magnussen H, Polkey MI, Wouters EFM. Physical activity monitoring in COPD: compliance and associations with clinical characteristics in a multicenter study. Respir Med 2012;106:522-30.

16. Orr R. Contribution of muscle weakness to postural instability in the elderly. A systematic review. Eur J Phys Rehabil Med 2010;46:183-220.

17. Horlings CG, van Engelen BG, Allum JH, Bloem BR. A weak balance: the contribution of muscle weakness to postural instability and falls. Nat Clin Pract Neurol 2008;4:504-15.

18. Bardin MG, Dourado VZ. Association between the occurrence of falls and the performance on the Incremental Shuttle Walk Test in elderly women. Rev Bras Fisioter 2012;16:275-80.

19. Weir JP. Quantifying test-retest reliability using the intraclass correlation coefficient and the SEM. J Strength Cond Res 2005;19:231-40.

20. Janssen DJ, Wouters EF, Schols JM, Spruit MA. Self-perceived symptoms and care needs of patients with severe to very severe chronic obstructive pulmonary disease, congestive heart failure or chronic renal failure and its consequences for their closest relatives: the research protocol. BMC Palliat Care 2008;7:5. 
21. Janssen DJ, Spruit MA, Uszko-Lencer NH, Schols JM, Wouters EF. Symptoms, comorbidities, and health care in advanced chronic obstructive pulmonary disease or chronic heart failure. J Palliat Med 2011;14:735-43.

22. Janssen DJ, Franssen FM, Wouters EF, Schols JM, Spruit MA. Impaired health status and care dependency in patients with advanced COPD or chronic heart failure. Qual Life Res 2011;20:1679-88.

23. Janssen DJ, Spruit MA, Wouters EF, Schols JM. Family caregiving in advanced chronic organ failure. J Am Med Dir Assoc 2012;13:394-9.

24. Janssen DJ, Spruit MA, Schols JM, Wouters EF. A call for high-quality advance care planning in outpatients with severe COPD or chronic heart failure. Chest 2011;139:1081-8.

25. Janssen DJ, Spruit MA, Schols JM, Cox B, Nawrot TS, Curtis JR, Wouters EFM. Predicting changes in preferences for life-sustaining treatment among patients with advanced chronic organ failure. Chest 2012;141:1251-9.

26. Janssen DJ, Curtis JR, Au DH, Spruit MA, Downey L, Schols JM, Wouters EFM, Engelberg RA. Patientclinician communication about end-of-life care for Dutch and US patients with COPD. Eur Respir J 2011;38:268-76.

27. Janssen DJ, Spruit MA, Schols JM, van der Sande FM, Frenken LA, Wouters EF. Insight Into Advance Care Planning for Patients on Dialysis. J Pain Symptom Manage 2013;45:104-13.

28. Janssen DJ, Spruit MA, Wouters EF, Schols JM. Symptom distress in advanced chronic organ failure: disagreement among patients and family caregivers. J Palliat Med 2012;15:447-56.

29. Wilke S, Janssen DJ, Wouters EF, Schols JM, Franssen FM, Spruit MA. Correlations between diseasespecific and generic health status questionnaires in patients with advanced COPD: a one-year observational study. Health Qual Life Outcomes 2012;10:98.

30. Janssen DJ, Wouters EF, Schols JM, Spruit MA. Care Dependency Independently Predicts Two-Year Survival in Outpatients With Advanced Chronic Organ Failure. J Am Med Dir Assoc 2013;14:194-8.

31. Global Initiative for Chronic Obstructive Lung Disease. Global strategy for the diagnosis, management andpreventionof chronic obstructive lung disease: revised 2011. Available at: http://www.goldcopd.org/uploads/ users/files/GOLD_Report_2011_Feb21.pdf. Accessed August 31, 2012.

32. Wilson RC, Jones PW. A comparison of the visual analogue scale and modified Borg scale for the measurement of dyspnoea during exercise. Clin Sci (Lond) 1989;76:277-82.

33. American Thoracic Society. Standardization of Spirometry, 1994 Update. Am J Respir Crit Care Med 1995;152:1107-36.

34. Knudson RJ, Burrows B, Lebowitz MD. The maximal expiratory flow-volume curve: its use in the detection of ventilatory abnormalities in a population study. Am Rev Respir Dis 1976;114:871-9.

35. Rutten FH, Cramer MJ, Zuithoff NP, Lammers JW, Verweij W, Grobbee DE, Hoes AW. Comparison of Btype natriuretic peptide assays for identifying heart failure in stable elderly patients with a clinical diagnosis of chronic obstructive pulmonary disease. Eur J Heart Fail 2007;9:651-9.

36. Charlson ME, Pompei $\mathrm{P}$, Ales KL, MacKenzie CR. A new method of classifying prognostic comorbidity in longitudinal studies: development and validation. J Chronic Dis 1987;40:373-83.

37. Charlson M, Szatrowski TP, Peterson J, Gold J. Validation of a combined comorbidity index. J Clin Epidemiol 1994;47:1245-51.

38. Dijkstra A, Tiesinga LJ, Goossen WT, Dassen TW. Further psychometric testing of the Dutch Care Dependency Scale on two different patient groups. Int J Nurs Pract 2002;8:305-14.

39. Dijkstra A, Tiesinga LJ, Plantinga L, Veltman G, Dassen TW. Diagnostic accuracy of the care dependency scale. J Adv Nurs 2005;50:410-6.

40. Dolan P. Modeling valuations for EuroQol health states. Med Care 1997;35:1095-108.

41. Shrout PE, Fleiss JL. Intraclass correlations: uses in assessing rater reliability. Psychol Bull 1979;86:420-8.

42. Huang SL, Hsieh CL, Wu RM, Tai CH, Lin CH, Lu WS. Minimal detectable change of the timed "up \& go" test and the dynamic gait index in people with Parkinson disease. Phys Ther 2011;91:114-21. 


\section{CHAPTER 5}

43. Hiengkaew V, Jitaree K, Chaiyawat P. Minimal detectable changes of the Berg Balance Scale, Fugl-Meyer Assessment Scale, Timed "Up \& Go" Test, gait speeds, and 2-minute walk test in individuals with chronic stroke with different degrees of ankle plantarflexor tone. Arch Phys Med Rehabil 2012;93:1201-8.

44. Bland JM, Altman DG. Statistical methods for assessing agreement between two methods of clinical measurement. Lancet 1986;1:307-10.

45. Vandenbroucke JP, von Elm E, Altman DG, Gotzsche PC, Mulrow CD, Pocock SJ, Poole C, Schlesselman JJ, Egger $M$; STROBE Initiative. Strengthening the Reporting of Observational Studies in Epidemiology (STROBE): explanation and elaboration. Epidemiology 2007;18:805-35.

46. Steffen TM, Hacker TA, Mollinger L. Age- and gender-related test performance in community-dwelling elderly people: Six-Minute Walk Test, Berg Balance Scale, Timed Up \& Go Test, and gait speeds. Phys Ther 2002;82:128-37.

47. Landis JR, Koch GG. The measurement of observer agreement for categorical data. Biometrics 1977;33:159-74.

48. Morris S, Morris ME, lansek R. Reliability of measurements obtained with the Timed "Up \& Go" test in people with Parkinson disease. Phys Ther 2001;81:810-8.

49. Smidt N, van der Windt DA, Assendelft WJ, Mourits AJ, Deville WL, de Winter AF, Bouter LM. Interobserver reproducibility of the assessment of severity of complaints, grip strength, and pressure pain threshold in patients with lateral epicondylitis. Arch Phys Med Rehabil 2002;83:1145-50.

50. Steffen T, Seney M. Test-retest reliability and minimal detectable change on balance and ambulation tests, the 36-item short-form health survey, and the unified Parkinson disease rating scale in people with parkinsonism. Phys Ther 2008;88:733-46.

51. Ling KW, Wong FS, Chan WK, Chan SY, Chan EP, Cheng YL, Yu WY. Effect of a home exercise program based on tai chi in patients with end-stage renal disease. Perit Dial Int 2003;23(Suppl 2):S99-S103.

52. Beauchamp MK, O'Hoski S, Goldstein RS, Brooks D. Effect of pulmonary rehabilitation on balance in persons with chronic obstructive pulmonary disease. Arch Phys Med Rehabil 2010;91:1460-5.

Reprinted from Archives of Physical Medicine and Rehabilitation, Vol. 94, Mesquita R, Janssen DJA, Wouters EFM, Schols JMGA, Pitta F, Spruit MA, Within-day test-retest reliability of the Timed "Up \& Go" test in patients with advanced chronic organ failure, pp. 2131-8, Copyright (2013), with permission from Elsevier. 


\section{SUPPLIERS' LIST}

a. IBM North America, 590 Madison Avenue, New York, NY 10022, United States.

b. GraphPad Software Inc, 2236 Avenida de la Playa, La Jolla, CA 92037, United States. 



\section{CHAPTER 6}

Measurement properties of the Timed Up \& Go test in patients with COPD

Rafael Mesquita, Sarah Wilke, Dionne E. Smid, Daisy J.A. Janssen, Frits M.E. Franssen, Vanessa S. Probst, Emiel F.M. Wouters, Jean W.M. Muris, Fabio Pitta, and Martijn A. Spruit

Chronic Respiratory Disease 2016; 13: 344-352 


\begin{abstract}
Objectives: We aimed to investigate the construct validity of the Timed Up \& Go (TUG) test in chronic obstructive pulmonary disease (COPD); to identify characteristics related to an abnormal TUG time; and to examine the responsiveness of the TUG to pulmonary rehabilitation (PR).
\end{abstract}

Methods: TUG time was assessed before and after comprehensive PR in 500 COPD patients; and compared cross-sectionally in 100 non-COPD subjects. Physical health outcomes, mental health outcomes, symptom-related outcomes and multidimensional indices were assessed in COPD patients only.

Results: Good convergent and discriminant validity was demonstrated by fair-tomoderate correlation with physical health outcomes, symptom-related outcomes, and multidimensional indices $\left(r_{s}=0.18-0.70\right)$, and by little correlation with mental health outcomes $\left(r_{s}=0.21-0.26\right)$. COPD patients had a worse TUG time than non-COPD subjects, demonstrating known-groups validity. A TUG time of $11.2 \mathrm{~s}$ had good sensitivity (0.75) and specificity (0.83) for identifying patients with a baseline six-minute walk distance $<350 \mathrm{~m}$. TUG time improved after PR $(P<0.0001)$ and a change of 0.9-1.4 s was identified as clinically important.

Conclusions: The TUG is valid and responsive in COPD. An abnormal result is indicative of poor health outcomes. This simple test provides valuable information and can be adopted in clinical and research settings. 


\section{A- INTRODUCTION}

The use of simple functional performance tests, such as the five-repetition sit-to-stand and the 4-metre gait speed has gained attention in chronic obstructive pulmonary disease (COPD) patients. Indeed, these tests are reliable, valid, and responsive to pulmonary rehabilitation $(P R)$ in COPD patients ${ }^{1-3}$.

Similarly, the Timed Up \& Go (TUG) test is also simple, cheap, and reliable ${ }^{4,5}$. Subjects are requested to stand up from a chair, walk a distance of $3 \mathrm{~m}$ at a comfortable and safe pace, turn and walk back to the chair to sit down again ${ }^{4}$. This test has been used for the assessment of functional mobility, walking ability, dynamic balance, and risk of falling in subjects with a variety of conditions ${ }^{4,6,7}$. Despite its simplicity, the TUG has been shown to predict morbidity and mortality in different populations ${ }^{8-10}$. The TUG is even suggested by the American Geriatrics Society and British Geriatrics Society as a gait and balance assessment tool to identify elderly patients who may benefit from a detailed fall risk assessment $^{11}$. In addition, the British Geriatrics Society, Age UK and Royal College of General Practitioners suggest it as a measure for the recognition of frailty in older peo$\mathrm{ple}^{12}$. The European Respiratory Society (statement on nutritional assessment and therapy in COPD) also suggest it as an appropriate measure of physical performance for research and clinical practice in COPD $^{13}$.

Even though COPD patients may present with lower-limb muscle dysfunction ${ }^{14}$, limited exercise capacity ${ }^{15}$, impaired balance ${ }^{16}$, and increased risk of falling ${ }^{17}$, the TUG has been used scarcely in this population. The TUG is reliable in COPD patients ${ }^{5}$, but other measurement properties, such as convergent discriminant and known-groups validity and responsiveness to PR, have not been comprehensively studied. Indeed, a recent systemic review on simple functional tests in COPD found no study investigating TUG's responsiveness and only one study investigating TUG's validity ${ }^{18}$.

Therefore, we aimed: 1) to investigate the construct validity of the TUG in COPD patients referred for PR; 2) to identify characteristics related to an abnormal TUG time; and 3) to examine the responsiveness of the TUG to a comprehensive PR programme.

\section{A- METHODS}

\section{B- Design and participants}

The present study includes data from the Chance study, a longitudinal observational study aiming to investigate the impact of cardiovascular comorbidities on health status in COPD patients ${ }^{19}$. Patients were assessed before and after a comprehensive PR pro- 
gramme between April 2012 and September 2014. Patients had to have stable disease (i.e., no exacerbation in the previous 4 weeks) and complete data on the TUG in order to be included in the current analysis. In addition, a sample of control subjects without COPD or any other debilitating disease was recruited in the primary care setting selected from the Registration Network Family Practices ${ }^{20}$. This sample is referred to as nonCOPD subjects and had to have a post-bronchodilator forced expiratory volume in the first second / forced vital capacity ratio $\left(\mathrm{FEV}_{1} / \mathrm{FVC}\right) \geq 70 \%$ and a healthy condition as determined by the investigator based on medical history and physical examination. The above-mentioned study was registered at the Dutch Trial Register (NTR 3416) and received approval from the Medical Ethical Committee of the Maastricht University Medical Center + (MUMC+), Maastricht, the Netherlands (METC 11-3-070). All participants gave formal written consent to participate.

\section{B- Outcome measures and PR programme}

The TUG was performed in all participants according to the original protocol: subjects are required to stand up from a chair, walk at a comfortable and safe speed a distance of 3 metres, turn and walk back to the chair to sit down again ${ }^{4}$. Participants wore their usual footwear and were allowed to use the chair's arms if they wanted to. If necessary, a demonstration and/or a practice trial were performed and the use of walking aids and/or oxygen was allowed. Participants on oxygen therapy were asked to carry the device for delivering oxygen only when using ambulatory oxygen. The time in seconds to perform the test was recorded by ordinary stopwatches and used as the main outcome of analysis. The timing started when the participant's back left the back of the chair and ended after the walk, when the participant's back was positioned against the back of the chair. Three trials were performed by the same assessor and the best trial (i.e., shortest duration) was used for analysis ${ }^{5}$. Participants were allowed to rest between the trials, if necessary.

Demographics, anthropometrics, lung function, and clinical data, such as comorbidities, smoking status and use of long-term oxygen therapy and/or walking aids, were assessed in all participants. In addition, the number of exacerbations in the previous 12 months (self-reported), physical health outcomes (six-minute walk test (6MWT), cardiopulmonary exercise test, and isokinetic quadriceps peak torque), symptom-related outcomes (modified Medical Research Council scale, COPD assessment test, and St. George's Respiratory Questionnaire), and mental health outcomes (Hospital Anxiety and Depression Scale) were assessed in COPD patients only. Furthermore, the Global Initiative for Chronic Obstructive Lung Disease (GOLD) 2007 and 2011 classifications were used, and the multidimensional severity indices Age, Dyspnoea, and Obstruction (ADO), and updated Body-mass index, airflow Obstruction, Dyspnoea, and Exercise capacity (BODE) 
were calculated. All details about each measurement can be found in the supplemental material.

Patients were enrolled in a 40-session PR programme (inpatient, 8 weeks, 5 days $\cdot$ week

${ }^{1}$; or outpatient, 8 weeks, 3 half days.week ${ }^{-1}$, followed by 8 weeks, 2 half days.week ${ }^{-1}$ ). In brief, the programme consisted of moderate-to-high-intensity progressive exercise training (i.e., endurance and strength training); nutritional support, occupational therapy and psychological counselling, if indicated; and twenty 1-hour educational group sessions. Exercise training prescription was based on a careful characterisation of the extra-pulmonary features of patients with COPD performed before PR. The training intensity increased during the rehabilitation period based on dyspnoea and fatigue symptom scores. Moreover, all patients underwent flexibility exercises, general physical exercise for lower and upper extremities, and daily supervised 30-min outdoor walks. Patients who were too dyspnoeic to perform endurance, interval or resistance training received lower-limb high-frequency neuromuscular electrical stimulation ${ }^{21}$. The programme was implemented by an interdisciplinary team including chest physician, respiratory nurses, dietician, occupational therapist, physiotherapist, psychologist and social worker.

\section{B- Statistical Analysis}

Data were expressed as relative frequency, mean \pm standard deviation, mean (95\% confidence intervals), or median (interquartile range), as appropriate. Construct validity was assessed in terms of convergent, discriminant and known-groups validity. Pearson or Spearman coefficient was used to investigate convergent and discriminant validity. We hypothesized that TUG time would correlate negatively and at least moderately (correlation coefficient $>0.40$ ) with physical health outcomes, and negatively and at least fairly (correlation coefficient $>0.30$ ) with symptom-related outcomes and multidimensional indices. We also hypothesized only positive, negligible-to-little correlation with mental health outcomes (correlation coefficient $<0.30$ ). Mann-Whitney $U$ test was used to investigate known-groups validity by comparing the TUG time between COPD and non-COPD subjects. The hypothesis was that COPD subjects would have a worse (i.e., longer) TUG time than non-COPD subjects.

For responsiveness, paired t test or Wilcoxon signed-rank test was used to compare pre- and post-PR measurements. In order to test whether changes in TUG time related to relevant changes in six-minute walk distance (6MWD), changes in TUG time were compared between patients with a clinically important change in 6MWD ( $\geq 30 \mathrm{~m})$ and patients with a non-clinically important change $(<30 \mathrm{~m})^{22}$. We hypothesized that a patient with a clinically meaningful change in 6MWD would have larger improvements in 
TUG time. Effect sizes were estimated with Cohen's d; logarithmic transformation was applied to substantially skewed data.

For minimal clinically important difference (MCID) estimation, anchor and distribution based methods were used. The 6MWT was used as anchor if there was a significant, fair correlation (correlation coefficient $\geq 0.30)^{23}$ between change in TUG time and change in anchor. Distribution based methods included the effect size and the minimal detectable change at $95 \%$ confidence $\left(\mathrm{MDC}_{95 \%}\right)$. A moderate effect size was calculated as half the standard deviation of the change scores ${ }^{24}$, while the MDC $_{95 \%}$ was calculated as 1.96 $\mathrm{x} V 2 \mathrm{x}$ standard error of measurement ${ }^{25}$. Kruskal-Wallis test (followed by Dunn post-hoc test), Mann-Whitney $U$ test, or chi-square test was used for other comparisons, and logistic regression analysis was used to investigate associations with an abnormal TUG time. Receiver operating characteristic curve analysis was used to identify the TUG time with best sensitivity and specificity for identifying patients with a $6 \mathrm{MWD}<350 \mathrm{~m}^{26}$. Statistical significance was considered as $\mathrm{P}<0.05$ and the analyses were performed using SPSS 17.0 (SPSS, Chicago, Illinois, United States of America (USA)) or GraphPad Prism 5 (GraphPad Software, La Jolla, California, USA). Details on sample size calculations according to the proposed objectives can be found in the supplemental material.

\section{A- RESULTS}

Of the 518 COPD patients included in the Chance study, 18 failed to perform the TUG test: $40 \%$ due to musculoskeletal problems, $10 \%$ due to injury, and $60 \%$ due to other medical reasons. Therefore, 500 COPD patients were included, together with 100 nonCOPD subjects. Patients had mild to very severe COPD. Patients had worse lung function and higher scores on the Charlson comorbidity index than non-COPD subjects (Table 1 ). In addition, patients had a slightly lower body mass index and a higher proportion of current smokers. 
Table 1 Characteristics of the samples

\begin{tabular}{|c|c|c|c|}
\hline Characteristic & COPD & Non-COPD & $\mathrm{P}$-value \\
\hline $\mathrm{N}$ & 500 & 100 & NA \\
\hline Male sex (\%) & 55 & 55 & 0.97 \\
\hline Age (years) & $64(57-71)$ & $64(60-69)$ & 0.64 \\
\hline $\mathrm{BMI}\left(\mathrm{kg} \cdot \mathrm{m}^{-2}\right)$ & $25.8(21.7-29.9)$ & $26.8(24.4-30.3)$ & 0.005 \\
\hline $\mathrm{FEV}_{1}(\%$ predicted $)$ & $46(32-63)$ & $113(101-123)$ & $<0.0001$ \\
\hline GOLD 1/2/3/4 (\%) & $7 / 36 / 38 / 19$ & NA & NA \\
\hline GOLD A/B/C/D (\%) & $1 / 22 / 2 / 75$ & NA & NA \\
\hline Charlson Comorbidity Index (points) & $1(1-2)$ & $0(0-2)$ & $<0.0001$ \\
\hline Smoking status N/F/C (\%) & $1 / 77 / 22$ & $25 / 63 / 12$ & $<0.0001$ \\
\hline Long-term oxygen therapy (\%) & 24 & 0 & $<0.0001$ \\
\hline$\geq 2$ exacerbations in the previous 12 months (\%) & 56 & NA & NA \\
\hline Walking aids during the TUG (\%) & 9 & 0 & $<0.0001$ \\
\hline
\end{tabular}

Data expressed as relative frequency or median (interquartile range). BMI = body mass index; $\mathrm{C}=$ current smoker; $\mathrm{F}=$ former smoker; $\mathrm{FEV}_{1}=$ forced expiratory volume in the first second; GOLD = Global Initiative for Chronic Obstructive Lung Disease; $N=$ never smoker; NA = not applicable. alncomplete data for GOLD groups, $n=488$.

\section{B- Construct validity}

Table 2 presents the correlations between TUG time and other health measures in patients with COPD. In general, fair-to-moderate correlations were found with physical health outcomes, symptom-related outcomes and multidimensional indices, according to convergent validity tests. The strongest correlation was found with functional exercise capacity (Figure 1; Table 2). Poor correlation was found with symptoms of anxiety and depression, according to discriminant validity tests. As part of known-groups validity analysis, COPD patients showed a worse TUG time (i.e., longer) compared with nonCOPD subjects $(9.8(8.5-11.8)$ versus $8.3(7.3-9.6)$ s, respectively; Figure 2$)$, further supporting construct validity. 


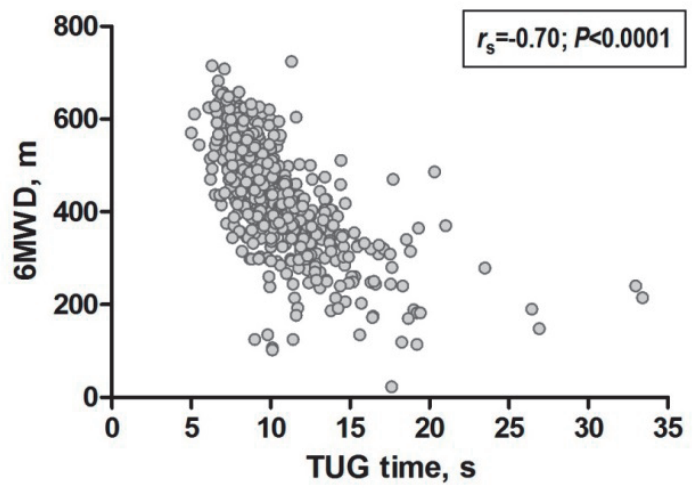

Figure 1 Correlation between Timed Up \& Go (TUG) time and six-minute walk distance (6MWD) in COPD patients.

Table 2 Correlations between TUG and other measures in COPD patients

\begin{tabular}{lcc}
\hline \multicolumn{1}{c}{ Outcome measure } & Median (IQR) & $\mathrm{r}_{\mathrm{s}}(95 \% \mathrm{CI})^{\mathrm{a}}$ \\
\hline Physical health outcomes & & \\
6MWT (m) & $435(354-512)$ & $-0.70(-0.75--0.65)$ \\
CPET (watts) & $64(47-87)$ & $-0.44(-0.52--0.37)$ \\
IQPT (joules) & $91.4(66.0-118.6)$ & $-0.33(-0.42--0.25)$ \\
Symptom-related outcomes & & \\
mMRC (points) & $2(2-3)$ & $0.49(0.42-0.56)$ \\
CAT (points) & $22(17-26)$ & $0.27(0.19-0.36)$ \\
SGRQ-C & & $0.18(0.09-0.27)$ \\
Symptoms (points) & $63.1(48.5-76.1)$ & $0.39(0.31-0.46)$ \\
Activity (points) & $83.6(67.8-92.2)$ & $0.40(0.32-0.47)$ \\
Impact (points) & $51.2(34.2-64.1)$ & $0.41(0.33-0.48)$ \\
Total (points) & $63.6(49.9-74.2)$ & \\
Multidimensional indices & & $0.52(0.45-0.58)$ \\
ADO index (points) & $4(3-6)$ & $0.55(0.49-0.61)$ \\
Updated BODE index (points) & $3(2-6)$ & $0.21(0.12-0.30)$ \\
Mental health outcomes & & $0.26(0.17-0.34)$ \\
HADS anxiety (points) & $7(4-11)$ & \\
HADS depression (points) & $7-11)$ & \\
\hline GWWT: six-minute walk test; BODE & & \\
\hline
\end{tabular}

6MWT: six-minute walk test; BODE = body-mass index, airflow obstruction, dyspnoea, and exercise capacity index; $C A T$ = COPD assessment test; $C P E T$ = cardiopulmonary exercise test; HADS = hospital anxiety and depression scale; IQPT: isokinetic quadriceps peak torque; $\mathrm{mMRC}=$ modified medical research council; SGRQ$C=$ COPD version of the St. George's respiratory questionnaire. Incomplete data for certain variables: 6MWT, $\mathrm{n}=497$; CPET, $\mathrm{n}=479 ;$ IQPT, $\mathrm{n}=452 ; \mathrm{mMRC}, \mathrm{n}=495 ; \mathrm{HADS}, \mathrm{n}=485$; CAT and $\mathrm{SGRQ}, \mathrm{n}=488$; $A D O$ index, $\mathrm{n}=495$; and updated BODE index, $n=492$. aAll P-values were $<0.0001$. 


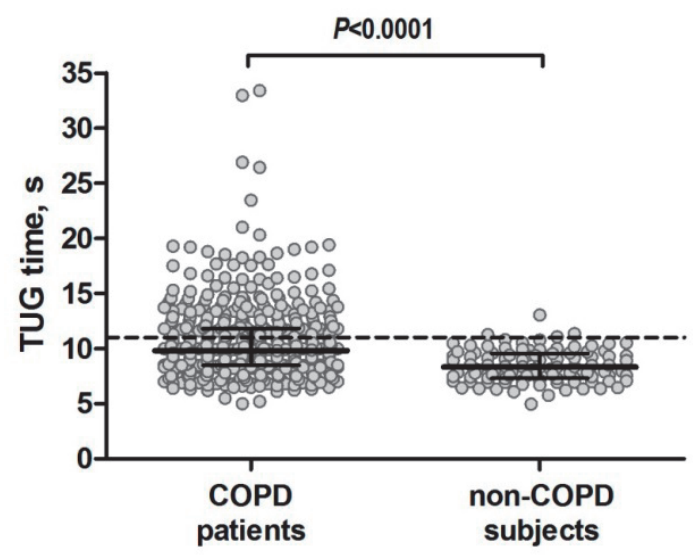

Figure 2 Timed Up \& Go (TUG) time in COPD patients and in non-COPD subjects. The horizontal bars represent median (interquartile range $25 \%-75 \%$ ). The dotted line corresponds to the $95^{\text {th }}$ percentile in the sample of non-COPD subjects (i.e., $11 \mathrm{~s}$ ), which represents the threshold adopted to identify an abnormal TUG time.

\section{B- Associations with abnormal TUG time}

Abnormal TUG time was defined as a TUG time $>11 \mathrm{~s}$, as this equals the $95^{\text {th }}$ percentile of the current non-COPD subjects. The proportion of COPD patients with an abnormal TUG time was 33\%. Patients with an abnormal TUG time were older, had higher body mass index and Charlson Comorbidity Index score, worse functional and maximal exercise capacity, worse quadriceps muscle function, more symptoms of dyspnoea, anxiety and depression, more impaired health status, worse disease severity based on multidimensional indices, and a higher proportion of former smokers, patients on long-term oxygen therapy and patients using walking aids during the TUG (Table 3). In general, significant associations between a worse TUG time and the above-mentioned outcomes were confirmed in logistic regression models and after stratification for these outcomes (e-Tables 1 and 2, respectively; supplemental material). Interestingly, a TUG time of $11.2 \mathrm{~s}$ had the best combination of sensitivity (0.75) and specificity $(0.83)$ for identifying patients with a baseline 6MWD $<350 \mathrm{~m}$ (area under the curve $=0.86(95 \% \mathrm{Cl} 0.82-$ $0.90), P<0.0001$; e-Figure 1 in the supplemental material). Similar analyses considering a baseline 6MWD $<200 \mathrm{~m}$ revealed that the cut-off with best combination of sensitivity (0.67) and specificity (0.90) was $13.8 \mathrm{~s}$ (area under the curve $=0.85(95 \% \mathrm{Cl} 0.77-$ 0.93), $\mathrm{P}<0.0001$; e-Figure 2 in the supplemental material). 
Table 3 Outcome measures in COPD patients with and without an abnormal baseline TUG time

\begin{tabular}{|c|c|c|c|}
\hline Characteristic & $\begin{array}{l}\text { Normal } \\
(n=333)\end{array}$ & $\begin{array}{c}\text { Abnormal } \\
(n=167)\end{array}$ & P-value \\
\hline Male sex (\%) & 56 & 53 & 0.63 \\
\hline Age (years) & $61(56-69)$ & $69(62-75)$ & $<0.0001$ \\
\hline $\mathrm{BMI}\left(\mathrm{kg} \cdot \mathrm{m}^{-2}\right)$ & $25.0(21.1-28.9)$ & $26.4(23.1-31.1)$ & 0.001 \\
\hline $\mathrm{FEV}_{1}(\%$ predicted) & $46(34-63)$ & $46(30-60)$ & 0.24 \\
\hline GOLD 1/2/3/4 (\%) & $8 / 35 / 40 / 17$ & $6 / 37 / 34 / 23$ & 0.34 \\
\hline GOLD A/B/C/D (\%) & $2 / 23 / 3 / 72$ & 0/19/0/81 & 0.06 \\
\hline Charlson Comorbidity Index (points) & $1(1-2)$ & $2(1-3)$ & $<0.0001$ \\
\hline Smoking status N/F/C (\%) & $1 / 74 / 25$ & $2 / 82 / 16$ & 0.02 \\
\hline Long-term oxygen therapy (\%) & 16 & 39 & $<0.0001$ \\
\hline$\geq 2$ exacerbations in the previous 12 months (\%) & 51 & 67 & 0.001 \\
\hline Walking aids during the TUG (\%) & 2 & 23 & $<0.0001$ \\
\hline 6MWT (m) & $480(415-545)$ & $340(273-396)$ & $<0.0001$ \\
\hline CPET (watts) & $69(54-97)$ & $51(41-66)$ & $<0.0001$ \\
\hline IQPT (joules) & $97.3(71.3-124.2)$ & $77.0(58.7-98.0)$ & $<0.001$ \\
\hline mMRC (points) & $2(1-3)$ & $3(2-4)$ & $<0.0001$ \\
\hline HADS anxiety (points) & $6(4-11)$ & $8(5-12)$ & 0.005 \\
\hline HADS depression (points) & $6(3-10)$ & $9(6-12)$ & $<0.0001$ \\
\hline CAT (points) & $21(17-25)$ & $23(19-28)$ & $<0.0001$ \\
\hline \multicolumn{4}{|l|}{ SGRQ-C } \\
\hline Symptoms (points) & $61.1(47.7-74.0)$ & $67.5(53.6-79.5)$ & 0.002 \\
\hline Activity (points) & $77.0(60.4-91.8)$ & $91.8(83.6-100.0)$ & $<0.0001$ \\
\hline Impact (points) & $46.3(29.0-60.3)$ & $59.9(45.3-74.4)$ & $<0.0001$ \\
\hline Total (points) & $58.6(43.7-70.2)$ & $68.8(59.5-79.9)$ & $<0.0001$ \\
\hline ADO index (points) & $4(3-5)$ & $6(5-7)$ & $<0.0001$ \\
\hline Updated BODE index (points) & $3(2-4)$ & $7(4-10)$ & $<0.0001$ \\
\hline TUG time (s) & $8.8(7.9-9.8)$ & $13.1(11.8-14.9)$ & $<0.0001$ \\
\hline
\end{tabular}

Data expressed as relative frequency or median (interquartile range). See tables 1 and 2 for definition of abbreviations and number of subjects with complete data.

\section{B- Responsiveness to PR and MCID estimation}

500 patients started PR, of which 378 patients (76\%) had complete data after PR. 92 patients dropped-out (18\%) and 30 patients (6\%) did not perform the TUG after PR. Patients who did not complete PR had worse baseline TUG time, a higher proportion of current smokers and tended to have worse forced expiratory volume in the first second at baseline (e-Table 3; supplemental material).

Mean TUG time improved significantly from $10.2 \pm 2.7$ to $9.7 \pm 2.3 \mathrm{~s}$ after PR (mean change $-0.5(95 \% \mathrm{Cl}-0.6--0.3) \mathrm{s}, \mathrm{P}<0.0001)$, with an effect size of 0.16 (Figure 3). Sig- 
nificant improvements in 6MWD (445 \pm 112 to $469 \pm 114 \mathrm{~m}, \mathrm{P}<0.0001$; effect size of 0.21 ), and St. George's Respiratory Questionnaire (COPD-version) total score (61.3 (49.0 - 72.3) to 50.7 (40.1 - 62.0) points, $P<0.0001$; effect size of 0.45 ) were also observed following PR. A significant correlation was observed between changes in TUG time and in 6MWD ( $r=-0.32 ; P<0.0001)$. Patients with a clinically important change in 6MWD of $30 \mathrm{~m}$ or more ${ }^{22}$ had a larger change in TUG time than patients with a non-clinically important change: $-1.0(95 \% \mathrm{Cl}-1.3--0.7)$ versus $-0.1(95 \% \mathrm{Cl}-0.3-0.2) \mathrm{s}$, respectively $(P<0.0001)$. The moderate effect size for the TUG time was $0.9 \mathrm{~s}$, while the absolute $\mathrm{MDC}_{95 \%}$ was $1.4 \mathrm{~s}$, which corresponds to a relative $\mathrm{MDC}_{95 \%}$ of $14 \%$. After stratifying for normal or abnormal baseline TUG time, only the latter group showed significant improvements after PR: $-1.5(95 \% \mathrm{Cl}-1.9--1.0)$ versus $0.01(95 \% \mathrm{Cl}-0.2-0.2) \mathrm{s}$ $(P<0.0001$; Figure 3).

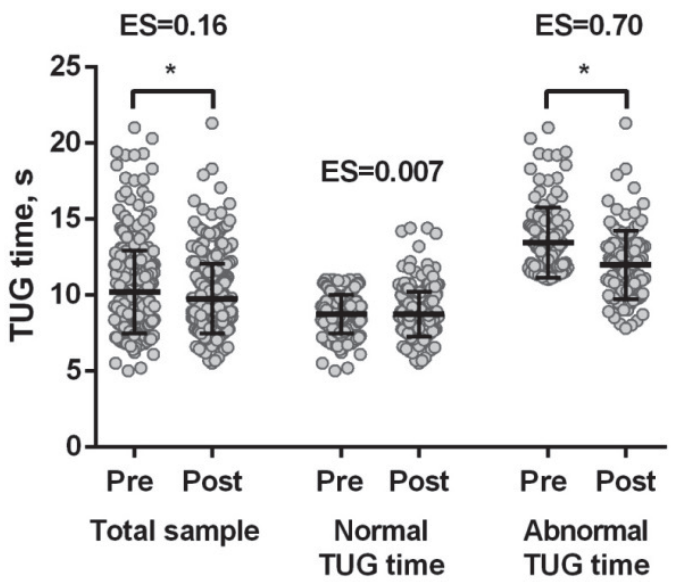

Figure 3 Timed Up \& Go (TUG) time before and after pulmonary rehabilitation in COPD patients. The horizontal bars represent mean (standard deviation). $\mathrm{ES}=$ effect size. ${ }^{*} \mathrm{P}<0.05$.

\section{A- DISCUSSION}

This is the first study to investigate different measurement properties of the TUG in COPD patients. The TUG was shown to be valid for the assessment of functional performance and responsive to PR. COPD patients with a baseline TUG time $>11 \mathrm{~s}$ showed poorer health outcome measures, but were more responsive to $P R$ in terms of the performance on the TUG. Moreover, a TUG time of $11.2 \mathrm{~s}$ had the best combination of sensitivity and specificity for identifying patients with a baseline 6MWD $<350 \mathrm{~m}$.

In the study of TUG's development ${ }^{4}$, the authors already identified this test as a valid outcome measure. Other studies have also supported the validity of TUG in different 
populations ${ }^{27-29}$, but no study so far has comprehensively investigated this property in patients with COPD. This gap was highlighted in a recent systemic review on simple functional tests in COPD which found only one study investigating the validity of the TUG in this population ${ }^{18}$. Nevertheless, only the correlation with lower limb maximal strength was investigated, and in fact this correlation did not reach statistical significance $^{18}$. Fair-to-moderate significant correlation was found between TUG time and other measures of functional performance (correlation coefficients $0.33-0.70$ ), suggesting that the test is indeed valid for the assessment of functional performance in COPD. Although the strongest correlation was found with the 6MWT $\left(r_{s}=0.70\right)$, we do not believe the TUG would be able to replace the 6MWT. These tests have different designs and the correlation coefficient between the tests was not excellent (i.e., >0.90). Nonetheless, a TUG time of $11.2 \mathrm{~s}$ had good sensitivity and specificity for identifying patients with a baseline 6MWD $<350 \mathrm{~m}$, the discriminatory threshold for mortality ${ }^{26}$. So, the TUG could be very useful to have a first insight into the patient's exercise capacity, especially in situations with limited time and space for a 6MWT. If a patient had a TUG time $>11 \mathrm{~s}$, then an additional 6MWT would be recommended. Of note, the 6MWT should be preferred if one wants to estimate specific parameters, such as exercise-induced desaturation and 6-minute walk work ${ }^{30}$. Further supporting construct validity and confirming our hypotheses, only weak correlations were found with mental health outcomes, and COPD subjects performed worse on the TUG than non-COPD subjects.

COPD is related to multi-systemic consequences, such as comorbidities and lower-limb muscle dysfunction ${ }^{14,31}$, which can compromise the performance on functional tests. Indeed, in our study COPD patients were found to have worse functional mobility (i.e., longer TUG time) when compared with healthy subjects. Although the median TUG time by COPD patients could be considered relatively low (i.e., good functional mobility) if compared with previous findings ${ }^{4}$, we believe this reflects the fact that not all COPD patients seem to have an impaired TUG performance since only around $1 / 3$ of the patients were found to have an abnormal TUG time, i.e., a TUG time $>11 \mathrm{~s}$. This threshold was derived from the sample of healthy subjects and is supported by the receiver operating characteristic curve analysis, which revealed a similar value (i.e., $11.2 \mathrm{~s}$ ) for identifying patients with a baseline 6MWD $<350 \mathrm{~m}$. Previous studies also suggest that 11 seconds is a reasonable cut-off point ${ }^{4,32}$. Nevertheless, if one aims to identify patients with a very poor baseline 6MWD $(<200 \mathrm{~m})$, a higher cut-off point should be used instead (i.e., 13.8 s). COPD patients with an abnormal TUG time showed worse health outcomes, but the opposite was also observed (i.e., patients with worse health outcomes showed a worse TUG time; e-Table 2). These associations support the ability of the TUG to reflect the multi-systemic consequences of COPD.

To date, no study has comprehensively investigated the responsiveness of the TUG to $P R$ in $C O P D^{18}$. TUG time improved significantly after PR, but a small effect size was ob- 
served (i.e., 0.16). Previous studies identified larger effects sizes (0.48 to 1.0) following $\mathrm{PR}^{33,34}$. However, these studies used the modified version of the test in which subjects are instructed to walk as fast as possible. This modified version seems to be more responsive to PR. When using the same version used in the current study, Beauchamp et al. also found a small/modest effect size (i.e., 0.30$)^{35}$. Nevertheless, the mean change found in their study (i.e., $1.5 \mathrm{~s}$ ) seems to be clinically relevant as it exceeds the MCID values identified in our study (0.9-1.4 s). The mean change in the total sample in our study did not exceed the identified MCID values, but this can also be found in other MCID studies ${ }^{1,3}$. Moreover, patients with an abnormal baseline TUG time did exceed the identified MCID values. The difference in the magnitude of change between our study and the study by Beauchamp et al. is probably due to the different baseline values, which are worse in the latter study ${ }^{35}$.

To the best of our knowledge, this is the first study to identify MCID values for the TUG in COPD. Importantly, these MCID values were derived according to anchor and distribution based methods. After performing a sub-analysis in patients with normal or abnormal TUG time at baseline we observed the greatest improvements in those with an abnormal TUG time (Figure 3). Although this could suggest a possible ceiling effect by this test, it does not diminish its clinical utility. In fact, this finding is more indicative that PR is especially indicated for patients with an abnormal baseline TUG time.

TUG is a simple, cheap and quick to perform test. Basically, only a chair, an ordinary stopwatch and a 3-metre course are necessary, which makes this test possible to be adopted in different settings (e.g., patient's home, hospitals). Moreover, considering that COPD patients take a median time of $9.8 \mathrm{~s}$ only to perform the test, that we have previously shown that only 2 trials are necessary in COPD patients ${ }^{5}$, and that most patients do not need any rest between the trials, the test itself can be done in less than 1 minute in the vast majority of patients. Besides being reliable ${ }^{5}$, valid, responsive to PR and able to identify patients with worse health outcomes in COPD, previous findings have shown that the TUG is also able to discriminate between fallers and non-fallers ${ }^{17}$. Other simple and quick functional performance tests, such as the five-repetition sit-tostand and the 4-metre gait speed, have also been explored and are increasingly being used in COPD ${ }^{1,2}$. Theoretically, the advantage of the TUG over the five-repetition sit-tostand and the 4-metre gait speed is that TUG includes both walking and sitting/standing manoeuvres, besides turning, which can challenge the balance of the patients. Nevertheless, to the best of our knowledge no study so far has performed a proper head-tohead comparison between these tests in COPD. Noteworthy, based on the current findings and on previous studies all these tests have been shown to be reliable, valid and responsive to $\mathrm{PR}$ in $\mathrm{COPD}^{1-3,5}$. 


\section{CHAPTER 6}

The current study has some methodological considerations. Probably the most important limitation is that other relevant outcomes, such as physical activity, falls and other measures of balance, were not available. Nevertheless, findings from previous studies do suggest that the TUG time is related to some of these outcomes in COPD ${ }^{17,36}$. Another limitation is that we were not able to include a measure of patient experience as an anchor for the calculation of the MCID. Nevertheless, the MCID of the six-minute walk test, which was used as anchor in our study, is in agreement with studies which took into account patient experience.

In conclusion, this study demonstrated that the TUG is valid for the assessment of functional performance in COPD. Patients with a TUG time $>11 \mathrm{~s}$ have poorer health outcomes than patients with a TUG time below this threshold. A very similar threshold had good sensitivity and specificity for identifying patients with a baseline 6MWD $<350 \mathrm{~m}$, which has been associated with worse prognosis in COPD. Moreover, the TUG is responsive to pulmonary rehabilitation, especially in patients with a baseline TUG time $>11 \mathrm{~s}$. The TUG is a simple functional performance test which provides valuable information and can be adopted in both clinical and research settings. 


\section{REFERENCES}

1. Jones SE, Kon SS, Canavan JL, et al. The five-repetition sit-to-stand test as a functional outcome measure in COPD. Thorax 2013; 68: 1015-1020.

2. Kon SS, Patel MS, Canavan JL, et al. Reliability and validity of 4-metre gait speed in COPD. Eur Respir J 2013; 42: 333-340.

3. Kon SS, Canavan JL, Nolan CM, et al. The 4-metre gait speed in COPD: responsiveness and minimal clinically important difference. Eur Respir J 2014; 43: 1298-1305.

4. Podsiadlo D and Richardson S. The timed "Up \& Go": a test of basic functional mobility for frail elderly persons. J Am Geriatr Soc 1991; 39: 142-148.

5. Mesquita R, Janssen DJ, Wouters EF, et al. Within-day test-retest reliability of the timed up \& go test in patients with advanced chronic organ failure. Arch Phys Med Rehabil 2013; 94: 2131-2138.

6. Flansbjer UB, Holmback AM, Downham D, et al. Reliability of gait performance tests in men and women with hemiparesis after stroke. J Rehabil Med 2005; 37: 75-82.

7. Resnik $L$ and Borgia M. Reliability of outcome measures for people with lower-limb amputations: distinguishing true change from statistical error. Phys Ther 2011; 91: 555-565.

8. Robinson TN, Wallace JI, Wu DS, et al. Accumulated frailty characteristics predict postoperative discharge institutionalization in the geriatric patient. J Am Coll Surg 2011; 213: 37-42.

9. Soubeyran P, Fonck M, Blanc-Bisson C, et al. Predictors of early death risk in older patients treated with first-line chemotherapy for cancer. J Clin Oncol 2012; 30: 1829-1834.

10. Laflamme GY, Rouleau DM, Leduc S, et al. The Timed Up and Go test is an early predictor of functional outcome after hemiarthroplasty for femoral neck fracture. J Bone Joint Surg Am 2012; 94: 1175-1179.

11. Panel on Prevention of Falls in Older Persons, American Geriatrics Society and British Geriatrics Society. Summary of the Updated American Geriatrics Society/British Geriatrics Society clinical practice guideline for prevention of falls in older persons. J Am Geriatr Soc 2011; 59: 148-157.

12. Turner G, Clegg A, British Geriatrics S, Age UK and Royal College of General P. Best practice guidelines for the management of frailty: a British Geriatrics Society, Age UK and Royal College of General Practitioners report. Age Ageing 2014; 43: 744-747.

13. Schols AM, Ferreira IM, Franssen FM, et al. Nutritional assessment and therapy in COPD: a European Respiratory Society statement. Eur Respir J 2014; 44: 1504-1520.

14. Maltais F, Decramer M, Casaburi R, et al. An official American Thoracic Society/European Respiratory Society statement: update on limb muscle dysfunction in chronic obstructive pulmonary disease. Am J Respir Crit Care Med 2014; 189: e15-e62.

15. Spruit MA, Franssen FM, Rutten EP, et al. Age-graded reductions in quadriceps muscle strength and peak aerobic capacity in COPD. Rev Bras Fisioter 2012; 16: 148-156.

16. Beauchamp MK, Sibley KM, Lakhani B, et al. Impairments in systems underlying control of balance in COPD. Chest 2012; 141: 1496-1503.

17. Beauchamp MK, Hill K, Goldstein RS, et al. Impairments in balance discriminate fallers from non-fallers in COPD. Respir Med 2009; 103: 1885-1891.

18. Bisca GW, Morita AA, Hernandes NA, et al. Simple lower limb functional tests in patients with Chronic Obstructive Pulmonary Disease: a systematic review. Arch Phys Med Rehabil 2015; 96: 2221-2230.

19. Smid DE, Wilke S, Jones PW, et al. Impact of cardiovascular comorbidities on COPD Assessment Test (CAT) and its responsiveness to pulmonary rehabilitation in patients with moderate to very severe COPD: protocol of the Chance study. BMJ open. 2015; 5: e007536.

20. Metsemakers JF, Hoppener P, Knottnerus JA, et al. Computerized health information in The Netherlands: a registration network of family practices. Br J Gen Pract 1992; 42: 102-106.

21. Spruit MA, Augustin IM, Vanfleteren L, et al. Differential response to pulmonary rehabilitation in COPD: multidimensional profiling. Eur Respir J 2015; 46: 1625-1635. 


\section{CHAPTER 6}

22. Holland AE, Spruit MA, Troosters T, et al. An official European Respiratory Society/American Thoracic Society Technical Standard: field walking tests in chronic respiratory disease. Eur Respir J 2014; 44: 14281446.

23. Revicki D, Hays RD, Cella D, et al. Recommended methods for determining responsiveness and minimally important differences for patient-reported outcomes. J Clin Epidemiol 2008; 61: 102-109.

24. Guyatt G, Walter S and Norman G. Measuring change over time: assessing the usefulness of evaluative instruments. J Chronic Dis 1987; 40: 171-178.

25. Weir JP. Quantifying test-retest reliability using the intraclass correlation coefficient and the SEM. J Strength Cond Res 2005; 19: 231-240.

26. Cote CG, Pinto-Plata V, Kasprzyk K, et al. The 6-min walk distance, peak oxygen uptake, and mortality in COPD. Chest 2007; 132: 1778-1785.

27. Schoppen T, Boonstra A, Groothoff JW, et al. The Timed "up and go" test: reliability and validity in persons with unilateral lower limb amputation. Arch Phys Med Rehabil 1999; 80: 825-828.

28. Ng SS and Hui-Chan CW. The timed up \& go test: its reliability and association with lower-limb impairments and locomotor capacities in people with chronic stroke. Arch Phys Med Rehabil 2005; 86: 16411647.

29. Lin MR, Hwang HF, Hu MH, et al. Psychometric comparisons of the timed up and go, one-leg stand, functional reach, and Tinetti balance measures in community-dwelling older people. J Am Geriatr Soc 2004; 52: 1343-1348.

30. Andrianopoulos V, Wouters EF, Pinto-Plata VM, et al. Prognostic value of variables derived from the sixminute walk test in patients with COPD: Results from the ECLIPSE study. Respir Med 2015; 109: 11381146.

31. Vestbo J, Hurd SS, Agusti AG, et al. Global strategy for the diagnosis, management, and prevention of chronic obstructive pulmonary disease: GOLD executive summary. Am J Respir Crit Care Med 2013; 187: 347-365.

32. Kamide N, Takahashi K and Shiba Y. Reference values for the Timed Up and Go test in healthy Japanese elderly people: determination using the methodology of meta-analysis. Geriatr Gerontol Int 2011; 11: 445-451.

33. Marques A, Jacome C, Cruz J, et al. Family-based psychosocial support and education as part of pulmonary rehabilitation in COPD: a randomized controlled trial. Chest 2015; 147: 662-672.

34. Marques A, Gabriel R, Jacome C, et al. Development of a family-based pulmonary rehabilitation programme: an exploratory study. Disabil Rehabil 2015; 37: 1340-1346.

35. Beauchamp MK, O'Hoski S, Goldstein RS, et al. Effect of pulmonary rehabilitation on balance in persons with chronic obstructive pulmonary disease. Arch Phys Med Rehabil 2010; 91: 1460-1465.

36. Marques A, Jacome C, Cruz J, et al. Effects of a pulmonary rehabilitation program with balance training on patients with COPD. J Cardiopulm Rehabil Prev 2015; 35: 154-158.

Reprinted from Chronic Respiratory Disease, Vol. 13, Mesquita R, Wilke S, Smid DE, Janssen DJA, Franssen FME, Probst VS, Wouters EFM, Muris JWM, Pitta F, Spruit MA, Measurement properties of the Timed Up \& Go test in patients with COPD, pp. 344-52, Copyright (2016), with permission from SAGE Publications. 


\section{SUPPLEMENTARY MATERIAL}

\section{MATERIAL AND METHODS}

\section{Assessment of other health outcome measures}

As part of demographics and anthropometrics age, sex, height, weight and body mass index (BMI) were assessed. BMI was calculated as weight per squared height $\left(\mathrm{kg} \cdot \mathrm{m}^{-2}\right)^{1}$. Abnormalities in weight were classified as underweight (BMI $<21 \mathrm{~kg} \cdot \mathrm{m}^{-2}$ ), normal weight (BMI 21-25 $\mathrm{kg} \cdot \mathrm{m}^{-2}$ ), pre-obese (BMI 25-30 $\mathrm{kg} \cdot \mathrm{m}^{-2}$ ), and obese $\left(\mathrm{BMI} \geq 30 \mathrm{~kg} \cdot \mathrm{m}^{-2}\right)^{2}$. For the assessment of smoking status patients were classified as never, former or current smokers. Yes-no questions were used for the use of long-term oxygen therapy and walking aids, and patients were also inquired about the number of exacerbations in the previous 12 months. If necessary, the sample was split into two groups according to the number of exacerbations: subjects with $0-1$ or $\geq 2$ exacerbations in the previous year. Two or more exacerbations in the preceding year has been suggested as an indication of higher exacerbation risk ${ }^{3}$.

The Charlson comorbidity index was used for the assessment of current self-reported comorbidities ${ }^{4}$. This instrument is composed by 19 categories of comorbidities and the total score reflects the cumulative increased likelihood of 1-year mortality ${ }^{5}$. Higher scores can be translated as a higher burden of comorbidities.

The modified Medical Research Council (MRC) scale was used to assess symptoms of dyspnoea ${ }^{6}$. This scale rates the intensity of dyspnoea during activities of daily living according to scores that range from 0 to 4, with higher scores indicating more dyspnoea. Symptoms of anxiety and depression were investigated using the Hospital Anxiety and Depression Scale (HADS), a validated and reliable self-administered questionnaire widely used for screening clinically relevant symptoms of anxiety and/or depression ${ }^{7}$. The HADS consists of 14 questions distributed in two subscales, 7 questions for the assessment of symptoms of anxiety (HADS anxiety subscale) and 7 questions for the assessment of symptoms of depression (HADS depression subscale). The total score in each subscale can range from 0 (optimal) to 21 (worst). Scores $\geq 10$ are suggestive of clinically relevant symptoms of anxiety and/or depression? ${ }^{7}$.

Two valid and reliable instruments were used for the assessment of disease-specific health status: the COPD assessment test (CAT), and the COPD-specific version of the St. George's Respiratory Questionnaire (SGRQ-C). The CAT is a questionnaire with 8 items 
raising questions regarding symptoms, energy, sleep and activity ${ }^{8}$. Scores can range from 0 (no impact) to 40 points (severe impact), and they can represent mild (0-10 points), moderate (11-20 points), severe (21-30 points) or very severe (31-40 points) impact on health status ${ }^{8}$. The SGRQ-C consists of a 40-item questionnaire divided in three domains (symptoms, activities and impact) ${ }^{9}$. Scores per domain and a total score are provided, and they can range from 0 (optimal) to 100 points (worst) ${ }^{9}$.

Lung function was measured with post-bronchodilator spirometry (Masterlab ${ }^{\circledR}$; Jaeger $A G$, Würzburg, Germany) following international recommendations ${ }^{10}$. The results were compared to reference values ${ }^{11}$ and the severity of airflow limitation was classified according to the Global Initiative for Chronic Obstructive Lung Disease (GOLD): GOLD 1 (forced expiratory volume in the first second $\left(\mathrm{FEV}_{1}\right) \geq 80 \%$ predicted); GOLD $2(50 \% \leq$ $\mathrm{FEV}_{1}<80 \%$ predicted); GOLD $3\left(30 \% \leq \mathrm{FEV}_{1}<50 \%\right.$ predicted) and; GOLD 4 (FEV $1<30 \%$ predicted) ${ }^{3}$.

Functional exercise capacity was measured with the six-minute walk test (6MWT), performed according to the European Respiratory Society (ERS) / American Thoracic Society (ATS) guidelines ${ }^{12}$. As this test is subjected to a learning effect ${ }^{13}$, two tests were performed and the test with the longest distance was considered for analysis. The cut-off point of 350 metres was used as it has been able to predict exacerbations and death ${ }^{14}$, 15. The cut-off point of 200 metres was also used to identify patients with very poor exercise capacity, as done in previous studies ${ }^{16,17}$. Maximal exercise capacity was measured with the cardiopulmonary exercise test (CPET), performed using a symptomlimited incremental protocol (+10 watts per minute) in a cycle ergometer and in accordance with the guidelines of the American Thoracic Society and the American College of Chest Physicians ${ }^{18}$. Single-leg isokinetic quadriceps peak torque (IQPT) was determined using a BIODEX computerized dynamometer (Biodex System 4 Pro, Biodex Medical Systems, Inc., New York, USA). Thirty volitional maximal contractions at an angular velocity of $90^{\circ}$ per second were performed twice, and the highest values were considered for further analyses ${ }^{19}$.

The GOLD 2011 classification was used to classify patients into groups A, B, C, or D ${ }^{3}$. GOLD grades 1-4 or exacerbations in the previous year were used to classify patients as low (group A or B) or high (group C or D) risk. Patients classified as GOLD grade 1 or 2, or with less than 2 exacerbations in the previous year, were classified as low risk, whilst those GOLD grade 3 or 4, or with 2 or more exacerbations in the previous year, were classified as high risk; the criterion with the worse score was used for analysis ${ }^{3}$. The SGRQ-C was used to classify patients as less (group A or C) or more (group B or D) symptoms $^{20}$. Patients with a SGRQ-C score lower than 25 were classified as less symptoms, whilst those with a score equal to or higher than 25 were classified as more symptoms $^{20}$. Different instruments have been proposed for the assessment of symptoms in 
the GOLD 2011 classification (modified MRC scale, CAT, Clinical COPD Questionnaire (CCQ) or SGRQ $)^{20}$. However, Wilke, Smid et al. have recently shown that the instrument of choice has no influence on the performance in the TUG ${ }^{21}$. The updated Body-mass index, airflow Obstruction, Dyspnoea, and Exercise capacity (BODE) index was calculated based on BMI, FEV 1 , modified MRC scale, and 6MWD. This index is a predictor of 3year mortality in COPD patients and its total score can range from 0 to 15 points, with higher scores meaning a worse prognosis ${ }^{22}$. The Age, Dyspnoea and Obstruction (ADO) index is a simpler severity index developed for use in primary-care settings ${ }^{22}$. It can range from 0 to 10 points and higher scores are also associated with worse prognosis ${ }^{22}$.

\section{Details on sample size calculations}

Considering the different objectives proposed in the study, different sample sizes would be required. In order to answer the objective on the validity of the TUG test, given that the minimal desired level of correlation for convergent and discriminant validity was defined as 0.3 , and considering a significance level of 0.05 with a 2-sided test and a power of $80 \%$, a sample size of 82 patients would be required in this part of the study. This number is far below the actual number of patients included (i.e., 500 patients; please see Results in the main text). For the analysis of known-groups validity, based on the results observed by Crişan et al. when assessing the TUG time in control subjects and stable COPD patients (TUG times of $8.6 \pm 2.5$ and $12.3 \pm 2.1$ seconds, respectively), and considering an alpha value of 0.05 with a 2-sided test and a power of 80\%, 16 patients and 16 healthy subjects would be needed in this part of the study ${ }^{23}$. Once again, these numbers are far below the actual number of participants included (i.e., 500 patients and 100 healthy subjects; please see Results in the main text).

To the best of our knowledge, no previous studies have investigated characteristics related to an abnormal TUG time in COPD patients. However, Jácome et al. performed similar analyses in patients with COPD using the modified version of the TUG test, which requires subjects to walk as fast as possible ${ }^{24}$. Considering the main differences found in that study between COPD patients with and without functional balance impairment, and based on an alpha value of 0.05 with a 2 -sided test and a power of $80 \%$, a sample size of 166 patients in each group (i.e., normal/abnormal TUG time) would be needed in this part of the study. This number is below the actual number of patients included (i.e., 333 patients with a normal TUG time and 167 patients with an abnormal TUG time; please see Results in the main text).

Responsiveness sample size calculation was based on the results observed by Beauchamp et al. in patients with COPD ${ }^{25}$. These authors observed TUG times of $15.7 \pm 5.3$ and $14.2 \pm 4.5$ seconds before and after pulmonary rehabilitation, respectively. Considering these findings, an alpha value of 0.05 with a 2 -sided test and a power of $80 \%$ add- 
ed by a drop-out rate of $32 \%^{2}$, a sample size of 124 patients would be required in this part of the study. The actual number of patients included was 378 patients (please see Results in the main text), which once again is above the number of patients needed.

Based on the above-mentioned calculations it can be observed that the number of participants included in the main analysis of the current study is actually above the number of participants required. All the above-mentioned analyses were performed with G*Power 3.1.9.2 (Düsseldorf, Germany). 


\section{RESULTS}

e-Table 1 Crude associations between an abnormal TUG time and other measures in COPD patients

\begin{tabular}{|c|c|c|c|c|}
\hline Measure & $\beta$ & SE & OR $(95 \% \mathrm{Cl})$ & P-value \\
\hline Sex (ref: female) & -0.09 & 0.19 & $0.91(0.63-1.33)$ & 0.63 \\
\hline Age (years) & 0.09 & 0.01 & $1.10(1.07-1.12)$ & $<0.0001$ \\
\hline $\mathrm{BMI}\left(\mathrm{kg} \cdot \mathrm{m}^{-2}\right)$ & 0.05 & 0.02 & $1.06(1.02-1.09)$ & 0.001 \\
\hline $\mathrm{FEV}_{1}(\%$ predicted $)$ & -0.01 & 0.01 & $1.00(0.99-1.00)$ & 0.29 \\
\hline \multicolumn{5}{|l|}{ GOLD grades } \\
\hline GOLD 1 (ref) & NA & NA & 1.00 & NA \\
\hline GOLD 2 & 0.37 & 0.40 & $1.44(0.66-3.17)$ & 0.36 \\
\hline GOLD 3 & 0.14 & 0.40 & $1.15(0.52-2.52)$ & 0.74 \\
\hline GOLD 4 & 0.60 & 0.42 & $1.82(0.79-4.17)$ & 0.16 \\
\hline GOLD groups (ref: A or B) & 0.33 & 0.24 & $1.40(0.88-2.22)$ & 0.16 \\
\hline Charlson Comorbidity Index (points) & 0.64 & 0.11 & $1.90(1.53-2.36)$ & $<0.0001$ \\
\hline \multicolumn{5}{|l|}{ Smoking status } \\
\hline Never smokers (ref) & NA & NA & 1.00 & NA \\
\hline Former smokers & -0.59 & 0.82 & $0.56(0.11-2.79)$ & 0.47 \\
\hline Current smokers & -1.12 & 0.85 & $0.33(0.6-1.71)$ & 0.18 \\
\hline Long-term oxygen therapy (ref: no) & 1.19 & 0.22 & $3.29(2.15-5.04)$ & $<0.0001$ \\
\hline Walking aids during the TUG (ref: no) & 3.00 & 0.54 & $20.17(6.98-58.30)$ & $<0.0001$ \\
\hline 6MWT (m) & -0.02 & 0.00 & $0.99(0.98-0.99)$ & $<0.0001$ \\
\hline CPET (watts) & -0.03 & 0.00 & $0.97(0.96-0.98)$ & $<0.0001$ \\
\hline IQPT (joules) & -0.02 & 0.00 & $0.98(0.97-0.99)$ & $<0.0001$ \\
\hline mMRC (points) & 1.11 & 0.12 & $3.04(2.40-3.86)$ & $<0.0001$ \\
\hline HADS anxiety (points) & 0.06 & 0.02 & $1.07(1.02-1.11)$ & 0.003 \\
\hline HADS depression (points) & 0.10 & 0.02 & $1.10(1.05-1.15)$ & $<0.0001$ \\
\hline CAT (points) & 0.08 & 0.02 & $1.08(1.05-1.12)$ & $<0.0001$ \\
\hline \multicolumn{5}{|l|}{ SGRQ-C } \\
\hline Symptoms (points) & 0.02 & 0.01 & $1.02(1.01-1.03)$ & 0.002 \\
\hline Activity (points) & 0.05 & 0.01 & $1.05(1.03-1.06)$ & $<0.0001$ \\
\hline Impact (points) & 0.04 & 0.01 & $1.04(1.03-1.05)$ & $<0.0001$ \\
\hline Total (points) & 0.05 & 0.01 & $1.05(1.04-1.07)$ & $<0.0001$ \\
\hline ADO index (points) & 0.68 & 0.08 & $1.97(1.70-2.28)$ & $<0.0001$ \\
\hline Updated BODE index (points) & 0.40 & 0.04 & $1.49(1.37-1.61)$ & $<0.0001$ \\
\hline
\end{tabular}

See tables 1 and 2 for definition of abbreviations and number of subjects with complete data. 
CHAPTER 6

e-Table 2 TUG time after stratification for other measures in COPD patients

\begin{tabular}{|c|c|c|c|}
\hline Measure & Cut-off points & Median (IQR) & P-value \\
\hline \multirow[t]{2}{*}{ Sex } & Male & $9.6(8.5-11.8)$ & 0.36 \\
\hline & Female & $10.0(8.5-11.8)$ & \\
\hline \multirow[t]{2}{*}{ Age (years) } & $<65$ & $9.0(7.9-10.6)$ & $<0.0001$ \\
\hline & $\geq 65$ & $10.8(9.2-13.4)$ & \\
\hline \multirow[t]{4}{*}{$\mathrm{BMI}\left(\mathrm{kg} \cdot \mathrm{m}^{-2}\right)$} & Underweight $(<21)$ & $9.1(7.9-10.5)$ & 0.001 \\
\hline & Normal weight (21-25) & $9.5(8.1-11.6)$ & \\
\hline & Pre-obese (25-30) & $10.0(8.6-12.1)^{\mathrm{a}}$ & \\
\hline & Obese $(\geq 30)$ & $10.5(8.9-12.5)^{\mathrm{a}}$ & \\
\hline \multirow[t]{4}{*}{$\mathrm{FEV}_{1}(\%$ predicted), GOLD grades } & GOLD 1 ( $\geq 80)$ & $9.4(8.6-11.3)$ & 0.19 \\
\hline & GOLD 2 (50-80) & $9.7(8.3-11.9)$ & \\
\hline & GOLD 3 (30-50) & $9.8(8.1-11.5)$ & \\
\hline & GOLD $4(<30)$ & $10.2(8.8-12.3)$ & \\
\hline \multirow[t]{4}{*}{$\mathrm{FEV}_{1}(\%$ predicted), GOLD groups } & GOLD A (0-1 exacerb. + SGRQ-C $<25$ points) & $8.2(7.6-9.1)$ & 0.001 \\
\hline & GOLD B (0-1 exacerb. +SGRQ-C $\geq 25$ points) & $9.3(8.0-11.3)$ & \\
\hline & GOLD C ( $\geq 2$ exacerb. + SGRQ-C $<25$ points) & $8.4(7.5-9.4)$ & \\
\hline & GOLD D ( $\geq 2$ exacerb. + SGRQ-C $\geq 25$ points) & $10.0(8.6-11.9)$ & \\
\hline \multirow[t]{2}{*}{ Charlson Comorbidity Index (points) } & 1 & $9.3(8.0-10.9)$ & $<0.0001$ \\
\hline & $\geq 2$ & $10.9(9.0-13.3)$ & \\
\hline \multirow[t]{3}{*}{ Smoking status } & Never smoker & $11.1(7.0-17.8)$ & 0.25 \\
\hline & Former smoker & $9.8(8.5-11.9)$ & \\
\hline & Current smoker & $9.5(8.0-11.0)$ & \\
\hline \multirow[t]{2}{*}{ Long-term oxygen therapy } & Yes & $11.4(9.5-13.8)$ & $<0.0001$ \\
\hline & No & $9.4(8.2-11.2)$ & \\
\hline \multirow[t]{2}{*}{ Walking aids during the TUG } & Yes & $14.7(12.3-18.4)$ & $<0.0001$ \\
\hline & No & $9.6(8.5-11.4)$ & \\
\hline \multirow[t]{2}{*}{ 6MWT (m) } & $<350$ & $13.0(11.2-15.6)$ & $<0.0001$ \\
\hline & $\geq 350$ & $9.2(8.0-10.7)$ & \\
\hline \multirow[t]{2}{*}{ CPET (watts) } & $<$ Median (64 watts) & $10.7(9.1-13.1)$ & $<0.0001$ \\
\hline & $\geq$ Median (64 watts) & $9.0(7.9-10.5)$ & \\
\hline \multirow[t]{2}{*}{ IQPT (joules) } & < Median (91.4 joules) & $10.4(9.0-12.5)$ & $<0.0001$ \\
\hline & $\geq$ Median (91.4 joules) & $9.0(7.9-10.6)$ & \\
\hline \multirow[t]{6}{*}{ mMRC (points) } & 0 & $8.6(7.2-9.3)$ & $<0.0001$ \\
\hline & 1 & $8.5(7.6-9.4)$ & \\
\hline & 2 & $9.3(8.2-10.9)^{c}$ & \\
\hline & 3 & $10.9(8.8-12.9)^{b, c, d}$ & \\
\hline & 4 & 12.0 & \\
\hline & & $(10.3-14.2)^{b, c, d, e}$ & \\
\hline \multirow[t]{2}{*}{ HADS anxiety (points) } & $<10$ & $9.5(8.1-11.3)$ & $<0.0001$ \\
\hline & $\geq 10$ & $10.2(8.8-13.1)$ & \\
\hline \multirow[t]{2}{*}{ HADS depression (points) } & $<10$ & $9.4(8.1-11.3)$ & $<0.0001$ \\
\hline & $\geq 10$ & $10.5(8.8-12.9)$ & \\
\hline \multirow[t]{3}{*}{ CAT (points) } & $0-10$ & $8.5(7.5-9.4)$ & $<0.0001$ \\
\hline & $11-20$ & $9.4(7.9-11.2)$ & \\
\hline & $21-30$ & $9.9(8.7-11.9)^{f, g}$ & \\
\hline
\end{tabular}


$31-40$

SGRQ-C Symptoms (points)

$<$ Median (63.1 points)

$\geq$ Median (63.1 points)

Activity (points)

$<$ Median (83.6 points)

$\geq$ Median (83.6 points)

Impact (points)

Total (points)

ADO index (points)

Updated BODE index (points)
$<$ Median (51.2 points)

$\geq$ Median (51.2 points)

$<$ Median (63.6 points)

$\geq$ Median (63.6 points)

$<$ Median (4 points)

$\geq$ Median (4 points)

$<$ Median (3 points)

$\geq$ Median (3 points)
13.2

$(10.8-15.3)^{f, g, h}$

$9.4(8.2-11.3)$

0.003

$10.1(8.6-12.5)$

$8.9(7.8-10.4) \quad<0.0001$

$10.7(9.0-12.7)$

$9.0(7.9-10.8)<0.0001$

$10.6(9.1-13.0)$

$9.0(7.9-10.8)<0.0001$

$10.6(9.1-12.9)$

$8.8(7.7-9.9) \quad<0.0001$

$10.6(9.0-12.9)$

$8.8(7.7-9.9) \quad<0.0001$ $10.4(8.8-12.8)$

Data expressed as median (interquartile range). See tables 1 and 2 for definition of abbreviations and number of subjects with complete data. ${ }^{a} \mathrm{P}<0.05$ vs Underweight; ${ }^{b} \mathrm{P}<0.05$ vs mMRC 0 ; ${ }^{\mathrm{C}} \mathrm{P}<0.05$ vs mMRC 1 ; ${ }^{d} \mathrm{P}<0.05$ vs mMRC 2; ${ }^{\mathrm{P}}<0.05$ vs mMRC $3 ;{ }^{\mathrm{f}} \mathrm{P}<0.05$ vs CAT $0-10$ points; ${ }^{\mathrm{g}} \mathrm{P}<0.05$ vs CAT $11-20$ points; ${ }^{\mathrm{h}} \mathrm{P}<0.05$ vs CAT $21-30$ points.

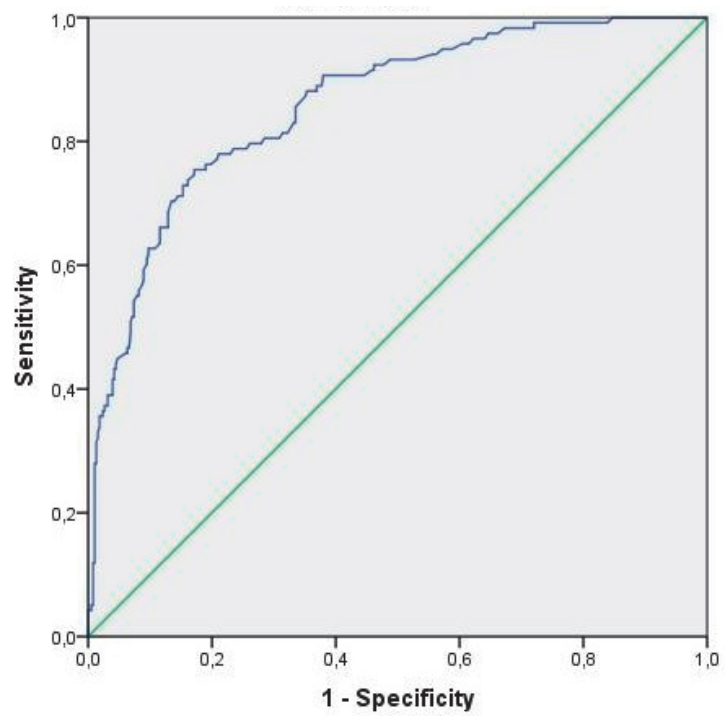

e-Figure 1 Receiver operating characteristic curves for Timed Up \& Go (TUG) time as predictor of poor sixminute walk distance (6MWD, <350 $\mathrm{m}$ ) in COPD patients 


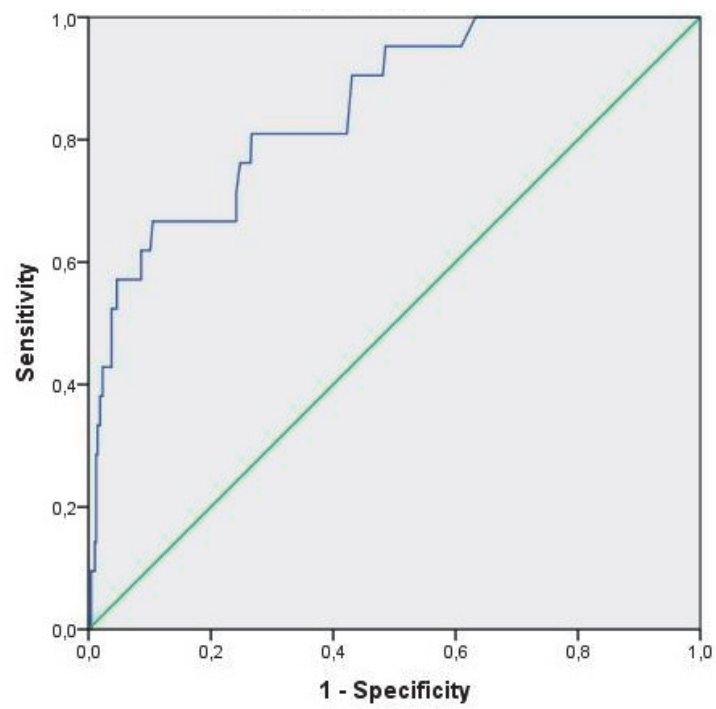

e-Figure 2 Receiver operating characteristic curves for Timed Up \& Go (TUG) time as predictor of very poor six-minute walk distance (6MWD, <200 m) in COPD patients

e-Table 3 General characteristics of COPD patients who completed and who did not complete pulmonary rehabilitation (PR)

\begin{tabular}{lccc}
\hline \multicolumn{1}{c}{ Characteristic } & Completed PR & Did not complete PR & P-value \\
\hline $\mathrm{N}$ & 378 & 122 & NA \\
Male sex (\%) & 55 & 54 & 0.86 \\
Age (years) & $64(58-71)$ & $64(56-72)$ & 0.69 \\
BMI (kg.m ${ }^{-2}$ ) & $25.7(21.7-29.9)$ & $25.8(21.4-29.9)$ & 0.75 \\
FEV $(\%$ predicted) & $47(34-64)$ & $42(31-59)$ & 0.05 \\
GOLD 1/2/3/4 (\%) & $8 / 37 / 36 / 19$ & $5 / 32 / 42 / 21$ & 0.12 \\
Charlson Comorbidity Index (points) & $1(1-2)$ & $1(1-2)$ & 0.21 \\
Smoking status N/F/C (\%) & $1 / 80 / 19$ & $2 / 67 / 31$ & 0.01 \\
Baseline TUG time (s) & $9.5(8.2-11.5)$ & $10.3(9.0-12.9)$ & 0.001 \\
\hline
\end{tabular}

Data expressed as as relative frequency or median (interquartile range). See tables 1 and 2 for definition of abbreviations. 


\section{References}

1. Keys A, Fidanza F, Karvonen MJ, Kimura N and Taylor HL. Indices of relative weight and obesity. J Chronic Dis 1972; 25: 329-343.

2. Spruit MA, Singh SJ, Garvey C, et al. An official american thoracic society/european respiratory society statement: key concepts and advances in pulmonary rehabilitation. Am J Respir Crit Care Med 2013; 188: e13-64.

3. Vestbo J, Hurd SS, Agusti AG, et al. Global strategy for the diagnosis, management, and prevention of chronic obstructive pulmonary disease: GOLD executive summary. Am J Respir Crit Care Med 2013; 187: 347-365.

4. Charlson ME, Pompei $\mathrm{P}$, Ales $\mathrm{KL}$ and MacKenzie CR. A new method of classifying prognostic comorbidity in longitudinal studies: development and validation. J Chronic Dis 1987; 40: 373-383.

5. Charlson M, Szatrowski TP, Peterson J and Gold J. Validation of a combined comorbidity index. J Clin Epidemiol 1994; 47: 1245-1251.

6. Bestall JC, Paul EA, Garrod R, Garnham R, Jones PW and Wedzicha JA. Usefulness of the Medical Research Council (MRC) dyspnoea scale as a measure of disability in patients with chronic obstructive pulmonary disease. Thorax 1999; 54: 581-586.

7. Zigmond AS and Snaith RP. The hospital anxiety and depression scale. Acta Psychiatr Scand 1983; 67: 361370.

8. Jones PW, Harding G, Berry P, Wiklund I, Chen WH and Kline Leidy N. Development and first validation of the COPD Assessment Test. Eur Respir J 2009; 34: 648-654.

9. Jones PW, Quirk FH, Baveystock CM and Littlejohns P. A self-complete measure of health status for chronic airflow limitation. The St. George's Respiratory Questionnaire. Am Rev Respir Dis 1992; 145: 1321-1327.

10. Miller MR, Hankinson J, Brusasco V, et al. Standardisation of spirometry. Eur Respir J 2005; 26: 319-338.

11. Knudson RJ, Burrows B and Lebowitz MD. The maximal expiratory flow-volume curve: its use in the detection of ventilatory abnormalities in a population study. Am Rev Respir Dis 1976; 114: 871-879.

12. Holland AE, Spruit MA, Troosters T, et al. An official European Respiratory Society/American Thoracic Society Technical Standard: field walking tests in chronic respiratory disease. Eur Respir J 2014; 44: 14281446.

13. Hernandes NA, Wouters EF, Meijer K, Annegarn J, Pitta F and Spruit MA. Reproducibility of 6-minute walking test in patients with COPD. Eur Respir J 2011; 38: 261-267.

14. Spruit MA, Polkey MI, Celli B, et al. Predicting outcomes from 6-minute walk distance in chronic obstructive pulmonary disease. J Am Med Dir Assoc 2012; 13: 291-297.

15. Cote CG, Pinto-Plata V, Kasprzyk K, Dordelly $L$ and Celli BR. The 6-min walk distance, peak oxygen uptake, and mortality in COPD. Chest 2007; 132: 1778-1785.

16. Szekely LA, Oelberg DA, Wright C, et al. Preoperative predictors of operative morbidity and mortality in COPD patients undergoing bilateral lung volume reduction surgery. Chest 1997; 111: 550-558.

17. Karpman C, DePew ZS, LeBrasseur NK, Novotny PJ and Benzo RP. Determinants of gait speed in COPD. Chest 2014; 146: 104-110.

18. American Thoracic Society (ATS)/American College of Chest Physicians (ACCP). ATS/ACCP Statement on cardiopulmonary exercise testing. Am J Respir Crit Care Med 2003; 167: 211-277.

19. Sillen MJ, Franssen FM, Delbressine JM, et al. Heterogeneity in clinical characteristics and co-morbidities in dyspneic individuals with COPD GOLD D: findings of the DICES trial. Respir Med 2013; 107: 1186-1194.

20. Global Initiative for Chronic Obstructive Lung Disease (GOLD). Global strategy for the diagnosis, management and prevention of COPD. http://www.goldcopd.org/ . Published 2014. Updated: Jan 2014. Date accessed: March 2014.

21. Wilke S, Smid DE, Spruit MA, et al. The 2014 Updated GOLD Strategy: A Comparison of the Various Scenarios. J COPD F 2014; 1: 212-220. 


\section{CHAPTER 6}

22. Puhan MA, Garcia-Aymerich J, Frey M, et al. Expansion of the prognostic assessment of patients with chronic obstructive pulmonary disease: the updated BODE index and the ADO index. Lancet 2009; 374: 704-11.

23. Crisan AF, Oancea C, Timar B, Fira-Mladinescu O and Tudorache V. Balance impairment in patients with COPD. PloS one 2015; 10: e0120573.

24. Jacome C, Cruz J, Gabriel R, Figueiredo D and Marques A. Functional balance in older adults with chronic obstructive pulmonary disease. J Aging Phys Act 2014; 22: 357-363.

25. Beauchamp MK, O'Hoski S, Goldstein RS and Brooks D. Effect of pulmonary rehabilitation on balance in persons with chronic obstructive pulmonary disease. Arch Phys Med Rehabil 2010; 91: 1460-1465. 


\section{CHAPTER 7}

\section{Physical activity patterns and clusters in 1001 patients with COPD}

Rafael Mesquita*, Gabriele Spina*, Fabio Pitta, David Donaire-Gonzalez, Brenda M. Deering, Mehul S. Patel, Katy E. Mitchell, Jennifer Alison, Arnoldus J.R. van Gestel, Stefanie Zogg, Philippe Gagnon, Beatriz Abascal-Bolado, Barbara Vagaggini, Judith Garcia-Aymerich, Sue C. Jenkins, Elisabeth A.P.M. Romme, Samantha S.C. Kon, Paul S. Albert, Benjamin Waschki, Dinesh Shrikrishna, Sally J. Singh, Nicholas S. Hopkinson, David Miedinger, Roberto P. Benzo, François Maltais, Pierluigi Paggiaro, Zoe J. McKeough, Michael I. Polkey, Kylie Hill, William D-C. Man, Christian F. Clarenbach, Nidia A. Hernandes, Daniela Savi, Sally Wootton, Karina C. Furlanetto, Li W. Cindy Ng, Anouk W. Vaes, Christine Jenkins, Peter R. Eastwood, Diana Jarreta, Anne Kirsten, Dina Brooks, David R. Hillman, Thaís Sant'Anna, Kenneth Meijer, Selina Dürr, Erica P.A. Rutten, Malcolm Kohler, Vanessa S. Probst, Ruth Tal-Singer, Esther Garcia Gil, Albertus C. den Brinker, Jörg D. Leuppi, Peter M.A. Calverley, Frank W.J.M. Smeenk, Richard W. Costello, Marco Gramm, Roger Goldstein, Miriam T.J. Groenen, Helgo Magnussen, Emiel F.M. Wouters, Richard L. ZuWallack, Oliver Amft, Henrik Watz, and Martijn A. Spruit

*Both authors contributed equally.

Chronic Respiratory Disease 2017; [Epub ahead of print] 


\section{CHAPTER 7}

\section{ABSTRACT}

Objectives: We described physical activity measures and hourly patterns in patients with COPD after stratification for generic and COPD-specific characteristics and, based on multiple physical activity measures, we identified clusters of patients.

Methods: 1001 patients with COPD (65\% men; age, 67 years; FEV 1 , 49\% predicted) were studied cross-sectionally. Demographics, anthropometrics, lung function, and clinical data were assessed. Daily physical activity measures and hourly patterns were analysed based on data from a multi-sensor armband. Principal component analysis (PCA) and cluster analysis were applied to physical activity measures to identify clusters.

Results: Age, body mass index (BMI), dyspnoea grade, and ADO index (including age, dyspnoea, and airflow obstruction) were associated with physical activity measures and hourly patterns. Five clusters were identified based on three PCA components, which accounted for $60 \%$ of variance of the data. Importantly, 'Couch Potatoes' (i.e., the most inactive cluster) were characterised by higher $\mathrm{BMI}$, lower $\mathrm{FEV}_{1}$, worse dyspnoea and higher ADO index compared to other clusters ( $P<0.05$ for all).

Conclusions: Daily physical activity measures and hourly patterns are heterogeneous in COPD. Clusters of patients were identified solely based on physical activity data. These findings may be useful to develop interventions aiming to promote physical activity in COPD. 


\section{A- INTRODUCTION}

Physical activity levels in patients with chronic obstructive pulmonary disease (COPD) are mostly presented as a total amount or as an average of multiple measurement days $^{1-6}$. Nevertheless, physical activity is a multi-dimensional construct which should be described by relevant constructs and measures ${ }^{7}$. A more detailed approach to physical activity data analysis could provide complementary information about the physical activity behaviour of patients with COPD.

Studies in different patient populations show that a more detailed analysis of physical activity can be achieved by plotting physical activity hourly patterns ${ }^{7-9}$ and by applying cluster analysis to physical activity measures ${ }^{8,10,11}$. Physical activity hourly patterns provide a graphic representation of the temporal trends of physical activity intensities over the course of a day ${ }^{7-9}$ and they can provide useful information. Rochester and colleagues $^{12}$, for example, observed a delayed morning start and a reduced peak of activity in patients with Parkinson's disease. Cluster analysis ${ }^{13}$, in turn, can be useful to identify subgroups of subjects with distinct physical activity characteristics. In middleaged Chinese adults, Lee and coworkers ${ }^{8}$ were able to identify two clusters of subjects, one more active than the other. Male subjects in the least active cluster had higher body fat percentage and older age than those in the active group. These detailed analyses could then lead to new insights regarding subgroups of patients with COPD with specific physical activity patterns, which may be used in further investigations and intervention strategies, ${ }^{6,14}$. Indeed, for specific groups of patients, greater and/or more sustainable results may be achieved if the focus shifts from an increase in moderate-tovigorous activities towards a reduction in sedentary time ${ }^{17-20}$.

Therefore, the main contributions provided by this work are: to describe physical activity measures and physical activity hourly patterns in patients with COPD after stratification for generic and COPD-specific characteristics, and to identify clusters of patients with COPD based on physical activity measures.

\section{A- METHODS}

Please, see the supplementary material file for all details.

\section{B- Study Design and Participants}

This is a pooled analysis of historical, cross-sectionally and objectively assessed physical activity data from ten countries (i.e., United Kingdom, Ireland, the Netherlands, Germany, Switzerland, Italy, Spain, the United States of America (USA), Brazil, and Australia). 
Published and/or unpublished physical activity data from previous studies as assessed by the SenseWear Armband or SenseWear Mini Armband activity monitors (both from BodyMedia Inc., Pittsburgh, PA, USA) were considered for analysis. A detailed description of the sources of the data included in the current analysis - including study registration number, study type, main publications, sites of recruitment, period of data collection, and number of subjects included-, is provided in the supplementary material (e-Table 1). Ethics Board approval was obtained from the local ethics committees/institutional review boards and written informed consent was provided by the participants. Subjects were included if they had: COPD with a post-bronchodilator forced expiratory volume in the first 1 second $\left(F V_{1}\right) /$ forced vital capacity (FVC) ratio $<0.70^{21}$, clinical stability at the time of physical activity assessment, and complete data for age, sex, body mass index (BMI) and daily physical activity measures.

\section{B- Assessments}

Demographics, anthropometrics, lung function, and clinical data were assessed. In order to investigate their association with physical activity measures and hourly patterns, these outcomes were stratified according to established criteria or according to the median value (i.e., above or below the median). The SenseWear Armband or SenseWear Mini Armband activity monitors, which use multisensory data in combination with pattern recognition algorithms to reliably estimate energy expenditure (EE) and metabolic equivalents of task (METs) ${ }^{22}$, were used to assess physical activity ${ }^{23-26}$. SenseWear Professional software versions 6.1 and 7.0 were used for data analysis. METs data are divided into activity intensity levels using the thresholds proposed by the American College of Sports Medicine ${ }^{27}$ : very light intensity, $<2.0$ METs; light intensity, 2.0 to $2.9 \mathrm{METs}$; and moderate-to-vigorous intensity, $\geq 3.0 \mathrm{METS}$.

Subjects with a minimum of four recorded days (two weekdays + Saturday + Sunday) ${ }^{1}$ with the device being used for $\geq 22$ hours $\cdot$ day $^{-1} 28$ were included in the analyses. Only recordings during waking hours of weekdays were considered for the main analysis, since physical activity measures during the weekend are known to be different ${ }^{1}$. The physical activity measures represent the average of all valid weekdays. Weekend days were used only for the presentation of daily physical activity hourly patterns. All values represent absolute values.

\section{B- Cluster Analysis}

Cluster analysis was used to identify subgroups of patients with distinct physical activity profiles. Firstly, minute-by-minute EE and METs data were stratified according to different criteria (and the combination of them): intensity (e.g., very light, light or moderateto-vigorous intensity), duration (e.g., bouts of activity; a bout was defined as a pre- 
defined minimum period of consecutive minutes (e.g., 10 minutes) in the same intensity), period of the day (e.g., before or after midday), frequency (e.g., number of bouts per day); and quantity (e.g., absolute numbers or percentage of total). These stratifications led to 180 distinct variables referred to as features (e-Table 2, supplementary material). Data regarding steps-per-day were not included in the current analysis as this outcome is subject to inaccuracy unless the subject walks at higher speeds ${ }^{29}$. Secondly, Principal Component Analysis (PCA) was used to compress the information contained in the high-dimensional feature set (180 dimensions) to a lower subspace (three dimensions) that is both convenient for data visualisation and able to account for the desired variance of the data (set to 60\%). The features were initially standardised using z-scores. All 180 features contributed to each principal component, but some contributed more than others. The most relevant features in each component are presented. Thirdly, a kmeans clustering algorithm with automatic selection of the number of clusters was applied to the three principal components to separate the subjects into groups with distinct characteristics. The algorithm selects the number of clusters in a way that the corresponding clustering results are the most stable under small perturbations of the input dataset ${ }^{13}$. The normalised mean over pairwise clustering distances was used as instability measure ${ }^{13}$. Feature extraction, PCA and cluster analysis were performed using Matlab R2012b (Mathworks Inc., USA).

\section{B- Statistical Analyses}

Continuous variables were expressed as median (interquartile range), as most variables showed non-normal distribution. Categorical variables were expressed as absolute and/or relative frequency. Mann-Whitney $U$ test or Kruskal-Wallis test (post hoc Dunn; significant if $\mathrm{P}<0.05$ ) was used for comparing continuous variables, while the chi-square test was used for categorical variables. The influence of seasons on daily physical activity measures was minimal (e-Table 3, supplementary material) and therefore this was not taken into consideration throughout the analyses. Spearman coefficient was used to investigate correlations, when appropriate. The area under each hourly pattern, named as the Area Under the Curve (AUC), was calculated and presented with its $95 \%$ confidence intervals to quantitatively represent time-varying averages of the hourly patterns. $\mathrm{P}<0.01$ was considered significant and all statistical analyses were performed using SPSS 17.0 (SPSS, Chicago, Illinois, USA) or GraphPad Prism 5 (GraphPad Software, La Jolla, California, USA). 


\section{A- RESULTS}

B- General Characteristics, Physical Activity Measures and Hourly Patterns of 1001 Patients with COPD

In total, 1001 patients with COPD were analysed (Table 1). The majority of patients were men, had a normal-to-overweight BMI, moderate-to-severe degree of airflow limitation, and only a small proportion used long-term oxygen therapy (LTOT). Regarding the assessment of physical activity levels, the median number of valid days analysed per patient was $6(6-6)$, resulting in a total of 6074 valid physical activity days, of which 4049 (67\%) were weekdays. The daily total time was smallest and the daily total EE was lowest in moderate-to-vigorous intensity (Table 1). Higher EE was observed during summer for very light and light intensities, but this did not translate into more time in these intensities (e-Table 3, supplementary material). Characteristics per country can be found in e-Table 4 (supplementary material). 
Table 1 General characteristics and daily physical activity measures of patients with COPD ( $n=1001)$

Characteristic/Physical activity measure

Value

General characteristics

Age, years

$67(61-72)$

Male, \%

65

$\mathrm{BMI}, \mathrm{kg} \cdot \mathrm{m}^{-2}$

$25.8(22.5-29.6)$

BMI classification - Underweight / Normal weight / Pre-obese / Obese, \%

$7 / 37$ / 34 / 22

mMRC dyspnoea grade*

$2(1-3)$

Long-term oxygen therapy, $\%^{\dagger}$

10

$\mathrm{FEV}_{1}, \mathrm{~L}$

$1.31(0.91-1.79)$

$\mathrm{FEV}_{1}, \%$ predicted

$49(34-64)$

$\mathrm{FEV}_{1} / \mathrm{FVC}, \%$

$45(35-56)$

$\mathrm{D}_{\text {LCO, }} \%$ predicted $^{\ddagger}$

$51(37-67)$

ADO index, points*

$4(3-5)$

GOLD 2007 classification - 1 / 2 / 3 / 4, \%

$9 / 40 / 34 / 17$

GOLD 2011 classification - A / B / C / D, \% ${ }^{*}$

Physical activity measures in very light intensity

Time, $\min \cdot$ day $^{-1}$

$803(710-901)$

EE, METs-min.day ${ }^{-1}$

$1032(822-1327)$

Physical activity measures in light intensity

Time, $\min \cdot$ day $^{-1}$

$142(92-194)$

EE, METs-min.day ${ }^{-1}$

$435(291-655)$

Physical activity measures in moderate-to-vigorous intensity ${ }^{\S}$

Time, $\min \cdot$ day $^{-1}$

$52(26-99)$

EE, METs-min $d a y^{-1}$

$267(132-550)$

Time in $\geq 10$ - $\min$ bouts, $\min \cdot \mathrm{day}^{-1}$

$6(0-22)$

Data expressed as relative frequency or median (interquartile range). BMI: body mass index; mMRC: modified Medical Research Council; $F E V_{1}$ : forced expiratory volume in the first second; FVC: forced vital capacity; $D_{\text {Lco: }}$ diffusion capacity of the lung for carbon monoxide; ADO: age, dyspnoea, and airflow obstruction index; GOLD: Global Initiative for Chronic Obstructive Lung Disease; EE: energy expenditure; MET: metabolic equivalent of task. "Data available for 868 subjects; ${ }^{\dagger}$ Data available for 707 subjects; ${ }^{\ddagger}$ Data available for 505 subjects; ${ }^{8}$ Time in bouts of physical activity were averaged out over the total number of valid days. 


\section{B- Stratification for Generic and COPD-specific Characteristics}

In general, patients of older age, female sex, LTOT users, lower diffusion capacity of the lung for carbon monoxide ( $\left.D_{L C O}\right)$, higher mMRC dyspnoea grade, higher BMI, higher ADO index (including age, dyspnoea, and airflow obstruction), higher GOLD grade and patients from GOLD group D spent the smallest daily total time and lowest daily total EE in moderate-to-vigorous intensity (e-Tables $5-13$, supplementary material). Daily physical activity hourly patterns after stratification for the abovementioned characteristics are presented in Figure 1 and e-Figure 1 (supplementary material), showing a significant influence of age, BMI, mMRC dyspnoea grades, and ADO index scores, as there was little or no overlap between the $95 \%$ confidence intervals of the hourly patterns. The AUC-values for these parameters varied between 0.25 and 0.36 (e-Table 14, supplementary material). The influence of GOLD grades or GOLD groups on these patterns was small. Moreover, only weak associations existed between $\mathrm{FEV}_{1}$ (\% predicted) and the time in activities of very light, light, and moderate-to-vigorous intensities (e-Figure 2). 

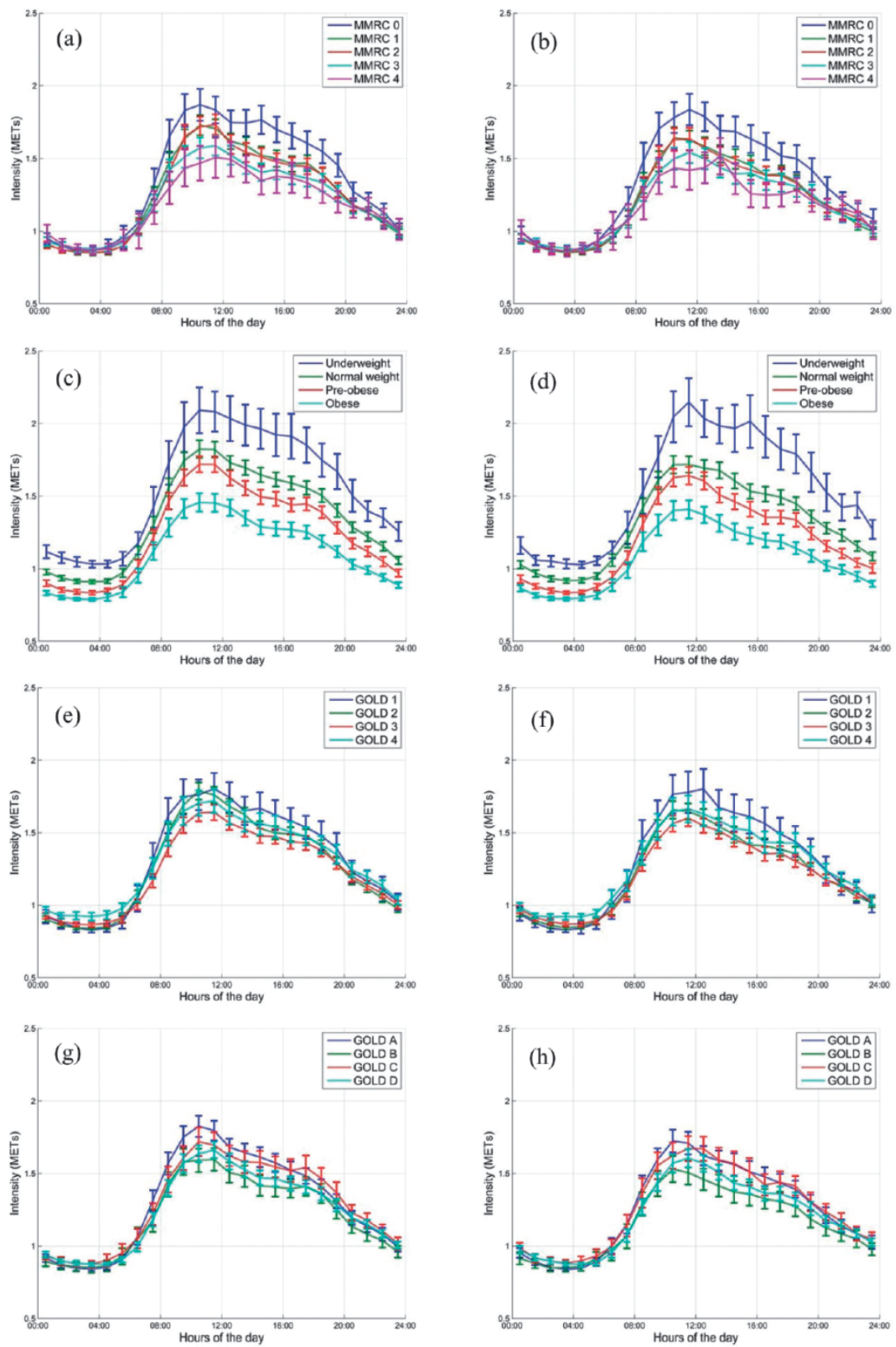

Figure 1 Daily physical activity hourly patterns of the patients with chronic obstructive pulmonary disease after stratification for: A and B - modified Medical Research Council (mMRC) grades, data available for 868 subjects only; C and D - body mass index (BMI) classification; E and F - Global Initiative for Chronic Obstructive Lung Disease (GOLD) grades (1 to 4); and G and H-GOLD groups ( $A$ to D). Figures $A, C$, E, and $G$ represent weekdays, while figures $B, D, F$, and $H$ represent weekend days. Data pooled per hour as mean $(95 \%$ confidence intervals). 


\section{B- Cluster Analysis of Daily Physical Activity Measures}

The PCA identified three components, which accounted for $60 \%$ of the total variance of the data (first component, 34\%; second component, 17\%; third component, $9 \%$ ). The most relevant features of each component were: $1^{\text {st }}$ component, daily time in $\geq 2$-min and $\geq 10$-min bouts of very light intensity; $2^{\text {nd }}$ component, total daily EE in moderate-tovigorous intensity and daily $E E$ in $\geq 2$-min and $\geq 10$-min bouts of moderate-to-vigorous intensity; $3^{\text {rd }}$ component, daily $E E$ in very light intensity after midday, total daily EE in very light intensity, and daily EE in $\geq 2$-min bouts of very light intensity after midday.

Cluster analysis performed on the three principal components identified five distinct clusters (Figure 2; see e-Video for a 3-dimensional video of Figure 2). Table 2 presents the characteristics and physical activity measures of these groups, which were named based on the time spent on each physical activity intensity, just like done previously ${ }^{30}$. 'Couch Potatoes' ( $n=216,22 \%$ ) were characterised by higher BMI, more dyspnoea, higher ADO index, more time in very light intensity, and less time in light and moderateto-vigorous intensities compared to other clusters. Similarly to 'Couch Potatoes', 'Highly Sedentary' ( $n=415,41 \%)$ spent more time in very light intensity and less time in moderate-to-vigorous intensity than other clusters. 'Sedentary Movers' ( $n=184,18 \%$ ) spent more time in light intensity and less time in moderate-to-vigorous intensity than 'Sedentary Exercisers' ( $n=165,17 \%$ ) and 'Busy Bees' ( $n=21,2 \%)$, while 'Sedentary Exercisers' in turn spent more time in light intensity compared to 'Busy Bees'. 'Busy Bees' were characterised by less time in very light intensity and more time in moderate-to-vigorous intensity compared to other clusters. Figure 3 presents the daily time in activities of different intensities by the clusters, highlighting the mixed arrangements of physical activity. Figure 4 presents the daily physical activity hourly patterns of the clusters. In all clusters the peak of intensity during the day occurred before midday, and in general weekdays and weekend days presented a similar pattern, especially in more inactive clusters. Hourly patterns after synchronisation of the waking up moment are presented in e-Figure 3 (supplementary material). Moreover, increasing AUC-values were found from 'Couch Potatoes' to 'Busy Bees' (e-Table 14, supplementary material). 

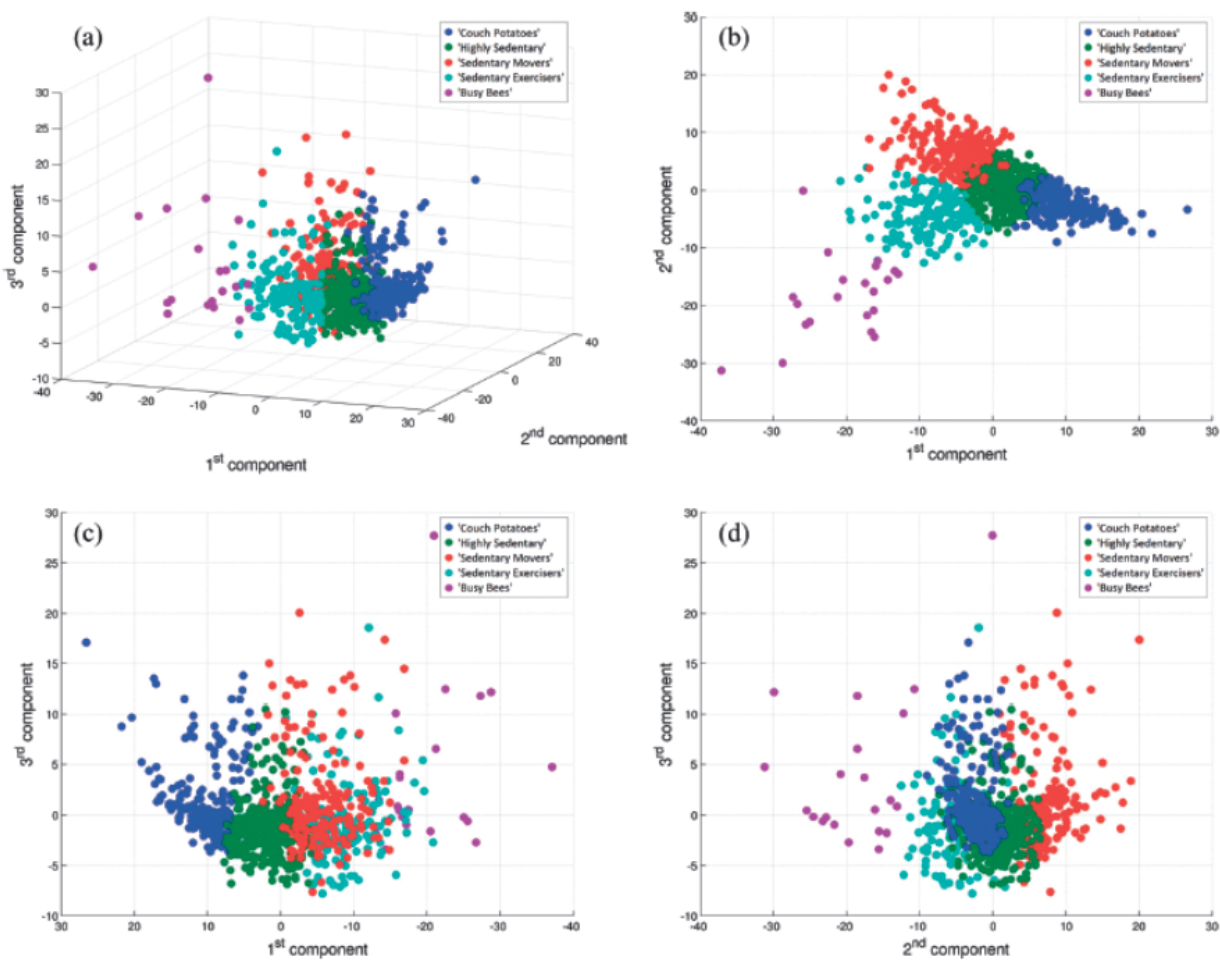

Figure 2 The five clusters identified. A: Graph in 3 dimensions presenting the three principal component analysis (PCA) components; B: Graph in 2 dimensions presenting the $1^{\text {st }}$ and $2^{\text {nd }}$ components; C: Graph in 2 dimensions presenting the $1^{\text {st }}$ and $3^{\text {rd }}$ components; and D: Graph in 2 dimensions presenting the $2^{\text {nd }}$ and $3^{\text {rd }}$ components. Details about the relationship between components and clusters can be found in the supplementary material. 


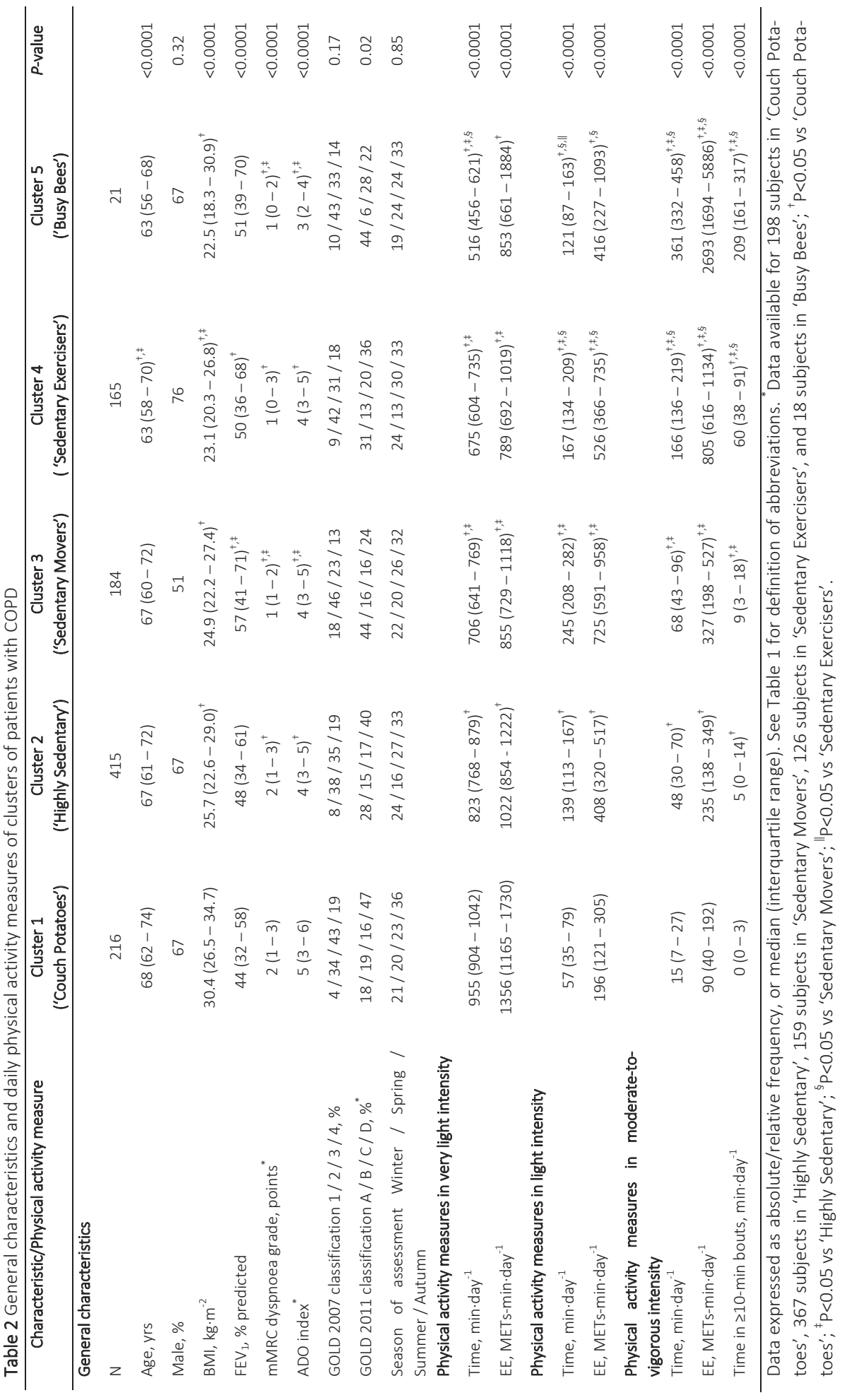


A)

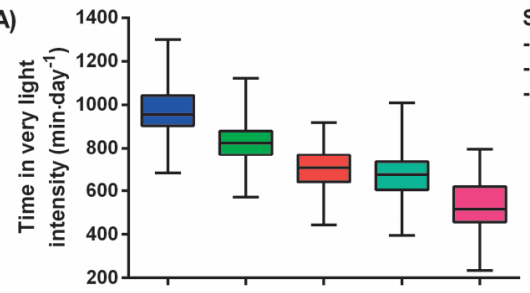

B)

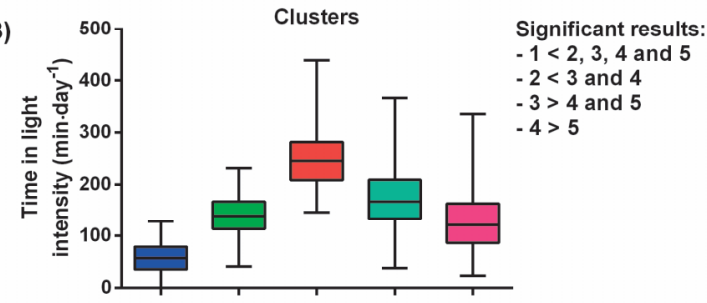

C)

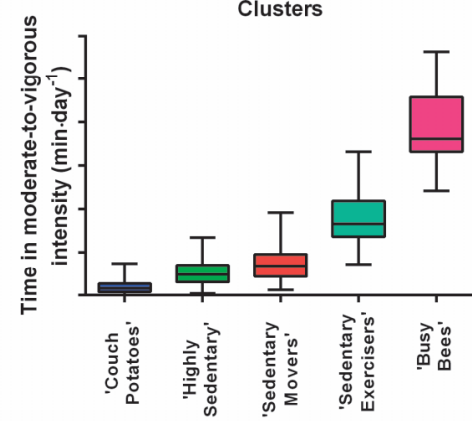

Significant results:

$1>2,3,4$ and 5

$-2>3,4$ and 5

$-3>5$ ignificant results:

$-1<2,3,4$ and 5

$-2<3,4$ and 5

$-3<4$ and 5

Figure 3 Daily time in activities of very light intensity (A), light intensity (B), and moderate-to-vigorous intensity (C) by clusters of patients with chronic obstructive pulmonary disease. Data presented as median (interquartile range).
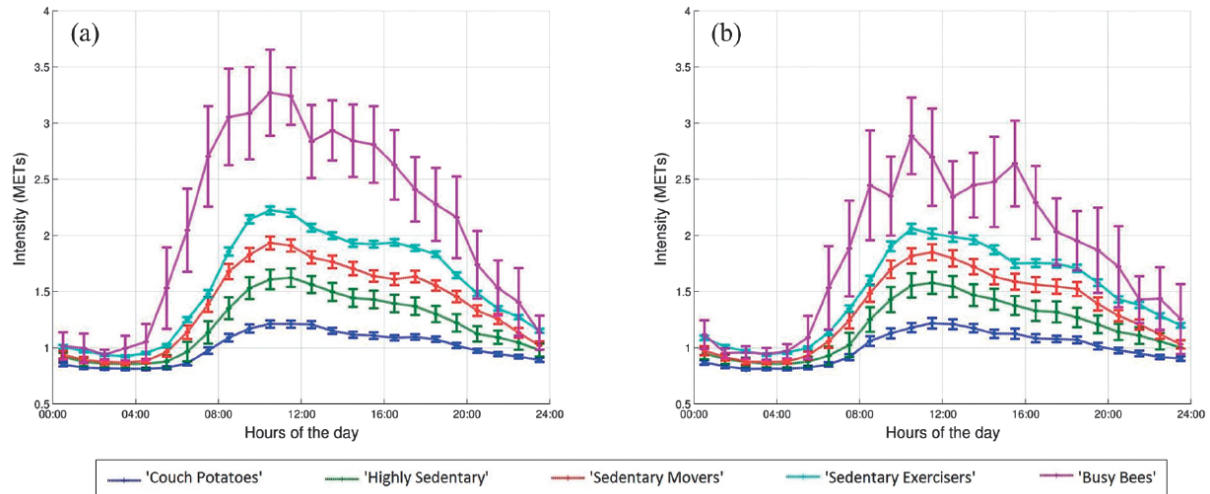

Figure 4 Daily physical activity hourly pattern of clusters of patients with chronic obstructive pulmonary disease during weekdays (A) and weekend days (B). Data pooled per hour as mean (95\% confidence intervals). 


\section{A- DISCUSSION}

The present study provides detailed analyses of objectively measured physical activity in a multinational sample of 1001 patients with COPD. The principal findings show that daily physical activity measures and hourly patterns vary considerably after stratification for generic and COPD-specific characteristics; and that patients with COPD can be clustered based on daily physical activity measures, with five clusters being identified, each with distinct physical activity measures and hourly patterns.

\section{B- Daily Physical Activity Measures and Physical Activity Hourly Patterns in COPD}

Our results clearly show that physical activity is a heterogeneous characteristic in patients with COPD, corroborating previous findings ${ }^{31,32}$. Distinct levels of physical activity were found after stratification for age (< or $\geq 67$ years), sex (male or female), BMI (underweight to obese), mMRC dyspnoea grade (0 to 4), LTOT (yes or no), $D_{\text {LCo }}(<$ or $\geq 51 \%$ predicted), ADO index (< or $\geq 4$ points), GOLD grades ( 1 to 4 ) and GOLD groups ( $A$ to $D$ ) (e-Tables 5-13, supplementary material). Interestingly, comparable time in very light and moderate-to-vigorous intensities was found between GOLD groups A and C, and between GOLD groups $B$ and $D$ (e-Table 13 ). This suggests that symptoms of dyspnoea, which discriminates between groups $A / C$ and $B / D$, are better associated with physical activity measures than the degree of airflow limitation, which discriminates between groups A/B and C/D. Demeyer and colleagues ${ }^{33}$ also investigated physical activity levels in patients with COPD after classification into GOLD groups. Our findings are in broad agreement with theirs. When using the $\mathrm{mMRC}$ scale to classify patients into GOLD groups, as done in our study, these authors found similar number of steps per day between GOLD groups $A$ and $C$, and between GOLD groups $B$ and $D^{33}$. In our study, despite the significant influence of GOLD groups on physical activity measures only little or no influence could be observed on physical activity hourly patterns. Few studies have investigated hourly patterns in COPD. In the study by Hecht and coworkers ${ }^{9}$, the authors observed that the highest activity level occurred during the late morning and early afternoon hours, which is corroborated by our findings. Together with our findings this suggests that hourly patterns have the potential to complement the information provided by summary values.

\section{B- Clusters of Patients with COPD Based on Daily Physical Activity Measures}

The present study is the first to apply cluster analysis using objectively assessed physical activity measures only to a large and diverse sample of patients with COPD. Indeed, five clusters were identified, each with distinct physical activity measures and hourly patterns. One very active cluster ('Busy Bees') and one very inactive cluster ('Couch Potatoes') were identified, but clusters in intermediate categories were also observed. 
Only a few studies have used objectively measured physical activity data solely to cluster subjects. In 10-to-12-year-old children, De Bourdeaudhuij and colleagues ${ }^{34}$ were able to identify a cluster with a mixed arrangement of physical activity (i.e., less time in moderate-to-vigorous intensity + less sedentary time). In our study, we also found a cluster with resembling characteristics (i.e., 'Sedentary Exercisers'), confirming that activities of moderate-to-vigorous intensity and sedentary activities are not two sides of one continuum ${ }^{34}$. Based on the amount of time in moderate-to-vigorous physical activity, 'Sedentary Exercisers' could be considered physically active ${ }^{27}$. Nonetheless, these patients spent over 11 hours in very light intensity (i.e., sedentary behaviour), which can have negative health consequences as previous studies have shown the detrimental effects of prolonged periods of sedentary behaviour on health outcomes ${ }^{35,36}$.

The characteristics of the clusters identified in our study can provide important insights on how to plan physical activity/exercise regimens for patients with COPD. For instance, 'Couch Potatoes' were characterised by worse airflow limitation and more debilitating symptoms compared to other clusters. Previous studies have shown that some interventions might be particularly useful in patients with these characteristics. For example, interval training has been show to result in lower symptom scores and fewer unintended breaks while reproducing the effects of endurance continuous training ${ }^{37}$. This type of training uses short bouts of high-intensity exercise interspersed regularly with periods of rest or lower intensity exercise. Another modality that is particularly useful in more symptomatic patients is transcutaneous neuromuscular electrical stimulation ${ }^{38}$, but new modalities are emerging such as downhill walking ${ }^{39}$. Moreover, based on their characteristics 'Couch potatoes' may benefit the most from pulmonary rehabilitation ${ }^{40}$.

'Couch potatoes' as well as 'Highly sedentary' were characterised by considerable amounts of time in very light physical activity (i.e., sedentary behaviour) and little time in moderate-to-vigorous physical activity. These clusters would probably benefit from an intervention focusing not only on increasing the time in moderate-to-vigorous physical activity, but also on reducing the time in sedentary behaviour. Potential ways to increase physical activity in COPD have been explored in a recent systematic review ${ }^{16}$. Although some intervention characteristics seemed to associate with greater improvements, such as interventions focusing specifically on increasing physical activity and longer pulmonary rehabilitation programmes ${ }^{16}$, to date there is little evidence that relevant and sustainable changes in physical activity are achievable in patients with COPD, especially physical activity in moderate-to-vigorous intensity ${ }^{20}$. Some patients might find it difficult to increase the time in moderate-to-vigorous physical activity, which suggests that it might be more realistic for these patients to increase the time in light activities ${ }^{17,19}$. In fact, there is emerging literature in other populations suggesting that health benefits can be achieved by decreasing time in sedentary behaviour and increasing the participation in light intensity physical activities ${ }^{41-43}$. In patients with 
COPD, low intensity exercises were able to improve health-related quality of life and functional status44. Moreover, a recent study demonstrated that greater quantity of low-intensity physical activity associates with a reduced risk of COPD hospitalisation ${ }^{18}$.

'Sedentary movers' and 'Sedentary exercisers' spent a considerable amount of time in moderate-to-vigorous intensity (i.e., 68 and 166 minutes, respectively), which is in agreement with the recommended by physical activity guidelines ${ }^{27}$. Nonetheless, these patients also spent important amounts of time in very light intensity (i.e., 706 and 675 minutes, respectively). Indeed, the daily amount of time spent in very light intensity (i.e., sedentary behaviour) by these groups is more than what other studies have considered as harmful (i.e., 7 hours) ${ }^{35,36}$. Sedentary behaviour has been the focus of recent literature in $\mathrm{COPD}^{20,45}$. Sedentary behaviour is defined as any waking behaviour characterised by an energy expenditure $\leq 1.5$ METs while in a sitting or reclining posture (e.g., watching television ${ }^{46}$. Epidemiological data in non-COPD populations have shown that engaging in moderate-to-vigorous intensity physical activity is not enough to fully protect against the detrimental consequences of prolonged periods of sedentary behaviour $^{47,48}$. This suggests that researchers and clinicians should also aim to reduce sedentary behaviour. Interventions focusing on sedentary behaviour in patients with COPD are scarce. Nevertheless, studies on other populations have shown promising results. In the 'Stand Up For Your Health' study ${ }^{49}$, a brief intervention based on goal setting and behavioural self-monitoring was shown to be feasible and able to reduce sedentary time and increase physical activity in Australian older adults. In the 'TView' study ${ }^{50}$, a three week programme using an electronic television lock-out system led to reductions in TV viewing time (a sedentary behaviour) of almost 3 hours per day in overweight and obese adults. Strategies based on the use of technology might also provide beneficial results, such as pads to measure sitting time ${ }^{51}$.

'Busy bees' were characterised by very high levels of moderate-to-vigorous physical activity. Not surprisingly, this group was formed by a very small number of patients (i.e., 21 patients, $2 \%$ of the whole sample). 'Busy bees' would probably benefit from strategies to maintain their very high physical activity levels. Home-based exercise training combined with monthly phone calls was able to maintain the gains in exercise capacity and health-related quality of life after a short pulmonary rehabilitation programme in patients with moderate COPD ${ }^{52}$. Strategies that can be easily incorporated into daily life, such as leisure walking, can produce more sustainable results. A randomised controlled trial including a 3-month programme of outdoor Nordic walking in patients with COPD resulted in significant improvements in exercise capacity and physical activity ${ }^{53}$. These improvements were sustained at 6 and 9 months after the initial intervention ${ }^{53}$. 


\section{B- Strengths and Methodological Considerations}

We have analysed a large and diverse sample of patients with COPD with objectively assessed physical activity data. This allowed detailed analyses of daily physical activity, even identifying clusters of patients with COPD with distinct physical activity measures, a novelty within the COPD literature. Physical activity hourly patterns were also investigated for the first time in a large-scale study in COPD, representing another important advance. All these analyses were only possible due to the use of objective methods to assess physical activity.

Some methodological considerations need to be taken into account. First, selection and information biases might be present, as the data were collected separately as part of different studies. Second, the clusters identified in our study were not validated as we were not able to show whether they relate to relevant clinical outcomes, such as COPDrelated hospitalisations and deaths due to the lack of follow-up assessments, or whether they could be replicated in another sample. Third, other characteristics which may influence physical activity levels in patients with COPD, such as comorbidities ${ }^{54}$, were not available.

To conclude, daily physical activity measures and hourly patterns in patients with COPD were found to vary considerably depending on the clinical characteristic. Moreover, five clusters of patients were identified, each with distinct physical activity measures and hourly patterns. The present data show that outcome measures need to be clearly delineated when evaluating interventions aiming to promote physical activity in patients with COPD. 


\section{REFERENCES}

1. Watz H, Waschki B, Meyer T, et al. Physical activity in patients with COPD. Eur Respir J 2009; 33: 262-272.

2. Pitta F, Takaki MY, Oliveira NH, et al. Relationship between pulmonary function and physical activity in daily life in patients with COPD. Respir Med 2008; 102: 1203-1207.

3. Hill K, Dolmage TE, Woon L, et al. Defining the relationship between average daily energy expenditure and field-based walking tests and aerobic reserve in COPD. Chest 2012; 141: 406-412.

4. Depew ZS, Karpman C, Novotny PJ, et al. Correlations between gait speed, 6-minute walk distance, physical activity, and self-efficacy in patients with severe chronic lung disease. Respir Care 2013; 58: 21132119.

5. Deering BM, Fullen B, Egan C, et al. Acupuncture as an adjunct to pulmonary rehabilitation. J Cardiopulm Rehabil Prev 2011; 31: 392-399.

6. Egan C, Deering BM, Blake C, et al. Short term and long term effects of pulmonary rehabilitation on physical activity in COPD. Respir Med 2012; 106: 1671-1679.

7. Bussmann JB and van den Berg-Emons RJ. To total amount of activity . . . . and beyond: perspectives on measuring physical behavior. Front Psychol 2013; 4: 463.

8. Lee PH, Yu YY, McDowell I, et al. A cluster analysis of patterns of objectively measured physical activity in Hong Kong. Public Health Nutr 2013; 16: 1436-1444.

9. Hecht A, Ma S, Porszasz J, et al. Methodology for using long-term accelerometry monitoring to describe daily activity patterns in COPD. COPD 2009; 6: 121-129.

10. Trilk JL, PateRR, PfeifferKA, et al.Acluster analysis of physical activity and sedentary behavior patterns in middle school girls. J Adolesc Health 2012; 51: 292-298.

11. Gubbels JS, Kremers SP, Stafleu A, et al. Clustering of energy balance-related behaviors in 5-year-old children: lifestyle patterns and their longitudinal association with weight status development in early childhood. Int J Behav Nutr Phys Act 2012; 9: 77.

12. Rochester L, Jones D, Hetherington V, et al. Gait and gait-related activities and fatigue in Parkinson's disease: what is the relationship? Disabil Rehabil 2006; 28: 1365-1371.

13. von Luxburg U. Clustering stability: an overview. Foundations and trends in machine learning 2010; 2: 235-274.

14. Cindy Ng LW, Mackney J, Jenkins S, et al. Does exercise training change physical activity in people with COPD? A systematic review and meta-analysis. Chron Respir Dis 2012; 9: 17-26.

15. Vaes AW, Cheung A, Atakhorrami M, et al. Effect of 'activity monitor-based' counseling on physical activity and health-related outcomes in patients with chronic diseases: a systematic review and meta-analysis. Ann Med 2013; 45: 397-412.

16. Mantoani LC, Rubio N, McKinstry B, et al. Interventions to modify physical activity in patients with COPD: a systematic review. Eur Respir J 2016; 48: 69-81.

17. Sparling PB, Howard BJ, Dunstan DW, et al. Recommendations for physical activity in older adults. BMJ 2015; 350: h100.

18. Donaire-Gonzalez D, Gimeno-Santos E, Balcells E, et al. Benefits of physical activity on COPD hospitalisation depend on intensity. Eur Respir J 2015; 46: 1281-1289.

19. Spruit MA, Pitta F, McAuley E, et al. Pulmonary rehabilitation and physical activity in patients with chronic obstructive pulmonary disease. Am J Respir Crit Care Med 2015; 192: 924-933.

20. Cavalheri V, Straker L, Gucciardi DF, et al. Changing physical activity and sedentary behaviour in people with COPD. Respirology 2016; 21: 419-426.

21. Vestbo J, Hurd SS, Agusti AG, et al. Global strategy for the diagnosis, management, and prevention of chronic obstructive pulmonary disease: GOLD executive summary. Am J Respir Crit Care Med 2013; 187: 347-365.

22. Jette M, Sidney $\mathrm{K}$ and Blumchen G. Metabolic equivalents (METS) in exercise testing, exercise prescription, and evaluation of functional capacity. Clin Cardiol 1990; 13: 555-565. 
23. Colbert LH, Matthews CE, Havighurst TC, et al. Comparative validity of physical activity measures in older adults. Med Sci Sports Exerc 2011; 43: 867-876.

24. Mackey DC, Manini TM, Schoeller DA, et al. Validation of an armband to measure daily energy expenditure in older adults. J Gerontol A Biol Sci Med Sci 2011; 66: 1108-1113.

25. Hill K, Dolmage TE, Woon L, et al. Measurement properties of the SenseWear armband in adults with chronic obstructive pulmonary disease. Thorax 2010; 65: 486-491.

26. Cavalheri V, Donaria L, Ferreira T, et al. Energy expenditure during daily activities as measured by two motion sensors in patients with COPD. Respir Med 2011; 105: 922-929.

27. Garber CE, Blissmer B, Deschenes MR, et al. American College of Sports Medicine position stand. Quantity and quality of exercise for developing and maintaining cardiorespiratory, musculoskeletal, and neuromotor fitness in apparently healthy adults: guidance for prescribing exercise. Med Sci Sports Exerc 2011; 43: 1334-1359.

28. Waschki B, Spruit MA, Watz $\mathrm{H}$, et al. Physical activity monitoring in COPD: compliance and associations with clinical characteristics in a multicenter study. Respir Med 2012; 106: 522-530.

29. Furlanetto KC, Bisca GW, Oldemberg N, et al. Step counting and energy expenditure estimation in patients with chronic obstructive pulmonary disease and healthy elderly: accuracy of 2 motion sensors. Arch Phys Med Rehabil 2010; 91: 261-267.

30. Bakrania $\mathrm{K}, \mathrm{Edwardson} \mathrm{CL}$, Bodicoat $\mathrm{DH}$, et al. Associations of mutually exclusive categories of physical activity and sedentary time with markers of cardiometabolic health in English adults: a cross-sectional analysis of the Health Survey for England. BMC Public Health 2016; 16: 25.

31. Garcia-Aymerich J, Serra I, Gomez FP, et al. Physical activity and clinical and functional status in COPD. Chest 2009; 136: 62-70.

32. Gimeno-Santos E, Frei A, Steurer-Stey C, et al. Determinants and outcomes of physical activity in patients with COPD: a systematic review. Thorax 2014; 69: 731-739.

33. Demeyer H, Gimeno-Santos E, Rabinovich RA, et al. Physical activity characteristics across GOLD quadrants depend on the questionnaire used. PLoS One 2016; 11: e0151255.

34. De Bourdeaudhuij I, Verloigne M, Maes L, et al. Associations of physical activity and sedentary time with weight and weight status among 10- to 12-year-old boys and girls in Europe: a cluster analysis within the ENERGY project. Pediatr Obes 2013; 8: 367-375.

35. Centers for Disease Control and Prevention. National health and nutrition examination survey data. CDC, 2011. www.cdc.gov/nchs/nhanes.htm (accessed 23 August 2015).

36. Chau JY, Grunseit AC, Chey T, et al. Daily sitting time and all-cause mortality: a meta-analysis. PLoS One 2013; 8: e80000.

37. Vogiatzis I, Nanas S and Roussos C. Interval training as an alternative modality to continuous exercise in patients with COPD. Eur Respir J 2002; 20: 12-19.

38. Sillen MJ, Franssen FM, Delbressine JM, et al. Efficacy of lower-limb muscle training modalities in severely dyspnoeic individuals with COPD and quadriceps muscle weakness: results from the DICES trial. Thorax 2014; 69: 525-531.

39. Camillo CA, Burtin C, Hornikx M, et al. Physiological responses during downhill walking: A new exercise modality for subjects with chronic obstructive pulmonary disease? Chron Respir Dis 2015; 12: 155-164.

40. Spruit MA, Augustin IM, Vanfleteren LE, et al. Differential response to pulmonary rehabilitation in COPD: multidimensional profiling. Eur Respir J 2015; 46: 1625-1635.

41. Pau M, Leban B, Collu G, et al. Effect of light and vigorous physical activity on balance and gait of older adults. Arch Gerontol Geriatr 2014; 59: 568-573.

42. Loprinzi PD, Lee H and Cardinal BJ. Daily movement patterns and biological markers among adults in the United States. Prev Med 2014; 60: 128-130.

43. Duvivier BM, Schaper NC, Bremers MA, et al. Minimal intensity physical activity (standing and walking) of longer duration improves insulin action and plasma lipids more than shorter periods of moderate to vigorous exercise (cycling) in sedentary subjects when energy expenditure is comparable. PLoS One 2013; 8: e55542. 


\section{CHAPTER 7}

44. Probst VS, Kovelis D, Hernandes NA, et al. Effects of 2 exercise training programs on physical activity in daily life in patients with COPD. Respir Care 2011; 56: 1799-1807.

45. Hill K, Gardiner PA, Cavalheri V, et al. Physical activity and sedentary behaviour: applying lessons to chronic obstructive pulmonary disease. Intern Med J 2015; 45: 474-482.

46. Sedentary Behaviour Research N. Letter to the editor: standardised use of the terms "sedentary" and "sedentary behaviours". Appl Physiol Nutr Metab 2012; 37: 540-542.

47. Healy GN, Dunstan DW, Salmon J, et al. Television time and continuous metabolic risk in physically active adults. Med Sci Sports Exerc 2008; 40: 639-645.

48. Healy GN, Matthews CE, Dunstan DW, et al. Sedentary time and cardio-metabolic biomarkers in US adults: NHANES 2003-06. Eur Heart J 2011; 32: 590-597.

49. Gardiner PA, Eakin EG, Healy GN, et al. Feasibility of reducing older adults' sedentary time. Am J Prev Med 2011; 41: 174-177.

50. Otten JJ, Jones KE, Littenberg B, et al. Effects of television viewing reduction on energy intake and expenditure in overweight and obese adults: a randomised controlled trial. Arch Intern Med 2009; 169 : 2109-2115.

51. Ryde GC, Gilson ND, Suppini A, et al. Validation of a novel, objective measure of occupational sitting. J Occup Health 2012; 54: 383-386.

52. du Moulin M, Taube K, Wegscheider K, et al. Home-based exercise training as maintenance after outpatient pulmonary rehabilitation. Respiration 2009; 77: 139-145.

53. Breyer MK, Breyer-Kohansal R, Funk GC, et al. Nordic walking improves daily physical activities in COPD: a randomised controlled trial. Respir Res 2010; 11: 112.

54. Watz H, Waschki B, Kirsten A, et al. The metabolic syndrome in patients with chronic bronchitis and COPD: frequency and associated consequences for systemic inflammation and physical inactivity. Chest 2009; 136: 1039-1046.

Reprinted from Chronic Respiratory Disease, Mesquita R, Spina G, Pitta F, Donaire-Gonzalez D, Deering BM, Patel MS, Mitchell KE, Alison J, van Gestel AJR, Zogg S, Gagnon P, Abascal-Bolado B, Vagaggini B, GarciaAymerich J, Jenkins SC, Romme EAPM, Kon SSC, Albert PS, Waschki B, Shrikrishna D, Singh SJ, Hopkinson NS, Miedinger D, Benzo RP, Maltais F, Paggiaro P, McKeough ZJ, Polkey MI, Hill K, Man WDC, Clarenbach CF, Hernandes NA, Savi D, Wootton S, Furlanetto KC, Cindy Ng LW, Vaes AW, Jenkins C, Eastwood PR, Jarreta D, Kirsten A, Brooks D, Hillman DR, Sant'Anna T, Meijer K, Dürr S, Rutten EPA, Kohler M, Probst VS, Tal-Singer R, Garcia Gil E, den Brinker AC, Leuppi JD, Calverley PMA, Smeenk FWJM, Costello RW, Gramm M, Goldstein R, Groenen MTJ, Magnussen H, Wouters EFM, ZuWallack RL, Amft O, Watz H, Spruit MA, Physical activity patterns and clusters in 1001 patients with COPD, 2017, [Epub ahead of print], with permission from SAGE Publications. 
SUPPLEMENTARY MATERIAL

\section{MATERIAL AND METHODS}

\section{Details of Data Sources}

The objectively assessed physical activity data used in the current analysis were collected as part of previous studies which were developed in 10 different countries (i.e., United Kingdom, Ireland, the Netherlands, Germany, Switzerland, Italy, Spain, the United States of America, Brazil, and Australia). The research groups that contributed to the current study were conveniently selected from recent publications (articles in peerreviewed journals and abstracts presented at major respiratory congresses) using the SenseWear Armband to assess physical activity in patients with COPD. A detailed description of the sources of the data included in the current analysis is provided in eTable 1. 


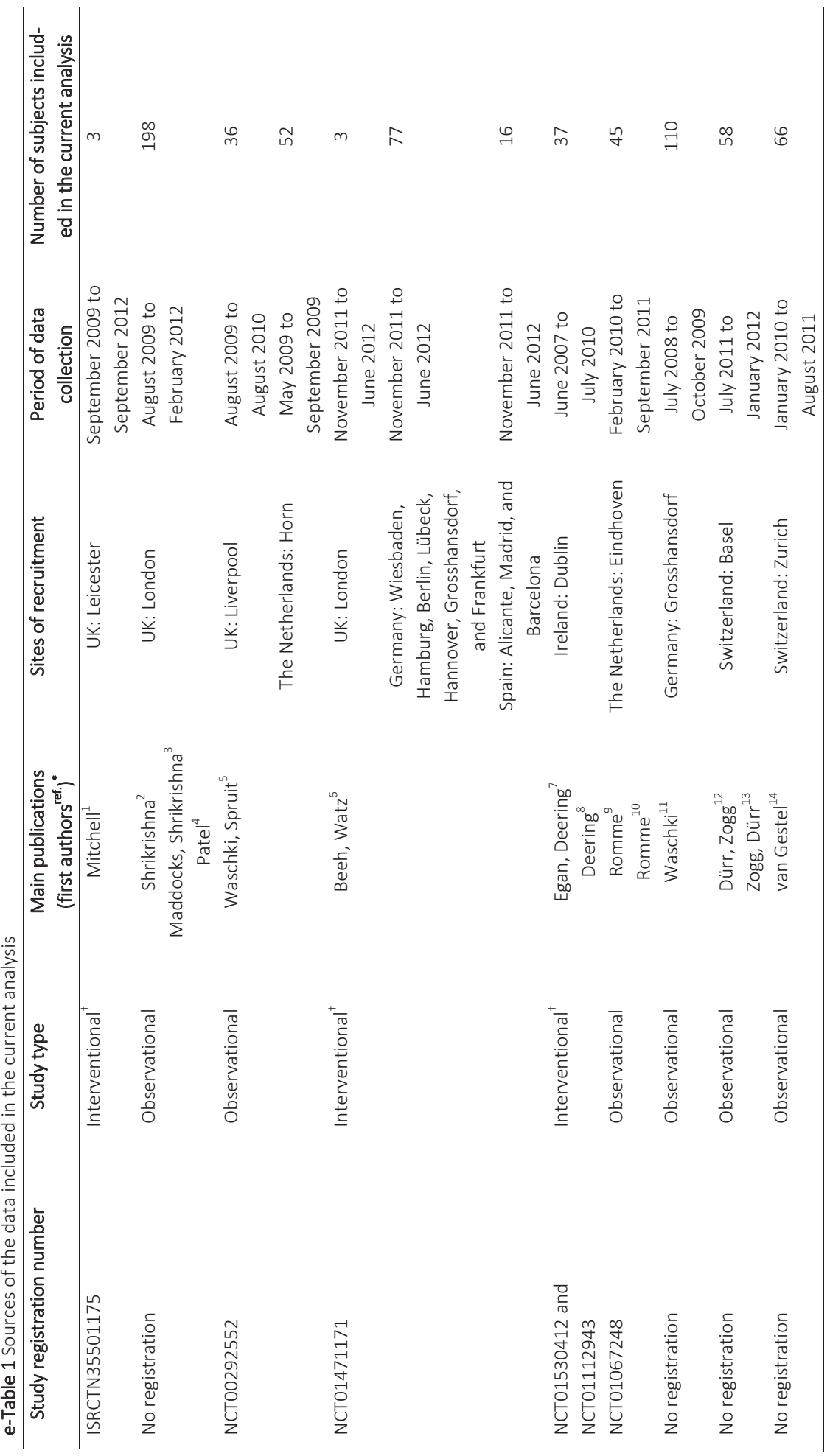




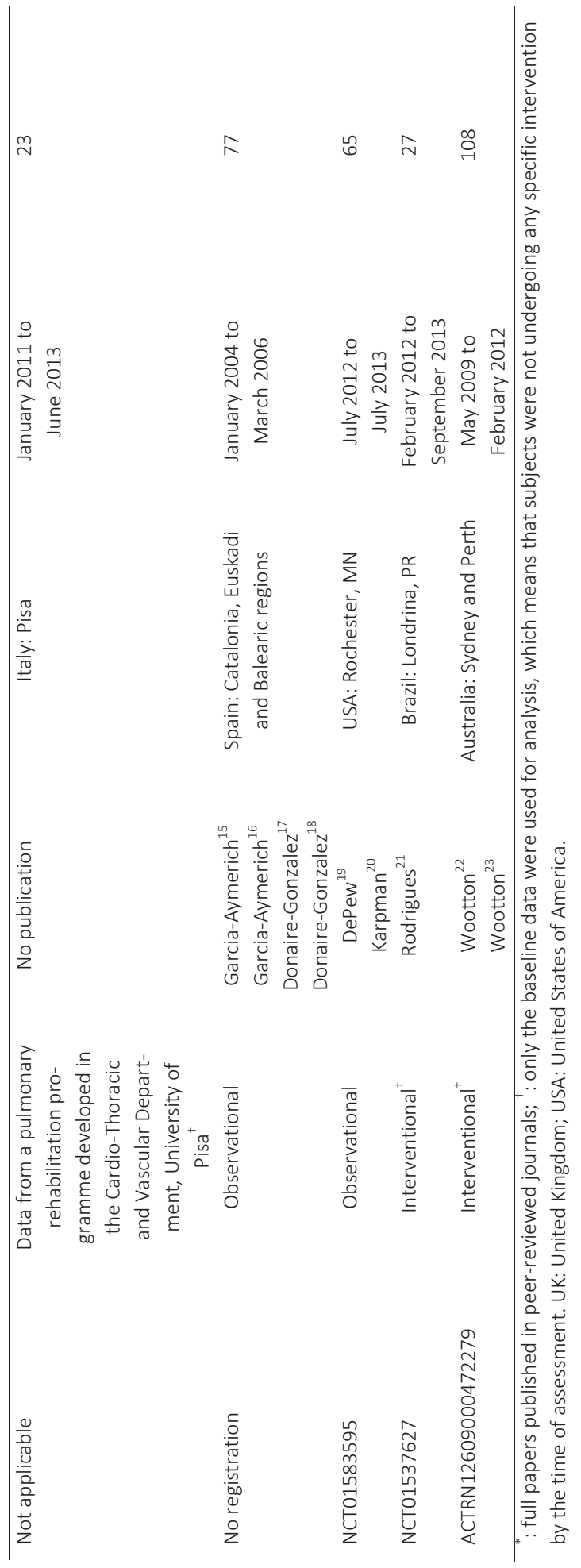




\section{Assessment of Demographics, Anthropometrics, Lung Function, and Clinical Data}

Age, sex, body mass index (BMI), post-bronchodilator forced expiratory volume in the first 1 second $\left(F E V_{1}\right.$, \% of predicted), post-bronchodilator $F V_{1} /$ forced vital capacity (FVC) ratio, diffusion capacity of the lung for carbon monoxide ( $D_{L C O}, \%$ of predicted), symptoms of dyspnoea by the modified Medical Research Council (mMRC) dyspnoea grade $^{24}$, and use of long-term oxygen therapy (LTOT, yes/no) were measured. In addition, the age, dyspnoea, and airflow obstruction (ADO) index was calculated, which predicts COPD mortality ${ }^{25}$, and participants were stratified by BMI (underweight, $<18.5$ $\mathrm{kg} \cdot \mathrm{m}^{-2}$; normal weight, 18.5 to $24.99 \mathrm{~kg} \cdot \mathrm{m}^{-2}$; pre-obese, 25 to $29.99 \mathrm{~kg} \cdot \mathrm{m}^{-2}$; or obese, $\geq 30 \mathrm{~kg} \cdot \mathrm{m}^{-2}$ ) and by Global Initiative for Chronic Obstructive Lung Disease (GOLD) classifications (2007, 1 to $4^{26}$; and 2011, A to $D^{27}$ ). GOLD 2011 classification (A to D) was based on the degree of airflow limitation (GOLD grades 1 to 4 ) and symptoms (mMRC dyspnoea grades 0 to 4 ).

\section{Selection of Waking Hour Recordings}

Firstly, the data collected with the SenseWear Armband devices were exported in the form of Microsoft Excel spreadsheets with one minute resolution. The data contains information about the sleeping time and, in particular, each minute assessed is marked by the SenseWear software as "sleeping" or "not sleeping" 28 . Then, in order to reduce the variability of the data, only minutes coded as "not sleeping" were selected for analysis. If a minute was coded as "sleeping" but had an intensity value higher than 2.0 metabolic equivalents of task (METs), which is compatible with light intensity, this minute was considered as "not sleeping" since it is very unlikely that a subject present such a high intensity whilst sleeping.

\section{Features used in the cluster analysis}

e-Table 2 presents the 180 features used for cluster analysis. 


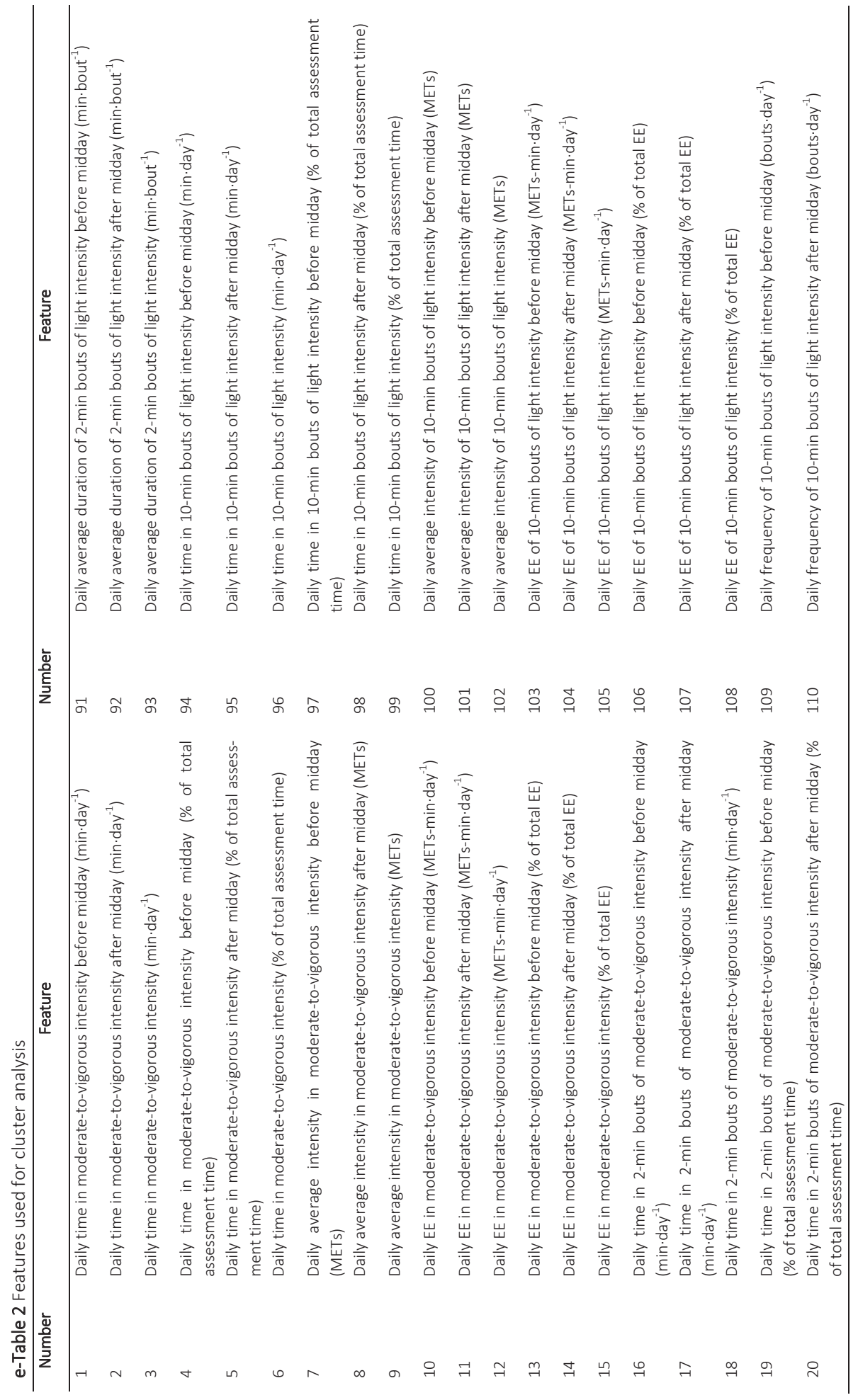




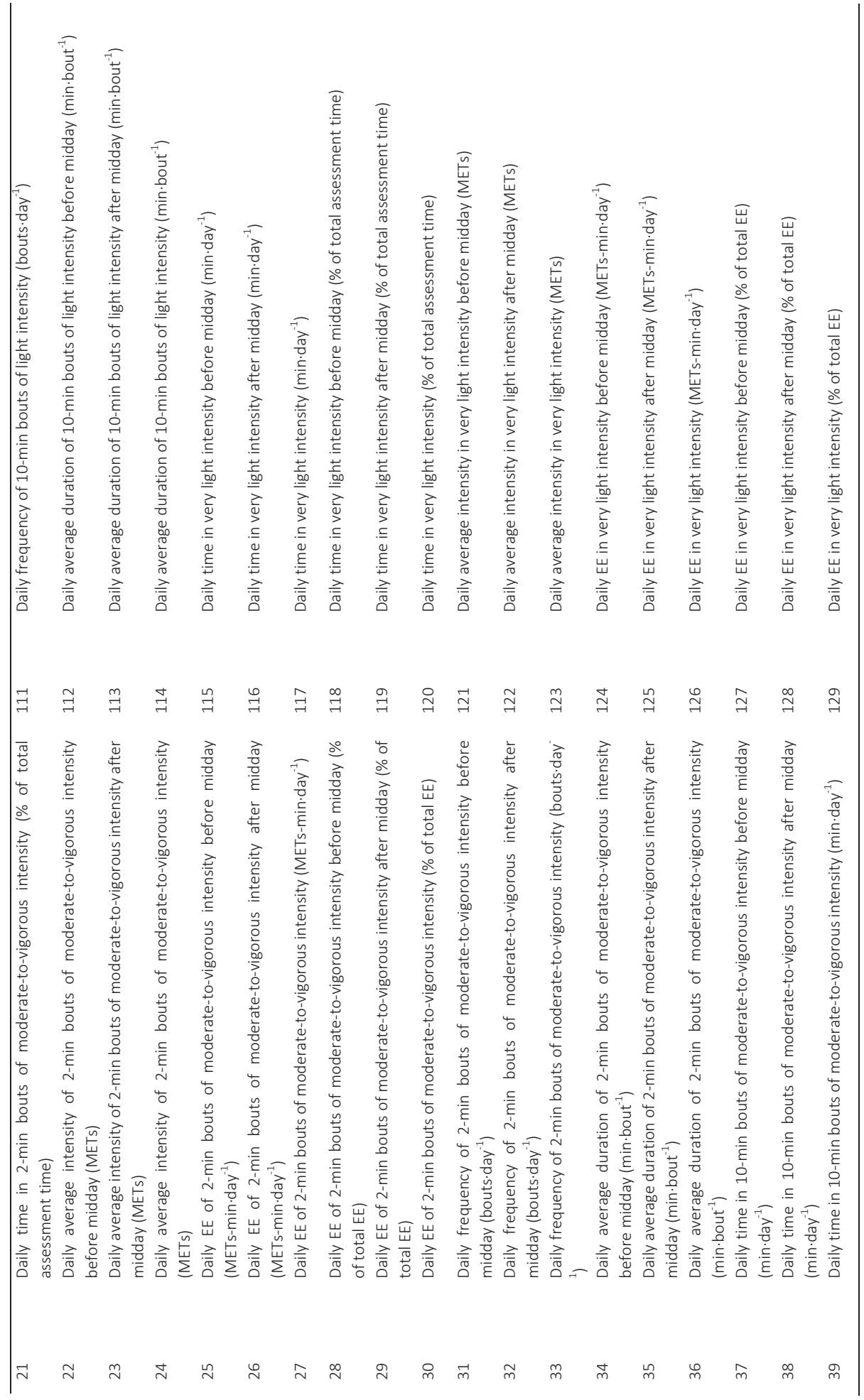




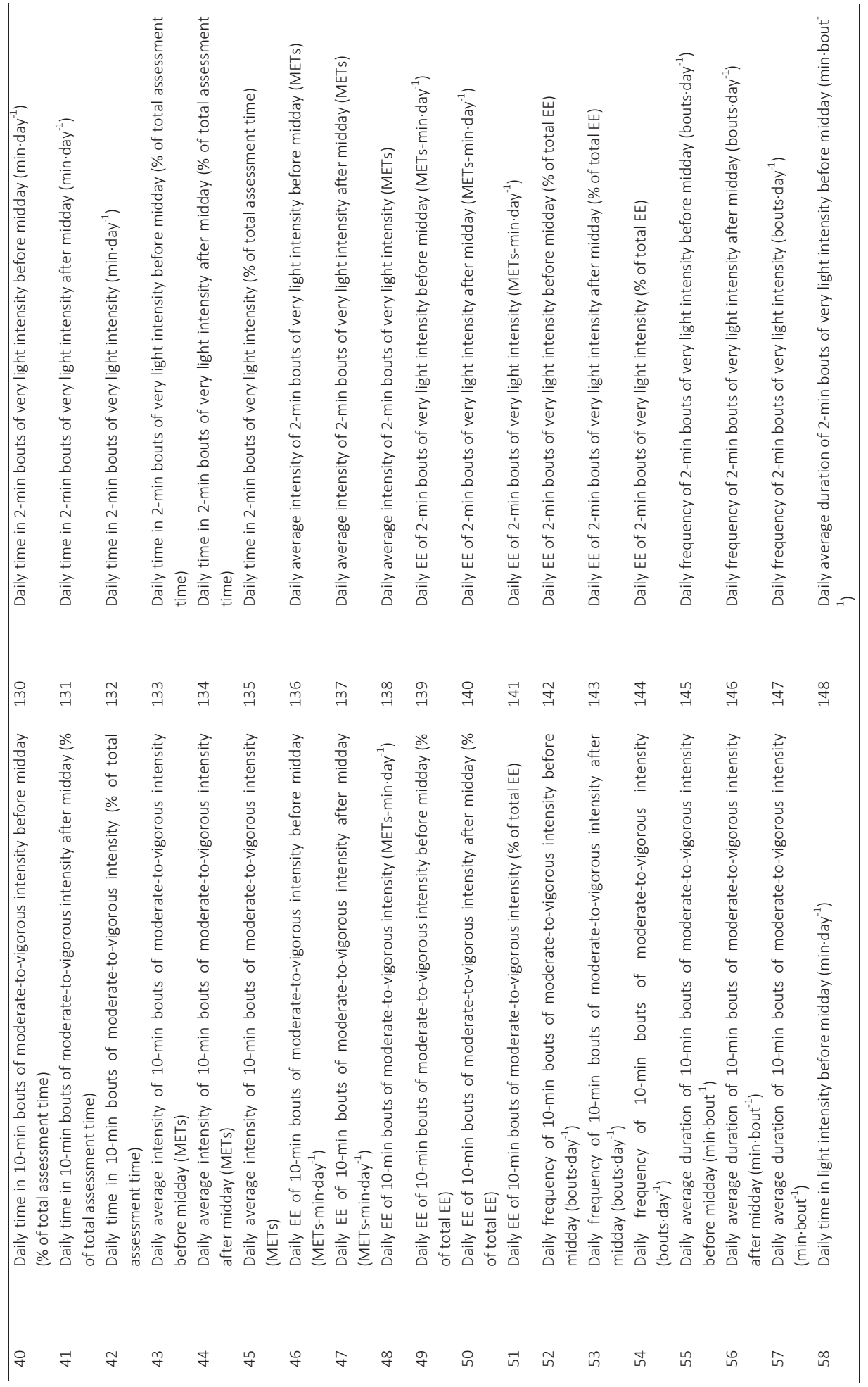




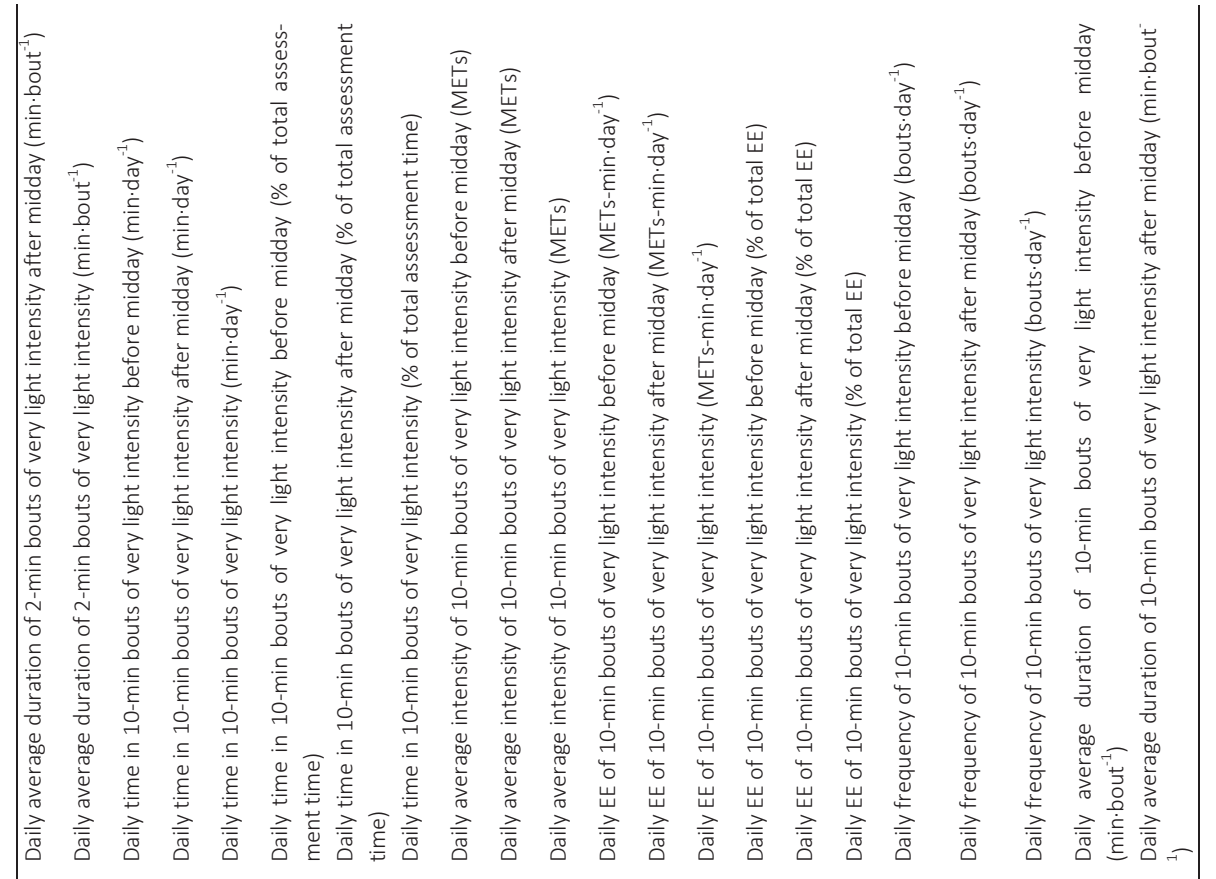

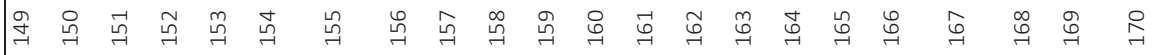

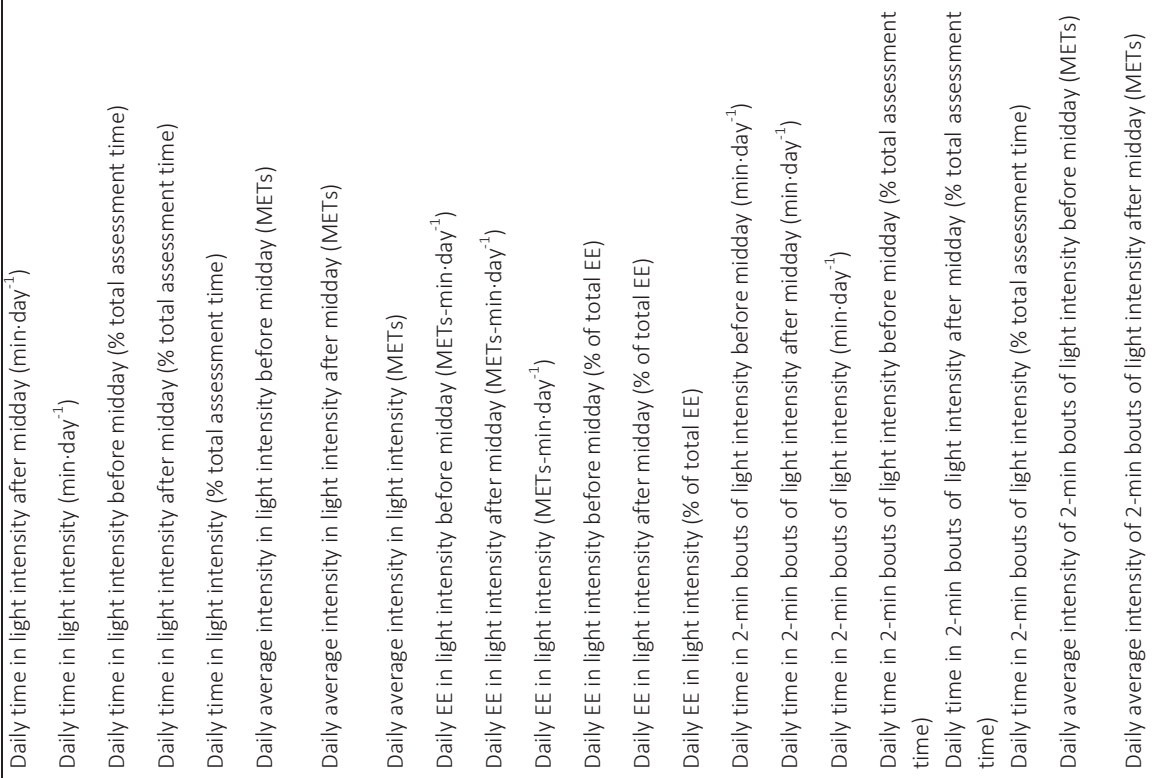

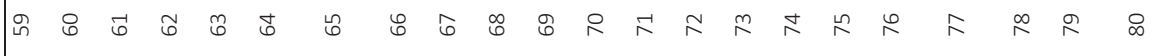




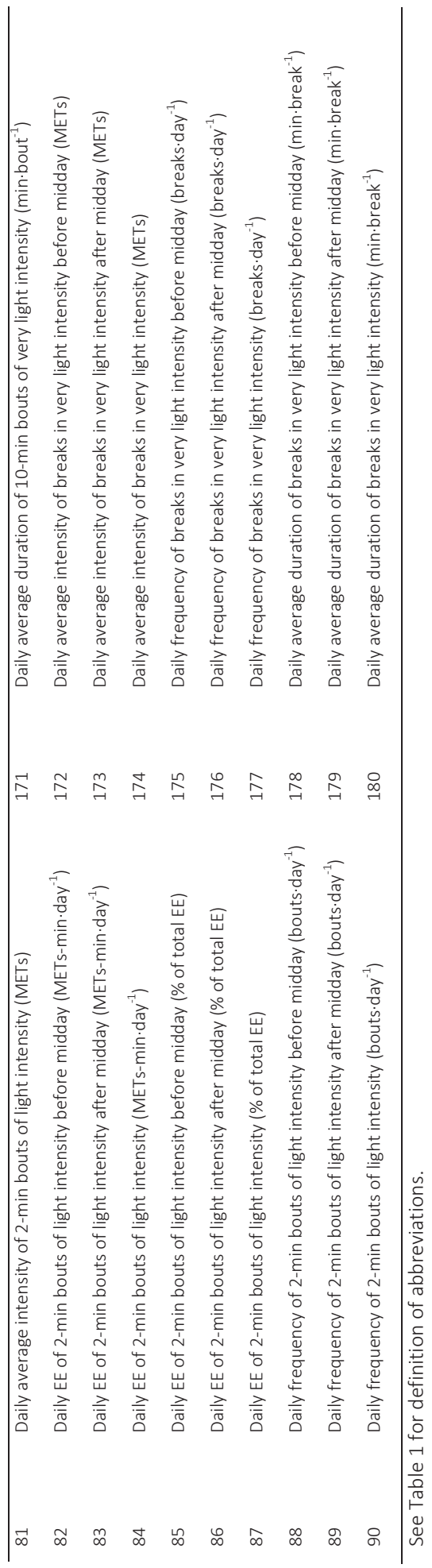




\section{Sample Size Calculation}

The main analysis in our study was the identification of clusters based on physical activity data. To the best of our knowledge, currently there are no sample size calculation formulas for cluster analysis as performed in our study. Some authors have suggested that the minimal sample size to include in studies using cluster analysis should be no less than $2 \mathrm{k}$ cases, preferably $5 \times 2 \mathrm{k}$, with $\mathrm{K}$ being the number of variables considered for analysis ${ }^{29,30}$. In our study, only 3 variables (i.e., the 3 components from the principal component analysis) were used for clustering. Therefore, the minimal sample size in our study should be 40 subjects, which is actually far below the actual number of participants included (i.e., 1001 subjects). Furthermore, our sample size is much larger than that of most previous studies using cluster analysis in COPD, which were still able to identify heterogeneous groups amongst different samples of patients with COPD ${ }^{16,31-34}$.

\section{Daily Physical Activity Measures after Stratification for Seasons of the Year}

Daily physical activity measures after stratification for seasons of the year can be found in e-Table 3. 


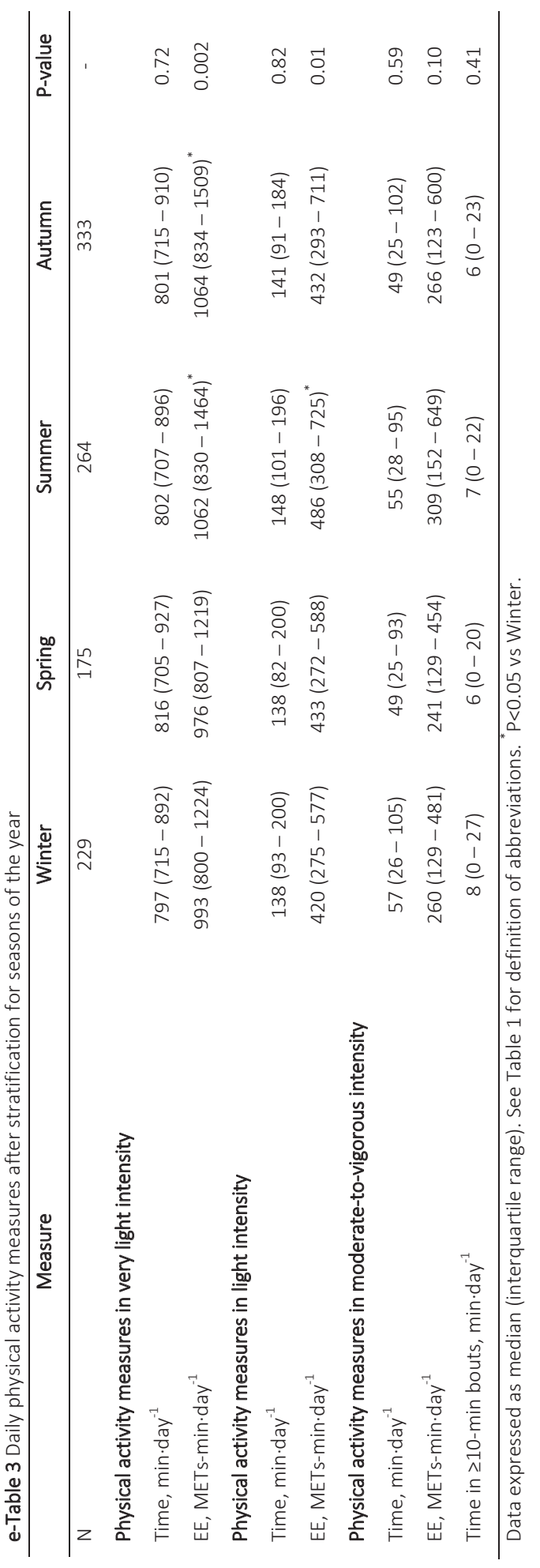




\section{RESULTS}

Characteristics after Stratification for Country of Assessment

General characteristics and physical activity measures after stratification per country of assessment are presented in e-Table 4. 


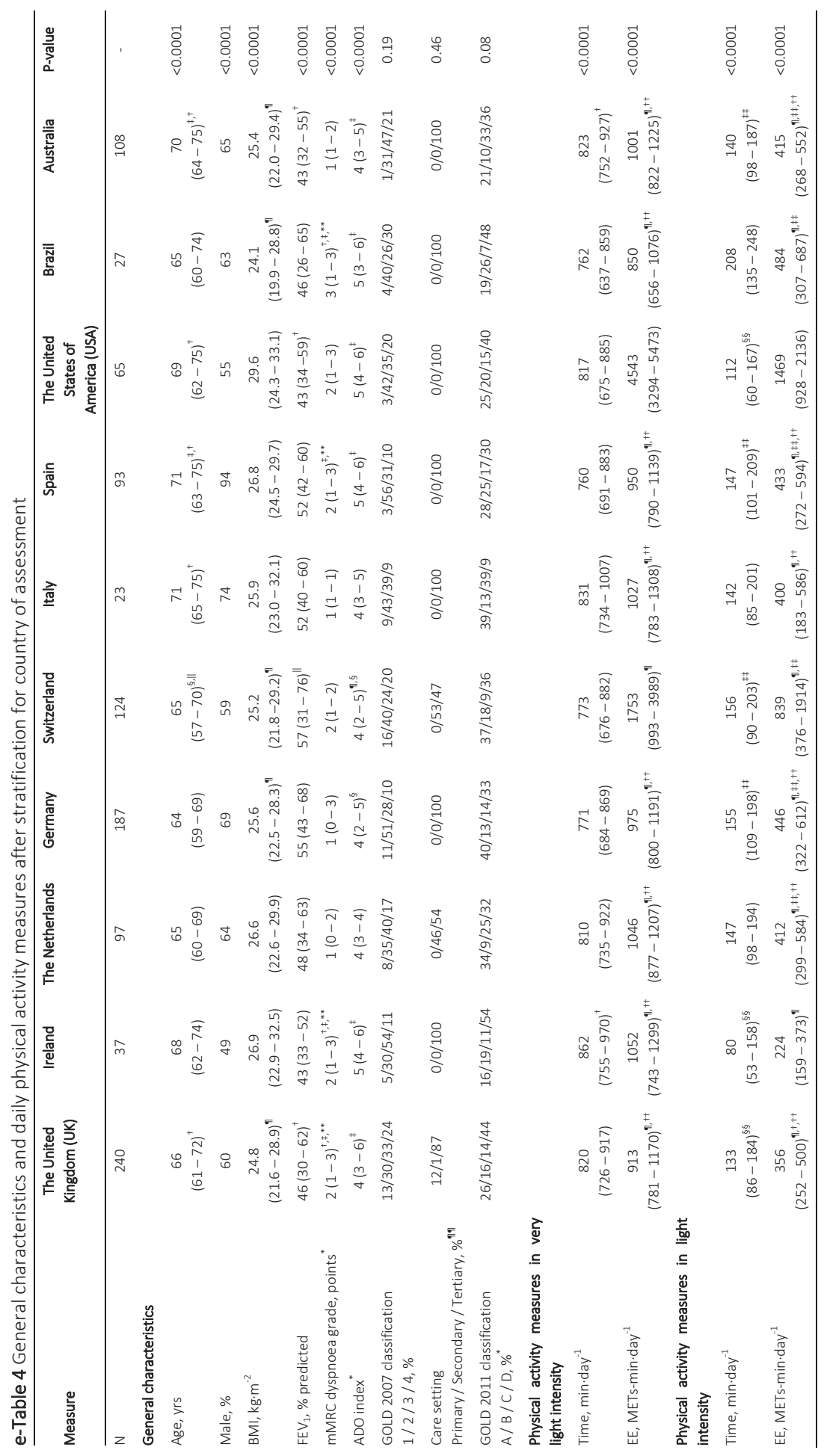




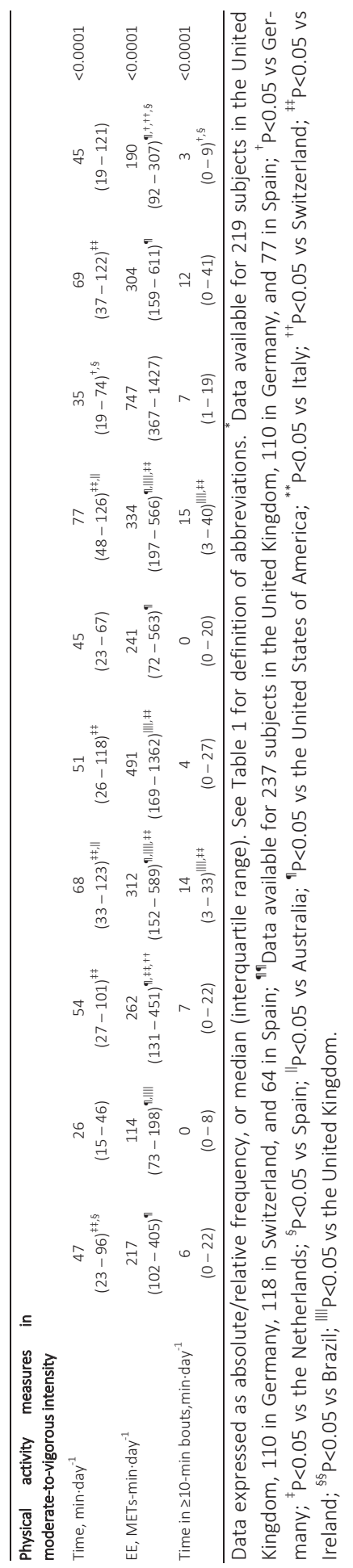


Daily Physical Activity Measures and Hourly Patterns after Stratification for Clinical Characteristics

Daily physical activity measures after stratification for clinical characteristics can be found in e-Tables 5-13. e-Figure 1 presents the daily physical activity hourly patterns after stratification for age groups, sex, long-term oxygen therapy use, diffusion capacity of the lung for carbon monoxide ( $\left.D_{L c O}\right)$ groups, and age, dyspnoea, and airflow obstruction (ADO) index groups. 
CHAPTER 7

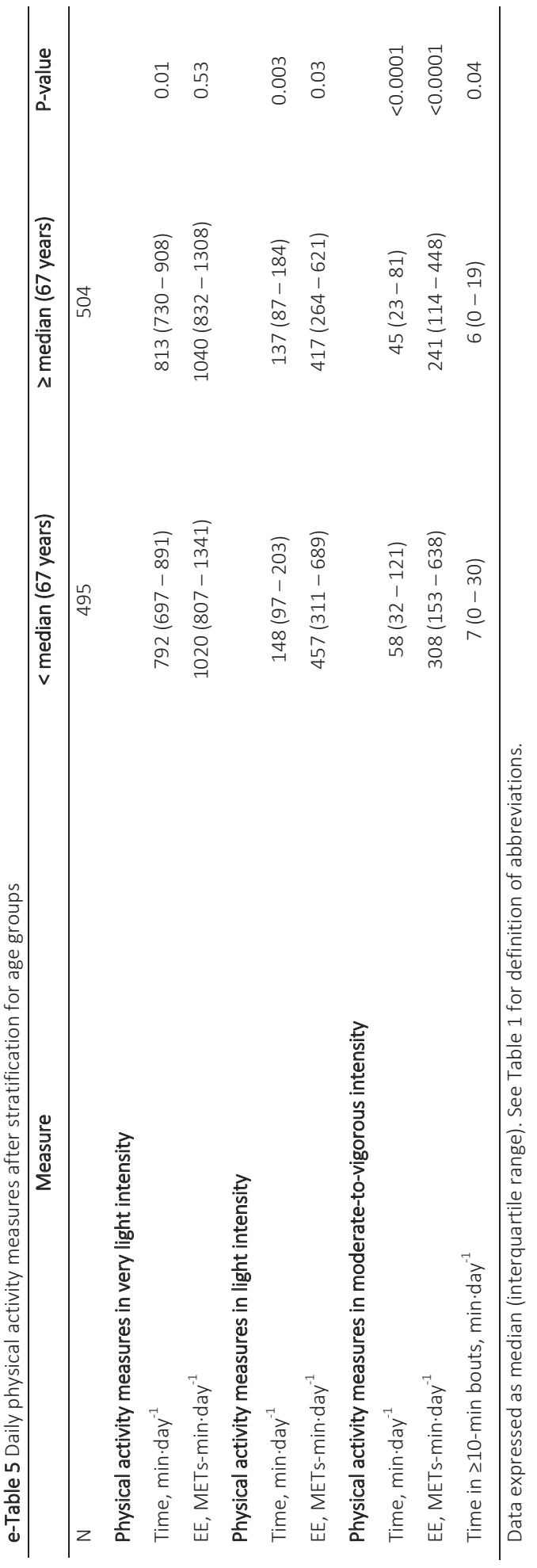




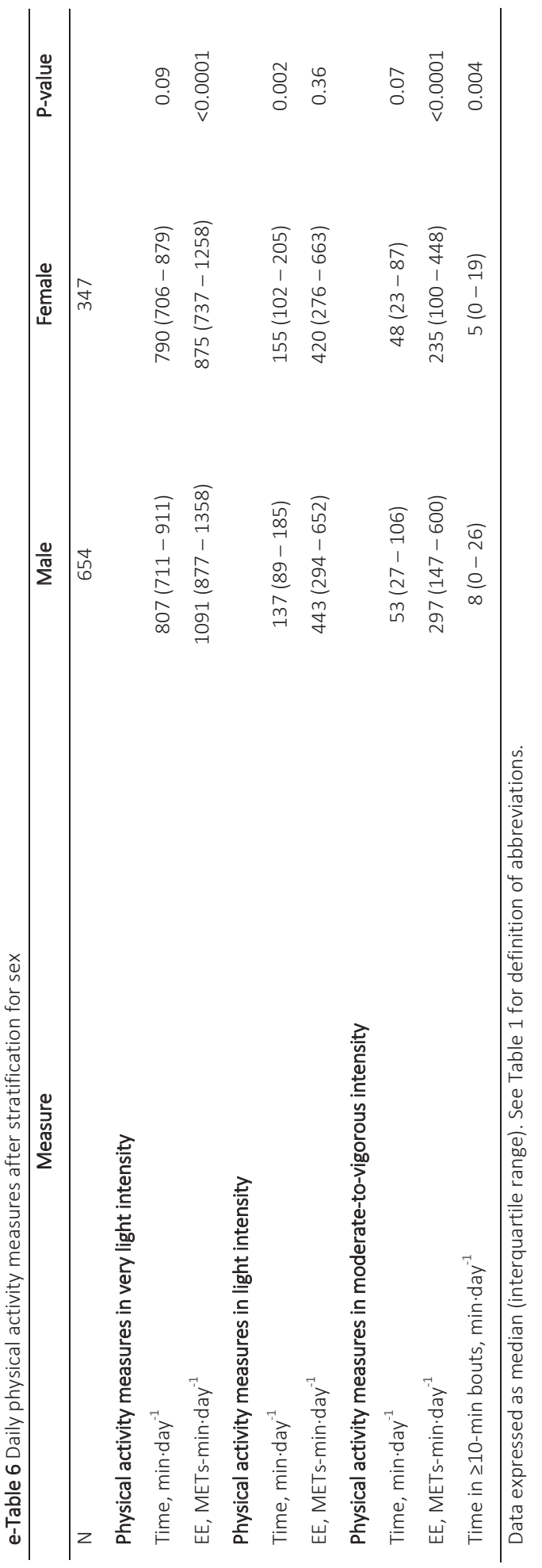


CHAPTER 7

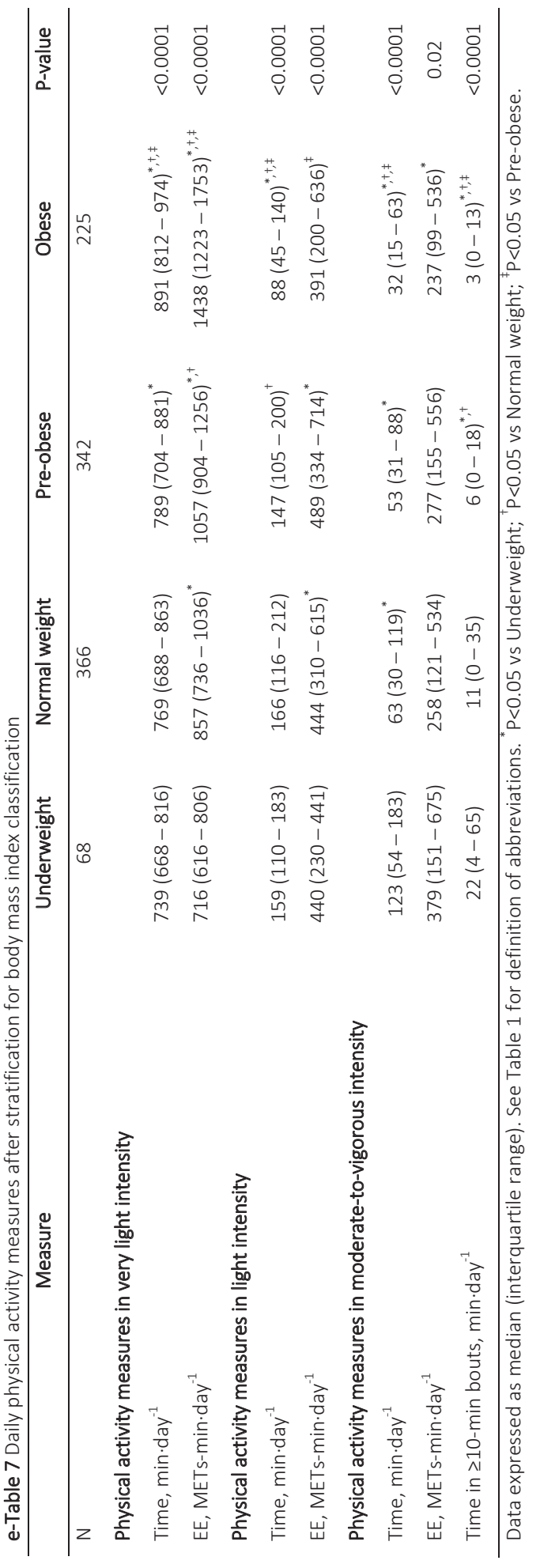




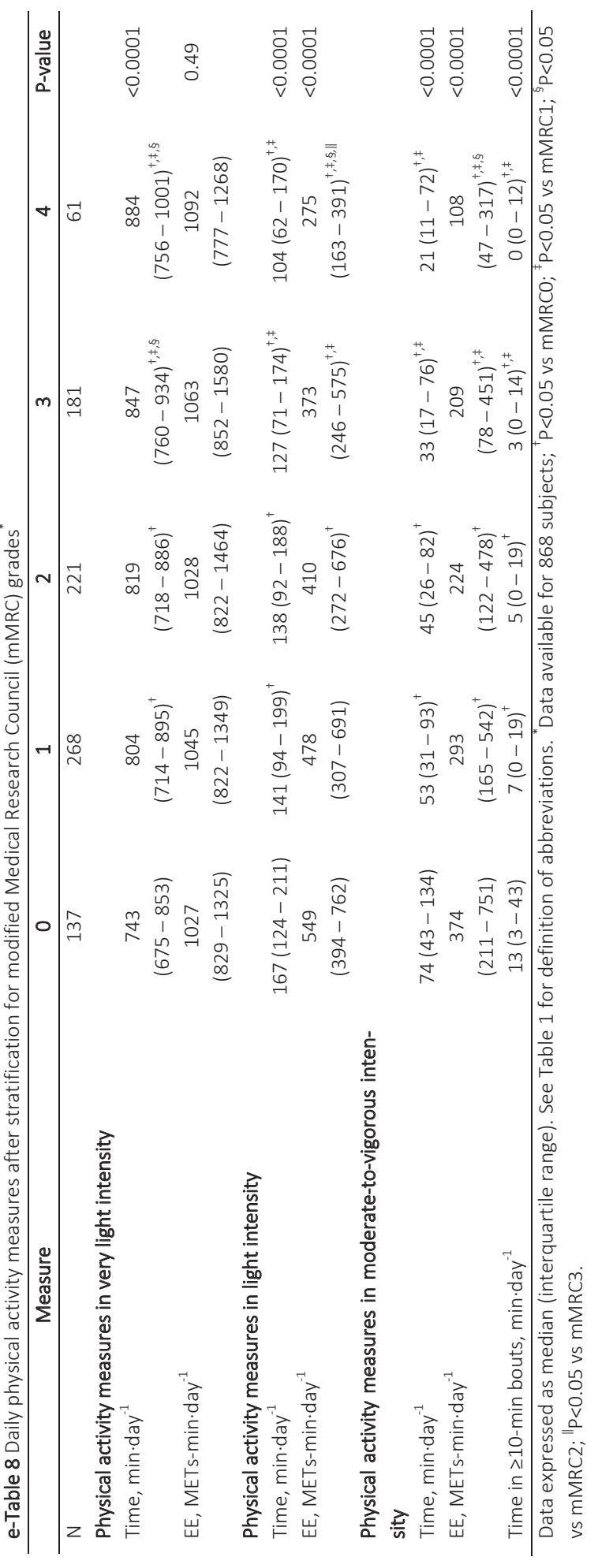


CHAPTER 7

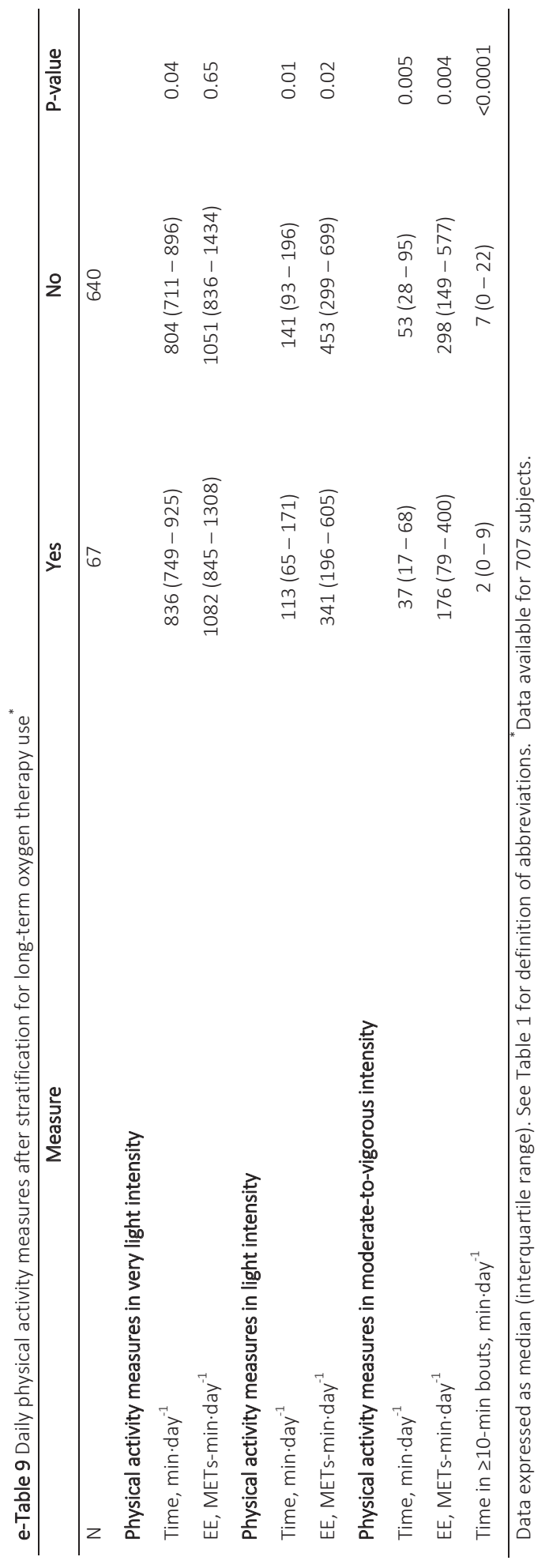




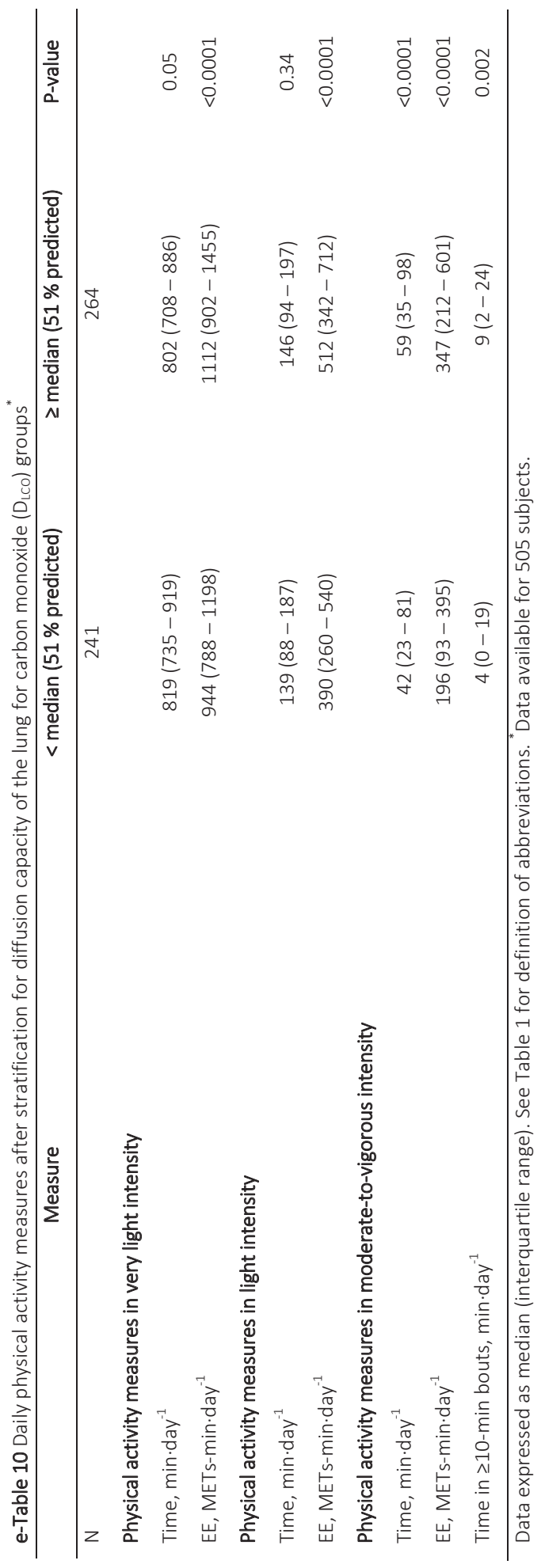


CHAPTER 7

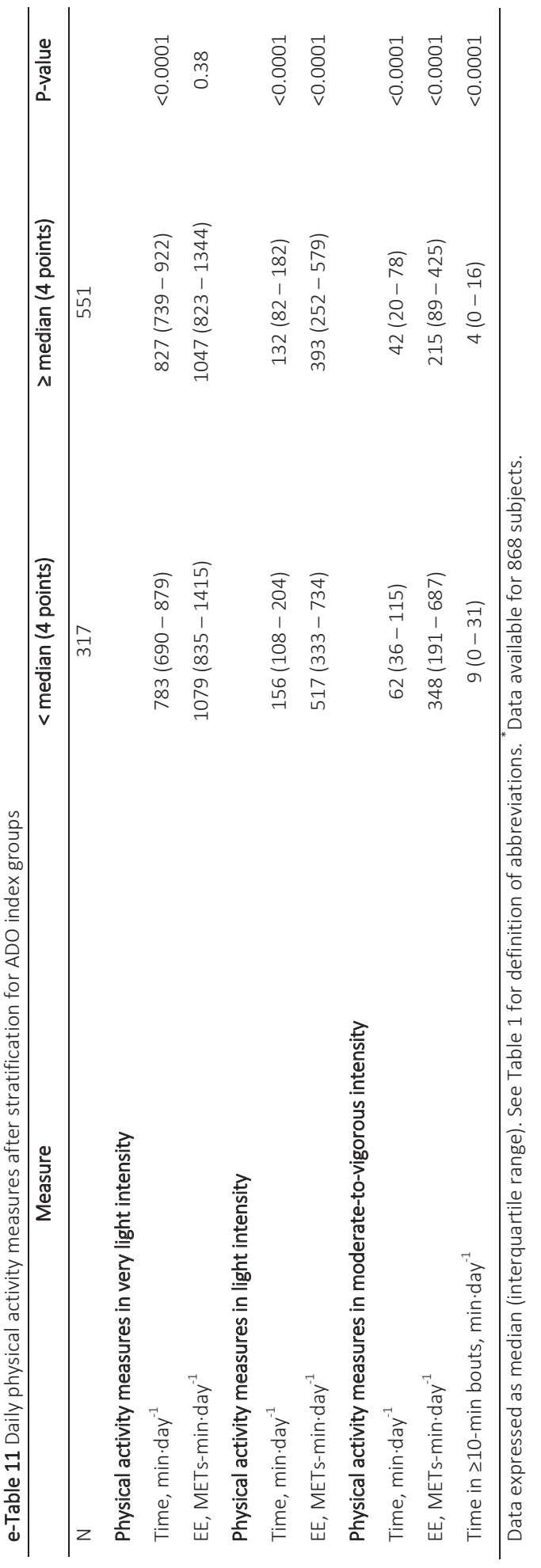




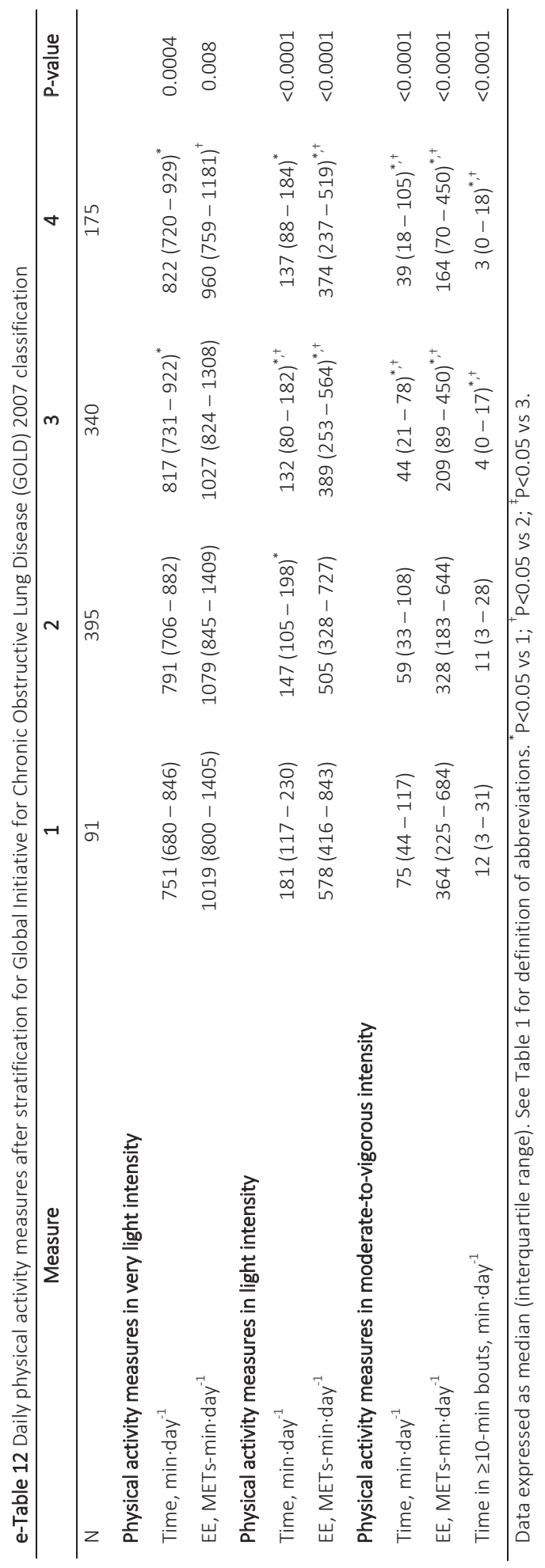


CHAPTER 7

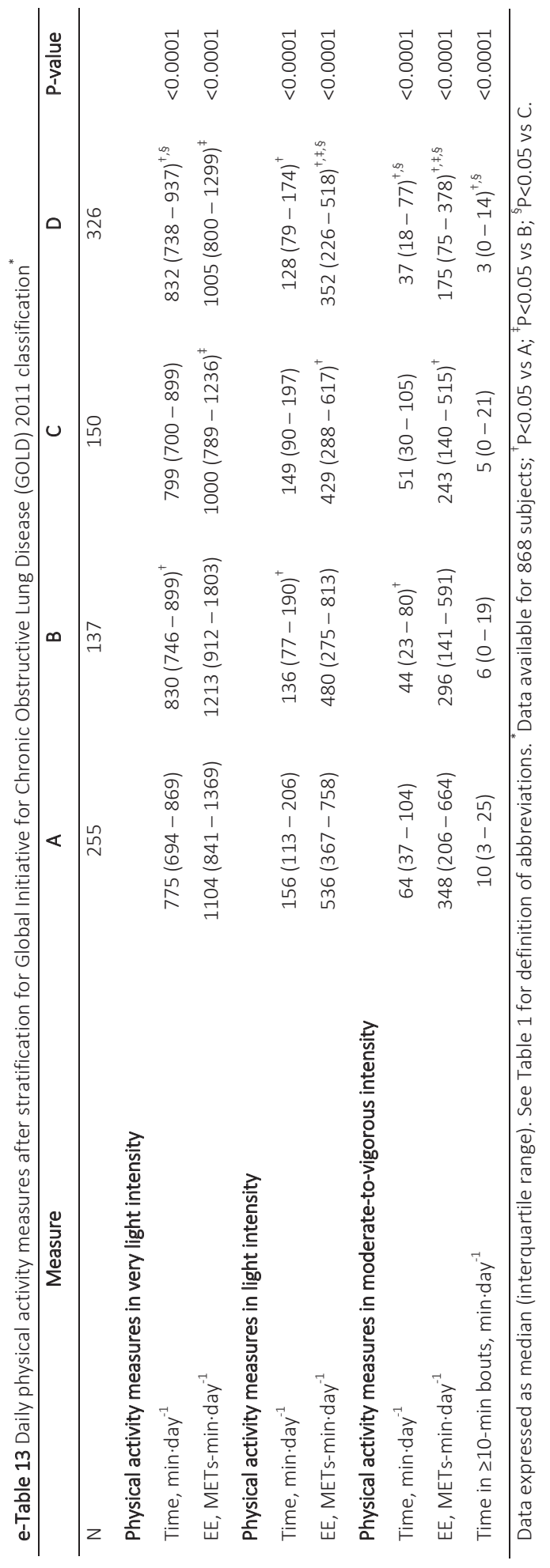



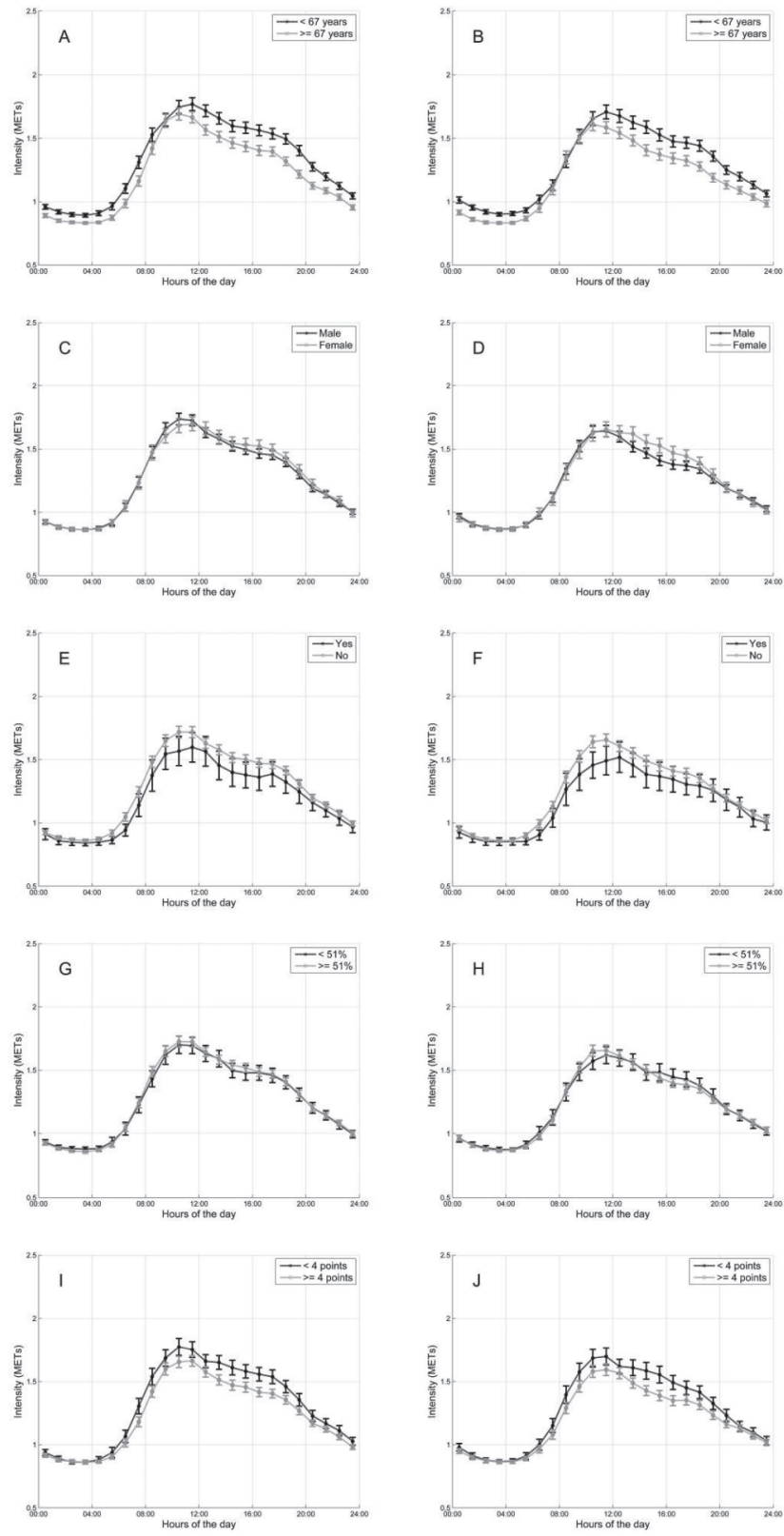

e-Figure 1 Daily physical activity hourly patterns of the patients with chronic obstructive pulmonary disease after stratification for: A and B - age groups (< or $\geq$ median, 67 years); $C$ and D - sex; E and F - long-term oxygen therapy (LTOT) use (yes or no), data available for 707 subjects only; $\mathrm{G}$ and $\mathrm{H}$ - diffusion capacity of the lung for carbon monoxide $\left(D_{\llcorner c o}\right)$ groups (< or $\geq$ median, $51 \%$ predicted), data available for 505 subjects; and I and $\mathrm{J}$ - age, dyspnoea, and airflow obstruction (ADO) index groups(< or $\geq$ median, 4 points). Figures A, C, E, G, and I represent weekdays, whilst figures B, D, F, H, and J represent weekend days. Data pooled per hour as mean ( $95 \%$ confidence intervals). 
Area Under the Curve (AUC) from Daily Physical Activity Hourly Patterns

e-Table 14 presents the AUC-values with their 95\% confidence intervals from daily physical activity hourly patterns. 


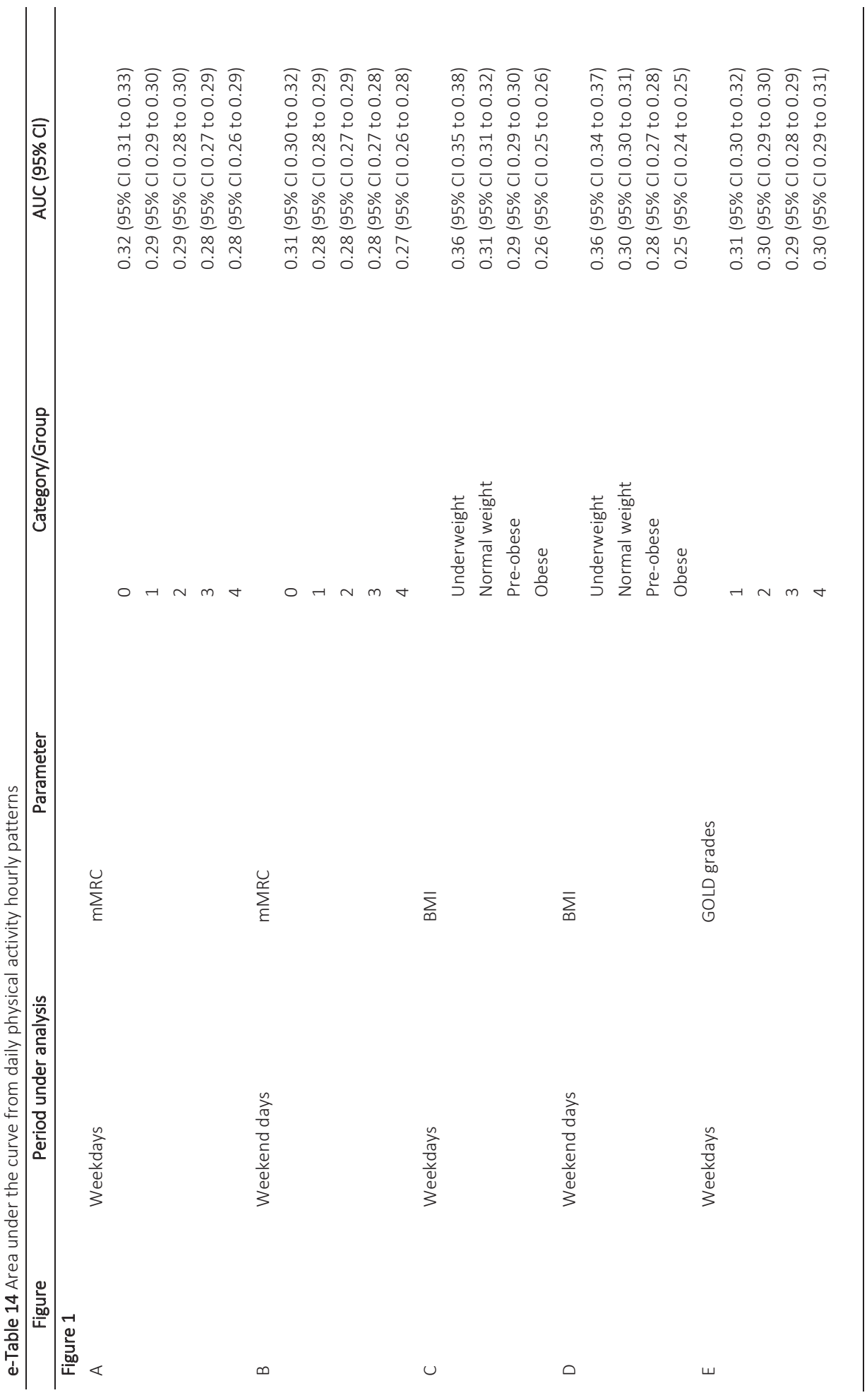




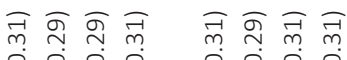

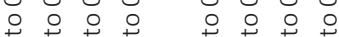

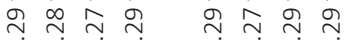

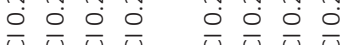
웅ㅇㅇ 우

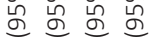
尚

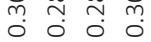
ํํํํํํํํ ํำ
วอ ว

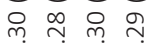

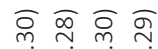

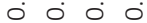
$+0+0$ $\stackrel{\infty}{\sim} \stackrel{\infty}{\sim} \stackrel{\infty}{\sim}$ $\circ \circ \div$ 웅

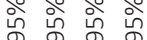
공 공

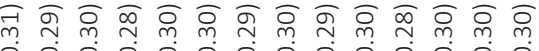

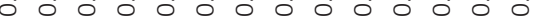

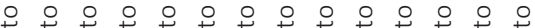

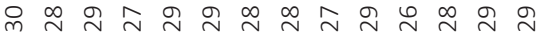

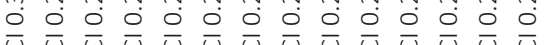
do do jo jo jo jo jo jo jo jo jo jo jo jo

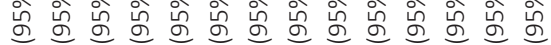

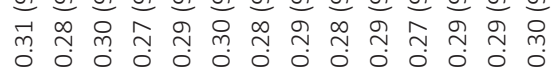

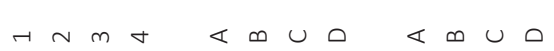

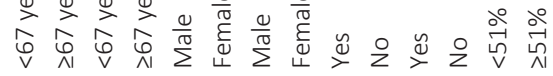

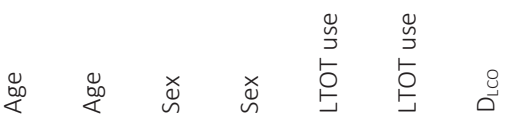

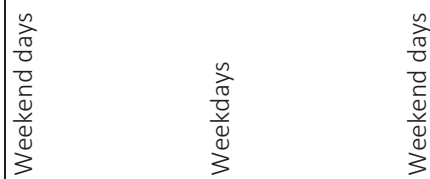

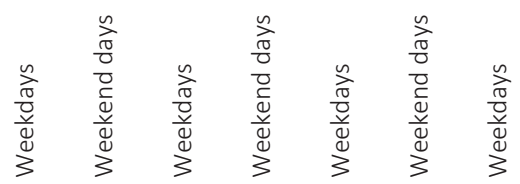

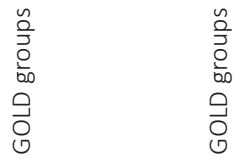

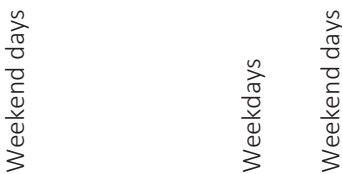

$\frac{\substack{\frac{\pi}{\pi} \\ \frac{0}{0}}}{3}$

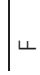

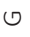

I

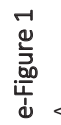




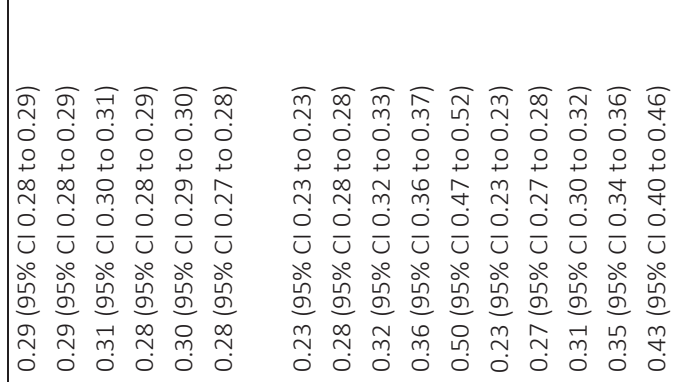

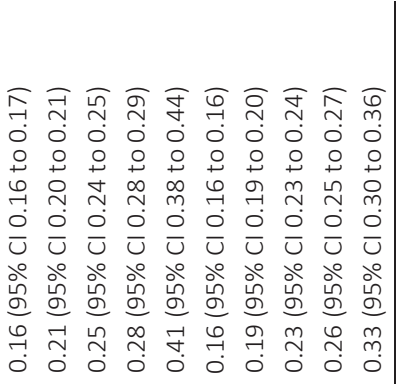

$\stackrel{\infty}{\stackrel{0}{5}}$

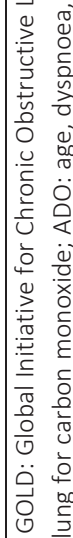

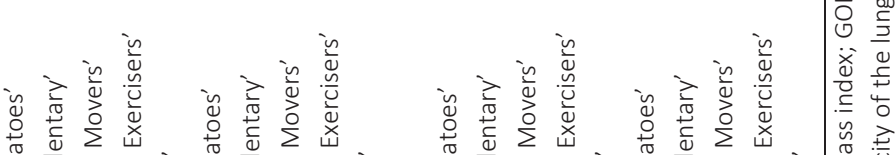

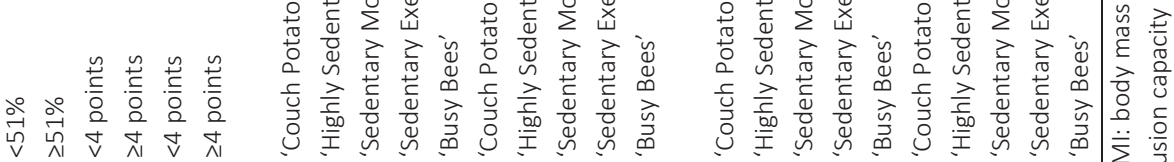

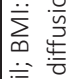

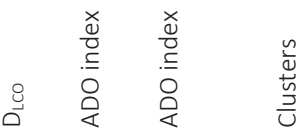

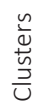

$\frac{n}{\dddot{U}}$
$\frac{\tilde{J}}{U}$

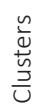

$\frac{n}{\pi}$
$\frac{\pi}{0}$
$\frac{0}{0}$
3

0
$\frac{\pi}{0}$
0
0
$\frac{0}{0}$
$\frac{0}{0}$
0
3

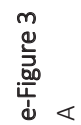

$\infty$

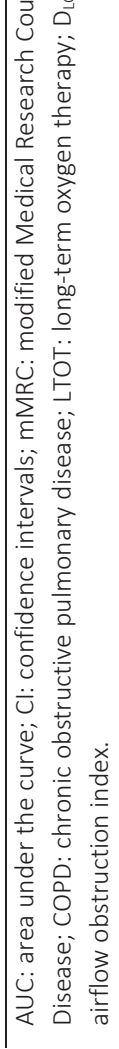


Association between Lung Function and Daily Physical Activity Measures

e-Figure 2 presents the correlation coefficients between forced expiratory volume in the first second (\% predicted) and the daily time in activities of very light intensity, light intensity, and moderate-to-vigorous intensity.
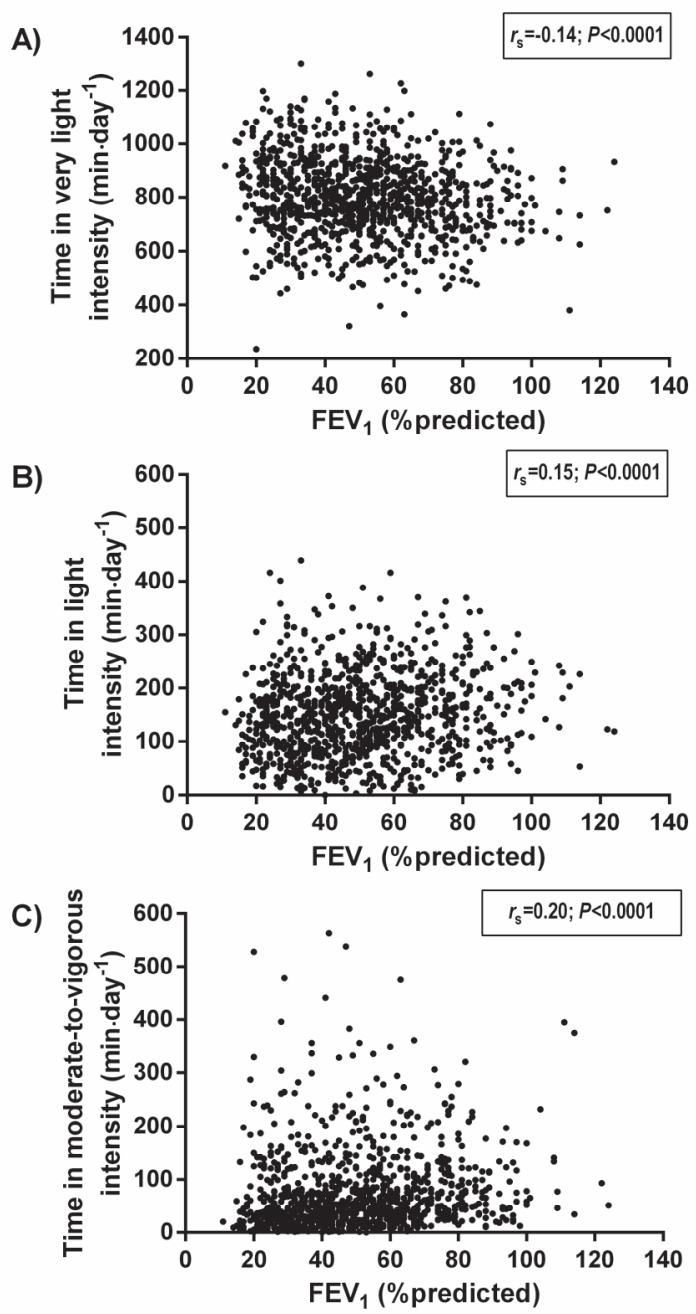

e-Figure 2 Spearman's correlation between forced expiratory volume in the first second (\% predicted) and the daily time in activities of very light intensity (A), light intensity (B), and moderate-to-vigorous intensity (C) for 1001 patients with chronic obstructive pulmonary disease. 


\section{Daily Physical Activity Hourly Patterns of Clusters of Patients with COPD}

e-Figure 3 presents the daily physical activity hourly pattern of the clusters of patients with chronic obstructive pulmonary disease after synchronisation of the waking up moment.
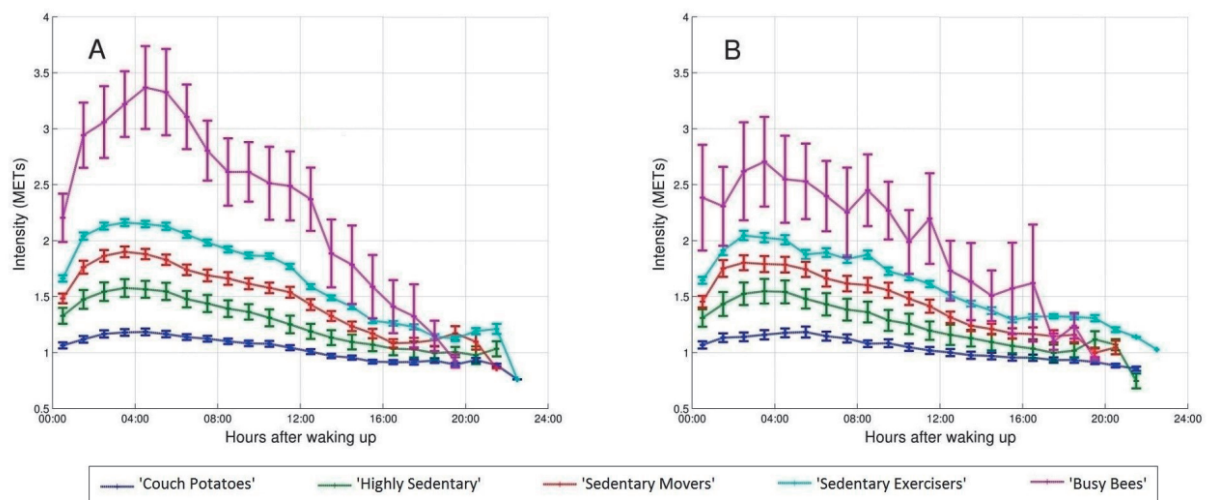

e-Figure 3 Daily physical activity hourly pattern of the clusters of patients with chronic obstructive pulmonary disease after synchronisation of the waking up moment during weekdays (A) and weekend days (B). Hourly patterns were presented before and after synchronisation to overcome the problem of subjects with different waking up times. Data pooled per hour as mean (95\% confidence intervals).

Detailed Analyses of the Components Identified in the Principal Component Analysis (PCA)

A detailed analysis of the relationship between the three components identified by PCA and the five clusters identified from these components is provided in the following paragraphs.

The first component was clearly able to discriminate between 'Couch Potatoes' and 'Highly Sedentary' (the most inactive clusters) from the three other clusters ('Sedentary Movers', 'Sedentary Exercisers', and 'Busy Bees') (Figures 2B and 2C). The second component was not really useful to identify more inactive patients ('Couch Potatoes' and 'Highly Sedentary'), but was able to discriminate the most active cluster ('Busy Bees') from the rest (Figures 2B and 2D). To discriminate 'Sedentary Movers' and 'Sedentary Exercisers' from the others it is important to consider the combination of the three components. Indeed, if only the first component was considered, for instance, these clusters would be added to the most active cluster ('Busy Bees') (Figures 2B and 2C). On the other hand, if only the second component was considered these clusters would be added to the inactive clusters (i.e., 'Couch Potatoes' and 'Highly Sedentary') (Figures 2B and 2D). 


\section{CHAPTER 7}

Having a closer look at the most relevant features of each component we can notice that the first component is related to the time spent in bouts of very light intensity, whilst the second component is related to the total daily EE in activities of moderate-tovigorous intensity, mostly in bouts of physical activity. Therefore, it can be suggested that the time in bouts of moderate-to-vigorous intensity can be a useful marker to discriminate patients who are very active from the others. 


\section{References}

1. Mitchell KE, Johnson-Warrington $\mathrm{V}$, Apps LD, et al. A self-management programme for COPD: a randomised controlled trial. Eur Respir J 2014; 44: 1538-1547.

2. Shrikrishna D, Patel M, Tanner RJ, et al. Quadriceps wasting and physical inactivity in patients with COPD. Eur Respir J 2012; 40: 1115-1122.

3. Maddocks M, Shrikrishna D, Vitoriano S, et al. Skeletal muscle adiposity is associated with physical activity, exercise capacity and fibre shift in COPD. Eur Respir J 2014; 44: 1188-1198.

4. Patel MS, Mohan D, Andersson YM, et al. Phenotypic characteristics associated with reduced short physical performance battery score in COPD. Chest 2014; 145: 1016-1024.

5. Waschki B, Spruit MA, Watz H, et al. Physical activity monitoring in COPD: compliance and associations with clinical characteristics in a multicenter study. Respir Med 2012; 106: 522-530.

6. Beeh KM, Watz H, Puente-Maestu L, et al. Aclidinium improves exercise endurance, dyspnea, lung hyperinflation, and physical activity in patients with COPD: a randomized, placebo-controlled, crossover trial. BMC Pulm Med 2014; 14: 209.

7. Egan C, Deering BM, Blake C, et al. Short term and long term effects of pulmonary rehabilitation on physical activity in COPD. Respir Med 2012; 106: 1671-1679.

8. Deering BM, Fullen B, Egan C, et al. Acupuncture as an adjunct to pulmonary rehabilitation. J Cardiopulm Rehabil Prev 2011; 31: 392-399.

9. Romme EA, Murchison JT, Phang KF, et al. Bone attenuation on routine chest CT correlates with bone mineral density on DXA in patients with COPD. J Bone Miner Res 2012; 27: 2338-2343.

10. Romme EA, Rutten EP, Geusens P, et al. Bone stiffness and failure load are related with clinical parameters in men with chronic obstructive pulmonary disease. J Bone Miner Res 2013; 28: 2186-2193.

11. Waschki B, Kirsten AM, Holz O, et al. Disease Progression and Changes in Physical Activity in Patients with Chronic Obstructive Pulmonary Disease. Am J Respir Crit Care Med 2015; 192: 295-306.

12. Durr S, Zogg S, Miedinger D, et al. Daily physical activity, functional capacity and quality of life in patients with COPD. COPD 2014; 11: 689-696.

13. Zogg S, Durr S, Miedinger D, et al. Differences in classification of COPD patients into risk groups A-D: a cross-sectional study. BMC Res Notes 2014; 7: 562.

14. van Gestel AJ, Clarenbach CF, Stowhas AC, et al. Predicting daily physical activity in patients with chronic obstructive pulmonary disease. PLoS One 2012; 7: e48081.

15. Garcia-Aymerich J, Gomez FP and Anto JM. Phenotypic characterization and course of chronic obstructive pulmonary disease in the PAC-COPD Study: design and methods. Arch Bronconeumol 2009; 45: 4-11.

16. Garcia-Aymerich J, Gomez FP, Benet M, et al. Identification and prospective validation of clinically relevant chronic obstructive pulmonary disease (COPD) subtypes. Thorax 2011; 66: 430-437.

17. Donaire-Gonzalez D, Gimeno-Santos E, Balcells E, et al. Physical activity in COPD patients: patterns and bouts. Eur Respir J 2013; 42: 993-1002.

18. Donaire-Gonzalez D, Gimeno-Santos E, Balcells E, et al. Benefits of physical activity on COPD hospitalisation depend on intensity. Eur Respir J 2015; 46: 1281-1289.

19. Depew ZS, Karpman C, Novotny PJ, et al. Correlations between gait speed, 6-minute walk distance, physical activity, and self-efficacy in patients with severe chronic lung disease. Respir Care 2013; 58: 21132119.

20. Karpman C, Lebrasseur NK, Depew ZS, et al. Measuring gait speed in the out-patient clinic: methodology and feasibility. Respir Care 2014; 59: 531-537.

21. Rodrigues A, Di Martino M, Nellessen AG, et al. Is the six-minute walk test a useful tool to prescribe highintensity exercise in patients with chronic obstructive pulmonary disease? Heart Lung 2016.

22. Wootton SL, Ng C, McKeough ZJ, et al. Estimating endurance shuttle walk test speed using the six-minute walk test in people with chronic obstructive pulmonary disease. Chron Respir Dis 2014; 11: 89-94.

23. Wootton SL, Ng LW, McKeough ZJ, et al. Ground-based walking training improves quality of life and exercise capacity in COPD. Eur Respir J 2014; 44: 885-894. 


\section{CHAPTER 7}

24. Bestall JC, Paul EA, Garrod R, et al. Usefulness of the Medical Research Council (MRC) dyspnoea scale as a measure of disability in patients with chronic obstructive pulmonary disease. Thorax 1999; 54: 581-586.

25. Puhan MA, Garcia-Aymerich J, Frey M, et al. Expansion of the prognostic assessment of patients with chronic obstructive pulmonary disease: the updated BODE index and the ADO index. Lancet 2009; 374 : 704-711.

26. Rabe KF, Hurd S, Anzueto A, et al. Global strategy for the diagnosis, management, and prevention of chronic obstructive pulmonary disease: GOLD executive summary. Am J Respir Crit Care Med 2007; 176: 532-555.

27. Vestbo J, Hurd SS, Agusti AG, et al. Global strategy for the diagnosis, management, and prevention of chronic obstructive pulmonary disease: GOLD executive summary. Am J Respir Crit Care Med 2013; 187: 347-365.

28. Sharif MM and Bahammam AS. Sleep estimation using BodyMedia's SenseWear armband in patients with obstructive sleep apnea. Ann Thorac Med 2013; 8: 53-57.

29. Dolnicar SA. Review of unquestioned standards in using cluster analysis for data-driven market segmentation. Proceedings of the Australian and New Zealand Marketing Academy Conference 2002 (ANZMAC 2002). Deakin University, Melbourne2002.

30. Formann AK. Die latent-class-analyse: einführung in die theorie und anwendung. Weinheim: Beltz, 1984.

31. Weatherall M, Travers J, Shirtcliffe PM, et al. Distinct clinical phenotypes of airways disease defined by cluster analysis. Eur Respir J 2009; 34: 812-818.

32. Burgel PR, Paillasseur JL, Caillaud D, et al. Clinical COPD phenotypes: a novel approach using principal component and cluster analyses. Eur Respir J 2010; 36: 531-539.

33. Vanfleteren LE, Spruit MA, Groenen M, et al. Clusters of comorbidities based on validated objective measurements and systemic inflammation in patients with chronic obstructive pulmonary disease. Am J Respir Crit Care Med 2013; 187: 728-735.

34. Harrison SL, Robertson N, Graham CD, et al. Can we identify patients with different illness schema following an acute exacerbation of COPD: a cluster analysis. Respir Med 2014; 108: 319-328. 


\section{CHAPTER 8}

Changes in physical activity and sedentary behaviour following pulmonary rehabilitation in patients with COPD

Rafael Mesquita, Kenneth Meijer, Fabio Pitta, Helena Azcuna, Yvonne M.J. Goërtz, Johannes M.N. Essers, Emiel F.M. Wouters, and Martijn A. Spruit

Respiratory Medicine 2017; 126: 122-129 


\title{
CHAPTER 8
}

\begin{abstract}
A more profound investigation about the responses in activity levels following pulmonary rehabilitation (PR) in patients with COPD is needed. We aimed to describe groups of patients with COPD according to patterns of change in physical activity and sedentary behaviour following PR.
\end{abstract}

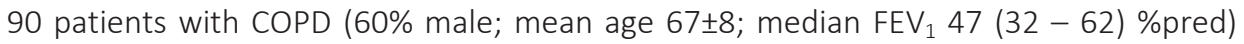
completed a comprehensive PR programme. A triaxial accelerometer was used to assess the time in sedentary behaviour, light activities and moderate-to-vigorous physical activity (MVPA). Additionally, exercise capacity, quality of life, and symptoms of anxiety and depression were assessed before and after PR.

Six groups with different patterns of change in physical activity and sedentary behaviour were identified. The two most prevalent patterns were represented by good responders (increase in physical activity and reduction in sedentary behaviour, 34\%) and poor responders (decrease in physical activity and increase in sedentary behaviour, 30\%). Good responders had greater improvements in six-minute walk distance (6MWD) and symptoms of depression than poor responders ( $\mathrm{P}<0.05$ for all). Strong correlation was found between changes in sedentary behaviour and changes in light activities $\left(r_{s}=-0.89\right.$; $P<0.0001)$. Changes in 6MWD correlated fairly with changes in sedentary behaviour $\left(r_{s}=-0.26\right)$, light activities $\left(r_{s}=0.25\right)$, and MVPA $\left(r_{s}=0.24\right) ; P<0.05$ for all.

Different patterns of change in activity levels following PR can be found in patients with COPD. Focusing on light physical activities might be a potential strategy to make patients less sedentary, but for this to be achieved prior (or at least parallel) improvements in functional capacity seem to be necessary. 


\section{INTRODUCTION}

Pulmonary rehabilitation (PR) generally reduces dyspnoea, increases exercise capacity and improves health status in patients with chronic obstructive pulmonary disease (COPD) [1]. Moreover, comprehensive PR has the great potential to increase daily physical activity [2]. Notwithstanding, the general impact of PR on physical activity levels has been shown to be very modest, if present at all $[3,4]$.

Recent literature has focused on the potential consequences of sedentary behaviour in COPD $[5,6]$, which is a concept distinct from physical activity. Sedentary behaviour can be defined as any waking behaviour characterised by an energy expenditure $\leq 1.5$ metabolic equivalents of task (METs) while in a sitting or reclining posture [7]. Although PR most probably can also contribute to a less sedentary lifestyle in patients with COPD, the impact of this intervention on measures of sedentary behaviour seems to be very modest, or even none. For example, Pitta et al. observed no changes in the time spent sitting or lying after a 3-month PR programme [8]. More recently, Egan et al. showed no reduction in the time spent sedentary after a 7-week PR programme [9].

The mean change in physical activity and sedentary behaviour following PR may not be significantly different compared to baseline. Then again, a broad range of change in physical activity and sedentary behaviour may exist amongst patients with COPD. This suggests that a subgroup of patients may increase physical activity levels and reduce sedentary behaviour. Similarly, a differential response following PR has been reported in patients with COPD for dyspnoea, exercise performance, problematic activities of daily life, mood status and health status [10]. Identifying and characterising groups with different patterns of change in physical activity and sedentary behaviour after PR may help clinicians to determine which groups benefit most from this intervention, as well as which groups could potentially benefit from additional therapies (e.g., behavioural intervention).

Therefore, the aim of this study was to characterise groups of patients with COPD according to their patterns of change in physical activity and sedentary behaviour after a comprehensive PR programme. In order to provide further insights on how to boost these changes, correlates of the changes were also investigated. 


\section{METHODS}

\section{Study design and participants}

In this retrospective observational study, patients with COPD being evaluated to participate in a comprehensive PR programme at CIRO, in Horn, the Netherlands, were screened for eligibility [11]. Inclusion criteria for the current analyses were: primary COPD diagnosis according to the Global Initiative for Chronic Obstructive Lung Disease (GOLD) [12]; complete physical activity assessment (please see below for details); and complete PR. Due to the use of de-identified pre-existing data, the current analyses are institutional review board exempt. The use of de-identified patients' records was approved by the Board of Directors of CIRO.

\section{Assessments}

As part of a 3-day pre-rehabilitation assessment performed at CIRO [11], patients were assessed concerning their demographics and clinical data, comorbidities, lung function, and body composition. As part of the initial assessment and of a 2-day postrehabilitation assessment, patients were also assessed concerning their exercise capacity (6-minute walk test (6MWT) [13] and constant work rate test (CWRT) [14]), quality of life (COPD-version of the St. George's Respiratory Questionnaire (SGRQ-C) [15]), and symptoms of anxiety and depression (Hospital Anxiety and Depression Scale (HADS) [16]). Details about these assessments can be found in the supplementary material. Immediately after the pre- and post-rehabilitation assessments, patients were sent home with an activity monitor for the assessment of physical activity and sedentary behaviour.

Either the CIRO activity monitor (CAM) or the MOX Activity Monitor (Maastricht Instruments B.V. in Maastricht, the Netherlands) were used to assess physical activity and sedentary behaviour during free-living conditions. The latter represents an upgraded version of the former. Both are lightweight, triaxial accelerometer devices which have shown to be valid in COPD $[17,18]$ and the results are interchangeable. Patients had the device attached to the frontal part of their right thigh and were instructed to keep it for at least 7 days. A valid activity levels assessment was defined as at least 5 days of assessment (3 weekdays + Saturday + Sunday) [19], each with at least $10 \mathrm{~h}$ of measurement. Data collected during waking hours were used for analysis. The average value of all valid assessment days was used to calculate each outcome measures: time in sedentary behaviour (<1.5 METs) [7], time in light activities (1.5-3.0 METs), and time in moderate-to-vigorous physical activity (MVPA, >3.0 METs). Moreover, characteristics of 10min bouts of activities in MVPA were estimated. In order to identify patterns of change following PR, patients were classified into groups according to the change (i.e., any 
increase or decrease) in sedentary behaviour, light activities, and MVPA. A reduction in the time spent sedentary or an increase in the time in light activities or in MVPA was classified as a good response, while any changes in the opposite directions were classified as a poor response. Detailed information about the assessment of physical activity and sedentary behaviour can be found online.

\section{Pulmonary rehabilitation programme}

All patients were enrolled in a comprehensive PR programme, as previously described [20]. Patients attended a 40-session programme which consisted, in brief, of highintensity progressive exercise training (i.e., endurance and strength training); nutritional support, occupational therapy and psychological counselling, if indicated; and 20 1-hour educational group sessions. The programme was implemented by an interdisciplinary team including chest physician, respiratory nurses, dietician, occupational therapist, physiotherapist, psychologist and social worker.

\section{Statistical analysis}

Data were expressed as relative frequency, mean \pm SD, mean (95\% confidence intervals, $\mathrm{CI}$ ), or median (interquartile range, IQR). The assumption of normality was tested by the Shapiro-Wilk test. Between-group comparisons were performed with unpaired t test or Mann-Whitney test (one-way ANOVA or Kruskal-Wallis, if more than 2 groups), whilst within-group comparisons were performed with paired t test or Wilcoxon test. Categorical variables were tested using Chi-squared test or Fisher exact test. Spearman coefficient was used to investigate correlations. Statistical analyses were performed using SPSS 22.0 (IBM, Armonk, NY, USA) or GraphPad Prism 6 (GraphPad Software, La Jolla, California, USA). A P-value $<0.05$ was considered significant.

\section{RESULTS}

\section{Baseline characteristics}

Ninety COPD patients assessed between March 2011 and November 2014 fulfilled all inclusion criteria and were included in the current analysis (Table 1). A flow-diagram shows the number of patients screened and excluded (e-Figure 1, supplementary material). Most patients included in the current study were male, former smokers, had at least 2 exacerbations in the previous last year, were in GOLD grade 2 or 3 , and belonged to GOLD group $D$ at baseline. Less than one third of the patients were on long-term oxygen therapy and hyperglycaemia, dyslipidaemia and low muscle mass were the most prevalent comorbidities. 


\section{CHAPTER 8}

Regarding the pre-rehabilitation assessment of physical activity and sedentary behaviour, a total of 533 valid days (353 weekdays) were analysed and the mean \pm SD number of valid days was $5.9 \pm 0.3$ days. On average, patients spent the vast majority of their time in sedentary behaviour (Table 2). Moreover, patients showed impaired exercise capacity, and poor health and mood status.

Table 1 Baseline general characteristics

\begin{tabular}{|c|c|c|}
\hline Characteristic & $\begin{array}{c}\text { Patients available for } \\
\text { analysis }(n)\end{array}$ & Value \\
\hline Male sex (\%) & 90 & 60 \\
\hline Age (years) & 90 & $67 \pm 8$ \\
\hline BMI $\left(\mathrm{kg} \cdot \mathrm{m}^{-2}\right)$ & 90 & $25.7(22.1-28.5)$ \\
\hline FFMI $\left(\mathrm{kg} \cdot \mathrm{m}^{-2}\right)$ & 88 & $17.1 \pm 2.4$ \\
\hline mMRC (points) & 90 & $2(2-3)$ \\
\hline Smoking status N/F/C (\%) & 88 & $6 / 78 / 16$ \\
\hline Pack-years & 75 & $40(30-50)$ \\
\hline Long-term oxygen therapy (\%) & 80 & 27 \\
\hline$\geq 2$ exacerbations in the previous 12 months (\%) & 80 & 59 \\
\hline $\mathrm{FEV}_{1}(\%$ predicted) & 90 & $47(32-62)$ \\
\hline $\mathrm{FEV}_{1} / \mathrm{FVC}(\%)$ & 90 & $36(28-44)$ \\
\hline RV (\% predicted) & 88 & $146(112-185)$ \\
\hline TLC (\% predicted) & 88 & $115 \pm 17$ \\
\hline RV/TLC (\%) & 88 & $50 \pm 12$ \\
\hline $\mathrm{D}_{\mathrm{LCO}}(\%$ predicted $)$ & 80 & $47(39-56)$ \\
\hline GOLD 1/2/3/4 (\%) & 90 & $12 / 31 / 37 / 20$ \\
\hline GOLD A/B/C/D (\%) & 90 & $9 / 21 / 3 / 67$ \\
\hline Updated BODE (points) & 90 & $3(2-6)$ \\
\hline \multicolumn{3}{|l|}{ Objectively identified comorbidities (\%) } \\
\hline Anaemia & 90 & 11 \\
\hline Dyslipidaemia & 90 & 40 \\
\hline Hyperglycaemia & 90 & 66 \\
\hline Renal impairment & 90 & 32 \\
\hline Symptoms of anxiety & 88 & 34 \\
\hline Symptoms of depression & 88 & 23 \\
\hline Underweight & 90 & 22 \\
\hline Obesity & 90 & 16 \\
\hline Low muscle mass & 88 & 43 \\
\hline
\end{tabular}

Data expressed relative frequency, mean \pm SD or median (IQR). BMI = body mass index; FFMI = fat-free mass index; $\mathrm{mMRC}=$ modified Medical Research Council; $\mathrm{N}=$ never smoker; $\mathrm{F}=$ former smoker; $\mathrm{C}=$ current smoker; $F_{E V}=$ forced expiratory volume in the first second; $F V C=$ forced vital capacity; $R V=$ residual volume; $T L C=$ total lung capacity; $D_{\mathrm{LCO}}=$ diffusing capacity for carbon monoxide; GOLD = Global Initiative for Chronic Obstructive Lung Disease; BODE = body mass index, airflow obstruction, dyspnoea, and exercise capacity index. 
Impact of pulmonary rehabilitation

Significant improvements in exercise capacity after PR were demonstrated by a longer 6-minute walk distance (6MWD) and CWRT time (Table 2). Moreover, 46\% and $56 \%$ of patients showed a clinically relevant improvement in 6MWD (i.e., $\geq 30 \mathrm{~m}$ ) [13] and CWRT time (i.e., $\geq 100 \mathrm{~s}$ ) [21], respectively. A significant improvement in quality of life was observed in the SGRQ-C total score, and $67 \%$ of patients showed a clinically relevant improvement (i.e., $\geq-4$ points) [22]. Significant improvements were also seen in symptoms of anxiety and depression, and the proportion of patients with a clinically relevant improvement (i.e., $\geq-1.5$ points) [23] was $61 \%$ and $60 \%$, respectively.

On average, no significant change was observed in any of the physical activity or sedentary behaviour measures following PR (Table 2; e-Figure 2, supplementary material). Baseline seasonal variation did not influence these results (e-Table 1). 


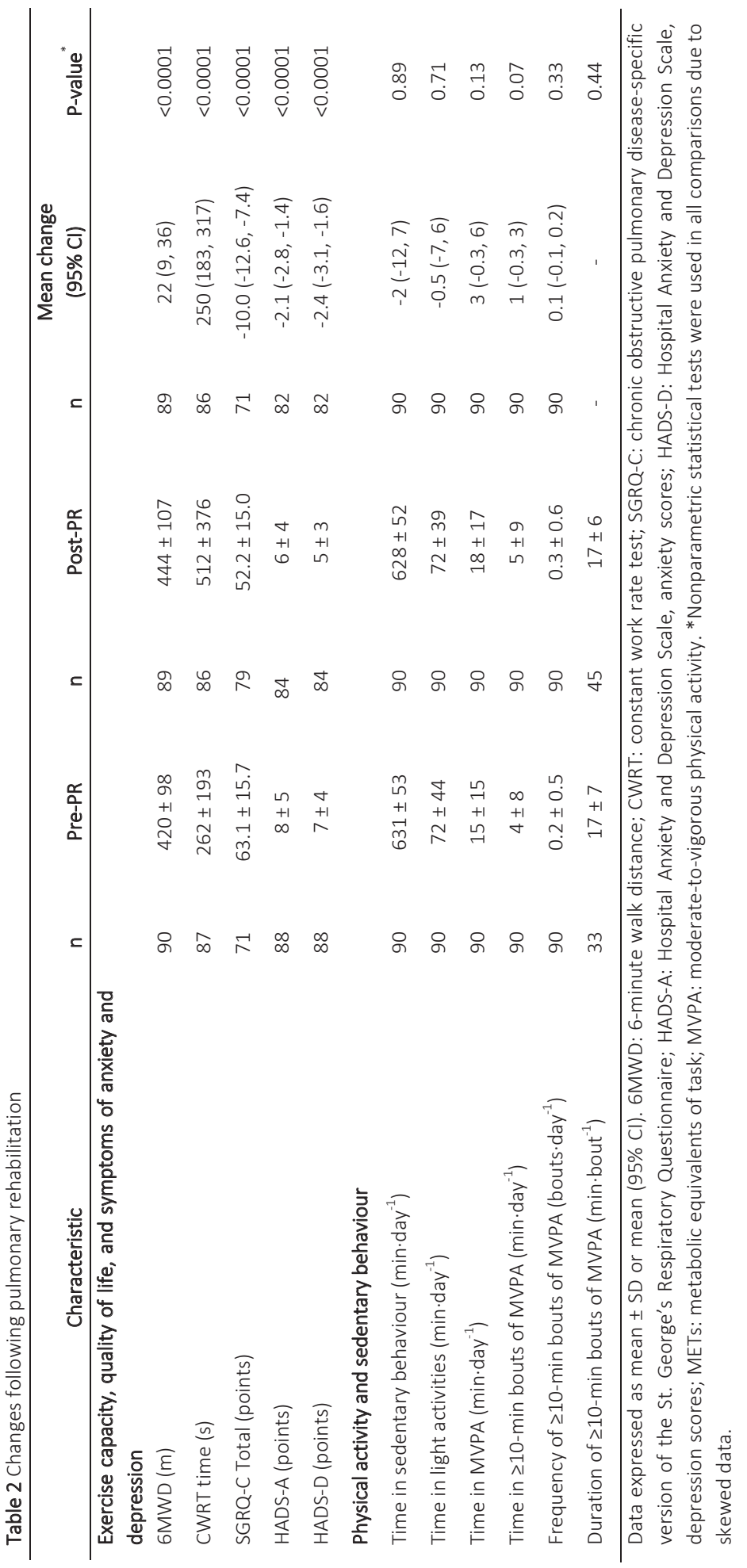




\section{Correlates of changes in sedentary behaviour and physical activity}

Table 3 presents the significant correlates of changes in sedentary behaviour, light activities and MVPA. All correlates of these changes (significant and non-significant ones) are presented in e-Table 2 (supplementary material). Changes in sedentary behaviour correlated fairly with baseline diffusing capacity for carbon monoxide $\left(D_{L C O}\right)$, updated body mass index, airflow obstruction, dyspnoea, and exercise capacity (BODE) index scores, time in sedentary behaviour, time in light activities, and time in MVPA. Little-to-fair correlations were found between changes in light activities and baseline body mass index (BMI), modified Medical Research Council (mMRC) scores, residual volume per total lung capacity ratio (RV/TLC), $D_{L C O}$, updated BODE scores, 6MWD, time in sedentary behaviour, time in light activities, and time in MVPA. Changes in MVPA correlated (negatively) only with baseline time in MVPA. There were no significant correlations between changes in sedentary behaviour, light activities or MVPA and other baseline characteristics (e-Table 2). Strong inverse correlations were found between changes in sedentary behaviour and changes in light activities (Figure 1A) and MPVA (Figure 1B), while changes in light activities correlated only fairly with changes in MVPA. There were fair correlations between changes in 6MWD and changes in sedentary behaviour, light activities and MVPA; in fact, changes in 6MWD was the only parameter to correlate with these three outcomes (e-Figure 3, supplementary material). When compared to patients with a change in $6 \mathrm{MWD}<30 \mathrm{~m}$, those with a clinically important change (i.e., $\geq 30 \mathrm{~m}$ [13]) more frequently decreased their time in sedentary behaviour (63\% vs 38\%, $\mathrm{P}=0.015)$ and increased their time in MVPA (66\% vs. 42\%, $\mathrm{P}=0.023)$. 


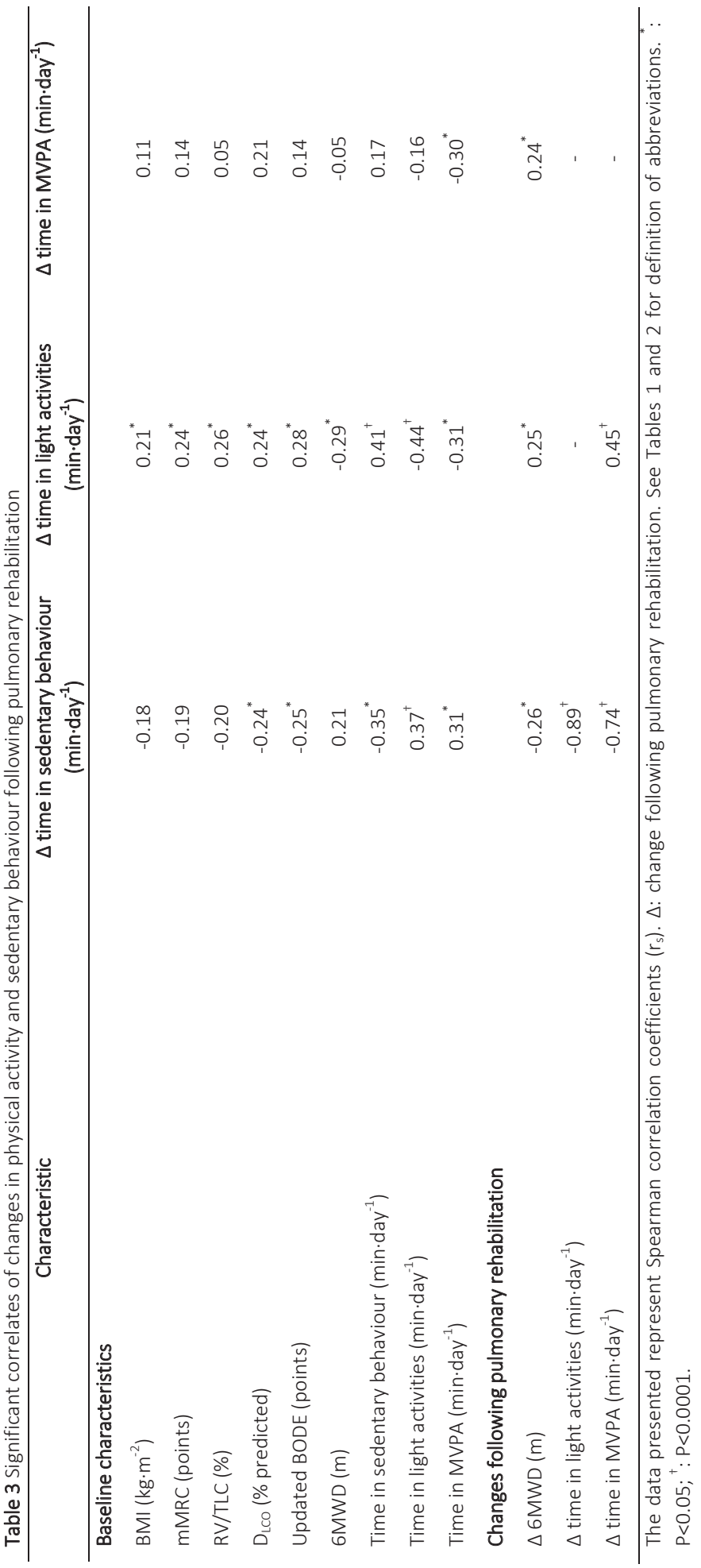



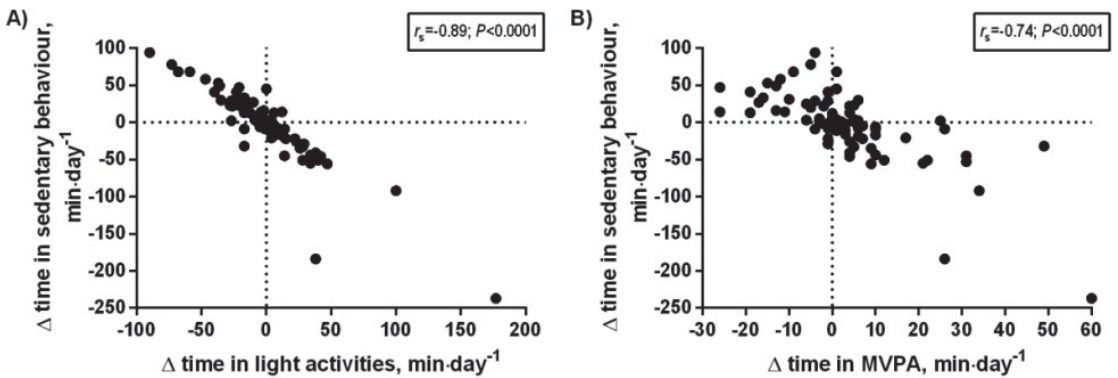

Figure 1 Correlations between changes $(\Delta)$ in sedentary behaviour and $\Delta$ in light activities $(A)$ and $\Delta$ in sedentary behaviour and $\Delta$ in moderate-to-vigorous physical activity (MVPA) (B) following pulmonary rehabilitation. Dotted lines correspond to the point of no change in each intensity.

\section{Patterns of change in physical activity and sedentary behaviour}

Based on changes in sedentary behaviour, light activities, and MVPA following PR, patients were classified into 6 groups: 1) poor responders (30\%); 2) poor responders with increase in light activities (6\%); 3) poor responders with increase in MVPA (14\%); 4) good responders (34\%); 5) good responders with decrease in light activities (7\%); and 6) good responders with decrease in MVPA (9\%) (Figure 2). Table 4 presents the characteristics of poor responders and good responders. Characteristics of other groups were not presented due to the small sample size. Baseline sex distribution, age, degree of airflow limitation, and Updated BODE index scores were comparable between poor responders and good responders. In contrast, good responders had higher BMI and lower time in MVPA at baseline. Greater improvements in 6MWD, HADS depression scores, sedentary behaviour, light activities, and MVPA following PR were found in good responders compared to poor responders. Also, cycle endurance time, health status and symptoms of anxiety tended to improve to a greater extent in good responders ( $P \leq 0.07$ for all). The behaviour of poor responders and good responders in terms of the changes in sedentary behaviour, light activities, and MVPA following PR can be found in Figure 3. 


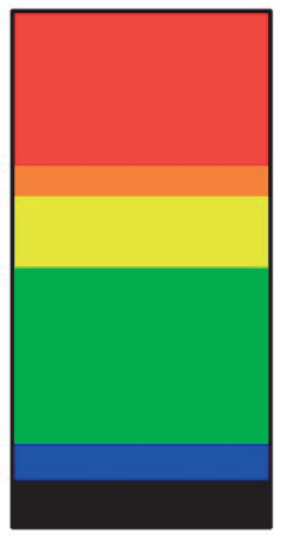

$\square$ Poor responders: increase in sedentary behaviour, decrease in light activities, and decrease in MVPA

Poor responders with increase in light activities: increase in sedentary behaviour, increase in light activities, and decrease in MVPA

$\square$ Poor responders with increase in MVPA: increase in sedentary behaviour, decrease in light activities, and increase in MVPA
$\square$ Good responders: reduction in sedentary behaviour, increase in light activities, and increase in MVPA

$\square$ Good responders with decrease in light activities: reduction in sedentary behaviour, decrease in light activities, and increase in MVPA

Good responders with decrease in MVPA: reduction in sedentary behaviour, increase in light activities, and decrease in MVPA

Figure 2 Proportion of COPD patients according to the pattern of change in physical activity and sedentary behaviour following pulmonary rehabilitation. MVPA: moderate-to-vigorous physical activity.

A)

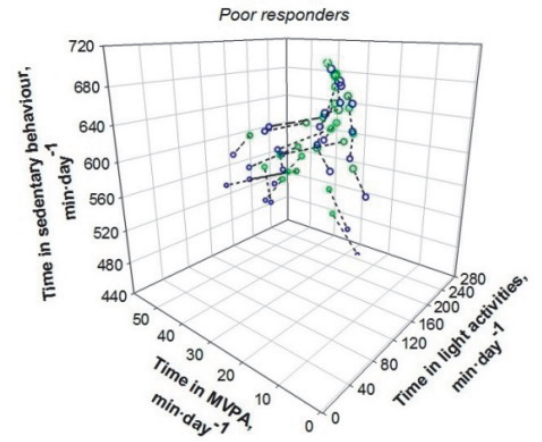

B)

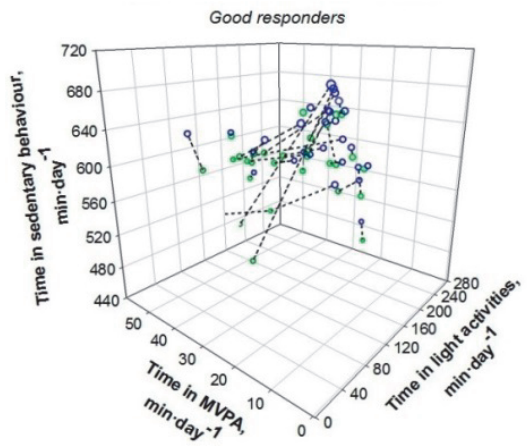

Figure 3 Changes in sedentary behaviour, light activities, and moderate-to-vigorous (MVPA) in poor responders (i.e., increase in sedentary behaviour, decrease in light activities, and decrease in MVPA; Figure A) and good responders (i.e., decrease in sedentary behaviour, increase in light activities, and increase in MVPA; Figure B) following pulmonary rehabilitation. Blue dots represent pre-rehabilitation values and green dots represent post-rehabilitation values. 


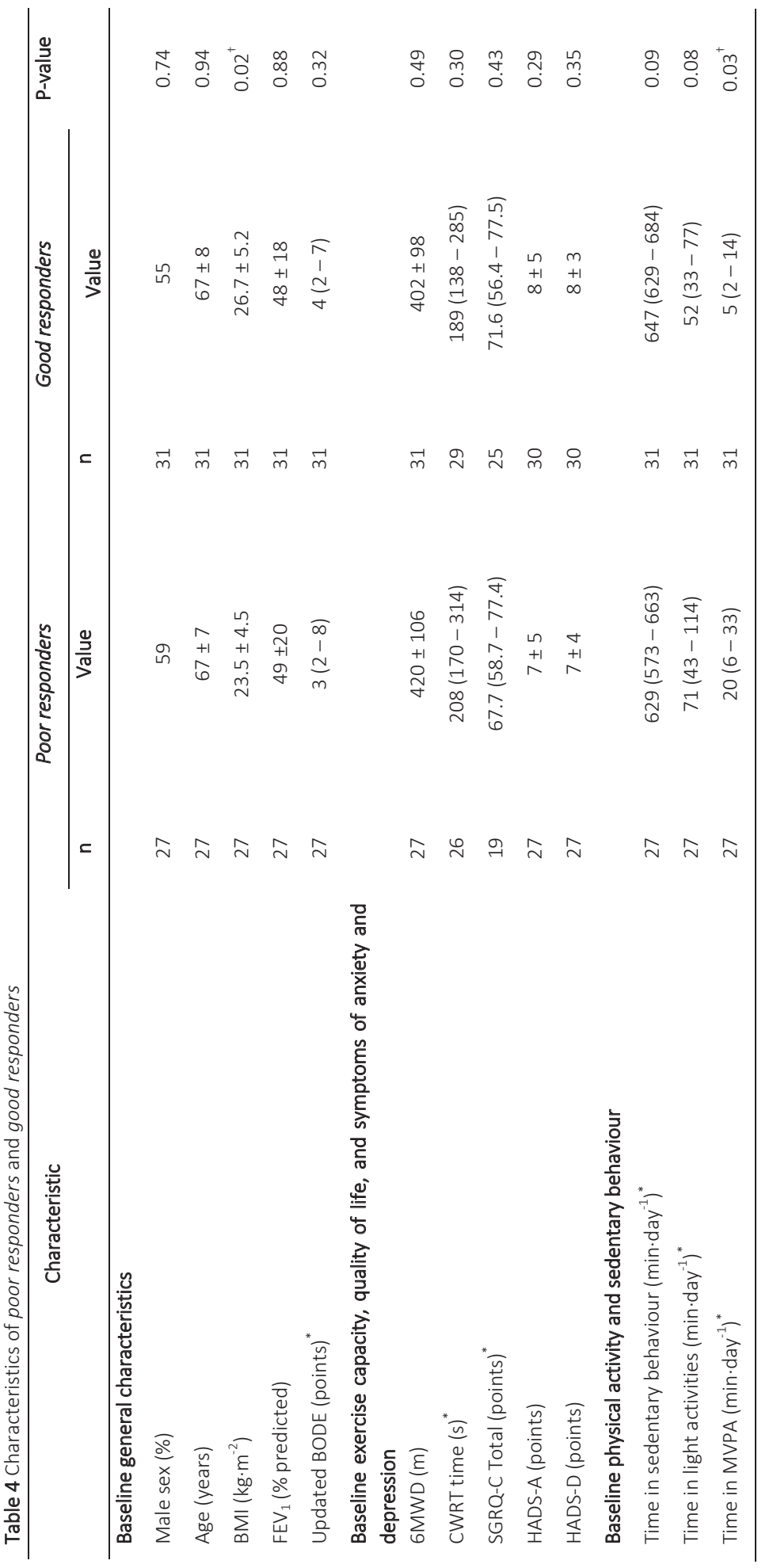




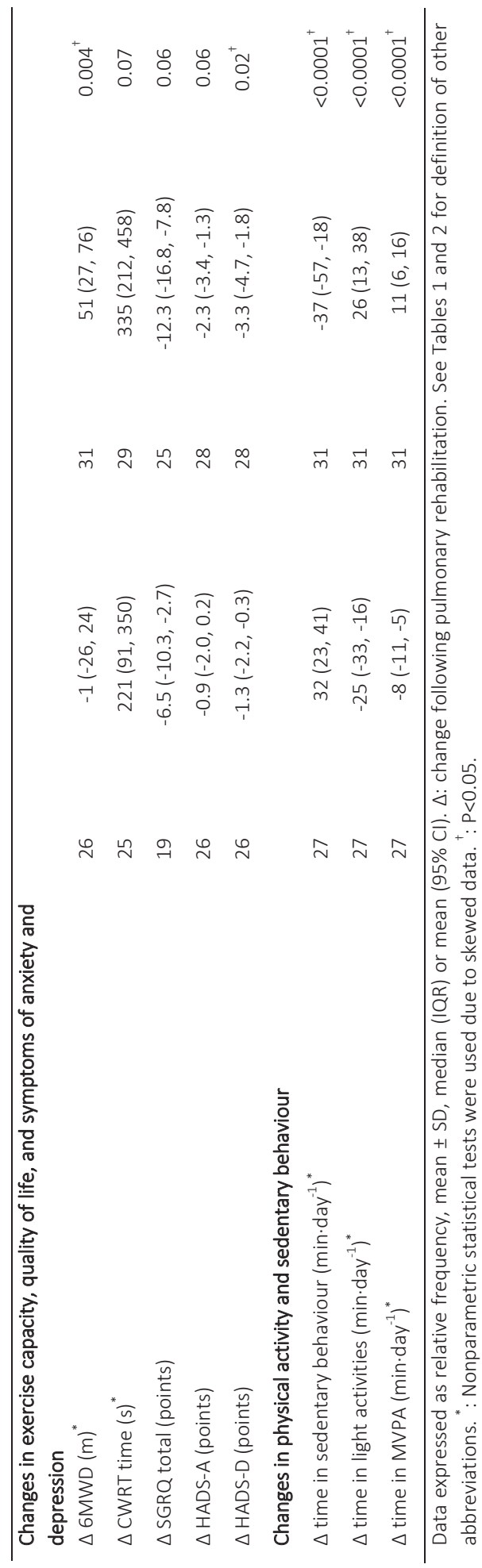




\section{DISCUSSION}

The present study corroborates previous studies by showing that, on average, sedentary behaviour and physical activity do not change following PR in patients with COPD. However, the response on these health outcomes is very heterogeneous, resulting in subgroups of patients with different patterns of change. While around $1 / 3$ of patients decreased their time in sedentary behaviour and increased their time in light activities and MVPA (i.e., good responders), a bit less than $1 / 3$ of patients had changes in the opposite direction (i.e., poor responders). Moreover, when analysing the whole sample we also observed that the strongest correlation was found between changes in sedentary behaviour and changes in light activities, but these changes also correlated with changes in 6MWD.

Over the last few decades the amount of research on the impact of interventions on activity levels in patients with COPD has increased considerably [4]. Nonetheless, much of this research has focused mainly on activities of at least moderate intensity [24-26], while our results clearly show that simplified activity monitoring focusing on MVPA alone may lead to an incomplete or incorrect picture of the impact of an intervention. While one patient may show a positive change in one intensity of activity following PR, the same patient may show negative changes in other intensities. This is exemplified in the study by Ramadi and co-workers [27]. These authors observed positive changes in sedentary time and light activities after an exercise rehabilitation programme in patients with cardiopulmonary diseases, but no significant changes were observed in the time in MVPA. Focusing on one intensity of activity alone may lead to oversimplification of reality and should therefore be avoided.

Overall, PR is thought to provide only modest improvements in physical activity in patients with COPD [4]. Nevertheless, a subgroup of patients (i.e., good responders) is actually able to show important improvements after a comprehensive PR programme. These patients showed a mean $(95 \% \mathrm{Cl})$ increase in light activities of $26(13,38)$ min.day ${ }^{-1}$ and in MVPA of $11(6,16)$ min.day ${ }^{-1}$ compared to baseline, which were accomplished through a reduction in sedentary behaviours of $-37(-57,-18)$ min day $^{-1}$. Recently, a reduction in sedentarism has gained attention in the COPD literature [2, 5, 6]. Patients with COPD spend the vast majority of their waking time in activities of very low energy expenditure or sedentary behaviours. We recently showed in a multinational sample of COPD patients that more than 13 hours of waking hours is spent in very light activities [28]. Evidence from epidemiological studies suggests that engaging in moderate-to-vigorous intensity physical activity may not be enough to fully protect against the detrimental consequences of prolonged periods of sedentary behaviour [29, 
30], which suggests that researchers and clinicians should also target reductions in sedentary behaviour.

A potential way to reduce the time spent sedentary in patients with COPD is by increasing the time in light activities. Indeed, around $70 \%$ of the reduction in sedentary time shown by good responders after PR was reallocated to light activities. Reductions in sedentary time by increases in light activities seem to be more easily achievable for patients with COPD than increases in MVPA [2]. A very strong correlation was found between changes in sedentary behaviour and changes in light activities in the whole sample $\left(r_{s}=-0.89\right)$, which was in fact stronger than the correlation with changes in MVPA $\left(r_{s}=-0.74\right)$. This suggests that it might be easier for patients to increase light activities than to increase MVPA. The increase in light activities can provide the basis for an active living [2]. This concept includes leisure, occupational, and household activities, as well as active transportation (walking and bicycling) [31]. For example, a more active living could be achieved by engaging in more household activities.

While around one third of patients improved after PR (good responders, 34\%), a slightly lower amount deteriorated instead (poor responders, 30\%). Patients who responded poorly showed, amongst other characteristics, considerably poorer changes in functional exercise capacity (i.e., 6MWD) compared to those who had a good response (Table 4). It seems that improvements in functional capacity are necessary to occur in order to allow for improvements in physical activity [32]. This is supported by the fact that good responders, on the other hand, showed a large improvement in 6MWD. Moreover, changes in 6MWD correlated significantly with changes in sedentary behaviour, light activities, and MVPA in the whole group, and patients with a change in 6MWD $<30 \mathrm{~m}$ [13] less frequently decreased their sedentary behaviour (38\% vs $63 \%, P=0.015$ ) and increased their MVPA (42\% vs 66\%, P=0.023). Just like patients have their lung function optimised with medical treatment before exercise training, maybe we need to optimise their functional capacity before (or at least at the same time) we aim at improvements in activity levels.

Some patients might find it easier to increase their time in light activities than their time in MVPA, as discussed earlier. Therefore, increasing the time in light activities specifically could be included as an aim of PR, especially in more deconditioned individuals. This is supported by the fact that good responders spent a lower baseline time in MVPA compared to poor responders (Table 4). Moreover, focusing on light activities is also an interesting option from a statistical point of view, since this outcome is more responsive than the time in MVPA [33]. Improving the physical condition of patients and promoting adherence to health-enhancing behaviours, including physical activity, are targets of PR [1]. Although previous studies have shown that PR is indeed able to improve the patient's physical condition, by showing improvements in functional capacity [34], not all 
studies have shown whether these improvements translate into increased physical activity [2]. Different factors have been suggested to explain this finding, such as the content and/or the duration of the PR programmes [2]. Nevertheless, our results suggest that maybe the outcome of study should also be considered. Increasing the patient's functional capacity automatically increases his/her functional reserve, which seems to be necessary to allow the patient to increase his/her functional performance (i.e., physical activity levels) [32].

Despite the comprehensive approach commonly used in PR [1], a poor response to this intervention is not uncommon [10]. Different factors may help explain the worsening showed by poor responders in our study. As suggested by Spruit et al. [10], it may be that a poor response to rehabilitation in a subgroup of patients is not COPD-specific and/or rehabilitation-specific. Poor responses can also be found after specialised rehabilitative interventions in other populations $[35,36]$ or in patients with COPD after interventions other than PR $[37,38]$. Regression towards the mean can also be thought as a possible explanation. However, the values from each assessment (i.e., pre- and postrehabilitation) were averaged among at least 5 days, and we believe this might have brought these values closer to the true mean of the sample. Other possible explanations may include poor motivation or exacerbations during the PR programme. Moreover, it cannot be neglected that maybe poor responders are doing less physical activity, but are doing it with less symptoms and/or more efficiently (i.e., with less energy expenditure). An important component of the PR programme performed at CIRO is the use of energy conservation techniques [11], which focuses on avoiding energy expenditure waste during activities of daily living. It is also part of this programme to introduce patients to activities such as outside walking, swimming and Nordic walking. It can also be that the patients did not have time to incorporate these activities into their daily life, as physical activity and sedentary behaviour were measured immediately after the PR programme. Finally, it can also be that being more physically active was not a personal goal by these patients [39]. Irrespective of the reasons why poor responders did not improve following a comprehensive $\mathrm{PR}$, recent literature suggests that some specific strategies and/or factors could be considered as when trying to make these patients less sedentary and/or more physically active. For example, our group has recently shown that patients with COPD who have a physically active loved one have a higher likelihood of being physically active themselves [40]. Still related to socialenvironmental factors, Arbillaga-Etxarri et al. [41] recently observed that dog walking and grandparenting are associated with a higher amount and intensity of physical activity in patients with COPD. In another recent study it was shown that patients with COPD who sleep better (e.g., non-fragmented sleep) are more physically active in the following day [42]. Finally, in a large multicentre randomised controlled trial, Demeyer and coworkers [43] have shown that the amount and intensity of physical activity can be 
significantly increased in patients with COPD using a 12-week semiautomated telecoaching intervention when compared to the usual care group.

Intriguingly, good responders had higher BMI and lower MVPA time at baseline compared to poor responders. However, results from previous studies may help to explain these findings. It is important to mention that good responders had a BMI of $26.7 \pm 5.2$ $\mathrm{kg} \cdot \mathrm{m}^{-2}$, which is compatible with overweight. O'Donnell and co-workers [44] have shown that an overweight BMI can bring some physiologic advantages to patients with airflow limitation, by reducing static lung hyperinflation and improving inspiratory capacity. Moreover, our results corroborate results of previous studies. For example, Spruit et al. [10] investigated groups of patients with COPD with distinct multidimensional responses to PR. Similarly, these authors observed that the group with the best response to PR (i.e., very good responders) had an overweight BMI $\left(26.3 \pm 5.6 \mathrm{~kg} \cdot \mathrm{m}^{-2}\right)$ at baseline, which was actually higher than the BMI of moderate and poor responders. Interestingly, very good responders had also a higher fat-free mass index, which might also help to explain their best performance. Unfortunately, activity levels were not investigated in their study. Regarding the low baseline MVPA time, we believe this was necessary to allow good responders to improve more, as there was more room for improvement. Likewise, in the study by Spruit et al. [10] very good responders had also the worst exercise performance at baseline, but showed the largest improvement. Finally, we cannot exclude the fact that maybe social (e.g., low socioeconomic status) or psychological (e.g., low self-efficacy) barriers have played a role.

\section{Methodological considerations}

A limited amount of patients was found in groups other than poor responders and good responders, which compromised a better characterisation of these groups. As activity monitoring was restricted to the leg, activities of the upper arms were probably underrepresented. Nevertheless, previous findings suggest that leg activities seem to be more impaired than arm activities in COPD $[45,46]$. Self-efficacy was not assessed in the current study. Noneevertheless, an improvement in self-efficacy, which has been reported following PR [47], may be a pre-requisite to change physical activity, reduce sedentarism and induce an active living [2]. Finally, it would be important to demonstrate that increased physical activity following PR is associated with improved outcomes, such as a reduction in health care resources utilisation. However, follow-up assessments were not part of our study design, therefore we were not able to address this question. 


\section{CONCLUSIONS}

In conclusion, one third of COPD patients are able to show improvements in physical activity and sedentary behaviour after a comprehensive PR programme. A potential strategy to make this group less sedentary and more physically active may be focusing on light rather than moderate-to-vigorous physical activities. Nevertheless, for this to be achieved prior (or at least parallel) improvements in functional capacity seem to be necessary. 


\section{REFERENCES}

1 M.A. Spruit, S.J. Singh, C. Garvey, R. Zuwallack, L. Nici, C. Rochester, K. Hill, A.E. Holland, S.C. Lareau, W.D. Man, et al., An official american thoracic society/european respiratory society statement: key concepts and advances in pulmonary rehabilitation, Am. J. Respir. Crit. Care Med. 188 (2013) e13e64.

2 M.A. Spruit, F. Pitta, E. McAuley, R.L. ZuWallack, L. Nici, Pulmonary rehabilitation and physical activity in patients with chronic obstructive pulmonary disease, Am. J. Respir. Crit. Care Med. 192 (2015) 924 e933.

3 H. Watz, F. Pitta, C.L. Rochester, J. Garcia-Aymerich, R. ZuWallack, T. Troosters, A.W. Vaes, M.A. Puhan, M. Jehn, M.I. Polkey, et al., An official European Respiratory Society statement on physical activity in COPD, Eur. Respir. J. 44 (2014) 1521e1537.

4 L.C. Mantoani, N. Rubio, B. McKinstry, W. MacNee, R.A. Rabinovich, Interventions to modify physical activity in patients with COPD: a systematic review, Eur. Respir. J. 48 (2016) 69e81.

5 V. Cavalheri, L. Straker, D.F. Gucciardi, P.A. Gardiner, K. Hill, Changing physical activity and sedentary behaviour in people with COPD, Respirology 21 (2016) 419e426.

6 K. Hill, P.A. Gardiner, V. Cavalheri, S.C. Jenkins, G.N. Healy, Physical activity and sedentary behaviour: applying lessons to chronic obstructive pulmonary disease, Intern Med. J. 45 (2015) 474e482.

7 Sedentary Behaviour Research N, Letter to the editor: standardized use of the terms "sedentary" and "sedentary behaviours", Appl. Physiol. Nutr. Metab. 37 (2012) 540e542.

8 F. Pitta, T. Troosters, V.S. Probst, D. Langer, M. Decramer, R. Gosselink, Are patients with COPD more active after pulmonary rehabilitation? Chest 134 (2008) $273 \mathrm{e} 280$.

9 C. Egan, B.M. Deering, C. Blake, B.M. Fullen, N.M. McCormack, M.A. Spruit, R.W. Costello, Short term and long term effects of pulmonary rehabilitation on physical activity in COPD, Respir. Med. 106 (2012) $1671 \mathrm{e} 1679$.

10 M.A. Spruit, I.M. Augustin, L.E. Vanfleteren, D.J. Janssen, S. Gaffron, H.J. Pennings, F. Smeenk, W. Pieters, J.J. van den Bergh, A.J. Michels, et al., Differential response to pulmonary rehabilitation in COPD: multidimensional profiling, Eur. Respir. J. 46 (2015) 1625e1635.

11 M.A. Spruit, I. Vanderhoven-Augustin, P.P. Janssen, E.F. Wouters, Integration of pulmonary rehabilitation in COPD, Lancet 371 (2008) 12e13.

12 J. Vestbo, S.S. Hurd, A.G. Agusti, P.W. Jones, C. Vogelmeier, A. Anzueto, P.J. Barnes, L.M. Fabbri, F.J. Martinez, M. Nishimura, et al., Global strategy for the diagnosis, management, and prevention of chronic obstructive pulmonary disease: GOLD executive summary, Am. J. Respir. Crit. Care Med. 187 (2013) 347 e365.

13 A.E. Holland, M.A. Spruit, T. Troosters, M.A. Puhan, V. Pepin, D. Saey, M.C. McCormack, B.W. Carlin, F.C. Sciurba, F. Pitta, et al., An official European Respiratory Society/American Thoracic Society Technical Standard: field walking tests in chronic respiratory disease, Eur. Respir. J. 44 (2014) 1428 e1446.

14 A. van 't Hul, R. Gosselink, G. Kwakkel, Constant-load cycle endurance performance: test-retest reliability and validity in patients with COPD, J. Cardiopulm. Rehabil. 23 (2003) 143e150.

15 M. Meguro, E.A. Barley, S. Spencer, P.W. Jones, Development and validation of an improved, COPDspecific version of the St. George respiratory questionnaire, Chest 132 (2007) 456e463.

16 A.S. Zigmond, R.P. Snaith, The hospital anxiety and depression scale, Acta Psychiatr. Scand. 67 (1983) $361 \mathrm{e} 370$.

17 J. Annegarn, M.A. Spruit, N.H. Uszko-Lencer, S. Vanbelle, H.H. Savelberg, A.M. Schols, E.F. Wouters, K. Meijer, Objective physical activity assessment in patients with chronic organ failure: a validation study of a new single-unit activity monitor, Arch. Phys. Med. Rehabil. 92 (2011), 1852e1857 e1851.

18 S. van der Weegen, H. Essers, M. Spreeuwenberg, R. Verwey, H. Tange, L. de Witte, K. Meijer, Concurrent validity of the MOX activity monitor compared to the ActiGraph GT3X, Telemed. J. E Health 21 (2015) $259 e 266$.

19 H. Watz, B. Waschki, T. Meyer, H. Magnussen, Physical activity in patients with COPD, Eur. Respir. J. 33 (2009) 262e272. 
20 R. Mesquita, S. Wilke, D.E. Smid, D.J. Janssen, F.M. Franssen, V.S. Probst, E.F. Wouters, J.W. Muris, F. Pitta, M.A. Spruit, Measurement properties of the Timed up \& Go test in patients with COPD, Chron. Respir. Dis. 13 (2016) 344e352.

21 L. Laviolette, J. Bourbeau, S. Bernard, Y. Lacasse, V. Pepin, M.J. Breton, M. Baltzan, M. Rouleau, F. Maltais, Assessing the impact of pulmonary rehabilitation on functional status in COPD, Thorax 63 (2008) $115 \mathrm{e} 121$.

22 P.W. Jones, St. George's respiratory questionnaire: MCID, COPD 2 (2005) 75e79.

23 M.A. Puhan, M. Frey, S. Buchi, H.J. Schunemann, The minimal important difference of the hospital anxiety and depression scale in patients with chronic obstructive pulmonary disease, Health Qual. Life Outcomes 6 (2008) 46.

24 T. Troosters, F.C. Sciurba, M. Decramer, N.M. Siafakas, S.S. Klioze, S.C. Sutradhar, I.M. Weisman, C. Yunis, Tiotropium in patients with moderate COPD naive to maintenance therapy: a randomised placebocontrolled trial, NPJ Prim. Care Respir. Med. 24 (2014) 14003.

25 V.S. Probst, D. Kovelis, N.A. Hernandes, C.A. Camillo, V. Cavalheri, F. Pitta, Effects of 2 exercise training programs on physical activity in daily life in patients with COPD, Respir. Care 56 (2011) 1799e1807.

26 H. Watz, F. Krippner, A. Kirsten, H. Magnussen, C. Vogelmeier, Indacaterol improves lung hyperinflation and physical activity in patients with moderate chronic obstructive pulmonary diseaseea randomized, multicenter, doubleblind, placebo-controlled study, BMC Pulm. Med. 14 (2014) 158.

27 A. Ramadi, M.K. Stickland, W.M. Rodgers, R.G. Haennel, Impact of supervised exercise rehabilitation on daily physical activity of cardiopulmonary patients, Heart Lung 44 (2015) 9e14.

28 R. Mesquita, G. Spina, F. Pitta, D. Donaire-Gonzalez, B.M. Deering, M.S. Patel, K.E. Mitchell, J. Alison, A.J.R. van Gestel, S. Zogg, et al., Physical activity patterns and clusters in 1001 patients with COPD, Chron. Respir. Dis. (2016) [Epub ahead of print]

29 G.N. Healy, D.W. Dunstan, J. Salmon, J.E. Shaw, P.Z. Zimmet, N. Owen, Television time and continuous metabolic risk in physically active adults, Med. Sci. Sports Exerc. 40 (2008) 639e645.

30 G.N. Healy, C.E. Matthews, D.W. Dunstan, E.A. Winkler, N. Owen, Sedentary time and cardio-metabolic biomarkers in US adults: NHANES 2003-06, Eur. Heart J. 32 (2011) 590e597.

31 J.F. Sallis, L.S. Linton, M.K. Kraft, C.L. Cutter, J. Kerr, J. Weitzel, A. Wilson, C. Spoon, I.D. Harrison, R. Cervero, et al., The Active Living Research program: six years of grantmaking, Am. J. Prev. Med. 36 (2009) S10es21.

32 D. Langer, H. Demeyer, T. Troosters, R. Gosselink, The importance of physical activity, in: A. Anzueto, Y. Heijdra, J.R. Hurst (Eds.), Controversies in COPD, vol. 69, 2015, pp. 224e239 (ERS Monograph).

33 H. Demeyer, C. Burtin, H. Van Remoortel, M. Hornikx, D. Langer, M. Decramer, R. Gosselink, W. Janssens, T. Troosters, Standardizing the analysis of physical activity in patients with COPD following a pulmonary rehabilitation program, Chest 146 (2014) 318 e327.

34 B. McCarthy, D. Casey, D. Devane, K. Murphy, E. Murphy, Y. Lacasse, Pulmonary rehabilitation for chronic obstructive pulmonary disease, Cochrane Database Syst. Rev. (2) (2015). CD003793.

35 J.P. Schmid, M. Zurek, H. Saner, Chronotropic incompetence predicts impaired response to exercise training in heart failure patients with sinus rhythm, Eur. J. Prev. Cardiol. 20 (2013) 585e592.

36 F. Cecchi, G. Pasquini, A. Paperini, R. Boni, C. Castagnoli, S. Pistritto, C. Macchi, Predictors of response to exercise therapy for chronic low back pain: result of a prospective study with one year follow-up, Eur. J. Phys. Rehabil. Med. 50 (2014) 143e151.

37 P.S. Burge, P.M. Calverley, P.W. Jones, S. Spencer, J.A. Anderson, Prednisolone response in patients with chronic obstructive pulmonary disease: results from the ISOLDE study, Thorax 58 (2003) $654 \mathrm{e} 658$.

38 G. Deslee, K. Klooster, M. Hetzel, F. Stanzel, R. Kessler, C.H. Marquette, C. Witt, S. Blaas, W. Gesierich, F.J. Herth, et al., Lung volume reduction coil treatment for patients with severe emphysema: a European multicentre trial, Thorax 69 (2014) 980e986.

39 H.F. van Stel, V.T. Colland, N.L. Heins, L.H. Rijssenbeek-Nouwens, W. Everaerd, Assessing inpatient pulmonary rehabilitation using the patient's view of outcome, J. Cardiopulm. Rehabil. 22 (2002) 201e210. 


\section{CHAPTER 8}

40 R. Mesquita, N. Nakken, D.J. Janssen, E.H. van den Bogaart, J.M. Delbressine, J.M. Essers, K. Meijer, M. van Vliet, G.J. de Vries, J.W. Muris, et al., Activity levels and exercise motivation in COPD patients and their resident loved ones, Chest (2017) [Epub ahead of print].

41 A. Arbillaga-Etxarri, E. Gimeno-Santos, A. Barberan-Garcia, M. Benet, E. Borrell, P. Dadvand, M. Foraster, A. Marin, M. Monteagudo, R. Rodriguez-Roisin, et al., Socio-environmental correlates of physical activity in patients with chronic obstructive pulmonary disease (COPD), Thorax (2017) [Epub ahead of print].

42 G. Spina, M.A. Spruit, J. Alison, R.P. Benzo, P.M. Calverley, C.F. Clarenbach, R.W. Costello, D. DonaireGonzalez, S. Durr, J. Garcia-Aymerich, et al., Analysis of nocturnal actigraphic sleep measures in patients with COPD and their association with daytime physical activity, Thorax (2017) [Epub ahead of print].

43 H. Demeyer, Z. Louvaris, A. Frei, R.A. Rabinovich, C. de Jong, E. Gimeno-Santos, M. Loeckx, S.C. Buttery, N. Rubio, T. Van der Molen, et al., Physical activity is increased by a 12-week semiautomated telecoaching programme in patients with COPD: a multicentre randomised controlled trial, Thorax (2017) [Epub ahead of print].

44 D.E. O'Donnell, A. Deesomchok, Y.M. Lam, J.A. Guenette, N. Amornputtisathaporn, L. Forkert, K.A. Webb, Effects of BMI on static lung volumes in patients with airway obstruction, Chest 140 (2011) 461e468.

45 P.P. Walker, A. Burnett, P.W. Flavahan, P.M. Calverley, Lower limb activity and its determinants in COPD, Thorax 63 (2008) 683e689.

46 K. Meijer, J. Annegarn, V. Lima Passos, H.H. Savelberg, A.M. Schols, E.F. Wouters, M.A. Spruit, Characteristics of daily arm activities in patients with COPD, Eur. Respir. J. 43 (2014) 1631e1641.

47 E. Vincent, L. Sewell, K. Wagg, S. Deacon, J. Williams, S. Singh, Measuring a change in self-efficacy following pulmonary rehabilitation: an evaluation of the PRAISE tool, Chest 140 (2011) 1534e1539.

Reprinted from Respiratory Medicine, Vol. 126, Mesquita R, Meijer K, Pitta F, Azcuna H, Goërtz YMJ, Essers JMN, Wouters EFM, Spruit MA, Changes in physical activity and sedentary behaviour following pulmonary rehabilitation in patients with COPD, pp. 122-9, Copyright (2017), with permission from Elsevier. 


\section{SUPPLEMENTARY MATERIAL}

\section{METHODS}

\section{Assessments}

As part of a 3-day initial assessment to participate in a comprehensive pulmonary rehabilitation (PR) programme at CIRO [1], patients had assessed their demographics and clinical data, comorbidities, lung function, and body composition. Demographics and clinical data included the assessment of age, sex, smoking status (never, former, or current), pack-years of smoking, use of long-term oxygen therapy (LTOT, yes or no), number of exacerbations in the previous 12 months $(<$ or $\geq 2)$ and symptoms of dyspnoea with the modified Medical Research Council (mMRC) scale [2]. The mMRC scale measures the intensity of dyspnoea during activities of daily living in scores from 0 to 4 , with higher scores indicating more dyspnoea [2].

The following comorbidities were objectively assessed in the current study: anaemia, dyslipidaemia, hyperglycaemia, renal impairment, symptoms of anxiety, symptoms of depression, underweight, obesity, and low muscle mass. Anaemia was defined as a haemoglobin level $<13 \mathrm{~g} / \mathrm{dL}(8.1 \mathrm{mmol} / \mathrm{L})$ in males or $<12 \mathrm{~g} / \mathrm{dL}(7.5 \mathrm{mmol} / \mathrm{L})$ in females, as proposed by the World Health Organization [3]. Dyslipidaemia was defined as either a triglyceride level $>1.7 \mathrm{mmol} / \mathrm{L}$ or a $\mathrm{HDL}$ cholesterol level $<1.03 \mathrm{mmol} / \mathrm{L}$ (in males) or $<1.29 \mathrm{mmol} / \mathrm{L}$ (in females) [4]. As suggested by the American Diabetes Association, hyperglycaemia was defined as a fasting glucose level $>5.6 \mathrm{mmol} / \mathrm{L}$ [5]. An estimated glomerular filtration rate (eGFR) $<60 \mathrm{ml} / \mathrm{min}$ was used to define renal impairment. According to the National Kidney Foundation Kidney Disease Outcome Quality Initiative (NKF KDOQI) guidelines this threshold corresponds to stage 3 chronic kidney disease [6]. eGFR was calculated with the Cockcroft-Gault formula based on sex, age, body weight and creatinine levels [7]. Symptoms of anxiety and depression were identified with the subscales of the Hospital Anxiety and Depression Scale (HADS) [8]. A score $\geq 10$ points in each subscale was indicative of abnormally high and clinically relevant symptoms [8]. Details about the HADS can be found in paragraphs ahead. Underweight and obesity were defined as a body mass index (BMI) $<21 \mathrm{~kg} \cdot \mathrm{m}^{-2}$ and $\geq 30 \mathrm{~kg} \cdot \mathrm{m}^{-2}$, respectively [9]. Low muscle mass was defined as a fat-free mass index (FFMI) below the $5^{\text {th }}$ percentile of reference values according to sex, age, and BMI [10]. Further details about the calculation of BMI and FFMI are provided in paragraphs ahead. 
Lung function was measured with spirometry, whole-body plethysmography, and the single breath method for diffusing capacity for carbon monoxide $\left(D_{L C O}\right)$, all following international recommendations [11-13]. Spirometry (Masterlab ${ }^{\circledR}$; Jaeger AG, Würzburg, Germany) was performed post-bronchodilator to measure forced vital capacity (FVC) and forced expiratory volume in the first second $\left(\mathrm{FEV}_{1}\right)$, to then calculate the $\mathrm{FEV} \mathrm{V}_{1} / \mathrm{FVC}$ ratio. The results were compared to reference values [14]. Based on the postbronchodilator $\mathrm{FEV}_{1}$, the severity of airflow limitation was classified according to the Global Initiative for Chronic Obstructive Lung Disease (GOLD): GOLD 1 (FEV $1 \geq 80 \%$ predicted); GOLD 2 (50\% $\mathrm{FEV}_{1}<80 \%$ predicted); GOLD 3 (30\% $\leq \mathrm{FEV}_{1}<50 \%$ predicted) and; GOLD 4 (FEV $1<30 \%$ predicted) [15]. Plethysmography was performed to measure residual volume (RV) and total lung capacity (TLC), to then calculate the RV/TLC ratio, while the single breath method (Masterlab ${ }^{\circledR}$; Jaeger AG, Würzburg, Germany) was used to measure $D_{\text {LCO }}$.

Body composition was assessed with BMI and FFMI. BMI was calculated as weight per squared height $\left(\mathrm{kg} \cdot \mathrm{m}^{-2}\right)$ [16], while FFMI was calculated as fat-free mass (FFM) per squared height $\left(\mathrm{kg} \cdot \mathrm{m}^{-2}\right)$. FFM was measured by whole-body dual energy X-ray absorptiometry (DEXA) using a Lunar Prodigy system (GE Healthcare, Piscataway, NJ, USA) and calculated as the sum of lean and mineral bone mass [17].

Both as part of the initial 3-day assessment and of a 2-day post-rehabilitation assessment, patients also had assessed their exercise capacity (6-minute walk test (6MWT) and constant work rate test (CWRT)), quality of life (COPD-version of the St. George's Respiratory Questionnaire (SGRQ-C)), and symptoms of anxiety and depression (HADS).

The 6MWT was used for the assessment of functional exercise capacity. This test followed the guidelines proposed by the European Respiratory Society (ERS) / American Thoracic Society (ATS) [18]. As the 6MWT is subjected to a learning effect [19], two tests were performed and the best test (i.e., longest distance) was used for analysis. Patients with an improvement in 6-minute walk distance (6MWD) $\geq 30$ metres after PR were deemed to have a clinically relevant improvement [18]. The CWRT was used for the assessment of submaximal exercise capacity. This test was performed on a cycle ergometer (Carefusion, Houten, the Netherlands) at $75 \%$ of the peak work rate identified during the symptom-limited incremental test (+10 watts per minute), which was also performed on a cycle ergometer (Carefusion, Houten, the Netherlands) [20]. An improvement in CWRT time $\geq 100$ seconds after PR was considered to be clinically relevant [21].

Quality of life was assessed with the SGRQ-C, which comprises 40 items [22]. A total score is provided, which can range from 0 (optimal) to 100 points (worst) [22]. Improvements (i.e., reductions) in SGRQ-C scores $\geq 4$ points after PR were considered to be 
clinically relevant [23]. As mentioned earlier, the HADS was used for the assessment of symptoms of anxiety and depression, which is a valid and reliable self-administered questionnaire widely used to screen clinically relevant symptoms of anxiety and/or depression [8]. The HADS consists of 14 questions distributed in two subscales, anxiety (HADS anxiety subscale, 7 questions) and depression (HADS depression subscale, 7 questions) [8]. The total score in each subscale can range from 0 (optimal) to 21 (worst) [8]. Improvements (i.e., reductions) in HADS scores $\geq 1.5$ points after PR were considered to be clinically relevant [24].

The GOLD 2011 classification was used to classify patients into groups A, B, C, or D based on the degree of airflow limitation (GOLD grades 1-4), the number of exacerbations in the previous 12 months, and symptoms as assessed by the mMRC scale [25]. GOLD grades 1-4 or the number of exacerbations in the previous 12 months were used to classify patients as low (group A or B) or high (group C or D) risk. Patients classified as GOLD grade 1 or 2 , or with $<2$ exacerbations in the previous 12 months, were classified as low risk, whilst those classified as GOLD grade 3 or 4 , or with $\geq 2$ exacerbations in the previous 12 months, were classified as high risk; the criterion with the worse score was used for analysis [25]. The mMRC scale was used to classify patients as less (group A or C) or more (group B or D) symptoms [25]. Patients with an mMRC score $<2$ points were classified as less symptoms, whilst those with a score $\geq 2$ points were classified as more symptoms [25].

The multidimensional severity index updated Body mass index, airflow Obstruction, Dyspnoea, and Exercise capacity (BODE) was calculated based on BMI, FEV 1, mMRC scale, and baseline 6MWD [26]. The updated BODE index is a predictor of 3-year mortality in COPD and its total score can range from 0 to 15 points, with higher scores indicating worse prognosis [26].

\section{Assessment of physical activity and sedentary behaviour}

Both CAM and MOX are one-sensor devices developed by Maastricht Instruments B.V. in Maastricht (the Netherlands). The latter represents an upgraded version of the former. The CAM contains a triaxial piezoresistive accelerometer, weighs $102 \mathrm{~g}$, and has a range of \pm 4 times gravity with a resolution of 0.02 gravity [27]. The measured signal is stored on a 2 gigabyte micro SD cart at a sample rate of $102 \mathrm{H}$ [27]. The MOX (model MMOXX1.01) is a small activity monitor $(4.5 \times 4.0 \times 1.4 \mathrm{~cm})$ which measures acceleration data $( \pm 6 \mathrm{G})$ in three axes with a sample frequency of $25 \mathrm{~Hz}$ [28]. Both CAM and MOX were worn on the frontal part of the right leg in line with the thigh midline, half way between the knee and hip. The CAM was attached to the patient's thigh with a lightweight elastic strap tightly secured, while the MOX was waterproofed in a finger cot and attached with a transparent dressing (i.e., Tegaderm ${ }^{\mathrm{TM}}$ transparent dressing), which 
allowed patients to wear it continuously even during water-based activities. Patients wearing the CAM were instructed to attach the device to their leg after waking up and remove it before going to bed. Although the devices were used during all waking hours or continuously, only data between 07:00 AM and 19:00 PM was used for analysis. After the 3-day initial assessment performed at CIRO and the 2-day assessment after pulmonary rehabilitation (PR), patients were sent home with the activity monitor and instructed to wear it for 7 consecutive days during their daily activities. After this period patients were instructed to send the activity monitor back by post mail with a selfaddressed pre-paid envelope.

Patients were instructed to register in a form non-wearing periods or any problems/adverse side effects while wearing the device. This form should be sent back to the researchers together with the activity monitors. After the researchers received the devices back from the patients, the data were downloaded using an application developed by Maastricht Instruments B.V. and exported to an EXCEL worksheet with an algorithm developed by the researchers using Matlab R2012b (Mathworks Inc., USA). Nonwearing periods were identified with this algorithm and defined as continuous periods of 2-h or more with no activity in all of the three axes.

Based on a study using activity categories of the ActiGraph GT3X accelerometer as standard [28], thresholds for sedentary behaviour ( $<1.5$ metabolic equivalents of task (METs)), light physical activity (between 1.5 and 3.0 METs), and moderate-to-vigorous physical activity (MVPA, >3.0 METs) were estimated and used to classify each 1-minute epoch of the data: sedentary behaviour, <220; light physical activity, between 220 and 480; and MVPA, >480 activity counts. Bouts of MVPA spent in $\geq 10$ minutes were also analysed. A bout of activity was defined as a period of consecutive minutes in MVPA with a minimum duration of 10 minutes. Time in $\geq 10$-min bouts of MVPA was calculated by summing up the time in bouts performed during the course of a day, and then averaging it among all valid days. As some patients had days with no bouts, the averaging process may have led to averaged values which are lower than 10 minutes. Frequency of $\geq 10$-min bouts of MVPA was calculated by counting the number of bouts performed during the course of a day, and then averaging it among all valid days, including those with no bouts. Duration of $\geq 10$-min bouts of MVPA was calculated by averaging the duration of all bouts performed during the course of a day, and then averaging it only among days which had bouts of activity. 
RESULTS

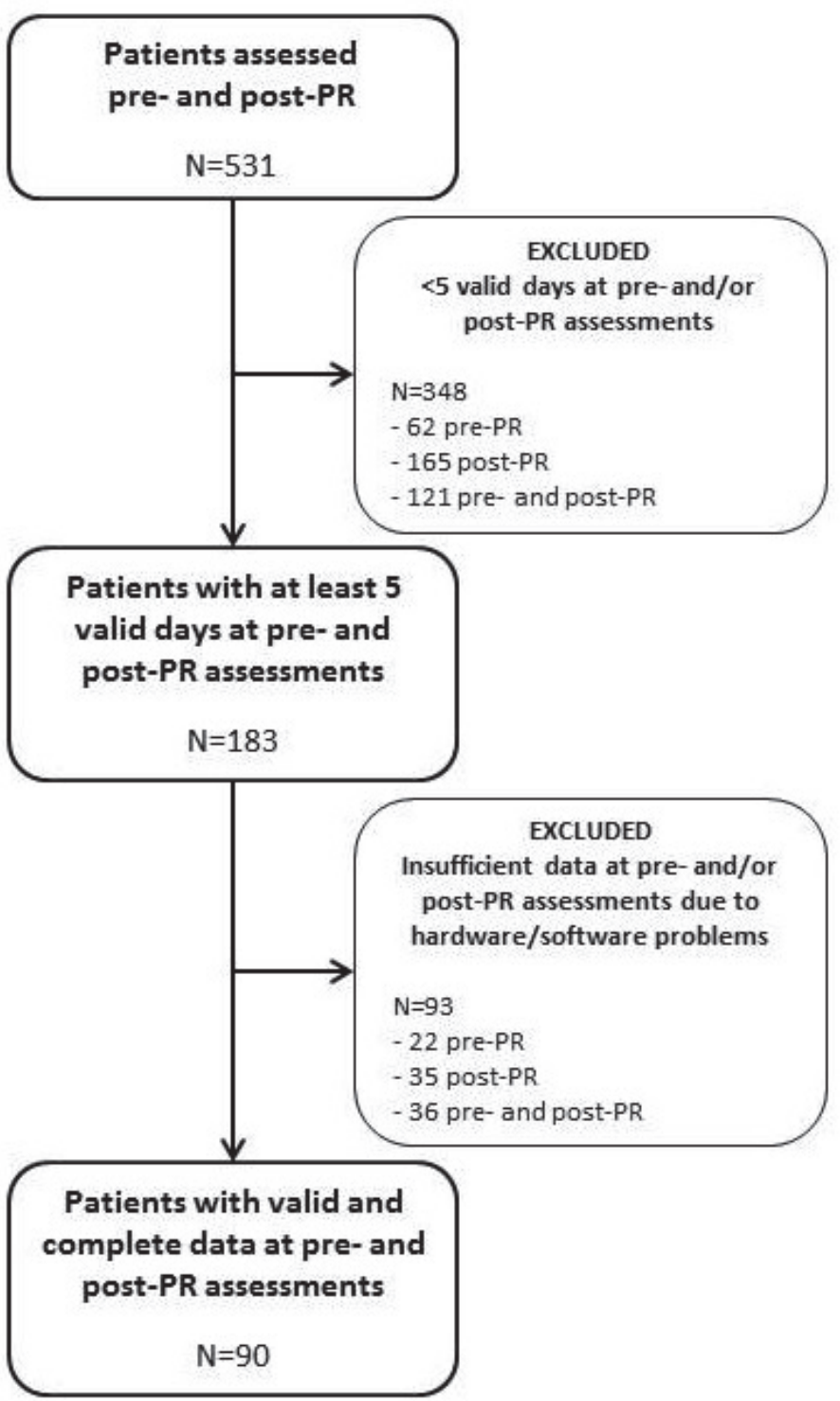

e-Figure 1 Flow-diagram of patients included in the current study. PR: pulmonary rehabilitation. 


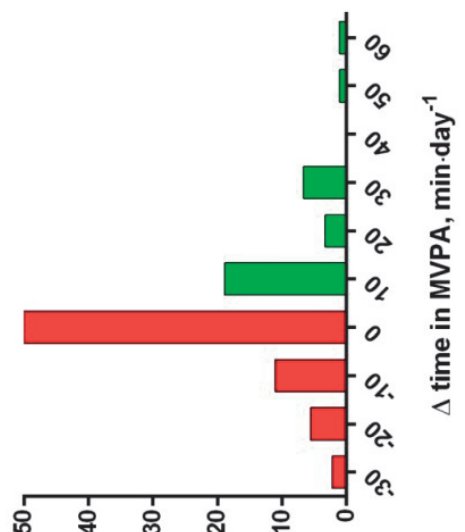

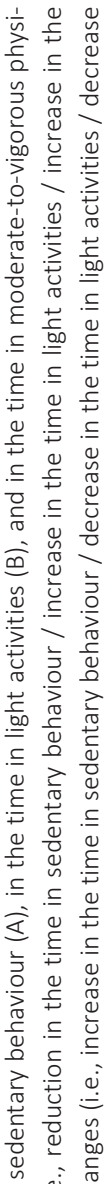

o

(\%) Кэuәnbədy

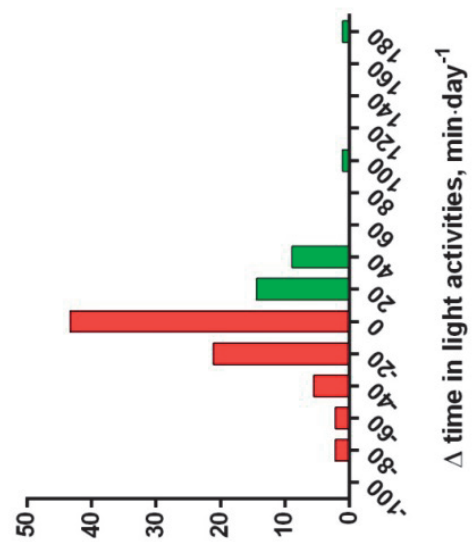

ต

(\%) Кวuәnbəג」

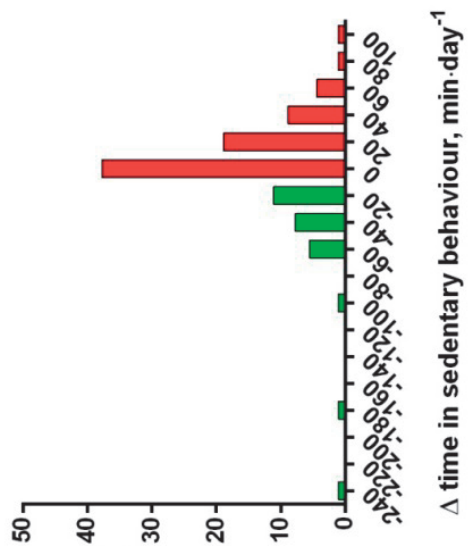

$\subseteq \dot{=}$

है

$\stackrel{0}{\frac{0}{5}} \stackrel{0}{\square}$

$\subseteq$ ○े

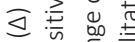

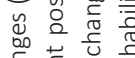

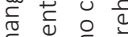

ธิ

\&

Ð

ᄃ $\frac{1}{0}$ 인

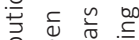

을 욜

离证 品

$\varepsilon \cup \stackrel{\sim}{\leftrightarrows}$

해용

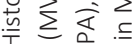

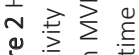

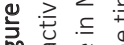

밍

ব

(\%) Кэuәnbəגコ

d $\overline{0} \cong$. 


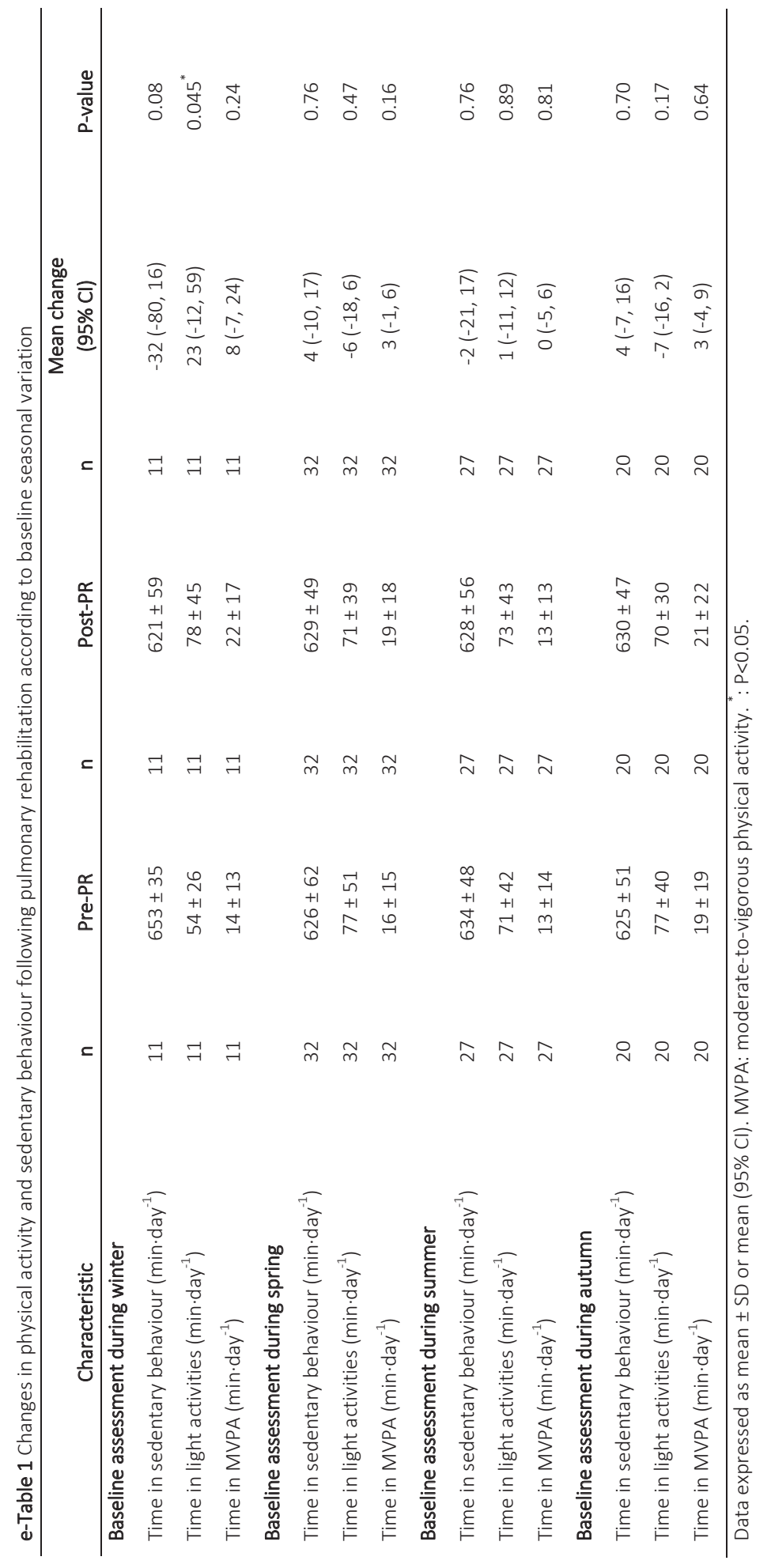


CHAPTER 8

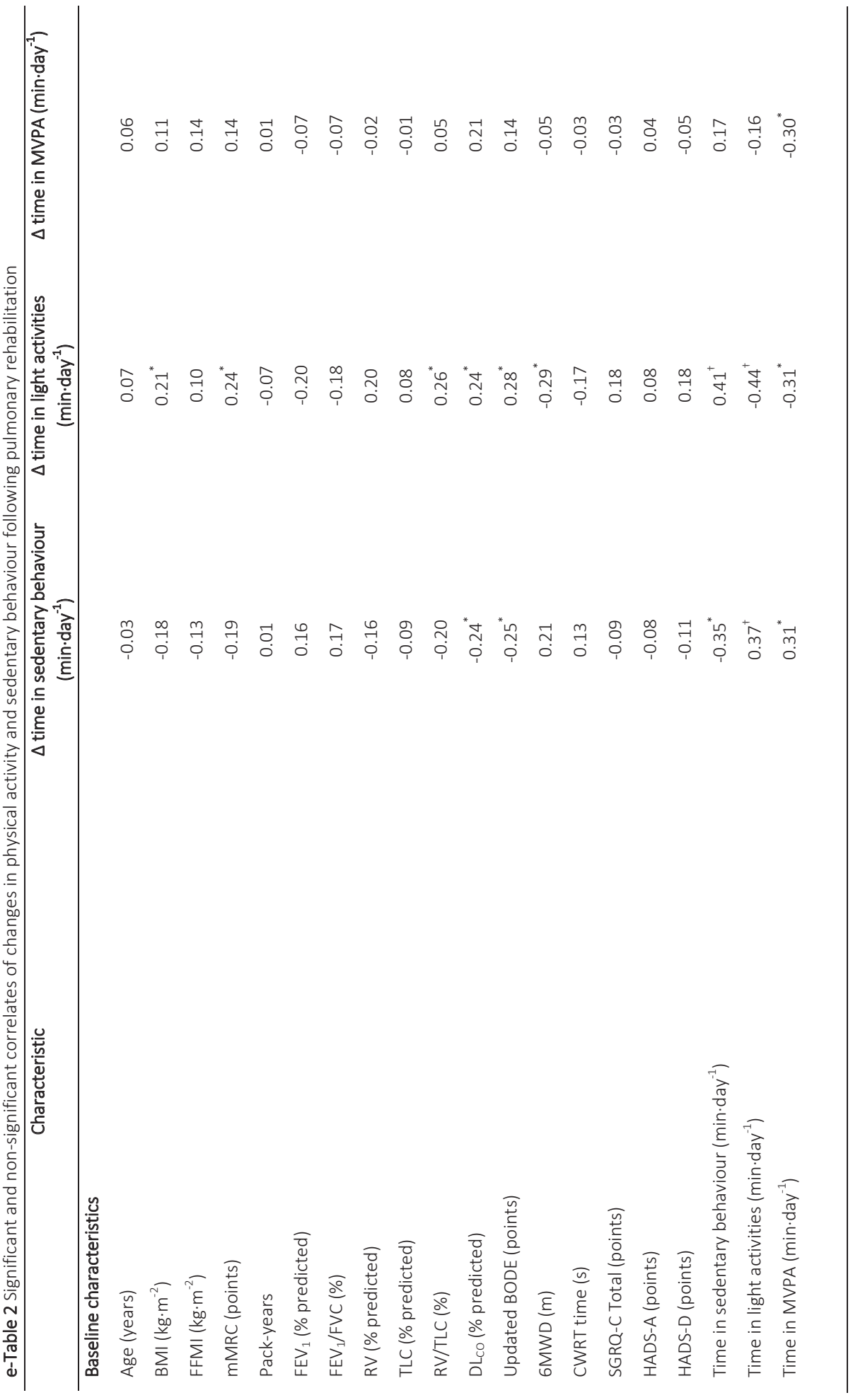


ACTIVITY LEVELS FOLLOWING PULMONARY REHABILITATION IN COPD

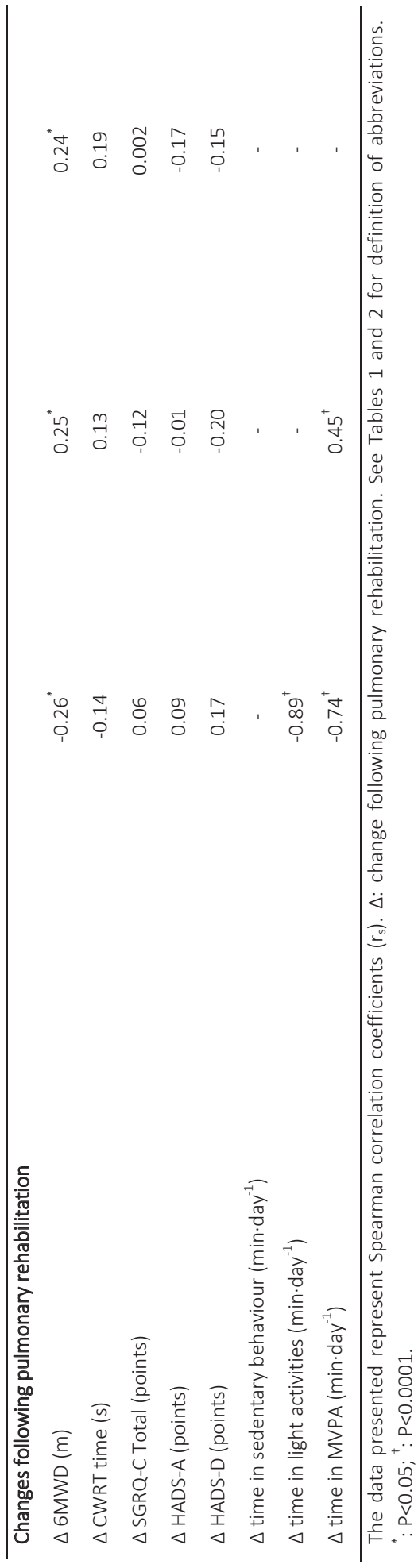




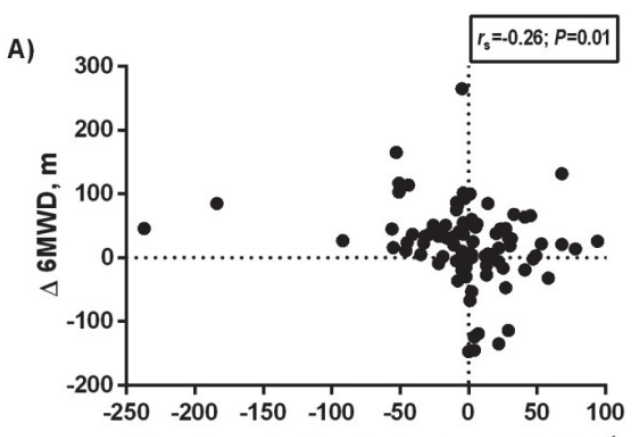

$\Delta$ time in sedentary behaviour, $\min \cdot$ day $^{-1}$
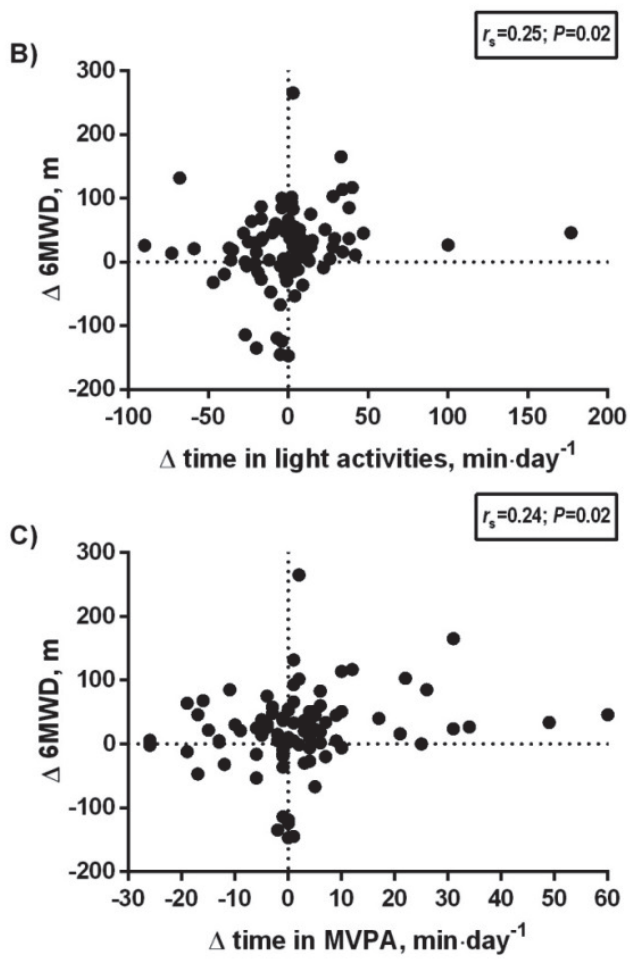

e-Figure 3 Correlations between changes $(\Delta)$ in the time in sedentary behaviour and $\Delta$ in 6 -minute walk distance (6MWD) (A), $\Delta$ in the time in light activities and $\Delta$ in 6MWD (B), and $\Delta$ in the time in moderate-tovigorous physical activity (MVPA) and $\triangle$ in 6MWD (C) following pulmonary rehabilitation. Dotted lines correspond to the point of no change in each intensity. 


\section{References}

1. Spruit MA, Vanderhoven-Augustin I, Janssen PP, Wouters EF: Integration of pulmonary rehabilitation in COPD. Lancet 2008, 371:12-13.

2. Bestall JC, Paul EA, Garrod R, Garnham R, Jones PW, Wedzicha JA: Usefulness of the Medical Research Council (MRC) dyspnoea scale as a measure of disability in patients with chronic obstructive pulmonary disease. Thorax 1999, 54:581-586.

3. Nutritional anaemias. Report of a WHO scientific group. World Health Organ Tech Rep Ser 1968, 405:537.

4. Alberti KG, Zimmet P, Shaw J, Group IDFETFC: The metabolic syndrome--a new worldwide definition. Lancet 2005, 366:1059-1062.

5. American Diabetes A: Diagnosis and classification of diabetes mellitus. Diabetes Care 2010, 33 Suppl 1:S62-69.

6. Kdoqi: KDOQI Clinical Practice Guidelines and Clinical Practice Recommendations for Diabetes and Chronic Kidney Disease. Am J Kidney Dis 2007, 49:S12-154.

7. Cockcroft DW, Gault MH: Prediction of creatinine clearance from serum creatinine. Nephron 1976, 16:3141.

8. Zigmond AS, Snaith RP: The hospital anxiety and depression scale. Acta Psychiatr Scand 1983, 67:361-370.

9. Spruit MA, Singh SJ, Garvey C, Zuwallack R, Nici L, Rochester C, Hill K, Holland AE, Lareau SC, Man WD, et al: An official american thoracic society/european respiratory society statement: key concepts and advances in pulmonary rehabilitation. Am J Respir Crit Care Med 2013, 188:e13-64.

10. Franssen FM, Rutten EP, Groenen MT, Vanfleteren LE, Wouters EF, Spruit MA: New reference values for body composition by bioelectrical impedance analysis in the general population: results from the UK Biobank. J Am Med Dir Assoc 2014, 15:448 e441-446.

11. Miller MR, Hankinson J, Brusasco V, Burgos F, Casaburi R, Coates A, Crapo R, Enright P, van der Grinten CP, Gustafsson P, et al: Standardisation of spirometry. Eur Respir J 2005, 26:319-338.

12. Wanger J, Clausen JL, Coates A, Pedersen OF, Brusasco V, Burgos F, Casaburi R, Crapo R, Enright P, van der Grinten CP, et al: Standardisation of the measurement of lung volumes. Eur Respir J 2005, 26:511522.

13. Macintyre N, Crapo RO, Viegi G, Johnson DC, van der Grinten CP, Brusasco V, Burgos F, Casaburi R, Coates $A$, Enright $P$, et al: Standardisation of the single-breath determination of carbon monoxide uptake in the lung. Eur Respir J 2005, 26:720-735.

14. Knudson RJ, Burrows B, Lebowitz MD: The maximal expiratory flow-volume curve: its use in the detection of ventilatory abnormalities in a population study. Am Rev Respir Dis 1976, 114:871-879.

15. Rabe KF, Hurd S, Anzueto A, Barnes PJ, Buist SA, Calverley P, Fukuchi Y, Jenkins C, Rodriguez-Roisin R, van Weel C, Zielinski J: Global strategy for the diagnosis, management, and prevention of chronic obstructive pulmonary disease: GOLD executive summary. Am J Respir Crit Care Med 2007, 176:532-555.

16. Keys A, Fidanza F, Karvonen MJ, Kimura N, Taylor HL: Indices of relative weight and obesity. J Chronic Dis 1972, 25:329-343.

17. Graat-Verboom L, Spruit MA, van den Borne BE, Smeenk FW, Martens EJ, Lunde R, Wouters EF: Correlates of osteoporosis in chronic obstructive pulmonary disease: An underestimated systemic component. Respir Med 2009, 103:1143-1151.

18. Holland AE, Spruit MA, Troosters T, Puhan MA, Pepin V, Saey D, McCormack MC, Carlin BW, Sciurba FC, Pitta F, et al: An official European Respiratory Society/American Thoracic Society Technical Standard: field walking tests in chronic respiratory disease. Eur Respir J 2014, 44:1428-1446.

19. Hernandes NA, Wouters EF, Meijer K, Annegarn J, Pitta F, Spruit MA: Reproducibility of 6-minute walking test in patients with COPD. Eur Respir J 2011, 38:261-267.

20. van 't Hul A, Gosselink R, Kwakkel G: Constant-load cycle endurance performance: test-retest reliability and validity in patients with COPD. J Cardiopulm Rehabil 2003, 23:143-150. 


\section{CHAPTER 8}

21. Laviolette L, Bourbeau J, Bernard S, Lacasse Y, Pepin V, Breton MJ, Baltzan M, Rouleau M, Maltais F: Assessing the impact of pulmonary rehabilitation on functional status in COPD. Thorax 2008, 63:115-121.

22. Meguro M, Barley EA, Spencer S, Jones PW: Development and Validation of an Improved, COPD-Specific Version of the St. George Respiratory Questionnaire. Chest 2007, 132:456-463.

23. Jones PW: St. George's Respiratory Questionnaire: MCID. COPD 2005, 2:75-79.

24. Puhan MA, Frey M, Buchi S, Schunemann HJ: The minimal important difference of the hospital anxiety and depression scale in patients with chronic obstructive pulmonary disease. Health Qual Life Outcomes 2008, 6:46.

25. Vestbo J, Hurd SS, Agusti AG, Jones PW, Vogelmeier C, Anzueto A, Barnes PJ, Fabbri LM, Martinez FJ, Nishimura $M$, et al: Global strategy for the diagnosis, management, and prevention of chronic obstructive pulmonary disease: GOLD executive summary. Am J Respir Crit Care Med 2013, 187:347-365.

26. Puhan MA, Garcia-Aymerich J, Frey M, ter Riet G, Anto JM, Agusti AG, Gomez FP, Rodriguez-Roisin R, Moons KG, Kessels AG, Held U: Expansion of the prognostic assessment of patients with chronic obstructive pulmonary disease: the updated BODE index and the ADO index. Lancet 2009, 374:704-711.

27. Annegarn J, Spruit MA, Uszko-Lencer NH, Vanbelle S, Savelberg HH, Schols AM, Wouters EF, Meijer K: Objective physical activity assessment in patients with chronic organ failure: a validation study of a new single-unit activity monitor. Arch Phys Med Rehabil 2011, 92:1852-1857 e1851.

28. van der Weegen S, Essers H, Spreeuwenberg M, Verwey R, Tange H, de Witte L, Meijer K: Concurrent Validity of the MOX Activity Monitor Compared to the ActiGraph GT3X. Telemed J E Health 2015, 21:259266. 


\section{CHAPTER 9}

Activity levels and exercise motivation in COPD patients and their resident loved ones

Rafael Mesquita, Nienke Nakken, Daisy J.A. Janssen, Esther H.A. van den Bogaart, Jeannet M.L. Delbressine, Johannes M.N. Essers, Kenneth Meijer, Monique van Vliet, Geeuwke J. de Vries, Jean W.M. Muris, Fabio Pitta, Emiel F.M. Wouters, and Martijn A. Spruit 


\title{
CHAPTER 9
}

\begin{abstract}
Background: Resident loved ones of patients with COPD can play an important role in helping patients to engage in physical activity. We aimed to compare activity levels and exercise motivation between COPD patients and their resident loved ones; to compare the same outcome measures in patients after stratification for the physical activity level of the loved ones; and to predict the likelihood of being physically active in patients with a physically active resident loved one.
\end{abstract}

Methods: 125 patient-loved one dyads were cross-sectionally and simultaneously assessed. Sedentary behaviour, light activities and moderate-to-vigorous physical activity (MVPA) were measured with a triaxial accelerometer during free-living conditions for at least 5 days. Five exercise motivation constructs were investigated: amotivation, external regulation, introjected regulation, identified regulation, and intrinsic regulation.

Results: Patients spent more time in sedentary behaviour and less time in physical activity than their loved ones $(P<0.0001)$. More intrinsic regulation was observed in loved ones compared to patients $(P=0.003)$, with no differences in other constructs. Despite similar exercise motivation, patients with an active loved one spent more time in MVPA

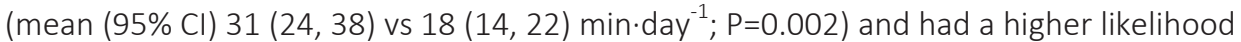
of being active $(\mathrm{OR}(95 \% \mathrm{Cl}) 4.36(1.41,13.30)$; $\mathrm{P}=0.01)$ than patients with an inactive loved one after controlling for age, body mass index and degree of airflow limitation.

Conclusions: COPD patients are more physically inactive and sedentary than their loved ones, despite relatively similar exercise motivation. Nevertheless, patients with an active loved one are more active themselves and have a higher likelihood of being active. 


\section{INTRODUCTION}

Chronic obstructive pulmonary disease (COPD) is characterised by breathlessness and fatigue that leads to problems with multiple activities of daily living ${ }^{1}$. Therefore, patients with COPD are less physically active ${ }^{2}$ and spend more time in sedentary behaviour ${ }^{3}$ compared to healthy subjects. This physically inactive/sedentary lifestyle can at least in part contribute to the development of extra-pulmonary features and comorbidities ${ }^{4}$.

Adopting a healthier lifestyle, including less sedentarism and more leisure-time physical activity, is one of the main goals of integrated COPD management ${ }^{5,6}$. Nevertheless, it seems difficult for patients to change their physical activity levels following structured pulmonary rehabilitation programmes (with or without individual physical activity counselling sessions) ${ }^{7,8}$ and/or using pedometer-based counselling programmes ${ }^{9}$.

Family members / resident loved ones may play an important role to achieve this goal ${ }^{10}$, for instance, by encouraging patients with COPD to have joint daily walks or to engage more in household activities. To consider family-based lifestyle interventions for patients with COPD and their (resident) family members, we first need a better understanding of the physical activity, sedentary behaviours and exercise motivation of the patients' family members. Indeed, 92\% of the resident loved ones of patients with COPD have one or more chronic conditions themselves (i.e., persistent airflow obstruction, obesity, and symptoms of anxiety/depression) ${ }^{11}$, which may all affect their daily physical activity levels. To the best of our knowledge, no previous study has ever specifically addressed physical activity levels, sedentary behaviour and exercise motivation of loved ones of patients with COPD.

Therefore, the aim of this study was threefold: 1) to compare physical activity, sedentary behaviour and exercise motivation between COPD patients and their resident loved ones; 2) to compare physical activity, sedentary behaviour and exercise motivation in patients after stratification for the physical activity level of the resident loved ones; and 3) to predict the likelihood of being physically active in patients with a physically active resident loved one. A priori, we hypothesised that patients are more physically inactive and sedentary than the resident loved ones; and that COPD patients with a physically active resident loved one have a higher likelihood of being physically active themselves. 


\section{MATERIAL AND METHODS}

\section{Study design and participants}

This is a cross-sectional analysis using baseline data of the Home Sweet Home study, an observational, longitudinal study on the home environment of COPD patients 12 . The Home Sweet Home study was approved by the Medical Ethics Committees United (MEC-U), the Netherlands (NL42721.060.12/M12-1280). Recruitment of participants was performed during hospital admission or at the outpatient respiratory clinic in 4 hospitals throughout the southern-eastern part of the Netherlands. Patients with COPD who participated in the Chance study ${ }^{13}$ and met the inclusion criteria of the Home Sweet Home study were also asked to participate in the study. Patients and resident loved ones who were included in the Home Sweet Home study were conveniently used in the current analysis. Therefore, the sample size calculation was based on the primary aims of that study ${ }^{12}$. Patients were included in the current analysis if they met the following criteria: Global Initiative for Chronic Obstructive Lung Disease (GOLD) COPD diagnosis with moderate-to-very-severe degree of airflow limitation (i.e., GOLD grades 2-4) ${ }^{5}$; no exacerbation or hospitalisation within the previous 4 weeks; having a resident loved one (i.e., a person living together with a COPD patient, regardless whether or not he or she provides informal care to the patient); and having a valid physical activity assessment (please see 'Assessments' subsection for details). Patients recruited during hospital admission were assessed only when in stable condition (i.e., no exacerbation or hospitalisation within the previous 4 weeks). Patients and/or loved ones were excluded in case of cognitive impairment or inability to speak and/or understand Dutch. All participants provided written informed consent. Inclusion period took place between July 2013 and December 2014.

\section{Assessments}

All assessments were performed during home visits. Assessments were made of demographics, clinical data, body composition (body impedance analysis), postbronchodilator lung function (spirometry), functional mobility (Timed Up \& Go test ${ }^{14,15}$ ), exercise motivation (Behavioural Regulation and Exercise Questionnaire $2^{16}$ ), generic and COPD-specific health status (EuroQol-5 Dimensions ${ }^{17}$ and COPD Assessment Test ${ }^{18}$, respectively), care dependency (Care Dependency Scale ${ }^{19}$ ), and symptoms of anxiety and depression (Hospital Anxiety and Depression Scale ${ }^{20}$ ). Further details can be found in the supplementary material. At the end of the home visit patients and their loved ones received an activity monitor for the assessment of physical activity and sedentary behaviour. 
These outcomes were assessed during free-living conditions with the MOX Activity Monitor (Maastricht Instruments B.V. in Maastricht, the Netherlands), a tri-axial accelerometer validated in patients with COPD and healthy subjects ${ }^{21,22}$. Patients and their loved ones wore their own monitor concomitantly and the accelerometer data of each patient-loved one dyad were synchronized. Participants had the device attached to the frontal part of their right thigh and were instructed to keep it for at least 7 days. A valid activity levels assessment was defined as at least 5 days of assessment (3 weekdays + Saturday + Sunday) ${ }^{23}$, each with at least 10 h of measurement. Data assessed during waking hours from the average value of all valid days were used to calculate each outcome measure: time in sedentary behaviour $(<1.5$ Metabolic Equivalents of Task (METs)), time in light activities (1.5-3.0 METs), time in moderate-to-vigorous physical activity (MVPA, >3.0 METs). Participants were considered physically active if they spent $\geq 30$ min in MVPA on at least 5 days per week ${ }^{24}$. Moreover, the time in weight-bearing, non-weight bearing and dynamic activities, as well as characteristics of 10-min bouts of activities in MVPA were estimated. Daily hourly patterns were also analysed (i.e., a graphic representation of the intensity of activity in counts $\cdot \mathrm{min}^{-1}$ during the course of a day), as was daily television-viewing time based on self-reports.

Exercise motivation was assessed with the Behavioural Regulation and Exercise Questionnaire 2 (BREQ-2) ${ }^{16}$. This instrument focuses on the reasons underlying peoples' decisions to engage or not in exercise based on principles of the self-determination theory ${ }^{16}$. The BREQ-2 is composed of 19 items distributed in five subscales: amotivation (e.g., "I don't see why I should have to exercise"), external regulation (e.g., "I exercise because other people say I should"), introjected regulation (e.g., "I feel guilty when I don't exercise"), identified regulation (e.g., "It's important to me to exercise regularly"), and intrinsic regulation (e.g., "I exercise because it is fun") ${ }^{16}$. These are different forms of regulation which lie along a continuum ranging from completely non-self-determined to completely self-determined regulation ${ }^{16}$. Scores per subscale are provided and can range from 0.00 (not true for the participant) to 4.00 (very true for the participant).

\section{Statistical analyses}

Continuous variables were tested for normality with the Shapiro-Wilk test, presented as mean \pm SD or median (IQR), and compared with unpaired t test or Mann-Whitney $U$ test, as appropriate. Categorical variables were presented as absolute and relative frequencies, and compared using Chi-squared test or Fisher's exact test. Analyses after controlling for age, body mass index (BMI), and degree of airflow limitation were performed with an analysis of covariance and the results were presented as mean $(95 \% \mathrm{Cl})$. Logistic regression models were generated to predict the likelihood (OR ( $95 \% \mathrm{CI})$ ) of being physically active for patients with physically active resident loved ones. Statistical analyses were performed using SPSS 22.0 (IBM, Armonk, NY, USA) or GraphPad Prism 6 


\section{CHAPTER 9}

(GraphPad Software, La Jolla, California, USA), and a P-value $<0.05$ was considered significant.

\section{RESULTS}

Of the 194 patient-loved one dyads who completed the baseline assessment of the Home Sweet Home study, 69 dyads could not be included in the current analysis due to an invalid activity levels assessment. Patients and loved ones from these dyads are referred to as non-included. e-Figure 1 summarises the reasons for ineligibility, while eTable 1 presents the comparison between included and non-included participants (supplementary material). Non-included patients were slightly younger, more often required care from relatives, had worse lung function and worse health status than included patients. Non-included loved ones used less medication, had slightly better functional mobility and had more anxiety symptoms than included loved ones. Details on participants screened for eligibility and willingness to participate in the Home Sweet Home study have been described elsewhere ${ }^{11,12}$.

\section{General characteristics}

Overall, COPD patients were characterised by moderate-to-severe degree of airflow limitation and an impaired health status (Table 1). Around one-fourth of the patients referred having a walking aid (i.e., rollator or cane) or using long-term oxygen therapy. Compared to their loved ones, patients more often received informal care from relatives, had higher burden of comorbidities, used more medication, had lower BMI and fat-free mass index, worse lung function, functional mobility, and health status, had more symptoms of depression and were more care dependent (Table 1). Ten patients $(8 \%)$ and one loved one (1\%) reported current participation in pulmonary rehabilitation. 
Table 1 General characteristic of the groups

\begin{tabular}{|c|c|c|c|}
\hline Characteristics & $\begin{array}{l}\text { COPD patients } \\
\quad(n=125)\end{array}$ & $\begin{array}{c}\text { Resident loved } \\
\text { ones } \\
(n=125)\end{array}$ & P-value \\
\hline Male sex, n (\%) & $69(55)$ & $54(43)$ & 0.06 \\
\hline Age, years & $67(62-74)$ & $66(61-73)$ & 0.48 \\
\hline Relationship between patient and loved one & & & 0.48 \\
\hline Partners (married or not), n (\%) & $122(97)$ & $123(98)$ & \\
\hline $\begin{array}{l}\text { Loved one is the son/daughter of the patier } \\
\mathrm{n}(\%)\end{array}$ & $2(2)$ & $2(2)$ & \\
\hline Friends, $\mathrm{n}(\%)$ & $1(1)$ & $0(0)$ & \\
\hline Working situation & & & 0.32 \\
\hline Paid job, n (\%) & $13(10)$ & $23(18)$ & \\
\hline Retired, n (\%) & $63(51)$ & $52(42)$ & \\
\hline Household work, n (\%) & $18(14)$ & $29(23)$ & \\
\hline Unable to work, n (\%) & $26(21)$ & $14(11)$ & \\
\hline Other (volunteer or unemployed), n (\%) & $5(4)$ & $7(6)$ & \\
\hline Current smoker, n (\%) & $23(18)$ & $36(29)$ & 0.05 \\
\hline Time living together, years & $41(32-47)$ & $42(32-47)$ & 0.92 \\
\hline Receiving informal care from relatives, $\mathrm{n}(\%)$ & $15(12)$ & $3(2)$ & 0.003 \\
\hline Rollator, n (\%) & $36(29)$ & - & \\
\hline Cane, n (\%) & $27(22)$ & - & \\
\hline Long-term oxygen therapy, $\mathrm{n}(\%)$ & $33(26)$ & - & \\
\hline Exacerbations last 12 months, $\mathrm{n}$ & $2(1-4)$ & - & \\
\hline Charlson Comorbidity Index, points & $2(1-3)$ & $1(0-2)$ & $<0.0001$ \\
\hline Medications in use, $\mathrm{n}$ & $7(5-10)$ & $4(1-6)$ & $<0.0001$ \\
\hline $\mathrm{BMI}, \mathrm{kg} \cdot \mathrm{m}^{-2}$ & $25.3(22.3-29.3)$ & $27.4(24.3-30.4)$ & 0.001 \\
\hline $\mathrm{BMI}<21 \mathrm{~kg} \cdot \mathrm{m}^{-2}, \mathrm{n}(\%)$ & $22(18)$ & $5(4)$ & 0.001 \\
\hline $\mathrm{BMI} \geq 30 \mathrm{~kg} \cdot \mathrm{m}^{-2}, \mathrm{n}(\%)$ & $25(20)$ & $36(29)$ & 0.10 \\
\hline $\mathrm{FEV}_{1}, \%$ predicted & $51(33-65)$ & $105(88-117)$ & $<0.0001$ \\
\hline $\mathrm{FEV}_{1} / \mathrm{FVC}, \%$ & $40(31-52)$ & $74(66-77)$ & $<0.0001$ \\
\hline $\mathrm{FEV}_{1} / \mathrm{FVC}<70 \%, \mathrm{n}(\%)$ & $125(100)$ & $39(32)$ & $<0.0001$ \\
\hline GOLD 2 / 3 / 4, n (\%) & $67(54) / 39(31) / 19(15)$ & - & \\
\hline GOLD A / B / C / D, n (\%) & $5(4) / 32(26) / 2(2) / 84(68)$ & - & \\
\hline $\mathrm{FFM}, \mathrm{kg}^{*}$ & $50.7(45.9-55.9)$ & $52.1(47.3-58.3)$ & 0.11 \\
\hline $\mathrm{FFMI}, \mathrm{kg} \cdot \mathrm{m}^{-2^{*}}$ & $18.0(17.2-19.3)$ & $19.1(18.1-20.4)$ & $<0.0001$ \\
\hline FFMI $<5^{\text {th }}$ percentile, $\mathrm{n}(\%)^{*}$ & $17(15)$ & $5(4)$ & 0.007 \\
\hline TUG time, s & $9.9(8.5-11.7)$ & $9.0(7.8-10.2)$ & 0.005 \\
\hline TUG time $>11 \mathrm{~s}, \mathrm{n}(\%)$ & $38(31)$ & $20(16)$ & 0.007 \\
\hline $\mathrm{mMRC}$, points & $2(2-4)$ & - & \\
\hline
\end{tabular}




\begin{tabular}{lccc}
\hline CAT, points & $20 \pm 7$ & - & \\
EQ-5D index score, points & $0.78(0.66-0.90)$ & $0.89(0.81-1.00)$ & $<0.0001$ \\
CDS, points & $71(66-74)$ & $75(74-75)$ & $<0.0001$ \\
Care dependent (CDS $\leq 68$ points), $n(\%)$ & $42(34)$ & $4(3)$ & $<0.0001$ \\
HADS Anxiety, points & $5(3-8)$ & $5(2-8)$ & 0.47 \\
HADS Anxiety $\geq 10$ points, $n(\%)$ & $18(14)$ & $20(16)$ & 0.73 \\
HADS Depression, points & $5(3-8)$ & $4(2-5)$ & $<0.0001$ \\
HADS Depression $\geq 10$ points, $n(\%)$ & $21(17)$ & $5(4)$ & 0.001 \\
\hline
\end{tabular}

Data expressed as absolute and relative frequency, mean $\pm \mathrm{SD}$, or median (IQR). BMI: body mass index; $\mathrm{FEV}_{1}$ : forced expiratory volume in the first second; FVC: forced vital capacity; GOLD: Global Initiative for Chronic Obstructive Lung Disease; FFM: fat-free mass; FFMI: fat-free mass index; TUG: Timed Up \& Go; mMRC: modified Medical Research Council scale; CAT: COPD assessment test; EQ-5D: EuroQol-5 Dimensions; CDS: care dependence scale; HADS: Hospital Anxiety and Depression Scale. " $\mathrm{n}=114$ for COPD patients and $n=115$ for resident loved ones.

\section{Physical activity and sedentary behaviour}

Both patients and loved ones had a median (interquartile range) of 6 ( $6-6)$ valid days of activity monitoring. The total number of valid days in each group was 737 days, of which 487 (66\%) were weekdays. Patients spent more time in sedentary behaviour and non-weight bearing activities than loved ones, whilst loved ones spent more time in light activities, MVPA, weight bearing and dynamic activities than patients (Table 2). The amount of time spent in $\geq 10$-min bouts of MVPA was similar between groups, as was the frequency and duration of these bouts. These results were observed irrespective of the day of the week (e-Table 2; supplementary material). More loved ones than patients were considered physically active, i.e., $\geq 30 \mathrm{~min}$ in MVPA on $\geq 5$ days ( $30 \%$ vs $17 \%$, $\mathrm{P}=0.01$ ). Daily hourly patterns revealed that resident loved ones performed their activities at higher intensities compared to patients, despite a similar activity pattern (Figure 1). The amount of self-reported time spent watching television was similar between groups (Table 2). 
Table 2 Physical activity and sedentary behaviour in COPD patients and their resident loved ones

\begin{tabular}{|c|c|c|c|}
\hline Characteristics & COPD patients & $\begin{array}{c}\text { Resident } \\
\text { loved ones }\end{array}$ & P-value \\
\hline Time in sedentary behaviour, $\min \cdot$ day $^{-1}$ & $616(566-663)$ & $558(498-606)$ & $<0.0001$ \\
\hline Time in light activities, min $\cdot$ day $^{-1}$ & $83(52-118)$ & $121(97-170)$ & $<0.0001$ \\
\hline Time in MVPA, min $\cdot d a y^{-1}$ & $12(2-41)$ & $31(16-52)$ & $<0.0001$ \\
\hline Proportion of daily time in sedentary behaviour, $\%$ & $86(79-92)$ & $78(69-84)$ & $<0.0001$ \\
\hline Proportion of daily time in light activities, $\%$ & $12(7-16)$ & $17(13-24)$ & $<0.0001$ \\
\hline Proportion of daily time in MVPA, \% & $2(0-6)$ & $4(2-7)$ & $<0.0001$ \\
\hline Time in $\geq 10$-min bouts of MVPA, min day $^{-1}$ & $0(0-10)$ & $2(0-11)$ & 0.35 \\
\hline Frequency of $\geq 10$-min bouts of MVPA, bouts. day ${ }^{-1}$ & $0(0-1)$ & $0(0-1)$ & 0.16 \\
\hline Duration of $\geq 10$-min bouts of MVPA, $\min \cdot$ bout $^{-1}$ & $14(12-18)$ & $15(12-20)$ & 0.24 \\
\hline Time in non-weight bearing activities, $\min \cdot$ day $^{-1}$ & $510 \pm 94$ & $427 \pm 86$ & $<0.0001$ \\
\hline Time in weight-bearing activities, min day $^{-1}$ & $153 \pm 71$ & $206 \pm 62$ & $<0.0001$ \\
\hline Time in dynamic activities, $\min \cdot$ day $^{-1}$ & $50(29-81)$ & $80(56-106)$ & $<0.0001$ \\
\hline Television-viewing time, $\mathrm{h} \cdot \mathrm{day}^{-1}$ & $3.5(2.5-5.0)$ & $3.0(2.0-4.5)$ & 0.09 \\
\hline
\end{tabular}

Data expressed as mean \pm SD or median (IQR). MVPA: moderate-to-vigorous physical activity.

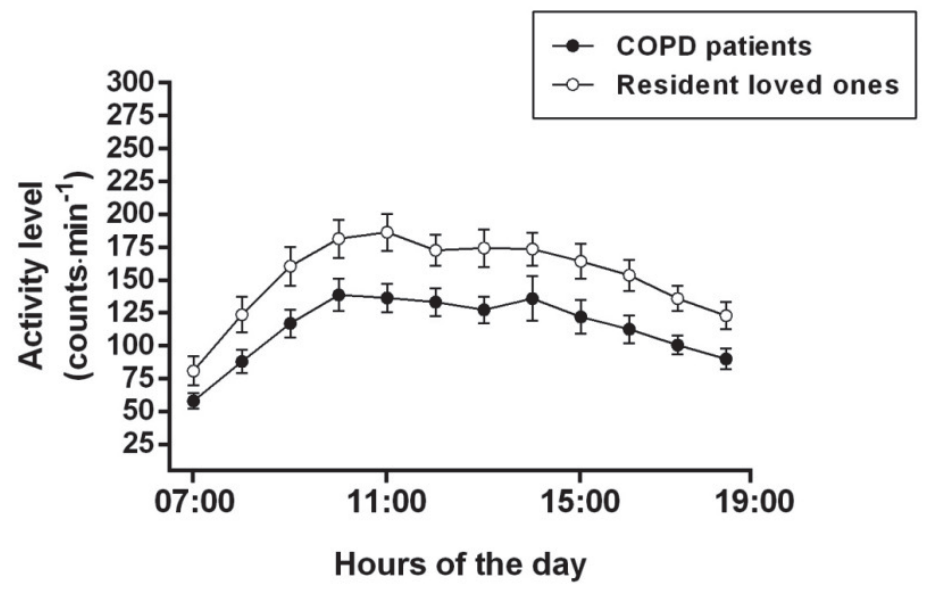

Figure 1 Daily physical activity hourly patterns of COPD patients and their resident loved ones. Data presented as mean (95\% confidence intervals).

\section{Exercise motivation}

Concerning exercise motivation, resident loved ones endorsed more intrinsic regulation than patients (Table 3). No differences were observed in other exercise motivation constructs. 
Table 3 Exercise motivation in COPD patients and their resident loved ones

\begin{tabular}{|c|c|c|c|}
\hline Characteristics & $\begin{array}{l}\text { COPD patients } \\
\quad(n=125)\end{array}$ & $\begin{array}{l}\text { Resident loved ones } \\
(n=125)\end{array}$ & P-value \\
\hline Amotivation, points & $0.25(0.00-1.13)$ & $0.25(0.00-1.25)$ & 0.90 \\
\hline External regulation, points & $0.63(0.00-1.25)$ & $0.25(0.00-1.50)$ & 0.15 \\
\hline Introjected regulation, points & $1.00(0.67-2.00)$ & $0.67(0.00-1.92)$ & 0.07 \\
\hline Identified regulation, points & $3.00(2.06-3.50)$ & $3.00(2.25-3.50)$ & 0.34 \\
\hline Intrinsic regulation, points & $3.00(2.00-3.75)$ & $3.25(2.50-4.00)$ & 0.003 \\
\hline
\end{tabular}

Data expressed as median (IQR).

\section{Physical activity in loved ones}

Comparisons between physically active $(n=38 ; 30 \%)$ and physically inactive loved ones ( $n=87 ; 70 \%$ ) can be found in e-Tables 3 and 4 (supplementary material). Active loved ones were younger, used less medication, had better functional mobility and showed stronger intrinsic motivation than the inactive ones. Tables 4 and 5 present the comparisons between the patients of these two groups. Patients with an active loved one were younger, had lower BMI, and worse airflow limitation than patients with an inactive loved one (Table 4). After controlling for age, BMI and degree of airflow limitation, patients with an active loved one were found to spend more time in MVPA than those with an inactive one (Table 5 and e-Figure 2 in the supplementary material). More time in $\geq 10$ min bouts of MVPA as well as a higher frequency and a longer duration of these bouts were also observed in patients with an active loved one (Table 5 and e-Figure 3 in the supplementary material). Sensitivity analyses revealed that these results remained unchanged after removing participants who reported current participation in pulmonary rehabilitation (e-Table 5, supplementary material). Moreover, patients with an active loved one were more often considered physically active than patients with an inactive loved one ( $29 \%$ vs $10 \%$, respectively, $P=0.02$; Table 5 ). No other differences were observed. Logistic regression showed a higher likelihood of being physically active among patients with an active loved one (OR (95\% Cl) $3.14(1.20,8.21)$; $P=0.02)$, even after adjusting for age, BMI and forced expiratory volume in the first second (OR (95\% CI) $4.36(1.41,13.30) ; P=0.01)$. Daily hourly patterns revealed a similar pattern and a similar intensity of activity in COPD patients irrespective of the physical activity level of their loved ones (Figure 2). On the other hand, physically active loved ones showed a different pattern with the peak of intensity during the morning and performed their activities at a higher intensity compared to physically inactive loved ones (e-Figure 4; supplementary material). 
Table 4 General characteristics of COPD patients with a physically active loved one and those with a physically inactive loved one

\begin{tabular}{|c|c|c|c|}
\hline Characteristics & $\begin{array}{c}\text { COPD patients with a } \\
\text { physically active loved } \\
\text { one }(n=38)\end{array}$ & $\begin{array}{c}\text { COPD patients with a } \\
\text { physically inactive loved } \\
\text { one }(n=87)\end{array}$ & P-value \\
\hline Male sex, $\mathrm{n}(\%)$ & $22(58)$ & $47(54)$ & 0.69 \\
\hline Age, years & $64 \pm 8$ & $68 \pm 9$ & 0.03 \\
\hline Relationship with the loved one & & & 0.28 \\
\hline Partners (married or not), $\mathrm{n}(\%)$ & $38(100)$ & $84(97)$ & \\
\hline $\begin{array}{l}\text { Loved one is the son/daughter of the } \\
\text { patient, } n(\%)\end{array}$ & $0(0)$ & $2(2)$ & \\
\hline Friends, n (\%) & $0(0)$ & $1(1)$ & \\
\hline Working situation & & & 0.60 \\
\hline Paid job, n (\%) & $6(16)$ & $7(8)$ & \\
\hline Retired, n (\%) & $17(45)$ & $46(53)$ & \\
\hline Household work, n (\%) & $5(13)$ & $13(15)$ & \\
\hline Unable to work, n (\%) & $10(26)$ & $16(18)$ & \\
\hline Other (volunteer or unemployed), n (\%) & $0(0)$ & $5(6)$ & \\
\hline Current smoker, n (\%) & $8(21)$ & $15(17)$ & 0.61 \\
\hline Time living together, years & $40(30-45)$ & $43(32-47)$ & 0.08 \\
\hline Receiving informal care from relatives, $\mathrm{n}(\%)$ & $6(16)$ & $9(10)$ & 0.39 \\
\hline Rollator, n (\%) & $9(24)$ & $27(31)$ & 0.40 \\
\hline Cane, n (\%) & $6(16)$ & $21(24)$ & 0.30 \\
\hline Long-term oxygen therapy, n (\%) & $11(29)$ & $22(25)$ & 0.67 \\
\hline Exacerbations last 12 months, $\mathrm{n}$ & $2(1-3)$ & $2(0-4)$ & 0.63 \\
\hline Charlson Comorbidity Index, points & $2(1-3)$ & $2(1-3)$ & 0.31 \\
\hline Medications in use, $\mathrm{n}$ & $6(4-10)$ & $7(5-11)$ & 0.15 \\
\hline $\mathrm{BMI}, \mathrm{kg} \cdot \mathrm{m}^{-2}$ & $23.7(21.5-27.4)$ & $25.8(22.8-29.4)$ & 0.03 \\
\hline $\mathrm{BMI}<21 \mathrm{~kg} \cdot \mathrm{m}^{-2}, \mathrm{n}(\%)$ & $9(24)$ & $13(15)$ & 0.24 \\
\hline $\mathrm{BMI} \geq 30 \mathrm{~kg} \cdot \mathrm{m}^{-2}, \mathrm{n}(\%)$ & $6(16)$ & $19(22)$ & 0.44 \\
\hline $\mathrm{FEV}_{1}, \%$ predicted & $40(30-61)$ & $54(36-65)$ & 0.01 \\
\hline GOLD $2 / 3 / 4, n(\%)$ & $16(42) / 13(34) / 9(24)$ & $51(59) / 26(30) / 10(11)$ & 0.046 \\
\hline GOLD A / B / C / D, n (\%) & $2(5) / 9(24) / 1(3) / 26(68)$ & $3(4) / 23(27) / 1(1) / 58(68)$ & 0.99 \\
\hline FFM, $\mathrm{kg}^{*}$ & $49.6(44.8-53.8)$ & $52.0(45.9-56.4)$ & 0.22 \\
\hline $\mathrm{FFMI}, \mathrm{kg} \cdot \mathrm{m}^{-2^{*}}$ & $17.9(16.4-19.0)$ & $18.1(17.5-19.5)$ & 0.19 \\
\hline $\mathrm{FFMI}<5^{\text {th }}$ percentile, $\mathrm{n}(\%)^{*}$ & $4(11)$ & $13(17)$ & 0.49 \\
\hline TUG time, s & $9.5(8.3-11.8)$ & $10.0(8.6-11.7)$ & 0.30 \\
\hline TUG time >11 s, n (\%) & $11(29)$ & $27(31)$ & 0.79 \\
\hline mMRC, points & $3(2-4)$ & $2(1-3)$ & 0.19 \\
\hline CAT, points & $20 \pm 6$ & $21 \pm 7$ & 0.35 \\
\hline
\end{tabular}




\begin{tabular}{lccc}
\hline EQ-5D index score, points & $0.78(0.59-0.93)$ & $0.78(0.69-0.86)$ & 0.90 \\
CDS, points & $71(64-75)$ & $71(68-74)$ & 0.83 \\
Care dependent (CDS $\leq 68$ points), $n(\%)$ & $14(38)$ & $28(32)$ & 0.54 \\
HADS Anxiety, points & $5(3-7)$ & $6(2-8)$ & 0.65 \\
HADS Anxiety $\geq 10$ points, $n(\%)$ & $5(13)$ & $13(15)$ & 0.79 \\
HADS Depression, points & $5(2-8)$ & $5(3-8)$ & 0.36 \\
HADS Depression $\geq 10$ points, $n(\%)$ & $5(13)$ & $16(18)$ & 0.47
\end{tabular}

Data expressed as absolute and relative frequency, mean $\pm S D$, or median (IQR). See Table 1 for definition of abbreviations. ${ }^{*}=35$ for COPD patients with a physically active loved one and $n=79$ for COPD patients with a physically inactive loved one.

Table 5 Physical activity, sedentary behaviour and exercise motivation of COPD patients with a physically active loved one and those with a physically inactive loved one

\begin{tabular}{|c|c|c|c|}
\hline Characteristics & $\begin{array}{l}\text { COPD patients with a } \\
\text { physically active loved } \\
\text { one }(n=38)\end{array}$ & $\begin{array}{l}\text { COPD patients with a } \\
\text { physically inactive loved } \\
\text { one }(n=87)\end{array}$ & P-value \\
\hline Season of assessment Winter / Spring / & $8(21) / 5(13) / 5(13) /$ & $19(22) / 8(9) / 22(25)$ & 0.78 \\
\hline Summer / Autumn, n (\%) & $20(53)$ & / 38 (44) & \\
\hline Time in sedentary behaviour, $\mathrm{min} \cdot \mathrm{day}^{-1}$ & $600(582,618)$ & $617(606,629)$ & 0.13 \\
\hline Time in light activities, min $\cdot d_{a y}{ }^{-1}$ & $88(75,101)$ & $84(75,92)$ & 0.60 \\
\hline Time in MVPA, min.day ${ }^{-1}$ & $31(24,38)$ & $18(14,22)$ & 0.002 \\
\hline$\geq 30$ min of MVPA on $\geq 5$ days, $n$ (\%) & $11(29)$ & $10(12)$ & 0.02 \\
\hline $\begin{array}{l}\text { Proportion of daily time in sedentary } \\
\text { behaviour, } \%\end{array}$ & $83(81,86)$ & $86(84,88)$ & 0.10 \\
\hline Proportion of daily time in light activities, $\%$ & $12(10,14)$ & $12(11,13)$ & 0.60 \\
\hline Proportion of daily time in MVPA, \% & $4(3,5)$ & $3(2,3)$ & 0.003 \\
\hline Time in $\geq 10$-min bouts of MVPA, min $\cdot$ day $^{-1}$ & $10(7,14)$ & $5(3,7)$ & 0.02 \\
\hline $\begin{array}{l}\text { Frequency of } \geq 10 \text {-min bouts of MVPA, } \\
\text { bouts.day }{ }^{-1}\end{array}$ & $0.6(0.4,0.8)$ & $0.3(0.2,0.4)$ & 0.004 \\
\hline $\begin{array}{l}\text { Duration of } \geq 10-\mathrm{min} \text { bouts of MVPA, } \\
{\mathrm{min} \cdot \text { bout }^{-1}}\end{array}$ & $19(16,23)$ & $14(12,17)$ & 0.03 \\
\hline $\begin{array}{l}\text { Time in non-weight bearing activities, } \\
\text { min.day }{ }^{-1}\end{array}$ & $512(484,540)$ & $509(491,527)$ & 0.86 \\
\hline Time in weight-bearing activities, $\min \cdot \mathrm{day}^{-1}$ & $143(121,166)$ & $157(143,172)$ & 0.31 \\
\hline Time in dynamic activities, min $\cdot$ day $^{-1}$ & $63(53,73)$ & $52(46,59)$ & 0.08 \\
\hline Television-viewing time, $\mathrm{h} \cdot \mathrm{day}^{-1}$ & $4.1(3.4,4.8)$ & $3.8(3.3,4.2)$ & 0.43 \\
\hline \multicolumn{4}{|l|}{ Exercise motivation, points } \\
\hline Amotivation & $0.70(0.41,1.00)$ & $0.63(0.44,0.82)$ & 0.70 \\
\hline External regulation & $0.84(0.55,1.14)$ & $0.82(0.63,1.01)$ & 0.90 \\
\hline Introjected regulation & $1.26(0.94,1.58)$ & $1.26(1.05,1.47)$ & 0.99 \\
\hline Identified regulation & $2.63(2.31,2.94)$ & $2.71(2.50,2.91)$ & 0.67 \\
\hline Intrinsic regulation & $2.80(2.46,3.13)$ & $2.72(2.50,2.94)$ & 0.72 \\
\hline
\end{tabular}

Data expressed as absolute and relative frequency or mean $(95 \% \mathrm{Cl})$. See Table 2 for definition of abbreviations. ": after controlling for age, body mass index and forced expiratory volume in the first second. 

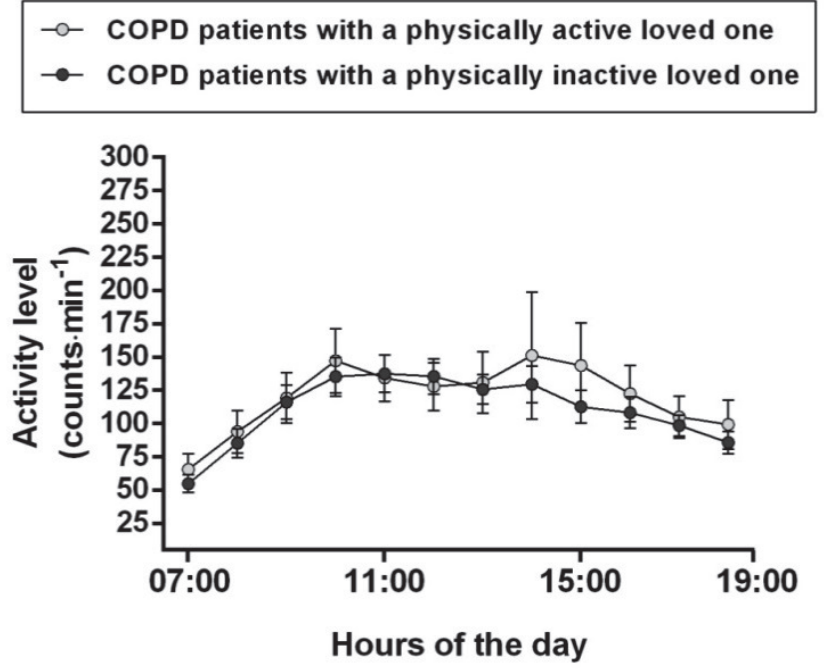

Figure 2 Daily physical activity hourly patterns of COPD patients with a physically active resident loved one and COPD patients with a physically inactive resident loved one. Data presented as mean ( $95 \%$ confidence intervals).

\section{DISCUSSION}

This is the first study to focus on physical activities, sedentary behaviour and exercise motivation in COPD patients and their resident loved ones. Despite relatively similar exercise motivation, COPD patients were found to be less active and more sedentary than their loved ones. Nevertheless, patients with an active loved one were more physically active than those with an inactive loved one.

We observed higher levels of physical activity and a higher likelihood of being physically active among patients with a physically active loved one, and these are probably the most relevant findings of our study. Resident loved ones can play an important role in helping patients to engage in healthy behaviours. Family-based interventions in other populations have shown to be useful to achieve healthy behaviours ${ }^{16,17}$. Family-based interventions target the family setting in which disease management takes place and address the educational, relational, and personal needs of all family members, and not only of the diseased subject ${ }^{18}$. Marques et al. ${ }^{19}$ recently showed that engaging family members of patients with COPD as part of a pulmonary rehabilitation programme led to improvements in coping strategies in both patients and family members. With the provision of appropriate information and instructions, resident loved ones can facilitate a more physically active lifestyle in patients with COPD, for example, by encouraging them to have joint daily walks or to engage more in household activities. We observed more 
physically active patients among those with a physically active loved one ( $29 \%$ vs $10 \%$ ). However, this means that $71 \%$ of these patients are still physically inactive while their resident loved ones are physically active. Patients in this subgroup are probably the ones who would benefit most from a family-based intervention by encouragements provided by their physically active loved ones. It is important to mention that familybased interventions alone are probably not enough to counteract the physical inactivity observed in COPD patients, as this is a complex behaviour influenced by different factors. Probably a combination of strategies is necessary to achieve greater and sustainable improvements ${ }^{20}$.

Although patients with a physically active loved one were found to be more active than those with a physically inactive loved one, similar levels of (high) sedentariness were observed in both the groups. This suggests that having a physically active loved one does not prevent patients from being sedentary, and that sedentariness should be reduced in both groups. Epidemiological data suggests that engaging in MVPA is not enough to fully protect against the detrimental consequences of prolonged periods of sedentary behaviour ${ }^{21,22}$. We have previously suggested that a useful strategy to increase MVPA in COPD patients might be to reduce the time in sedentary behaviour by increasing the time in light activities ${ }^{6,23}$. Reductions in sedentary time by increases in light activities could help pave the way to posterior increases in MVPA ${ }^{6,24}$. This strategy could be useful for resident loved ones as well, as $70 \%$ of them failed to reach the minimal level to be considered as physically active. This amount is higher than that reported previously for European older adults based on data from self-reports (30-50\%) ${ }^{25}$, but similar to more recent findings using objectively measured data (around $72 \%)^{26}$.

In our study we also investigated daily activity hourly patterns, which have not been widely explored in COPD. We observed a similar pattern between patients and their loved ones, although the latter group performed their activities at a higher intensity. When we compared groups of patients according to the physical activity level of their loves ones, a similar flattened pattern was observed in both groups suggesting more sustained activity throughout the day. We believe that the higher level of MVPA observed in patients with a physically active loved one (please see Table 5) is probably spread throughout the day, which possibly explains why this was not observed in the hourly patterns. Nevertheless, as highlighted previously, daily hourly patterns seem to provide complementary information to activity measures ${ }^{23}$.

Despite presenting with different levels of physical activity and sedentariness, COPD patients and their loved ones showed relatively similar exercise motivation. Although an association between activity levels and exercise motivation could be hypothesised, a very poor relationship between these outcomes was previously reported ${ }^{27}$. Intrinsic regulation was the only construct to differ between these groups, with COPD patients 
showing less of this type of regulation (Table 3). Intrinsic regulation refers to engaging in exercise for the enjoyment or inherent satisfaction of itself ${ }^{15}$. We believe COPD patients showed less of this type of regulation because of the discomfort caused by shortness of breath during exercise ${ }^{5}$, which prevents them from enjoying this activity. Regardless of the reason, a previous study observed that intrinsic motivation for exercise in COPD can be improved with daily physical activity counselling ${ }^{28}$.

Strengths from the present study include the considerable number of participants (125 COPD patients and 125 loved ones) with objective measurement of activity levels and simultaneous assessment during a full week. Nevertheless, some limitations are acknowledged. Our sample is composed mainly by patients from secondary care, which may limit extending our findings to primary or tertiary care patients ${ }^{29}$. Moreover, activity monitoring was restricted to the leg, which means that activities of the upper arms were underrepresented. Nevertheless, previous findings suggest that leg activities seem to be more impaired than arm activities in COPD ${ }^{30}$.

In summary, COPD patients are less physically active and more sedentary than their resident loved ones, despite relatively similar exercise motivation. Nonetheless, patients with a physically active loved one spend more time in physical activity and have a higher likelihood of being physically active than those with a physically inactive loved one. This suggests that patients' resident loved ones could be used as a way to facilitate a more physically active lifestyle by patients. Future studies are warranted on the efficacy of family-based physical activity counselling in patients with COPD and their resident loved ones. 


\section{REFERENCES}

1. Annegarn J, Meijer K, Passos VL, et al. Problematic activities of daily life are weakly associated with clinical characteristics in COPD. J Am Med Dir Assoc. 2012;13(3):284-290.

2. Pitta F, Troosters T, Spruit MA, Probst VS, Decramer M, Gosselink R. Characteristics of physical activities in daily life in chronic obstructive pulmonary disease. Am J Respir Crit Care Med. 2005;171(9):972-977.

3. Cavalheri V, Straker L, Gucciardi DF, Gardiner PA, Hill K. Changing physical activity and sedentary behaviour in people with COPD. Respirology. 2016;21(3):419-426.

4. Van Remoortel $H$, Hornikx M, Langer $D$, et al. Risk factors and comorbidities in the preclinical stages of chronic obstructive pulmonary disease. Am J Respir Crit Care Med. 2014;189(1):30-38.

5. Vestbo J, Hurd SS, Agusti AG, et al. Global strategy for the diagnosis, management, and prevention of chronic obstructive pulmonary disease: GOLD executive summary. Am J Respir Crit Care Med. 2013;187(4):347-365.

6. Vanfleteren LE, Spruit MA, Wouters EF, Franssen FM. Management of chronic obstructive pulmonary disease beyond the lungs. Lancet Respir Med. 2016;4(11):911-924.

7. Spruit MA, Pitta F, McAuley E, ZuWallack RL, Nici L. Pulmonary Rehabilitation and Physical Activity in Patients with Chronic Obstructive Pulmonary Disease. Am J Respir Crit Care Med. 2015;192(8):924-933.

8. Burtin C, Langer D, van Remoortel H, et al. Physical Activity Counselling during Pulmonary Rehabilitation in Patients with COPD: A Randomised Controlled Trial. PLoS One. 2015;10(12):e0144989.

9. Vaes AW, Cheung A, Atakhorrami M, et al. Effect of 'activity monitor-based' counseling on physical activity and health-related outcomes in patients with chronic diseases: A systematic review and meta-analysis. Ann Med. 2013;45(5-6):397-412.

10. King AC. Interventions to promote physical activity by older adults. J Gerontol A Biol Sci Med Sci. 2001;56 Spec No 2:36-46.

11. Nakken N, Spruit MA, van den Bogaart EH, et al. Health Status and Morbidities in Resident Relatives of Patients With COPD. J Am Med Dir Assoc. 2016;17(3):276 e271-278.

12. Nakken $N$, Janssen DJ, van den Bogaart EH, Vercoulen JH, Wouters EF, Spruit MA. An observational, longitudinal study on the home environment of people with chronic obstructive pulmonary disease: the research protocol of the Home Sweet Home study. BMJ Open. 2014;4(11):e006098.

13. Smid DE, Wilke S, Jones PW, et al. Impact of cardiovascular comorbidities on COPD Assessment Test (CAT) and its responsiveness to pulmonary rehabilitation in patients with moderate to very severe COPD: protocol of the Chance study. BMJ Open. 2015;5(7):e007536.

14. Mesquita R, Janssen DJ, Wouters EF, Schols JM, Pitta F, Spruit MA. Within-day test-retest reliability of the timed up \& go test in patients with advanced chronic organ failure. Arch Phys Med Rehabil. 2013;94(11):2131-2138.

15. Mesquita R, Wilke S, Smid DE, et al. Measurement properties of the Timed Up \& Go test in patients with COPD. Chron Respir Dis. 2016;13(4):344-352.

16. Markland D, Tobin V. A modification to the Behavioural Regulation in Exercise Questionnaire to include an assessment of amotivation. J Sport Exerc Psychol. 2004;26(2):191-196.

17. Dolan P. Modeling valuations for EuroQol health states. Med Care. 1997;35(11):1095-1108.

18. Jones PW, Harding G, Berry P, Wiklund I, Chen WH, Kline Leidy N. Development and first validation of the COPD Assessment Test. Eur Respir J. 2009;34(3):648-654.

19. Dijkstra A, Tiesinga LJ, Goossen WT, Dassen TW. Further psychometric testing of the Dutch Care Dependency Scale on two different patient groups. Int J Nurs Pract. 2002;8(6):305-314.

20. Zigmond AS, Snaith RP. The hospital anxiety and depression scale. Acta Psychiatr Scand. 1983;67(6):361370.

21. Annegarn J, Spruit MA, Uszko-Lencer NH, et al. Objective physical activity assessment in patients with chronic organ failure: a validation study of a new single-unit activity monitor. Arch Phys Med Rehabil. 2011;92(11):1852-1857 e1851. 
22. van der Weegen S, Essers $H$, Spreeuwenberg M, et al. Concurrent Validity of the MOX Activity Monitor Compared to the ActiGraph GT3X. Telemed J E Health. 2015;21(4):259-266.

23. Watz H, Waschki B, Meyer T, Magnussen H. Physical activity in patients with COPD. Eur Respir J. 2009;33(2):262-272.

24. Garber CE, Blissmer B, Deschenes MR, et al. American College of Sports Medicine position stand. Quantity and quality of exercise for developing and maintaining cardiorespiratory, musculoskeletal, and neuromotor fitness in apparently healthy adults: guidance for prescribing exercise. Med Sci Sports Exerc. 2011;43(7):1334-1359.

25. Viitasalo A, Eloranta AM, Lintu N, et al. The effects of a 2-year individualized and family-based lifestyle intervention on physical activity, sedentary behavior and diet in children. Prev Med. 2016;87:81-88.

26. Hu J, Wallace DC, McCoy TP, Amirehsani KA. A family-based diabetes intervention for Hispanic adults and their family members. Diabetes Educ. 2014;40(1):48-59.

27. Fisher L, Weihs KL. Can addressing family relationships improve outcomes in chronic disease? Report of the National Working Group on Family-Based Interventions in Chronic Disease. J Fam Pract. 2000;49(6):561-566.

28. Marques A, Jacome C, Cruz J, Gabriel R, Brooks D, Figueiredo D. Family-based psychosocial support and education as part of pulmonary rehabilitation in COPD: a randomized controlled trial. Chest. 2015;147(3):662-672.

29. Thorpe O, Kumar S, Johnston K. Barriers to and enablers of physical activity in patients with COPD following a hospital admission: a qualitative study. Int J Chron Obstruct Pulmon Dis. 2014;9:115-128.

30. Mesquita RB, Morano MT, Landim FL, Collares PM, Pinto JM. Social support network and health of elderly individuals with chronic pneumopathies [Article in Portuguese]. Cien Saude Colet. 2012;17(5):1125-1133.

31. Mantoani LC, Rubio N, McKinstry B, MacNee W, Rabinovich RA. Interventions to modify physical activity in patients with COPD: a systematic review. Eur Respir J. 2016;48(1):69-81.

32. Healy GN, Dunstan DW, Salmon J, Shaw JE, Zimmet PZ, Owen N. Television time and continuous metabolic risk in physically active adults. Med Sci Sports Exerc. 2008;40(4):639-645.

33. Healy GN, Matthews CE, Dunstan DW, Winkler EA, Owen N. Sedentary time and cardio-metabolic biomarkers in US adults: NHANES 2003-06. Eur Heart J. 2011;32(5):590-597.

34. Mesquita R, Spina G, Pitta F, et al. Physical activity patterns and clusters in 1001 patients with COPD [published online ahead of print February 24, 2017]. Chron Respir Dis. http://dx.doi.org/ 10.1177/1479972316687207.

35. Sparling PB, Howard BJ, Dunstan DW, Owen N. Recommendations for physical activity in older adults. BMJ. 2015;350:h100.

36. Hallal PC, Andersen LB, Bull FC, et al. Global physical activity levels: surveillance progress, pitfalls, and prospects. Lancet. 2012;380(9838):247-257.

37. Lohne-Seiler H, Hansen BH, Kolle E, Anderssen SA. Accelerometer-determined physical activity and selfreported health in a population of older adults (65-85 years): a cross-sectional study. BMC Public Health. 2014;14:284.

38. Altenburg WA, Bossenbroek L, de Greef MH, Kerstjens HA, ten Hacken NH, Wempe JB. Functional and psychological variables both affect daily physical activity in COPD: a structural equations model. Respir Med. 2013;107(11):1740-1747.

39. Hospes G, Bossenbroek L, Ten Hacken NH, van Hengel P, de Greef MH. Enhancement of daily physical activity increases physical fitness of outclinic COPD patients: results of an exercise counseling program. Patient Educ Couns. 2009;75(2):274-278.

40. Smid DE, Spruit MA, Houben-Wilke $S$, et al. Burden of COPD in patients treated in different care settings in the Netherlands. Respir Med. 2016;118:76-83.

41. Shrikrishna D, Patel M, Tanner RJ, et al. Quadriceps wasting and physical inactivity in patients with COPD. Eur Respir J. 2012;40(5):1115-1122.

42. Meijer K, Annegarn J, Lima Passos V, et al. Characteristics of daily arm activities in patients with COPD. Eur Respir J. 2014;43(6):1631-1641.

43. Perneger TV. What's wrong with Bonferroni adjustments. BMJ. 1998;316(7139):1236-1238. 


\section{CHAPTER 9}

Reprinted from Chest, Vol. 151, MesquitaR, Nakken N, Janssen DJA, van den Bogaart EHA, Delbressine JML, Essers JMN, Meijer K, van Vliet M, de Vries GJ, Muris JWM, Pitta F, Wouters EFM, Spruit MA, Activity levels and exercise motivation in COPD patients and their resident loved ones, pp. 1028-38, Copyright (2017), with permission from Elsevier. 


\section{SUPPLEMENTARY MATERIAL}

\section{MATERIALS AND METHODS}

\section{Assessments}

As part of demographics, chronic obstructive pulmonary disease (COPD) patients and their resident loved ones had recorded their age, sex, relationship with the patient/loved one, working situation (paid job, retired, household work, unable to work, or other), time living together with the patient/loved one, and if they were receiving informal care from relatives at the time of the assessment (yes or no). Clinical data included the assessment of smoking status (current smoking, yes or no), number of medications in use, and comorbidity burden using the Charlson Comorbidity Index. This index is used for the assessment of current self-reported comorbidities and is composed by 19 categories of comorbidities ${ }^{1}$. The total score reflects the cumulative increased likelihood of 1-year mortality and a higher score means a higher burden of comorbidities ${ }^{2}$. Patients only were also inquired if they were using long-term oxygen therapy or if they had walking aids (rollator or cane), as well as the number of exacerbations (with or without hospitalisation) in the previous 12 months. Also in patients only, symptoms of dyspnoea were assessed with the modified Medical Research Council (mMRC) scale, which measures the intensity of dyspnoea during activities of daily living in scores from 0 to 4 with higher scores indicating more dyspnoea ${ }^{3}$.

Lung function was assessed with post-bronchodilator spirometry using a handheld spirometer (SpiroPro ${ }^{\circledR}$ Viasys, Jaeger, Hoechberg, Germany). Forced vital capacity (FVC) and forced expiratory volume in the first second $\left(\mathrm{FEV}_{1}\right)$ were measured and the ratio between $\mathrm{FEV}_{1}$ and FVC calculated. Persistent airflow limitation was defined as a postbronchodilator $\mathrm{FEV}_{1} / \mathrm{FVC}$ ratio $<70 \%{ }^{4}$. International recommendations were followed ${ }^{5}$ and the results were compared to reference values ${ }^{6}$. Additionally, based on the degree of airflow limitation (i.e., $\mathrm{FEV}_{1}$ in \% predicted) COPD patients were classified into Global Initiative for Chronic Obstructive Lung Disease (GOLD) grades 2 to 4 according to the 2007 GOLD classification ${ }^{7}$ : GOLD grade 2 (50\% $\mathrm{FEV}_{1}<80 \%$ predicted); GOLD grade 3 ( $30 \% \leq \mathrm{FEV}_{1}<50 \%$ predicted); and GOLD grade 4 ( $\mathrm{FEV}_{1}<30 \%$ predicted). GOLD grade 1 patients were not considered in the current analyses since patients with a $\mathrm{FEV}_{1} \geq 80 \%$ predicted were not included in the Home Sweet Home study. COPD patients were also classified into GOLD groups A to D according to the 2011 GOLD classification ${ }^{4}$. For this classification the degree of airflow limitation (GOLD grades), the number of exacerbations in the previous 12 months, and symptoms as assessed by the COPD assessment 
test (CAT) were taken into account. GOLD grades 2-4 or exacerbations in the previous 12 months were used to classify patients as low (group A or B) or high (group C or D) risk. Patients classified as GOLD grade 2 or with $<2$ exacerbations in the previous year were classified as low risk, whilst those with GOLD grade 3 or 4 or with $\geq 2$ exacerbations in the previous year were classified as high risk; the criterion with the worse score was used for further analyses ${ }^{4}$. The CAT was used to classify patients as less (group A or C) or more (group B or D) symptoms ${ }^{4}$. Patients with a CAT score $<10$ points were classified as less symptoms, whilst those with a score $\geq 10$ points were classified as more symptoms ${ }^{4}$. Further details about the CAT are provided in paragraphs ahead.

Body composition was assessed based on body mass index (BMI), fat-free mass (FFM) and fat-free mass index (FFMI). BMI was calculated as weight per squared height $\left(\mathrm{kg} \cdot \mathrm{m}^{-}\right.$ $\left.{ }^{2}\right)^{8}$. Underweight was defined as a BMI $<21 \mathrm{~kg} \cdot \mathrm{m}^{-2}$ as this cut-off has been used in multidimensional indices to predict mortality in $\mathrm{COPD}^{9,10}$. Obesity was defined as a $\mathrm{BMI} \geq 30$ $\mathrm{kg} \cdot \mathrm{m}^{-2}$ according to the World Health Organization definition ${ }^{11}$. FFM was measured using body impedance analysis, as previously described ${ }^{12}$. FFMI was calculated as FFM per squared height $\left(\mathrm{kg} \cdot \mathrm{m}^{-2}\right)$ and low muscle mass was defined as a FFMI $<5^{\text {th }}$ percentile according to the reference values of Franssen et al. ${ }^{13}$.

The Timed Up \& Go (TUG) test was used for the assessment of functional mobility. This test has been shown to be reliable and valid in COPD ${ }^{14,15}$. As described in the original protocol, participants were required to stand up from a chair, walk at a comfortable and safe speed a distance of 3 metres, turn and walk back to the chair to sit down again ${ }^{16}$. During the test participants were instructed to wear their usual footwear, and walking aids and/or oxygen were allowed, if necessary. The time in seconds was recorded by ordinary stopwatches and used as the main outcome of analysis. Two trials were performed by the same assessor and the trial with the shortest duration was used for further analyses ${ }^{14}$. The cut-off point of $11 \mathrm{~s}$ was used in the current study as we previously observed worse health outcomes in patients with a TUG time higher than this threshold 15

Health status was assessed with two instruments, the generic EuroQol-5 Dimensions (EQ-5D) questionnaire and the disease-specific CAT. The EQ-5D is composed of 5 items (mobility, self-care, usual activity, pain/discomfort, anxiety/ depression) each with 3 levels (no problems, some problems, extreme problems) ${ }^{17}$. A final index score is provided and can range from -0.59 (worst) to 1.0 (best) ${ }^{17}$. The CAT comprises eight items raising questions regarding symptoms, energy, sleep and activity ${ }^{18}$. Scores can range from 0 (no impact) to 40 points (severe impact) ${ }^{18}$.

Care dependency was assessed with the care dependency scale (CDS). This instrument is composed of 15 items related to basic and instrumental activities of daily living ${ }^{19}$. A 
final score is provided and can range from 15 (worst) to 75 (best) points ${ }^{19}$. The cut-off of $\leq 68$ points was used to identify care dependent patients ${ }^{20}$.

Symptoms of anxiety and depression were assessed with the Hospital Anxiety and Depression Scale (HADS). This instrument has been used to screen clinically relevant symptoms of anxiety and/or depression ${ }^{21}$. It consists of 14 questions distributed in two subscales, 7 for the assessment of anxiety (HADS anxiety subscale) and 7 for the assessment of depression (HADS depression subscale) ${ }^{21}$. A total score in each subscale can range from 0 (optimal) to 21 (worst) ${ }^{21}$. In addition, a score $\geq 10$ points for each subscale was considered as clinically relevant symptoms of anxiety or depression.

Exercise motivation was assessed with the Behavioural Regulation and Exercise Questionnaire 2 (BREQ-2), which comprises 19 items distributed in five subscales: amotivation, external regulation, introjected regulation, identified regulation, and intrinsic regulation ${ }^{22}$. Amotivation is a completely non-self-determined regulation and can be understood as a state of lacking any intention to engage in exercise. External regulation relates to engaging in exercise in order to satisfy external pressures (e.g., from friends, family) or in order to achieve externally imposed rewards. Introjected regulation is observed when external controls are internalised and translated as self-imposed pressures in order to avoid guilt or to maintain self-esteem. Identified regulation is the conscious acceptance of exercising as an important behaviour to achieve personally valued outcomes. Finally, intrinsic regulation refers to engaging in exercise for the enjoyment, fun, interest, or inherent satisfaction of itself. Scores per subscale are provided and can range from 0.00 (not true for the participant) to 4.00 (very true for the participant) ${ }^{22}$.

Physical activity and sedentary behaviour were assessed with the MOX Activity Monitor (Maastricht Instruments B.V., Maastricht, the Netherlands), which has been validated in patients with COPD and healthy subjects ${ }^{23,24}$. The MOX (model MMOXX1.01) is a small activity monitor $(4.5 \times 4.0 \times 1.4 \mathrm{~cm})$ which measures acceleration data $( \pm 6 \mathrm{G})$ in three axes with a sample frequency of $25 \mathrm{~Hz}$. This device was attached to the frontal part of the participant's right leg, in line with the thigh midline and half way between the knee and hip. First, the device was waterproofed by the researcher in a finger cot and then

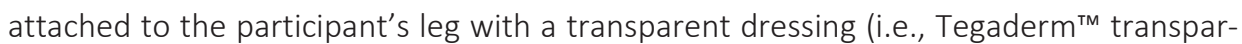
ent dressing). This allowed continuous wearing, even during water-based activities. Although participants used the device continuously, only data between 07:00 AM and 19:00 PM were used for analysis. Participants were instructed to keep the device for 7 consecutive days and send it back to the researchers by post mail with a self-addressed pre-paid envelope. Participants were also instructed to register in a form non-wearing periods or any problems/adverse side effects while wearing the device. This form was sent back to the researchers together with the activity monitors. After the devices were received back from the participants, the data were downloaded using an application 
developed by Maastricht Instruments B.V. and exported to an EXCEL worksheet with a Matlab R2012b (Mathworks Inc., USA) algorithm developed by the researchers. Nonwearing periods were identified using this algorithm and defined as continuous periods of $\geq 2-h$ with no activity in all three axes of the device.

Based on a study that used activity categories of the ActiGraph GT3X accelerometer as standard ${ }^{24}$, thresholds for sedentary behaviour $(<1.5$ metabolic equivalents of task (METs) 25), light physical activity (between 1.5 and 3.0 METs), and moderate-tovigorous physical activity (MVPA, >3.0 METs 26) were estimated and used to classify each 1-minute epoch of the data: sedentary behaviour, <220 activity counts; light physical activity, between 220 and 480 activity counts; and MVPA, >480 activity counts. Bouts of MVPA spent in $\geq 10$ minutes were also analysed. A bout of activity was defined as a period of consecutive minutes in MVPA with a minimum duration of 10 minutes. Time in $\geq 10$-min bouts of MVPA was calculated by summing up the time in $\geq 10$-min bouts performed during the course of a day, and then averaging it among all valid days. As some patients had days with no bouts, the averaging process may have led to averaged values which are lower than 10 minutes. Frequency of $\geq 10$-min bouts of MVPA was calculated by counting the number of $\geq 10$-min bouts performed during the course of a day, and then averaging it among all valid days (including those with no bouts). Duration of $\geq 10$-min bouts of MVPA was calculated by averaging the duration of all $\geq 10$ min bouts performed during the course of a day, and then averaging it only among days with bouts of activity. Daily physical activity hourly patterns were also analysed in the current study. They provide a graphic representation of the temporal trends of activity intensities over the course of a day ${ }^{27-29}$. First, the minute-by-minute data for each participant were averaged to one value representing all valid days (weekdays and weekend days combined). Then the 60 values from each hour were averaged to one value representing each hour. These values were then used to plot activity hourly patterns. Data in the hourly patterns graphs are presented as mean ( $95 \%$ confidence intervals). Daily television-viewing time was also analysed based on self-reports. 


\section{RESULTS}

\section{Complete baseline assessment HSH study}

194 patients with COPD and 194 loved ones

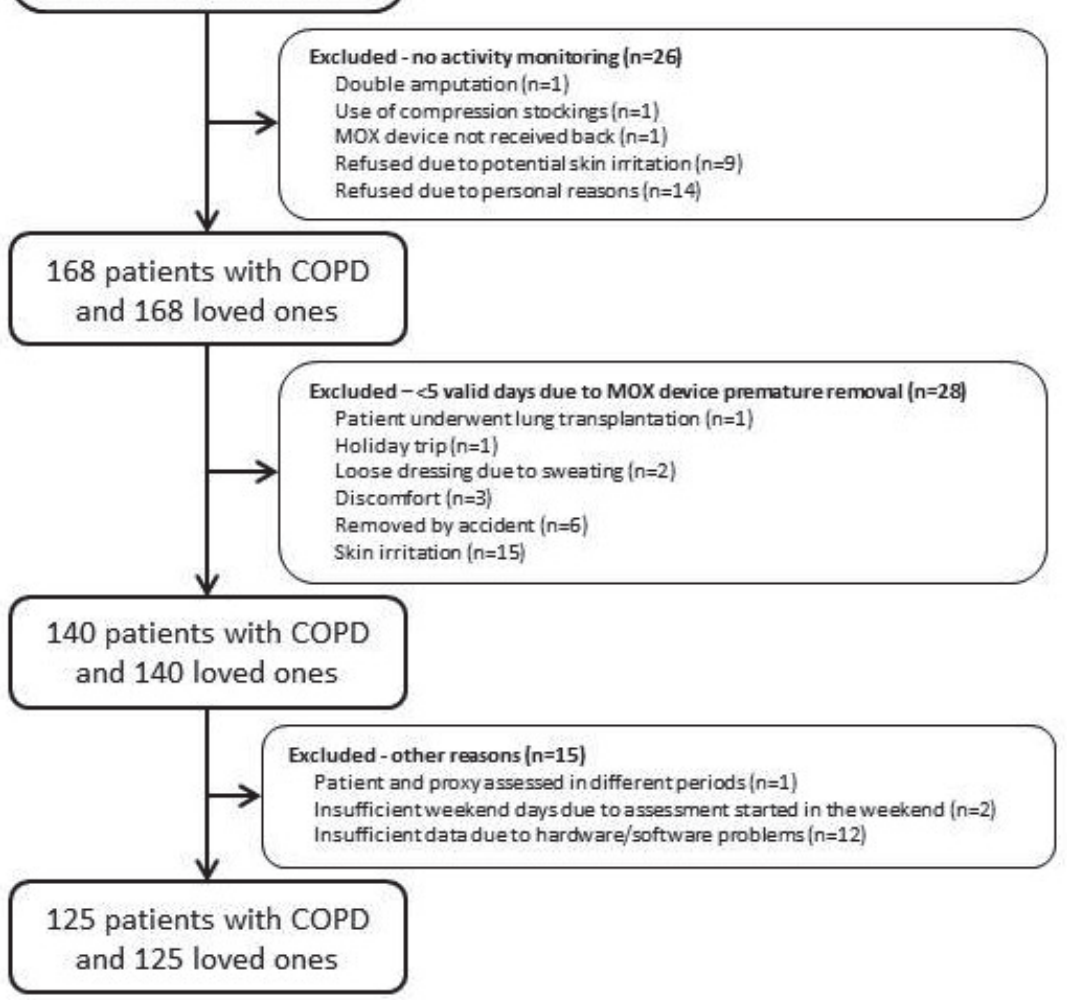

e-Figure 1 Flow-diagram of participants included in the current study. HSH: Home Sweet Home. 


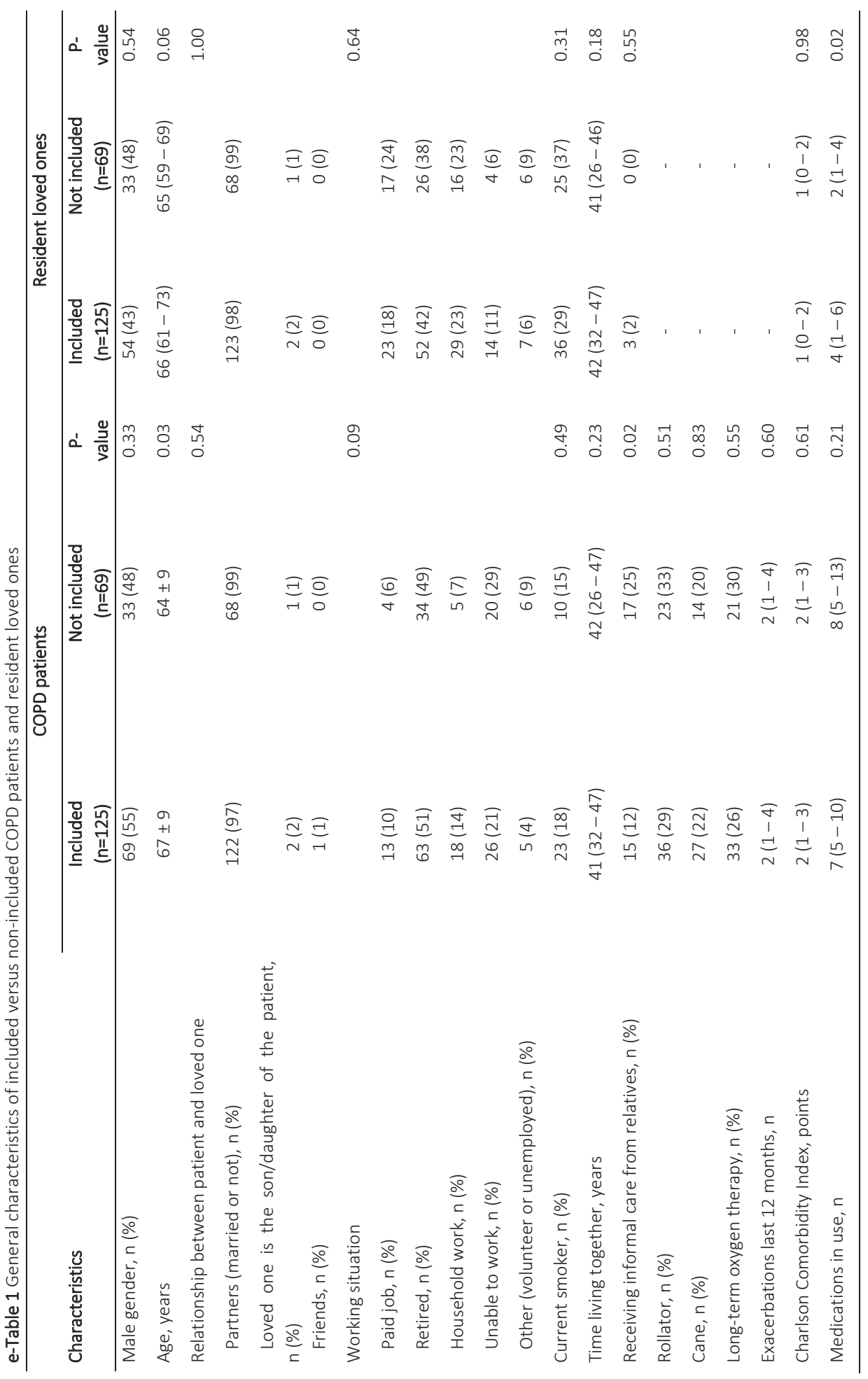




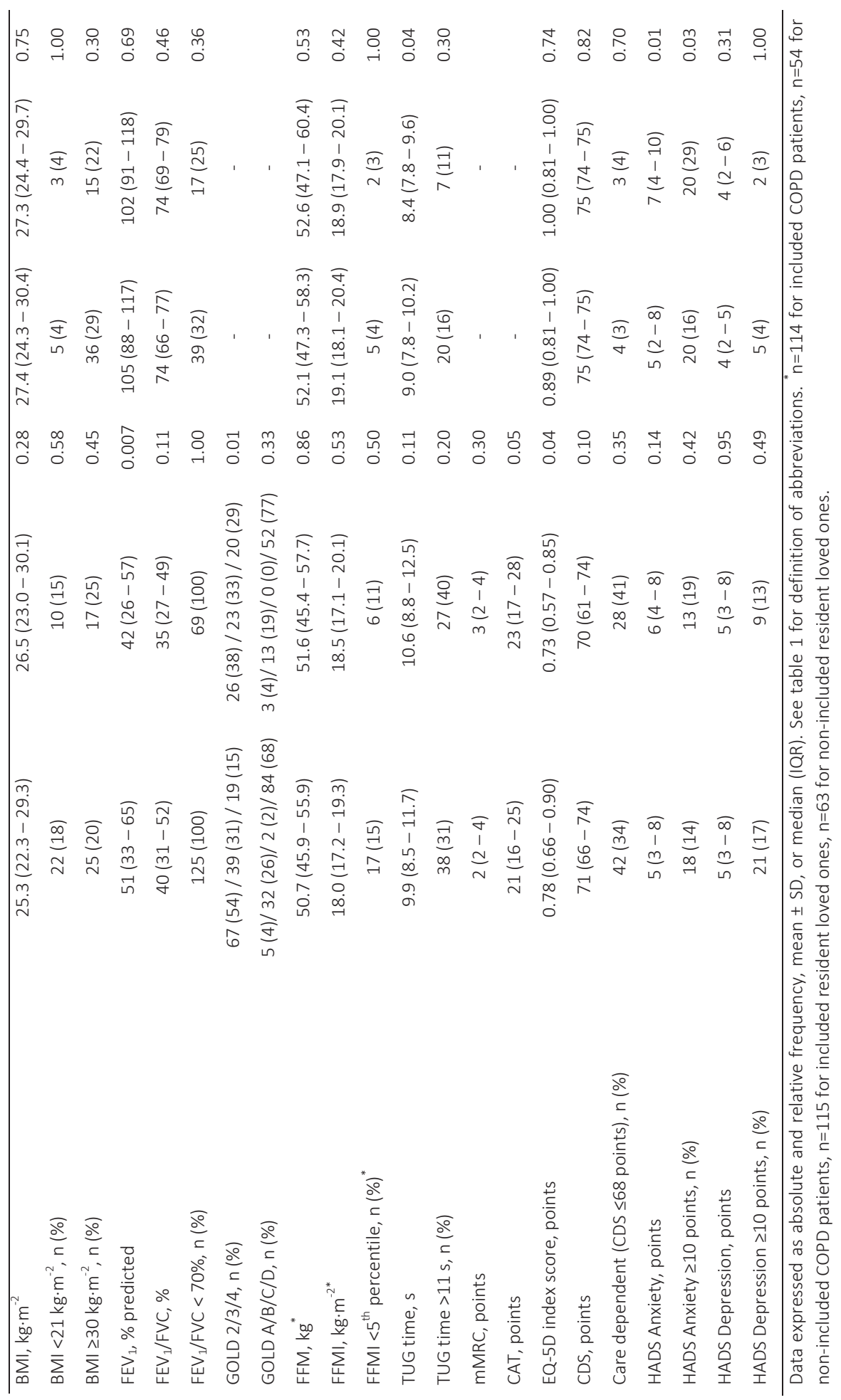




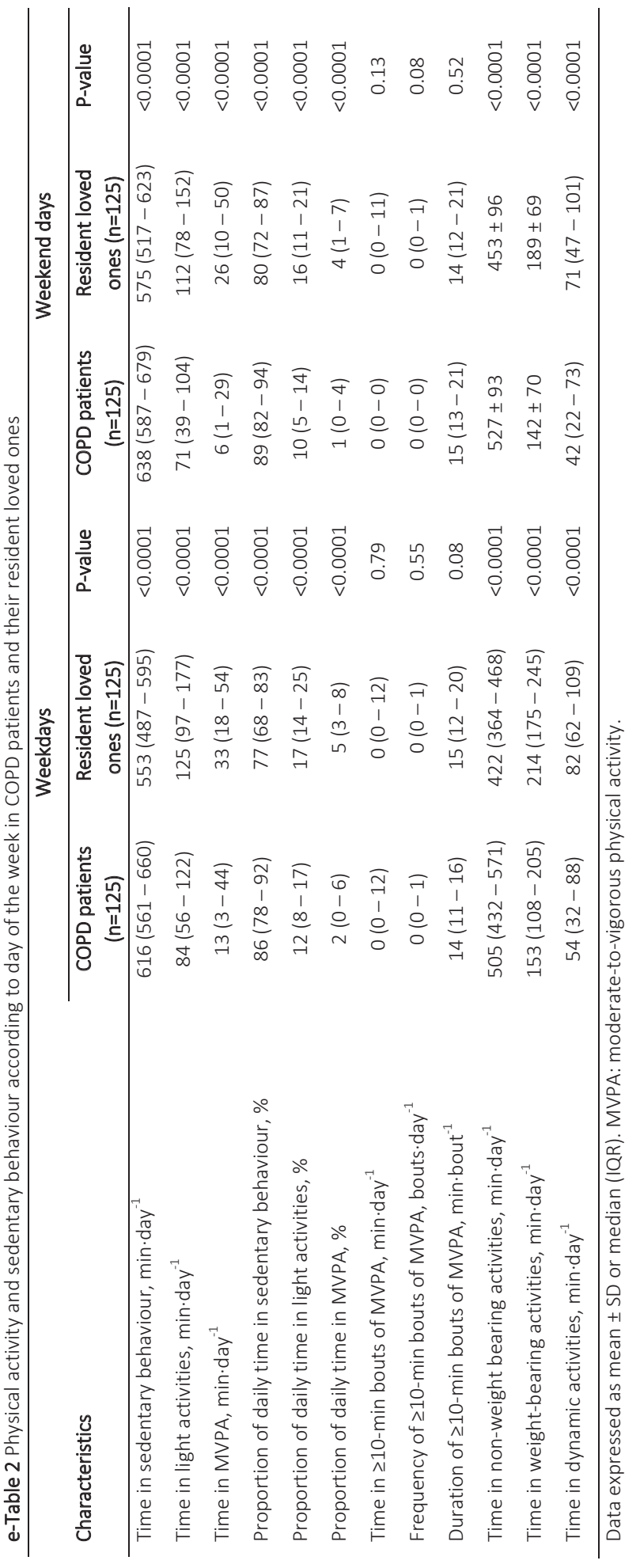


e-Table 3 General characteristics of physically active and physically inactive resident loved ones

\begin{tabular}{|c|c|c|c|}
\hline Characteristics & $\begin{array}{c}\text { Physically active loved } \\
\text { one }(n=38)\end{array}$ & $\begin{array}{l}\text { Physically inactive loved } \\
\text { one }(n=87)\end{array}$ & P-value \\
\hline Male sex, $\mathrm{n}(\%)$ & $16(42)$ & $38(44)$ & 0.87 \\
\hline Age, years & $64(61-68)$ & $67(62-74)$ & 0.03 \\
\hline Relationship with the patient & & & 1.00 \\
\hline Partners (married or not), n (\%) & $38(100)$ & $85(98)$ & \\
\hline $\begin{array}{l}\text { Loved one is the son/daughter of the } \\
\text { patient, } \mathrm{n}(\%)\end{array}$ & $0(0)$ & $2(2)$ & \\
\hline Friends, n (\%) & $0(0)$ & $0(0)$ & \\
\hline Working situation & & & 0.40 \\
\hline Paid job, n (\%) & $13(34)$ & $10(12)$ & \\
\hline Retired, n (\%) & $10(26)$ & $42(48)$ & \\
\hline Household work, n (\%) & $8(21)$ & $21(24)$ & \\
\hline Unable to work, n (\%) & $4(11)$ & $10(12)$ & \\
\hline Other (volunteer or unemployed), n (\%) & $3(8)$ & $4(4)$ & \\
\hline Current smoker, n (\%) & $7(18)$ & $29(34)$ & 0.08 \\
\hline Time living together, years & $40(29-45)$ & $43(34-48)$ & 0.08 \\
\hline Receiving informal care from relatives, $\mathrm{n}(\%)$ & $1(3)$ & $2(2)$ & 1.00 \\
\hline Charlson Comorbidity Index, points & $0(0-2)$ & $1(0-2)$ & 0.24 \\
\hline Medications in use, $\mathrm{n}$ & $2(1-4)$ & $4(2-6)$ & 0.006 \\
\hline $\mathrm{BMI}, \mathrm{kg} \cdot \mathrm{m}^{-2}$ & $27.6(24.8-30.2)$ & $27.4(23.8-30.5)$ & 0.85 \\
\hline $\mathrm{BMI}<21 \mathrm{~kg} \cdot \mathrm{m}^{-2}, \mathrm{n}(\%)$ & $1(3)$ & $4(4)$ & 1.00 \\
\hline $\mathrm{BMI} \geq 30 \mathrm{~kg} \cdot \mathrm{m}^{-2}, \mathrm{n}(\%)$ & $10(27)$ & $26(30)$ & 0.75 \\
\hline $\mathrm{FEV}_{1}, \%$ predicted & $103 \pm 20$ & $102 \pm 25$ & 0.91 \\
\hline $\mathrm{FEV}_{1} / \mathrm{FVC}, \%$ & $75(71-79)$ & $73(64-77)$ & 0.06 \\
\hline $\mathrm{FEV}_{1} / \mathrm{FVC}<70 \%, \mathrm{n}(\%)$ & $8(22)$ & $31(36)$ & 0.12 \\
\hline FFM, $\mathrm{kg}^{*}$ & $51.8(47.0-56.6)$ & $52.6(48.0-60.0)$ & 0.31 \\
\hline $\mathrm{FFMI}, \mathrm{kg} \cdot \mathrm{m}^{-2^{*}}$ & $19.1(18.2-19.9)$ & $19.0(18.0-20.4)$ & 0.76 \\
\hline FFMI $<5^{\text {th }}$ percentile, $\mathrm{n}(\%)^{*}$ & $0(0)$ & $5(6)$ & 0.32 \\
\hline TUG time, s & $8.5(7.4-10.0)$ & $9.1(8.4-10.6)$ & 0.03 \\
\hline TUG time $>11 \mathrm{~s}, \mathrm{n}(\%)$ & $3(8)$ & $17(20)$ & 0.11 \\
\hline EQ-5D index score, points & $0.95(0.81-1.00)$ & $0.89(0.81-1.00)$ & 0.75 \\
\hline CDS, points & $75(74-75)$ & $75(74-75)$ & 0.99 \\
\hline Care dependent (CDS $\leq 68$ points), $\mathrm{n}(\%)$ & $0(0)$ & $4(5)$ & 0.31 \\
\hline HADS Anxiety, points & $5(3-7)$ & $4(2-8)$ & 0.52 \\
\hline HADS Anxiety $\geq 10$ points, $n(\%)$ & $5(13)$ & $15(17)$ & 0.57 \\
\hline HADS Depression, points & $4(1-6)$ & $3(2-5)$ & 0.79 \\
\hline HADS Depression $\geq 10$ points, $n(\%)$ & $0(0)$ & $5(6)$ & 0.32 \\
\hline
\end{tabular}

Data expressed as absolute and relative frequency, mean \pm SD, or median (IQR). See Table 1 for definition of abbreviations. ${ }^{*} \mathrm{n}=34$ for physically active loved ones and $\mathrm{n}=81$ for physically inactive loved ones. 


\section{CHAPTER 9}

e-Table 4 Physical activity, sedentary behaviour and exercise motivation of physically active and physically inactive resident loved ones

\begin{tabular}{|c|c|c|c|}
\hline Characteristics & $\begin{array}{l}\text { Physically active loved } \\
\text { one }(n=38)\end{array}$ & $\begin{array}{l}\text { Physically inactive } \\
\text { loved one }(n=87)\end{array}$ & P-value \\
\hline Time in sedentary behaviour, $\min \cdot$ day $^{-1}$ & $475 \pm 59$ & $580 \pm 53$ & $<0.0001$ \\
\hline Time in light activities, min $\cdot$ day $^{-1}$ & $170(125-203)$ & $103(86-147)$ & $<0.0001$ \\
\hline Time in MVPA, min $\cdot d_{a y}{ }^{-1}$ & $72(46-97)$ & $22(13-32)$ & $<0.0001$ \\
\hline $\begin{array}{l}\text { Proportion of daily time in sedentary } \\
\text { behaviour, } \%\end{array}$ & $66 \pm 8$ & $81 \pm 7$ & $<0.0001$ \\
\hline Proportion of daily time in light activities, $\%$ & $24(17-28)$ & $14(12-20)$ & $<0.0001$ \\
\hline Proportion of daily time in MVPA, \% & $10(6-13)$ & $3(2-4)$ & $<0.0001$ \\
\hline Time in $\geq 10$-min bouts of MVPA, min day $^{-1}$ & $10(0-38)$ & $0(0-8)$ & $<0.0001$ \\
\hline $\begin{array}{l}\text { Frequency of } \geq 10-\min \text { bouts of MVPA, } \\
\text { bouts.day }{ }^{-1}\end{array}$ & $1(0-2)$ & $0(0-1)$ & $<0.0001$ \\
\hline $\begin{array}{l}\text { Duration of } \geq 10-\min \text { bouts of MVPA, } \\
\text { min'bout }^{-1}\end{array}$ & $17(13-22)$ & $15(11-19)$ & 0.23 \\
\hline $\begin{array}{l}\text { Time in non-weight bearing activities, } \\
\text { min.day }{ }^{-1}\end{array}$ & $360 \pm 77$ & $457 \pm 73$ & $<0.0001$ \\
\hline Time in weight-bearing activities, $\min \cdot$ day $^{-1}$ & $234 \pm 65$ & $193 \pm 57$ & 0.001 \\
\hline Time in dynamic activities, min day $^{-1}$ & $123(99-143)$ & $64(52-83)$ & $<0.0001$ \\
\hline Television-viewing time, $h \cdot$ day $^{-1}$ & $2.5(1.4-4.0)$ & $4.0(3.0-5.0)$ & 0.002 \\
\hline \multicolumn{4}{|l|}{ Exercise motivation, points } \\
\hline Amotivation & $0.00(0.00-1.31)$ & $0.50(0.00-1.25)$ & 0.34 \\
\hline External regulation & $0.13(0.00-1.50)$ & $0.25(0.00-1.44)$ & 0.95 \\
\hline Introjected regulation & $0.67(0.00-1.67)$ & $0.67(0.00-2.00)$ & 0.46 \\
\hline Identified regulation & $3.13(2.50-3.56)$ & $3.00(2.00-3.44)$ & 0.07 \\
\hline Intrinsic regulation & $3.88(3.00-4.00)$ & $3.25(2.25-4.00)$ & 0.01 \\
\hline
\end{tabular}

Data expressed as mean \pm SD or median (IQR). MVPA: moderate-to-vigorous physical activity. 
e-Table 5 Physical activity, sedentary behaviour and exercise motivation of COPD patients with a physically active loved one and those with a physically inactive loved one after removing patients who reported current participation in pulmonary rehabilitation $(n=10)$

\begin{tabular}{|c|c|c|c|}
\hline Characteristics & $\begin{array}{l}\text { COPD patients with a } \\
\text { physically active loved } \\
\text { one }(n=32)^{*}\end{array}$ & $\begin{array}{c}\text { COPD patients with a } \\
\text { physically inactive loved } \\
\text { one }(n=82)^{+}\end{array}$ & P-value \\
\hline Season of assessment Winter / Spring / & 6 (19) / 3 (9) / 5 (16) / & $17(21) / 8(10) / 20(24)$ & 0.53 \\
\hline Summer / Autumn, n (\%) & $18(56)$ & / 37 (45) & \\
\hline Time in sedentary behaviour, min $\cdot$ day $^{-1}$ & $600(580,620)$ & $614(602,626)$ & 0.26 \\
\hline Time in light activities, $\mathrm{min} \cdot \mathrm{day}^{-1}$ & $87(72,101)$ & $86(77,95)$ & 0.93 \\
\hline Time in MVPA, min $\cdot$ day $^{-1}$ & $32(25,40)$ & $19(15,24)$ & 0.005 \\
\hline$\geq 30$ min of MVPA on $\geq 5$ days, $n(\%)$ & $10(31)$ & $10(12)$ & 0.02 \\
\hline $\begin{array}{l}\text { Proportion of daily time in sedentary } \\
\text { behaviour, } \%\end{array}$ & $83(81,86)$ & $85(84,87)$ & 0.21 \\
\hline Proportion of daily time in light activities, $\%$ & $12(10,14)$ & $12(11,13)$ & 0.93 \\
\hline Proportion of daily time in MVPA, \% & $5(3,6)$ & $3(2,3)$ & 0.007 \\
\hline Time in $\geq 10$-min bouts of MVPA, min $\cdot$ day $^{-1}$ & $11(7,15)$ & $6(3,8)$ & 0.02 \\
\hline $\begin{array}{l}\text { Frequency of } \geq 10-\text { min bouts of MVPA, } \\
\text { bouts.day }{ }^{-1}\end{array}$ & $0.7(0.5,0.9)$ & $0.3(0.2,0.4)$ & 0.005 \\
\hline $\begin{array}{l}\text { Duration of } \geq 10-\min \text { bouts of MVPA, } \\
\text { min.bout }^{-1}\end{array}$ & $20(16,24)$ & $14(12,17)$ & 0.03 \\
\hline $\begin{array}{l}\text { Time in non-weight bearing activities, } \\
\text { min.day }{ }^{-1}\end{array}$ & $515(485,545)$ & $505(487,524)$ & 0.60 \\
\hline Time in weight-bearing activities, min $\cdot$ day $^{-1}$ & $139(115,163)$ & $159(145,174)$ & 0.17 \\
\hline Time in dynamic activities, min $\cdot$ day $^{-1}$ & $65(54,76)$ & $54(48,61)$ & 0.12 \\
\hline Television-viewing time, $\mathrm{h} \cdot \mathrm{day}^{-1}$ & $4.3(3.6,5.1)$ & $3.8(3.4,4.3)$ & 0.31 \\
\hline \multicolumn{4}{|l|}{ Exercise motivation, points } \\
\hline Amotivation & $0.71(0.38,1.03)$ & $0.62(0.42,0.81)$ & 0.65 \\
\hline External regulation & $0.83(0.51,1.15)$ & $0.78(0.59,0.98)$ & 0.82 \\
\hline Introjected regulation & $1.17(0.83,1.52)$ & $1.26(1.05,1.47)$ & 0.68 \\
\hline Identified regulation & $2.56(2.22,2.91)$ & $2.73(2.52,2.95)$ & 0.42 \\
\hline Intrinsic regulation & $2.76(2.39,3.13)$ & $2.76(2.53,2.98)$ & 0.98 \\
\hline
\end{tabular}

Data expressed as absolute and relative frequency or mean $(95 \% \mathrm{Cl})$. See Table 2 for definition of abbreviations. ${ }^{*}: \mathrm{n}=32$ due to missing values from 6 patients; ${ }^{\dagger}: \mathrm{n}=82$ due to missing values from 5 patients; ${ }^{\ddagger}:$ after controlling for age, body mass index and forced expiratory volume in the first second. 
A)

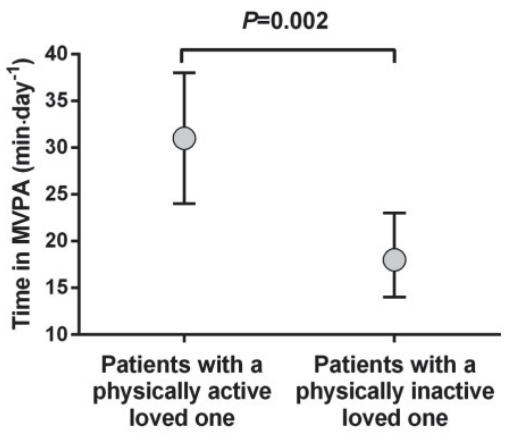

B)

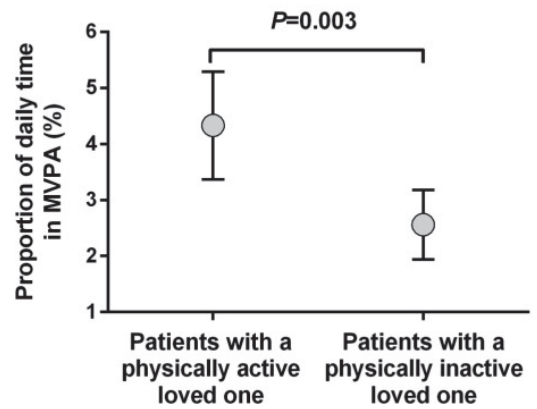

e-Figure 2 Time spent in moderate-to-vigorous physical activity (MVPA) in COPD patients with a physically active resident loved one and COPD patients with a physically inactive resident loved one after controlling for age, body mass index and degree of airflow limitation. A: absolute values; B: proportion of total assessment time. Data presented as mean (95\% confidence intervals). 


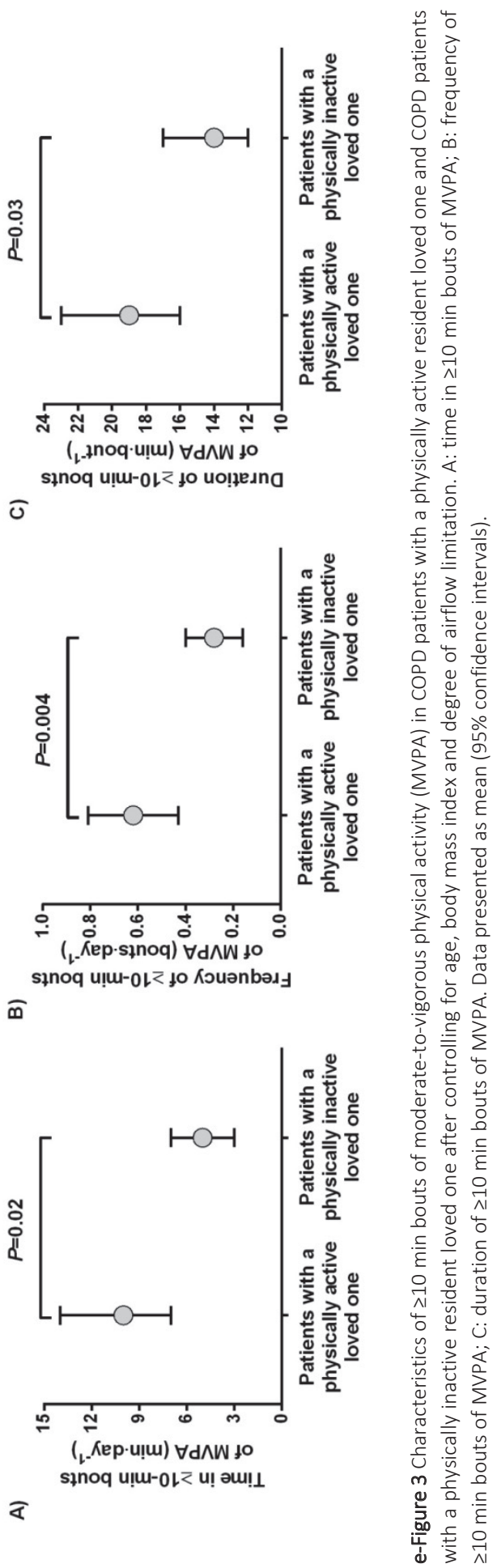




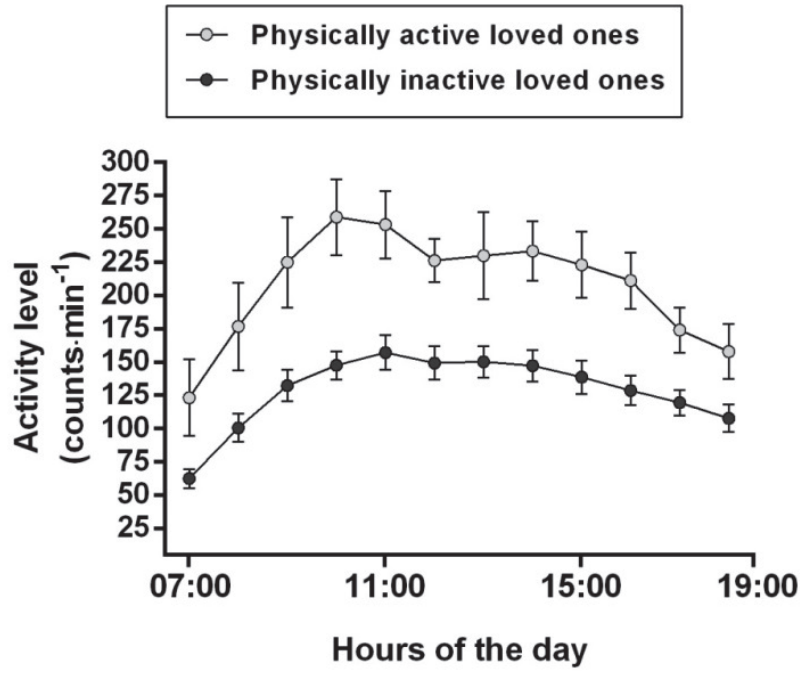

e-Figure 4 Daily physical activity hourly patterns of physically active and physically inactive resident loved ones. Data presented as mean (95\% confidence intervals). 


\section{References}

1. Charlson ME, Pompei P, Ales KL, MacKenzie CR. A new method of classifying prognostic comorbidity in longitudinal studies: development and validation. J Chronic Dis. 1987;40(5):373-383.

2. Charlson M, Szatrowski TP, Peterson J, Gold J. Validation of a combined comorbidity index. J Clin Epidemiol. 1994;47(11):1245-1251.

3. Bestall JC, Paul EA, Garrod R, Garnham R, Jones PW, Wedzicha JA. Usefulness of the Medical Research Council (MRC) dyspnoea scale as a measure of disability in patients with chronic obstructive pulmonary disease. Thorax. 1999;54(7):581-586.

4. Vestbo J, Hurd SS, Agusti AG, et al. Global strategy for the diagnosis, management, and prevention of chronic obstructive pulmonary disease: GOLD executive summary. Am J Respir Crit Care Med. 2013;187(4):347-365.

5. Miller MR, Hankinson J, Brusasco V, et al. Standardisation of spirometry. Eur Respir J. 2005;26(2):319-338.

6. Knudson RJ, Burrows B, Lebowitz MD. The maximal expiratory flow-volume curve: its use in the detection of ventilatory abnormalities in a population study. Am Rev Respir Dis. 1976;114(5):871-879.

7. Rabe KF, Hurd S, Anzueto A, et al. Global strategy for the diagnosis, management, and prevention of chronic obstructive pulmonary disease: GOLD executive summary. Am J Respir Crit Care Med. 2007;176(6):532-555.

8. Keys A, Fidanza F, Karvonen MJ, Kimura N, Taylor HL. Indices of relative weight and obesity. J Chronic Dis. 1972;25(6):329-343.

9. Celli BR, Cote CG, Marin JM, et al. The body-mass index, airflow obstruction, dyspnea, and exercise capacity index in chronic obstructive pulmonary disease. N Engl J Med. 2004;350(10):1005-1012.

10. Puhan MA, Garcia-Aymerich J, Frey M, et al. Expansion of the prognostic assessment of patients with chronic obstructive pulmonary disease: the updated BODE index and the ADO index. Lancet. 2009;374(9691):704-711.

11. Physical status: the use and interpretation of anthropometry. Report of a WHO Expert Committee. World Health Organ Tech Rep Ser. 1995;854:1-452.

12. Rutten EP, Spruit MA, Wouters EF. Critical view on diagnosing muscle wasting by single-frequency bioelectrical impedance in COPD. Respir Med. 2010;104(1):91-98.

13. Franssen FM, Rutten EP, Groenen MT, Vanfleteren LE, Wouters EF, Spruit MA. New reference values for body composition by bioelectrical impedance analysis in the general population: results from the UK Biobank. J Am Med Dir Assoc. 2014;15(6):448 e441-446.

14. Mesquita R, Janssen DJ, Wouters EF, Schols JM, Pitta F, Spruit MA. Within-day test-retest reliability of the timed up \& go test in patients with advanced chronic organ failure. Arch Phys Med Rehabil. 2013;94(11):2131-2138.

15. Mesquita R, Wilke S, Smid DE, et al. Measurement properties of the Timed Up \& Go test in patients with COPD. Chron Respir Dis. 2016;13(4):344-352.

16. Podsiadlo D, Richardson S. The timed "Up \& Go": a test of basic functional mobility for frail elderly persons. J Am Geriatr Soc. 1991;39(2):142-148.

17. Dolan P. Modeling valuations for EuroQol health states. Med Care. 1997;35(11):1095-1108.

18. Jones PW, Harding G, Berry P, Wiklund I, Chen WH, Kline Leidy N. Development and first validation of the COPD Assessment Test. Eur Respir J. 2009;34(3):648-654.

19. Dijkstra A, Tiesinga LJ, Goossen WT, Dassen TW. Further psychometric testing of the Dutch Care Dependency Scale on two different patient groups. Int J Nurs Pract. 2002;8(6):305-314.

20. Dijkstra A, Tiesinga LJ, Plantinga L, Veltman G, Dassen TW. Diagnostic accuracy of the care dependency scale. J Adv Nurs. 2005;50(4):410-416.

21. Zigmond AS, Snaith RP. The hospital anxiety and depression scale. Acta Psychiatr Scand. 1983;67(6):361370.

22. Markland D, Tobin V. A modification to the Behavioural Regulation in Exercise Questionnaire to include an assessment of amotivation. J Sport Exerc Psychol. 2004;26(2):191-196. 


\section{CHAPTER 9}

23. Annegarn J, Spruit MA, Uszko-Lencer NH, et al. Objective physical activity assessment in patients with chronic organ failure: a validation study of a new single-unit activity monitor. Arch Phys Med Rehabil. 2011;92(11):1852-1857 e1851.

24. van der Weegen S, Essers H, Spreeuwenberg M, et al. Concurrent Validity of the MOX Activity Monitor Compared to the ActiGraph GT3X. Telemed J E Health. 2015;21(4):259-266.

25. Sedentary Behaviour Research N. Letter to the editor: standardized use of the terms "sedentary" and "sedentary behaviours". Appl Physiol Nutr Metab. 2012;37(3):540-542.

26. Garber CE, Blissmer B, Deschenes MR, et al. American College of Sports Medicine position stand. Quantity and quality of exercise for developing and maintaining cardiorespiratory, musculoskeletal, and neuromotor fitness in apparently healthy adults: guidance for prescribing exercise. Med Sci Sports Exerc. 2011;43(7):1334-1359.

27. Bussmann JB, van den Berg-Emons RJ. To total amount of activity..... and beyond: perspectives on measuring physical behavior. Front Psychol. 2013;4:463.

28. Lee PH, Yu YY, McDowell I, Leung GM, Lam TH. A cluster analysis of patterns of objectively measured physical activity in Hong Kong. Public Health Nutr. 2013;16(8):1436-1444.

29. Hecht A, Ma S, Porszasz J, Casaburi R. Methodology for using long-term accelerometry monitoring to describe daily activity patterns in COPD. COPD. 2009;6(2):121-129. 
CHAPTER 10

General Discussion 


\section{Overview of findings}

The current thesis provides several novel insights. Patients with chronic obstructive pulmonary disease (COPD) and cardiac impairments were found to have worse physical and psychological status than those without cardiac impairments. Nevertheless, comorbid conditions (including cardiac ones) do not seem to preclude patients with COPD from showing significant and clinically meaningful improvements in exercise capacity and health status following a comprehensive pulmonary rehabilitation (PR) programme. Moreover, the 6-minute walk test (6MWT) was found to be valid and reliable in patients with chronic heart failure (CHF), while similar results were found for the Timed Up \& Go (TUG) test in patients with COPD. Moreover, the TUG test was also found to be responsive to PR in COPD. Physical activity clusters could be identified in patients with COPD and the characteristics of these clusters might be useful to help delineate interventions focusing on the adoption of a more physically active and/or less sedentary lifestyle. Moreover, around one-third of patients with COPD are able to show improvements in physical activity and sedentary behaviour following PR and this may be achieved mainly by reductions in sedentary time and increases in light-intensity physical activity (LPA) rather than moderate to vigorous-intensity physical activity (MVPA). Finally, having a physically active resident loved one seems to be beneficial for a more physically active lifestyle in patients with COPD.

\section{Comorbidities and physical capacity in patients with chronic cardiopulmonary diseases}

Comorbidities are a common finding in patients with chronic cardiopulmonary diseases $[1,2]$. Cardiovascular comorbidities in patients with pulmonary diseases, and pulmonary diseases in patients with cardiovascular conditions are amongst the most common comorbidities [1, 2]. In Chapter 2, 16.5\% of the patients with COPD considered for analysis were found to have a left ventricular ejection fraction (LVEF) lower than 50\%, which has been used to diagnose heart failure with reduced ejection fraction [3]. This prevalence is similar to that reported by previous studies [4, 5]. Interestingly, recent reports suggest that pulmonary comorbidities in patients with cardiac conditions seem to be an even more common finding. In a recent European multicentre study, Franssen et al. [6] observed that lung function abnormalities compatible with a diagnosis of COPD were found in $30.5 \%$ of (former) smoking patients with ischaemic heart disease, which is one of the main causes of CHF. Importantly, the authors also observed that the lung function abnormalities were largely undiagnosed and untreated, and that they were associated with increased symptom burden, reduced health status, and increased health risk [6]. Comorbidities in patients with chronic cardiopulmonary diseases are also associated with impaired physical capacity [1, 2]. Physical capacity (fitness) refers to the ability to 
carry out daily tasks with vigour and alertness, without undue fatigue and with energy to enjoy pursuits and to meet emergencies; and can be operationalised as a set of measurable attributes such as exercise tolerance, functional mobility, and muscular strength and endurance [7]. In Chapter 2, patients with COPD and an objectively identified impaired LVEF had worse exercise capacity, quadriceps muscle function, and functional mobility than patients with COPD with a normal LVEF who were matched for sex, age, body mass index (BMI) and the degree of airflow limitation. Moreover, patients with impaired LVEF also had more symptoms of anxiety and depression. Previous studies have already suggested a potential negative impact of cardiac conditions on physical capacity measures in patients with COPD [8-10]. Nevertheless, in most of these studies the comorbid condition was identified based on a non-objective measurement and in none of them the groups were matched for potential confounders, such as sex, age, $\mathrm{BMI}$, and airflow limitation. As highlighted in an accompanying editorial [11], data from Chapter 2 are important for two main reasons: "first, to verify that the seemingly obvious link between left ventricular dysfunction and impaired performance of COPD patients indeed exists, and second, to highlight the importance of diagnosing and treating cardiac disorders in patients with COPD". Oliveira and colleagues recently extended the findings of Chapter 2 by showing a reduction in peripheral muscle perfusion during exercise in patients with COPD and heart failure compared to patients with each condition alone [12]. Moreover, patients with COPD and heart failure were found to have an impaired cerebral blood flow at rest and during exercise in comparison to patients with COPD alone [13]. These findings suggest that active screening for cardiac comorbidities in patients with COPD seems reasonable. Moreover, some interventions have been suggested as potentially beneficial for this subgroup of patients with COPD [12, 13]. For example, non-invasive ventilation [14] and/or heliox [15] could improve cardiac output by addressing the negative interaction between the heart and the lungs. Adopting training regimens that are suitable for patients with severe central cardiorespiratory constraints can also be beneficial, such as training of small muscle groups [16] or neuromuscular electrical stimulation [17]. For example, one-legged exercise training has been suggested to induce the same metabolic and functional demands as those induced by two-legged training, however at a lower total metabolic load, and hence a lower ventilatory load [16, 18]. Similarly, high-frequency neuromuscular electrical stimulation has showed to induce significantly lower metabolic response (i.e., lower peak oxygen uptake and minute ventilation) compared to resistance exercise training [19], while being equally effective in strengthening the quadriceps muscles of patients with COPD [17]. Of note, patients with heart failure who have comorbid COPD might also benefit from these treatment strategies as they are associated with a lower burden on the cardiopulmonary system.

While patients with COPD and cardiac impairments had the worst physical and psychological status, it was not clear whether and to what extent cardiac and other comorbid 
conditions could influence responses to a comprehensive PR programme. Chapter 3 showed that neither the presence of individual or clusters of objectively identified comorbidities, nor the presence of self-reported comorbidities importantly affected the likelihood of achieving clinically meaningful changes in exercise capacity and health status following PR. The influence of comorbidities on responses to PR in patients with COPD has been investigated in previous studies, but conflicting results have been reported $[20,21]$. In a large $(n=2962)$ retrospective study investigating the role of comorbidities retrieved from medical files on PR outcomes in patients with COPD, Crisafulli and colleagues [22] observed that metabolic diseases were inversely related to improvements in six-minute walk distance (6MWD), while cardiac diseases were inversely related to improvements in health status. Some years later the same research group showed in a prospective study that self-reported osteoporosis was inversely related to improvements in 6MWD in patients with COPD following the same intervention [23]. Interestingly, outcomes of exercise-based rehabilitation in patients with cardiac diseases do not seem to be importantly affected by pulmonary conditions. In a recent study in patients with heart failure, Mentz et al. [24] observed no differences in changes in health status or exercise capacity between patients with or without comorbid COPD following an exercise programme using aerobic training. Differences between the results presented in Chapter 3 and those reported by previous studies may be explained by differences in study characteristics, such as the method of analysing the results. For example, while the two studies by Crisafulli and colleagues $[22,23]$ focused on the impact of comorbidities on the likelihood of having meaningful improvements, Mentz and co-workers [24] focused on comparing between groups the changes in each outcome following the exercise programme. In Chapter 3, both type of analyses were used and similar results were found compared to those reported by Mentz et al. [24], but different results were observed when compared to those reported by Crisafulli and colleagues [22, 23], which may be a consequence of the threshold used to define a clinical meaningful change in 6MWD (i.e., $54 \mathrm{~m}$ [25] in the previous studies and $30 \mathrm{~m}$ [26] in Chapter 3 ). Of note, the method used to identify the comorbidities may also have played a role. While most previous studies used data from self-report or medical records, Triest et al. [27] recently reported that poor agreement exists between objectively identified and chart-based comorbidities in patients with COPD, in general with a lower prevalence of chart-based when compared to objectively identified comorbidities. The abovementioned findings suggest that, in general, an objective diagnosis should be preferred over a chart-based diagnosis and that an active search for comorbidities (at least for the ones with important negative impact) may prove beneficial for a better disease management, and hence a better prognosis. The findings by Triest and colleagues [27] might also suggest that patients with COPD and unknown comorbidities may have been included in many previous PR studies, but were still able to successfully complete the programme. 
Despite the considerable prevalence of comorbidities in patients with chronic cardiopulmonary diseases $[1,2]$, contemporary rehabilitation programmes may not be fully prepared to deal with these conditions. For example, a recent survey about the content and organisational aspects of PR programmes across the world revealed that, while $62 \%$ of the programmes in North America had a chest physician as part of their team, only $20 \%$ and $8.6 \%$ had a cardiologist and a psychologist, respectively [28]. Additionally, 17 'PR teams' consisted of only one type of healthcare professional [28]. These findings might be a consequence of the adoption of a disease-based rather than a patient-based approach. An individualised (patient-based) approach as part of an integrated disease management programme could achieve greater success and help improve prognosis [3, 29-31]. Indeed, such approach is in agreement with the definition of PR as established in the recent 2013 Statement on PR from the American Thoracic Society (ATS) and European Respiratory Society (ERS) [32], which considers PR as a comprehensive intervention based on a thorough patient assessment followed by patient-tailored therapies designed to improve the physical and psychological condition of people with chronic pulmonary disease and to promote the long-term adherence to health-enhancing behaviours. Therefore, PR provides a great opportunity to coordinate the patient's care throughout the clinical course of the disease [32]. Adaptations to the model of PR haven been proposed to attend patients with conditions other than chronic pulmonary diseases. For example, Man and co-workers [33] have recently suggested the use of 'breathlessness rehabilitation services', which according to the authors are generic rehabilitation programmes designed for patients with chronic conditions such as COPD and CHF, who frequently develop symptoms of exertional dyspnoea and fatigue and have lowerlimb muscle dysfunction and exercise intolerance [33]. Although such approach may seem promising, some shortcomings can be highlighted. Patients with COPD and patients with CHF have different educational needs in terms of their medications and the pathophysiology of the disease, which would complicate the educational component of the rehabilitation programme. Moreover, adjunct therapies which have shown to be beneficial in patients with COPD, such as oxygen and helium-hyperoxic gas mixtures and one-legged exercise training, cannot be directly adopted with patients with cardiac diseases since they have been less explored in this population [33]. Additionally, it is even questionable whether 'breathlessness rehabilitation services' should be regarded as rehabilitation since such approach focuses mainly on symptoms without the analysis of the underlying pathology. Instead of becoming more generic to include different populations, rehabilitation programmes in the future should become more specialised to treat better the complex patients with chronic cardiopulmonary diseases. 


\section{Measurement properties of physical capacity tests in patients with chronic cardiopulmonary diseases}

Patients with chronic cardiopulmonary diseases are characterised by physiological impairments that can compromise the ability of these patients to undertake physically demanding activities. Adequately measuring physical capacity of these patients is important for estimating physical status, for assessing prognosis, and for evaluation of treatment response [34-36]. Different tests can be used for the measurement of physical capacity, ranging from complex and time-consuming tests, such as the incremental cardiopulmonary exercise tests [36], to more simple and quick-to-perform tests, such as field walking tests (e.g., 6MWT [26], the TUG test [37]). Although tests such as the cardiopulmonary exercise test are able to provide detailed information on the cardiovascular, pulmonary, and muscular systems, they require state-of-the-art equipment and skilled staff, which can compromise their use in daily clinical practice on a broad scale. Field walking tests on the other hand have the advantage of being easy to administer and inexpensive to complete, in addition to better mimicking activities of daily living [38]. In order to increase the uptake of field walking tests in patients with chronic cardiopulmonary diseases in clinical and research settings, a comprehensive investigation of the measurement properties of these tests needs to be performed. The reliability and validity of the 6MWT in patients with CHF was investigated in Chapter 4, while the same measurement properties for the TUG test in patients with COPD were investigated in Chapters 5 and 6 . Moreover, Chapter 6 also investigated the responsiveness of the TUG test in patients with COPD.

Reliability refers to the extent to which a measurement is consistent and free from error [39]. Chapter 4 showed that the 6MWT is reliable when performed two times on subsequent days in patients with CHF, while Chapter 5 showed that the TUG test is reliable when performed at least two times on the same day in patients with advanced COPD, $\mathrm{CHF}$ or chronic renal failure. These findings corroborate findings from previous studies. In patients with cardiac diseases entering a rehabilitation programme, Hamilton and colleagues [40] observed good reliability between three 6MWT trials performed on different days. Moreover, these authors also observed statistical difference between trials [40]. In Chapter 4, only 2 trials were performed and there was a statistically significant mean difference of $31 \mathrm{~m}(95 \% \mathrm{Cl} 27,35 \mathrm{~m})$ between the trials. Interestingly, Hernandes et al. [41] observed a relatively similar mean difference of $27 \mathrm{~m} \mathrm{(95 \%} \mathrm{Cl} \mathrm{37,} 107$ $\mathrm{m}$ ) in patients with COPD, which were also tested in the CIRO setting according to the exactly same protocol. This might suggest that the learning effect between two 6MWT trials is not disease-specific, at least not for chronic cardiopulmonary diseases. Concerning the TUG test, Bellet and co-workers [42] observed good reliability (i.e., intraclass correlation coefficient (ICC) of 0.85 ) between two trials after one practice trial when using a modified version of the test (i.e., patients were asked to walk as quickly as pos- 
sible) in patients with cardiac diseases, but there was a statistically significant reduction (i.e., improvement) of $5 \%$ between the trials. In patients with COPD, Marques et al. [43] also observed good reliability (i.e., ICC of 0.92) when using the same modified version, but in this case no statistical difference was observed. In terms of validity, which refers to the degree to which a test is measuring what it is intended to measure [39], Chapter 4 demonstrated that the 6MWT is valid in terms of convergent, discriminant and known-groups validity, which are all different aspects of construct validity [39]. Previous studies already investigated the validity of the 6MWT in patients with cardiac diseases. However, in most of them the analyses were restricted to convergent validity against measures of peak exercise capacity. For example, Hamilton et al. [40] reported a valid test based on a moderate-to-good correlation ( $r=0.69)$ with maximum metabolic equivalents (METs) from a symptom-limited exercise test. Concerning the TUG test, its validity has been the focus of recent studies in patients with COPD. In the study by Albarrati and colleagues [44], a good correlation ( $r=-0.71$ ) with the 6MWD was reported, which supports convergent validity, and a significantly worse TUG time in patients compared to a control group was observed, which supports known-groups validity. Nevertheless, discriminant validity was not investigated. Importantly, the different aspects of validity are necessary to inform about the robustness of a test. In terms of responsiveness, which in brief refers to the ability of a test to demonstrate change [39], this property was also investigated in Chapter 6 . There was a significant reduction (i.e., improvement) in TUG time in patients with COPD following a comprehensive PR programme. Importantly, minimal clinically important difference (MCID) values of 0.9-1.4 seconds were also reported in Chapter 6, which are necessary to facilitate the interpretation of the clinical relevance of changes in outcomes of PR or other therapies $[45,46]$. Collectively, the abovementioned findings suggest that the 6MWT and the TUG test are reliable and valid in patients with chronic cardiopulmonary diseases. Moreover, the TUG test is responsive to change with PR in patients with COPD, especially in those with an abnormally high result at baseline.

Other physical capacity tests that are similar to the 6MWT and TUG test are also reliable, valid and responsive to change in patients with chronic cardiopulmonary diseases. An example of such test is the Incremental Shuttle Walk test (ISWT), in which subjects are requested to walk around an elliptical 10-m course at increasing speeds dictated by signals generated from an audio; the test is terminated when the participant is not able to keep up with the speeds in the allowed time [47]. Recent systematic reviews revealed that this test is valid, reliable and responsive to change in patients with chronic cardiopulmonary diseases $[34,48]$. The MCID for the ISWT was described as $48 \mathrm{~m}(95 \%$ $\mathrm{Cl} 38.6,56.5 \mathrm{~m})$ in patients with COPD [49] and $70 \mathrm{~m} \mathrm{(95 \%} \mathrm{Cl} \mathrm{51.5,} 88.5 \mathrm{~m})$ in patients with cardiac diseases [50]. A variation of the ISWT for the assessment of endurance exercise capacity is the Endurance Shuttle Walk Test (ESWT) [51]. In the ESWT participants are requested to walk at a predetermined percentage of maximum walking per- 
formance as assessed by the ISWT for as long as possible [51]. The ESWT is also reliable, valid and responsive to change in patients with chronic pulmonary diseases [34], but studies in patients with cardiac diseases are scarce. Other field physical capacity tests have recently been explored in patients with chronic cardiopulmonary diseases, such as the 4-metre gait speed test [52-55], the 5-repetition or 1-min sit-to-stand test [54-57], and steps tests $[55,58,59]$. Most of these tests have also shown to be reliable, valid and responsive to change. Nevertheless, a head-to-head comparison between the different tests in patients with chronic cardiopulmonary diseases is yet to be performed.

With different field tests being available for the assessment of physical capacity, the question of which test is the best one remains. In the context of exercise-based rehabilitation programmes, one of the most important measurement properties to consider is the responsiveness of the test. Moreover, choosing a test with established MCID values is also important for its interpretability. Different studies have shown that 6MWT and TUG test are responsive to change with exercise-based rehabilitation in patients with chronic cardiopulmonary diseases [25, 42, 60-62]. When aiming to compare different tests in terms of their responsiveness, a useful measure is the effect size, which is unit free and allows quantifying the magnitude of change with an intervention [39]. Previous studies in patients with chronic cardiopulmonary diseases have reported effect sizes of 0.49-0.65 for the 6MWT following an exercise-based rehabilitation programme [25, 42, $60]$, while lower values were observed for the TUG test when the original version of the test was used (i.e., 0.16 as reported in Chapter 6 and 0.30 as found by Beauchamp et al. [61]). Nevertheless, when the modified version of the TUG test was used, effect sizes that are similar or higher to those found for the 6MWT were observed (i.e., 0.51-1.0 $[42,62])$. Moreover, a larger effect size (i.e., 0.70) was observed in Chapter 6 in patients with COPD who had an abnormal TUG time at baseline (i.e., $>11$ seconds). Concerning MCID values, recent studies in patients with chronic cardiopulmonary diseases have suggested values for the 6MWT between 25 and 35 metres [34, 63, 64], while Chapter 6 is the only study investigating values for the TUG test, suggesting values of 0.9-1.4 seconds. The abovementioned findings are indicative that both 6MWT and TUG test are suitable for outcome assessment before and after rehabilitation programmes. However, the 6MWT and the modified version of the TUG test, where subjects are requested to walk as quickly as possible, seem to be more responsive for the majority of patients with chronic cardiopulmonary diseases, while the original version of the TUG seems to be particularly more useful in patients with more compromised mobility. Other field capacity tests can also be useful. For example, the ESWT seems to be more sensitive to change after a course of rehabilitation than the ISWT and the 6MWT in patients with COPD $[51,65]$. Additionally, other characteristics, such as feasibility, ease of administration, and patient tolerance, are important to be considered [66]. 


\section{Physical activity and sedentary behaviour in patients with chronic cardiopulmonary diseases}

Patients with chronic cardiopulmonary diseases are characterised by symptoms such as exertional dyspnoea and fatigue, which can restrict physical activity in daily life. Previous research has shown that impairments in physical activity start early in the disease process [67] and further decline with advancing disease [68, 69]. Importantly, low levels of physical activity in patients with these diseases are associated with poor prognosis. In patients with chronic heart failure, Walsh et al. [70] observed that the daily amount of steps as assessed with a pedometer was a strong predictor of death and appears to be more powerful than laboratory-based exercise tests. In the study by Waschki and colleagues [71], physical activity level as assessed by a multisensory armband was identified as the strongest predictor of all-cause mortality in patients with stable COPD, even when compared with established predictors such as lung function and exercise capacity. Thus, improving physical activity in these populations becomes crucial. Recent literature in other populations has focused on the negative impact of sedentary behaviour, which is a concept distinct from physical activity. In a population-representative sample of individuals from the United States, Healy and co-workers [72] observed deleterious associations between prolonged sedentary time and cardio-metabolic and inflammatory biomarkers, independently of the time spent in moderate-to-vigorous exercise. In another study, adults with diabetes who were in the highest quartile of sedentary time (i.e., more time in sedentary behaviour) had a significantly increased mortality risk compared to those in the lowest quartile, even after adjusting for physical activity [73]. Therefore, not only improving physical activity, but also reducing sedentary behaviour becomes an important target.

Previous studies investigating physical activity in patients in chronic cardiopulmonary diseases have frequently described this outcome in terms of measures such as the time spent in specific activities (e.g., walking) [74, 75], daily number of steps [76], and time in activities of different intensities [76]. The same applies for studies investigating sedentary behaviour, most of which have described this outcome as the time in activities with very low activity counts or energy expenditure $[77,78]$. While the use of a few measures only might be important for statistical analyses and to provide a more straightforward message, this approach might compromise the understanding of these two complex behaviours. For example, it can be reasoned that the lack of significant improvements in physical activity and/sedentary behaviour measures after exercise training programmes observed in previous studies is related to the use of few and/or poorly responsive measures $[79,80]$. This is exemplified in the study by Demeyer and colleagues [81], who observed no significant change in the time spent in MVPA in patients with COPD following a comprehensive PR programme, but did find a significant increase in the daily number of steps [81]. These findings suggest that a more detailed 
approach to activity data analysis is necessary when aiming to make patients more physically active and less sedentary in their daily life. Chapter 7 advanced towards this approach by investigating physical activity measures and hourly patterns in patients with COPD after stratification for different characteristics, and by identifying clusters of patients based on multiple physical activity measures. It was found that physical activity measures and hourly patterns very considerably and differently depending on the characteristic. More importantly, five clusters with different physical activity characteristics were identified: 'Couch potatoes', 'Highly sedentary', 'Sedentary movers', 'Sedentary exercisers', and 'Busy bees'. These clusters were identified using robust statistical methods and based on 180 physical activity parameters generated by the stratification of energy expenditure and METs data. This allowed identifying clusters with different arrangements of physical activity, which are important to provide insights on the prognosis of the clusters and on how improve their physical activity levels. For example, 'Couch potatoes' were characterised by higher dyspnoea grades and worse disease severity, which are all related to a poor prognosis [82, 83]. Moreover, the same cluster as well as 'Highly sedentary' spent considerable amounts of time in very light-intensity physical activity (i.e., sedentary behaviour) and very little time in MVPA, which might have occurred because these patients find it difficult to engage in MVPA and therefore spend most of their time in sedentary behaviour. A potential strategy to make these patients more active is reducing their sedentary time by increasing the time in LPA [84, 85]. This is supported by previous studies suggesting benefits with spending more time in LPA. As an example, in a landmark study in patients with COPD, Garcia-Aymerich et al. [86] showed that even those reporting low levels of physical activity (i.e., LPA for less than 2 hours per week) have a lower risk of all-cause mortality, death from respiratory causes, and death from cardiovascular causes than those reporting a very low level of physical activity (excessive sitting time, no activity during leisure time, and no jogging or cycling). Nevertheless, the volume of physical activity also seems important. In another study in patients with COPD, Vaes et al. [87] reported that those who decreased their physical activity levels from moderate (i.e., LPA for 2-4 hours per week) or high (i.e., LPA for more than 4 hours per week or vigorous-intensity physical activity for more than 2 hours per week) to low (i.e., LPA for less than 2 hours per week) had the highest hazard ratios of mortality. LPA is also important in cardiac patients. In a recent study in patients with congestive heart failure it was observed that even in those with high levels of MVPA, LPA still associates significantly and positively with health-related quality of life [88]. Increases in the time spent in LPA may indeed prove to be more realistic and may pave the way to more intense physical activities [84, 85].

Detailed analyses of physical activity data were also the focus of Chapter 8 . This chapter aimed to explore changes in physical activity and sedentary behaviour in patients with COPD following a comprehensive PR programme. It was shown that around 1/3 of patients were able to significantly improve activity levels following PR (i.e., good respond- 
ers), while a slightly lower proportion did not change or even declined in terms of activity levels (i.e., poor responders). While much of the research on physical activity in patients with COPD has focused mainly on activities of at least moderate intensity [89-91], the results of Chapter 8 suggest that simplified activity monitoring focusing on MVPA alone may lead to an incomplete or incorrect picture of the impact of PR. This is supported by the study of Ramadi and co-workers [92], in which the authors observed beneficial changes in sedentary time and light activities after an exercise rehabilitation programme in patients with different cardiopulmonary diseases, but no significant changes in the time in MVPA [92]. Findings from Chapter 8 also suggest that focusing on sedentary behaviour and LPA rather than MVPA can be a useful strategy. This chapter showed that most part of the change in sedentary time showed by good responders,

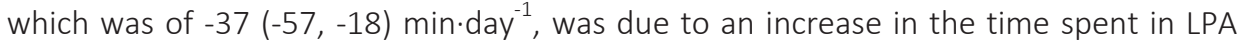
(i.e., $26(13,38)$ min $\cdot$ day $^{-1} ; 70 \%$ of the change in sedentary time). A similar finding was observed by Louvaris and colleagues [93], who reported a significant reduction in sedentary time of around 46 min.day ${ }^{-1}$ in patients with COPD following a PR programme

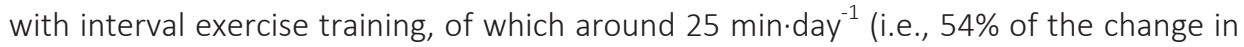
sedentary time) was due to an increase in the time spent in LPA [93]. These findings suggest that increases in LPA might be more easily achievable than increases in MVPA. As highlighted by Spruit et al. [85], increases in LPA during PR are potentially mediated by increases in self-efficacy (Figure 1). Self-efficacy refers to an individual's beliefs in his/her capabilities to organise and execute the course of action required to produce given attainments [94]. The importance of self-efficacy for improvements in physical activity in patients with COPD is demonstrated in a recent study. Larson and colleagues [95] observed a mean increase of almost 21 minutes in LPA following an exercisespecific self-efficacy enhancing intervention in patients in COPD [95]. Interestingly, although this intervention focused on meeting physical activity guidelines and increasing MVPA, no statistical change was observed for the time in MVPA [95] once again suggesting that increases in LPA are more easily achievable. Importantly, examples of LPA include self-care and household activities. Considering that COPD and CHF are more frequently found in older individuals, stimulating LPA in patients with these conditions can also contribute to an independent living. 


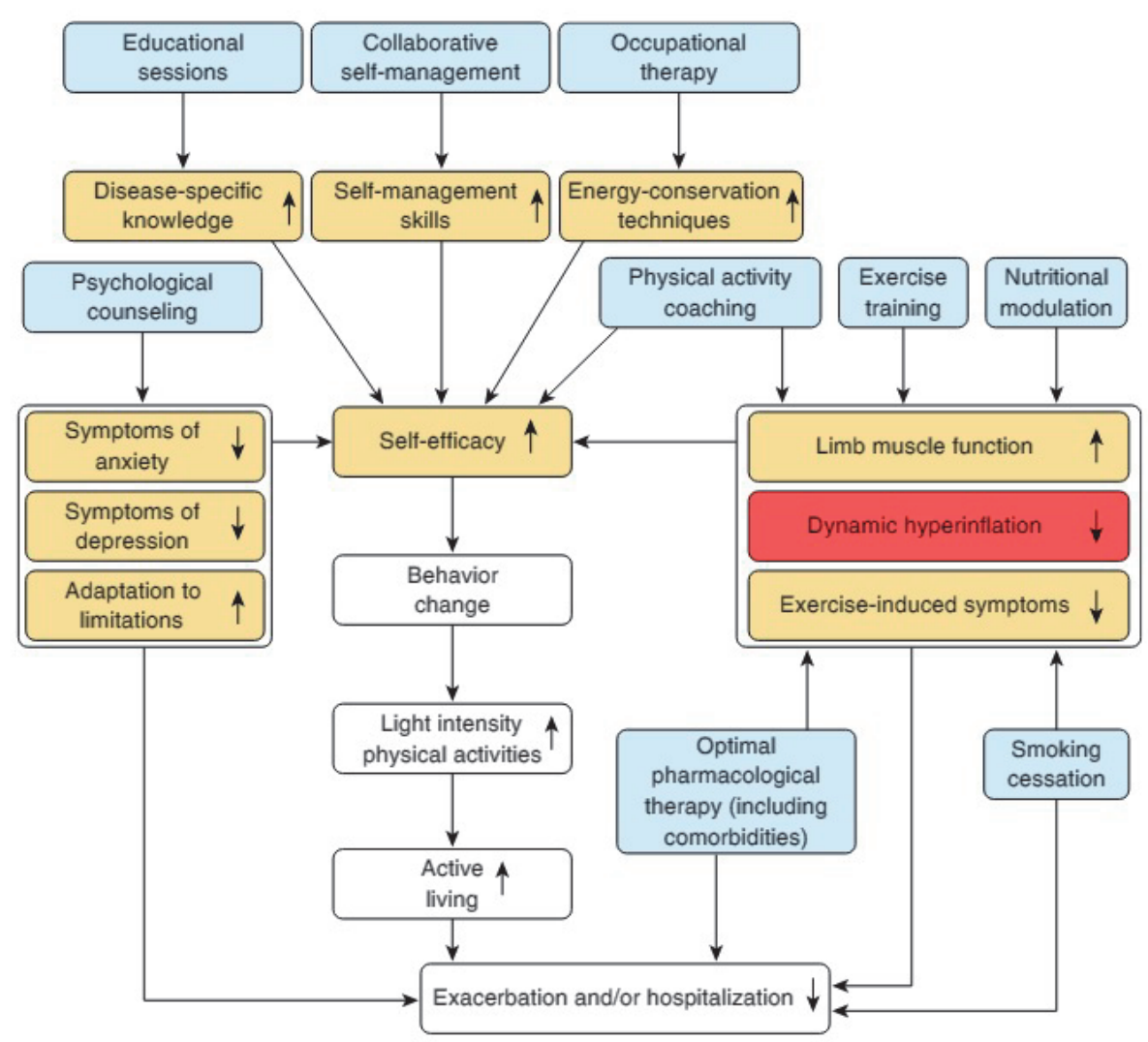

Figure 1 Impact of pulmonary rehabilitation components (blue rectangles) on pulmonary (red rectangles) and extra-pulmonary (yellow rectangles) features in patients with COPD. This figure highlights the influence of increases in self-efficacy on increases in light-intensity physical activity. Reprinted with permission of the American Thoracic Society. Copyright (C) 2016 American Thoracic Society. Spruit MA, Pitta F, McAuley E, ZuWallack RL, Nici L (2015). Pulmonary rehabilitation and physical activity in patients with chronic obstructive pulmonary disease. Am J Respir Crit Care Med;192:924-933. The American Journal of Respiratory and Critical Care Medicine is an official journal of the American Thoracic Society.

Changing the focus from increases in MVPA to reductions in sedentary behaviour by increases in LPA has a great potential to pave the way for a more physically active lifestyle in patients with chronic cardiopulmonary diseases. Nevertheless, not all patients may benefit from this strategy, as is the case with many other interventions in these patients [96], and therefore other strategies should be considered. Involving family members/loved ones in physical activity enhancing interventions may also be of value. Chapter 9 investigated focused on investigating activity levels in patients with COPD and their loved ones. Besides showing that these patients are more sedentary and physically inactive than their loved ones, Chapter 9 also showed that patients with COPD who have a physically active loved one are more physically active and have a higher chance of being physically active than those with an inactive loved one. As suggested in previous 
studies, social support from family and friends can be important for a more physically active lifestyle [97, 98]. In patients participating cardiac rehabilitation, Franks et al. [99] observed that spouses' provision of support was positively associated with patients' health behaviour, including engaging in physical activity or exercise. When investigating potential correlates of physical activity in patients with COPD, Hartman et al. [100] observed very weak and non-significant correlations between locomotion time and social support for exercise from family and friends. Nevertheless, as highlighted by the authors, patients with COPD in their study reported low social support scores, which therefore may explain the weak association with physical activity. Interestingly, in the same study self-efficacy was positively and independently associated with physical activity. Loved ones could help patients to be more active not only by verbally encouraging them, but also by joining them in physical activities, such as having joint daily walks. Cobb et al. [101] recently observed a positive association between individuals' changes in the sport/exercise and leisure indices and their spouses' changes. Moreover, higher odds of meeting physical activity recommendations were observed if the spouse met recommendations. Patients with chronic cardiopulmonary diseases may also prefer to engage in physical activity with others rather than alone. Hartman and colleagues [102] observed that $40 \%$ of patients with COPD prefer to be physically active with others and $30 \%$ a combination of with others and alone. Of note, encouraging patients to be active together with their loved ones will certainly have benefits for both groups. This is particularly important when considering that (co)morbidities are a common finding not only in COPD, but also among their loved ones [103]. It is also important to underline that using patients' loved ones to facilitate a more physically active lifestyle or reducing sedentary behaviour by increasing LPA may not work as an effective strategy in all patients. Therefore, future studies should investigate which groups of patients may benefit more from each intervention.

\section{Limitations and future directions}

Some limitations should be acknowledged when interpreting the results of this PhD thesis. Although Chapters 2 and 3 include as strengths the fact that comorbid conditions were identified objectively, a number of comorbidities that can be found in patients with COPD were not investigated. For example, Houben-Wilke et al. [104] recently observed that peripheral artery disease is found in almost $9 \%$ of patients with COPD and is associated with worse functional exercise capacity and more impaired health status, even after correcting for confounders. Moreover, while comorbid conditions were the main focus of Chapters 2 and 3, other health outcome measures that could have strengthened the findings were not included, such as physical activity. Contrarily, in Chapter 7 for example, physical activity was the outcome, but comorbidities were not investigated. Importantly, previous research suggested a negative association between 
comorbidities and physical activity levels in patients with COPD. In the study by Watz and colleagues [105], patients with COPD and metabolic syndrome were found to have a significantly decreased physical activity level compared to patients with COPD without metabolic syndrome. No or only a few patients with COPD who were classified as GOLD grade 1 were included in Chapters 3, 5, 6, 7, and 9, which constitutes another limitation. In Chapter 5, actually only severe or very severe patients with COPD were included. Moreover, the samples included in Chapters 2, 3, 4, 6, and 8 were recruited from tertiary care (i.e., when entering a rehabilitation programme), while patients from Chapter 9 were recruited mainly from secondary care. Combined these limitations may compromise the generalisability of the findings. Additionally, the sample size in Chapters 2, 3,8 , and 9 might have limited some analyses and also compromised the generalisability of the findings. Although there will never be evidence that is generalisable for all situations, researchers in future studies should try to include more representative populations. Finally, the cross-sectional design adopted in Chapters 2, 4, 5, 7, and 9 jeopardised a deeper comprehension of their findings. For example, it is not possible to know whether the impact of an impaired LVEF on physical and psychological status observed in Chapter 2 or the physical activity clusters identified in Chapter 7 relate to the prognosis of the disease in patients with COPD. Importantly, previous studies do suggest that cardiac conditions in patients with COPD are associated with poor prognosis $[106,107]$; however, in most studies non-objective measurements were used to identify the comorbid condition.

While this PhD thesis provides important insights on how to assess, interpret and improve physical functioning in patients with chronic cardiopulmonary diseases, several important questions remain to be investigated. Given the relevance of comorbidities in the management of chronic cardiopulmonary diseases, more work is necessary to investigate the potential impact of integrated disease management interventions in patients with chronic cardiopulmonary diseases. Recent initiatives in this direction have been developed to specifically address comorbidities, such as the Programme of Integrated Care for Patients With Chronic Obstructive Pulmonary Disease and Multiple Comorbidities (PICCOPD+) [108]. PICCOPD+ is an ongoing randomised controlled trial to investigate the impact of an intervention including an individualised care plan for the management of COPD and comorbidities, among other strategies, on the need for emergency department visits and hospital admission [108]. Results are not yet available. In order to increase the uptake and contribute to the interpretability of the 6MWT in patients with $\mathrm{CHF}$, more studies are necessary to investigate in detail its responsiveness to commonly used intervention, such as exercise-based rehabilitation, and to identify changes that are clinically relevant. Only a few studies investigated MCID values for the 6MWT in this population. In Chapter 4, the upper limit of the MCID value of $30.1 \mathrm{~m}$ (95\% Cl 20.8, $39.4 \mathrm{~m}$ ) was used in the analyses based on the findings from Shoemaker and colleagues [63]. However, in their study only 22 patients with CHF were included. In 
another study by the same research group, the MCID value of $45 \mathrm{~m}$ is suggested [109]. Nevertheless, this value was estimated based on data from a systematic review. A randomised controlled trial should be planned to test the effectiveness of an intervention that takes into account the characteristics of the patients in terms of physical activity and sedentary behaviour. Moreover, it should also be tested the impact of an intervention focusing on reducing sedentary behaviour by increasing the time LPA and of a physical activity intervention that involves the patient's loved ones. Interventions focusing on reducing sedentary behaviour in patients with chronic cardiopulmonary diseases are starting to emerge. For example, the Chronic Obstructive Pulmonary Disease-Sitting and ExacerbAtions Trial (COPD-SEAT) study is a randomised controlled trial to evaluate the feasibility and acceptability of a 2-week at-home intervention with education and selfmonitoring to reduce periods of sedentary behaviour in patients with COPD after an acute exacerbation [110]. Results of this study are expected to be available soon. Recent research also suggests that sleep quality is an important outcome to take into account when investigating physical activity in patients with chronic cardiopulmonary diseases. In patients with COPD, Spina et al. [111] observed that sleep impairment was greatest in patients with severe airway limitation and worse exertional dyspnoea, and was associated with the amount of physical activity the patients undertook during the following waking day. Finally, the studies included in this thesis should be replicated in patients with chronic cardiopulmonary diseases other than COPD and CHF, preferably after taking into account the limitations mentioned in the beginning of this section.

\section{Concluding remarks}

In summary, this thesis demonstrated that impairments in physical functioning in patients with chronic cardiopulmonary diseases are complex and should be carefully assessed in clinical and research settings. Cardiac impairments in patients with COPD were found to associate with poor physical and psychological status. Nevertheless, neither this nor other comorbid conditions seem to preclude patients with COPD from obtaining significant and clinically meaningful improvements in physical capacity and health status following PR. Moreover, for a proper assessment of physical capacity in patients with chronic cardiopulmonary diseases, such as COPD and CHF, tests that provide valid, reliable, and responsive results should be used and this can be achieved by using the 6MWT or the TUG test. Patients with COPD can be clustered based on their activity levels and the characteristics of the clusters are useful to provide insights on how to improve activity in this population. Specifically, reducing sedentary behaviour by increasing LPA can be an important strategy. This is also supported by the study of changes in activity levels after a comprehensive PR programme, where a group of patients with COPD were able to reduce their time in sedentary behaviour mainly by increases in the time in LPA. Moreover, using different outcome measures of physical activity and 
sedentary behaviour seems necessary in order to avoid oversimplification of reality. Finally, involving loved ones of patients with COPD in interventions for a more physically active and/or less sedentary lifestyle can also be a promising strategy. 


\section{REFERENCES}

1. Cavailles A, Brinchault-Rabin G, Dixmier A, Goupil F, Gut-Gobert C, Marchand-Adam S, Meurice JC, Morel H, Person-Tacnet C, Leroyer C, Diot P: Comorbidities of COPD. Eur Respir Rev 2013, 22:454-475.

2. Dahlstrom U: Frequent non-cardiac comorbidities in patients with chronic heart failure. Eur J Heart Fail 2005, 7:309-316.

3. Ponikowski P, Voors AA, Anker SD, Bueno H, Cleland JG, Coats AJ, Falk V, Gonzalez-Juanatey JR, Harjola VP, Jankowska EA, et al: 2016 ESC Guidelines for the diagnosis and treatment of acute and chronic heart failure: The Task Force for the diagnosis and treatment of acute and chronic heart failure of the European Society of Cardiology (ESC). Developed with the special contribution of the Heart Failure Association (HFA) of the ESC. Eur J Heart Fail 2016, 18:891-975.

4. Rutten FH, Moons KG, Cramer MJ, Grobbee DE, Zuithoff NP, Lammers JW, Hoes AW: Recognising heart failure in elderly patients with stable chronic obstructive pulmonary disease in primary care: cross sectional diagnostic study. BMJ 2005, 331:1379.

5. Rutten FH, Cramer MJ, Grobbee DE, Sachs AP, Kirkels JH, Lammers JW, Hoes AW: Unrecognized heart failure in elderly patients with stable chronic obstructive pulmonary disease. Eur Heart J 2005, 26:18871894.

6. Franssen FM, Soriano JB, Roche N, Bloomfield PH, Brusselle G, Fabbri LM, Garcia-Rio F, Kearney MT, Kwon N, Lundback B, et al: Lung Function Abnormalities in Smokers With Ischemic Heart Disease. Am J Respir Crit Care Med 2016, 194:568-576.

7. Caspersen CJ, Powell KE, Christenson GM: Physical activity, exercise, and physical fitness: definitions and distinctions for health-related research. Public Health Rep 1985, 100:126-131.

8. Miller J, Edwards LD, Agusti A, Bakke P, Calverley PM, Celli B, Coxson HO, Crim C, Lomas DA, Miller BE, et al: Comorbidity, systemic inflammation and outcomes in the ECLIPSE cohort. Respir Med 2013, 107:13761384.

9. Putcha N, Han MK, Martinez CH, Foreman MG, Anzueto AR, Casaburi R, Cho MH, Hanania NA, Hersh CP, Kinney GL, et al: Comorbidities of COPD have a major impact on clinical outcomes, particularly in African Americans. Chronic Obstr Pulm Dis (Miami) 2014, 1:105-114.

10. Patel AR, Donaldson GC, Mackay AJ, Wedzicha JA, Hurst JR: The impact of ischemic heart disease on symptoms, health status, and exacerbations in patients with COPD. Chest 2012, 141:851-857.

11. Stankovic I, Neskovic AN, Lainscak M: Physical and psychological status of patients with COPD and impaired LVEF: Two bads don't make a good. Int J Cardiol 2017, 227:249-250.

12. Oliveira MF, Arbex FF, Alencar MC, Souza A, Sperandio PA, Medeiros WM, Mazzuco A, Borghi-Silva A, Medina LA, Santos R, et al: Heart Failure Impairs Muscle Blood Flow and Endurance Exercise Tolerance in COPD. COPD 2016, 13:407-415.

13. Oliveira MF, Alencar MC, Arbex F, Souza A, Sperandio P, Medina L, Medeiros WM, Hirai DM, O'Donnell $D E$, Neder JA: Effects of heart failure on cerebral blood flow in COPD: Rest and exercise. Respir Physiol Neurobiol 2016, 221:41-48.

14. Borghi-Silva A, Oliveira CC, Carrascosa C, Maia J, Berton DC, Queiroga F, Jr., Ferreira EM, Almeida DR, Nery LE, Neder JA: Respiratory muscle unloading improves leg muscle oxygenation during exercise in patients with COPD. Thorax 2008, 63:910-915.

15. Vogiatzis I, Habazettl H, Aliverti A, Athanasopoulos D, Louvaris Z, LoMauro A, Wagner H, Roussos C, Wagner PD, Zakynthinos S: Effect of helium breathing on intercostal and quadriceps muscle blood flow during exercise in COPD patients. Am J Physiol Regul Integr Comp Physiol 2011, 300:R1549-1559.

16. Dolmage TE, Goldstein RS: Effects of one-legged exercise training of patients with COPD. Chest 2008, 133:370-376.

17. Sillen MJ, Franssen FM, Delbressine JM, Vaes AW, Wouters EF, Spruit MA: Efficacy of lower-limb muscle training modalities in severely dyspnoeic individuals with COPD and quadriceps muscle weakness: results from the DICES trial. Thorax 2014, 69:525-531. 
18. Dolmage TE, Goldstein RS: Response to one-legged cycling in patients with COPD. Chest 2006, 129:325332.

19. Sillen MJ, Janssen PP, Akkermans MA, Wouters EF, Spruit MA: The metabolic response during resistance training and neuromuscular electrical stimulation (NMES) in patients with COPD, a pilot study. Respir Med 2008, 102:786-789.

20. Hornikx M, Van Remoortel H, Demeyer H, Marcal Camillo CA, Decramer M, Janssens W, Troosters T: The influence of comorbidities on outcomes of pulmonary rehabilitation programs in patients with COPD: a systematic review. Biomed Res Int 2013, 2013:146148.

21. Franssen FM, Rochester CL: Comorbidities in patients with COPD and pulmonary rehabilitation: do they matter? Eur Respir Rev 2014, 23:131-141.

22. Crisafulli E, Costi S, Luppi F, Cirelli G, Cilione C, Coletti O, Fabbri LM, Clini EM: Role of comorbidities in a cohort of patients with COPD undergoing pulmonary rehabilitation. Thorax 2008, 63:487-492.

23. Crisafulli E, Gorgone P, Vagaggini B, Pagani M, Rossi G, Costa F, Guarriello V, Paggiaro P, Chetta A, de Blasio $F$, et al: Efficacy of standard rehabilitation in COPD outpatients with comorbidities. Eur Respir J 2010, 36:1042-1048.

24. Mentz RJ, Schulte PJ, Fleg JL, Fiuzat M, Kraus WE, Pina IL, Keteyian SJ, Kitzman DW, Whellan DJ, Ellis SJ, O'Connor CM: Clinical characteristics, response to exercise training, and outcomes in patients with heart failure and chronic obstructive pulmonary disease: findings from Heart Failure and A Controlled Trial Investigating Outcomes of Exercise TraiNing (HF-ACTION). Am Heart J 2013, 165:193-199.

25. Redelmeier DA, Bayoumi AM, Goldstein RS, Guyatt GH: Interpreting small differences in functional status: the Six Minute Walk test in chronic lung disease patients. Am J Respir Crit Care Med 1997, 155:12781282.

26. Holland AE, Spruit MA, Troosters T, Puhan MA, Pepin V, Saey D, McCormack MC, Carlin BW, Sciurba FC, Pitta F, et al: An official European Respiratory Society/American Thoracic Society Technical Standard: field walking tests in chronic respiratory disease. Eur Respir J 2014, 44:1428-1446.

27. Triest FJ, Franssen FM, Spruit MA, Groenen MT, Wouters EF, Vanfleteren LE: Poor agreement between chart-based and objectively identified comorbidities of COPD. Eur Respir J 2015, 46:1492-1495.

28. Spruit MA, Pitta F, Garvey C, ZuWallack RL, Roberts CM, Collins EG, Goldstein R, McNamara R, Surpas P, Atsuyoshi $\mathrm{K}$, et al: Differences in content and organisational aspects of pulmonary rehabilitation programmes. Eur Respir J 2014, 43:1326-1337.

29. Vanfleteren LE, Spruit MA, Wouters EF, Franssen FM: Management of chronic obstructive pulmonary disease beyond the lungs. Lancet Respir Med 2016, 4:911-924.

30. Vestbo J, Hurd SS, Agusti AG, Jones PW, Vogelmeier C, Anzueto A, Barnes PJ, Fabbri LM, Martinez FJ, Nishimura $M$, et al: Global strategy for the diagnosis, management, and prevention of chronic obstructive pulmonary disease: GOLD executive summary. Am J Respir Crit Care Med 2013, 187:347-365.

31. Roversi S, Fabbri LM, Sin DD, Hawkins NM, Agusti A: Chronic Obstructive Pulmonary Disease and Cardiac Diseases: An Urgent Need for Integrated Care. Am J Respir Crit Care Med 2016.

32. Spruit MA, Singh SJ, Garvey C, Zuwallack R, Nici L, Rochester C, Hill K, Holland AE, Lareau SC, Man WD, et al: An official american thoracic society/european respiratory society statement: key concepts and advances in pulmonary rehabilitation. Am J Respir Crit Care Med 2013, 188:e13-64.

33. Man WD, Chowdhury F, Taylor RS, Evans RA, Doherty P, Singh SJ, Booth S, Thomason D, Andrews D, Lee C, et al: Building consensus for provision of breathlessness rehabilitation for patients with chronic obstructive pulmonary disease and chronic heart failure. Chron Respir Dis 2016, 13:229-239.

34. Singh SJ, Puhan MA, Andrianopoulos V, Hernandes NA, Mitchell KE, Hill CJ, Lee AL, Camillo CA, Troosters T, Spruit MA, et al: An official systematic review of the European Respiratory Society/American Thoracic Society: measurement properties of field walking tests in chronic respiratory disease. Eur Respir J 2014, 44:1447-1478.

35. Fleg JL, Pina IL, Balady GJ, Chaitman BR, Fletcher B, Lavie C, Limacher MC, Stein RA, Williams M, Bazzarre $\mathrm{T}$ : Assessment of functional capacity in clinical and research applications: An advisory from the Committee on Exercise, Rehabilitation, and Prevention, Council on Clinical Cardiology, American Heart Association. Circulation 2000, 102:1591-1597. 
36. ATS/ACCP Statement on cardiopulmonary exercise testing. Am J Respir Crit Care Med 2003, 167:211-277.

37. Podsiadlo D, Richardson S: The timed "Up \& Go": a test of basic functional mobility for frail elderly persons. J Am Geriatr Soc 1991, 39:142-148.

38. Guyatt GH, Thompson PJ, Berman LB, Sullivan MJ, Townsend M, Jones NL, Pugsley SO: How should we measure function in patients with chronic heart and lung disease? J Chronic Dis 1985, 38:517-524.

39. Portney LD, Watkins MP: Foundations of clinical research: applications to practice. 3rd edn. Upper Saddle River Prentice Hall; 2008.

40. Hamilton DM, Haennel RG: Validity and reliability of the 6-minute walk test in a cardiac rehabilitation population. J Cardiopulm Rehabil 2000, 20:156-164.

41. Hernandes NA, Wouters EF, Meijer K, Annegarn J, Pitta F, Spruit MA: Reproducibility of 6-minute walking test in patients with COPD. Eur Respir J 2011, 38:261-267.

42. Bellet RN, Francis RL, Jacob JS, Healy KM, Bartlett HJ, Adams L, Morris NR: Timed Up and Go Tests in cardiac rehabilitation: reliability and comparison with the 6-Minute Walk Test. J Cardiopulm Rehabil Prev 2013, 33:99-105.

43. Marques A, Cruz J, Quina S, Regencio M, Jacome C: Reliability, Agreement and Minimal Detectable Change of the Timed Up \& Go and the 10-Meter Walk Tests in Older Patients with COPD. COPD 2016, 13:279-287.

44. Albarrati AM, Gale NS, Enright S, Munnery MM, Cockcroft JR, Shale DJ: A simple and rapid test of physical performance inchronic obstructive pulmonary disease. Int J Chron Obstruct Pulmon Dis 2016, 11:17851791.

45. Puhan MA, Mador MJ, Held U, Goldstein R, Guyatt GH, Schunemann HJ: Interpretation of treatment changes in 6-minute walk distance in patients with COPD. Eur Respir J 2008, 32:637-643.

46. Laviolette L, Bourbeau J, Bernard S, Lacasse Y, Pepin V, Breton MJ, Baltzan M, Rouleau M, Maltais F: Assessing the impact of pulmonary rehabilitation on functional status in COPD. Thorax 2008, 63:115-121.

47. Singh SJ, Morgan MD, Scott S, Walters D, Hardman AE: Development of a shuttle walking test of disability in patients with chronic airways obstruction. Thorax 1992, 47:1019-1024.

48. Parreira VF, Janaudis-Ferreira T, Evans RA, Mathur S, Goldstein RS, Brooks D: Measurement properties of the incremental shuttle walk test. a systematic review. Chest 2014, 145:1357-1369.

49. Singh SJ, Jones PW, Evans R, Morgan MD: Minimum clinically important improvement for the incremental shuttle walking test. Thorax 2008, 63:775-777.

50. Houchen-Wolloff L, Boyce S, Singh S: The minimum clinically important improvement in the incremental shuttle walk test following cardiac rehabilitation. Eur J Prev Cardiol 2015, 22:972-978.

51. Revill SM, Morgan MD, Singh SJ, Williams J, Hardman AE: The endurance shuttle walk: a new field test for the assessment of endurance capacity in chronic obstructive pulmonary disease. Thorax 1999, 54:213222.

52. Kon SS, Patel MS, Canavan JL, Clark AL, Jones SE, Nolan CM, Cullinan P, Polkey MI, Man WD: Reliability and validity of 4-metre gait speed in COPD. Eur Respir J 2013, 42:333-340.

53. Kon SS, Canavan JL, Nolan CM, Clark AL, Jones SE, Cullinan P, Polkey MI, Man WD: The 4-metre gait speed in COPD: responsiveness and minimal clinically important difference. Eur Respir J 2014, 43:1298-1305.

54. Puthoff ML, Saskowski D: Reliability and responsiveness of gait speed, five times sit to stand, and hand grip strength for patients in cardiac rehabilitation. Cardiopulm Phys Ther J 2013, 24:31-37.

55. Bisca GW, Morita AA, Hernandes NA, Probst VS, Pitta F: Simple lower limb functional tests in patients with Chronic Obstructive Pulmonary Disease: a systematic review. Arch Phys Med Rehabil 2015.

56. Jones SE, Kon SS, Canavan JL, Patel MS, Clark AL, Nolan CM, Polkey MI, Man WD: The five-repetition sitto-stand test as a functional outcome measure in COPD. Thorax 2013, 68:1015-1020.

57. Ozalevli S, Ozden A, Itil O, Akkoclu A: Comparison of the Sit-to-Stand Test with 6 min walk test in patients with chronic obstructive pulmonary disease. Respir Med 2007, 101:286-293.

58. Andrade $\mathrm{CH}$, Cianci RG, Malaguti C, Corso SD: The use of step tests for the assessment of exercise capacity in healthy subjects and in patients with chronic lung disease. J Bras Pneumol 2012, 38:116-124. 
59. Wegrzynowska-Teodorczyk K, Mozdzanowska D, Josiak K, Siennicka A, Nowakowska K, Banasiak W, Jankowska EA, Ponikowski P, Wozniewski M: Could the two-minute step test be an alternative to the sixminute walk test for patients with systolic heart failure? Eur J Prev Cardiol 2016, 23:1307-1313.

60. Verrill DE, Barton C, Beasley W, Lippard M, King CN: Six-minute walk performance and quality of life comparisons in North Carolina cardiac rehabilitation programs. Heart Lung 2003, 32:41-51.

61. Beauchamp MK, O'Hoski S, Goldstein RS, Brooks D: Effect of pulmonary rehabilitation on balance in persons with chronic obstructive pulmonary disease. Arch Phys Med Rehabil 2010, 91:1460-1465.

62. Marques A, Gabriel R, Jacome C, Cruz J, Brooks D, Figueiredo D: Development of a family-based pulmonary rehabilitation programme: an exploratory study. Disabil Rehabil 2015, 37:1340-1346.

63. Shoemaker MJ, Curtis AB, Vangsnes E, Dickinson MG: Clinically meaningful change estimates for the sixminute walk test and daily activity in individuals with chronic heart failure. Cardiopulm Phys Ther J 2013, 24:21-29.

64. Gremeaux V, Troisgros O, Benaim S, Hannequin A, Laurent Y, Casillas JM, Benaim C: Determining the minimal clinically important difference for the six-minute walk test and the 200-meter fast-walk test during cardiac rehabilitation program in coronary artery disease patients after acute coronary syndrome. Arch Phys Med Rehabil 2011, 92:611-619.

65. Eaton $T$, Young $P$, Nicol K, Kolbe J: The endurance shuttle walking test: a responsive measure in pulmonary rehabilitation for COPD patients. Chron Respir Dis 2006, 3:3-9.

66. Solway S, Brooks D, Lacasse Y, Thomas S: A qualitative systematic overview of the measurement properties of functional walk tests used in the cardiorespiratory domain. Chest 2001, 119:256-270.

67. Van Remoortel H, Hornikx M, Demeyer H, Langer D, Burtin C, Decramer M, Gosselink R, Janssens W, Troosters T: Daily physical activity in subjects with newly diagnosed COPD. Thorax 2013, 68:962-963.

68. Dontje ML, van der Wal MH, Stolk RP, Brugemann J, Jaarsma T, Wijtvliet PE, van der Schans CP, de Greef MH: Daily physical activity in stable heart failure patients. J Cardiovasc Nurs 2014, 29:218-226.

69. Watz H, Waschki B, Meyer T, Magnussen H: Physical activity in patients with COPD. Eur Respir J 2009, 33:262-272.

70. Walsh JT, Charlesworth A, Andrews R, Hawkins M, Cowley AJ: Relation of daily activity levels in patients with chronic heart failure to long-term prognosis. Am J Cardiol 1997, 79:1364-1369.

71. Waschki B, Kirsten A, Holz O, Muller KC, Meyer T, Watz H, Magnussen H: Physical activity is the strongest predictor of all-cause mortality in patients with COPD: a prospective cohort study. Chest 2011, 140:331342.

72. Healy GN, Matthews CE, Dunstan DW, Winkler EA, Owen N: Sedentary time and cardio-metabolic biomarkers in US adults: NHANES 2003-06. Eur Heart J 2011, 32:590-597.

73. Glenn KR, Slaughter JC, Fowke JH, Buchowski MS, Matthews CE, Signorello LB, Blot WJ, Lipworth L: Physical activity, sedentary behavior and all-cause mortality among blacks and whites with diabetes. Ann Epidemiol 2015, 25:649-655.

74. van den Berg-Emons H, Bussmann J, Balk A, Keijzer-Oster D, Stam H: Level of activities associated with mobility during everyday life in patients with chronic congestive heart failure as measured with an "activity monitor". Phys Ther 2001, 81:1502-1511.

75. Pitta F, Troosters T, Spruit MA, Probst VS, Decramer M, Gosselink R: Characteristics of physical activities in daily life in chronic obstructive pulmonary disease. Am J Respir Crit Care Med 2005, 171:972-977.

76. Nguyen HQ, Steele BG, Dougherty CM, Burr RL: Physical activity patterns of patients with cardiopulmonary illnesses. Arch Phys Med Rehabil 2012, 93:2360-2366.

77. McNamara RJ, McKeough ZJ, McKenzie DK, Alison JA: Physical comorbidities affect physical activity in chronic obstructive pulmonary disease: a prospective cohort study. Respirology 2014, 19:866-872.

78. Edwards MK, Loprinzi PD: Sedentary behavior \& health-related quality of life among congestive heart failure patients. Int J Cardiol 2016, 220:520-523.

79. van den Berg-Emons R, Balk A, Bussmann H, Stam H: Does aerobic training lead to a more active lifestyle and improved quality of life in patients with chronic heart failure? Eur J Heart Fail 2004, 6:95-100.

80. Pitta F, Troosters T, Probst VS, Langer D, Decramer M, Gosselink R: Are patients with COPD more active after pulmonary rehabilitation? Chest 2008, 134:273-280. 
81. Demeyer H, Burtin C, Van Remoortel H, Hornikx M, Langer D, Decramer M, Gosselink R, Janssens W, Troosters T: Standardizing the analysis of physical activity in patients with COPD following a pulmonary rehabilitation program. Chest 2014, 146:318-327.

82. Nishimura K, Izumi T, Tsukino M, Oga T: Dyspnea is a better predictor of 5-year survival than airway obstruction in patients with COPD. Chest 2002, 121:1434-1440.

83. Puhan MA, Garcia-Aymerich J, Frey M, ter Riet G, Anto JM, Agusti AG, Gomez FP, Rodriguez-Roisin R, Moons KG, Kessels AG, Held U: Expansion of the prognostic assessment of patients with chronic obstructive pulmonary disease: the updated BODE index and the ADO index. Lancet 2009, 374:704-711.

84. Sparling PB, Howard BJ, Dunstan DW, Owen N: Recommendations for physical activity in older adults. BMJ 2015, 350:h100.

85. Spruit MA, Pitta F, McAuley E, ZuWallack RL, Nici L: Pulmonary Rehabilitation and Physical Activity in Patients with Chronic Obstructive Pulmonary Disease. Am J Respir Crit Care Med 2015, 192:924-933.

86. Garcia-Aymerich J, Lange P, Benet M, Schnohr P, Anto JM: Regular physical activity reduces hospital admission and mortality in chronic obstructive pulmonary disease: a population based cohort study. Thorax 2006, 61:772-778.

87. Vaes AW, Garcia-Aymerich J, Marott JL, Benet M, Groenen MT, Schnohr P, Franssen FM, Vestbo J, Wouters EF, Lange P, Spruit MA: Changes in physical activity and all-cause mortality in COPD. Eur Respir J 2014, 44:1199-1209.

88. Loprinzi PD: Implications of light-intensity physical activity in improving health-related quality of life among congestive heart failure patients. Int J Cardiol 2016, 212:16-17.

89. Troosters T, Sciurba FC, Decramer M, Siafakas NM, Klioze SS, Sutradhar SC, Weisman IM, Yunis C: Tiotropium in patients with moderate COPD naive to maintenance therapy: a randomised placebo-controlled trial. NPJ Prim Care Respir Med 2014, 24:14003.

90. Probst VS, Kovelis D, Hernandes NA, Camillo CA, Cavalheri V, Pitta F: Effects of 2 exercise training programs on physical activity in daily life in patients with COPD. Respir Care 2011, 56:1799-1807.

91. Watz H, Krippner F, Kirsten A, Magnussen H, Vogelmeier C: Indacaterol improves lung hyperinflation and physical activity in patients with moderate chronic obstructive pulmonary disease--a randomized, multicenter, double-blind, placebo-controlled study. BMC Pulm Med 2014, 14:158.

92. Ramadi A, Stickland MK, Rodgers WM, Haennel RG: Impact of supervised exercise rehabilitation on daily physical activity of cardiopulmonary patients. Heart Lung 2015, 44:9-14.

93. Louvaris Z, Spetsioti S, Kortianou EA, Vasilopoulou M, Nasis I, Kaltsakas G, Koulouris NG, Vogiatzis I: Interval training induces clinically meaningful effects in daily activity levels in COPD. Eur Respir J 2016, 48:567570.

94. Bandura A: Self-efficacy: toward a unifying theory of behavioral change. Psychol Rev 1977, 84:191-215.

95. Larson JL, Covey MK, Kapella MC, Alex CG, McAuley E: Self-efficacy enhancing intervention increases light physical activity in people with chronic obstructive pulmonary disease. Int J Chron Obstruct Pulmon Dis 2014, 9:1081-1090.

96. Spruit MA, Augustin IM, Vanfleteren LE, Janssen DJ, Gaffron S, Pennings HJ, Smeenk F, Pieters W, van den Bergh JJ, Michels AJ, et al: Differential response to pulmonary rehabilitation in COPD: multidimensional profiling. Eur Respir J 2015, 46:1625-1635.

97. Sherwood NE, Jeffery RW: The behavioral determinants of exercise: implications for physical activity interventions. Annu Rev Nutr 2000, 20:21-44.

98. Marquez B, Dunsiger SI, Pekmezi D, Larsen BA, Marcus BH: Social Support and Physical Activity Change in Latinas: Results From the Seamos Saludables Trial. Health Psychol 2016.

99. Franks MM, Stephens MA, Rook KS, Franklin BA, Keteyian SJ, Artinian NT: Spouses' provision of healthrelated support and control to patients participating in cardiac rehabilitation. J Fam Psychol 2006, 20:311318.

100. Hartman JE, Boezen HM, de Greef MH, Ten Hacken NH: Physical and psychosocial factors associated with physical activity in patients with chronic obstructive pulmonary disease. Arch Phys Med Rehabil 2013, 94:2396-2402 e2397. 
101.Cobb LK, Godino JG, Selvin E, Kucharska-Newton A, Coresh J, Koton S: Spousal Influence on Physical Activity in Middle-Aged and Older Adults: The ARIC Study. Am J Epidemiol 2016, 183:444-451.

102. Hartman JE, ten Hacken NH, Boezen HM, de Greef MH: Self-efficacy for physical activity and insight into its benefits are modifiable factors associated with physical activity in people with COPD: a mixed-methods study. J Physiother 2013, 59:117-124.

103. Nakken N, Spruit MA, van den Bogaart EH, van Vliet M, de Vries GJ, Custers FL, Vercoulen JH, Asijee GM, Muris JW, Vanfleteren LE, et al: Health Status and Morbidities in Resident Relatives of Patients With COPD. J Am Med Dir Assoc 2016, 17:276 e271-278.

104. Houben-Wilke S, Jorres RA, Bals R, Franssen FM, Glaser S, Holle R, Karch A, Koch A, Magnussen H, Obst A, et al: Peripheral Artery Disease and its Clinical Relevance in Patients with COPD in the COSYCONET Study. Am J Respir Crit Care Med 2016.

105. Watz H, Waschki B, Kirsten A, Muller KC, Kretschmar G, Meyer T, Holz O, Magnussen H: The metabolic syndrome in patients with chronic bronchitis and COPD: frequency and associated consequences for systemic inflammation and physical inactivity. Chest 2009, 136:1039-1046.

106. Mannino DM, Thorn D, Swensen A, Holguin F: Prevalence and outcomes of diabetes, hypertension and cardiovascular disease in COPD. Eur Respir J 2008, 32:962-969.

107.Curkendall SM, DeLuise C, Jones JK, Lanes S, Stang MR, Goehring E, Jr., She D: Cardiovascular disease in patients with chronic obstructive pulmonary disease, Saskatchewan Canada cardiovascular disease in COPD patients. Ann Epidemiol 2006, 16:63-70.

108. Toronto East General Hospital; Southlake Regional Health Centre; University of Toronto; Ontario Ministry of Health and Long Term Care. Program of Integrated Care for Patients With Chronic Obstructive Pulmonary Disease and Multiple Comorbidities: A Randomized Controlled Trial. In: ClinicalTrials.gov [Internet]. Bethesda (MD): National Library of Medicine (US). 2000- [cited 2016 Nov 03]. Available from: https://clinicaltrials.gov/ct2/show/NCT01648621 NLM Identifier: NCT01648621.

109. Shoemaker MJ, Curtis AB, Vangsnes E, Dickinson MG: Triangulating Clinically Meaningful Change in the Six-minute Walk Test in Individuals with Chronic Heart Failure: A Systematic Review. Cardiopulm Phys Ther J 2012, 23:5-15.

110.Orme M, Weedon A, Esliger D, Saukko P, Morgan M, Steiner M, Downey J, Singh S, Sherar L: Study protocol for Chronic Obstructive Pulmonary Disease-Sitting and ExacerbAtions Trial (COPD-SEAT): a randomised controlled feasibility trial of a home-based self-monitoring sedentary behaviour intervention. BMJ Open 2016, 6:e013014.

111.Spina G, Spruit MA, Alison J, Benzo RP, Calverley PMA, Clarenbach CF, Costello RW, Donaire-Gonzalez D, Dürr S, Garcia-Aymerich J, et al: Analysis of nocturnal actigraphic sleep measures in patients with COPD and their association with daytime physical activity. Thorax 2017, 72:694-701. 
Summary 
Chronic cardiopulmonary diseases, such as chronic obstructive pulmonary disease (COPD) and chronic heart failure (CHF), are responsible for high levels of morbidity and mortality worldwide. Although these diseases have historically been described in terms of the function of the main organ affected, they are nowadays known to be associated with systemic manifestations, such as comorbidities and peripheral muscle abnormalities, which contribute importantly to their overall severity. Together with symptoms like dyspnoea and fatigue, systemic manifestations like peripheral muscle abnormalities can importantly affect physical functioning in these patients, for example by leading to reduced physical capacity (e.g., reduced exercise tolerance and functional mobility) and a physically inactive and/or sedentary lifestyle. These features can ultimately lead to premature mortality. Therefore, a more profound investigation of physical functioning measures in patients with chronic cardiopulmonary diseases could provide important insights about how to facilitate disease management and improve prognosis. The main aim of this thesis was to provide a better understanding of physical functioning measures in patients with chronic cardiopulmonary diseases by exploring three main themes: 1) the investigation of the impact of comorbidities on relevant outcome measures, including measures of physical capacity, in patients with COPD (Chapters 2 and 3 ); 2) the investigation of measurement properties of field physical capacity tests in patients with COPD or CHF (Chapters 4, 5 and 6); and 3) the investigation of patterns of physical activity and sedentary behaviour in patients with COPD (Chapters 7, 8 and 9). Additionally, Chapter 10 generally discusses in a broader perspective these three main themes, presents the main limitations of the previous chapters, gives future directions, and provides concluding remarks.

Chapters 2 and 3 focused on the impact of comorbidities on relevant outcome measures in patients with COPD. The aim of Chapter 2 was to compare health outcome measures, including measures of physical capacity, between two groups of patients with COPD, one with and the other one without an impaired left ventricular ejection fraction (LVEF), which was defined as a LVEF $<50 \%$. These groups were compared after controlling for relevant confounders, namely age, sex, body mass index and degree of airflow limitation. Patients with an impaired LVEF had worse functional and maximal exercise capacity, weaker quadriceps muscles, besides more symptoms of anxiety and depression than those with a normal LVEF, but health status was not statistically different between these groups. These findings highlight the clear impact of an impaired LVEF on physical and psychological status in patients with COPD, reinforcing the importance of assessing and treating cardiac problems in patients with COPD. In Chapter 3 the aim was to investigate whether both individual as well as clusters of objectively identified comorbidities could have an impact on changes in exercise capacity and health status following a comprehensive pulmonary rehabilitation (PR) programme. Individual comorbidities did not influence improvements in exercise capacity and health status, but when comorbidity clusters were considered in the analyses only the "psychologic" cluster showed a 
higher likelihood of achieving a meaningful improvement in the 6-minute walk test (6MWT). These findings suggest that patients with COPD should not be withheld from rehabilitation programmes based on stable comorbidities. Collectively, the findings of Chapters 2 and 3 suggest that the presence of cardiac abnormalities can lead to impaired physical and psychological status in patients with COPD, but these impairments do not seem to prevent patients from showing clinically meaningful improvements in exercise capacity and health status following PR.

In Chapters 4, 5 and 6 the focus was shifted to the investigation of the measurement properties of field physical capacity tests in patients with COPD or CHF. In Chapter 4 the aim was to investigate measurement properties of the 6MWT in patients with CHF. Specifically, the reproducibility, construct validity, and determinants of the distance covered during this test were investigated. The 6MWT was found to be reproducible between two tests performed on subsequent day, but a learning effect of 31 metres was observed. Older age, lower lung diffusing capacity and higher New York Heart Association (NYHA) class were associated with a lower likelihood of having a meaningful increase in the second test. Moreover, the best 6-minute walk distance (6MWD) had moderate-to-good correlations with peak exercise capacity and no-to-fair correlations with body composition, lung function, ejection fraction, and symptoms of anxiety and depression. Additionally, the best 6MWD was independently associated with maximal power output during a maximal cardiopulmonary exercise test, estimated glomerular filtration rate and age, which accounted for $51.7 \%$ of the variability. These findings strengthen the clinical utility of the 6MWT in CHF. Chapters 5 and 6 focused on the measurement properties of the Timed Up \& Go (TUG) test in patients with chronic cardiopulmonary diseases. In Chapter 5 the aim was to investigate the test-retest reliability of the TUG performed three times on the same day in patients with advanced COPD, $\mathrm{CHF}$, or chronic renal failure. In general, good agreement was observed in the total sample and in the subgroups. However, statistical improvement occurred in the total sample from the first to the second trial, but the third trial added little or no information to the first two trials. These findings suggest that the TUG test is reliable in patients with different advanced chronic diseases after two trials. Complementing the previous chapter, Chapter 6 aimed to investigate TUG's construct validity, to identify characteristics related to an abnormal result, and to examine TUG's responsiveness to PR in patients with COPD. Good convergent and discriminant validity was demonstrated by fair-to-moderate correlation with physical health outcomes, symptom-related outcomes and multidimensional indices and by little correlation with mental health outcomes. A TUG time of around 11 seconds was able to identify patients with worse health outcomes. Moreover, TUG time improved following PR and a change of 0.9-1.4 seconds was identified as clinically important. These findings indicate that this simple test provides valuable information and can be adopted in clinical and research settings. 
Chapters 7, 8 and 9 focused on investigating patterns of physical activity and sedentary behaviour in patients with COPD. Specifically, the main aim of Chapter 7 was to identify clusters of patients with COPD based on physical activity measures assessed objectively with an activity monitor in a multinational sample. Five clusters were identified: 'couch potatoes', 'highly sedentary', 'sedentary movers', 'sedentary exercisers', and 'busy bees'. These clusters had distinct characteristics, which can be crucial information to plan tailored interventions. For example, 'couch potatoes' as well as 'highly sedentary' (i.e., the most inactive clusters) were characterised by considerable amounts of time in very light physical activity (i.e., mainly sedentary behaviour) and little time in moderate to vigorous-intensity physical activity (MVPA). These patients could benefit from an intervention focusing not only on increasing the amount of time in MVPA, but also on reducing the time in very light intensity. Chapter 8 also focused on investigating subgroups with particular characteristics, but in this case the groups were identified according to their pattern of change in activity levels following a comprehensive PR programme. Six groups were identified. The most prevalent patterns were represented by good responders (increase in physical activity and reduction in sedentary behaviour, $34 \%$ of patients) and poor responders (decrease in physical activity and increase in sedentary behaviour, $30 \%$ of patients). Strong correlation was found between changes in sedentary behaviour and changes in light activities, suggesting that light activities might be the focus of future interventions. Moreover, changes in six-minute walk distance correlated significantly with changes in physical activity and sedentary behaviour, suggesting that improvements in functional capacity seem necessary to achieve improvements in activity levels. Chapter 9 aimed to investigate activity levels and exercise motivation in patients with COPD and their resident loved ones. Patients spent more time in sedentary behaviour and less time in physical activity than their loved ones. Moreover, despite similar exercise motivation patients with an active loved one spent more time in MVPA and had a higher likelihood of being active than patients with an inactive loved one after controlling for age, body mass index and degree of airflow limitation. These findings suggest that resident loved ones of patients with COPD could be used as a way to make patients more physically active.

Finally, Chapter 10 provided a general discussion of the findings of the previous chapters. Limitations were also presented and discussed in this chapter, such as the investigation of a limited number of comorbidities in the chapters investigating comorbidities, or samples with specific characteristics that could limit the generalisability of the findings. Moreover, this chapter also gave future directions suggesting questions to be answered in future studies. For example, it would be interesting to investigate the impact of integrated disease management interventions in patients with chronic cardiopulmonary diseases and relevant comorbidities. Lastly, this chapter provided concluding remarks showing that impairments in physical functioning in patients with chronic car- 
diopulmonary diseases are complex and should be carefully assessed in clinical and research settings. 

Samenvatting 
Chronische cardiopulmonaire aandoeningen, zoals chronisch obstructief longlijden (COPD) en chronisch hartfalen (CHF), zijn belangrijke oorzaken van morbiditeit en mortaliteit wereldwijd. Hoewel deze aandoeningen in het verleden werden beschreven op basis van het aangedane orgaan, worden ze tegenwoordig geassocieerd met systemische verschijnselen die een grote bijdrage leveren aan de ernst van de ziekte, zoals comorbiditeiten en afwijkingen in de perifere spieren. De systemische verschijnselen, zoals afwijkingen in de perifere spieren, kunnen samen met symptomen als kortademigheid en vermoeidheid het fysiek functioneren van patiënten sterk beïnvloeden door bv. afname van fysieke capaciteit (bv. afgenomen inspanningstolerantie en functionele mobiliteit) en een inactieve en/of sedentaire leefstijl. Deze factoren kunnen uiteindelijk leiden tot premature mortaliteit. Verder onderzoek naar parameters die het fysiek functioneren van patiënten met cardiopulmonaire aandoeningen in kaart brengen, kan daarom nieuwe inzichten bieden over ziektemanagement en het verbeteren van de prognose. Het hoofddoel van deze thesis was om meer inzicht te krijgen in de parameters van het fysiek functioneren van patiënten met cardiopulmonaire aandoeningen aan de hand van de volgende drie thema's: 1) het onderzoeken van de impact van comorbiditeiten op relevante uitkomstmaten bij patiënten met COPD, inclusief parameters van het fysiek functioneren (Hoofdstuk 2 en 3); 2) het onderzoeken van de meeteigenschappen van veldtesten die de fysieke capaciteit meten bij patiënten met COPD of CHF (Hoofdstuk 4, 5 en 6); 3) en het onderzoeken van patronen van fysieke activiteit en sedentair gedrag bij patiënten met COPD (Hoofdstuk 7, 8 en 9). In hoofdstuk 10 worden de drie bovenstaande thema's in een breder perspectief besproken. Tevens worden de beperkingen van de voorgaande hoofdstukken beschreven, worden aanbevelingen gedaan voor de toekomst en worden conclusies getrokken.

Hoofdstuk 2 en 3 beschrijven de impact van comorbiditeiten op relevante uitkomstmaten bij patiënten met COPD. Het doel van hoofdstuk 2 was het vergelijken van gezondheidsgerelateerde uitkomstmaten, inclusief het meten van fysieke capaciteit, tussen twee groepen van patiënten met COPD. Bij de ene groep was sprake van een verminderde linker ventrikelejectie fractie, gedefinieerd als LVEF $<50 \%$, en de andere groep had een normale LVEF. Deze groepen werden vergeleken na correctie voor relevante verstorende factoren, namelijk leeftijd, geslacht, body mass index en mate van luchtwegobstructie. Bij patiënten met een verminderde LVEF was er sprake van een verminderd functionele- en maximale inspanningscapaciteit, verminderde quadriceps spierkracht en daarnaast meer symptomen van angst en depressie in vergelijking met patiënten met een normale LVEF. De gezondheidsstatus was niet significant verschillend tussen beide groepen. Deze bevindingen benadrukken de impact van een verminderde LVEF op de fysieke- en psychologische status bij patiënten met COPD. Dit onderstreept de noodzaak van het vaststellen en behandelen van cardiale problemen bij patiënten met COPD. Het doel van hoofdstuk 3 was het onderzoeken van de invloed van zowel individuele comorbiditeiten als clusters van comorbiditeiten op veranderingen in inspanningscapaciteit en 
gezondheidsstatus, na het voltooien van een allesomvattend longrevalidatieprogramma (PR). Individuele comorbiditeiten hadden geen invloed op verbeteringen van inspanningscapaciteit en gezondheidsstatus. Echter, wanneer de comorbiditeiten geclusterd werden geanalyseerd, werd alleen bij het "psychologische" cluster een verhoogde kans op het bereiken van een noemenswaardige verandering in de 6-minuten wandeltest (6MWT) gevonden. Deze bevindingen laten zien dat patiënten met COPD én stabiele comorbiditeiten niet weerhouden moeten worden van een revalidatieprogramma. Samenvattend laten de bevindingen van hoofdstuk 2 en 3 zien dat de aanwezigheid van cardiale afwijkingen kan leiden tot een verminderde fysieke en psychologische status bij patiënten met COPD, maar deze afwijkingen lijken geen invloed te hebben op het bereiken van een klinisch relevante vooruitgang in inspanningscapaciteit en gezondheidsstatus na het volgen van PR.

In hoofdstuk 4, 5 en 6 lag de nadruk op het onderzoeken van de meeteigenschappen van veldtesten die de fysieke capaciteit meten bij patiënten met COPD of CHF. Het doel van hoofdstuk 4 was het onderzoeken van de meeteigenschappen van de 6MWT bij patiënten met CHF. Hierbij is specifiek gekeken naar de reproduceerbaarheid, constructvaliditeit en determinanten van de afgelegde afstand tijdens de test. Er werd gevonden dat de 6MWT reproduceerbaar is als deze wordt afgenomen op achtereenvolgende dagen, maar dat er een leereffect is van $31 \mathrm{~m}$. Een hogere leeftijd, een lagere diffusiecapaciteit van de longen en een hogere New York Heart Association (NYHA) klasse werden geassocieerd met een grotere kans op een betekenisvolle verbetering in de tweede test. Verder bleek dat beste 6-minuten wandelafstand (6MWD) een gemiddeld tot goede correlatie had met de maximale inspanningscapaciteit en geen tot matige correlatie met lichaamssamenstelling, longfunctie, ejectiefractie en symptomen van angst en depressie. Ook werd de beste 6MWD geassocieerd met het maximale vermogen gegenereerd gedurende een maximale cardiopulmunaire inspanningstest, het geschatte glomerulaire filtratietempo en de leeftijd, waarbij deze 51,7\% van de variabiliteit verklaarden. Deze bevindingen versterken de klinische bruikbaarheid van de 6MWT in CHF. Hoofdstuk 5 en 6 waren gericht op de meeteigenschappen van de Timed Up \& Go (TUG) test bij patiënten met chronische cardiopulmonaire aandoeningen. In hoofdstuk 5 werd de test-hertest betrouwbaarheid van de TUG test onderzocht, door het afnemen van drie testen op dezelfde dag bij patiënten met gevorderd COPD, CHF of chronisch nierfalen. In het algemeen werd er een goede overeenstemming gevonden in zowel de totale groep als de subgroepen. Er werd wel een statische verbetering gevonden in de tweede test in vergelijking met de eerste test, maar de derde test leverde weinig nieuwe informatie op ten opzichte van de eerste twee testen. Deze bevindingen suggereren dat de TUG test betrouwbaar is wanneer deze twee maal wordt afgenomen bij patiënten met verschillende chronische aandoeningen. Aanvullend op het vorige hoofdstuk, werd in hoofdstuk 6 de constructvaliditeit van de TUG test onderzocht, om eigenschappen te identificeren die gerelateerd zijn aan een afwijkend resultaat en aan 
het effect van PR bij patiënten met COPD. Er werd een goede convergente en discriminante validiteit gevonden bij een matig tot gemiddelde correlatie met fysieke uitkomstmaten, symptoom-gerelateerde uitkomstmaten en multidimensionale indexen en bij een kleine correlatie met psychische uitkomstmaten. Patiënten met slechte uitkomstmaten waren te identificeren door een TUG-tijd van ongeveer 11 seconde. Verder verbeterde de TUG tijd na PR en een verandering van 0,9-1,4 seconde werd geïdentificeerd als klinisch relevant. Deze bevindingen laten zien dat deze simpele test belangrijke informatie verschaft en gebruikt kan worden in zowel de klinische praktijk als in wetenschappelijk onderzoek.

In hoofdstuk 7, 8 en 9 is gekeken naar patronen van fysieke activiteit en sedentair gedrag bij patiënten met COPD. Meer specifiek was het doel van hoofdstuk 7 om clusters van patiënten te identificeren, gebaseerd op fysieke activiteit die objectief gemeten werd met een activiteitenmonitor in een multinationale groep. Er waren vijf clusters te onderscheiden: "bank hangers", "zeer sedentaire personen", "sedentaire bewegers", "sedentaire sporters", "bezige bijen". Deze clusters hadden specifieke eigenschappen, die belangrijke informatie bevatten voor het plannen van op maat gemaakte interventies. Zo gold voor de "bank hangers" en "zeer sedentaire personen" (de meest inactieve clusters) dat deze per dag veel tijd zeer licht fysiek actief doorbrachten (d.w.z. dat ze voornamelijk sedentair gedrag vertoonden) en uitermate weinig tijd matig tot sterk fysiek actief waren (MVPA). Deze patiënten kunnen baat hebben bij een interventie die niet alleen gericht is op het verhogen van de tijd waarin ze matig tot sterk fysiek actief zijn, maar ook gericht is op het beperken van de tijd waarin ze slechts zeer licht fysiek actief zijn. Ook in hoofdstuk 8 werd gekeken naar subgroepen met specifieke eigenschappen, maar in dit geval werden de groepen gedefinieerd op basis van het patroon van veranderingen in activiteit na het volgen van een allesomvattend PR programma. Hierbij werden zes groepen gedefinieerd. De meest uitgesproken patronen werden weergegeven door de patiënten die goed reageerden op PR (toename van fysieke activiteit en afname van sedentair gedrag, $34 \%$ van de patiënten) en de patiënten die slecht reageerden (afname van fysieke activiteit en toename van sedentair gedrag, 30\% van de patiënten). Er werd een sterke correlatie gevonden tussen veranderingen in sedentair gedrag en veranderingen in licht intensieve activiteiten. Dit suggereert dat toekomstige interventies zich zouden kunnen richten op licht intensieve activiteiten. Verder werd er een significante correlatie gevonden tussen veranderingen in 6MWD en veranderingen in fysieke activiteit en sedentair gedrag. Het lijkt er op dat verbeteringen in functionele capaciteit noodzakelijk zijn om verbeteringen te kunnen bereiken in activiteitenniveau. In hoofdstuk 9 werden fysieke activiteit en motivatie om in te spannen bij patiënten met COPD en hun inwonende naasten onderzocht. Patiënten brengen meer tijd sedentair door en zijn minder tijd fysiek actief vergeleken met hun inwonende naaste. Verder blijkt dat, ondanks gelijkwaardige motivatie om in te spannen, patiënten met een actieve naaste meer tijd besteden in MVPA en een grotere kans hadden om fysiek actief te 
zijn vergeleken met patiënten met een inactieve naasten, na correctie voor leeftijd, body mass index en mate van luchtwegobstructie. Deze bevindingen suggereren dat inwonende naasten van patiënten met COPD een rol kunnen spelen bij het verbeteren van de fysieke activiteit van patiënten met COPD.

Ten slotte werd in hoofdstuk 10 een algemene discussie over de bevindingen uit de voorgaande hoofdstukken beschreven. Tekortkomingen werden benoemd en bediscussieerd in dit hoofdstuk, zoals het beperkte aantal comorbiditeiten of onderzochte populaties met specifieke eigenschappen die zouden kunnen zorgen voor een beperkte generaliseerbaarheid van de resultaten. Verder werden in dit hoofdstuk ook aanbevelingen gedaan voor toekomstig onderzoek, het zou bijvoorbeeld interessant kunnen zijn om de impact te onderzoeken van geïntegreerde interventies gericht op ziektemanagement bij patiënten met chronische cardiopulmonaire aandoeningen en relevante comorbiditeiten. Ook beschreef dit hoofdstuk conclusies waarin aangegeven werd dat beperkingen in het fysiek functioneren bij patiënten met cardiopulmonaire aandoeningen complex zijn en met voorzichtigheid beoordeeld moeten worden, zowel in de klinische praktijk als in wetenschappelijk onderzoek. 

Valorisation 
Valorisation can be understood as a process of assuring that scientific knowledge can be translated into clinical practice; as an act of making research results appropriate and useful to enhance opportunities for others to use them. Knowledge valorisation, specifically, refers to the process of creating value from knowledge by making knowledge suitable and available for social and/or economic use, and/or for translation into competitive products or services, for example. The findings of the present thesis aimed to provide a better understanding of physical functioning in patients with chronic cardiopulmonary diseases. The present chapter will focus on the valorisation of these findings.

\section{Relevance}

Chronic cardiopulmonary diseases, such as chronic obstructive pulmonary disease (COPD) and chronic heart failure (CHF), are associated with high morbidity and mortality worldwide. Patients with these diseases frequently present with important impairments in physical functioning, which in turn will contribute to even higher morbidity and mortality. These findings can be further worsened by the presence of comorbidities. In the present thesis, physical functioning in patients with chronic cardiopulmonary diseases was investigated by exploring three main themes: the impact of comorbidities on relevant outcome measures in patients with COPD, including measures of physical capacity; measurement properties of field physical capacity tests in patients with COPD or CHF; and patterns of physical activity and sedentary behaviour in patients with COPD. Findings related to these themes can be of relevance from a social and economic point of view, as discussed below.

Firstly, the findings presented in the current thesis demonstrated that patients with COPD and cardiac impairments have worse physical and psychological status than those without cardiac impairments. However, comorbid conditions do not seem to preclude patients with COPD from showing significant and clinically meaningful improvements following a comprehensive pulmonary rehabilitation (PR) programme. Given the negative impact of comorbidities in patients with COPD, an active search for these conditions could prove beneficial for a better disease management, and hence a better prognosis. Potentially, this could positively impact the patient's quality of life and lead to lower health care costs. Secondly, the present thesis' findings revealed that the 6-minute walk test (6MWT) is both valid and reliable in patients with $\mathrm{CHF}$, with similar results for the Timed Up \& Go (TUG) test in patients with COPD. Besides, the TUG test was also found to be responsive to PR in patients with COPD. Using these simple and quick-to-perform tests to investigate the physical capacity of patients with chronic cardiopulmonary diseases, when compared to more complex tests (e.g., incremental cardiopulmonary exercise test), could facilitate the assessment of physical capacity on a broader scale, and could lead to lower costs. Thirdly, the present thesis demonstrated that detailed inves- 
tigations of activity levels in patients with COPD can be useful to help delineate more successful interventions/strategies to increase physical activity and decrease sedentary behaviour in this population. Moreover, the present thesis demonstrated that having a physically active resident loved one seems to be beneficial for a more physically active lifestyle. Investing on interventions/strategies to increase physical activity and reduce sedentary behaviour, which are important modifiable risk factors, could result in a longer life with better quality of life in patients with COPD.

\section{Target groups}

\section{Health care provides}

Comorbidities in patients with COPD were the main outcome of two chapters in the present thesis. The results of these chapters highlight the importance of assessing comorbidities in this population. For example, it was shown that patients with COPD and cardiac impairments have worse physical and psychological status than those without cardiac impairments. Health care providers should be aware of the negative impact of comorbidities in patients with COPD in order to establish the best treatment available for these conditions. The present thesis also showed that 6MWT and TUG are reliable and valid physical capacity tests, which reinforces their utility in both clinical and research settings. Moreover, clinicians could use patients with a TUG time higher than 11 $s$ as a target for therapies such as exercise training, since these patients were found to have worse physical and psychological health outcomes. It was also found that a reduction in TUG time of 0.9-1.4 s following PR was identified as clinically important. This will help researchers and clinicians in the interpretation of changes as a result of intervention trials or rehabilitation programmes. In three chapters of the present thesis activity levels of patients with COPD were investigated. The findings of these chapters are important for clinicians to help delineate interventions/strategies to increase physical activity and reduce sedentary behaviour. One important finding in all three chapters was that patients with COPD spent the majority of their time in sedentary behaviour. One way to reduce the amount of sedentary behaviour, as suggested in one of the papers, could be by increasing the amount of time in light activities, such as household activities. Another way to make patients less sedentary and/or more physically active, as suggested in another chapter, could be by supporting joint activities with physically active loved ones.

\section{Patients with chronic cardiopulmonary diseases}

Many findings of the present thesis are of interest for patients with chronic cardiopulmonary diseases. A deeper understanding of the negative impact of comorbidities in 
patients with COPD was achieved. Patients should be aware of this impact and talk to their family and physicians about it in order to facilitate an early diagnosis, which would allow an early treatment and prevent negative consequences. Another finding of the current thesis is that physical capacity is an important outcome in patients with chronic cardiopulmonary diseases. Once patients have been diagnosed with the cardiac and/or pulmonary disease, many of them tend to see the primary impairment (i.e., cardiac or pulmonary dysfunction) as the main problem and cause of symptoms. The present thesis reinforces that patients should be aware that their physical capacity can be reduced and associated with symptoms, but they should also be aware that this reduction can be restored with interventions such as PR. The present thesis also demonstrated that patients with COPD are physically inactive and/or sedentary. This highlights the importance of raising awareness about the negative impact and potential consequences of a physically inactive and/or sedentary lifestyle, and about the benefits of an active lifestyle.

\section{Industry}

The vast majority of studies that investigated comorbidities in patients with chronic cardiopulmonary diseases used medical charts or self-reports. Previous studies have shown that poor agreement exists between objectively identified and non-objectively identified comorbidities. Non-objective measurements are usually preferred due to their simplicity and low cost. Therefore, the industry should work on developing new diagnosing tools that are simple to use and able to provide accurate results at reasonably low costs. Three chapters of the present thesis investigated the measurement properties of relatively simple physical capacity tests, such as the 6MWT and TUG. One of the advantages of these tests is that they mimic basic activities of daily living, such as walking and rising from a chair. Recent research has investigated the potential of devices that could be used during these tests to provide further information, such as accelerometers and gas analysers. This could be potential targets for the industry. Activity monitoring was used in three chapters of the present thesis. In two of them, activity monitoring was restricted to the leg, while in the other one activity monitoring was restricted to the arm. The industry could work on developing an activity monitor that would measure motion accurately of both upper and lower limbs. Moreover, one of the findings of the present thesis was that patients with COPD spend the vast majority of their time in sedentary behaviour. The industry could work on developing and improving technologies to control sedentary behaviour, such as an electronic television lockout system or pads to measure sitting time. 


\section{Activities and products}

The findings of the present thesis were translated mainly as presentations in international congresses and publication of manuscripts in high impact journals in the field of expertise. These findings were presented at the 2013 and 2015 American Thoracic Society (ATS) International Conference; 2013, 2014, 2015, and 2016 European Respiratory Society (ERS) International Congress; and 2015 CIRO pre-ERS meeting. During both 2013 and 2015 ATS International Conferences, the abstracts submitted were granted with the International Trainee Development Scholarship. Moreover, the abstract presented at the 2015 CIRO pre-ERS meeting was awarded with $2^{\text {nd }}$ place for best abstracts in rehabilitation and chronic care. The findings obtained with the current thesis also led to the publication of original manuscripts and a research letter in high impact peer-reviewed scientific journals. This thesis' findings were also presented during a summer course in 2014, and a workshop and a national congress in 2015. All the above mentioned activities allowed the findings of the present thesis to be made available to a wider public, which may provoke further discussions and be the basis for future studies on physical functioning in patients with chronic cardiopulmonary diseases. 

Acknowledgments 
I would like to acknowledge here many people without whom this thesis would not have been possible; people who contributed not only academically but also to my personal growth. First, I would like to thank my beloved parents Parsifal and Marilene, who sometimes did not really understand why I would prefer to travel so far away to study, when I could study in my hometown while having all my family and friends around me. Even without really understanding why, they always supported me in my dreams and decisions. I am and will always be grateful for this unconditional support. Now a few words in Portuguese so that this message can reach them: "Pai, mãe, muito obrigado pelo apoio incondicional de sempre! Amo muito vocês e tenho muito orgulho de vocês! Sem vocês com certeza não teria chegado até aqui! Mãe, obrigado por esse amor e cuidado independente de onde eu estivesse, a senhora é meu anjo nesse mundo!". Thank you to my siblings Parsifal Júnior and Ana Racquel as well, who were always a source of support despite the distance.

My sincere gratitude to my supervisors, Prof. Dr. Martijn Spruit and Prof. Dr. Emiel Wouters, who welcomed me as a PhD candidate and supported me in many different ways during these almost 4 years of PhD. Prof. Wouters, thank you for all your support and for all the knowledge shared during this period. It was an honour to work with you and to be part of the CIRO team. Martijn, thank you very much for your support, for the knowledge shared, for your patience, and for all the professional opportunities I was given. I learnt a lot from you. You are not only a very bright researcher, but also very focused and determined; traits that have been very inspiring. My deepest gratitude to my co-supervisor Prof. Dr. Fabio Pitta, without whom I would not be where I am today. Thank you very much for your long-lasting support and for being not only a mentor, but also a friend. Your support was essential in many difficult moments, and I am and will always be grateful for that. I am very happy that you contributed and were part of many of my academic achievements, from the moment I arrived in Londrina, Paraná, Brazil, until the moment of my PhD defence.

I would like to acknowledge Prof. Dr. M.K.C. Hesselink, Dr. J.B.J. Bussmann, Prof. E.M. Clini, Prof. Dr. R.A. de Bie, and Prof. Dr. L.W. van Rhijn, who kindly agreed to be part of my thesis review committee. To all the co-authors of the manuscripts that formed this thesis, thank you very much for taking a moment to lend your expertise, insight and support. My gratitude to all co-authors who provided the data for the manuscript including physical activity data from different parts of the world, to Gabriele Spina, for the help with organising and analysing these data, and to Henrik Watz, for the valuable insights to make sense of the data. I would also like to thank Helena Azcuna, Yvonne Goërtz, Nienke Nakken, Jeannet Delbressine, Johannes Essers, and Kenneth Meijer for their help with organising and analysing physical activity data as part of other manuscripts. 
I would like to acknowledge my funder, the National Council for Scientific and Technological Development (CNPq/Brazil). I am grateful for the financial support to develop my PhD activities in The Netherlands. I would also like to acknowledge the financial and academic support from CIRO (Horn, The Nertherlands) and Maastricht University (Maastricht, The Netherlands).

To all my colleagues at $\mathrm{CIRO}$, thank you very much for all your encouragement and for the many enjoyable moments in- and outside CIRO. Thank you to Dr. Frits Franssen, Dr. Lowie E.G.W. Vanfleteren, Dr. Daisy Janssen, Dr. Nicole Uszko-Lencer, and Miriam Groenen, for your help with organising, analysing and making sense of the data used in many of my studies. My gratitude to the nursing team for the help with collecting data for the "religiosity project". Thank you to Ingrid Augustin for her valuable support and assistance, to Dr. Maurice Sillen for his kind support, and to Gerrie Janssen for the help with organising issues related to my PhD. A special thank you to Sarah, Dionne Smid, Jeannet, Carmen, Wai-Yan, Fiona, Nienke, Esther, Anouk, Dionne Braeken, Cindy, Coby, and Vasilis, colleagues with whom I was in daily contact. Thank you very much for all the translations, breaks with fun moments, wall-sit challenges, lactose-free food during celebrations, and even for the sometimes deep/weird/non-sense discussions. I owe a more than special thank you to Sarah for her friendship in- and outside CIRO, from the moment I arrived in The Netherlands and we could bike to CIRO together, until the current days.

Thank you to many of my friends in Brazil who even with the distance and lack of daily contact were always available for a chat and to support me when I most needed. Thank you to very dear friends from my hometown, Glaucus, Alisson, Henrique, Nathália, and Juliana Montenegro. Also from my hometown, to my cousins Patrícia Collares, with whom I started my career as a physiotherapist and researcher, and Raphael Moura, who was an important source of support specially in the final phase of my PhD. Thank you to dear mentors Fátima Luna, Maria Tereza Morano, and Daniela Gardano as well, who highly contributed to the development of my career as a physiotherapist and researcher, and became very dear friends. A special thank you to all my friends and colleagues who I met during my time in Londrina, Paraná, Brazil, and with whom I started my career as a respiratory physiotherapist and researcher: Juliana Zabatiero, Leila Donária, Nidia Hernandes, Thaís Sant'Anna, Karina Furlanetto, Gianna Bisca, Mahara Proença, Aline Nellessen, Larissa Martinez, Alexandre Salomão, Carlos Augusto, Leandro Mantoani, and Vinícius Cavalheri. Many of you were very important sources of support during my PhD. A more than special thank you to my almost-twin-sister Ju Zabatiero, for her unconditional support and love, and for the help with reading some parts of my thesis giving her valuable insights. Thank you to Dr. Vanessa Probst for the continuing support since my masters, and to the colleagues from my time participating in the projects at UNOPAR, Laís Ribeiro, Débora Rafaelli, and Josiane Felcar. 
Last but definitely not least, thank you to the very few but true friends I made during my time living in Maastricht, The Netherlands. Thank you to Frank and Halla, for their support and for every single moment spent together, for the many chats, nights out, coffees/teas, trips etc. You both contributed to make the experience of living in The Netherlands much more remarkable. Thank you also for accepting to be my paraninfos. Thank you to Gesiele, a Brazilian treasure I found in The Netherlands. Thank you for all your love and support. Thank you to Irena and Nanca for the fun moments and deep discussions, to Ramon for the enjoyable moments, and to Terezinha and Geraldo for their support. Probably many other people contributed somehow to this achievement, but were not mentioned here. To these people I express my deepest gratitude as well. 
Curriculum Vitae 
Rafael B. de Mesquita was born on March 14th 1986 in Fortaleza, Ceará, Brazil. He graduated from High School at September 7th School in Fortaleza, Ceará, Brazil in 2003. In 2008 he graduated as a Bachelor in Physiotherapy at the University of Fortaleza (Fortaleza, Ceará, Brazil). In 2011 he completed a Residency in Respiratory Physiotherapy at the University Hospital of Londrina, which is affiliated with the State University of Londrina (Londrina, Paraná, Brazil). In 2013 he received his MSc degree in Rehabilitation Sciences from the collaborative programme between the State University of Londrina and the North of Paraná University, in Londrina, Paraná, Brazil. Before finishing his studies to receive his MSc degree, he was granted with the CIRO+ Short-Term Research Training Fellowship 2012, which allowed him to spend 3 months at CIRO, in Horn, The Netherlands, developing a research project. In 2013 he started his PhD at Maastricht University, in Maastricht, The Netherlands, being affiliated with the Department of Respiratory Medicine, Maastricht University Medical Center+ (MUMC+), in Maastricht, The Netherlands, and the Department of Research \& Education, CIRO, Center of Expertise for Chronic Organ Failure, in Horn, The Netherlands. During his PhD, entitled "Physical functioning in patients with chronic cardiopulmonary diseases", he was supervised by Prof. Dr. Martijn A. Spruit (supervisor), Prof. Dr. Emiel F.M. Wouters (supervisor), and Prof. Dr. Fabio Pitta (co-supervisor). Findings of his thesis were presented at international congresses and these presentations allowed him to be granted twice (2013 and 2015) with the International Trainee Development Scholarship by the American Thoracic Society (ATS), during the annual congress of this society. 
List of Publications 
Mesquita, R., Spina, G., Pitta, F., Donaire-Gonzalez, D., Deering, B.M., Patel, M., Mitchell, K.E., Alison, J., Van Gestel, A.J.R., Zogg, S., Gagnon, P., Abascal-Bolado, B., Vagaggini, B., Garcia-Aymerich, J., Jenkins, S.C., Romme, E., Kon, S., Albert, P., Waschki, B., Shrikrishna, D., Singh, S.J., Hopkinson, N.S., Miedinger, D., Benzo, R.P., Maltais, F., Paggiaro, P., Mckeough, Z.J., Polkey, M.I., Hill, K., Man, W.D.-C., Clarenbach, C.F., Hernandes, N.A., Savi, D., Wootton, S., Furlanetto, K.C., Cindy Ng, L.W., Vaes, A.W., Jenkins, C., Eastwood, P.R., Jarreta, D., Kirsten, A., Brooks, D., Hillman, D.R., Sant'anna, T., Meijer, K., Dürr, S., Rutten, E.P.A., Kohler, M., Probst, V.S., Tal-Singer, R.M., Garcia Gil, E., Den Brinker, A.C., Leuppi, J.D., Calverley, P.M.A., Smeenk, F.W., Costello, R.W., Gramm, M., Goldstein, R., Groenen, M.T.J., Magnussen, H., Wouters, E.F.M., ZuWallack, R., Amft, O., Watz, H., Spruit, M.A. (2017). Physical activity patterns and clusters in 1001 patients with COPD. Chron Respir Dis, Published Online First: 24 February 2017. doi: 10.1177/1479972316687207.

Mesquita, R., Meijer, K., Pitta, F., Azcuna, H., Goërtz, Y.M.J., Essers, J.M.N., Wouters, E.F.M., Spruit, M.A. (2017). Changes in physical activity and sedentary behaviour following pulmonary rehabilitation in patients with COPD. Respir Med, 126, 122-129.

Uszko-Lencer, N.H.M.K., Mesquita, R., Janssen, E., Werter, C., Brunner-La Rocca, H.P., Pitta, F., Wouters, E.F.M., Spruit, M.A. (2017). Reliability, construct validity and determinants of 6-minute walk test performance in patients with chronic heart failure. Int J Cardiol, 240, 285-290.

Spina, G., Spruit, M.A., Alison, J., Benzo, R.P., Calverley, P.M.A., Clarenbach, C.F., CostelIo, R.W., Donaire-Gonzalez, D., Dürr, S., Garcia-Aymerich, J., Van Gestel, A.J.R., Gramm, M., Hernandes, N.A., Hill, K., Hopkinson, N.S., Jarreta, D., Kohler, M., Kirsten, A., Leuppi, J.D., Magnussen, H., Maltais, F., Man, W.D.-C., Mckeough, Z.J., Mesquita, R., Miedinger, D., Pitta, F., Singh, S.J., Smeenk, F.W., Tal-Singer, R., Vagaggini, B., Waschki, B., Watz, H., Wouters, E.F., Zogg, S., den Brinker, A.C. (2017). Analysis of nocturnal actigraphic sleep measures in patients with COPD and their association with daytime physical activity. Thorax, 72(8), 694-701.

Mesquita, R., Nakken, N., Janssen, D.J.A., Van Den Bogaart, E., Delbressine, J.M.L., Essers, J.M.N., Meijer, K., Van Vliet, M., De Vries, G.J., Muris, J.W.M., Pitta, F., Wouters, E.F.M., Spruit, M.A. (2017). Activity levels and exercise motivation in COPD patients and their resident loved ones. Chest, 151(5), 1028-1038.

De Castro, L.A., Ribeiro, L.R., Mesquita, R., De Carvalho, D.R., Felcar, J.M., Merli, M.F., Fernandes, K.B., Da Silva, R.A., Teixeira, D.C., Spruit, M.A., Pitta, F., Probst, V.S. (2016). Static and Functional Balance in Individuals With COPD: Comparison With Healthy Con- 
trols and Differences According to Sex and Disease Severity. Respir Care, 61(11), 14881496.

Mesquita, R., Franssen, F.M.E., Houben-Wilke, S., Uszko-Lencer, N.H.M.K., Vanfleteren, L.E.G.W., Goërtz, Y.M.J., Pitta, F., Wouters, E.F.M., Spruit, M.A. (2016). What is the impact of impaired left ventricular ejection fraction in COPD after adjusting for confounders? Int J Cardiol, 225, 365-370.

Mesquita, R., Wilke, S., Smid, D.E., Janssen, D.J., Franssen, F.M., Probst, V.S., Wouters, E.F., Muris, J.W., Pitta, F., Spruit, M.A. (2016). Measurement properties of the Timed Up \& Go test in patients with COPD. Chron Respir Dis, 13(4), 344-352.

Spina, G., Casale, P., Albert, P.S., Alison, J., Garcia-Aymerich, J., Costello, R.W., Hernandes, N.A., van Gestel, A.J., Leuppi, J.D., Mesquita, R., Singh, S.J., Smeenk, F.W., TalSinger, R., Wouters, E.F., Spruit, M.A. \& den Brinker, A.C. (2015). Identifying Physical Activity Profiles in COPD Patients Using Topic Models. IEEE J Biomed Health Inform, 19(5), 1567-1576.

Gonçalves, C.G., Mesquita, R., Hayashi, D., Merli, M.F., Vidotto, L.S., Fernandes, K.B. \& Probst, V.S. (2015). Does the Incremental Shuttle Walking Test require maximal effort in healthy subjects of different ages? Physiotherapy, 101(2), 141-146.

Mesquita, R., Gonçalves, C.G., Hayashi, D., Costa, V.S., Teixeira, D.C., de Freitas, E.R., Felcar, J.M., Pitta, F., Molari, M. \& Probst, V.S. (2015). Smoking status and its relationship with exercise capacity, physical activity in daily life and quality of life in physically independent, elderly individuals. Physiotherapy, 101(1), 55-61.

Ribeiro, L.R., Mesquita, R.B., Vidotto, L.S., Merli, M.F., Carvalho, D.R., de Castro, L.A. \& Probst, V.S. (2015). Are 30 minutes of rest between two incremental shuttle walking tests enough for cardiovascular variables and perceived exertion to return to baseline values? Braz J Phys Ther, 19(2), 105-113.

Mesquita, R., Vanfleteren, L.E., Franssen, F.M., Sarv, J., Taib, Z., Groenen, M.T., Gaffron, S., Bruijnzeel, P.L., Pitta, F., Wouters, E.F. \& Spruit, M.A. (2015). Objectively identified comorbidities in chronic obstructive pulmonary disease: impact on pulmonary rehabilitation outcomes. Eur Respir J, 46(2), 545-548.

Oliveira, L.A., Mesquita, R., Brito, I.L., Laburú, V.M., Pitta, F. \& Probst, V.S. (2014). Relationship between the work developed in maximal and submaximal exercise capacity tests and the degree of airflow obstruction in individuals with Chronic Obstructive Pulmonary Disease. Fisioter Pesqui, 21(1), 81-86. 
Donária, L., Carvalho, M.Y.L., Mesquita, R., Martinez, L., Silva, M.M.M., Ribeiro, L.R.G., Felcar, J.M., Hernandes, N.A., Pitta, F. \& Probst, V.S. (2014). Pressão inspiratória nasal e hiperinsuflação pulmonar estática em indivíduos com DPOC. Conscientiae saúde, 13(1), 47-53.

Morano, M.T., Mesquita, R.B., Da Silva, G.P., Araújo, A.S., Pinto, J.M., Neto, A.G., Viana, C.M., Moraes, M.O. \& Pereira, E.D. (2014). Comparison of the effects of pulmonary rehabilitation with chest physical therapy on the levels of fibrinogen and albumin in patients with lung cancer awaiting lung resection: a randomized clinical trial. BMC Pulm Med, 14, 121-127.

Donária, L., Mesquita, R., Martinez, L., Sípoli, L., Felcar, J.M., Probst, V.S., Hernandes, N.A. \& Pitta, F. (2014). Relationship Between Sniff Nasal Inspiratory Pressure and BODE Index in Patients with COPD. Lung, 192(6), 897-903.

Mesquita, R., Melo, L.T.M., Vasconcelos, R.S., Soares, D.M., Félix, G.A.A., Férrer, L.P.A. \& Abdon, A.P.V. (2014). Avaliação neurofuncional em pacientes com hanseníase. Rev bras promoç saúde, 27(2), 247-255.

Morano, M.T., Araújo, A.S., Nascimento, F.B., Da Silva, G.F., Mesquita, R., Pinto, J.S., de Moraes Filho, M.O. \& Pereira, E.D. (2013). Preoperative Pulmonary Rehabilitation Versus Chest Physical Therapy in Patients Undergoing Lung Cancer Resection: A Pilot Randomized Controlled Trial. Arch Phys Med Rehabil, 94(1), 53-58.

Mazzarin, C.M., Mesquita, R., Donária, L., Genz, I.C.H., Faria, L.M.M., Hernandes, N.A., Pitta, F. \& Probst, V.S. (2013). Capacidade inspiratória e sua relação com diferentes medidas de capacidade de exercício em indivíduos com DPOC. ASSOBRAFIR Ciência, 4(1), 9-19.

Mesquita, R., Donária, L., Genz, I.C.H., Pitta, F. \& Probst, V.S. (2013). Respiratory muscle strength during and after hospitalization for copd exacerbation. Respir Care, 58(12), 2142-2149.

Mesquita, R., Janssen, D.J.A., Wouters, E.F.M., Schols, J.M.G.A., Pitta, F. \& Spruit, M.A. (2013). Within-Day Test-Retest Reliability of the Timed Up \& Go Test in Patients with Advanced Chronic Organ Failure. Arch Phys Med Rehabil, 94(11), 2131-2138.

Mesquita, R.B., Morano, M.T.A.P., Landim, F.L.P., Collares, P.M.C. \& Pinto, J.M.S. (2012). Rede de apoio social e saúde de idosos pneumopatas crônicos. Cien Saude Colet, 17(5), 1125-1133. 
Probst, V.S., Hernandes, N.A., Teixeira, D.C., Felcar, J.M., Mesquita, R.B., Gonçalves, C.G., Hayashi, D., Singh, S. \& Pitta, F. (2012). Reference values for the incremental shuttle walking test. Respir Med, 106(2), 243-248.

Araújo, E.C.L.S., Freitas, E.R.F.S., Mesquita, R., Probst, V.S. \& Atallah, A.N. (2012). Efeitos agudos da oscilação oral de alta frequência sobre parâmetros cardiorrespiratórios na DPOC: comparação entre os equipamentos Flutter VRP1 e Shaker. ASSOBRAFIR Ciência, 3(3), 9-18.

Marques, A.K.M.C., Landim, F.L.P., Collares, P.M. \& Mesquita, R.B. (2011). Apoio social na experiência do familiar cuidador. Cien Saude Colet, 16(suppl 1), 945-955.

Silva, G.P.F., Silveira, J.M., Mesquita, R.B., Olegário, N.B.C. \& Morano, M.T.A.P. (2011). Efeitos de um Programa de Reabilitação Pulmonar Domiciliar na Qualidade de Vida em Pneumopatas Crônicos - Estudo Piloto. Revista Inspirar, 3(5), 12-15.

Landim, F.L.P., Fernandes, A.M., Mesquita, R.B., Collares, P.M.C. \& Frota, M.A. (2010). Análise das redes interpessoais: aplicação na realidade de uma equipe de enfermagem atuando em unidade de hematologia. Saúde soc, 19(4), 828-837.

Mesquita, R.B., Ribeiro, L.R.G., Dias, M.C., Avelar, T.B. \& Probst, V.S. (2010). Relação entre o índice de massa corpórea e a capacidade máxima de exercício em homens e mulheres. ASSOBRAFIR Ciência, 1(1), 23-33.

Moura, C.V.A., Landim, F.L.P., Collares, P.M.C., Mesquita, R.B. \& Valdés, M.T.M. (2010). Rede de apoio social ao familiar cuidador de pessoa com atrofia muscular espinhal I e II. Rev bras promoç saúde, 23(2), p. 126-135.

Mesquita, R.B., Collares, P.M., Landim, F.L.P. \& Peixoto, A.C.R. (2009). Apoio social na inclusão de crianças com necessidades educacionais especiais: a perspectiva dos professores. Ciênc cuid saúde, 8(1), 34-41, 2009.

Collares, P.M.C., Magalhães, M.S., Landim, F.L.P., Mesquita, R.B. \& Marques, A.K.M.C. (2009). Desenvolvimento de recurso de animação como suporte informativo na incontinência urinária. Acta Fisiatr, 16(3), 110-115.

Mesquita, R.B., Landim, F.L.P., Collares, P.M. \& Luna, C.G. (2008). Análise de redes sociais informais: aplicação na realidade da escola inclusive. Interface Comunic, Saúde, Educ, 12(26), 549-562. 
Cunha, M.L., Landim, F.L.P., Lima, M.F.C., Vieira, L.J.E.S., Mesquita, R.B. \& Collares, P.M. (2008). Dança de salão: repercussões nas atividades de vida diária. Cad saúde colet, 16(3), 559-568.

Oliveira, V.L.B., Landim, F.L.P., Collares, P.M., Mesquita, R.B. \& Santos, Z.M.S.A. (2007). Modelo explicativo popular e profissional das mensagens de cartazes utilizados nas campanhas de saúde. Texto contexto - enferm, 16(2), 287-293.

Landim, F.L.P., Comaru, J.L., Mesquita, R.B. \& Collares, P.M. (2006). Redes sociais informais no cotidiano de uma comunidade da periferia de Fortaleza. Cogitare Enferm, 11(1), 16-23. 